Hos on is

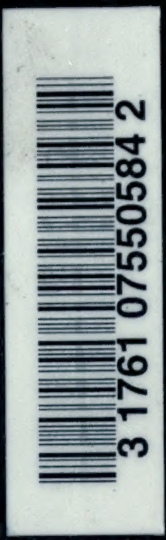

was

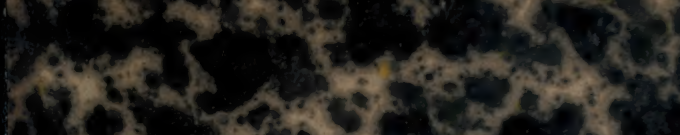

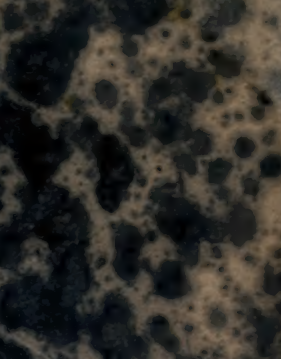

1.

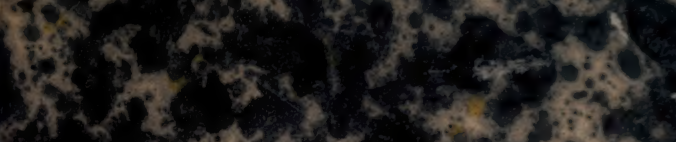

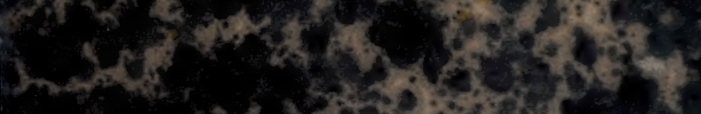

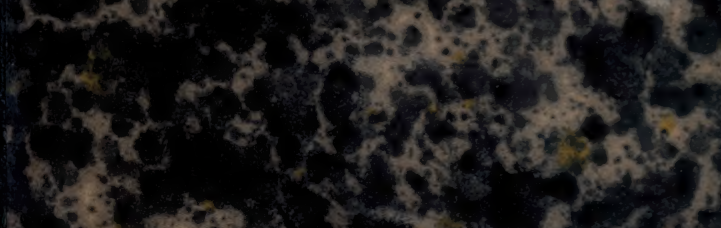

12

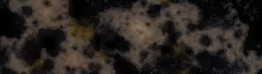<smiles>C1CCCCC1</smiles>

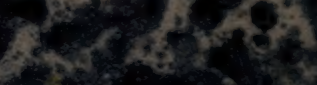

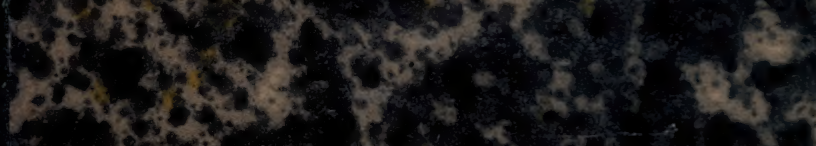

15.

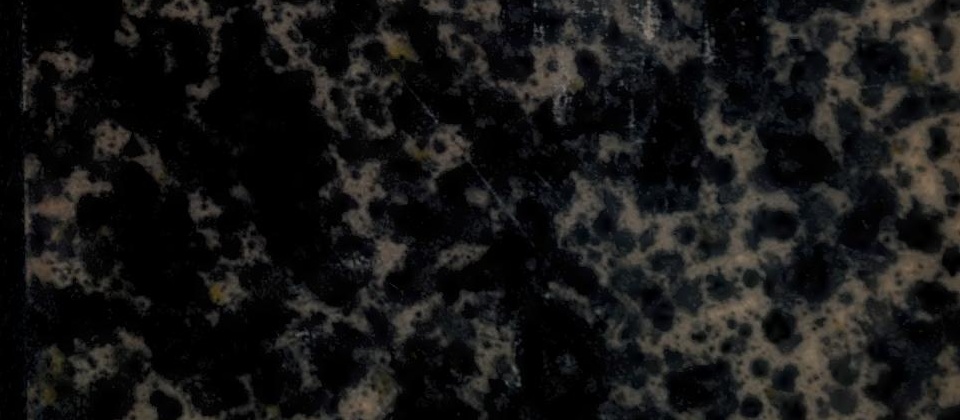

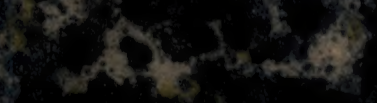

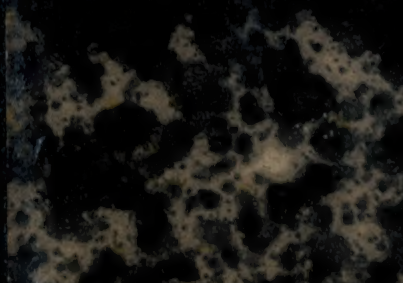
a 


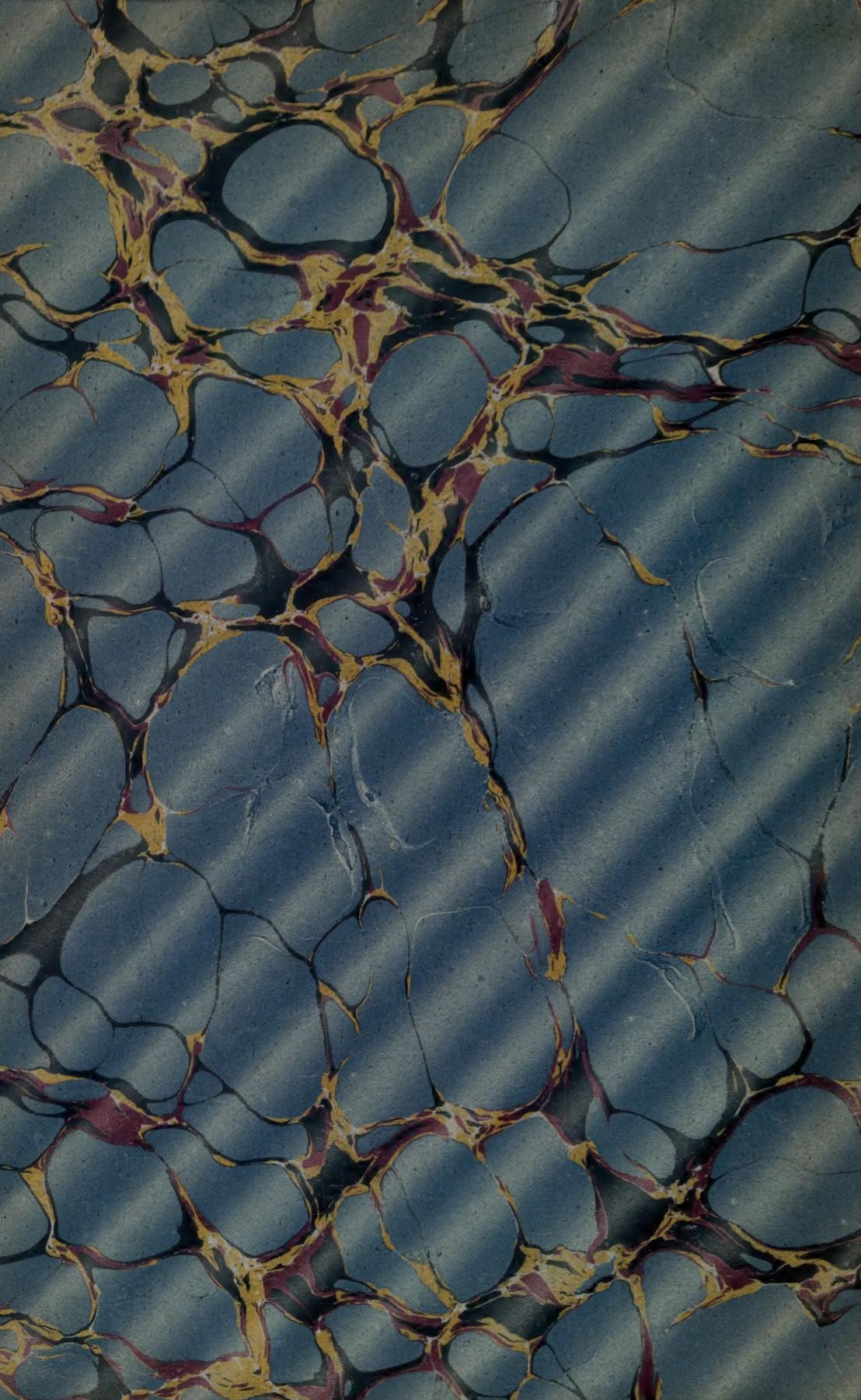




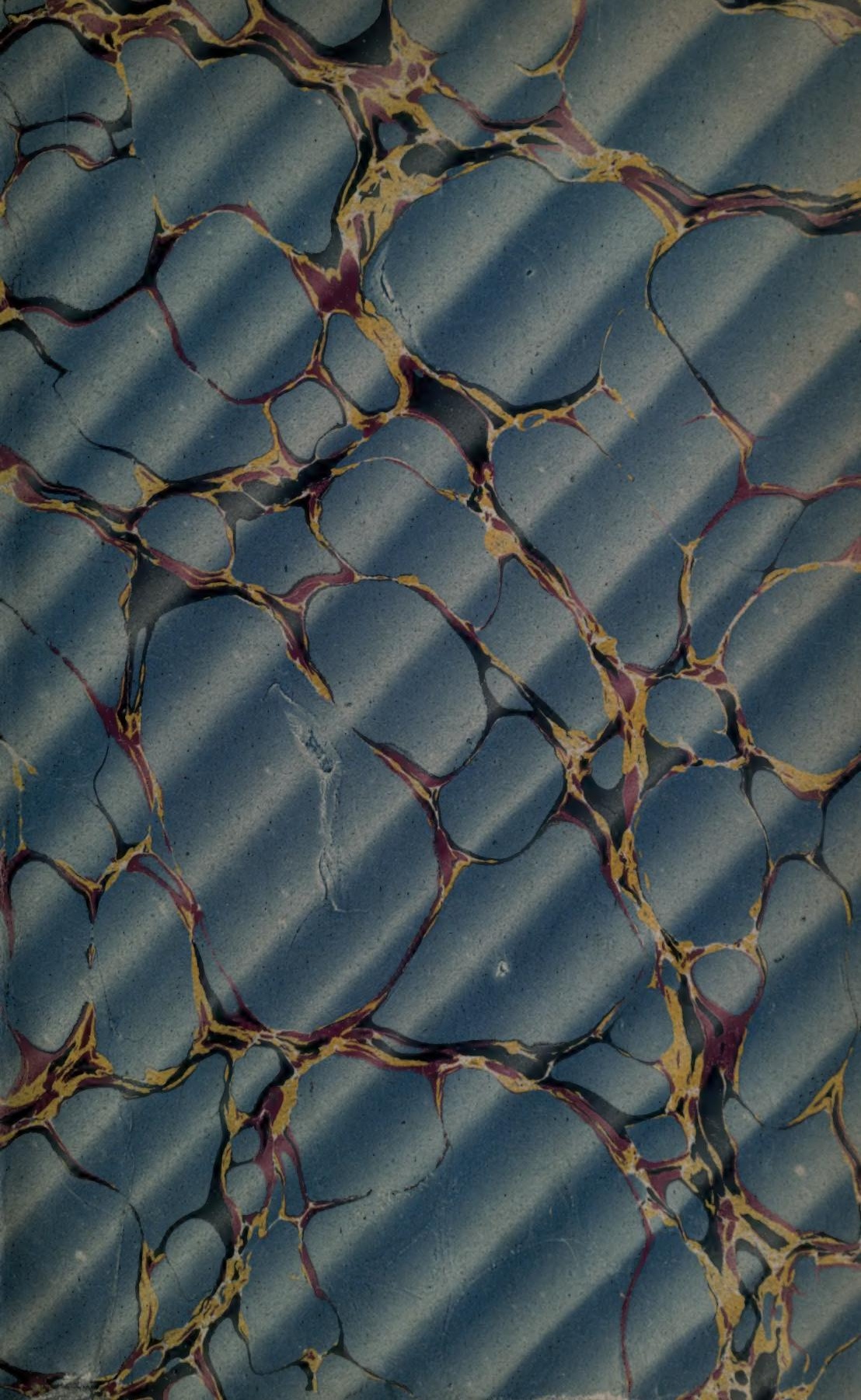



$\sqrt{3}$

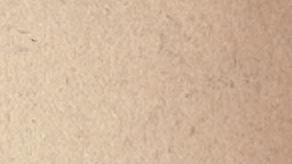





\section{LES FORETS}




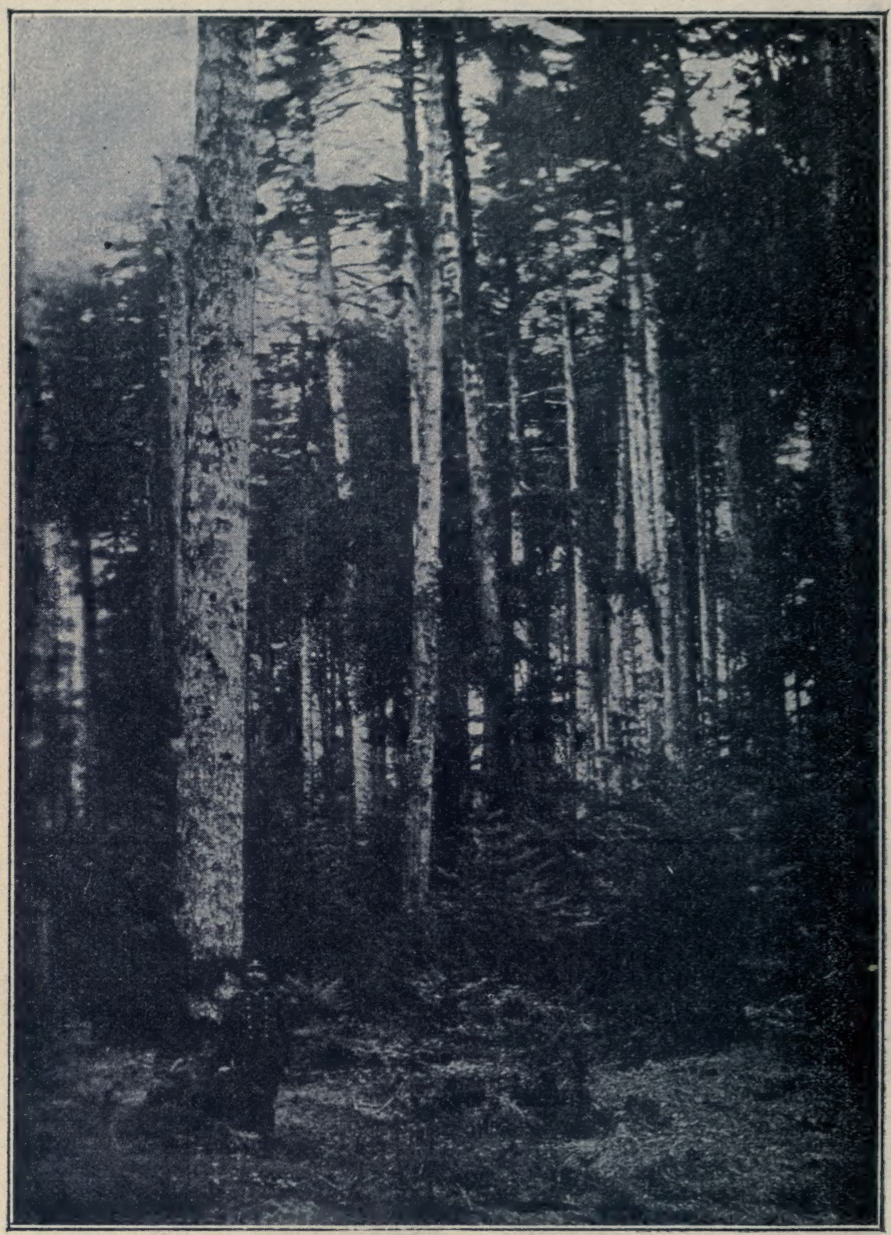

Une Sapinière près de Leviers (Doubs), (photographie de M. Juvanon du Vachat). 


\section{TRAITÉ PRATIQUE DE SYLVICULTURE}
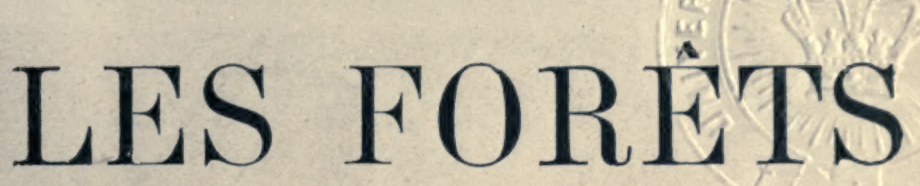

P A R

\section{BOPPE}

Directeur honoraire de l'École Nationale des Eaux et Forêts de Nancy, Membre du Conseil supérieur de l'Agriculture,

Membre correspondant de la Société Nationale d'Agriculture,

Officier de la Légion d'honneur.

E T

\section{Ant. JOLYET}

Inspecteur adjoint des Eaux et Forèts,

Chargé de Cours à l'Ecole Nationale des Eaux et Forêts de Nancy.

Avec 95 photogravures intercalées dans le texte.

\section{LIBRARY}

PARIS

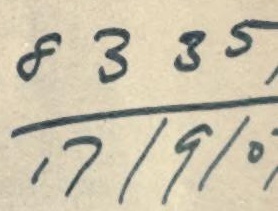

\section{LIBRAIRIE J.-B. BAILLIÈRE ET FILS}

19, rue Hautefeuille, près du Boulevard Saint-Germain

\section{1}

Tous droits réservés. 


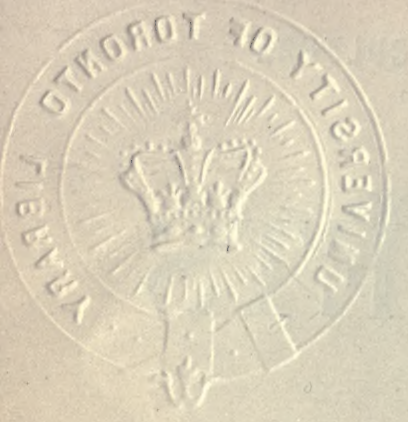

SD
391
$B 6$

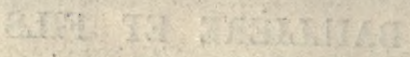




\section{AVANT-PROPOS}

Sollicité de toutes parts de publier les leçons que j’ai longtemps professées à l'École Nationale des Eaux et Forêts, j'ai pensé devoir donner à ce volume un sens pratique qui le mit à la portée de tous les représentants de la richesse forestière en France, aussi bien des propriétaires particuliers que des fonctionnaires de l'État.

Pour m'aider dans cette tâche, j'ai eu la bonne fortune de rencontrer dans M. l'Inspecteur adjoint Antoine Jolyet, un collaborateur des plus dévoués, dont l'érudition m'a permis de mettre au niveau du jour toutes les données empruntées directement aux sciences naturelles.

Il nous a paru utile d'éclairer le texte par des paysages forestiers. De ceux-ci, beaucoup ont été empruntés à l'album des Élèves de l'École Nationale des Eaux et Forêts; en effet, chaque promotion compte ses artistes, qui, pendant les tournées, ont la bonne 
habitude de prendre l'objet de la démonstration sur le point précis où elle vient d'être faite.

Nous en devons d'autres à d'aimables Correspondants, qui nous ont gracieusement confié leurs clichés.

Enfin, l'obligeance de M. Fournel, adjudant à l'École, nous en a grandement facilité la mise en œuvre.

Que tous reçoivent ici nos meilleurs remercîments.

\section{Boppe.}

Nancy, le $1^{\text {er }}$ juillet 1900. 


\section{PLAN DE L'OUVRAGE}

Imiter la nature, hater son cuvre, telle est la maxime fondamenta!e de la sylviculture.

PARAde.

La culture forestière a toujours occupé une place spéciale à côté des autres exploitations agricoles; et cela pour deux motifs.

Le premier, c'est que les végétaux forestiers ne donnent des produits rémunérateurs qu'au bout d'un nombre d'années d'existence plus ou moins considérable. Si le fait a des conséquences dans l'ordre économique, il en a surtout dans l'ordre naturel.

La forêt, en effet, - obligée de se suffire à ellemême pendant un demi siècle, un siècle et même davantage, dans les terrains généralement médiocres que l'agriculture lui abandonne, de braver les hivers rigoureux et les étés excessifs, - se constitue et se perpétue en harmonie intime avec les conditions de sol et de climat du lieu. Les plantes agricoles, au contraire, sont des hôtes de passage, auxquels la vie est rendue possible, d'une manière plus ou moins 
factice, par les soins constants dont on les entoure. Une forêt n'est pas non plus, comme un champ de blé, un simple groupement d'individus de même espèce croissant côte à côte jusqu'à l'époque oủ le bûcheron juge à propos d'y porter la hache : des essences forestières, aux exigences souvent les plus diverses, s'y trouvent réunies, et, de leur naissance à leur vieillesse, non seulement elles grandissent, mais encore elles modifient leurs besoins, quant à l'état physique du sol qui les porte ou quant à l'espacement qui leur est dévolu; elles-mêmes, en épaississant ou en éclaircissant leur feuillage, ont une action considérable sur ce sol, sur la quantité de lumière qu'elles y laissent arriver, sur les détritus qu'elles lui restituent; toujours en lutte les unes avec les autres, elles se prêtent ou s'opposent à l'existence de toute une population d'arbres nouveauvenus, d'arbustes, de plantes basses, ou d'animaux qui, à leur tour, réagissent sur elles. En un mot, la forêt, bien que constituée par un assemblage d'êtres vivants, soumis à toutes les vicissitudes du climat, est un organisme unique, éminemment variable et complexe, ayant sa vie propre, ses périodes de régénération, ses phases d'évolution, et ses crises souvent mortelles.

La forêt, c'est l'ensemble des végétaux qui occupent le sol, - lichens, mousses et autres cryptogames qui tapissent la terre, - herbes, graminées et autres, qui disparaissent en hiver, sauf à renaître de leurs souches ou de leurs semences, -ronces, airelles, broussailles, morts-bois, formant sous-étage avec les jeunes plants des bonnes essences forestières qui s'y trouvent mêlées et qui sont l'espoir de la régénération, - enfin, arbres forestiers plus ou moins élancés, plus ou moins gros, plus ou moins serrés, en taillis ou en futaie (1).

(1) E. Guinier. Le mouton. Revue des Eaux et Forêts, 16 juillet 1897. 
Le second motif réside dans le caractère extensif de la culture forestière, qui réduit à peu de chose l'intervention humaine. Bien rares sont les cas ou la pioche ameublit les sols forestiers, plus rares encore ceux où un apport d'engrais augmente sa fertilité. En fait, la forêt est un bien naturel, que l'homme se contente de domestiquer à son profit.

La sylviculture est donc pour nous: la science qui étudie les phénomènes relatifs à la végétation de la forét sauvage, et l'art d'exploiter celle-ci sans entraver son fonctionnement physiologique.

En d'autres termes, le sylviculteur a comme principaux ouvriers les agents qui président aux phénomènes dont le sol et l'atmosphère sont le théâtre.

A lui de maintenir l'harmonie dans ces forces mises gratuitement à sa disposition; à lui de les diriger vers la production de la matière ligneuse et de tous les avantages que procure l'état boisé d'une contrée. Ia besogne est intéressante; elle est aussi des plus délicates, car, parfois, des accidents de force majeure, trop souvent aussi des fautes commises par une imprévoyance coupable ou par ignorance, suffisent à détruire tout l'équilibre du système.

C'est dire qu'avant tout le sylviculteur devra observer, chercher dans l'étude de chaque station, de chaque massif, les renseignements nécessaires à sa gestion. Si la chose est vraie d'une façon générale, dans un pays comme la France, où, elle est justifiée plus que partout ailleurs, des hautes chênaies des vallées de la Loire et de la Seine aux sapinières des Vosges, du Jura et des Pyrénées, - des taillis 
sous futaies du Nord et de l'Est aux taillis simples de chêne vert de la Provence, - la forêt se présente sous des aspects si divers, en conservant partout son utilité et son charme.

Évitant donc avec soin de poser à priori des règles, des systèmes auxquels devraient se plier toutes les forêts, nous adopterons la méthode d'observation que nous venons de recommander, considérant d'abord l'arbre au strict point de vue forestier; puis, nous étudierons l'espèce qui s'affirme par son tempérament, et nous la suivrons dans ses rapports avec les phénomènes météoriques et avec le sol. Ce sera l'occasion de passer en revue les différentes essences qui peuplent nos plaines et nos montagnes, en donnant l'aire d'habitation de chacune avec les lois qui président à cette distribution.

Ensuite, nous verrons comment ces essences se comportent quand elles sont à l'état isolé, ou réunies en massifs pour former les peuplements, dont l'ensemble constitue la forét. Celle-ci, influencée par le sol et le climat, change d'aspect dans chaque station et nous en montrerons les principaux types.

Après avoir établi les exigences de la forêt spontanée dans chaque station, nous dirons d'une manière générale par quel genre de culture la forêt aménagée, c'est-à-dire économiquement constituée, sera régénérée et améliorée, en vue de diriger la fabrication de la matière bois vers telle ou telle qualité de marchandise.

Seulement alors, nous aborderons l'étude détaillée des régimes et des modes de traitement en usage avec leur application en toutes circonstances. 
Après ces six premiers Chapitres consacrés à la vieille forêt en état de rendement, il nous restera deux questions importantes à examiner : $1^{\circ}$ la protection de la forét, contre les dommages qu'elle peut subir et d'où qu'ils lui viennent; $-2^{\circ}$ le boisement des terrains nus, partout où l'exploitation rationnelle du sol le commande, qu'il s'agisse de satisfaire à des intérêts publics ou à des intérêts privés; elles feront l'objet des Chapitres VII et VIII.

Notre tâche sera bien remplie si nous arrivons à faire comprendre aux propriétaires de forêts que le meilleur moyen de les bien cultiver est de s'y promener souvent, de les interroger toujours sur leur état de santé, sur leurs besoins. Ils seront surpris de la facilité avec laquelle on comprend le langage des arbres, et du plaisir qu'on éprouve à leur répondre. 



\title{
LES FORETS
}

\section{TRAITÉ PRATIQUE DE SYLVIGULTURE}

\section{CHAPITRE PREMIER}

\section{L'A R B RE}

\author{
ARTICLE PREMIER
}

\section{PARTIES CONSTITUTIVES D'UN ARBRE}

Partie aérienne. - Enracinement. - Structure du bois.

Écorce.

Partie aérienne. - La partie aérienne d'un arbre comprend la tige, c'est-à-dire l'axe du végétal, qui va du niveau du sol jusqu'au bourgeon terminal, - et les branches, insérées sur cette tige; celles-ci se divisent à leur tour en branches principales ou maitresses branches, en rameaux et en ramules.

Il est plus intéressant dans la pratique de distinguer le fat, portion de la tige allant du sol au niveau des premières branches, - et la cime ou houppier englobant le reste de la tige et les branches. C'est le fût qui fournit, en effet, le bois propre au plus grand nombre d'usages, par suite, le plus précieux. D'ailleurs, si dans un sapin on peut suivre facilement la tige jusqu'au dernier bourgeon, il n'en est pas de même chez toutes les espèces : bien souvent cette tige se bifurque, se divise en un nombre de plus en plus grand de ramifications et n'existe plus qu'à l'état théorique.

Il peut arriver que brusquement, pour une raison ou pour 
une autre, des branches de faible grosseur naissent sur le fût d'un arbre qui jusque-là en était dépourvu. On les appelle branches gourmandes ou gourmands.

Enracinement. - Le collet de la racine est la ligne qui sépare la portion inférieure du fût d'une part, et les racines de l'autre : le microscope accuse des différences sensibles

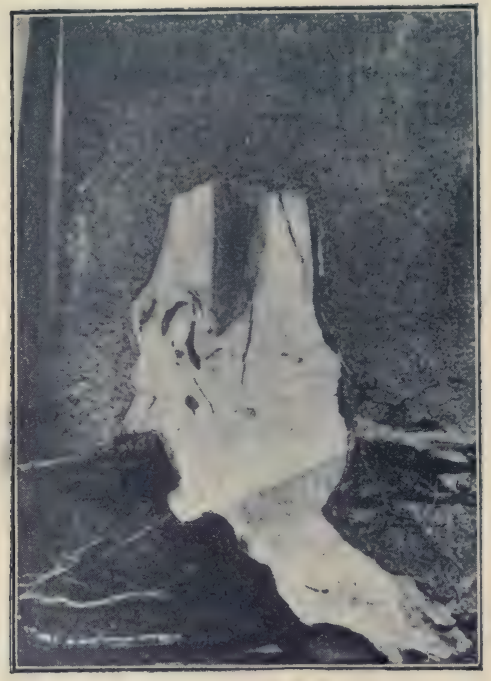

Fig. 1. - Souche de chêne : à droite et à gauche, aubier; au milieu, bois parfait; en bas, collet de la racine. dans la structure du bois de tige et celle du bois de racine; c'est au niveau du collet que finit, dans les chênes, la distinction entre l'aubier et le bois parfait (fig. 1).

Dans les racines nous ne trouvons rien d'analogue au fût; mais il peut se faire que l'une d'elles prolonge directement la tige, et s'affirme parmi toutes les autres; c'est le pivot. Le développement du pivot n'est prononcé que pendant la jeunesse de l'arbre; plus tard il s'arrête dans sa croissance, et, alors même qu'il ne rencontre pas d'obstacle à son allongement, il s'atrophie, ou se ramifie en racines nouvelles qui s'enfoncent plus ou moins obliquement dans la terre.

Par analogie, quand les racines d'un arbre ont une tendance à prendre une direction verticale, on dit que l'enracinement est pivotant ou mieux profond. Lorsqu'au contraire elles ont une propension à s'étaler horizontalement, on dit qu'il est traçanl ou superficiel. Cette question de l'enracinement est très importante : on conçoit combien il est peu indiqué de cultiver des arbres à enracinement profond dans un terrain superficiel. 
Les ramifications extrêmement fines des racines se nomment le chevelu.

Structure du bois. - L'arbre s'accroît en hauteur par le développement du bourgeon terminal, lequel s'ouvre au printemps et donne une pousse qui grandit généralement jusque vers le milieu de l'été. Il s'organise alors un nouveau bourgeon terminal, qui passera l'hiver à l'état latent. Les branches s'allongent par un processus analogue.

L'arbre s'accroît en grosseur par la multiplication des cellules dans la zone génératrice. Cette multiplication se fait, chez les essences indigènes du moins, de l'intérieur vers l'extérieur dans le sens du rayon; sur une section transversale du fût; d'autre part, elle est suspendue durant l'hiver; il en résulte que la masse de bois fabriquée chaque année forme une couche ligneuse bien distincte, qui se superpose à la précédente et l'enveloppe de toutes parts. On peut donc avec raison appeler aussi couches annuelles ces couches ligneuses, et déduire de leur nombre l'âge de l'arbre.

Il est en général facile, sur la tranche d'une tige exploitée, de distinguer les différentes couches ligneuses. Tantôt, en effet, le bois fabriqué au début de la saison de végétation, dit bois de printemps, est franchement distinct du bois d'élé, qui se forme plus tard. Et, comme dans ce cas la caractéristique du bois de printemps est d'être constitué par des éléments à parois minces et à grosses cavités intérieures, il apparaît tou-

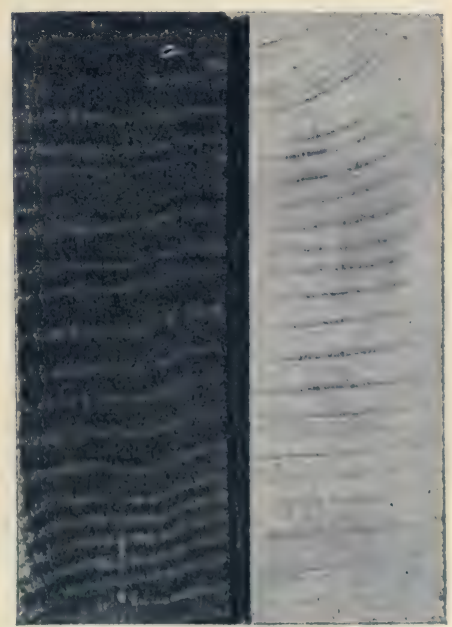

Fig. 2. - A gauche, bois de chêne; à droite, bois de hêtre. jours sous l'aspect d'un tissu tendre ou blanchâtre, par opposition au tissu plus dur et plus coloré du bois d'été (chêne (fig. 2), sapin, etc.). 
Tantôt, au contraire, bois de printemps et bois d'été se ressemblent; mais alors les derniers éléments de celui-ci, ceux qui bordent la couche ligneuse vers l'extérieur, sont très minces et souvent colorés en brun, ce qui rend encore les formations annuelles distinctes l'une de l'autre, avec plus de difficulté toutefois (hêtre (fig. 2), bouleau, charme, fruitiers, etc.).

Des accidents de végétation peuvent déterminer une formation prématurée et temporaire de bois d'été. Un peu de pratique permet de reconnaître ces fausses limites d'accroissement souvent interrompues et à bords toujours indécis.

Chez certains arbres on distingue un aubier et un bois parfait, le premier de coloration pâle, riche en amidon, le second plus foncé, pauvre en cette matière, mais chargé de tanin qui imprègne les parois des éléments, donnant aux tissus, en même temps qu'une coloration spéciale, des qualités particulières de résistance à la décomposition. La couche d'aubier est épaisse chez le chêne rouvre, le chêne pédonculé, les pins, mince chez

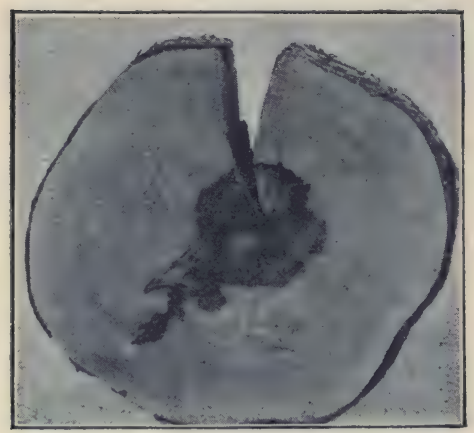

Fig. 3. - Poirier (coloration centrale). le mélèze, très mince ehcz le châtaignier, le robinier, etc.

Parfois aussi, au centre de la tige apparaissent des taches, des zones ou des flammes noires, rouges ou brunes, qu'on pourrait à première vue prendre pour un bois parfait plus accentué encore, quand, en réalité, elles ne sont dues qu'à un commencement d'altération. Et si ces colorations spéciales peuvent être recherchées pour l'ébénisterie, du moins ne faut-il pas en conclure à une supériorité du bois au point de vue de sa conservation, bien au contraire. Les chênes à feuilles persistantes, le poirier (fig. 3), le lprunier, présentent fréquemment cet accident. Dans le même ordre d'idées nous citerons le hois rouge, 
PARTies CONSTitutives d'UN ARBRE.

malheureusement trop fréquent chez les vieux hêtres.

Écorce. - L'écorce est pour les arbres ce qu'est la peau pour les animaux; une enveloppe étanche protégeant les régions plus profondes contre les agents extérieurs. Toute lésion de l'écorce est donc une plaie avec ses conséquences funestes, une porte ouverte aux germes infectieux.

Parmi les tissus constituant l'écorce, un des plus impro-

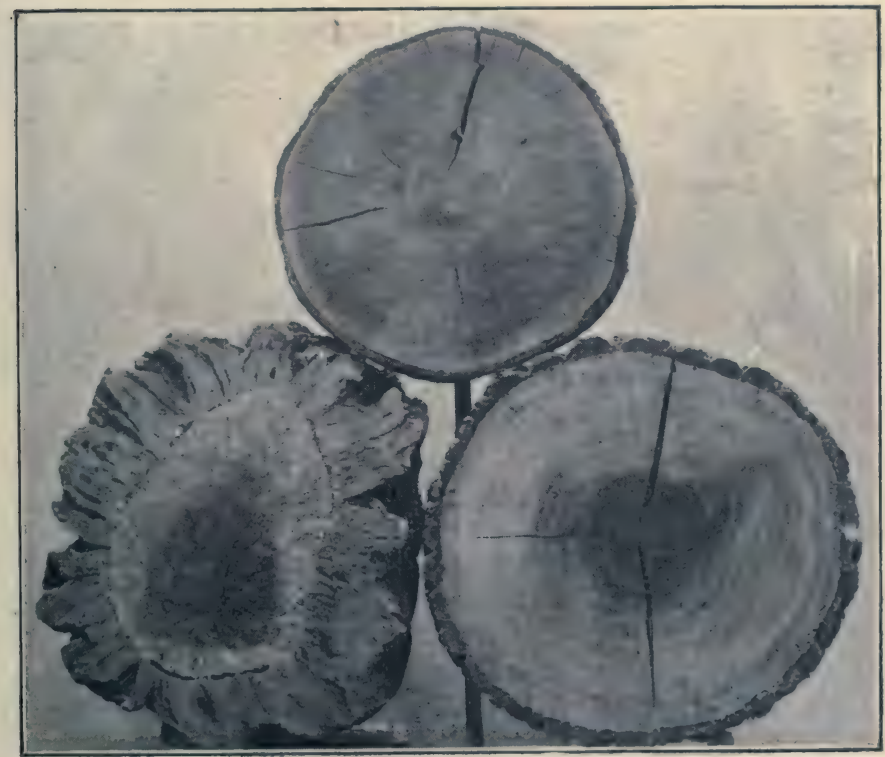

Fig. 4. - En haut, cerisier merisier (liège externe mince); à gauche, chêne liège (liège externe épais); à droite, chêne rouvre (liège interne), rhy tidome gerçuré).

tants est le liège, caractérisé par son imperméabilité. Tantôt le liège est externe, et peut alors acquérir des épaisseurs considérables, comme dans le chêne-liège, ou rester très mince comme dans le hêtre et le charme, tous deux remarquables par leurs écorces fines et luisantes (fig. 4). Tantôt, au contraire, se formant au milieu des autres tissus de l'écorce, il en isole des lames qui finissent par mourir : liège et tissus morts constituent une croûte rugueuse, sèche, appelée rhytidome. 
Tout le monde connaît le rhytidome gerçuré (1) des chênes, des ormes, des peupliers, qui s'effrite par l'usure dans ses parties extérieures, tandis qu'il s'accroît dans les parties profondes par la formation incessante de nouvelles lames de liège; ou encore le rhytidome des pins, des mélèzes dont les larges écailles se détachent facilement. Chez les vieux mélèzes ce rhytidome acquiert souvent une épaisseur d'un décimètre et plus.

Nous mentionnerons aussi le liber. C'est par les tubes criblés du liber que descendent les matières nutritives élaborées par les feuilles et qui vont se diffuser dans le corps de l'arbre et dans les racines. Une blessure annulaire de l'écorce interrompant à un niveau quelconque la continuité de ces tubes criblés a pour résultat d'affamer (2) toutes les régions du végétal situées au-dessous d'elle et occasionne la mort de l'individu.

Les fibres du liber sont parfois assez résistantes pour être utilisées comme matières textiles (tilleul).

ARTICLE II

\section{FORME DES ARBRES}

Élagage naturel. - Forme spécifique. - Influence du sol et du climat. - Forme forestière. - Généralités sur l'accroissement. Accroissement en hauteur. - Accroissement en diamètre; ses variations suivant les espèces et les individus; ses variations dans un même arbre; anomalies diverses.

Élagage naturel. - Pour nous rendre compte de la forme d'un arbre, suivons le développement d'un chêne depuis ses premières années jusqu'à sa veillesse. Au début, il est formé d'une tige principale peu ramifiée, dont le bourgeon terminal, plus gros et mieux nourri que celui des ramilles grêles qui l'entourent, donne naissance à une pousse vigou-

(1) Ces gerçures se produisent inévitablement dans une enveloppe rigide, contrainte de s'étendre pour permettre le grossissement de l'arbre.

(2) L'eau puisée dans le sol par les racines, continue à monter aux feuilles par le corps de l'arbre. 
reuse. L'arbre s'élève alors rapidement. Mais, au fur et à mesure que la tige grandit, elle produit des rameaux nombreux, qui, en vieillissant, se couvrent d'une abondante frondaison, et prennent des dimensions de plus en plus fortes. L'importance de la tige diminue progressivement; le plus souvent son bourgeon terminal s'étiole, et l'arbre cesse de croître en hauteur.

D'autre part, les branches inférieures, que dominent de plus en plus celles qui se développent au-dessus d'elles, se dessèchent et finissent par tomber. La tige se dénude de la sorte de bas en haut, tandis que la cime s'élève. Un moment vient, toutefois, où la croissance en hauteur se ralentit beaucoup ; la production d'axes nouveaux, dans les régions élevées, se réduisant à très peu de chose, les rameaux qui ont pu se maintenir vivants jusque là ont dès lors leur existence assurée : ils s'affirment, s'allongent, grossissent et se constituent en branches principales : le fût est parvenu à sa longueur maxima.

Ce phénoméne est ce que l'on nomme l'élagage naturel. C'est un de nos adjuvants les plus précieux.

D'ailleurs les branches dominées, longtemps avant de mourir, ne s'accroissent plus en longueur ni en grosseur; lorsqu'elles tombent, leur diamètre est généralement très faible relativement à celui de la tige : il ne reste ainsi sur le tronc que des cicatrices insignifiantes, à peine visibles dans le débit. Au contraire, l'amputation de main d'homme des branches vivantes ou mortes, même les plus petites, occasionne toujours une blessure et une solution de continuité dont la trace ne s'efface jamais dans le bois.

Forme spécifique. - La forme de l'arbre varie suivant les espèces, et, chez une même espèce, suivant l'âge du sujet. Elle varie également avec la nature du sol ou la rigueur du climat. Enfin les conditions plus ou moins factices dans lesquelles nous plaçons les végétaux de nos forêts leur créent, à côté de la forme spécifique, une forme forestière du plus haut intérêt à considérer.

La forme spécifique dépend du degré de résistance des branches inférieures au couvert des branches supérieures. Elle dépend aussi de l'ouverture de l'angle sous lequel les 
branches sont insérées sur la tige. Ainsi les cimes sont d'autant plus longues et étroites que les branches sont plus redressées (arbres pyramidaux) ou plus abaissées (formes à branches retombantes). Elles sont au contraire d'autant plus courtes et étalées que les branches, plus horizontales, s'éloignent davantage du fût (la plupart des feuillus, certains résineux).

C'est à une cause semblable qu'il faut attribuer les modifications dues à l'âge. Les

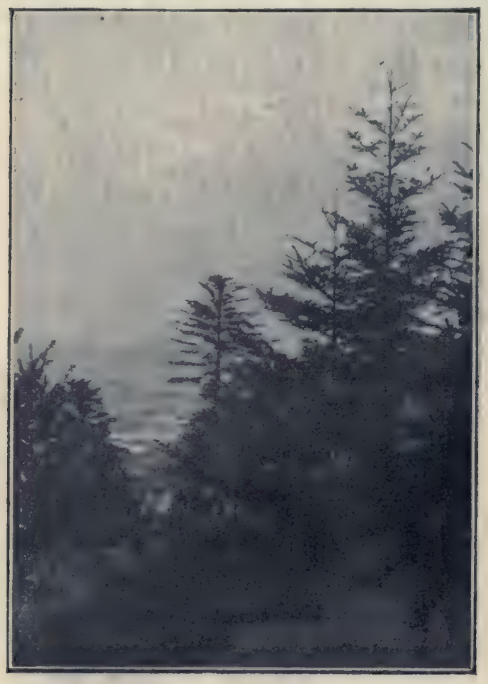

Fig. 5. - Au milieu : sapinfaisant la table; à droite, jeunes sapins à cime pyramidale. (Photographie de M. P. Hirsch.) branches sont, à l'origine, redressées comme les pousses qui les ont produites. En s'allongeant elles fléchissent sous leur propre poids, et sous celui de la neige, des feuilles et des fruits. La transformation des bourgeons de prolongement en boutons à fruits modifie, d'autre part, la ramification de certains arbres dès qu'ils deviennent fertiles. Une fructification abondante est toujours une cause d'éclaircissement de la cime.

Les arbres ont donc une physionomie, un port spécial qu'ils doivent à leur tempérament propre et à la disposition naturelle de leurs rameaux. La cime du chêne est irrégulière et ovoïde, celles du hêtre et du tilleul sont pleines et sphériques, etc. C'est surtout dans la vieillesse que certains arbres prennent leur facies particulier. Il suffit de parcourir une futaie mélangée de sapins et d'épicéas pour constater la différence entre le port de ces deux essences. Tandis que l'épicéa garde toujours sa forme de pyramide aigue pointant vers le ciel, chez les vieux sapins la flèche s'oblitère, et les 
branches voisines du sommet s'étendent horizontalement comme celles du cèdre (fig. 5) (1). Les pins piniers sont célèbres par leur cime en parasol, les vieux pins laricios par l'inclinaison accentuée de l'extrémité de leur tige (fig. 6), etc.

Influence du sol et du climat. - Le sol agit surtout par son plus ou moins de profondeur. Tous les forestiers savent que dans les terrains meubles et profonds du grès vosgien

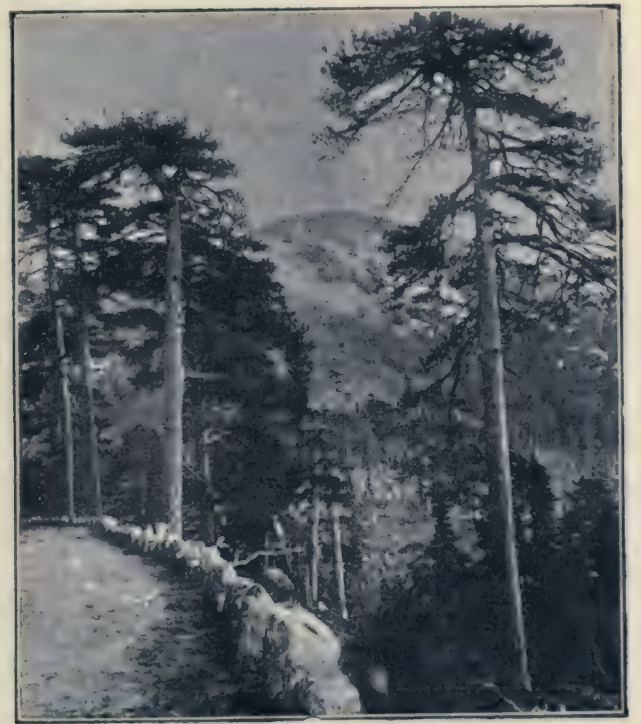

Fig. 6. - Vieux pins laricios, forêt d'Aitone (Corse).

(Photographie de M. H. Bregeault.)

les sapins sont plus élevés que sur les granits. Il en est de même du chêne qui n'acquiert jamais une grande hauteur dans les sols superficiels.

L'influence du climat est peut être moins nette. Pourtant il est bien certain qu'un arbre placé sous un climat qui ne lui convient qu'à demi, pour un motif ou pour un autre, se rapetisse, se rapproche de la forme buissonnante, tels les derniers

(1) On dit alors qu'un sapin fait la table. 
spécimens de la végétation forestière dans les régions montagneuses et arctiques. On constate aussi qu'aux expositions du Nord et de l'Est les axes se développent plus en hauteur qu'aux expositions Sud et Ouest moins fraîches et plus ensoleillées.

Les phénomènes météoriques peuvent avoir une action toute spéciale. Les vents violents déjettent la cime des arbres (fig. 7); le fait est très visible sur les bords de l'Atlantique où

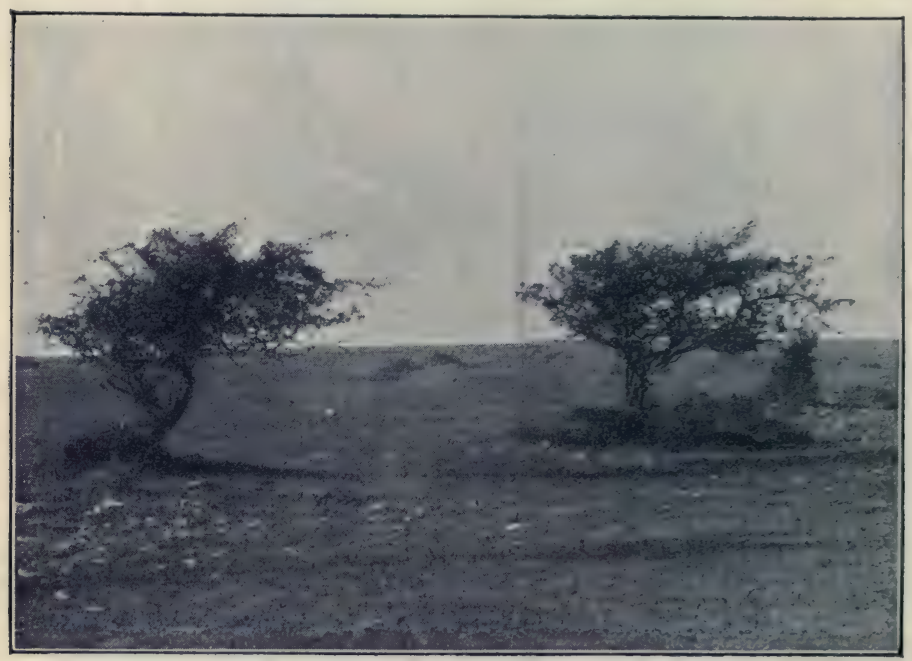

Fig. 7. - Aubépines déjetées par le vent, Chargey-lès-Port (Haute-Saône).

les arbres n'ont de branches que du côté de la terre. C'est l'inverse qui a lieu sur le littoral de la Provence, le mistral ne permettant aux branches de se développer que du côté de la mer. Même chose se passe vers la cime de nos montagnes sous l'action des vents dominants de l'Ouest.

Dans les belles futaies des plateaux du Risoux ou du Massacre, dans le Jura, les épicéas affectent la forme columnaire pour résister au poids des neiges (fig. 8). Tantôt les branches sont courtes, toutes de même longueur; tantôt ces branches longues, mais flexibles, coulent jusque près du sol en s'appli- 
quant contre le tronc; dans l'un et l'autre cas, l'épicéa de ces hautes régions se présente sous la forme d'un long fuseau

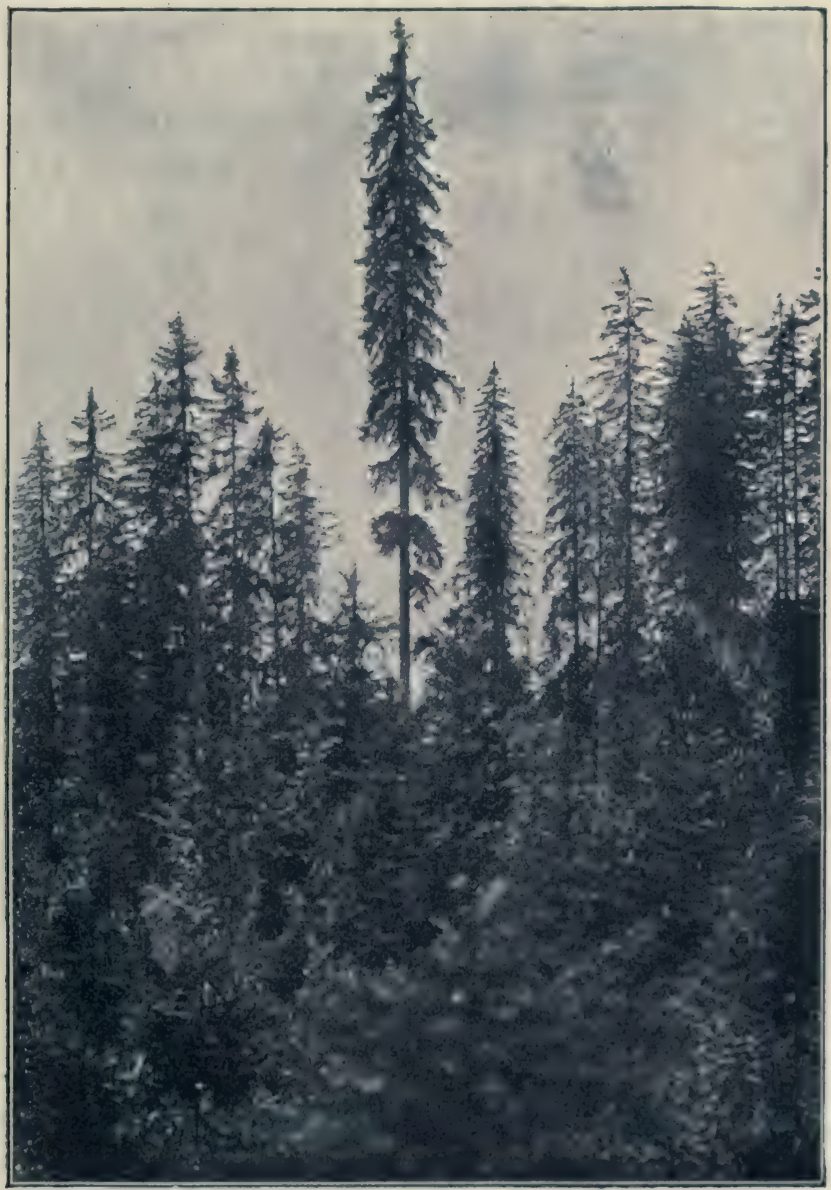

Fig. 8. - Épicéa columnaire, forêt du Risoux (Jura). (Photographie de M. P. Galland.)

de verdure qui laisse tomber indifférent les masses de neige s'abattant dans ces forêts.

Forme forestière. - La forme spécifique ne conserve sa 
constance que chez l'arbre isolé dont les branches se développent librement (fig. 9). Dans nos massifs forestiers, il n'en est plus de même. La forme forestière apparaît.

Si l'arbre est entouré de végétaux qui, recépés périodiquement, n'arrivent jamais à dépasser une certaine hauteur inférieure à celle à laquelle il peut prétendre, le couvert latéral vient augmenter l'effet de l'élagage naturel. Alors sa tige se dénude jusqu'au niveau le plus élevé atteint par les sujets

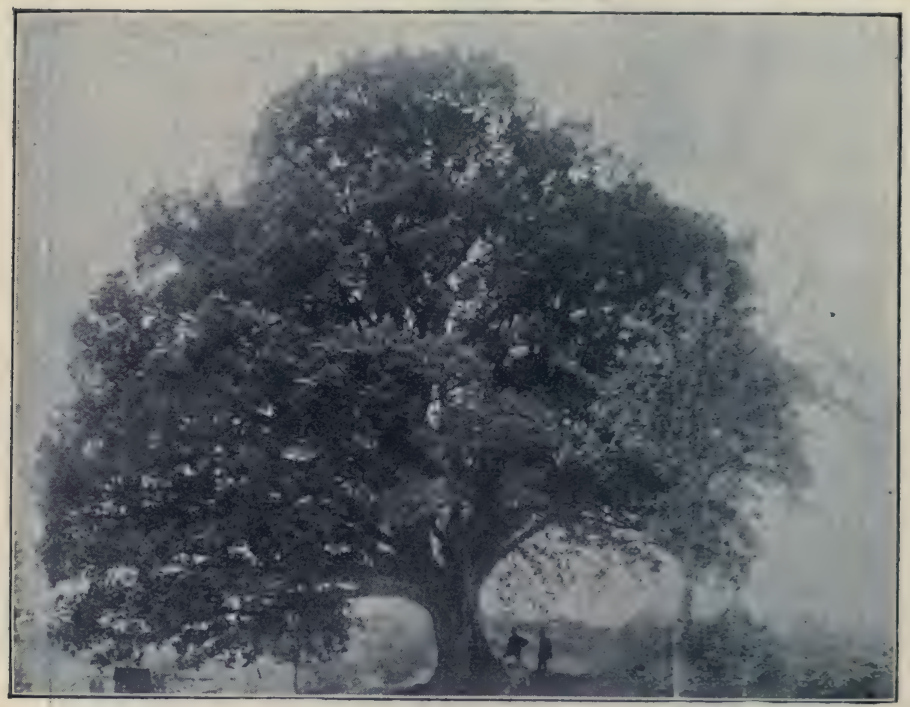

Fig. 9. - Chêne isolé (forme spécifique), Etival (Vosges). (Photographic de M. J. George.)

environnants et sa cime se constitue au-dessus de l'océan de verdure dans lequel le fût reste noyé. Le fût est donc plus long que dans l'arbre crû en rase campagne. Mais cette forme forestière reste voisine de la forme spécifique, la cime ayant encore toute latitude pour se développer. C'est le cas des réserves de nos taillis sous futaie (fig. 10).

Il en est autrement quand l'arbre fait partie intégrante d'un massif serré, dont tous les sujets se poussent en hauteur. Il y a, dès lors, lutte pour la lumière, c'est-à-dire pour la vie. 
Les dernières branches seules étant assez éclairées pour demeurer vivantes, la tige se dégarnit de plus en plus; d'ailleurs ces branches elles-mêmes ne peuvent s'allonger

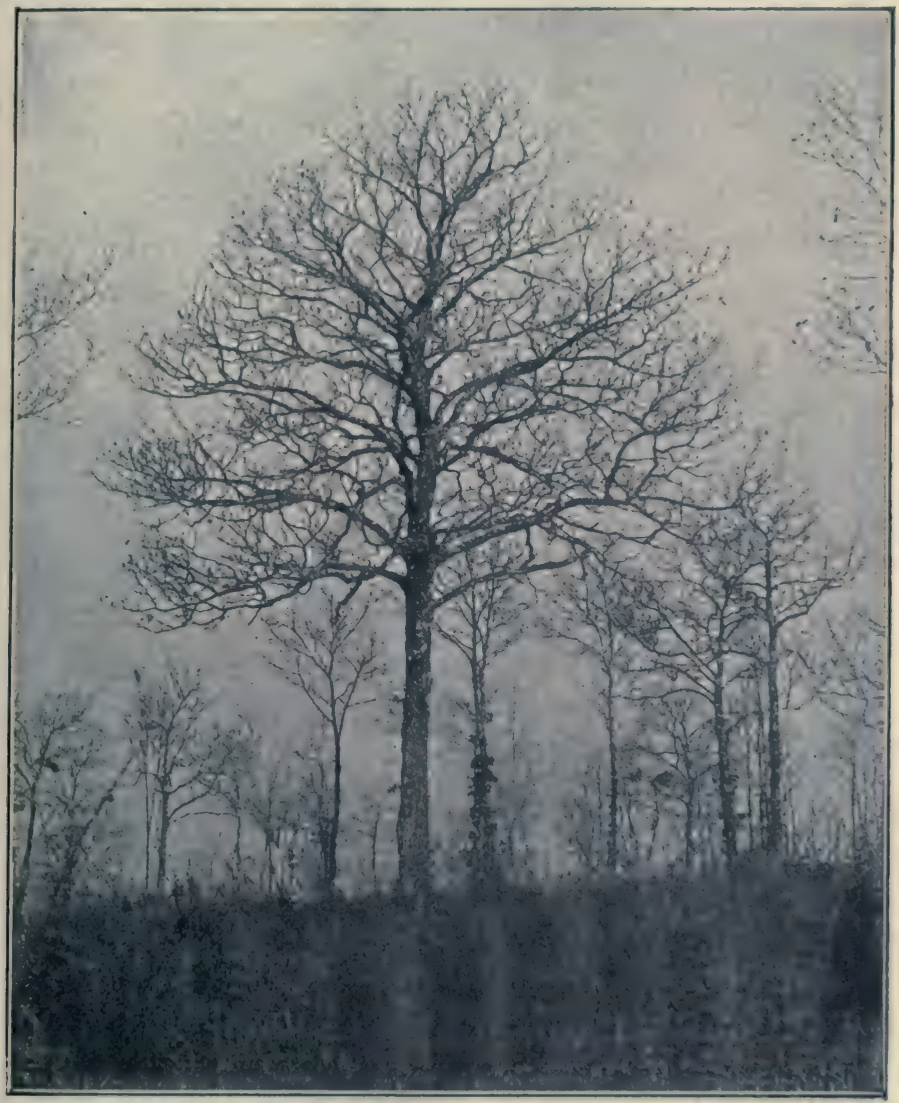

Fig. 10. - Chêne de taillis sous futaie (forme forestière), forêt de Chargey-lès-Port (Haute-Saône).

sans se heurter à celles des arbres voisins. Aussi la forme spécifique disparaît-elle ou à peu près; dans les vieux massifs, chênes, hêtres ou sapins prennent une forme analogue : ce sont toujours les mêmes fûts démesurément longs, surmontés par une cime grêle (fig. 11). 
Mais ces formes anormales ne se maintiennent que sous l'influence persistante des causes qui les ont fait naître. Isole-

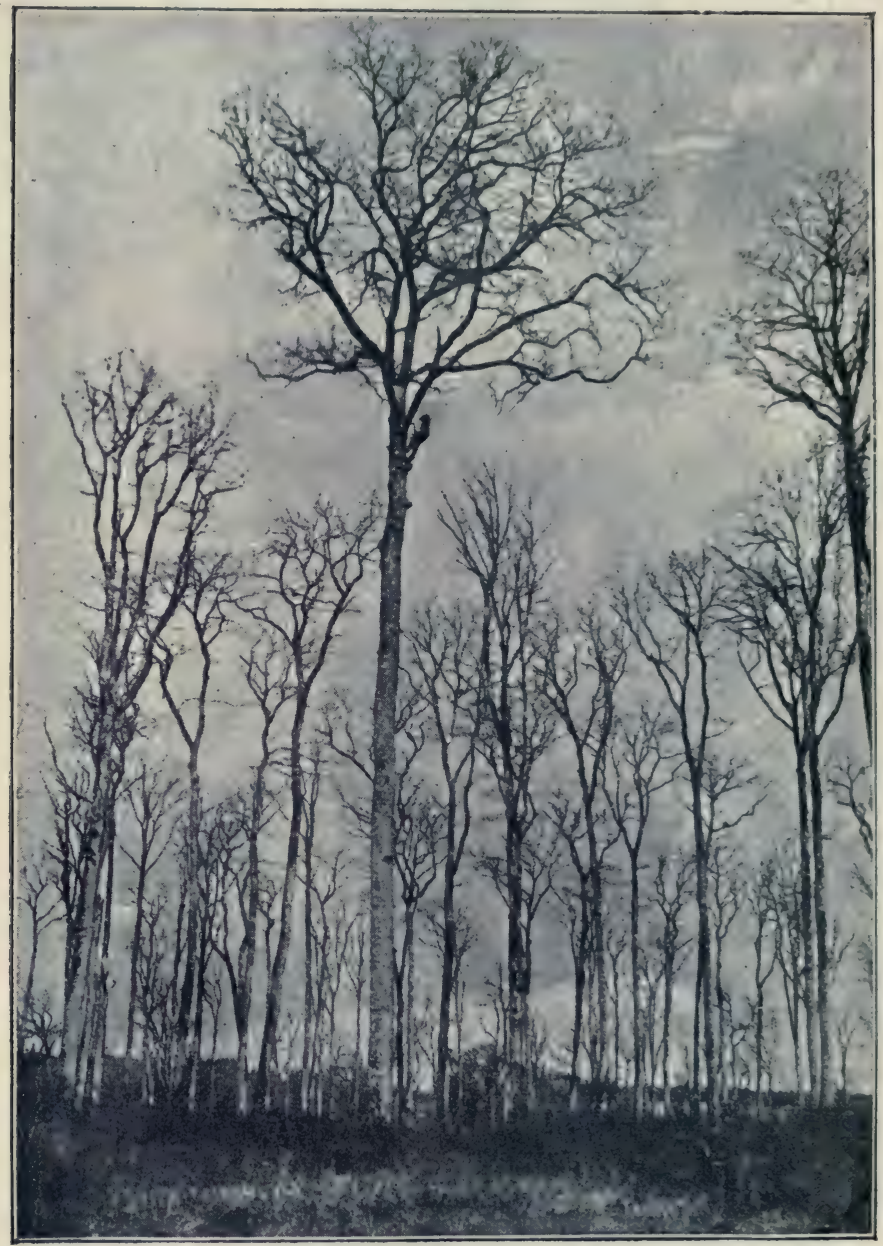

Fig. 11. - Chêne de futaie (forme forestière), avec un ébrancheur s'apprêtant à en couper la cime, forêt de Bercé (Sarthe). (Photographie de M. Couturier, photographe au Mans.)

t-on un arbre crû en massif, on voit bientôt sa cime dépérir. Si l'espèce est douée de la faculté d'émettre des rejets, et si 
l'individu n'est pas trop âgé, il peut, dans des conditions favorables, se reformer une nouvelle tête au dessous de la première; dans le cas contraire, il est voué à une mort certaine.

De même, quand un arbre se trouve peu à peu englobé dans un massif plus jeune qui s'élève autour de lui, ses branches les plus basses périssent, successivement étouffées par le couvert latéral qui se crée autour d'elles. L'arbre recommence à croitre en hauteur et se constitue une nouvelle cime dans l'espace qui lui resle disponible au milieu du peuplement dont il fait désormais partie. Toutefois la mort des grosses branches basses entraîne des nécroses qui dégradent son bois et abrègent la durée de son existence.

Généralités sur l'accroissement. - L'activité physiologique plus ou moins grande des feuilles et des racines, la répartition inégale dans l'arbre des matériaux élaborés, se traduisent par des variations dans l'accroissement en hauteur et dans l'accroissement en diamètre, par suite, dans la forme de la tige.

Pour étudier ces variations, deux procédés sont possibles: soit prendre tous les ans, ou mieux, à plus longs intervalles, des mesures extérieures sur un arbre vivant, mesures que l'on compare entre elles, soit abattre l'arbre et le disséquer pour se rendre compte de la manière dont il s'est accrû.

La grosseur d'un arbre sur pied s'évalue à l'aide de chaînes ou de rubans donnant la circonférence, et à l'aide de compas, d'une forme analogue à celle des compas de cordonniers, qui donnent le diamètre (fig. 12) (1).

Diamètres et circonférences se mesurent le plus souvent à hauteur d'homme, c'est-à-dire à $1^{\mathrm{m}}, 30$ (parfois $1^{\mathrm{m}}, 50$ ) audessus du sol (2). La surface d'une section horizontale de la tige à ce niveau est dile surface terrière.

(1) Quand on utilise la chaine, il faut éviter les protubérances accidentelles de l'écorce, les plaques de mousse, etc. Quand on se sert du compas, il est prudent de mesurer deux diamètres perpendiculaires et de prendre la moyenne des lectures.

En général, le diamètre calculé d'après la circonférence est un peu plus fort que le diamètre mesuré directement au compas.

(2) Il est sage de repérer sur l'arbre, par un cercle de couleur noire ou rouge, le niveau précis oủ la mesure a été prise, afin de ne pas s'en écarter dans l'avenir. 
La hauteur s'évalue à l'œil ou avec l'aide de l'un des nombreux instruments inventés à cet effet. Un des plus pratiques

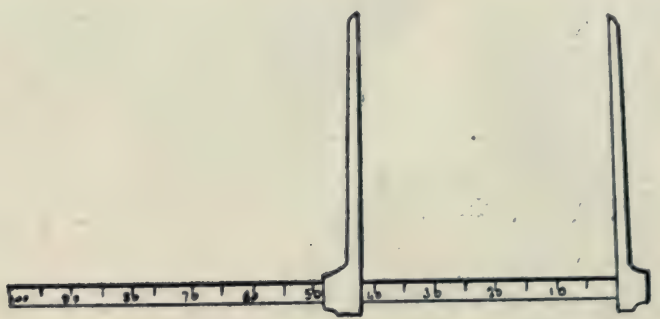

Fig. 12. - Compas forestier.

parmi ces dendromètres est le clisimètre Goulier, adapté à cet emploi par M. Belliéni, ingénieur opticien à Nancy et basé

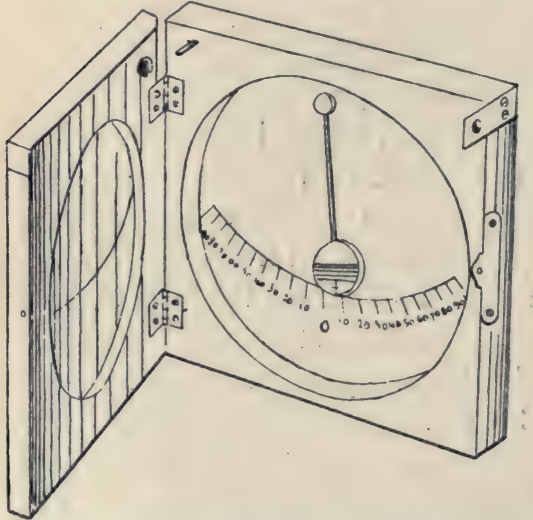

Fig. 13. - Clisimètre Goulier. surles propriétés destangentes trigonométriques (fig. 13). Mais, quelle que soit la perfection du dendromètre utilisé, avec les données qu'il fournit, il n'est pas possible de suivre d'une façon sérieuse la marche de l'allongement des arbres sur pied. Il est préférable d'attribuer à ceux-ci des hauteurs mesurées sur des sujets d'expérience de même grosseur et même végétation préalablement abattus.

Le procédé par dissection, ou procédé par analyses de tiges, est de beaucoup le plus précis.

Sur l'arbre exploité, on prélève des rondelles de distance en distance, par exemple : au niveau du sol, à $1 \mathrm{~m}, 30$ au-dessus du sol, puis à $3^{\mathrm{m}}, 30,5^{\mathrm{m}}, 30,7^{\mathrm{m}}, 30$, etc. Comptant sur la rondelle de base, puis sur toutes les autres, le nombre de. 
couches ligneuses (1), on en déduit l'âge total du sujet et, par de simples soustractions, ses âges successifs quand il avait les hauteurs de $1^{\mathrm{m}}, 30,3^{\mathrm{m}}, 30$, etc. On est ainsi en possession de données précises sur la marche de la croissance en hauteur. Il ne reste plus qu'à mesurer le diamètre de chaque rondelle (2) pour avoir tous renseignements quant à la croissance en grosseur.

Le plus commode est d'exprimer par des graphiques les résultats obtenus.

Des variations du diamètre et de la hauteur, on déduit celles de la surface de section (accroissement circulaire) et celles du volume.

Accroissement en hauteur. - L'accroissement en hauteur est surtout rapide pendant la jeunesse (3); il passe bientôt par un maximum, puis diminue et se réduit à néant chez les vieux arbres. Le pin sylvestre, le mélèze, l'épicéa, s'allongent très vite au début de leur existence; le hêtre, le sapin, et surtout l'épicéa grandissent plus régulièrement et jusqu'à un âge plus avancé. Nous avons parlé déjà de l'influence du sol et du climat et de celle de l'état isolé ou de l'état en massif sur le point qui nous occupe.

Ajoutons ce fait très constant que la croissance en hauteur des rejets de souche est toujours bien différente de celle des brins de semence. Beaucoup plus active pendant les dix ou vingt premières années, elle se ralentit en même temps que la vitalité du sujet. Vers trente ans, les brins de semence reprennent un avantage qui va toujours en s'accentuant.

(1) Quand les couches annuelles sont peu distinctes, on frotte la section avec de la terre, ou mieux, on a recours à un colorant pour les rendre plus visibles. Dans tous les cas, la section est rabotée et passée au râcloir.

(2) La surface de la section doit être rigoureusement normale à l'axe de l'arbre : si les accroissements étaient coupés obliquement, on mesurerait des épaisseurs trop fortes. - Quand la section n'est pas un cercle, on prend la moyenne de deux diamètres perpendiculaires, ou même de trois ou quatre diamètres convenablement choisis.

(3) Nous ne pouvons que résumer brièvement ces questions si importantes de l'accroissement des arbres. Au lecteur désireux de plus amples détails, nous recommanderons le Traité de M. le Professcur Hüfrel : Les Arbres et les Peuplements forestiers; formation de leur volume et de leur valeur, Nancy, 1893. 
Accroissement en diamètre. - L'accroissement en diamètre varie dans d'énormes proportions suivant les essences (1) et surtout suivant les conditions dans lesquelles l'arbre s'est accrû. Ainsi les pins sylvestres de nos plaines françaises pourront avoir des accroissements de 5 millimètres d'épaisseur et plus, tandis que leurs congénères de Finlande fourniront ces bois si recherchés dont les couches annuelles ne sont pour ainsi dire distinctes qu'à la loupe. Il en est de même pour l'épicéa et pour le mélèze, hôtes habituels de stations froides, mais fréquemment introduits sous des climats beaucoup plus doux.

L'influence de la profondeur et de la richesse du sol est tout aussi considérable. On devine que dans les terrains limoneux qui bordent nos rivières, les arbres grossiront plus vite que sur des plateaux calcaires sans profondeur et exposés au desséchement, ou sur des sables grossiers et stériles.

Il y a lieu de tenir compte enfin de l'état de massif plus ou moins serré dans lequel l'arbre a vécu. Les chênes de taillis sous futaie, dont la ramure puissante est toujours baignée delumière, ont les accroissements larges; ils sont minces, au contraire, chez les sujets de même essence, mais à cimes réduites, qui peuplent les massifs serrés de nos vieilles futaies (fig. 14)(2).

Sur un arbre donné on observe le plus souvent:

1. Que l'épaisseur des anneaux ligneux d'une même section transversale est variable; la rapidité de la croissance en diamètre n'a donc pas été constante pendant toute la vie du sujet. En général l'accroissement du diamètre, considérable au début, diminue de plus en plus à partir d'un certain âge.

$2^{\circ}$ Que le manchon ligneux fabriqué au cours d'une année quelconque a une épaisseur variable aux différents niveaux

(1) Ainsi le pin cembro a toujours des couches ligneuses remarquablement minces, aussi bien dans les jardins botaniques de la plaine que dans les régions montagneuses où il est spontané.

(2) L'épaisseur du bois de printemps des essences feuillues varie peu. Donc, quand les accroissements sont larges, c'est le bois d'été qui domine; aussi les chênes qui ont crû vite ont-ils un bois lourd et nerveux. Dans les résineux, au contraire, c'est le bois d'été qui reste constant en épaisseur. La largeur des accroissements correspond à une plus forte proportion de bois de printemps, c'est-à-dire de tissus légers et mous. 
FORME DES ARBRES.

de la tige, depuis le sol jusqu'au bourgeon terminal. Ainsi

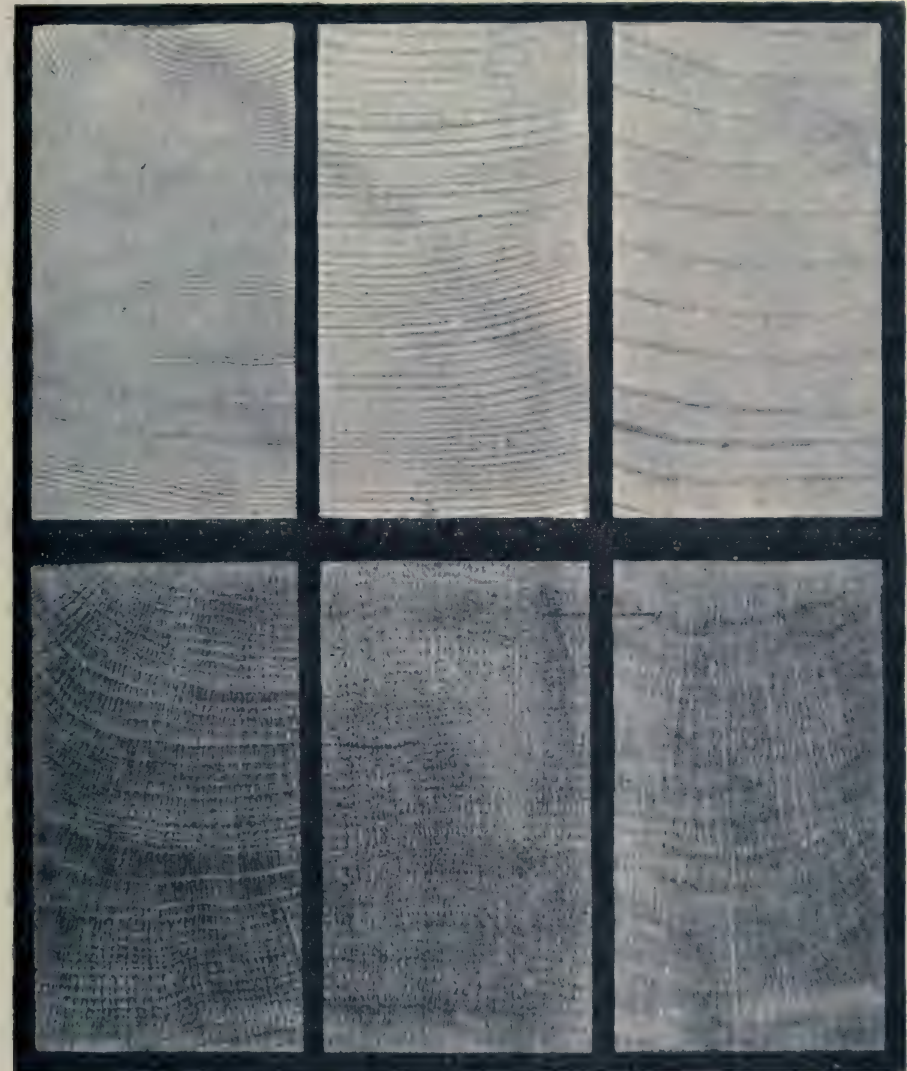

Fig. 14. - Types de bois de chêne et d’épicéa : 1. Épicea (Chamounix, alt. $1400 \mathrm{~m}$.). - 2. Épicéa (Grande Chartreuse, alt. $1360 \mathrm{~m}$.). - 3. Épicéa (Saint-Laurent, alt. $450 \mathrm{~m}$.). - 4. Chêne rouvre (Vosges, taillis sous futaie). -5 . Chêne rouvre (Allier, futaie pleine). -6 . Chêne pédonculé (Landes, arbre isolé). (Le no 1 est en haut et à gauche.)

la croissance en diamètre n'a jamais eu la même intensité sur toute la longueur de cette tige (1).

(1) L'accroissement en diamètre peut diminuer sans que cela implique nécessairement une diminution dans l'accroissement circulaire. En effet, si le rayon du cercle intérieur est plus grand, l'anneau ligneux aura parfois, avec une épaisseur moindre, une surface plus considérable. 
Cette inégalité de l'épaisseur des accroissements aux différents niveaux se traduit pas des modifications importantes dans la forme de la tige considérée dans son ensemble. Celle-ci se rapproche plus ou moins de solides géométriques variant du cylindre à la neiloïde, en passant par le cône et le paraboloïde (1).

A ce sujet, M. le Dr Nordlinger (2) cite les faits suivants : $1^{\circ}$ Dans un jeune arbre garni de ses branches depuis le sol, conséquemment isolé, les accroissements s'amincissent régulièrement de la base au sommet : la forme de la tige est conique ; $2^{\circ}$ quand les branches inférieures sèchent naturellement, les accroissements présentent leur plus grande épaisseur dans le voisinage et au-dessous des premières branches vives; ils s'amincissent de là jusqu'au pied : la tige se rapproche du paraboloïde; $3^{\circ}$ à l'état de massif, les couches deviennent de plus en plus larges par le haut, souvent deux ou trois fois plus larges qu'au pied : elles donnent à la tige une forme plus cylindrique; $4^{\circ}$ l'arbre en massif qu'on isole, s'accroît dans l'ordre inverse; c'est-à-dire que les grossissements supérieurs s'amincissent, tandis que les inférieurs s'élargissent; la tige a des tendances à revenir à la forme conique qui est la plus générale chez les arbres crûs isolément.

Donc, comme le fait observer M. Hüffel (3), quand par suite de circonstances favorables les fûts sont élevés, ils sont aussi, le plus souvent, très voisins de la forme cylindrique.

Signalons enfin l'évasement prononcé habituel dans les régions tout à fait basses de la tige et qui tient à l'empattement fơrmé par la naissance des grosses racines. C'est en partie pour éviter les erreurs que ferait commettre cette déformation, que

(1) Cette forme s'exprime soit par un coefficient de forme, facteur plus petit que l'unité, par lequel, pour avoir le volume vrai, il faut multiplier le volume d'un cylindre ayant comme base la surface terrière de l'arbre et comme hauteur celle de ce dernier; ou par un coefficient de décroissance, facteur également plus petit que l'unité, par lequel il faut multiplier le diamètre à hauteur d'homme pour avoir le diamètre au milieu de la tige.

(2) Nordlinger, Die technischen Eigenschaften der Hölzer. Stuttgard, 1860.

(3) Hüffel, loc, cit. 
l'on a l'habitude de mesurer les arbres à $1^{\mathrm{m}}, 30$ au-dessus du sol.

Nous avons dit que, parfois, un ou plusieurs anneaux ligneux se trouvent, sur tout leur pourtour, plus épais ou plus minces que ceux qui les avoisinent. Les augmentations d'épaisseur sont dues à des conditions particulièrement favorables à la vie de l'arbre: abondance des pluies d'été dans un climat har bituellement sec, pléthore de nourriture, etc... A l'appui de ces faits, plusieurs auteurs, notamment M. l'Inspecteur Bartet (1) pour les chênes, et M. le Professeur Henry (2) pour les hêtres, ont constaté qu'après une exploitation, les arbres réservés dans les taillis sous futaie accusent soudain une brusque augmentation dans l'accroissement en diamètre. "Les convives étant moins nombreux à table, dit M. Henry, sont plus copieusement servis... " Cette augmentation ne se maintient d'ailleurs que pendant quelques années (3).

Les diminutions sont liées à des causes contraires. Ce peut être un hiver rigoureux, qui, sans tuer un chêne, lui occasionne un tort suffisant pour que sa croissance soit ralentie pendant quelques années; ce peut être un été exceptionnellement sec (4), ou au contraire, dans les régions monta-

(1) Bartet, Recherches sur l'accroussement des chênes de taillis sous futaie. (Revue des Eaux et Forêts, 1891.)

(2) E. Henry, Accroissement des arbres de réserve après l'exploitation du taillis. (Bull. Société des Sciences de Nancy, 1899.)

(3) Elle parait être surtout sensible dans les régions basses de la tige : M. Henry le constate notamment pour le hêtre.- M. Mer signale un fait analogue chez des sapins après une éclaircie. (Revue des Eaux et Foréts, 1889, page 72.)

(4) M. Henry signale qu'en 1893, année célèbre par la sécheressé de l'été, l'accroissement des hêtres n'a été qu'environ 37 p. 100 de l'accroissement pendant l'année 1891 dans la forêt de Ilaye, et n'a même pas atteint 26 p. 100 dans certaines forêts des environs d'Hẻricourt (Ilaute-Saône), d'oú une perte considérable pour les propriétaires de forèts. Les chênes à enracinement plus profond ont moins souffert. - Influence de la sécheresse de 1893 sur la végélation forestière. (Bull. Société des Sciences de Nincy, 1897.)

M. Mer a constaté un fait analogue pour le sapin dans les Vosges à la suite de cette même sécheresse, qui avait eu pour autre conséquence de réduire chez le sapin la longueur de la pousse annuelle. - Influence de l'élat climatérique sur la croissance des sapins. (Ext. Journal de Botanique, $n .10,11,12,13$ el 14, année 1895.) 
gneuses, un été trop pluvieux (1); ou bien encore une invasion d'insectes (2), qui détruit le feuillage, une gelée tardive au printemps, etc.

Quand les anneaux ligneux n'ont qu'une épaisseur très

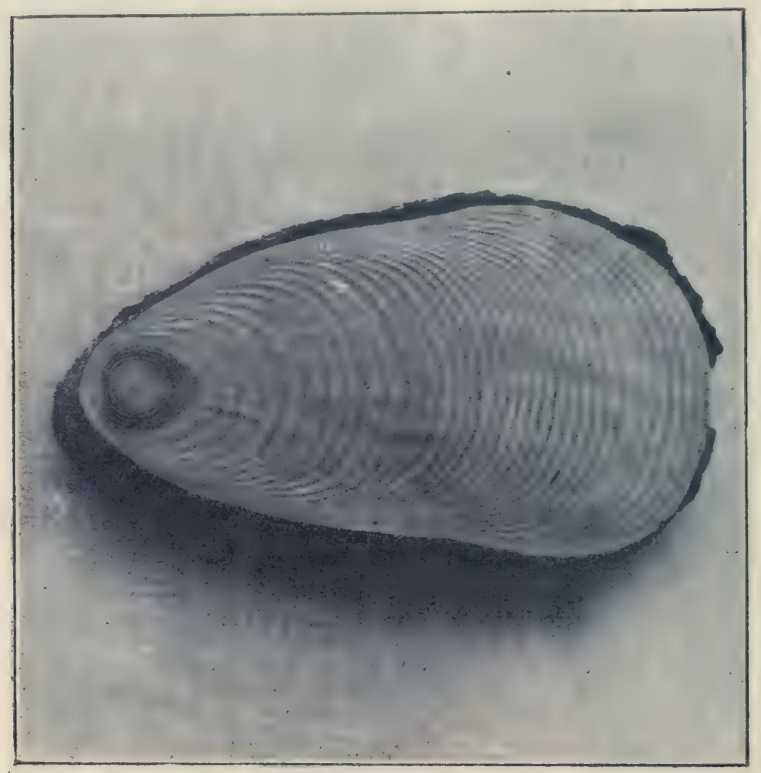

Fig. 15. - Pin sylvestre (moelle excentrique).

réduite sur certaines régions de leur pourtour, la tige devient excentrique, phénomène fréquent chez les arbres qui croissent sur des terrains très inclinés ou sur les lisières des forêts et qui reçoivent ainsi plus de nourriture d'un côté que de l'autre (fig. 15).

Le fait est normal chez les espèces à contours sinueux et irréguliers comme les génévriers, les ifs, les thuias, les oliviers, etc.

(1) MM. Henry et Mer, dans les travaux cités, établissent que pendant l'été froid de 1888, la croissance des hêtres et des sapins a été sensiblement ralentie.

(2) Aux invasions périodiques des hannetons correspondent, chez les chênes, des accroissements très minces, souvent presque nuls. 
REPRODUCTION.

ARTICLE III

\section{REPRODUCTION}

Semis et rejets. - Fertilité des arbres. - Germination des graines et installation des semis. - Rejets proventifs et rejets adventifs. Influence de l'époque de l'exploitation sur l'évolution des rejets. Influence du mode d'abatage. - Drageons. - Rejets de racine. Modes spéciaux d'exploitation.

Semis et rejets. - L'arbre peut se reproduire par voie sexuée, c'est-à-dire par la germination de graines qui donnent naissance à de nouveaux individus, libres, dès le premier jour, de toute attache avec le pied-mère, - ou par voie asexuée, c'est-à-dire par l'évolution de bourgeons se développant en drageons ou en rejets, qui ne prennent que peu à peu une individualité plus ou moins complète.

Sans doute, des rejets de charme ou de chêne peuvent, à la longue, s'isoler de la souche dont ils dépendaient à l'origine; sans doute, des drageons de tremble ou de robinier peuvent percer le sol très loin de l'arbre sur les racines duquel ils se sont constitués; il n'en est pas moins vrai que la régénération par voie sexuée est le véritable mode de reproduction; la régénération par voie asexuée n'est qu'un procédé de rajeunissement; elle produit des descendants qui n'ont pas, en général, la vitalité et la longévité des individus nés de semence.

Tout sujet issu d'une graine porte, au début, le nom de semis, plus tard celui de brin de semence, ou simplement de brin. Les forêts traitées en futaie sont toujours régénérées par voie de semis.

Les différents modes de rajeunissement par les axes (c'està-dire par l'évolution de bourgeons) sont la bouture, la marcotte, le rejet et le drageon. La bouture et la marcotte sont surtout utilisées en arboriculture; les rejets et les drageons prennent, au contraire, une importance capitale daus la régénération des forêts traitées en taillis.

Fertilité des arbres. - Pour qu'un arbre donne naissance à des semis, la première condition est qu'il porte des graines fertiles en quantité appréciable. Or, toutes les essences ne 
sont pas également fécondes. On peut les classer en deux groupes; celles à semences lourdes (1) et celles à semences légères. La fructification absorbe chez les premières une beaucoup plus grande quantité de matières nutritives que chez les secondes, et, tandis que l'arbre à semences légères fournit presque tous les ans celles-ci en très grande quantité, il est rare que, si la semence est lourde, le même sujet soit fertile deux années de suite.

La fécondité varie non seulement avec l'espèce, mais encore, pour chaque espèce, avec les conditions plus ou moins favorables de climat et de sol. Le bourgeon à fleur étant formé dès l'année qui précède celle de la fructification, une récolte de semence est le résultat du concours de deux années favorables consécutives : circonstances qui diminuent singulièrement les chances de l'obtenir. Les gelées printanières qui détruisent les bourgeons floraux, les temps froids qui nuisent à la fécondation, les insectes dont les larves minent les bourgeons avant la floraison ou vivent à l'intérieur des semences une fois formées, comptent parmi les causes de destruction les plus fréquentes. Si le climat est doux, si l'arbre est placé dans sa zone naturelle d'expansion, la fécondité sera, la chose va sans dire, plus grande que dans les conditions inverses (2). A côté de la clémence de la température, qui diminue les chances d'accidents, il faut constater l'effet direct de la lumière et de la chaleur. Aussi dans les régions méridionales, les arbres forestiers fructifient-ils plus tôt et plus abondamment que dans les contrées du Nord, froides et brumeuses.

A ce même point de vue, les arbres isolés ou les réserves des taillis sous futaie, dont la cime est bien étalée au soleil, sont toujours beaucoup plus fertiles que ceux qui croissent en

(1) Nous employons le terme semence de préférence au terme graine. Sous ce nom de semences, nous confondrons, en effet, des fruits comme le gland et des graines comme celles du sapin.

(2) Tandis que dans le bassin de l'Adour les chênes donnent des semences tous les deux ou trois ans, dans les régions du Centre et de l'Ouest les glandées se produisent seulement tous les quatre à huit ans ét dans le Nord et l'Est de la France elles se font atterrdre parfois quinze ans et plus. De même, la fertilité du sapin diminue sensiblement aux grandes altitudes. 
massif serré. D'ailleurs lés premiers, qui ont une ramification puissante et touffue, portent en abondance des bourgeons floraux, lesquels naissent en pleine lumière et à l'extrémité des rameaux; il n'en est pas de même chez les seconds, dont la cime est toujours réduite et pauvre en menues branches.

La profondeur et la richesse du sol permettent aux arbres de réparer plus vite les fatigues des années de semence : celles-ci se succéderont donc d'autant plus fréquemment que les conditions seront meilleures.

En général (1) les arbres forestiers ne commencent à porter des graines fertiles, que lorsqu'ils ont à peu près atteint leur hauteur normale. Ils sont alors adultes et fructifient avec autant d'abondance et de régularité que le comportent leur situation et l'espèce à laquelle ils appartiennent. Toutefois, cette fécondité diminue progressivement quand l'arbre devient vieux et très vieux.

Germination des graines et installation des semis. Supposons réunies toutes les conditions voulues pour qu'un chêne ou un sapin donne de bonnes semences; la naissance de semis nombreux ne sera pas encore certaine. Que l'hiver, en effet, soit trop froid ou trop humide, les semences gèlent ou pourrissent sur le sol; qu'un brusque refroidissement survienne au moment de la germination; les jeunes plantules sont détruites dès. leur formation. D'autre part, et c'est là peut-être une des causes les plus fréquentes d'insuccès, le sol n'est toujours pàs en état de recevoir utilement les fruits ou les graines. Les semis de sapin ne réussissent que si la graine tombe sur du terreau; ceux du pin maritime préfèrent un sable meuble et presque dépourvu de toute autre végétation; les racines dès jeunes plants d'épicéas ne peuvent arriver à percer les couches d'aiguilles mortes qui jonchent le terrain; beaucoup d'essences réclament de l'abri dans leur jeunesse, d'autres le plein découvert, etc... En somme, chaque espèce a ses

(1) Accidentellement des arbres poussant en sol maigre ou superficiel, ou bien encore des sujets fatigués par une transplantation récente ou pour tout autre motif, se mettent à fruits de très bonne heure; mais ce n'est là qu'un effet dû à leur situation précaire. Aussi leur's semences sont-elles généralement stériles ou tout au moins de qualité très médiocre; il faut bien se garder de les récolter. 
exigences souvent très absolues; mais presque tontes demandent que le sol soit meuble : quand il est dur, tassé, desséché, la germination peut se faire, mais le jeune plant ne parvient pas à se créer un enracinement suffisant avant les chaleurs de l'été, alors qu'il peut être tué par la sécherese. Cet état malencontreux du sol est une conséquence inévitable du pâturage; il est également fréquent sous les vieux massifs dont le couvert est élevé et déjà éclairci ; cet inconvénient, ajouté au fait que les arbres sont âgés et par suite moins fertiles, rend la régénération par la semence beaucoup plus aléatoire qui dans les massifs d'âge moyen.

Le remède existe-t-il? Oui heureusement, et la chose a trop d'importance pour qu'au risque de nous répéter nous ne l'indiquions pas dès maintenant. Notre règle habituelle sera de maintenir, ou de créer au besoin, en-dessous des vieilles futaies, un sous-étage d'essences acceptant le couvert, que maintiendra le sol meuble et riche en terreau jusqu'au moment où l'on voudra installer les semis. Dans le cas où cette précaution n'aura pas été prise ou n'aura pas produit un effet suffisant, il restera la ressource de recourir à la pioche et de donner au sol une légère façon par bandes ou en plein : un tel travail exécuté avec intelligence est peu coûteux et donne les meilleurs résultats.

Rejets proventifs et rejets adventifs. - Certaines espèces ne drageonnent pas et ne rejettent pas de souches, d'autres jouissent de ces facultés à des degrés très divers.

Parmi les rejets, il faut distinguer ceux de bourgeons proventifs et ceux de bourgeons adventifs.

Les bourgeons proventifs, ou bourgeons dormants, sont des bourgeons qui, au lieu de se développer tout de suite en rameaux, restent dans l'écorce à l'état rudimentaire, et ne s'allongent chaque saison que d'une quantité égale à l'épaisseur de l'anneau ligneux formé. Ils vivent à l'état latent pendant de longues années, toujours prêts à se développer aussitôt qu'une cause accidentelle leur en fournit l'occasion. Ainsi une blessure grave, une incision annulaire profonde, la suppression ou la mort naturelle de branches principales, l'amputation du tronc en un point quelconque de sa hauteur, font naître, 
immédiatement au-dessous de la blessure ou de la section, des rameaux plus ou moins nombreux. De même, après l'isolement brusque d'un arbre, on voit apparaître ces mêmes productions aux emplacements qu'aurait occupés la cime chez un individu de même espèce normalement constitué. De même encore, quand un arbre est dominé ou dépérissant, des brindilles nombreuses se développent sur les parties dénudées de sa tige et de ses grosses branches. Tous ces effets ont une seule et même cause : l'évolution de bourgeons proventifs localement réveillés par un apport plus considérable de matières nutritives ou par l'influence d'une lumière plus abondante.

Les rejets d'origine proventive appartiennent donc toujours à la formation primitive del'axe sur lequel ils sont implantés; ils sont en correspondance directe avec la moelle centrale : en un mot, à leur point d'insertion sur la tige, ils ont le même âge que celle-ci. On comprend dès lors que ces bourgeons, qui à la longue perdent leur vitalité, soient plus nombreux sur les parties jeunes ou d'âge moyen que sur celles qui sont plus âgées.

Du reste, la persistance de cette vitalité diffère beaucoup. suivant les essences: chezle hêtre et le bouleau, par exemple, elle s'éteint de bonne heure, après une vingtaine d'années au maximum; chez le chêne, chez le charme, elle se maintient jusqu'à un âge très avancé, quatre-vingts ans et même davantage. En résumé, plus le sujet mutilé est vieux, moins nombreux sont les bourgeons qui évoluent; certes, il n'est pas rare, dans les coupes, de voir des rejets apparaître sur les souches de modernes et d'anciens, mais ils s'étiolent bientôt, et sont étouffés par ceux qui sont issus des souches plus jeunes les avoisinant.

Quoi qu'il en soit, parmi les essences feuillues existe une grande inégalité dans l'aptitude à donner des rejets d'origine proventive. Par opposition au hêtre et au bouleau, nous venons de citer le chêne et le charme, dont les bourgeons dormants sont bien plus longévifs et aussi bien plus nombreux. A ces deux essences nous pouvons ajouter entre beaucoup d'autres: le tilleul, qui donne des touffes de rejets à végétation parfois exubérante, les érables, etc...

Les bourgeons adventifs s'organisent dans le tissu cicatri- 
ciel, ou bourrelet de recouvrement, qui se forme sur les bords de toutes les blessures ou sections faites sur la tige. Les rejets auxquels ils donnent naissance n'apportent qu'un faible appoint à la reproduction des. essences riches en bourgeons proventifs. Chez le hêtre et le bouleau, mal partagés à ce dernier point de vue, ils sont au contraire assez abondants et surtout très précieux (1). Dans tous les cas, ils sont mal soudés à la souche et il suffit d'un choc ou d'un vent violent pour les détacher.

Il ne saurait être question de reproduction par rejets pour les conifères indigènes.

Quelle que soit l'origine des rejets, la lumière et la chaleur jouent un rôle important dans leur développement. Ils seront d'autant plus abondants que la lumière sera plus vive et le climat plus chaud; c'est dire que leur évolution se fera mieux en plein découvert que sous un massif, en plaine qu'en montagne, à l'exposition Sud qu'à l'exposition Nord, enfin dans les régions méridionales que dans les régions septentrionales. Il faut ensuite que les rejets naissants puissent vivre et, pour cette.raison encore, un climat suffisamment chaud est indispensable; en effet, poussant tard au printemps qui suit le recépage, renfermant des tissus gorgés d'eau, ils sont détruits par les gelées de l'hiver, si la saison de végétation n'a pas été assez longue, si l'automne n'a pas été assez chaud pour qu'ils puissent s'aoûter.

\section{Influence de l'époque de l'exploitation sur l'évolution} des rejets. - Le sylviculteur peut intervenir efficacement pour favoriser ou entraver l'évolution des rejets. Il y a lieu de considérer à cet égard l'époque de l'année où se pratique le recépage et les conditions dans lesquelles il est exécuté; c'est la base fondamentale de tout traitement en taillis.

Les rejets, avons-nous dit, seront d'autant plus abondants

(1) M. Bartet a observé que, dans les environs de Nancy, les souches de hêtre engendrent un peu plus de rejets adventifs que de rejets proventifs, tandis que sur les souches de charme, les proventifs sont environ huit fois plus nombreux que les adventifs. - De l'Influence exercée par l'époque de l'abatage sur la produclion et le développement des rejets de souches. (Annales de la Science agronomique française et étrangère, 1891.) 
que l'arbre sera plus jeune. Il faut toutefois tenir compte de l'épuisement du sol et de la souche qu'entraînent infailliblement des exploitations souvent répétées. En effet, comme nous le verrons dans la suite, les axes jeunes sont les plus riches en matières minérales. Il en résulte qu'on prend beaucoup plus au sol en y coupant cinq fois de suite des taillis de vingt ans qu'en y exploitant une fois des arbres de cent ans. Aussi croyons-nous téméraire de compter sur un taillis pour fournir d'une manière permanente des récoltes bi ou trisannuelles de menues ramilles utilisables comme fourrage. La chose peut se faire par extraordinaire une fois ou deux après une révolution normale, mais, répétées trop souvent, ces exploitations ruineraient le sol à bref délai. Malgré tout, les exploitations à court terme sont à la rigueur possibles dans les sols très riches en matières nutritives, comme les limons et certaines argiles. Elles sont, au contraire, désastreuses dans les sables peu fertiles et les calcaires superficiels.

Pour des raisons économiques, l'exploitation des taillis a presque toujours lieu pendant la morte saison, de la fin de l'automne au premier printemps, la main-d'œuvre étant alors moins chère. C'est également l'époque la plus avantageuse au point de vue cultural : pendant l'hiver, en effet, les matériaux de réserve destinés à la nourriture des bourgeons proveritifs, à la formation et au développement des bourgeons adventifs, se trouvent en quantité maxima accumulés dans les souches. D'autre part, les rejets pouvant évoluer dès le premier printemps auront toute la bonne saison pour grandir et s'aoûter.

Mais certaines considérations peuvent conduire à n'exploiter que beaucoup plus tard: à la fin du printemps par exemple. Il en est ainsi dans les taillis de chêne destinés à la production des écorces : la levée de l'écorce ne s'opérant bien qu'en temps de sève, force est de reculer l'abatage des perches jusqu'aux mois de mai et de juin. Ce retard n'a pas d'inconvénient sérieux quant au nombre et à la vigueur des rejets, comme il résulte de recherches faites à la station d'expériences de l'École forestière par M. Bartet (1), et dont nous résumerons

(1) E. Bartet, loc. cit. 
ainsi les conclusions : pour le chêne les meilleurs résultats sont obtenus par l'exploitation en mars, avril et mai (la coupe en juin occasionne un déchet déjà très appréciable); - pour le charme en mars et avril; - pour le hêtre la coupe en juin est la plus favorable au point de vue du nombre des rejets, celle en avril active leur croissance. D'ailleurs, chez le charme comme chez le hêtre, la coupe en pleine foliaison augmente la proportion des rejets d'origine adventive par rapport à ceux d'origine proventive. Pour toutes les essences, l'époque la plus défavorable est le milieu d'août: les exploitations à la fin d'août et en septembre sont moins dangereuses, car les rejets n'apparaissent qu'au printemps suivant.

Dans les climats doux, on peut sans inconvénient couper dès novembre; mais, dans les contrées où l'hiver est habituellement rude, il vaut mieux attendre le printemps, les souches fraîchement recépées pouvant souffrir des grands froids. Dans tous les cas, il est bon de suspendre la coupe pendant le fort de l'hiver: le bois gelé éclate sous la hache, et les sections d'abatage ne présentent plus toute la netteté désirable.

En Sologne (1), on a l'habitude d'exploiter les bouleaux en automne (novembre et décembre); quand on coupe au printemps, la sève inonde les souches et peut, paraît-il, les faire pẻrir. On abat, au contraire, le chêne en cette dernière saison pour éviter l'action nocive du froid, et retarder l'évolution des rejets, qui échappent ainsi à l'effet désastreux des gelées printanières.

Influence du mode d'abatage. - L'abatage doit se faire avec des outils bien tranchants, d'un poids proportionné à la grosseur des sujets à couper : les perches ayant un décimètre de tour et au-dessus sont coupées à la hache; pour les brins plus faibles, il est préférable d'employer la serpe, afin d'éviter l'ébranlement et surtout la rupture des racines. L'usage de la scie doit être rigoureusement proscrit, et même avec la serpe ou la hache, il faut veiller à ne pas déchirer l'écorce au-dessous de la section.

On donne à celle-ci un léger-bombement vers le haut, de

(1) Observation de M. l'Inspecteur Croizette-Desnoyers. 
mảnière à faciliter l'écoulement des eaux pluviales; c'est l'exploitation dite en talus, par opposition à l'exploitation en gouttière très défavorable à la vitalité des souches (fig. 16).

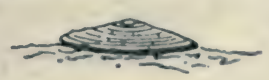

1

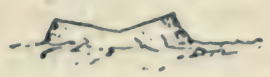

$\ell$

Fig. 16. - Exploitations en talus et en gouttière.

En thèse générale, la section sera opérée aussi près de terre que possible pour forcer les rejets à naître au niveau du sol et même au-dessous; c'est la condition indispensable pour perpétuer les peuplements traités en taillis. Il faut, en effet, établir une distinction entre les rejets de souche, d'origine proventive ou adventive, et les rejets de tige.

Le véritable rejet de souche naissant en contact avec la terre, ses jeunes tissus peuvent émettre des racines qui lui sont propres, ce qui lui permet de se marcotter et par suite de s'isoler, de former un sujet indépendant. Sans doute, ùne souche après l'exploitation est vouée à la pourriture; mais celle-ci n'apparaît d'abord que dans les régions supérieures et centrales; les zones qui avoisinent l'écorce, c'est-à-dire le point d'insertion des rejets, présentent, il est vrai, des signes manifestes d'altération tels que colorations diverses, lignes en zigzag noires ou très foncées dues à des transports de matières oxydées, etc., mais la nécrose ne les gagne qu'assez lentement, et la patte du rejet a le temps de se recouvrir d'un tissu cicatriciel qui intercepte toute communication avec le bois mort (1).

Le rejet de tige, au contraire, reste directement attaché à l'axe mutilé qui le porte. Il ne s'affranchit jamais et n'assure pas la perpétuation de l'individu; celui-ci est toujours constitué par la tige ou portion de tige centrale, dont les productions nouvelles ne sont que des ramifications. Tôt ou tard cette tige meurt de caducité, entraînant avec elle la perte de tous les rejets auxquels elle a servi de centre de végétation, et il faut pourvoir à son remplacement.

(1) Théodore Hartig. Vollstandige Naturgeschichte der forstlichen Culturpflanzen Deutschlands. Berlin, 1852. 
Quoi qu'il en soit, l'évolution des rejets de tige a servi de point de départ à plusieurs modes de traitement: ainsi les têtards et les arbres d'émonde, dont la tige est recepée à une hauteur variant de un à plusieurs mètres, ainsi encore les taillis furetés, où la section est généralement beaucoup plus rapprochée du sol. Il faut, en effet, considérer comme rejets de tige tous ceux qui, sur une souche, naissent à une hauteur telle qu'ils ne puissent se créer un enracinement propre, si faible d'ailleurs que soit cette hauteur. Dans les taillis furetés et même dans des taillis simples exploités trop haut, le cas se présente toujours. Ce ne sont plus alors que des vieux étocs bosselés, chancreux, dépassant le sol de 10 à 50 centimètres et qui s'acheminent vers la stérilité. On ne peut mieux les comparer qu'à la tête mutilée des saules si communs dans nos prairies.

L'ensemble des rejets nés sur une même souche porte le nom de cépée ou de trochée. Ces cépées sont caractérisées par le groupement de plusieurs individus autour d'un centre commun et aussi par la courbure en forme de crosse que présente individuellement chaque rejet à sa base.

Drageons. - Les bourgeons à drageons s'organisent spontanément sur les racines saines et intactes d'un certain nombre d'arbres et d'arbustes feuillus. Citons parmi les arbres: le peuplier tremble, l'aune blanc, le robinier faux acacia, le chêne tauzin, le chêne yeuse, l'orme champêtre. Les drageons naissent plus communément sur les racines horizontales et superficielles, sans qu'il faille généraliser cette faculté à toutes les essences à racines traçantes. Ils sont le résultat d'un fait physiologique normal localisé chez des espèces déterminées; on n'est pas libre de les faire naître, comme les rejets de souche, sur tous les arbres feuillus. Les drageons évoluent de préférence sur les racines d'arbres isolés, comme les peupliers et les ormes plantés le long des routes, les robiniers qui ornent les jardins ou encore sur celles de sujets récemment exploités. Un exemple très fréquent de ce dernier cas nous est donné par les drageons de peuplier tremble qui poussent par myriades dans certaines coupes de taillis après le recépage des quelques individus de cette essence qui pouvaient s'y trouver. 
Le drageon s'affranchit facilement pour former bientôt une tige indépendante; mieux donc que le rejet, il assure la reproduction et même l'expansion de l'arbre, les drageons pouvant percer le sol très loin du pied qui lui sert d'origine.

Rejets de racine. - Sur les racines d'espèces qui d'ailleurs ne sont pas drageonnantes, notamment sur les racines du bouleau, peuvent s'organiser des bourgeons qui évoluent, le cas échéant, en rejets sorlant de terre à une très faible distance ( 1 centimètre à peine) du pied-mère.

Les jeunes racines du bouleau forment des bourgeons d'un ordre tout spécial, qui, sans s'être produits à l'aisselle des feuilles, rappellent beaucoup les bourgeons proventifs. Comme ces derniers, les bourgeons de racines, une fois formés, restent latents et peuvent se multiplier en nombre tel qu'ils rendent parfois le bois de souche madré (1).

Quiconque a planté des bouleaux a éprouvé l'agréable surprise de voir un de ces rejets naître au pied d'un plant qu'il croyait mort.

Ajoutons enfin qu'une section, une blessure, peuvent provoquer sur toutes les racines d'arbres feuillus l'évolution de rejets d'origine adventive, souvent appelés faux drageons. Ils n'offrent rien de bien spécial, si ce n'est que, naissant très près de terre, demeurant longtemps grêles et chétifs, ils ont, en général, le temps de se souder solidement à la racine, et même de s'individualiser avant qu'ils soient de taille à offrir prise au vent ou aux chocs, qui décollent si souvent les rejets adventifs ordinaires.

Procédés spéciaux d'exploitation. - La coupe entre deux terres, c'est-à-dire la coupe exécutée en dessous du niveau du sol, provoque naturellement l'évolution des rejets de racines et des drageons.

Un des procédés les plus connus, spécialement appliqué à une espèce drageonnante, le chêne yeuse, est appelé le saut de piquet. Pour faire sauter le piquet, on coupe chaque brin d'une cépée à 25 centimètres au-dessus du sol; puis, avec une forte cognée, nommée passe-partout, dont le tranchant est mal avivé, on fend la souche entre les tiges principales. Avec la

(1) Flore forestière, par A. Mathieu, quatrième édition, revue par P. Fliche.

Bopps et Jolyir. 
tête de la cognée, on frappe ensuite sur chaque brin et on l'ébranle jusqu'à pouvoir l'arracher à la main. L'opération est bonne ou désastreuse : bonne, si elle est faite dans un but cultural, celui de favoriser la naissance des drageons; désastreuse, au contraire, si le bûcheron n'a d'autre objectif que d'extraire le plus possible de bois de racines, dont l'écorce, très riche en tanin, se vend à un prix élevé. Elle équivaut alors à un véritable défrichement.

Il est, en cela comme en toutes choses, une mesure à garder et des conditions locales à observer. Ainsi, dans les régions méridionales, quelles que soient les essences, i] sera bon de couper les tiges un peu au-dessous du niveau du sol, afin d'éviter que les souches exploitées trop haut se dessèchent à l'ardeur du soleil et que l'écorce se détache du bois. Si la nature pierreuse du terrain empêche de ravaler les étocs, on peut avec avantage préserver la section en la recouvrant de quelques centimètres de terreau mélangé de broussailles.

Inversement, en Sologne (1), on a l'habitude de couper le chêne un peu plus haut que la règle dans les terrains où l'eau séjourne lors des grandes pluies; - dans les cantons oú la sécheresse et le froid sont à craindre; - enfin sur les sols peu profonds. En effet, en exploitant ainsi, on ne coupe que le ou les rejets, sans entamer complètement la souche; la surface de section est donc bien moins considérable, et, par suite, les effets de la sécheresse ou des gelées sont moins redoutables. D'autre part, l'abatage ne nécessite pas des coups de hache aussi nombreux, aussi violents, ce qui peut avoir de l'importance dans des sols légers et peu profonds où l'ébranlement des racines est à craindre.

De même, sur le bord des rivières, il est permis de couper à 1 ou 2 centimètres au-dessus du sol, pour que les souches ne se trouvent pas complètement enfoncées sous les dépôts possibles de limon.

(1) Observations de M. l'inspecteur Croizette-Desnoyers. 


\title{
CHAPITRE II \\ LES ESSENCES
}

\author{
ARTICLE PREMIER
}

GÉNÉRALITES

Définitions. - Tempérament. - Influence de la lumière. - Influence de l'humidité atmosphérique. - Influence de la température et influences diverses. - Aire forestière. - Influence des qualités chimiques et physiques du sol - Influence de la fertilité. - Essences sociales et disséminées. - Dissémination. - Longévité.

Définitions. - Dans le langage forestier, le mot essence est synonyme d'espéce.

Les essences qui peuplent les forêts peuvent être rapportées à deux groupes, suivant leurs dimensions: l'arbre et l'arbrisseau.

M. Mathieu (1) a défini chacun de ces deux types comme il suit :

Arbre, végétal ligneux, à tige simple et unie, et s'élevant à 7 mètres au moins. - Arbrisseau, végétal ligneux, rameux dès la base, dont la hauteur totale va de 1 à 7 mètres.

Le chêne, le hêtre, le frêne, l'orme, le sapin, le mélèze sont des arbres. Le houx, le noisetier, le fusain, etc., sont des arbrisseaux. Parmi ces derniers, on distingue, sous le nom de sous-arbrisseaux, ceux qui restent dans les plus petites dimensions et atteignent rarement 1 mètre de hauteur, comme la bruyère, le genêt, l'airelle, etc.

On confond sous le nom de morts bois tous les arbrisseaux. Ils sont sans valeur marchande, à moins qu'ils ne puissent servir à quelques usages spéciaux, tels : la bourdaine, dont le charbon est recherché pour la fabrication des

(1) Mathieu, Flore forestière. 
poudres noires, le cornouiller mâle, dont on fait des manches d'outils, les épines, utilisées comme cannes, manches de parapluie, etc.

La dénomination de bois blancs, impropre, mais consacrée par l'usage, indique, parmi les essences feuillues, les bois d'une contexture molle, quelle qu'en soit d'ailleurs la couleur (aunes, tilleuls, peupliers, saules....) Nous emploierons à son lieu et place l'expression de bois tendres ou bois moux (1).

Par opposition á cetle désignation des bois blancs ou bois tendres, on comprend sous le nom de bois durs les autres feuillus (chêne, charme, hêtre, etc....)

Les résineux ou conifères, dont le bois a des qualités spéciales, ne rentrent pas dans ces catégories.

Tempérament. - Les grandes espèces ligneuses se comportent différemment en présence des agents naturels de la production. Les unes affirment des exigences spéciales; les autres marquent de simples préférences; d'autres enfin, et ce ne sont pas les moins utiles, s'accommodent des conditions mauvaises qui seraient fatales à la majorité. Si, en même temps que de ces aptitudes diverses, on tient compte de la longévité, on obtiendra toutes les données qui, réunies, constituent le tempérament des essences.

Influence de la lumière. - Dans leur entier développement, toutes les espèces recherchent la lumière; mais, dans leur jeune âge, quelques-unes demandent le plein découvert, quand les autres ont besoin d'un abri. Entre ces deux extrêmes, la faculté de supporter l'ombrage se trouve développée à des degrés variables. Parmi les premières on peut citer : le pin sylvestre, le mélèze, le chêne, l'orme, le bouleau, le tremble; ... parmi les secondes, le hêtre et le sapin se rangent en première ligne; le charme et l'épicéa ont des allures intermédiaires.

De cette aptitude toute spéciale qui dispose le jeune plant à craindre ou à supporter les effets de l'ombrage, découlent des conséquences permanentes pendant toute l'existence de l'arbre. Il ne saurait d'ailleurs en être autrement, car cette organisation, et par suite ces exigences du bourgeon extrême

(1) Autrefois le " mol bois ". 
de la jeune tige, se retrouveront plus tard dans tous les bourgeons de l'arbre constitué, qu'ils terminent la tige ou les rameaux. Aussi les espèces dont les bourgeons se développent à l'ombre ont-elles un feuillage abondant, dont tous les éléments fonctionnent avec activité. Elles peuvent se maintenir longtemps vivantes sous un massif d'autres arbres plus élevés et attendre patiemment qu'une trouée faite dans l'étage supérieur leur permette de reprendre essor. Toutefois l'épanouissement simultané des bourgeons et, par suite, le nombre très restreint de ceux qui restent à l'état dormant, est, en diverses circonstances, une cause d'infériorité pour ces espèces feuillues ; après une gelée printanière ou une invasion d'insectes, par exemple, elles sont dans l'impossibilité de se reconstituer une frondaison suffisante. De même, si on les mutile ou les recèpe, elles émettent peu de rejets. C'est le cas du hêtre.

Inversement, chez les essences qui ont besoin de la pleine lumière pour ouvrir leurs bourgeons, bon nombre de ceux-ci restent à l'état latent, même sur les ramifications les plus élevées de l'arbre ; dans tous les cas, les branches inférieures ne donnent que des feuilles rares et chlorotiques. Dominées, ces espèces s'étiolent et meurent bientôt : par contre, celles qui appartiennent à la classe des feuillus, comme le chêne, sont toujours prêtes, le cas échéant, à émettre des rejets ou à réparer les accidents dont nous parlions quelques lignes plus haut.

On voit toute l'importance que prend en sylviculture la manière dont le jeune plant se comporte à l'égard de la lumière. Il n'est donc pas surprenant que les auteurs forestiers soient partis de cette donnée, à l'exclusion de toutes les autres, pour caractériser le tempérament d'une essence. C'est ainsi qu'en Allemagne on les distingue en essences d'ombre et essences de lumière. En France, on appelle souvent délicates les essences qui ont besoin d'un abri dans leur jeunesse et robustes celles qui demandent la pleine lumière dès leur naissance. Les expressions allemandes sont peut-être préférables, en ce sens qu'elles ne préjugent en rien du plus ou moins de résistance d'une espèce à l'égard des influences mauvaises. 
Toutefois cette division ne reste absolument vraie que dans les régions moyennes de l'Europe, et, même dans ces limites, l'intensité plus ou moins grande de la lumière atténue ou exagère les aptitudes qui caractérisent chacun des deux types.

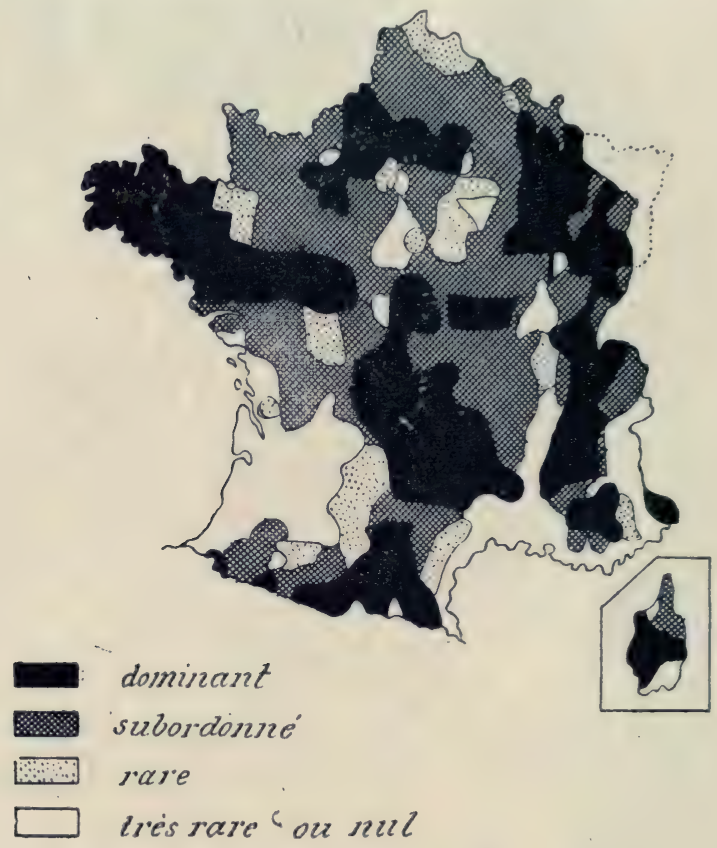

Fig. 17. - Distribution du Hêtre (1).

En effet, les jeunes plants d'essence de lumière supportent mieux le couvert dans les régions chaudes et bien ensoleillées que dans les stations froides et brumeuses; dans les premières, certains arbres dits à couvert léger ont une frondaison plus épaisse que ne le comporterait cette qualification et les

(1) L'Atlas de Statistique forestière de 1878, auquel nous empruntons les cartes ci-jointes, donne la distribution des essences par cantonnement. Les limites des zones sont donc bien souvent des limites administratives, qu'il ne faut point considérer comme rigoureusement exactes : tout un cantonnement peut être ombré d'une certaine façon, alors que l'essence en question fait défaut sur certains points. 
espèces douées de la faculté de repousser de souche y fournissent des rejets plus abondants. C'est ainsi que les jeunes plants de chêne, que l'on considère comme très robustes dans le Nord de la France, où ils ne résistent pas plus de trois ou

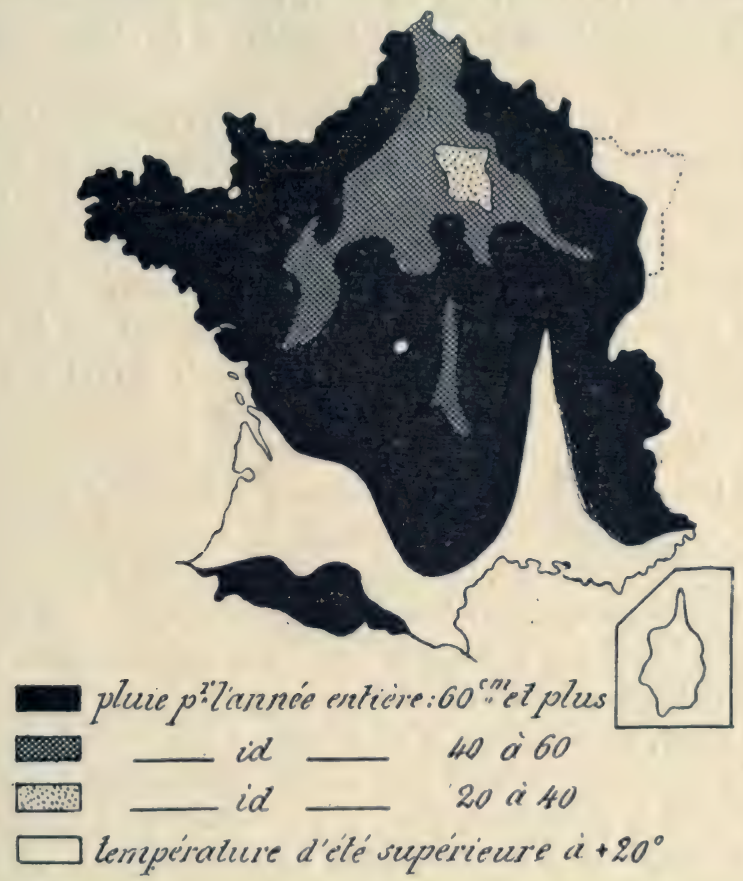

Fig. 18. - Carte météorologique, d'après la France météorologique, par E. Levasseur. Paris, Ch. Delagrave.

quatre ans à l'action du couvert, se maintiennent, dans les forêts du bassin de l'Adour, en fourrés vigoureux sous des massifs complets et bien feuillés. C'est ainsi encore qu'en Provence, en Italie et en Espagne, on laisse grimper la vigne contre les arbres fruitiers, et les raisins mûrissent dans leurs cimes malgré l'ombre du feuillage.

Influence de l'humidité atmosphérique. - Les essences à frondaison abondante, comme le hêtre et le sapin, ont une transpiration très active. Il en résulte que, sous un climat sec 
leurs racines sont parfois impuissantes à rétablir l'équilibre et l'arbre est exposé à périr de soif. L'humidité de l'atmosphère leur semble pourtant plus indispensable que celle du sol ; ainsi les hêtres et les sapins acceptent un terrain relativement sec, si le climat est humide, tandis qu'ils dépérissent, bien que plantés dans un sol humide, si le climat est trop sec.

En France, le hêtre est rare et fait même totalement défaut dans les stations dont l'atmosphère est desséchée en été par une température moyenne supérieure à $+20^{\circ}$. Partout ailleurs il existe en quantité variable, excepté cependant aux environs de Fontainebleau, sur certains cantons disjoints où la hauteur d'eau fournie par les pluies annuelles n'atteint pas $\mathbf{4 0}$ centimètres. En fait, pour qu'il soit dominant, il faut que cette quantité atteigne et dépasse 60 centimètres.

Le hêtre existe donc, en plaine et en montagne, partout où il trouve le minimum nécessaire d'humidité atmosphérique, qu'il le doive au voisinage de la mer, à la latitude, ou à l'altitude. D'ailleurs, bien souvent, on le voit plus abondant dans l'intérieur des grands massifs forestiers, à cause de l'humidité relative que produit la forêt, que sur les lisières exposées aux vents secs soufflant des plaines voisines.

Le sapin, au contraire, n'est spontané que dans les régions à relief montagneux, ou, à tout le moins, accidenté. Toutes nos montagnes le possèdent, c'est là seulement qu'il trouve, en même temps que la fraîcheur, sans doute aussi certaines autres conditions dont nous ne nous rendons pas très bien compte.

Il ne faudrait pas toutefois spécialiser aux essences d'ombre ce besoin d'humidité atmosphérique; car le mélèze, essence de lumière, redoute encore plus la sécheresse de l'air que le hêtre et le sapin.

D'une manière générale, d'ailleurs, on peut dire que la vapeur d'eau, quand elle ne se présente pas sous la forme de brouillards trop fréquents qui interceptent la chaleur et la lumière, est toujours favorable à la végétation forestière. Néanmoins certaines espèces sont très exigeantes à cet égard, d'autres beaucoup moins; il en est même, comme le pin 
sylvestre, et surtout le pin d'Alep qui font preuve d'une extrême résistance à la sécheresse.

Influence de la température et influences diverses. Le plus ou moins de sensibilité aux excès de température, aussi bien en dessus qu'en dessous du 0 centigrade, est encore

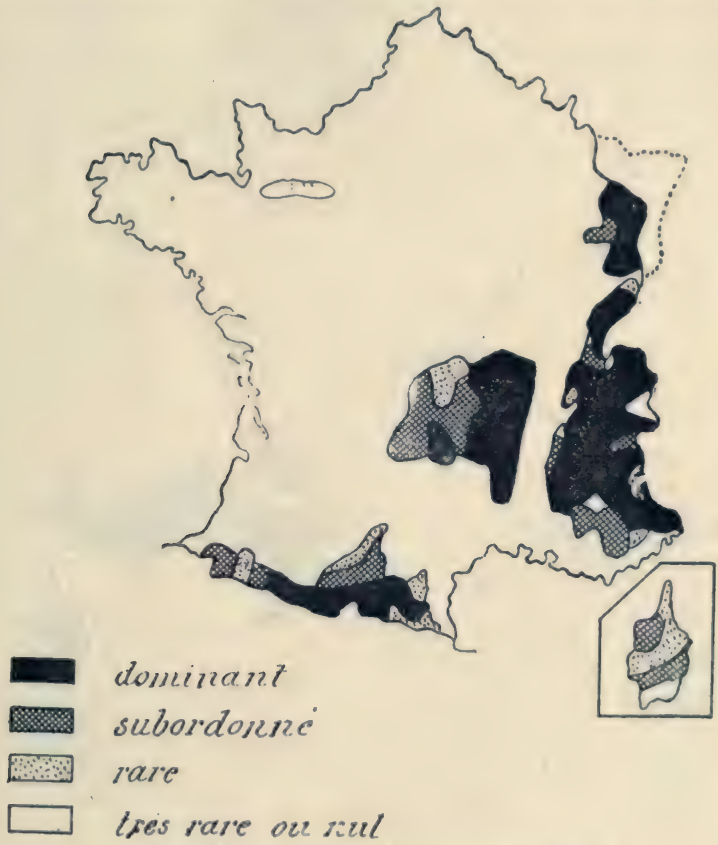

Fig. 19. - Distribution du Sapin.

une des principales causes de la distribution géographique des espèces.

Pour chacune, on constate une limite méridionale de son aire d'habitation fixée par un degré de chaleur qu'elle ne peut plus supporter, et une limite septentrionale où un froid trop intense met obstacle à son expansion. Pourtant, il faut bien reconnaître, en ce qui concerne la limite méridionale, que si la chaleur agit directement, elle intervient surtout, et d'une façon préjudiciable, par les sécheresses exagérées qui en sont le corollaire habituel. 
Les espèces indigènes peuvent être classées comme il suit, eu égard à la manière dont elles se comportent en présence du froid.

$1^{\circ}$ Le pin d'Alep, le chêne liège, le chêne occidental, périssent quand le thermomètre descend au-dessous de

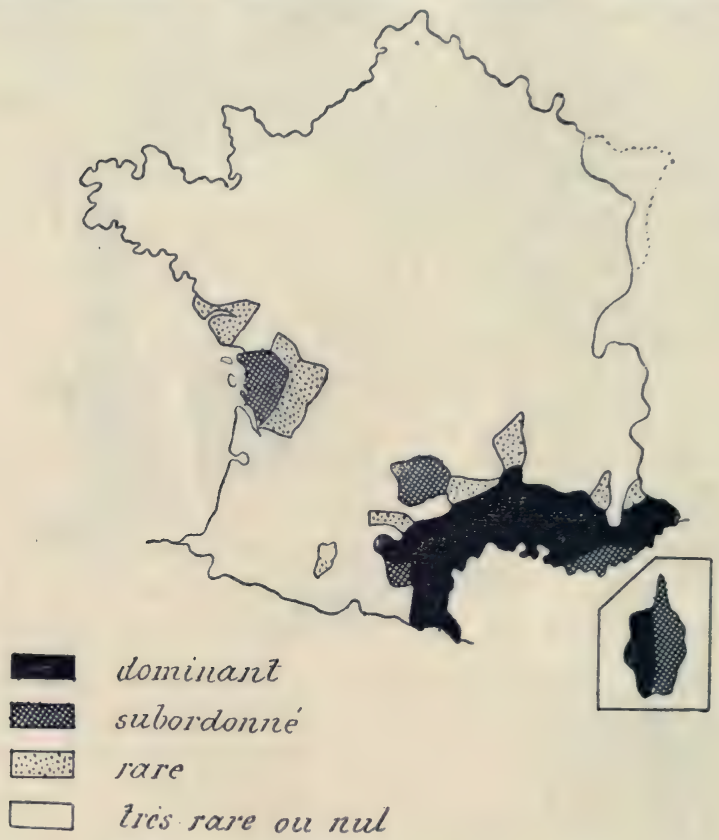

Fig. 20. - Distribution du Chêne vert.

$-6^{\circ}$, ou du moins quand il se maintient quelque temps à ces basses températures.

$2^{\circ}$ Un froid prolongé de $-20^{\circ}$ tue le chêne yeuse et le pin maritime.

Ces deux essences, malgré leurs allures franchement méridionales, peuvent remonter assez haut vers le Nord sur les côtes de l'Océan, où le voisinage de la mer atténue les effets de la latitude. Au contraire, les espèces du premier groupe restent confinées dans les parties chaudes de la Provence (1).

(1) Exception doit être faite pour le chêne occidental qui habite le 
Cette même température de $-20^{\circ}$ endommage ou tue les tiges du chêne tauzin et du châtaignier, mais les souches sont rarement atteintes et leur régénération par rejets demeure le plus souvent assurée.

$3^{\circ}$ Si le thermomètre descend à $-30^{\circ}$, on voit disparaître

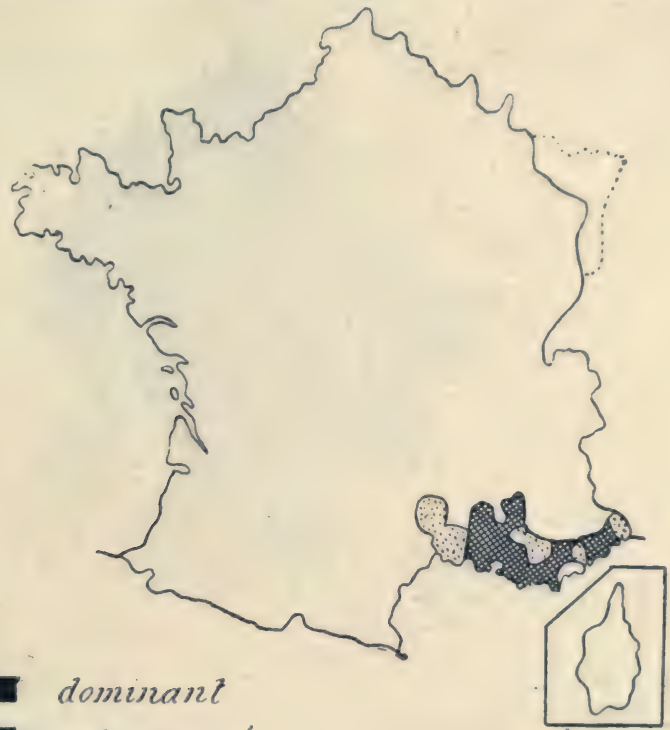

subordoridé

rine

$\square$ lies rave ou nul

Fig. 21. - Distribution du Pin d'Alep.

un certain nombre de tiges de chêne rouvre, de chêne pédonculé, d'érable champêtre, d'orme, de charme, de hêtre et de sapin.

$4^{\circ}$ Enfin le pin de montagne, le pin sylvestre, le mélèze, l'épicéa, le pin cembro, parmi les résineux; les grands érables, le bouleau, le sorbier des oiseleurs, etc..., parmi les feuillus, semblent pouvoir supporter les froids les plus vifs dans bassin de la Garonne, mais est un arbre de verger, plutôt qu'une essence forestière. 
les stations les plus extrêmes, sous le climat de la France.

On ne saurait donner trop d'attention - surtout quand on constitue artificiellement de nouvelles forêts - à la résistance au froid des différentes espèces. Dans notra pays, si on excepte les régions montagneuses où les saisons offrent une certaine

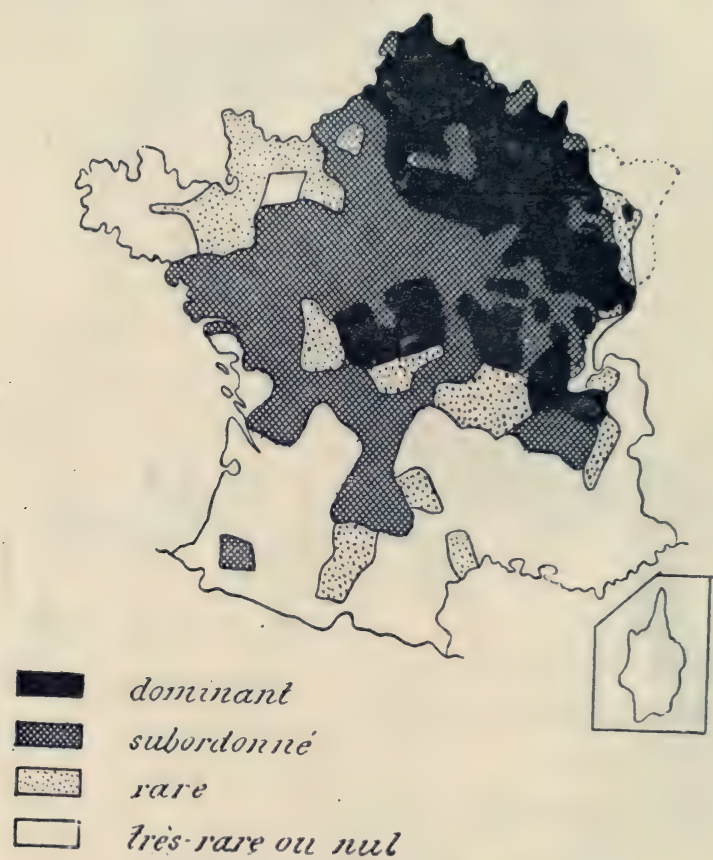

Fig. 22. - Distribution du Charme.

régularité, on est exposé à supporter de temps à autre un hiver exceptionnel par sa rigueur et qui suffit à anéantir des plantations déjà anciennes. La dure expérience faite en 18791880 a permis de classer les essences indigènes et exotiques suivant le degré de résistance dont elles ont fait preuve (1).

(1) Charles Baltet, De l'action du froid sur les végétaux pendant l’hiver 1879-80. Paris, impr. J. Tremblay 1882. Extrait des mémoires de la Société nationale d'agriculture de France, t. CXXVII. 
Nous en dirons quelques mots au chapitre des repeuplements artificiels.

En dehors de l'action des gelées d'hiver, le chêne, le hêtre, le sapin et aussi l'épicéa, sont souvent atteints par les gelées printanières. Dans certaines stations (vallons particulièrement

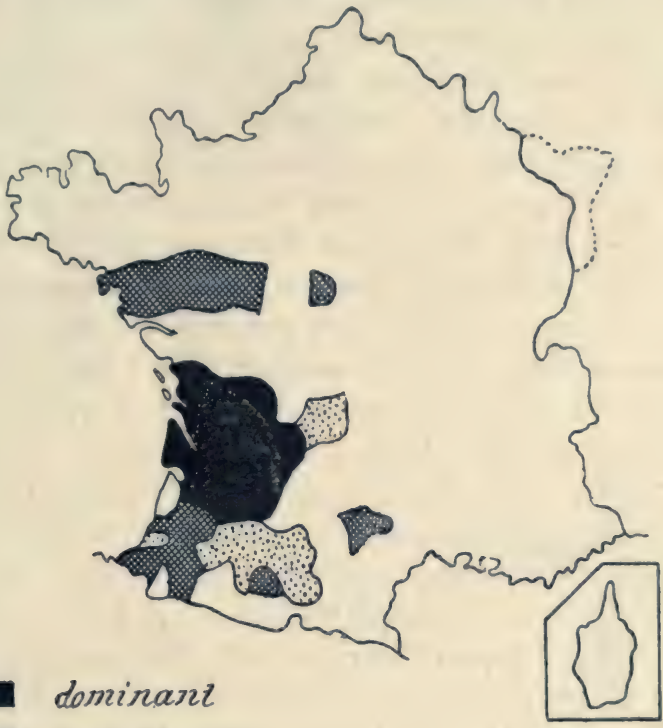

Dominant

subordonzé

rare

$\square$ Zres rare ou nul

Fig. 23. - Distribution du Chêne tauzin.

froids, voisinage des étangs, etc...) les dégats causés par cet accident météorique, se répètent assez fréquemment pour rendre impossible la culture de ces espèces. Au contraire, les jeunes pousses du pin sylvestre et du charme supportent sans danger un froid persistant de plusieurs degrés.

Enfin certaines essences ont une aire d'habitation restreinte sans que les causes qui en fixent les limites soient bien apparentes. Ainsi, l'ostrya à feuilles de charme est localisé dans le Sud-Est, le chêne tauzin dans le Sud-Ouest, le 
charme est un arbre du Nord-Est ; Pourquoi?... Nous citons le fait sans le discuter.

Aire forestière. - Il y a lieu de distinguer l'aire botanique d'une espèce, de son aire forestière. L'aire botanique, toujours la plus étendue, comprend toutes les régions où cette espèce peut vivre et se reproduire. Or, dans le voisinage des limites de cette aire, soit en latitude, soit en altitude, non seulement le végétal considéré peut devenir plus rare, mais souvent encore il se modifie. Tous les observateurs connaissent les changements, parfois très sensibles, qui affectent les caractères de la plante quand le climat se refroidit ou se réchauffe à l'excès : sa taille diminue, sa floraison est moins abondante, ses fruits demeurent stériles, ses feuilles se rapetissent comme cela arrive chez le hêtre aux grandes altitudes et dans les stations sèches; dans le midi, le chêne rouvre garnit de poils ses feuilles et ses rameaux, de là son aspect tomenteux et son nom de chêne blanc.....

Or, si certaines de ces modifications ne sont intéressantes que pour le botaniste, d'autres peuvent avoir une importance forestière considérable. A quoi bon cultiver telle ou telle essence, si, sous un climat trop froid, elle devient chétive et buissonnante? A quoi bon remplacer des essences s'accommodant d'hivers rigoureux par d'autres qui ont théoriquement plus de valeur, si, sous la latitude du lieu, elles ne doivent donner qu'un bois industriellement déclassé par les tares de toutes sortes qui dégradent les arbres mal venants?

Nous appellerons donc aire forestière d'une essence, l'étendue des régions oủ elle peut, non seulement vivre et se reproduire, mais encore donner un bois d'œuvre sain et capable de jouer un rôle économique utile.

\section{Influence des qualités chimiques et physiques du sol. -} En général, les essences forestières se montrent assez indifférentes quant aux qualités chimiques du sol. Néanmoins il résulte d'analyses faites par MM. Fliche et Grandeau (1) que certaines d'entre elles, sans indiquer une préférence pour les autres éléments minéralogiques, manifestent une évidente

(1) Annales de la station agronomique de l'Est. Quatre mémoires de recherches chimiques et physiologiques sur la végétation forestière. 
répulsion pour la chaux. Ainsi le pin maritime refuse de se développer dans les terrains qui renferment plus de 4 p. 100 de calcaire (1). La carte ci-jointe fait voir que non seulement ce pin recherche les climats méridionaux, mais qu'il évite les calcaires de la Provence, et reste cantonné sur les sables de

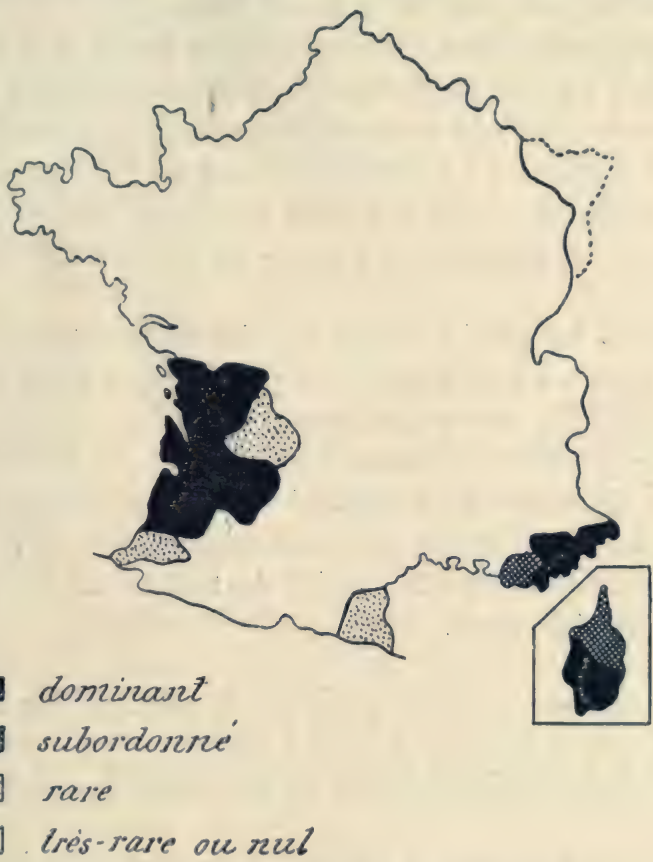

Fig. 24. - Distribution du Pin maritime.

l'Océan ou sur les terrains non calcaires des Maures et de l'Esterel. On nomme ces essences calciluges. Beaucoup d'arbustes et de sous-arbrisseaux sont dans le même cas : la callune (vulgairement bruyère), l'airelle myrtille, la fougère impériale, etc...

(1) On s'exposerait à de grosses erreurs en se basant sur la nature de la roche sous-jacente pour qualifier un sol de calcaire ou de siliceux. Par lixiviation ou par apport de matériaux, un terrain reposant sur des dalles calcaires ne contient souvent que des traces infinitésimales de chaux. Une analyse rapide au calcimétre s'impose. 
D'autres espèces affectionnent aussi les sols siliceux, mais sans qu'on puisse affirmer que cette préférence soit due à l'absence de chaux, plutôt qu'aux qualités physiques propres aux terres de cette nature. C'est le cas du pin sylvestre, qui se plaît dans les terrains divisés et notamment dans les sols graveleux provenant de roches dolomitiques.

Quelques essences, comme le chêne yeuse et le pin d'Alep, affectent, au contraire, des allures calcicoles. Mais ici encore, c'est une propriété accessoire des sols calcaires, leur plus grande aptitude à l'échauffement, qui les leur fait rechercher. Ainsi le chêne yeuse, qui ne se rencontre en France que sur les calcaires, est beaucoup moins exclusif en Corse et en Algérie (1).

L'aune, l'orme, le frêne, le pin de montagne, peuvent vivre dans les sols aquatiques pourvu que l'eau ne soit pas stagnante; les terres simplement humides conviennent à ces mêmes espèces et au chêne pédonculé ; le pin maritime, le pin sylvestre, le pin noir d'Autriche, le pin d'Alep comptent parmi les essences qui s'accommodent le mieux des terrains secs (2); le bouleau a la faculté de tolérer les excès d'humidité et de sécheresse.

La consommation plus ou moins grande d'eau que font les végétaux ligneux, comme toutes les plantes à chlorophylle, dépend essentiellement de la transpiration. Plus les feuilles perdent d'eau par la transpiration, plus elles en extraient du sol si elles fonctionnent normalement...

Les résineux forment un contraste bien net avec les feuillus : leurs feuilles aciculaires, étroites et raides, couvertes d'un épiderme épais, fortement cuticularisé et imprégné de résine, provoquent une telle diminution dans la transpiration qu'elles évaporent, d'après les recherches de von Hœnel, 6 à 10 fois moins d'eau que celles des feuillus; en conséquence, les résineux ont des exigences moindres en eau et en

(1) P. Fliche in A. Mathieu, Flore Forestière, quatrième édition, Paris, J.-B. Baillière et fils, 1897.

(2) Certains sols sont évidemment humides, d'autres évidemment secs. Pourtant, il ne faut pas se fier à une simple apparence : la partie superficielle de certaines argiles, de certaines marnes prend un aspect de sécheresse extrême, après quelques jours de soleil, alors que les couches profondes tiennent à la disposition des racines plus d'eau qu'on se l'imagine. M. le professeur Fliche explique de cette façon la réussite de l'aune dans les craies de la Champagne. 
principes nutritifs que les arbres feuillus croissant avec eux sur le même sol (1).

Le frêne, le chêne n'atteignent de belles dimensions qu'en terrain profond; le hêtre, l'épicéa et quelques autres essences à enracinement traçant acceptent volontiers des sols superficiels. Certaines espèces sont plus accommodantes que d'autres, mais il reste acquis, en principe, que la végétation forestière sera toujours plus belle sur les sols frais et profonds que sur ceux qui présentent les qualités inverses.

Influence de la fertilité. - De même, tous les arbres, comme les hommes et les animaux, préfèrent une nourriture abondante à un régime trop maigre; et, si l'on voit des essences se développer convenablement dans les sols les plus pauvres, c'est que, douées d'un tempérament frugal, elles résistent dans un milieu où d'autres mourraient d'inanition.

A la suite d'analyses qu'il a fait porter sur des arbres de la forêt de Haye, en terrain calcaire jurassique, M. le Professeur Henry classe nos principales espèces de la manière suivante, en commençant par les plus exigeantes $(2): 1^{\circ}$ frêne et érable ; $2^{\circ}$ tremble; $3^{\circ}$ chêne, orme de montagne et alisier; $4^{\circ}$ hêtre et charme.

Le frêne enlève au sol deux fois plus de potasse et d'acide phosphorique que le hêtre.

Dans le même ordre d'idées, M. le Professeur Ebermayer (3) distingue :

$1^{\circ}$ Les feuillus, à grande consommation, demandant plus que tous les autres, de la potasse et de l'acide phosphorique; ils ne prospèrent que dans le lehm, les marnes et les calcaires argileux. Citons par ordre d'exigence: le frêne, le peuplier pyramidal, l'orme champêtre, les tilleuls, le sorbier des oiseleurs, le robinier, le marronnier d'Inde et les érables plane et sycomore;

$2^{\circ}$ Les feuillus à consommation moyenne : tremble, saules

(1) Ebermayer, La nutrition minérale des arbres des Foréts. (Traduction par E. Henry, Annales de la station agronomique de l'Est.)

(2) E. Henry, Etudes chimiques sur les principales essences de la forêt de Haye et sur leurs cendres. (Annales de la station agronomique de l'Est.)

(3) Ebermayer, loc. cit.

Boppe et Joryet. 
et chênes, peuplier blanc, charme, hêtre, érable champêtre;

$3^{\circ}$ Les feuillus à faible consommation : bouleau, aune glutineux et aune blanc;

$4^{\circ}$ Les résineux : le moins frugal est le sapin, qui exige sensiblement plus de potasse et d'acide phosphorique que les suivants; puis viennent le pin. cembro, le mélèze, l'épicéa (ce dernier demande une notable quantité de chaux, autant que le hêtre), le pin Weymouth, enfin le pin sylvestre et, pour terminer la série, le pin noir et le pin de montagne. Après cette dernière espèce, l'auteur ne range que la callune et un lichen.

Il faut toutefois remarquer, avec M. Ebermayer, qu'une espèce peut avoir des cendres très riches en certaines substances, sans exiger un sol qui les renferme en quantité notable; elle dispose quelquefois, en effet, de moyens spéciaux qui lui permettent de ... gagner sa vie. Ainsi le frêne " aime à prendre une partie de sa nourriture à l'état de dissolution dans les eaux de ruissellement ou d'infiltration; dans ces cas, il se contente de sols assez pauvres ". L'aune, les saules agissent de même. Le robinier, en sa qualité de légumineuse, vit en symbiose avec un bacille, grâce auquel il transforme directement l'azote de l'air en matière albuminoïde : malgré ses exigences, il doit être rangé parmi les espèces améliorantes. Les protubérances des racines de l'orme glutineux renferment un schizomycète, qui joue, sans doute, le rôle du bacille chez le robinier. Le hêtre s'accommode de sols très superficiels, une couche de terreau lui suffit : en effet ses radicelles sont entourées de filaments mycéliens, ou mycorhizes, " qui apportent l'eau et les matières nutritives et par l'intermédiaire desquelles les arbres se nourrissent bien mieux dans les sols humiques qu'ils ne le feraient à l'aide de poils radicaux dans les sols minéraux (Franck). " L'épicéa est dans le même cas.

Quoi qu'il en soit, l'extrême frugalité des résineux, jointe à leur moindre besoin d'eau, fait que ces essences sont toutes désignées pour rétablir l'état boisé dans les forêts épuisées et pour constituer, par voie artificielle, un premier peuplement dans les friches et les landes stériles. Cette observation s'applique également au bouleau.

Essences sociales et essences disséminées. - Dans 
le travail cité plus haut, le $D^{\text {r }}$ Ebermayer fait une intéressante comparaison entre les végétaux forestiers et les plantes agricoles. Seuls, dit-il, les feuillus du premier groupe, le robinier excepté, exigent des sols assez bons pour la culture des prairies et des céréales. Les autres se contentent tous de terrains que l'agriculture ne pourrait utiliser à moins de fortes fumures.

Or, dans un pays de vieille civilisation comme le nôtre, les bonnes terres à blé sont généralement employées.... à donner du blé ; il est rare que le sylviculteur en dispose. Le cas peut se présenter, cependant, motivé par des raisons spéciales : déclivité du terrain, fréquence des inondations, etc. Alors des terres excellentes reviennent à la culture forestière; nous en avons vu, en Bavière, sur des terrains basaltiques dans la région de Rhön; nous en connaissons également sur les bords de la Saône, de l'Oignon, de l'Adour; il doit s'en rencontrer le long de la plupart de nos grands cours d'eau.

Mais, ailleurs, c'est-à-dire dans la majorité des cas, le sol des forêts est trop pauvre pour nourrir un grand nombre de végétaux exigeants. De même que les animaux de proie vivant de chair, c'est-à-dire d'une nourriture de luxe, sont moins nombreux que le peuple des granivores ou des rongeurs, de même les gros mangeurs de potasse et d'acide phosphorique doivent rester clairsemés. Les nombreuses familles sont l'apanage des petites gens, a dit quelque part Toussenel.

Ceci nous amène à classer nos arbres forestiers en deux grands groupes: les essences sociales et les essences disséminées.

Les premières, grâce à leur frugalité et à une certaine flexibilité de tempérament, semblent se plier au plus grand nombre des circonstances locales; aussi constituent-elles la partie principale, souvent même la totalité des peuplements. Le chêne, le hêtre, le sapin, l'épicéa, les pins, sont des essences sociales.

Les essences disséminées, au contraire, affectent de vivre, soit par pieds isolés, soit par bouquets épars. Cette répulsion apparente pour leurs semblables résulte d'exigences trop spéciales de leur part au point de vue de la fertilité du sol. 
Réunies en grand nombre sur un même point, à moins qu'il s'agisse de l'un de ces terrains d'une fertilité exceptionnelle que nous citions plus haut, elles s'affament entre elles, et l'individu le mieux doué résiste seul. Les frênes, les ormes, les érables, les fruitiers, les bois blancs, font partie de ce groupe, qui ne contient que des espèces feuillues.

La variété de la flore forestière sur un même point est donc un indice certain de la fertilité du sol, et aussi de la douceur du climat. Car, plus les circonstances sont mauvaises, plus est restreint le nombre des espèces douées d'une force de résistance suffisante pour s'y maintenir. C'est ainsi que les essences disséminées sont toutes, ou à peu près toutes, cantonnées dans les régions de plaine. En montagne, au contraire, on rencontre surtout des espèces sociales; parfois même, dans les stations les plus rudes, une seule forme occupe toute la forêt.

Dissémination. - La nature semble d'ailleurs avoir pris soin de régler la répartition des essences sociales et des essences disséminées par la manière dont elle assure leur multiplication.

Toutes les espèces à semences lourdes sont sociales par la force des choses et aussi par nécessité : d'une part, en effet, ces semences tombent au pied de l'arbre qui les porte, ce qui dispose les nouveaux individus par bouquets et même par masses considérables; d'autre part, elles ont besoin, pour se protéger contre le froid et la dessiccation, d'unecouverture de feuilles mortes qui ne se rencontre que dans les massifs forestiers ; tombant en automne, avant la chute des feuilles, qui les recouvrent quelques jours plus tard, elles sont, en outre, recherchées par les rongeurs (1), par les sangliers, et ces animaux en enfouissent autant qu'ils en mangent.

(1) Bien que sociales, ces essences ne sont pas dépourvues de tout moyen de dissémination au loin. Ce sont les petits rongeurs, en accumulant des provisions d'hiver exagérées, les geais, en transportant des glands ou des faines qu'ils oublient ou laissent tomber, qui se chargent de cette mission. Les chênes nés de glands réunis par les rongeurs naissent par paquets; ceux qui proviennent des semences jetées çà et.là par les oiseaux germent isolés. 
Toutes les espèces disséminées ont des semences légères, souvent ailées, ou munies d'aigrettes rendant plus facile leur transport par le vent, qui les disperse au loin (1). D'ailleurs elles sont, non plus charnues, mais sèches, et supportent sans en souffrir de fortes gelées. Elles germent, enfin, sur un sol nu, pourvu qu'elles y rencontent la faible quantité d'humidité qui leur est nécessaire. Une épaisse couverture morte est, pour beaucoup d'entre elles, plus nuisible qu'utile à leur installation; car, en raison de l'extrême ténuité de leurs organes naissants, elles recherchent, avant tout, une surface meuble (2) et riche en terreau; aussi, pour en faciliter l'installation, la nature fait-elle tomber leurs semences pendant l'hiver, après la chute des feuilles, quand celles-ci sont déjà tassées et en voie de décomposition. Ajoutons qu'elles sont presque toutes d'une exubérante fécondité et que leurs jeunes semis, dont la croissance est des plus rapides, luttent avantageusement contre la végétation herbacée au milieu de laquelle ils sont jetés.

Tous les résineux ont des semences ailées, dont la dissémination se fait au loin sous l'action du vent, comme chez les espèces précédentes. Et, pourlant, ce sont des essences sociales. Mais, en général cantonnées dans les régions montagneuses et dans les mauvais sols de la plaine, elles ont peu à redouter la compétition d'autres espèces. Elles donnent d'ailleurs des graines en quantité suffisante pour assurer, tout à la fois, la régénération des massifs qu elles constituent, et leur installation dans les pâturages avoisinants, qu'elles auraient bien vite envahis, si l'homme et les troupeaux n'y mettaient obstacle (3). Enfin, les résineux sont tous plus ou moins des

(1) Les fruiliers ont des semences lourdes et charnues; mais les graines sont transporlées de côtés et d'autres par les oiseaux et se retrouvent dans leurs déjections : le résultat est le même.

(2) Aussi, exception faite des forêts en terrain sablonneux, où le peuplement n'est pas très dense, où le sol n'est jamais compact, les semis des saules et des bouleaux n'apparaissent-ils nombreux que dans les clairières et principalement sur les anciennes places à charbon, dont l'aire est couverte de fraisil. Ils sont plus que rares dans le massif lui-mème.

(3) C'est ainsi que la dispersion de leurs graines légères fait remonter certaines espèces au delà des limites de l'aire où elles mûrissent leurs 
essences de lumière, leurs jeunes plants naissent dans des clairières, dans des vides, plutôt que sous la projection immédiate des vieux arbres. Le sapin seul fait exception, par le poids de sa graine plus grosse que celle des épicéas et de la plupart des pins de nos forêts, par la désarticulation de son cône, dont toutes les graines tombent en bloc sur le sol; cette essence d'ombre par excellence n'est pas sans présenter quelques analogies avec les espèces feuillues sociales, le hêtre notamment.

En résumé, si l'on considère l'énorme production des semences forestières, les procédés multiples mis en œuvre pour assurer leur dispersion et leur adaptation aux milieux propices, il semble que la nature ait voulu rétablir, au profit de l'espèce végétale, la faculté de locomotion qu'elle a refusée à l'individu. Un arbre périra, plusieurs fois séculaire, sur le point même où le hasard a fixé la graine dont il est sorti; mais, longtemps avant sa mort, sa descendance, qui constitue le massif, se meut sans cesse dans toutes les directions. Cette progression continue, pour être plus lente, n'est pas moins assurée que celle des animaux doués de mouvement, et bientôt la forêt aurait reconquis son ancien domaine, si, à tout instant, l'homme ne venait pas l'arrêter dans sa marche envahissante.

Longévité. - Dans la pratique forestière, par longévité, il faut entendre non pas la durée absolue de la vie des arbres, mais le temps pendant lequel leur bois reste généralement sain. D'ailleurs chaque espèce est douée d'une vitalité qui lui est propre, mais dont un concours de circonstances plus ou moins favorables peut avancer ou reculer le terme dans des limites très étendues.

Les conifères et toutes les essences de bois dur, peut-être le charme excepté, sont susceptibles de s'accroître sans dépérir pendant cent cinquante ans et même davantage. Certaines

fruits. On remarque, en effet, que, dans la haute montagne, vers les confins supérieurs de leur habitat, les forêts résineuses se prolongent et s'égrènent en arbres épars, qui sont, en général, stériles. Ils proviennent des semences apportées des massifs inférieurs. Que ceux-ci soient imprudemment détruits, les arbres des pâtures, n'étant plus remplacés, disparaîtront après eux.-A. Mathey, Inspecteur-adjoint des Eaux et Forêts, le Pâturage en forêt. Besançon, imp. Paul Jacquin, 1900. 
espèces, notamment le chêne et le mélèze, se maintiennent en massif pendant trois cents ans et plus. En général, les bois tendres et les arbustes ont une durée beaucoup moindre.

Un climat favorable augmente la longévité; aussi les espèces naturalisées ou introduites vivent-elles moins longtemps que dans leur station d'origine; mais l'influence de la fertilité du sol est capitale : sur un terrain pauvre ou manquant de profondeur, les arbres tombent en décrépitude bien avant l'âge normal. On chercherait vainement à les maintenir sur pied au delà de ces limites; tous périraient sans profit.

Dans une même station, l'état de massif augmente ou diminue la longévité d'un sujet considéré isolément; tel arbre appartenant à une espèce plus longévive que ses voisins, sera fatalement entraîné par le dépérissement du massif avec lequel il fait corps : il partagera le sort commun quand, seul, ou associé à ses semblables, il aurait pu vivre plus longtemps; tel autre, au contraire, verra son existence prolongée par les conditions meilleures de protection et d'humidité que lui assure l'état de massif.

C'est, d'ailleurs, sous l'influence de l'énergie vitale propre à chaque individu que la sélection s'opère dans les forêts naturelles d'une manière plus parfaite que dans les milieux cultivés. En effet, la nature jette à profusion les germes des arbres forestiers; mais il n'en survit qu'un petit nombre, appartenant aux types les plus parfails, lesquels, en arrivant à l'âge de la fertilité, fourniront les germes les mieux préparés pour la propagation de l'espèce. Cet argument doit être invoqué en faveur de la régénération nalurelle; car, lorsqu'on élève les plants destinés à la régénération artificielle dans des pépinières où, par des soins constants, on en sauve le plus grand nombre, on constitue les peuplements avec une foule de tiges de vigueur au-dessous de la moyenne, et que la nature aurait éliminées. Il en est de même pour les semis de main d'homme, dans lesquels on est obligé d'épargner la graine par raison d'économie. Semis et plantation donneront des massifs moins résistants que ceux de la forêt naturelle, et, dans l'avenir, les races rront en s'affaiblissant. 


\section{MONOGRAPHIES DES PRINGIPALES ESSENGES (1)}

\section{Le chêne rouvre et le chêne pédonculé.}

Aire et Station. - Indifférents à la composition minérale du terrain, ces deux chênes se plaisent surtout dans les sols profonds, argileux sans trop de compacité. Bien que, pendant longtemps, on les ait considérés comme pouvant impunément se substituer l'un à l'autre, ils manifestent des préférences distinctes (2). Le pédonculé exige un sol humide ou, tout ou moins, frais; les terrains argilo-sablonneux, fussent-ils submergés en certaines saisons, lui sont très favorables, c'est, par excellence, l'espèce des grandes plaines et des vallées. Au contraire, les sols bas, argileux, humides conviennent peu au chêne rouvre; celui-ci préfère les terrains plus meubles, graveleux, sablonneux, calcaires, pourvu qu'une certaine quantité d'argile y maintienne la fraîcheur dont il ne peut se passer; les collines, les plateaux, les contreforts des montagnes, où il trouve plus habituellement toutes ces conditions réunies, forment sa station de prédilection. On ne saurait attacher trop d'importance, dans les travaux de repeuplement, à cette différence fondamentale, afin de placer chacune des deux formes dans la station qui lui est propre.

Sous ces réserves, le chêne pédonculé et le chêne rouvre, se rencontrent en France à peu près partout, sauf dans la haule montagne et dans les stations les plus chaudes du littoral de la Méditerranée. Le premier est très abondant dans les régions du sud-ouest où il peuple, presque à lui seul, les forêts feuillues des Landes et du bassin de l'Adour. C'est lui qu'on plante dans les haies de la Normandie, c'est lui qu'on retrouve dans

(1) Nous indiquerons sommairement la distribution géographique des essences et nous prierons le lecteur de se reporter pour plus de détails à la Flore forestière, par A. Mathieu, $4^{\mathrm{e}}$ édition, revue par M. Fliche, Paris, J.-B. Baillière et fils, 1897. C'est à cette flore que nous empruntons toutes les données botaniques.

(2) Dubois, Considérations culturales sur les futaies du chêne du Blésois, Blois, Lexesne, 1856. 
les alluvions de la Saône et de nos grandes vallées. Le second domine dans le centre de la France et dans les régions accidentées; il s'élève même jusqu'à 1000 mètres d'altitude et au delà. Ces chênes atteignent leur maximum d'expansion dans la zone tempérée, où ils caractérisent, par leur abondance, une région se confondant souvent avec celle de la vigne; au-dessus d'eux on trouve le sapin.

Tempérament. - Les deux espèces ont un jeune plant robuste; leur couvert est léger, souvent même incomplet pour le pédonculé. Ils résistent bien à la chaleur, assez bien aux grands froids de l'hiver, mais leur feuillage est très sensible aux gelées printanières; le pédonculé, qui entre en végétation plus tard que le rouvre, est moins exposé à ces accidents (1). L'un et l'autre repoussent parfaitement de souche et conservent cette faculté jusqu'à un âge très avancé.

Enracinement, fructification. - La racine de ces deux chênes est essentiellement pivotante. Le gland est un fruit lourd; les années de semence, ou glandées, sont surtout fréquentes dans la région du Sud-Ouest, où l'on peut récolter des fruits presque tous les ans; plus on s'avance vers le Nord, plus les glandées sont rares; dans la France septentrionalc, elles se font parfois attendre dix et même quinze ans.

Croissance, longévité. - La croissance de ces chênes. lente au début, devient bientôt assez active dans les bons sols ; elle se soutient jusqu'à un âge très avancé, car leur longévité est très considérable et dépasse plusicurs siècles. L'accroissement en hauteur des arbres en massif, comme des sujets isolés, s'arrête vers l'âge de cent ans.

Bois et usages. - Le bois de chêne doit être mis au premier rang pour l'ensemble des qualités qu'on y rencontre; mais, suivant la nature du sol et du climat, qui active ou ralentit la végétation, il est plus ou moins nerveux ou plus ou moins tendre. Le bois du pédonculé est, en général, plus

(1) Cette observation de M. l'Inspecteur Bartet dans la forêt de Haye (Observations phénologiques sur les chênes rouvre et pédonculé), (Ann. Sc. agronom. française et étrangère), suffirait à expliquer ce fait souvent constaté que les chênes pédonculés donnent des glands plus fréquemment que les chênes rouvres. 
dense que celui du rouvre, il convient mieux comme bois de construction. Le rouvre est plus estimé pour le travail et pour la fente; le pédonculé de Hongrie donne, néanmoins du merrain très apprécié.

Produits accessoires. - L'écorce de ces deux chênes est utilisée comme écorce à tan; celle du rouvre, qui croît sur les coteaux chauds et bien éclairés, est généralement plus riche et plus recherchée.

Allures forestières. - " Le grand mérite du chêne pour les propriétaires de bois, dit M. Broilliard (1), c'est qu'il abonde dans les forêts, qu'il croît dans presque tous les sols, qu'il prospère à l'état de massif ou d'arbre isolé, en futaie comme en taillis, et qu'il joint à la rusticité une longévité permettant à la plupart des sujets d'arriver à de grandes dimensions. "

Le chêne s'accommode très bien du traitement en taillis simple et fournit un chauffage estimé, surtout quand il provient de taillis écorcés; on le vend alors sous le nom de bois pelard.

Mais le principal mérite du chêne consiste dans ses qualités comme bois d'œuvre et dans les grandes dimensions qu'il est susceptible d'acquérir. Traité en futaie, il devient le géant des forêts de la plaine; par contre, ce n'est pas avant l'âge de deux cents à trois cents ans qu'il acquiert son maximum de valeur. Les massifs qu'il forme ont besoin d'être desserrés de bonne heure; mais alors ils entretiennent mal la fertilité du sol. Il ne convient donc pas d'élever le chêne à l'état pur et on doit le conduire en mélange avec le hêtre ou avec le charme; à leur défaut, il est indispensable de conserver avec soin toute la végétation arbustive qui se développe en sousbois, grâce à la demi-lumière tamisée à travers sa cime (2). A ce point de vue, l'exercice du pâturage sous les vieilles futaies de chêne est particulièrement nuisible. En général, le feuillage plus fourni du chêne rouvre lui permet de mieux accepter ce genre de traitement.

Leur tempérament robuste, leurs exigences spéciales au

(1) Broilliard, Traitement des bois en France.

(2) Dubois, loc. cit. 
point de vue de la lumière et de l'espace, disposent naturellement ces deux chênes et, plus spécialement le pédonculé, à rechercher l'état isolé (1), aussi les élève-t-on avec avantage sous forme de réserves dans les taillis sous futaie. Ils donnent alors une proportion de bois d'œuvre moins forte que dans les massifs de futaie, mais, grâce à un grossissement plus rapide, leur bois acquiert la plus grande dureté. Isolés dans les campagnes, ils fournissaient autrefois des pièces courbes recherchées pour les constructions navales.

Ces deux chênes, le rouvre surtout, possèdent la précieuse faculté de se plier aux conditions les plus diverses que le traitement leur impose; ils vivent même longtemps en forme de têtards et d'arbres d'émonde. Dans les sols les plus maigres et les plus secs, le rouvre végète sous les dimensions réduites d'un simple buisson ; c'est sous cette forme qu'on le voit persister dans certaines forêts ruinées, comme le dernier représentant des anciennes richesses. En un mot, suivant la fertilité des milieux, le chêne se montre exigeant ou frugal; mais, c'est seulement dans les bons sols, qu'il mérite sa réputation et que les dépenses pour le multiplier se justifient.

Variétés. - Le chêne rouvre et le chêne pédonculé, comme beaucoup d'espèces dont les aires sont étendues, présentent d'assez nombreuses variétés.

Nous en citerons deux :

$1^{\circ}$ Le chêne pubescent, variété du chêne rouvre : c'est le chêne blanc du midi, arbre généralement de petite taille, souvent tortueux ou même simple buisson, mais qui n'en est pas moins précieux par la complaisance avec laquelle il accepte de croître dans les pierrailles calcaires brûlées par le soleil.

$2^{\circ}$ Le chêne de juin ou tardif, variété du chêne pédonculé : cette intéressante variété, signalée en France par M. le Conservateur de Béer dès $187 \%$, est surtout connue depuis l'étude

(1) Le chêne pédonculé constitue rarement des massifs de futaie pleine : ainsi, dans certaines régions de la Normandie, il est très commun dans les haies qui séparent les héritages et fait totalement défaut, à quelques pas de là, dans les futaies domaniales. Il est, au contraire, l'arbre des taillis sous futaie, dont il fournit les réserves idéales (quand la nature du sol s'y prête, bien entendu). 
très complète qu'en a faite M. le Conservateur Gilardoni (1). L'épanouissement tardif de ses bourgeons (ils s'ouvrent un

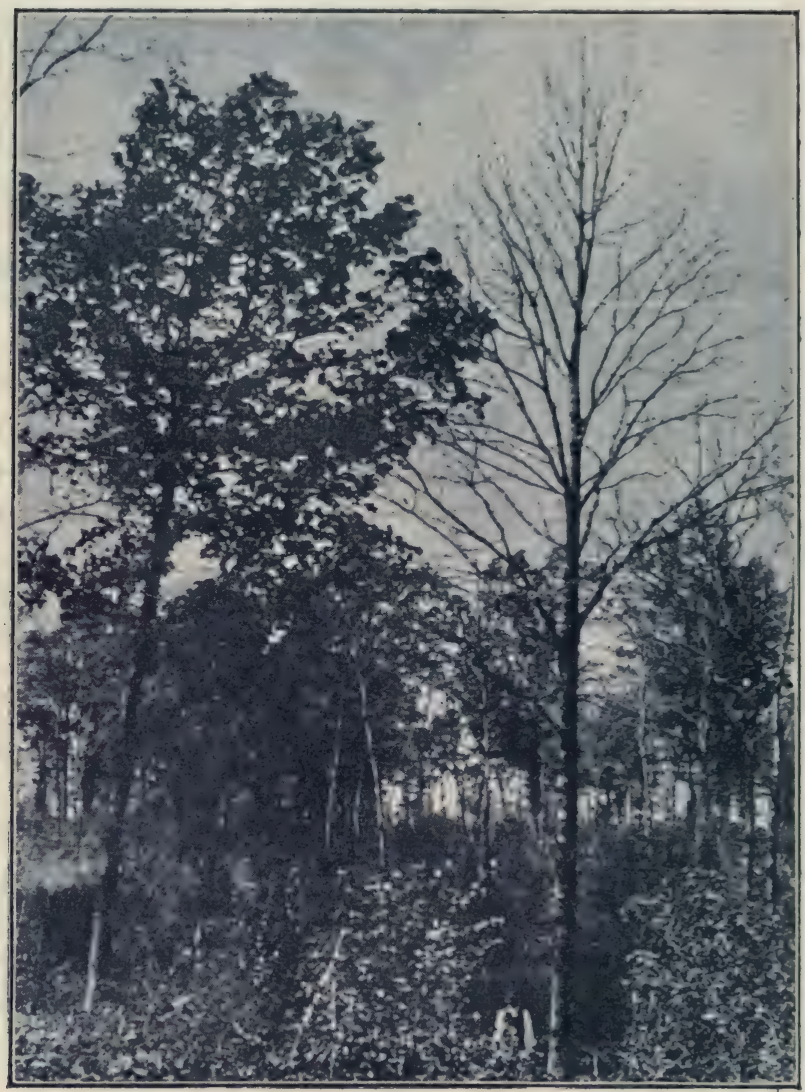

Fig. 25. - Forêt du Petit-Noir (Jura). Vue prise le 10 mai 1891: à droite Chêne de juin. (Photographie de M. Gilardoni.)

mois, quelquefois deux mois plus tard que ceux des chênes pédonculés ordinaires) (fig. 25), met cet arbre à l'abri des ge-

(1) Le chêne de juin, notice sur une variété bressane du chêne pédonculé, par M. Gilardoni, inspecteur des Forêts. Nancy, impr. Berger-Levrault et Cie, 1895. 
LE CHÊNE ROUVRE ET LE CIÊNE PÉDONCULí.

lées printanières. Il est surtout commun dans la vallée de la Saône (départements de Saône-et-Loire et du Jura). M. le Conservateur Duchalais l'a retrouvé dans la vallée de la Loire et M. Földes en Hongrie. Des expériences faites à l'École Nationale des Eaux et Forêts à Nancy (fig. 26), ont

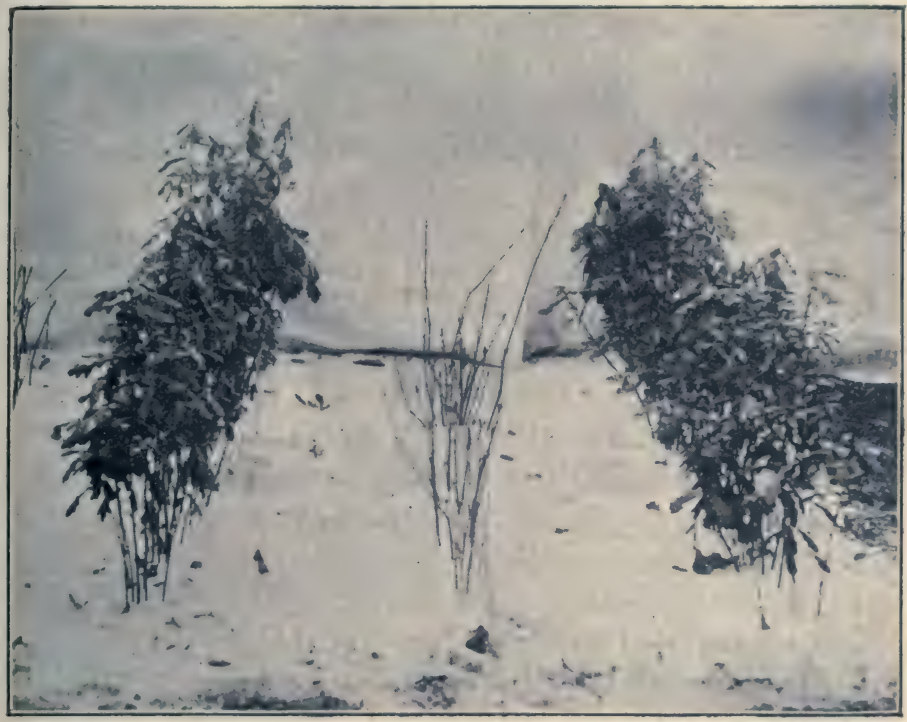

Fig. 26. - A gauche Chênes de juin, glands de la forêt du Petit-Noir (Jura). - A droite Chênes pédonculés ordinaires, glands de la forêt du Petit-Noir (Jura). Semis en automne 1896, dans le jardin de l'École Nationale des Eaux et Forêts. Aspect le 4 mai 1898. (Photographie de M. l'Adjudant Fournel.)

montré que le caraclère particulier à cet arbre, le relard dans la foliaison et la floraison, élait complètement héréditaire (1).

Le port des chênes tardifs est particulièrement élancé, avantage dû sans doute à ce que la croissance n'est pas entravée par les accidents de gelée.

(1) Bulletin Société des Sciences de Nancy, 1899. 


\section{Le chêne tauzin.}

Aire et station. - Cette essence, propre au Sud-Ouest de la France, caractérise la région océanique qui s'étend des Pyrénées à la Loire et même un peu plus au Nord, et du littoral à une ligne tirée d'Orléans à Tulle et à Toulouse. Elle semble préférer les sols siliceux et croît plutôt dans les stations du chêne pédonculé que dans celles du chêne rouvre.

Tempérament. - Le tempérament du jeune plant est robuste; le couvert de l'arbre est léger; on ne rencontre le chêne tauzin que dans les stations où les grands froids de l'hiver et les gelées printanières sont peu à craindre. D'ailleurs, comme il entre en végétation très tardivement, il est moins exposé que les deux espèces précédentes à ces derniers accidents. Il repousse très bien de souches.

Enracinement, fructification. - Bien que pourvu d'un pivot, ce chêne est particulièrement remarquable par ses racines traçantes, qui possèdent au plus haut degré la faculté de drageonner.

La production des glands est assez abondante chaque année.

Croissance. - Le chêne tauzin est, le plus souvent, un arbre tortueux; il est loin d'atteindre les belles dimensions du rouvre ou du pédonculé.

Bois et usages. - Son bois raide, se gerçant et se tourmentant beaucoup, peu estimé comme bois de construction ou de travail, est, au contraire, recherché comme bois de feu.

Produits accessoires. - L'écorce jeune renferme beaucoup de tanin et fournit du tan d'une qualité supérieure. Dans les départements du Sud-Ouest, ses glands sont récoltés pour l'engraissement des porcs.

Allures forestières. - Le traitement en taillis convient à tous égards au chêne tauzin; il doit même lui être exclusivement appliqué, car ses dimensions toujours restreintes et ses médiocres qualités comme bois d'œuvre, font qu'on n'aurait aucun intérêt à le traiter en futaie. 


\section{Le chêne-liège et le chêne occidental.}

Le chêne-liège est une essence exclusivement méditerranéenne. Répandu en Corse et en Algérie, il occupe dans la France continentale trois régions disjointes : les Maures et l'Esterel, - le Gard et l'Hérault, - les Pyrénées-Orientales. Cette essence calcifuge se trouve cantonnée sur les sols granitiques, porphyriques et schisteux. Dans les Maures, elle monte jusqu'à 700 mètres d'altitude ; dans les Pyrénées, jusqu’à 500 .

Tempérament. - Le feuillage du chêne-liège est persistant, mais grêle, rare; son couvert est léger, son jeune plant très robuste. Il repousse bien de souches.

Enracinement, fructification. - Les racines sont, à la fois, pivotantes et traçantes, suivant la profondeur du sol; elles ont aussi la faculté de drageonner, notamment quand les souches ont été atteintes par les incendies qui ravagent les forêts dans sa région. La fructification est précoce et se produit dès l'âge de quinze ans ; ce n'est guère qu'à trente ans qu'elle est abondante et soutenue; en France, elle n'est le plus souvent qu'intermittente.

Croissance, longévité. - Arbre de deuxième grandeur, d'une croissance assez rapide dans le jeune âge, mais prenant bientôt une forme trapue. Sa longévité est grande.

Bois et ses usages. - Son bois est peu estimé comme bois de service, parce qu'il est très lourd et se tourmente trop; il fournit un très bon combustible et d'excellent charbon.

Produits accessoires. - Le liège qu'on tire de son écorce a plus de valeur que le bois lui-même; il donne une très grande importance à cette précieuse essence, dont on ne saurait trop recommander la multiplication partout où elle peut prospérer. Les glands, sans être très âpres, ne sont pas ordinairement comestibles. En Algérie, le liber est très recherché à cause de sa richesse en tanin; les vieux arbres sont exploités en vue de cette récolte.

Le chêne occidental. - Il ressemble en tous points au chêne-liège : pour le tempérament, l'enracinement, la crois- 
sance, les qualités de son bois et de son écorce, ses exigences calcifuges. Il n'en diffère que par la maturation bisannuelle de ses glands et sa localisation sur les côtes de l'Océan.

Allures forestières. - Le chêne-liège et le chêne occidental n'ont d'autre importance forestière que celle de leur écorce.

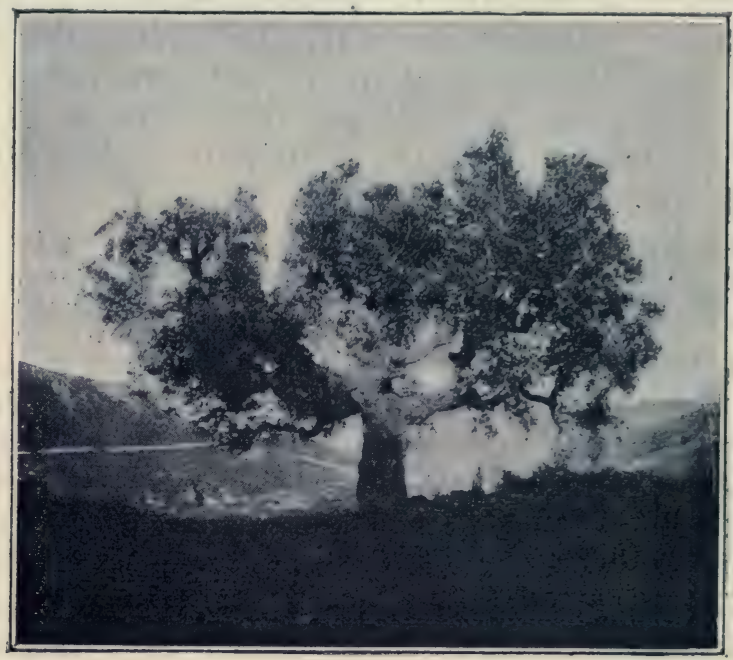

Fig. 27. - Chêne-liège près de Bastia. - Photographie de M. H. Bregeault.

A ce point de vue, on en obtient le meilleur rendement en les disposant par pieds isolés, à la façon des arbres fruitiers dans les vergers (fig. 27).

\section{Le chêne yeuse ou chêne vert.}

Aire et station. - Cette espèce méridionale est rare dans la région océanique, très commune, au contraire, sur le versant méditerranéen, de Menton à Céret, - de la mer à Digne, Sisteron et Montélimart; elle remonte même au nord de Valence. Elle manifeste, dans la France continentale du moins, une préférence pour les sols calcaires, de là son 
abondance sur le littoral méditerranéen, sauf dans les régions granitiques des Maures et de l'Esterel; de là aussi sa rareté sur le littoral de l'Océan, si ce n'est dans les stations calcaires de la Charente; de là enfin, son apparition dans le Plateau central, par les cañons des Causses. Elle parvient dans les Alpes et en Provence jusqu'à l'altitude de 700 à 800 mètres, dans les Pyrénées à celle de 600 mètres.

Tempérament. - L'yeuse est très robuste et résiste aux expositions méridionales les plus chaudes; son feuillage, persistant, est assez léger. Il fournit des rejets de souches jusqu'à un âge avancé.

Enracinement, fructification. - Bien que solidement (1) ancré dans le sol, l'yeuse possède des racines latérales drageonnantes.

La fructification est précoce et se produit dès huit à dix ans; elle se soutient abondante et régulière jusqu'à un âge avancé.

Croissance, longévité. - Sa croissance est assez rapide dans le jeune âge, pourtant il ne dépasse pas les dimensions d'un arbre de troisième grandeur. Sa longévité atteint trois siècles et plus.

Bois et ses usages. - Le bois du chêne yeuse est très dur très lourd et extrêmement compact; il est fort sujet à se tourmenter et peu convenable au travail; son poids exagéré et ses faibles dimensions en longueur limitent ses emplois comme bois de construction. Il n'a point d'égal comme combustible.

Produits accessoires. - L'écorce du chêne yeuse donne un tan très estimé, supérieur à celui des chênes à feuilles caduques. Les glands, lorsqu'ils sont doux, ont une saveur agréable et servent à la nourriture de l'homme, qu'ils soient crus ou cuits. On les récolte pour cet usage dans quelques départements du Midi.

Allures forestières. - Comme le chêne tauzin et pour des causes identiques, le chêne yeuse ne comporte pas d'au.

(1) Les pivots du chêne vert pénètrent dans certaines crevasses jusqu'à 15 et 20 mètres de profondeur. Beaucoup de végétaux des stations sèches agissent ainsi pour se procurer l'eau qui a disparu des régions superficielles du terrain.

Bopps et JouxET. 
tre traitement que celui du taillis simple. Sous cette forme, il peut être utilement associé au pin d'Alep; il fournit à celui-ci d'excellents sous-bois peu sujets à être détruits par les incendies.

\section{Le châtaignier.}

Station. - Le châtaignier, répandu sur beaucoup de points de la Franee, ne paraît cependant pas y être spontané, et c'est une erreur populaire de croire que l'hiver de 1709 l'a fait disparaître des régions de la Loire. Il recherche les terrains siliceux.

Tempérament. - Le châtaignier exige moins de lumière et forme, en conséquence, des massifs plus serrés que les chênes à feuilles caduques; il tient assez bien le milieu, pour le tempérament, entre le hêtre et ces chênes, avec lesquels il peut être maintenu en sous-étage. Il repousse très bien de souches.

Fructification. - Le châtaignier fructifie vers vingt-cinq ou trente ans, s'il est isolé - vers quarante ou soixante ans, s'il est en massif ; ses années de semences se succèdent à deux ou trois ans d'intervalle et sont généralement abondantes.

Enracinement. - L'enracinement est formé d'un pivot assez allongé et de nombreuses et fortes racines latérales.

Croissance, longévité. - C'est un arbre de première grandeur, à croissance rapide et d'une grande longévité, malgré les tares centrales qu'il contracte d'assezbonne heure.

Bois et ses usages. - Le bois de châtaignier a beaucoup d'analogie avec celui du chêne (1); il lui est cependant inférieur en qualité pour les constructions; même dans les contrées les plus méridionales de la France, l'arbre se creuse avant d'avoir atteint les dimensions qui le rendraient propre à ce service. Il est estimé comme bois de merrain et fournit également de très bons échalas; en effet, son aubier étant toujours très mince, il y a une forte proportion de bois de

(1) La confusion est toutefois impossible entre ces deux bois : le bois du chêne a de larges rayons médullaires; celui du châtaignier, au contraire, a des rayons invisibles à l'œil nu : il n'est jamais maillé. 
cœur, même dans les jeunes sujets; il est aussi recherché pour la fabrication des cercles de tonneau.

Produits accessoires. - Son fruit est comestible; sous le nom de châtaigne, il sert à l'alimentation dans les départements du Centre et du Midi. Le marron comestible n'est autre chose que le fruit d'un châtaignier amélioré et traité à la façon des arbres fruitiers. Cette culture, très répandue dans la région des Maures, fournit des fruits très estimés, connus dans le commerce sous les noms de marrons du Luc ou de Lyon.

Allures forestières. - Le châtaignier exige un climat doux. En France, du moins, il constitue rarement des massifs de quelque étendue. Il est souvent introduit artificiellement, sur de petites surfaces, dans les sols fertiles que leur déclivité rend impropres à l'agriculture. Traité en taillis, il donne alors des produits très abondants et recherchés comme menu bois d'œuvre. Pour constituer un massif de futaie, le chêne devra toujours lui être préféré.

\section{Le hêtre ou foyard.}

Aire et station. - Il se rencontre partout, en France, si ce n'est dans la haute région des Alpes, sur le littoral méditerranéen de Nice à Perpignan, et sur les côtes de l'Océan de Bayonne aux Sables d'Olonne; il abonde dans les régions de collines et dans les montagnes de moyenne élévation. Le hêtre, assez indifférent à la composition minéralogique du terrain, préfère, néanmoins, les sols légers et surtout calcaires, il redoute les terres fortes et mouillées et craint la trop grande chaleur.

Tempérament. - Le jeune plant de hêtre est très délicat, le couvert de l'arbre très épais. Il supporte les plus grands froids de l'hiver, mais son jeune feuillage, bien qu'un peu moins sensible aux gelées printanières que celui du chêne, est souvent atteint à cause de sa précocité. Le hêtre repousse mal de souches dans les climats froids, mieux et même bien dans les régions méridionales, notamment dans les Pyrénées; partout il perd d'assez bonne heure, à vingt-cinq ou trente ans, la faculté d'émettre des rejets de bourgeons proven- 
tifs; cẹux d'origine adventive sont alors plus fréquents.

Enracinement, fructification. - Dans les premières années, la racine pivote,reste simple et s'enfonce à peu près autant dans le sol que la tige s'élève dans l'air ; vers douze ou quinze ans, le pivot s'arrête et les racines latérales prennent une grande extension; à trente ans, celles-ci cessent de croître et sont alors remplacées par des racines superficielles, traçantes, souvent en partie saillantes hors du sol jusqu'à une certaine distance du pied de l'arbre. En somme, l'enracinement total est peu profond, mais très étendu en. surface, sans pour cela donner de drageons.

Le hêtre appartient à la catégorie des arbres à semences lourdes; il ne fructifie qu'à un âge avancé, vers soixante ou quatre-vingts ans en massif, quarante ou cinquante ans, quand il est isolé. Les faînes ne sont abondantes que tous les cinq ou six ans dans les circonstances les plus favorables, et, quelquefois, tous les quinze ou vingt ans seulement. Les fainées complètes sont généralement plus communes dans les plaines et sur les coteaux que dans les régions montagneuses; mais, en retour, les annéés de disette absolue n'y sont pas rares : en montagne, au contraire, les faînées partielles se succèdent à peu près sans interruption.

Groissance, longévité. - Le hêtre atteint de très grandes dimenșions, sans parvenir cependant à celles du chêne et du sapin, en raison de sa longévité bien moins élevée et qui dépasse rarement deux cents ou deux cent cinquante ans. Cette longévité décroît d'une façon notable au fur et à mesure qu'il quitte la région des collines pour s'abaisser dans la plaine; dans les forêts situées sur les bords de la Loire, il dépérit, en massif, avant l'âge de cent cinquante ans.

Au début de son existence, le plant s'accroît peu, environ d'un décimètre de hauteur annuellement ; mais, passé cinq ans, il prend son essor, écrasant autour de lui les espèces de lumière qu'on voudrait lui associer; vers quarante ou cinquante ans, il parvient à son maximum d'allongement annuel; comme le chêne, entre quatre-vingts et cent, il ne s'accraît plus sensiblement en hauteur.

Bois et ses usages. - Les emplois du hêtre sont nombreux 
comme bois d'industrie. Il est très estimé commé chauffage et donne un charbon lourd et de bonne qualité.

Produits accessoires. - La faîne contient une huile grasse, comestible quand elle est exprimée à froid; en tous cas, très propre à l'éclairage. La récolte des faînes devient, de ce fait, dans les années d'abondance, une source importante de produit pour les propriétaires ou pour les habitants qui achètent le droit de' les ramasser.

Allures forestières. - L'état de massif plein est celui qui convient le mieux au hêtre; par son couvert épais et ses détritus abondants, il fertilise le sol. Les produits les plus précieux sont aussi tirés des arbires dont le fût élancé et lisse s'est constitué dans les futaies régulières. Il se régénère facilement par la semence, et la faculté qu'ont ses jeunes plants de supporter un couvert prolongé, permet d'user de tous les délais nécessaires pour obtenir un ensemencement naturel complet. Il peut être élevé à l'état pur, mais il est avantageux de lui associer d'autres essences à croissance aussi rapide que la sienne : un massif de hêtre est, en effet, le milieu fécondant le plus favorable à leur bon développement. Par contre; il faut surveiller ses tendances à devenir dominant et, lorsqu'on ne veut lui réserver qu'un rôle secondaire, c'est à l'état. de sous-étage qu'il rendra le plus de services, sans devenir jamais nuisible. Le hêtre accepte le jardinage, mais alors les arbres fournissent moins de bois d'œuvre que dans les futaies régulières. Cette essence conserve trop peu de temps la faculté de rejeter de souches pour être avantageusement traitée en taillis simple. Sous cette forme, le peuplement ne se maintient complet que sur les versants bien éclairés et à la condition expresse d'être exploité très jeune. Parmi toutes les essences forestières, c'est à peu près la seule qui puisse s'accommoder du traitement en taillis d'âges mêlés ou furetés. Maintenu à l'état de réserve dans les taillis composés, le hêtre se constitue une cime ample, épaisse, qui écrase toute la végétation inférieure; on n'obtient ainsi que des arbres à fût court, donnant un bois de travail de médiocre qualité et en faible quantité.

En résumé le hêtre est l'espèce par excellence à cultiver 
dans les sols sans profondeur; on ne saurait trop le propager dans les collines jurassiques du Nord et de l'Est de la France; sa frugalité le désigne également pour remettre en état les terres sableuses fatiguées par le pâturage, par le maintien abusif du chêne à l'état pur, ou par le traitement en taillis.

\section{Le charme.}

Aire et station. - C'est un arbre de plaines et de coteaux : en montagne, qu'il s'agisse des Vosges, du Jura ou du Plateau central, il disparaît brusquement, sans passer par l'état buissonnant qu'affectent beaucoup d'essences à la limite supérieure de leur aire. Il occupe toute la partie de la France située au nord d'une ligne tirée de Grenoble à l'embouchure de la Gironde; il est surtout commun dans les départements du Nord et de l'Est. Le charme accepte tous les terrains, mais il accuse une préférence marquée pour les sols frais et profonds (1).

Tempérament. - Le charme, bien que son tempérament soit robuste, a besoin d'abri dans sa jeunesse, en raison de la faiblesse de l'enracinement des plants naissants et des dangers auxquels les exposerait le desséchement d'un sol découvert. Il demande bientôt de la lumière, qu'il faut lui ménager prudemment. En sols secs, pierreux, les chaleurs exceptionnelles de certains étés lui sont parfois fur estes; en revanche, il présente, en toutes circonstances, une résistance remarquable à l'action des gelées printanières. Le charme rejette parfaitement de souche.

Enracinement, fructification. - L'enracinement est faible et superficiel. Il se compose de nombreuses racines latérales, issues d'un pivot bientôt oblitéré, et l'ensemble pénètre rarement à plus de 50 centimètres de profondeur.

Malgré leur disposition traçante, ces racines n'émettent pas de drageons; mais les brins traînants du charme se mar-

(1) C'est, du moins, en pareille station qu'il constitue les arbres les plus longévifs et les plus beaux; mais ses taillis couvrent des étendues considérables de terrains calcaires superficiels. 
cottent avec la plus grande facilité. Dans la forêt de Champenoux (Meurthe et Moselle) d'épais fourrés de cette essence se constituent ainsi, et s'étendent de proche en proche, constituant un obstacle sérieux à la régénération du chêne par la semence. Il en est ainsi sur beaucoup d'autres points.

La fécondité du charme se manifeste de bonne heure, à vingt ans et même au-dessous : il est peu de végétaux forestiers qui aient des années de semences aussi abondantes et en même temps aussi répétées. Le fruit du charme est un gland peu volumineux, dont la dissémination est encore facilitée par la cupule légère à laquelle il est soudé et qui joue le rôle d'une aile offrant une large prise au vent. Ce gland ne germe que le deuxième printemps après sa maturité.

Croissance, longévité. - La croissance du charme est toujours lente et, à toutes les époques de sa vie, elle est de beaucoup inférieure à celles du chêne et du hêtre. La végétation des rejets de souches est, au contraire, assez active pendant les vingt ou trente premières années : à cette époque, elle se ralentit pour devenir assez semblable à celle des sujets de franc-pied. Quelle que soit son origine, le charme reste toujours un arbre de deuxième grandeur. Il peut atteindre cent à cent vingt ans, et même dépasser cent cinquante ans, dans de bonnes conditions et quand, au point de vue cultural, on a intérêt à le maintenir.

Bois et ses usages. - Le charme fournit un chauffage de toute première qualité; il n'est pas employé comme bois de construction, en raison de son peu de durée ; sa fibre, souvent entrelacée, le rend d'un travail peu facile et les menuisiers ne l'utilisent pas communément ; cependant sa dureté, son homogénéité, sa tenacité, le rendent précieux pour la fabrication de formes et d'outils divers et pour la confection de certaines pièces de machines, qui ont à subir des frottements, telles que dents d'engrenages, etc. On ne tire du charme aucun produit accessoire.

Allures forestières. - Le charme ne se rencontre à l'état pur, dans la futaie, que par suite d'erreurs culturales. A cause de la lenteur de sa croissance, les espèces qui l'accompagnent parviennent toujours à le dominer; mais, dans le rôle secon- 
daire où il y a lieu de le confiner, il peut rendre les plus grands services, comme remplissage.

Traité en taillis, il donne jusqu'à un âge assez avancé (cinquante à soixante ans) des rejets très abondants, dont on obtient de bons produits. Dans son aire, il se perpétue par rejets de souche, même dans les conditions les plus mauvaises, et c'est grâce à sa présence que certains taillis, en sol presque stérile, conservent quelque valeur.

Sa végétation est trop lente et son couvert est trop bas, pour qu'on puisse l'élever avec profit sous forme de réserve dans les taillis composés ; c'est donc seulement à titre exceptionnel, et quand on n'a rien de mieux à attendre du terrain, qu'on aura recours à lui. Si on n'a jamais l'occasion d'élever le charme en futaie pour lui-même, il est toujours fort utile de l'associer au chêne; car il augmente la densité du massif dans sa jeunesse; plus tard, il protège les fûts des arbres, en même temps qu'il entretient la fertilité du sol: ce qui permet de maintenir, sans inconvénient, les cimes des chênes dans l'état clair qui leur convient spécialement.

\section{Le frêne.}

Aire et station. - Le frêne vit à l'état disséminé dans presque toutes les régions de la France, où il croît dans les conditions les plus diverses. C'est dans les plaines basses, dans les grandes vallées, dans les vallons à sol frais et fertile qu'on le rencontre le plus communément. Il est habituellement le compagnon de l'orme, de l'aune, du chêne pédonculé. Mais on le trouve aussi dans les montagnes, sans qu'il atteigne cependant l'altitude du hêtre, et dans les sols secs des collines, pourvu qu'ils sóient suffisamment meubles.

Tempérament. - Le feuillage léger du frêne, sa cime peu ramifiée sont les indices d'un tempérament robuste; c'est donc un arbre de pleine lumière, qui demande à être maintenu à l'état isolé. Nous avons vu, cependant, des jeunes plants de frêne résister au couvert; aussi serions-nous tentés de croire qu'ils le supportent mieux que les jeunes chênes. Ils ont, en tout cas, cet avantage, que grâce à leur tendance à 
pousser en hauteur, à filer, ils percent beaucoup mieux que les chênes les fourrés de ronces, de rejets ou d'épines dans lesquels ils peuvent se trouver englobés. Le frêne rejette bien de souches.

Enracinement, fructification. - La racine s'enfonce profondément dans la jeunesse et ne tarde pas d'émettre des ramifications latérales qui, se développant beaucoup en longueur et en grosseur, constituent à l'arbre une assiette très puissante. Sa fructification est abondante et assez régulière.

Croissance, longévité. - Le frêne est un arbre de première grandeur, qui peut atteindre les plus belles dimensions si les conditions lui sont favorables; il reste, au contraire, étiolé et chétif dans les sols médiocres. En tout état de choses, sa croissance, relativement rapide dans sa jeunesse, en fait partout une essence précieuse. Sa longévité est au moins égale à celle du hêtre.

Bois. - Le bois du frêne, peu propre à la construction, est, à cause de son élasticité, très recherché comme bois de travail. Il donne un bon combustible.

Produits accessoires. - A l'état vert, comme à l'état sec, les feuilles fournissent un bon fourrage; aussi, en pays de basses montagnes, rencontre-t-on souvent des frênes dans le voisinage des habitations, où ils sont exploités en têtards et surtout en arbres d'émonde.

\section{Les érables.}

Observations générales. - On compte en France cinq espèces d'érables : l'érable sycomore, l'érable plane, l'érable à feuilles d'obier, l'érable champêtre et l'érable de Montpellier.

Les trois premières forment la catégorie des grands érables et présentent entre elles une grande analogie, au double point de vue des conditions d'existence et de la qualité des produits. Elles sont exigeantes sous le rapport de la fertilité du sol et vivent dans les forêts à l'état disséminé. Le feuillage de ces arbres est assez épais. Leurs jeunes plants peuvent être qualifiés de robustes; pourtant ils se maintiennent longtemps sous le couvert, et, hors forêt, s'installent de préférence au 
milieu de buissons, ou encore sous des pins sylvestres, sous des bouleaux, qui leur donnent un premier abri. Les grands érables ont donc un tempérament mixte, intermédiaire entre celui des essences de lumière, et celui des essences d'ombre.

Ils fructifient régulièrement et leur samare légère, disséminée au loin, germe en abondance dans les clairières des forêts, sur les places privées d'herbes.

Les souches produisent des rejets abondants et vigoureux.

Leur bois, recherché comme bois de travail, fournit un très bon comestible.

L'érable sycomore se rencontre, le plus souvent, mélangé au hêtre, dont il a toutes les exigences climatériques; il préfère les pays accidentés aux plaines et, dans les montagnes, dépasse la région des sapins. Dans les Alpes, il parvient jusqu'à 1500 mètres d'altitude. Sa croissance est très rapide dans la jeunesse et devient parfois gênante, comme celle des bois blancs, pour les autres essences avec lesquelles il s'associe naturellement. A l'état isolé, le sycomore développe une belle cime, fortement étalée. Il peut vivre de cent cinquante à deux cents ans.

L'érable plane accompagne souvent le sycomore, bien que, plus délicat, il soit plus rare et s'élève moins que lui dans les montagnes. En forêt, son aire d'habitation semble limitée à quelques départements du Nord et de l'Est de la France. Sa croissance est aussi plus lente que celle du sycomore. Le plane est souvent utilisé, et à juste titre, comme arbre de parc ou d'avenue.

L'érable à feuilles d'obier joue un rôle assez important dans les forêts du Jura, des Alpes, des Cévennes, des Pyrénées, où il vit, en plus ou moins grand nombre, à l'état disséminé; par sa forme, son couvert et ses exigences, il se rapproche beaucoup du sycomore; son bois est également recherché par l'industrie et fournit un bon combustible.

L'érable champêtre est plutôt un grand arbrisseau qu'un arbre; à cause de sa croissance très lente, il disparaît de bonne heure au milieu des grandes essences qu'on laisse vieillir à l'état de massif ; par contre, il est très commun dans les taillis de la région parisienne, où il accompagne le charme et 
forme avec lui un utile mélange. Son bois, toujours rare sous des dimensions un peu fortes, présente une ténacité spéciale, qui le fait rechercher par les tourneurs; il est d'ailleurs très bon comme combustible.

L'érable de Montpellier, malgré ses petites dimensions et la lenteur de son accroissement, est précieux par la qualité qu'il possède de croître dans les sols les plus secs, jusque dans les fissures des rochers. Il est assez répandu dans les forêts des zones girondine et provençale, où il se comporte en tous points comme l'érable champêtre dans les stations qui lui sont propres.

\section{Les ormes.}

L'orme champêtre recherche, avant tout, les sols meubles et fertiles; il peut devenir un arbre de la plus grande taille, c'est dans les terrains d'alluvions récentes, sur les bords submersibles des fleuves et des rivières, qu'on le trouve le plus abondant à l'état spontané, aussi bien au nord qu'au midi de la France. Il devient alors envahissant par ses racines traçantes, qui se développent en rayonnant et par ses semences nombreuses et légères, qui se disséminent au loin; les semis naturels qui en résultent, grâce à la rapidité de leur végétation, risquent peu d'être étouffés par les herbes. En terrain sec, ses dimensions sont bien réduites, il donne des tiges irrégulières à fibre tourmentée : de là le nom d'orme tortillard, qui lui est alors appliqué. Cette espèce est très fréquemment et, avec raison, plantée le long des routes, des avenues, sur les promenades publiques et sur les glacis des places fortes. Bien que l'orme se rencontre à peu près partout, il semble préférer les climats doux et même chauds du Midi à ceux plus rudes et surtout plus froids du nord-est, où son bois est souvent altéré par les gélivures.

Son bois parfait, dur, élastique, extrêmement tenace, est d'une durée au moins égale à celle du chêne; aussi est-il recherché pour une foule d'ouvrages; l'aubier se dégrade rapidement et n'est pas employé. Les feuilles d'orme constituent le meilleur fourrage que puissent fournir les arbres forestiers; desséchées à l'air libre, c'est-à-dire fanées, elles sont presque 
aussi riches en azote que la luzerne et le tréfle des prairies artificielles et sont supérieures à cet égard au foin des prairies naturelles.

L'orme de montagne, facilement reconnaissable à ses feuilles plus larges, à sa cime plus ample, n'atteint jamais les grandes dimensions du précédent. Il est fréquemment disséminé au milieu des ehênes, des hêtres et même des sapins, dans les forêts des coteaux et des basses montagnes ; malgré ses préférences pour les terrains frais et légers, il se trouve en assez bon état de végétation sur les sols secs des calcaires jurassiques et jusque dans les éboulis rocheux.

Quoique doué par ses racines et par sa fécondité de la même faculté d'expansion que l'orme champêtre, en forêt, du moins, l'orme de montagne est beaucoup moins intéressant que ce dernier; car son bois est beaucoup plus léger, plus mou, moins tenace et moins durable. Jues charrons savent très bien le distinguer; ils le désignent sous le nom d'orme blanc et refusent habituellement de l'employer.

Citons, pour mémoire; l'orme diffus, peu répandu et donnant un bois des plus médiocres.

\section{Allures forestières des grandes essences disséminées.} - Al'exclusion des érables champêtres et de Montpellier, essences n'entrant que dans la composition des taiilis, les autres formes des trois genres précédents offrent entre elles assez d'analogie pour qu'on puisse les considérer comme un groupe à part.

Frêne, orme et grands érables constituent d'excellentes réserves dans les taillis sous futaie. L'orme par ses drageons, le frêne et les érables par leurs nombreux semis, donnent d'excellents baliveaux ayant une longévité assurée. Le frêne et l'orme champêtre, ce dernier surtout, veulent toutefois des sols profonds et riches. Les grands érables, bien moins exigeants, constituent une ressource précieuse dans des taillis sous futaie à sol superficiel, où les chênes ne poussent qu'à regret. Ils ont cet avantage de ne rien craindre des gelées printanières; l'orme est dans le même cas, qualité précieuse qu'il ne partage malheureusement pasavec le frêne.

Peut-être l'orme champêtre pourrait-il se maintenir dans 
des massifs de futaie? Nous avouons n'en point connaitre d'exempie. Au contraire, le frêne existe fréquemment dans les futaies de chêne. Plus souvent encore, on trouve le sycomore dans les sapinières ou les hètraies : il s'accommode très bien d'un pareil voisinage.

Dans la basse montagne, les essences disséminées sont parfois traitées en têtards et en arbres d'émonde. Leur feuillage est alors dorıné aux bestiaux comme fourrage vert.

\section{Le micocoulier.}

Aire et station. - Celte espéce habite les climats méridionaux. Elle prospère en plaine, en coteaux et même en montagne, à toutes les expositions; elle réussit bien dans tous les terrains, pourvu qu ils ne soient ni trop légers, ni trop humides ou marécageux; on la voit végéter jusque dans les pierrailles et sur les ruines; ce sont néanmoins les sols gras et frais qu'elle préfère.

Tempẻrament. - Son feuillage est très léger; son enracinement est puissant, pivotant et traçant. Ses racines drageonnent; les souches produisent des rejets abondants et d'une grande vigueur.

La croissance du micocoulier est rapide, et, bien qu'il n'acquière pas les dimensions d'un arbre de première grandeur, il peut atteindre 20 mètres de hauteur totale et 3 mètres de circonférence à la base, à cause de sa grande longévité. II fructifie assez jeune, mais il reste quelquefois deux années sans rien produire.

Bois et usages. - Son bois ressemble à celui du frêne, dont il a toutes les qualités, à un degré plus élevé encore.

Comme produits accessoires, on tire de ses racines une matière tinctoriale jaune. Ses feuilles donnent aussi un bon fourrage pour le bétail.

Allures forestières. - Le micocoulier se rencontre rarement en massif. Il se plaît mieux à l’état isolé; aussi, son rôle est-il plus important comme arbre d'alignement, d'agrément ou de culture industrielle, que comme arbre forestier proprement dit. On le rencontre, néanmoins, disséminé dans les 
terrains vagues, pauvres, livrés au pâturage ou couverts dé broussailles. En somme, dans sa station, il se comporte à la façon des ormes dans les régions plus septentrionales.

\section{Les fruitiers.}

Observations gẻnérales. - Les fruitiers se rencontrent, en France, à l'état disséminé, dans presque toutes les forêts de plaines ef de coteaux, où, à cause de la lenteur de leur végétation et de leurs faibles dimensions, ils sont surtout cantonnés dans les peuplements traités en taillis simple ou en taillis sous futaie. Parmi les nombreuses espèces qui composent ce groupe, l'alisier torminal, le sorbier cormier, le cerisier merisier et le poirier sauvage méritent d'être signalés.

Les deux premiers préfèrent les terrains calcaires; mais l'alisier torminal n'atteint de belles dimensions que si le sol présente suffisamment de profondeur et de fraîcheur. Ils ont une assez grande longévité et se signalent par les qualités exceptionnelles de leur bois ; on a donc un avantage réel àles laisser vieillir.

Le cerisier merisier, commun en plaine, est beaucoup plus rare en montagne, où il s'étend dans la région du hêtre, sans, toutefois, la dépasser. Il n'est pas très exigeant sous le rapport des qualités du sol, il préfère néanmoins ceux qui sont frais et meubles. Sa croissance est assez rapide jusqu'à l'âge de trente ou quarante ans, puis il dépérit d'assez bonne heure pour qu'il soit imprudent de le laisser vivre au-delà de soixante à soixante-dix ans.

Son bois s'altère rapidement à l'air ; sa couleur acajou et ses qualités spéciales, le font rechercher des tourneurs pour la fabrication des bois de chaises et de fauteuils ; c'est un combustible de médiocre qualité.

Allures forestières. - En futaie, ces espèces ne peuvent jouer qu'un rôle de remplissage dans les jeunes peuplements. Dans les taillis sous futaie, il n'y a jamais lieu de se préoccuper de leur multiplication; on se contentera de réserver les sujetsles mieux venants, partout où il ne se présentera rien de meilleur pour occuper leur place; quoi qu'il en soit, on 
devra toujours les préférer au charme, non seulement à cause des qualités précieuses de leur bois, dont la valeur, celle du sorbier surtout, dépasse parfois celle du chêne, mais aussi parce que leurs baies servent de nourriture à des oiseaux utiles.

\section{Le boulean (1).}

Aire et station. - Le bouleau se distingue par son extrême rusticité. Il vit dans toutes les stations, aussi bien en plaine qu'en montagne, pourvu que l'atmosphère ne soit pas trop sèche. Peu exigeant sur la nature du sol et réussissant mềme dans les tourbières, il préfère néanmoins les terres légères et sablonneuses.

Tempérament. - Essence de pleine lumière, le bouleau a le couvert extrêmement léger; à l'état pur, il ne donne que des massifs très clairs, sous l'ombrage desquels le sol se dégrade. Il émet des rejets de souche et des rejets de racine assez nombreux, mais il est rare que, dans la suite, un ou deux brins de la cepée ne prennent pas l'avantage sur tous les autres, qui demeurent chétifs. L'énorme quantité de semis que donne cette essence dans les terrains sablonneux, supplée, d'ailleurs, le cas échéant, à l'insuffisance des rejets. Par contre, le bouleau n'atteint pas de bien grandes dimensions; son fût dépasse rarement 50 centimètres de diamètre. Il vit cent à cent-vingt ans et peut aller exceptionnellement jusqu'à cent cinquante.

Bois et usages. - Son bois, demi dur, blanc, soyeux, est d'assez bonne qualité; impropre à la construction, il sert à la menuiserie, au tour et à la fabrication des sabots; il fournit un assez bon combustible.

Son écorce renferme une huile essentielle, qui donne au cuir de Russie son odeur spéciale. Elle n'est pas utilisée en France pour cet usage.

Allures forestières. - La cime du bouleau a un tel besoin de lumière qu'elle n'accepte pas l'état de massif plein. C'est seu-

(1) Il n'y a pas lieu de distinguer ici le bouleau verruqueux et le bouleau pubescent, ce dernier plus commun dans les stations froides et humides. 
lement dans les sols pauvres, ou même ruinés, qu'il forme des peuplements purs; il prend alors des allures franchement sociales; partout ailleurs les espèces spontanées s'associent naturellement à lui.

Il est indifférent de le voir mélangé avec d'autres espèces feuillues, mais il est nuisible aux résineux, dont les bourgeons sont brisés lorsque, par un grand vent, les rameaux flexibles du bouleau viennent fouetter leur cime.

Le bouleau est souvent envahissant, parfois mème un peu gênant. Sa présence est pourtant justifiée dans les jeunes régénérations, car les espèces précieuses se développent sous son abri protecteur. Le mélange force les sujets d'élite à s'élever et, dès que ceux-ci ont acquis une résistance suffisante, le moment est seulement venu de couper les bouleaux, qui donneront alors de bons produits. On commet trop souvent l'erreur de les extirper radicalement avant cette époque.

D'ailleurs, grâce à son extrême frugalité, comparable à celle des résineux, le bouleau reste souvent la seule essence forestière de quelque valeur peuplant les forêts en voie de dégradation; pour le même motif, il est utilisé comme première essence de boisement dans les terrains pauvres.

\section{Les aunes.}

L'aune commun se rencontre dans les stations humides de toute la France; il affectionne les talus des rivières et des ruisseaux, dont il sert à fixer les berges. Il semble d'ailleurs assez accommodant sous le rapport du sol, car il résiste en même temps sur les terrains marécageux ou tourbeux et sur les craies de la champagne (1); néanmoins, les argiles trop compactes et le séjour prolongé des eaux stagnantes à la surface du sol lui sont préjudiciables.

Sa croissance est rapide, mais il vit rarement au-delà de soixante-dix à quatre-vingts ans; il repousse parfaitement de souches, Son bois, demi dur, coloré en rouge, se dégrade

(1) Nous avons eu l'occasion de dire que ces craies conservent une dose d'humidité plus considérable qu'on ne se l'imagine souvent (M. Fliche). 
rapidement quand il est employé à l'air; au contraire, sous l'eau, il a une durée au moins égale à celle du chêne. Il est utilisé par la menuiserie pour certains usages, et, comme bois de chauffage, recherché pour la boulangerie.

L'aune blanc abonde dans le lit des torrents des Alpes; il se propage sur les berges des cours d'eau, les éboulis et les cônes de déjection, où il ne craint pas les sols couverts de gros galets amenés par les torrents. Également représenté dans le Jura, il ne se rencontre pas dans les sols siliceux et granitiques des Vosges et du Plateau central. Son bois ressemble à celui du précédent.

L'aune vert appartient, en France, à la région alpine; c'est le dernier représentant de la forêt dans les hautes stations, sur le confin des clappes rocheuses et des neiges éternelles. Il recherche les sols siliceux, frais ou humides et réussit sur les pentes les plus rapides, oú il est très propre à fixer le sol et à le garantir contre les affouillements et les éboulements. Ce feuillu est la meilleure essence à propager pour garnir les hauts couloirs des avalanches, car, il peut être recepé souvent et possède la précieuse faculté de se redresser, après avoir été écrasé sous le poids de la neige; il est regrettable que ses exigences trop spéciales au point de vue du sol ne permettent pas de l'utiliser dans les montagnes calcaires, où aucune forme analogue ne se présente pour le remplacer. L'aune vert n'est, à proprement parler, qu'un arbrisseau, dont le bois ne peut servir qu'au chauffage; il est d'ailleurs très utile, dans les hautes stations qu'il habite, pour fournir le combustible nécessaire aux fromageries.

Allures forestières. - Les aunes ne se rencontrent qu'accidentellement dans les futaies; exploités en taillis, ils rendent d'excellents services et, dans des sols trop humides pour beaucoup d'autres espèces, on a un avantagre sérieux à les propager. On les introduit alors par plantation, car les semis, naturels ou artificiels, ne pourrraient réussir au milieu des hautes herbes qui envahissent ces terrains. 


\section{Les bois tendres.}

Le tilleul (1) est un arbre de très grande taille, qui, à l'état isolé, atteint les plus belles dimensions; il se rencontre dans les plaines et sur les coteaux, où il ne dépasse pas l'altitude du chêne. Il recherche les sols frais. - Son bois blanc, léger, mou, de peu de durée, mais d'un travail facile et se gerçant peu, est apprécié des tourneurs et des sculpteurs. C'est un assez médiocre combustible. Le liber de l'écorce du tilleul, fournit la matière filamenteuse connue sous le nom de tille.

Le peuplier tremble (2) se rencontre en France dans tous les sols et dans toutes les stations; de la plaine, il s'élève dans la montagne jusqu'aux altitudes de 1600 à 1700 mètres. Il recherche les sols frais, humides, riches en terreau, pourvu qu'ils ne soient pas trop compacts, et prend alors un développement convenable; partout ailleurs il reste chétif. L'accroissement du tremble est très rapide, mais sa faible longévité ne lui permet pas de fournir de gros arbres; pourtant, dans certaines stations qui lui sont particulièrement favorables, on peut voir des tiges qui mesurent $0^{\mathrm{m}}, 80$ de diamètre. En général, il atteint tout son développement vers cinquante ou soixante ans; passé cet âge, son bois est presque toujours dégradé par la pourriture centrale. Son couvert très léger, et son tempérament robuste ne lui permettent pas de supporter le moindre abri. Il pousse assez mal de souches, mais drageonne très abondamment. - Le bois de tremble est léger, tendre, de consistance homogène; il est recherché pour la fabrication des allumettes et des pâtes à papier. Chauffage médiocre, il est cependant d'un bon usage comme bois de boulangerie.

Les Saules comprennent un grand nombre d'espèces, qui, presque toutes, habitent les bords des grands fleuves et des rivières, ou les terrains submersibles :

Le saule blanc est l'espèce la plus importante du genre par sa taille et sa longévité; croissant à l'état libre dans les sols

(1) Nous réunissons le tilleul à grandes feuilles et le tilleul à petites feuilles : ce dernier est le plus commun en forêt.

(2) Les autres peupliers indigènes sont très rares en forèt. 
frais et fertiles, il peut donner un grand arbre. Il est toujours assez rare dans les forêts, où son couvert léger est peu nuisible aux espèces plus précieuses. Le saule blanc se bouture très facilement. - Son bois, faiblement teinté en rose, est léger et très homogène; il sert à la sculpture et se débite en voliges pour la fabrication des caisses d'emballage. Comme chauffage, il peut être assimilé au tremble.

Le saule marceau est bien plus répandu que le précédent; il accompagne partout le tremble; comme lui, il se bouture assez mal. Sa longévité est d'ailleurs très restreinte. Croissant en liberté, il vit à peine quarante à cinquante ans; englobé dans un massif, il périt vers vingt-cinq ans. - Son bois, plus dur, plus lourd que celui du tremble, présente, employé à l'air, une certaine résistance à l'altération et fournit, pour ce motif, des perches à houblon et des échalas recherchés. C'est un mauvais combustible.

Allures forestières des bois tendres. - En général, les bois tendres ne sont appelés qu'à jouer un rôle secondaire dans le traitement des forêts; ce sont surtout des essences accessoires et de remplissage. Toutefois chacune d'elles a une manière d'être spéciale.

On trouve rarement le tilleul dans les vieux massifs de futaie. Mais, abondant dans certains taillis, il fournit des rejets nombreux et d'une croissance extrêmement rapide dans les débuts; toutefois, sa production moyenne en argent ne dépasse pas celles des espèces plus précieuses, à moins qu'on tire parti de son écorce, comme dans les environs de Chantilly. Sa présence est même nuisible sur les points où l'on cherche une régénération par la semence; il y a souvent lieu, dans ce cas, de l'extirper radicalement, car ses énormes cépées étouffent les semis qui les environnent.

Le tremble se multiplie très abondamment dans les jeunes futaies; mais il est surtout commun dans les taillis, où, par ses nombreux drageons, il envahit quelquefois toutes les surfaces récemment exploitées. Malgré cette extrême abondance, le tremble devient rarement nuisible, car la vigueur de ses drageons s'épuise vite et beaucoup d'entre eux disparaissent des les premières années. On commet souvent la faute de les 
détruire trop tôt, sous prétexte d'amélioration culturale; il est facile, en effet, de constater que leurs tiges grêles et élancées servent de tuteurs et d'abri à celles des espèces plus délicates, qu'elles soutiennent et protègent sans les entraver. En les laissant grossir, on en obtiendra, plus tard, des produits abondants, qui feront largement compensation à des dommages éventuels. En un mot, il en est du tremble comme du bouleau; il faut les compter l'un et l'autre comme remplissage, tant qu'on ne peut obtenir mieux à leur place; sans chercher à les propager, on ne doit pas les expulser systématiquement des forêts; il suffit de les surveiller et d'en tirer 'profit, dès qu'ils deviennent gênants, en tous cas, avant leur complet dépérissement. Le couvert très léger du tremble, sa reproduction pardrageons, le rangent au nombre des essences dont le choix est logique comme arbre de réserve dans les taillis sous futaie, où il ne dépasse pas la dimension de moderne.

Malgré sa longévité assez grande, le saule blanc dépérit de très bonne heure dans les massifs forestiers; c'est lui qui contribue pour la plus forte part à former, dans certaines forêts, la charge de bois sec des délinquants. Dans les campagnes, il est surtout planté sur le bord des ruisseaux, où on le traite en têtard. Il est aussi cultivé pour la production de l'osier. La facilité avec laquelle il se bouture permet de l'utiliser sous forme de plançons pour soutenir les talus et former les barrages vivants dans le lit des torrents.

Le saule marceau est une espèce envahissante au premier chef et qui, par sa croissance rapide et son couvert, assez épais dans le jeune âge, est souvent préjudiciable aux bonnes essences. Il faut alors l'extirper sans ménagement. Le saule marceau peut néanmoins rendre des services, à cause de sa rusticité; on l'emploie avec succès pour former un premier broussaillement dans les terrains pauvres à reboiser.

D'autres saules, dont la taille ne dépasse pas celle des arbustes, sont localisés sur les bords des grands fleuves, dans les zones périodiquement inondées. Exploités sous forme de $m e$ nus taillis, on en tire un parti très avantageux. 


\section{Le sapin.}

Aire et station. - Le sapin constitue, en France, de grandes forêts dans les régions montagneuses des Vosges, du Jura, des Alpes, des Pyrénées et du Plateau Central ; rare dans les colines de la Bourgogne, il manque dans les Maures et l'Esterel et dans les Ardennes, mais on le trouve sur quelques points des collines de Normandie à la limite des bassins de la Seine et de la Loire. Partout, le sapin recherche le climat montagneux et il n'apparaît dans les faibles altitudes (200 ou 250 mètres) que sur les coteaux, au pied de montagnes, du climat desquelles ces derniers participent. Il caractérise le commencement de la région froide qui succède à celle du charme et du chêne.

Le sapin prospère dans les Vosges jusqu'à 1100 mètres, jusqu'à 1300 dans le Jura; il atteint 1500 mètres dans le Plateau central, 1700 dans les Alpes, et près de 2000 dans les Pyrénées. La nature minéralogique du sol lui est indifférente, pourvu que le terrain soit meuble, profond et simplement frais (1).

Tempérament. - Le jeune plant du sapin est très délicat; c'est l'arbre d'ombre par excellence et, plus que toute autre espèce, il conserve la faculté de prendre son essor après avoir été longtemps gêné par le couvert. Il résiste assez bien aux grands froids de l'hiver, mais il est très sensible aux gelées printanières.

Enracinement, fructification. - L'enracinement du sapin est profond. Toutefois cette essence, profitant des crevasses qui sillonnent les sous-sols calcaires, acquiert de fort belles dimensions sur des terrains qu'à première vue on lui aurait jugé contraires. Les sapins du Jura sont là pour en témoigner. La fructification, assez abondante et assez régulière, ne présente point les intermittences et les inégalités que l'on observe chez les pins, les chênes et les hêtres.

Croissance, longévité. - Le sapin est un arbre de première

(1) Ou tout au moins que le sous-sol soit fissuré dans le seṇs vertical. 
grandeur, qui peut parvenir à $\mathbf{4 0}$ mètres d'élévation (1) sur $1^{\mathrm{m}}, 20$ à 2 mètres de diamètre. Il croît rapidement en hauteur jusque vers l'âge de cent à cent vingt ans, en conservant une cime pyramidale aiguë. A un âge plus avancé, l'axe principal cesse de s'allonger et la cime s'aplatit de plus en plus au sommet; c'est alors l'époque de la pleine fructification.

Sa longévité est très considérable.

Bois et ses usages. - Bois de construction et de travail de premier ordre, souvent utilisé pour la fente, il donne un chauffage médiocre, sauf son écorce et ses branches, ou raies, qui sont gorgées de résine et lancent une flamme claire et vive.

Produits accessoires. - L'écorce du sapin renferme une certaine quantité de tanin et pourrait servir à la préparation des cuirs. L'arbre ne fournit que peu de résine; celle-ci est localisée dans l'écorce et on a perdu l'habitude d'en retirer ce produit, qui était connu dans les Vosges sous le nom de térébenthine de Strasbourg.

Allures forestières. - Le sapin, comme toutes les conifères indigènes, ne peut être traité autrement qu'en futaie. Il constitue les forêts les plus sombres et affectionne l'état de massif très serré. Un peuplement de sapin abandonné à luimême comporte toujours plusieurs étages de végétation; le feuillage se continue pour ainsi dire sans interruption, depuis les plus hautes cimes jusqu'au niveau du sol, et le couvert épais qui en résulte entretient la fertilité du terrain, en même temps que l'humidité constante dont cette espèce ne peut se passer. Aussi, le sapin semble-t-il né pour le traitement en futaie jardinée. Du reste, dans toutes les forêts qu'on ne fatigue pas par le pâturage ou par des exploitations abusives, le semis naturel ne fait jamais défaut, même sous les massifs intacts.

Si les circonstances climatériques locales ne s'y opposent pas, le sapin accepte la forme de futaie régulière; mais à cette condition expresse qu'on respecte les différents étages superposés qu'il réclame d'une façon impérative, surtout dans les sols exposés à se dessécher. En effet, quand le sapin a cessé

(1) La hauteur du "Président " dans la forêt de la Joux (Jura) dépasse 49 mètres. 
de croître en hauteur, sa cime s'étale et s'éclaircit; le sol, mal protégé par le couvert d'un peuplement réduit à un étage unique, va sans cesse en se dégradant; il est envahi par une épaisse couverture vivante, au milieu de laquelle la régénération naturelle devient toujours difficile et parfois même impossible.

Sous cette réserve, le sapin peut former de bonnes forêts à l'état pur; mais il est préférable de lui associer d'autres essences, notamment le hêtre et l'épicéa. Le mélange avec les formes qui le précèdent ou qui le suivent en altitude, s'impose dès qu'on approche des limites inférieures ou supérieures de la zone montagneuse relativement étroite qu'il habite. Le sapin fait d'ailleurs excellent ménage avec les feuillus, arbres ou arbustes, dont il recherche le terreau. C'est la raison pour laquelle il envahit les taillis avoisinant la sapinière.

\section{L'épicêa.}

Aire et station. - Bien que très répandu dans les plaines du nord de l'Europe, l'épicéa ne se rencontre spontané en France que dans une zone supérieure à celle du sapin. Assez rare dans les Vosges, où il monte jusqu'à 1300 mètres d'altitude, il couvre des étendues considérables sur les deuxième et troisième plateaux du Jura, jusqu'à 1500 mètres, et acquiert une importance toute spéciale dans les Alpes septentrionales et centrales, oúlil parvient à 2400 mètres. Moins commun déjà dans les Alpes méridionales, il est remplacé dans les Pyrénées par le Pin de montagne, et manque dans le Plateau central. Dans ces différentes régions, l'épicéa recherche surtout les stations froides et fraiches. Malgré son adaptation toute spéciale aux sols calcaires, l'épicéa ne se montre nullement exclusif à cet égard et accepte tous les terrains, même mouilleux.

Tempérament. - Le tempérament de l'épicéa est assez robuste. Son jeune plant ne résiste pas à un ombrage épais. Au contraire, on le voit s'installer en plein découvert, dans l'herbe des clairières ou des pâturages. Toutefois, à cause de son faible enracinement, le léger couvert du bouleau ou du 
sorbier des oiseleurs, l'abri d'une roche ou d'un mur de clôture, celui de quelques broussailles lui conviennent à merveille. Partout, il craint la sécheresse. Il résiste indifférent aux hivers les plus rigoureux et souffre moins que le sapin des gelées printanières. Il présente, sous tous ces rapports, une certaine analogie avec le charme.

Enracinement, fructification. - L'enracinement de l'épicéa consiste essentiellement en une souche dépourvue de pivot et garnie de racines traçantes.

Sur les plateaux calcaires du Jura, il est curieux de voir ces racines ramper sur le sol jusqu'à ce qu'elles rencontrent une fente, une lésine, dans laquelle elles s'engagent aussitôt; elles arrivent a faire ainsi corps avec les blocs de rocher. Cela permet à cette espèce traçante de lutter contre les terribles coups de vent qui balayent ces régions. Souvent, on la voit s'installer dans le bois pourri, sur les souches exploitées, à plusieurs décimètres au-dessus du sol; de là ses racines gagnent la terre en lui donnant pour base une sorte de candélabre renversé. La fructification est sujette à plus d'intermittences et d'irrégularités que celle du sapin et, suivant les contrées, n'est abondante que tous les deux, six et même huit ans; elle se produit normalement à cinquante ans.

Croissance, longévité. - L'épicéa est un arbre de très grande dimension, à tige droite, élancée, pouvant atteindre jusqu'à 50 mètres d'élévation, mais dont le diamètre reste généralement inférieur à celui du sapin. Son couvert est assez épais; il conserve une cime touffue, pyramidale et qui jamais ne prend la forme tabulaire, naturelle chez les sapins qui ont dépassé cent ans.

Sa longévité atteint quatre cents à cinq cents ans.

Bois et usages. - Le bois d'épicéa, plus léger, en général plus blanc que celui du sapin, s'emploie aux mêmes usages et, suivant sa provenance, se paie plus ou moins cher que ce dernier. Il est mou, spongieux, de qualité inférieure dans les stations basses, en raison de la rapidité de sa croissance; vers les limites supérieures de sa zone, au contraire, il acquiert d'excellentes qualités et sa valeur dépasse du quart au cinquième, celle du bois de sapin. C'est ainsi que, dans les 
Alpes, il est toujours préféré à ce dernier, quel que soit l'emploi auquel on le destine. En résumé, bois de construction et de travail de première ordre, il peut même être employé pour la mâture.

Produits accessoires. - L'écorce contient du tanin et sert, dans les régions oủ le chêne fait défaut, à la préparation du cuir. Il faut $10 \mathrm{kilogr}$. d'écorce d'épicéa pour remplacer 3 kilogr. d'écorce de chêne. En France, on a renoncé à extraire la résine de l'épicéa ; cet arbre est, en effet, trop précieux comme bois d'œuvre, pour qu'on lui fasse subir les dégradations que comporte cette pratique, d'ailleurs peu lucrative.

Allures forestières. - Comme le sapin, l'épicéa demande à être maintenu en massif sombre, mais il s'y comporte d'une manière différente; végétant mal à l'état dominé, il se constitue naturellement en un seul étage, auquel le grand nombre des tiges conserve toute sa densité. Grâce à la forme toujours conique de sa cime, il possède, plus qu'aucune autre essence, la faculté de vivre à l'état très serré, ce qui lui permet de former les peuplements les plus riches en matériel.

Il est avantageusement traité en futaie régulière; mais comme il est très exposé à être renversé par le vent, on fera bien de le mélanger avec le hêtre, le sapin ou le mélèze : les peuplements acquièrent ainsi plus de solidité et se régénèrent plus facilement. Il se montre d'ailleurs très envahissant dans les pâtures et les taillis qui avoisinent les forêts résineuses; on dirait que, sous l'influence d'un traitement méthodique, il a des tendances à déserter les grands massifs. A cause de son tempérament robuste, il est une des rares essences pour lesquelles on a pu tenter avec succès la régénération naturelle par coupe à blanc.

Aucune espèce ne supporte mieux la transplantation. La souplesse de son tempérament lui permet de vivre à peu près partout; cette faculté d'adaptation se traduit par de perpétuels changements dans sa forme, changements qui ont donné naissance aux nombreuses races souvent décrites (1).

(1) Brenot, Remarques sur les deux variétés de l'épicéa commun. Paris, Imprimerie nationale, 1878; - Revue des Eaux el Foréts, 1870.

- Dr Schröter, Vielgestaltigkeit der Fitchte. Zurich, 1898. 


\section{Le mélèze.}

Aire et station. - En France, le mélèze n'apparaît spontané que dans les régions élevées des Alpes. Il y vit en massif mélangé avec le sapin, l'épicéa, le pin sylvestre ou le pin de montagne, à partir de 1200 mètres d'altitude. Pur ou mélangé avec le pin cembro, il peut s'élever jusqu'à 2900 mètres. Par contre, on le voit s'installer naturellement, même audessous de 1000 mètres d'altitude, dans les friches mises en défens, quand elles sont exposées au Nord ou à l'Est.

Le mélèze ne marque aucune préférence pour les qualités minéralogiques des sols, pourvu qu'ils soient suffisamment meubles, légers et profonds. L'espace et la lumière lui sont indispensables.

Tempérament. - Le jeune plant de mélèze ne supporte pas le moindre couvert; ce qu'il craint surtout, c'est la sécheresse. Naissant en pleine lumière, sa graine ne peut germer que dans un terrain toujours humide. Le feuillage, qui se renouvelle en totalité chaque année, fournit un terreau mieux constitué que celui d'aucune autre conifère; il ne forme d'ailleurs qu'un couvert assez léger, sous lequel s'installe un gazon continu, généralement pâturé ou fauché.

Enracinement, fructification. - L'enracinement, très solide, se fait par plusieurs racines principales, obliquement et profondément enfoncées, desquelles partent un grand nombre d'autres petites racines plus ou moins traçantes; le pivot véritable s'est oblitéré dès les premières années.

La fécondité du mélèze est précoce et la fructification s'opère assez régulièrement tous les ans; mais la récolte donne toujours une forte proportion de graines vaines.

Croissance, longévité. - La croissance du mélèze, rapide dans sa jeunesse, devient extrêmement lente à un âge plus avancé.

Dans sa station, il peut vivre plus de cinq siècles et donner des arbres énormes. Transporté à de faibles altitudes, sa longévité est fort réduite.

Bois et ses usages. - Le bois du mélèze est d'excellente 
qualité, c'est un des plus précieux que produisent les forêts indigènes; on a pu dire de lui avec raison qu'il est le chêne de la montagne. Dans les Alpes, il passe pour imputrescible dans toutes les conditions d'emploi. Descendu sur les collines par la culture artificielle, il donne encore un très bon bois; malgré la rapidité de sa végétation, il conserve son aubier mince, et son bois parfait reste préférable à celui de toutes les autres espèces résineuses qui pourraient lui être associées.

Produits accessoires. - En France, les forêts de mélèze ne sont pas soumises au résinage. Les feuilles de cette essence sécrètent une gomme particulière, que la médecine utilise, comme purgatif, sous le nom de manne de Briançon L'écorce du jeune mélèze est très riche en tanin.

Allures forestières. - Dans les hautes régions habitées par le mélèze, les peuplements ont à lutter contre la rigueur du climat, l'âpreté du sol, la déclivité des pentes et le voisinage des pâtures. Alors, le traitement d'une forêt est toujours chose fort délicate, car la moindre imprudence peut-être l'occasion de désastres irréparables.

A cause de son extrême besoin de lumière à tous les âges, le mélèze végète mal en étages superposés; les massifs clairs, composés d'arbres de même hauteur, sont de beaucoup préférables. Pour perpétuer cet état, il suffit de mettre scrupuleusement en défens, non seulement les bois très jeunes, mais encore les parties exploitables que l'on veut régénérer. Dès que le découvert est suffisant, le semis s'installe au milieu du gazon peu touffu qui tapisse toujours le sol de la forêt.

A la limite inférieure de son aire, le mélèze se mélange avantageusement avec l'épicéa, le pin de montagne ou même le pin sylvestre. Il accepte alors le traitement jardiné adapté à ces espèces. Conservé en groupe dans les hauts gazons, il abrite le bétail, en même temps qu'il fournit d'excellents produits: aucune espèce ne convient mieux pour la création de prés-bois alpestres. 


\section{Le pin sylvestre.}

Aire et station. - Le pin sylvestre est l'arbre des plaines et des montagnes sablonneuses.

En France, on le trouve spontané dans les Vosges (1), où il peut être cultivé jusqu'à l'altitude de 1100 mètres, dans le Plateau Central jusqu'à 1500 mètres, dans les Alpes jusqu'à 1700 , dans les Pyrénées jusqu'à 2000. Partout il recherche les expositions chaudes. Excepté dans les régions du pin d'Alep et du pin maritime, le pin sylvestre a été propagé comme principale essence de reboisement; en plaine comme én montagne, il a ainsi reçu une très grande expansion artificielle. Sans refuser de croître dans les sols compacts, il préfère de beaucoup ceux qui sont meubles et divisés.

Tempérament. -- Le pin sylvestre a le couvert léger, surtout à un âge avancé ; son jeune plant est des plus robustes ; l'arbre constitué, très avide de lumière, ne comporte pas l'état de massif serré. Il exige de l'espace pour développer en largeur une cime qui ne peut rester feuillée sur une grande hauteur; aussi est-il indispensable de lui en ménager, sans quoi l'on s'expose à ne produire que des arbres grêles, qui se plient sous leur propre poids, sous celui de la neige ou du givre, et à voir, chaque année, les peuplements se clairiérer d'eux-même par la rupture des tiges dont la cime est trop réduite. Cette essence n'a rien à redouter des gelées d'hiver, ni de celles du printemps; par contre, plus que toutes les autres, elle est exposée aux ravages des insectes et des champignons : dangers qui sont surtout à redouter en dehors de ses stations naturelles. Le pin sylvestre craint aussi les hauts sommets exposés à des vents violents; alors, il semble résister tant qu'il est assez jeune pour passer l'hiver sous la neige; mais, dès que, en grandissant, sa cime dépasse cette zone, il perd ses feuilles et ne tarde pas à périr, plutôt sous l'action mécanique des vents, que par l'effet d'un climat trop rigoureux .

(1) Dans les Vosges, l'origine spontanée du pin sylvestre n'est certaine qu'en dehors de nos frontières. 
Enracinement, fructification. - L'enracinement est assez variable; en sol léger et profond, le pivot se développe beaucoup et forme la partie essentielle de la racine jusque vers trente ou quarante ans; passé ce terme, les racines latérales s'accroissent en vigueur, mais ont aussi une tendance à s'enfoncer. Dans les terrains liants, au contraire, le pivot s'arrête de très bonne heure et des racines, peu profondément situées, ne lardent pas à le remplacer.

La fécondité du pin sylvestre est très précoce et l'on voit des arbres isolés qui, à quinze ans, produisent déjà des cônes et de bonnes graines; régulièrement, dans les massifs, ce n'est guère que vers cinquante ans, et même au delà, qu'elle se prononce. En général, on trouve au moins quelques cônes chaque année, mais il n'y a abondance que tous les trois à cinq ans.

Croissance, longévité. - Dans ses stations naturelles, le pin sylvestre est un arbre de grande taille, qui atteint 30 ou 40 mètres d'élévation, mais dépasse rarement 4 mètres de circonférence et reste, à cet égard, bien en arrière du sapin. Sa croissance, très lente dans le Nord, est infiniment plus rapide dans les climats tempérés.

Sa longévité est également très grande. Dans les plaines où il a été introduit, il vit beaucoup moins longtemps.

Bois et ses usages. - Le bois du pin sylvestre, de toute première qualité sous les climats septentrionaux et montagneux, devient médiocre, au fur et à mesure qu'un ciel plus doux lui donne une croissance plus rapide. Dans ce dernier cas, la proportion d'aubier est considérable, relativement à celle du bois parfait, qui, lui-même, bien que d'assez bonne qualité, est loin de valoir le bois connu dans le commerce sous le nom de sapin rouge, et qui n'est autre que le pin sylvestre de Norwège ou de Finlande. Comme chauffage, il est meilleur que le sapin et l'épicéa; il est surtout recherché par la boulangerie. Ainsi que la plupart des résineux, il entre aujourd'hui dans la fabrication des pâtes à papier.

Produits accessoires. - On ne résine pas le pin sylvestre, autrement qu'en délit. Ses souches, distillées en vase clos, donnent du goudron et du charbon de bonne qualité. 
Allures forestières. - Le pin sylvestre doit être conduit en futaie régulière. En massif pur et dans les stations convenables, les peuplements jeunes entretiennent assez bien le sol jusque vers l'âge de vingt-cinq à trente ans; plus tard, l'état superficiel se dégrade et, sous les vieux massifs purs, la régénération naturelle devient difficile. C'est ce qui explique pourquoi, dans les forêts fatiguées, on est souvent obligé d'avoir recours à la régénération artificielle, qui s'obtient d'ailleurs facilement, soit par plantations, soit par semis. Le pin sylvestre se mélange naturellement avec le hêtre, le sapin ou l'épicéa, au milieu desquels, grâce à sa naissance rapide, il se maintient à l'état dominant et fournit d'excellents produits. Ces mélanges sont malheureusement beaucoup trop rares en France, et, dans bien des circonstances, on aurait intérêt à les créer par voie artificielle. Dans la haute montagne, il accepte un jardinage adapté à son tempérament.

Souvent cette essence se rencontre dans les sols trop pauvres pour qu'on puisse lui associer aucune autre espèce de grande taille; dans ce cas, il est indispensable de respecter scrupuleusement toute la végétation arbustive qui buissonne à ses pieds. Qu'ils soient purs ou mélangés, les pins sylvestres sont faciles à traiter; il suffit, à tous les âges, de ménager aux plus belles tiges l'espace nécessaire pour développer librement leur cime.

La frugalité de cette forme et sa faculté de s'adapter aux conditions les plus diverses, en font une espèce précieuse entre toutes pour la mise en valeur des terrains incultes, dans les régions de collines et de basses montagnes.

\section{Le pin de montagne ou pin à erochets.}

Aire et station. - Dans les Alpes et, surtout, dans les Pyrénées, le pin de montagne constitue, seul ou mélangé, des forêts étendues. Dans ces deux stations, il apparaît vers 1500 mètres, s'élève en massif jusqu'à 2200 et se maintient même à 2500 mètres d'altitude. Jamais il ne descend dans les plaines, même dans les plus septentrionales (1).

(1) Le pin de montagne existe sur quelques sommets des Hautes- 
Il croît sur tous les sols, qu'ils soient secs, humides, marécageux ou tourbeux. Outre les qualités de son bois, cette propriété de végéter dans les conditions où aucune autre essence ne pourrait se maintenir, le rend précieux pour assurer la permanence de l'état boisé dans les régions alpestres.

Tempérament. - Dans les hautes stations oú il est cantonné, son jeune plant ne demande aucun abri; néanmoins le pin de montagne résiste mieux que le pin sylvestre à l'action du couvert, sa cime reste beaucoup plus longue, et il peut reprendre une belle végétation une fois qu'il a été dégagé. Le feuillage de l'arbre est assez épais.

Enracinement, fructification. - L'enracinement est composé de plusieurs maîtresses racines traçantes; on n'y remarque pas de pivot principal.

La fructification se produit de bonne heure, vers dix ans, et se maintient abondante et continue.

Croissance, longévité. - La croissance du pin de montagne est lente, jamais il n'atteint les dimensions d'arbre de première grandeur; cependant, les belles formes de sa tige et la régularité de ses anneaux ligneux en font une espèce intéressante à tous égards. Sa longévité peut dépasser deux cents ans.

Bois et ses usages. - Le pin de montagne est estimé pour la charpente et le sciage et, à l'exception du mélèze, on le préfère, dans les Alpes, aux autres essences résineuses. Le grain de son bois est très fin et très égal; aussi se fend-il et se travaille-t-il très bien. Comme chauffage, il est aussi plus apprécié que les autres conifères.

Allures forestières. - Le pin de montagne offre la plus grande analogie avec l'épicéa; cependant on ne rencontre presque jamais ces deux espèces associées; au contraire, le pin de montagne semble se substituer complètement à l'épicéa, dès que le climat devient trop sec pour ce dernier. Quoi qu'il en soit, l'un et l'autre peuvent être traités d'une façon identique, soit à l'état pur, soit en mélange avec le sapin ou le mélèze.

Vosges, et dans les tourbières du Jura. Mais son importance forestière $y$ est à peu près nulle. 
Dans les hauts couloirs, il maintient l'état boisé, grâce à sa forme en candélabre et à ses branches flexibles, qui se courbent sous la neige. Il y faut abandonner ces massifs à leur rôle de forêts de protection.

\section{Le pin maritime.}

Aire et station. - Le pin maritime a son maximum d'expansion dans les Landes et sur les côtes de l'Océan jusqu'à Bayonne. Il existe le long des Pyrénées, et se retrouve abondant sur le littoral méditerranéen, dans les Maures et l'Esterel, et en Corse ou il atteint l'altitude de 1000 mètres. Il est rare en Algérie. La culture l'a répandu dans tout l'ouest de la France, et même dans le centre (Sologne). Cette essence calcifuge accepte tous les sols, même les plus médiocres, pourvu qu'ils soient siliceux.

Tempérament. - Arbre de pleine lumière, à couvert léger, le pin maritime résiste très bien à la chaleur et à la sécheresse. Il supporte mal des froids prolongés et dépassant $-15^{\circ}$.

Enracinement, fructification. - Les racines sont développées, pivotantes et traçantes à la fois. Cette disposition, jointe à une croissance très rapide dans la jeunesse et au peu d'exigence de l'espèce à l'égard de la fertilité du sol, a fait choisir avec succès le pin maritime pour fixer les sables mouvants des dunes et pour boiser ceux des Landes.

La fructification est précoce ; dans les sables des dunes, elle apparaît quelquefois à quinze ans, mais alors les graines sont souvent vaines. Elle devient très abondante et presque continue vers quarante ou cinquante ans.

Croissance, longévité. - Le pin maritime est un arbre de grande taille et de végétation rapide, longtemps soutenue, qui peut, à cent ans, atteindre plus de 3 mètres de circonférence; il parvient à de plus grandes dimensions encore à la faveur de sa longévité plusieurs fois séculaire et arrive à 30 mètres de haut sur 4 à 5 mètres, et même plus, de tour.

Bois et ses usages. - Le bois du pin maritime est à grain grossier; ses accroissements sont épais et très apparents. Purgé de son aubier, il donne des bois de construction et 
de sciage de qualité moyenne. Le gemmage diminue considérablement le développement des arbres et enlève toute homogénéité au bois dans la région des carres; il faut néan-

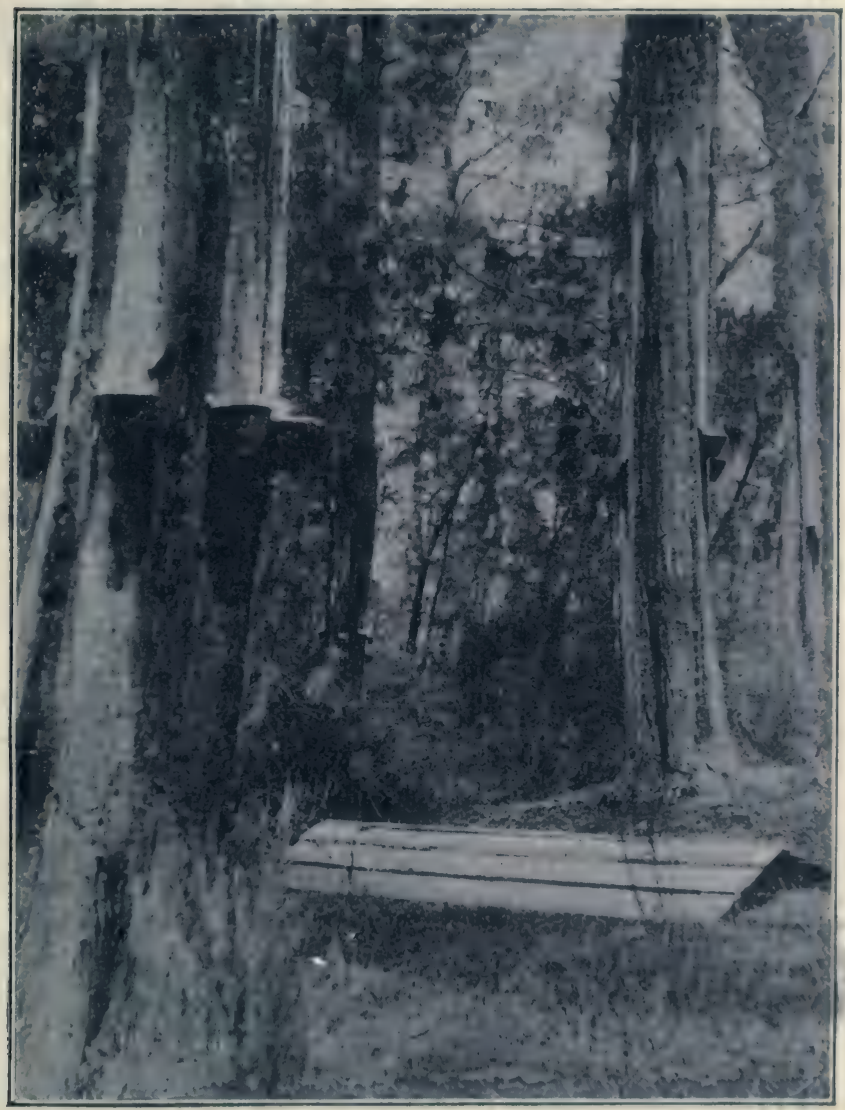

Fig. 28. - Vieux pins maritimes gemmés. - Pots, système Hugues, recueillant la résine qui découle des carres; - par terre : un barcous, réservoir à résine, recouvert d'un abri en planches. (D'après une photographie achetée au commerce.)

moins reconnaître que cette opération, en ralentissant la croissance, donne, dans les portions restées intactes, un bois plus lourd, plus résineux, beaucoup plus résistant et plus 
durable. C'est un combustible très recherché par la boulangerie; dans les foyers ouverts, il a l'inconvénient d'éclater et de projeter au loin des étincelles.

Produits accessoires. - Surtout exploité au point de vue de la gemme (fig. 28), le pin maritime fournit en résine un revenu qui peut varier de 25 à 40 francs par hectare.

Allures forestières. - Dans ce cas, le pin maritime est soumis à un traitement spécial; en toute autre circonstance, il doit être conduit comme le pin sylvestre, peut-être même en massif plus clair. Il se régénère très facilement, à cause de sa fertilité précoce et abondante, aussi bien que par sa faculté de s'installer sur les sols nus.

\section{Le pin d'Alep.}

Aire et station. - Essence méditerranéenne au premier chef, très sensible aux gelées d'hiver, le pin d'Alep reste, en France, confiné sur les terrains calcaires des régions chaudes de la Provence. Il prospère sur les versants rocheux les plus dépourvus de terre végétale, les plus brûlés par le soleil.

Tempérament. - Son jeune plant est très robuste, son couvert très léger; sa résistance considérable à la sécheresse en fait un arbre des plus utiles.

Enracinement, fructification. - L'enracinement est pivotant et, en même temps, largement développé; il reste parfois superficiel, au détriment de la bonne végétation de l'arbre. La fructification est précoce et abondante; l'arbre est caractérisé par la persistance des cônes ouverts, qui restent indéfiniment attachés aux rameaux.

Croissance, longévité. - Le pin d'Alep a une croissance assez rapide; cependant, il ne dépasse pas les dimensions de seconde grandeur; vers vingt ans, il forme un arbre de tige grêle, flexueuse, dont la végétation se ralentit; à un âge avancé, la cime s'étale largement et prend la forme en parasol, analogue à celle du pin pinier.

Bois et ses usages. - Son bois, d'assez médiocre qualité, sert néanmoins dans la menue charpente, et fournit une grande quantité de sciage, employé à la confection de caisses 
d'emballage ou de tonneaux pour le transport des matières sèches. C'est un combustible flambant bien et recherché dans certaines usines.

Allures forestières. - Le pin d'Alep se rencontre peu en massif pur. Le plus souvent, il constitue des forêts livrées au pâturage, en mélange avec le chêne yeuse et d'autres espèces feuillues. On exploite ces dernières en taillis, et on réserve les pins pour les réaliser dès qu'ils ont atteint des dimensions convenables. Dans ces conditions, le pin d'Alep se régénère très bien; il mériterait cependant, à cause de sa rusticité et des produits qu'il donne, un traitement plus rationnel.

\section{Les pins larieios.}

Le pin laricio a donné naissance à des races diverses, comme le laricio de Corse, le laricio des Cévennes, celui des Pyrénées, spontanés dans ces trois localités; - comme le pin de Calabre et le pin noir d'Autriche, artificiellement introduits en France.

Le pin laricio de Corse, que nous prendrons pour type, ne se rencontre que dans cette île; il habite les régions montagneuses, où sa station commence vers 1000 mètres, c'est-àdire au-dessus de celle du pin maritime et s'élève jusque vers 1700 mètres. Il recherche les sols graveleux qui proviennent de la décomposition des granits.

Le tempérament du pin laricio est loin d'être aussi robuste que celui du pin sylvestre; son couvert est assez épais et, dans les hautes altitudes qu'il habite, le semis naturel s'installe et résiste sous le couvert des grands arbres. Il supporte assez bien le froid, même dans les régions septentrionales de la France. Cependant, l'hiver de 1879-1880 a fait périr, en grand nombre, ceux qu'on y avait introduits.

L'enracinement est généralement faible et pivotant dans l'origine; il n'est représenté, plus tard, que par quelques racines traçantes, peu allongées comparativement aux dimensions de l'arbre.

La fructification se produit dans des conditions analogues à celles du pin sylvestre qui se trouverait dans les mêmes stations. 
Le pin laricio de Corse est un arbre de première grandeur, qui parvient quelquefois à une hauteur de 45 mètres et à une circonférence de $5^{\mathrm{m}}$ à $6^{\mathrm{m}}$. Ja tige, bien plutôt cylindrique que conique, se dépouille rapidement de ses branches inférieures, et reste complètement nue jusqu'au-dessous de la cime, qui, dès quatre-vingts ou cent ans, est courte, aplatie, peu développée et formée de quelques grosses branches irrégulièrement ramifiées. L'accroissement en diamètre est peu rapide; vers cent cinquante ans, il se ralentit encore et devient très faible; sa longévité dépasse cinq cents ans.

Le pin laricio de Corse, caractérisé par l'épaisseur de son aubier, possède un bois parfait, dont les qualités toutes spéciales le font rechercher pour la construction, malgré son poids exagéré.

Bien que ce pin soit. assez riche en résine, il ne peut pas être gemmé avantageusement.

Le laricio des Cévennes est tortueux et sans élévation sur les sols peu profonds du calcaire corallien ou du grès houiller de l'Hérault, du Gard et de l'Ardèche, où il est, en outre, fatigué par la violence des vents de la vallée du Rhône, mais il s'élance dès que le terrain devient frais et profond. M. l'Inspecteur-Adjoint Calas l'a trouvé aux environs de Confolens (Pyrénées-Orientales), seule station française de laricio dans les Pyrénées françajses. Il ne semble pas, dès lors, qu'il y ait lieu de faire une race distincte du laricio des Pyrénées, connu sur le versant Espagnol de la chaîne.

Le pin laricio d'Autriche, ou pin noir, est fréquemment employé, pour sa rusticité, dans les boisements des terrains calcaires; il donne des détritus abondants, qui améliorent le sol. Son introduction et sa culture en France, de date relativement récente, ne permettent pas de juger comment se comporteront des massifs âgés, en état de donner du bois de service. En Autriche, notamment dans le Wienerwald, ses produits sont préférés, pour tous usages, à ceux du pin sylvestre croissant dans les mêmes conditions. Il y est de plus soumis au gemmage.

Il existe dans le domaine des Barres-Vilmorin (Loiret) des massifs de laricio de Calabre, aussi remarquables par leur 
rusticité que par la beauté de leur port, l'exiguilté et la régularité de leurs branches, la forme étroite, allongée et aiguë de leur cirne. Ce serait une race intéressante à propager.

Allures forestières. - Le pin laricio de Corse, n'a pas pas encore été soumis depuis assez longtemps à un traitement méthodique, pour qu'on soit bien fixé sur la manière de le conduire. Néanmoins, toutes les races de pins laricios affectent plutôt les allures de l'épicéa que celles du pin sylvestre. Ils semblent donc pouvoir être traités sans inconvénients à la façon de la première de ces essences; c'est d'ailleurs ainsi qu'on procède, pour le pin noir, dans son pays d'origine.

\section{Le pin cembro.}

Aire et station. - Le pin cembro est peu répandu en France, il croît uniquement dans les Alpes, en mélange avec le mélèze, le pin de montagne et l'épicéa ; c'est le dernier des arbres résineux de la montagne: au-dessus de lui, la végétation arborescente n'est plus représentée que par un petit nombre d'espèces feuillues, dont la plus intéressante est l'aune vert. Il s'accommode de tous les terrains, pourvu qu'ils soient assez profonds et frais.

Tempérament. - Le jeune plant craint surtout la sécheresse, l'arbre constitué a le couvert assez épais, mais les branches restent, en général, ccurtes, et la projection de la cime sur le sol est réduite à très peu de chose.

Enracinement, fructification. - L'enracinement est largement développé; il se fait, dans la jeunesse, par un pivot et de fortes racines latérales; vers quinze ou vingt ans, le premier s'atrophie et les secondes seules continuent à s'accroître avec vigueur, en traçant au loin.

La fructification commence vers cinquante ans et n'est abondante que tous les quatre à six ans.

Croissance, longévité. - La croissance du pin cembro est extrêmement lente; néanmoins, par sa très grande longévité, cet arbre parvient à d'assez fortes dimensions en grosseur, mais sa hauteur dépasse rarement quinze ou vingt mètres. 
Il est du reste remarquable que sa croissance reste la même lorsqu'on le cultive dans les régions basses; il est bien différent en cela du mélèze, qui, dans de semblables circonstances, se développe avec une rapidité extraordinaire.

Bois et ses usages. - Son boís est tendre et peu recherché pour les constructions; mais, à cause de la lenteur de sa croissance, il est d'une homogénéité parfaite, et, comme tel, très recherché pour la fabrication des petites figurines en bois sculpté, qui forment une des industries des hautes vallées de la Suisse.

Produits accessoires. - Le pin cembro ne se résine pas. Sa graine, assez grosse et remplie d'une amande comestible et savoureuse, se vend sur les marchés du Briançonnais.

Allures forestières. - Le pin cembro ne comporte pas d'autre traitement que celui que la nature veut bien lui donner. Cependant, s'il était mieux conservé, il rendrait encore d'importants services pour la protection des massifs inférieurs; il maintiendrait le sol, arrêterait les avalanches et servirait de refuge au bétail. Malheureusement, cette essence tend à disparaître sous la hache du montagnard, qui l'accuse à tort d'occuper une place exclusivement réservée à l'herbe. C'est à peine si, dans les Alpes françaises, on rencontre encore quelques rares peuplements de Cembro à qui on puisse donner le nom de forêt. Ce ne sont même, le plus souvent, comme dans la forêt des Ailles (Briançonnais), que de petits massifs disposés en la forme de prés-bois.

Le pin cembro n'accepte le mélange avec l'épicéa et avec le mélèze qu'à l'état de massif clair. 


\title{
CHAPITRE III \\ LES PEUPLEMENTS
}

\author{
ARTTICLE PREMIER
}

\section{GÉNÉRALITÉS}

\begin{abstract}
Définition. - Genèse d'un peuplement. - Situation particulière du peuplement forestier.
\end{abstract}

Définition. - On nomme peuplement une association de végétaux ligneux vivant sur un espace déterminé de terrain forestier.

Genèse d'un peuplement. - De quelle manière les peuplements se sont-ils formés sur les terres vierges, à l'aurore des époques géologiques? On peut s'en rendre comple en suivant la série des phénomènes qui accompagnent, sous nos yeux, l'installation de la forêt sur les rochers nus, qu'il s'agisse de moraines granitiques, d'éboulis calcaires, ou de cônes de déjection accumulés par le temps aux pieds d'une falaise. L'algue et le lichen apparaissent les premiers sur la pierre nue, qu'ils attaquent et désorganisent comme le font, d'autre part, les actions atmosphériques. Les débris de ces végétaux inférieurs se mélangent aux éléments sableux provenant de l'usure et constituent la première terre végétale, que le vent et les pluies emploient à combler les vides béants entre les blocs de rochers. Alors s'installent les plantes d'un ordre plus élevé, d'abord les espèces herbacées annuelles ou vivaces, puis les espèces ligneuses, arbrisseaux et arbustes, et enfin les arbres.

A partir de cet instant, le peuplement s'organise, et de proche en proche, en gagnant sur l'espace, il devient la forêt.

Situation particulière du peuplement forestier. - Cet état de peuplement, dont la situalion est perçue d'instinct au 
moment où l'on quitte les vergers ou autres terrains plantés d'arbres, pour pénétrer sous les ombrages forestiers, crée un être à part, un organisme complexe, dont l'individualité s'affirme dans ses fonctions, comme dans son évolution.

Les fonctions du peuplement se rapportent aux effels qu'il produit : d'une part sur les arbres qui le composent, d'autre part sur le sol qui le nourrit; c'est après avoir étudié cette double action, que nous parlerons de l'évolution.

ARTICLE II

\section{AGTION RÉGIPROQUE DES ARBRES LES UNS SUR LES AUTRES}

Les peuplements dans la forêt sauvage. - Perturbations apportées par l'intervention de l'homme. - Origine des peuplements. - Leur forme. - Leur consistance. - Leur constitution en étages de végétation. - Leur composition; avantages des peuplements mélangés. - Mélanges rationnels. - Le hêtre. - Conduite des peuplements mélangés. - État de végétation des peuplements.

\section{Les peuplements dans la forêt sauvage. - Dans la forêt} primitive, dans la forêt vierge, les arbres atteignent le dernier terme de leur longévité; ils ne tombent que par vétusté et enrichissent le sol de leurs débris. Partout se trouvent des sujets fertiles, dont les graines se disséminent à profusion sur une terre toujours prête à les recevoir. En sorte que, sur chaque point découvert par les hasards du destin, l'individu doué de la plus grande énergie vitale parvient, à son tour et à son heure, à se rendre maître de la place qu'il occupera jusqu'à la fin de son existence. Naturellement, suivant les climats et, sous chaque climat, suivant les stations, telles ou telles essences seront prédominantes. Mais il y aura place pour toutes les espèces de la région, qu'elles soient d'ombre ou de lumière. De là, cette variété sans fin dans l'aspect de la forêt sauvage, ces éternels changements de situation et de groupement dans une liste plus ou moins longue d'espèces spontanées, changements qui prendraient bien d'autres proportions encore si, au lieu de limiter nos observations à des espaces de temps assez courts pour être comparés à la vie humaine, on voulait tenir compte des modifications plus 
profondes qui ont accompagné les évolutions géologiques du globe.

\section{Perturbations apportées par l'intervention de l'homme.}

- Mais, dès que l'homme est intervenu, variant ses procédés d'exploitation de façon à approprier les produits de la forêt à la diversité de ses besoins, il a modifié les actions naturelles. Le traitement appliqué à chaque forêt varie avec les essences cultivées et avec la nature des marchandises que l'on se propose d'en tirer : bois à brûler, bois de travail, écorces, résines, etc... Il s'établit, dès lors, entre le traitement et la manière d'être du peuplement, une relation nécessaire et constante.

Ces effets du traitement se manifestent sur l'origine, la forme, la consistance, la composition et l'état de végétation du peuplement et lui donnent le type dans lequel il se maintiendra.

Origine des peuplements. - L'origine d'un peuplement peut être naturelle ou artificielle : naturelle, quand la régénération est obtenue sous l'influence des seules actions de la nature, artificielle, lorsqu'elle provient de plantations ou de semis exécutés de main d'homme.

En France, le principe de la régénération naturelle est généralement appliqué; aussi, l'origine de la grande majorité des peuplements se rattache-t-elle à l'une des causes suivantes : soit la dissémination naturelle des graines, qui donne naissance à des peuplements formés de brins de semence; - soit la reproduction par les axes, qui rajeunit les peuplements au moyen des rejets de souches ou des drageons.

Quel que soit l'âge des peuplements, on est convenu de les appeler des futaies (1) dans le premier cas; des taillis simples dans le second. On a donné à chacun de ces groupes fondamentaux le nom de régime, qui est synonyme de: mode de régénération. On dit : le régime de la futaie, le régime du taillis simple.

(1) Autrefois, en France, on appelait futaies tous les gros arbres, tous ceux dont la tige mesurait à hauteur d'homme trois pieds de tour, quelle que fût leur origine. La définition actuelle date de la publication du magistral traité de MM. Lorentz et Parade : la Cullure des hois. 
Lorsqu'on cherche à réunir sur une même surface les avantages du régime de la futaie à ceux du régime du taillis simple, on obtient des peuplements mixtes, qui, dans la pratique, acquièrent une importance suffisante pour qu'on ait cru devoir en faire un troisième régime, celui du taillis sous futaie, dit aussi régime du taillis composé.

Forme des peuplements. - Dans chacun de ces régimes, suivant qu'on fait porter les exploitations sur des surfaces continues ou sur des tiges considérées individuellement, ou bien que l'on adopte toute autre combinaison pour récolter les produits, on applique des modes de traitements différents. On appelle forme d'un peuplement le facies qu'il revêt sous l'influence du traitement qu'on lui applique.

A ce point de vue, les peuplements se divisent en deux classes principales : ceux qui sont composés de tiges ayant sensiblement même âge et, par suite, mêmes dimensions - et ceux qui sont formés de tiges de différents âges et conséquemment de hauteurs et de grosseurs différentes. Les premiers sont les peuplements d'un seul âge ou uniformes; les seconds sont dits d'âges multiples ou inégaux; on les appelle encore mêlés, en ce. sens que les plus jeunes sujets et les plus âgés sont confusément agencés sur des espaces restreints.

Quand une série (1) de futaie est composée d'une suile non interrompue de peuplements ayant même âge chacun, on dit qu'elle est traitée en futaie régulière ou futaie pleine; de même pour la série de taillis, qui sera traitée en taillis simple régulier. - Quand la série de futaie est composée de groupes de peuplements d'âges mêlés, on dit qu'elle est jardinée; dans les mêmes conditions un taillis simple est fureté. Le jardinage et le furetage sont les modes de traitement qui donnent naissance à ces formes. Le régime du taillis composé ne comporte nécessairement que des peuplements mêlés.

Quand, pour des causes d'un ordre cultural ou économique,

(1) On appelle série d'exploitation ou simplement série, toute forêt ou portion de forêt, disposée de façon à fournir, durant la révolution, une série de coupes successives et annuelles (Lorentz et Parade, Culture des bois). La notion de la série, conception toute française, doit être attribuée à ces auteurs. 
on est amené à changer le mode de traitement appliqué jusqu'alors à une forêt, on lui fait subir un traitement temporaire, qui prend le nom de conversion, quand on passe d'un régime à un autre régime, - et de transformation, quand on change simplement le mode de traitement dans le même régime.

Consistance des peuplements. - La consistance d'un peuplement se rapporte au nombre des tiges qu'il renferme et à la densité de son feuillage.

Le nombre des tiges peut varier à l'infini en raison de l'âge des peuplements, des essences qui le composent et de la fertilité locale. Mais, en tenant compte de ces différents éléments, on peut admettre que, pour chaque état particulier, il existe un nombre maximum de tiges qui n'est jamais dépassé. Plus on se rapproche de ce maximum, plus le peuplement est plein.

On dit qu'un peuplement est un massif, dès que les branches des arbres se touchent sans être agitées par le vent. Le massif est serré, quand les branches s'entrelacent. Dès que les cimes des arbres sont isolées, l'état de massif n'existe plus: on obtient alors un peuplement d'arbres isolés ou clairplanté.

On nomme clairières les surfaces de peu d'étendue peuplées d'arbres épars et, dans lesquelles, sous l'influence d'un couvert insuffisant, l'état superficiel du sol est dégradé. Les vides sont des espaces plus grands, entièrement dépourvus de bonnes essences et tout au plus couverts de mort bois. Le freuplement est clairièré, s'il y existe de nombreuses clairières; entrecoupé, quand il présente des vides.

La densité du feuillage, par suite l'obscurité plus ou moins grande du couvert, dépend avant tout de la frondaison des essences du massif. A ce point de vue, chaque espèce se constitue d'une manière différente et, dès que l'état maximum de densité qu'elle comporte est atteint, il disparaît autant de feuillage dans le dessous qu'il s'en produit dans le dessus. Nul n'ira chercher, sous les pins sylvestres ou les bouleaux, les épais ombrages des sapins ou des hêtres (fig. 29 et 30). Entre ces types extrêmes, tous les intermédiaires existent. 


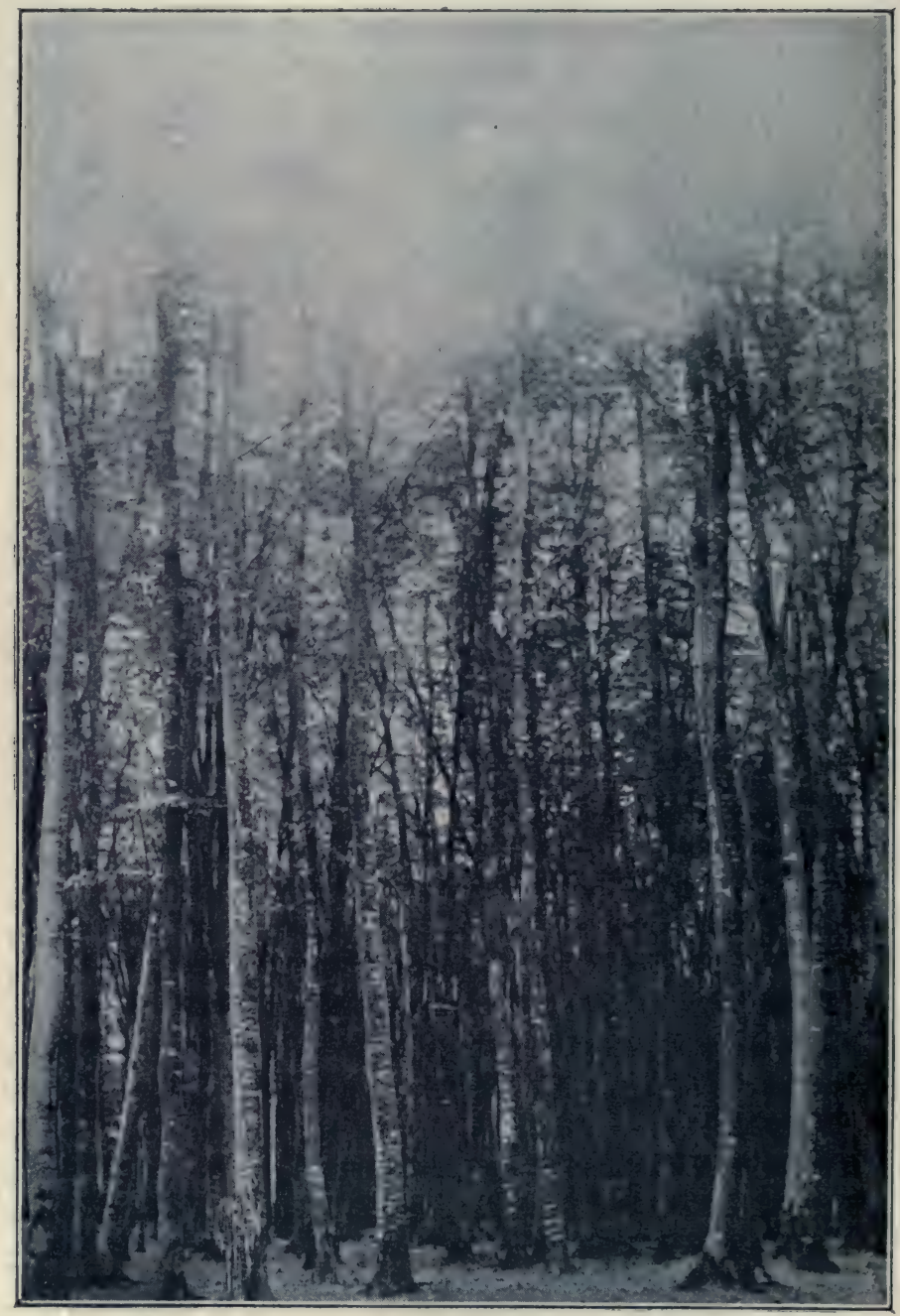

Fig. 29. - Vieille futaie de hêtre en massif très serré, canton de la Mare-aux-Bourres (Lyons-la-Forêt). (Photographie de M. J. George.) 
ACTION DES ARBRES LES UNS SUR LES AUTRES.

D'ailleurs, en sol fertile et sous un climat doux, la végétation des arbres est plus luxuriante, les limbes des feuilles

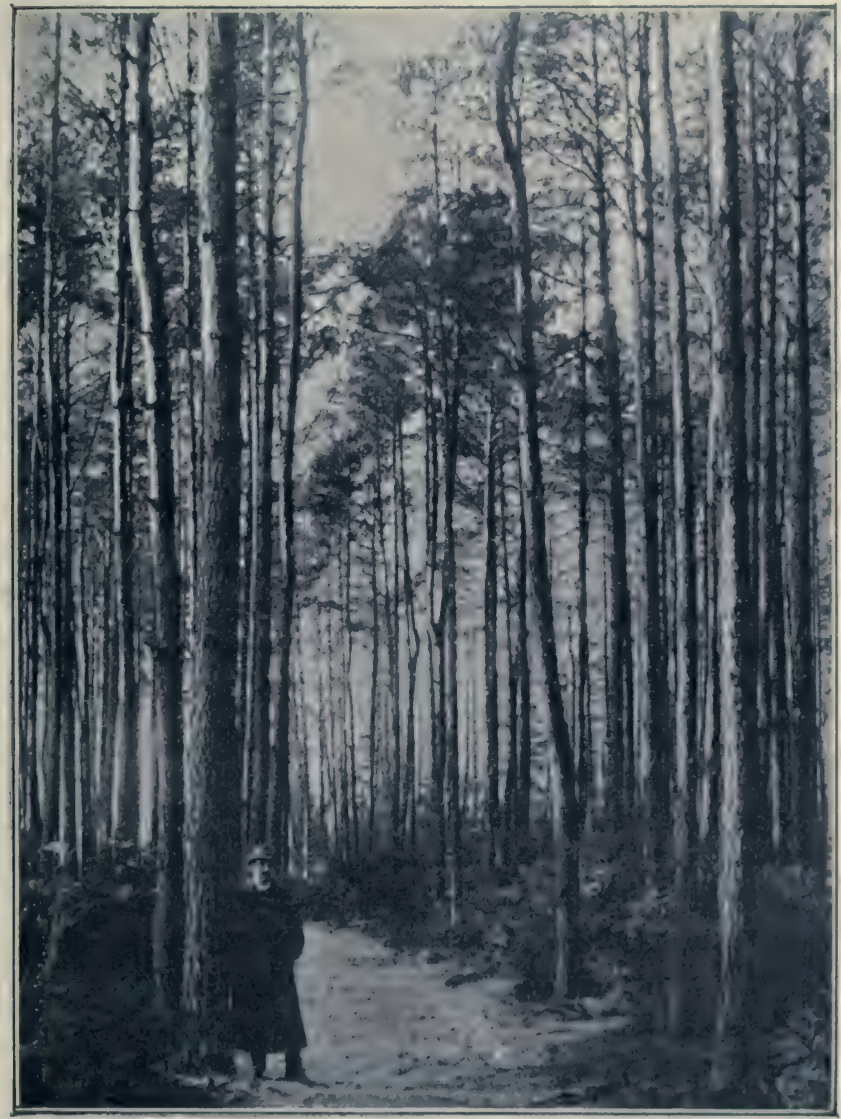

Fig. 30. - IJaut perchis de pins sylvestres formant massif clair, canton des Rappes, forêt de Bertrichamps (Meurthe-et-Moselle. (Photographie de M. l'Ingénieur Guéroult.)

sont plus développés, par suite le couvert est plus dense, plus noir, que dans les sols médiocres et sous les climats rudes.

Étages de végétation. - Jusqu'alors nous avons considéré 
le peuplement à l'état simple, c'est-à-dire, formé par un seul étage de végétation, fourni par des arbres semblables les uns aux autres, dont les cimes s'étalent parallèlement au sol dans une même zone de hauteur. Mais, souvent, on rencontre sur un même point plusieurs de ces étages ; il est rare, cependant, que ces peuplements composés en présentent plus de deux. On appelle, alors, étage dominant, celui qui est formé par la masse des cimes les plus élevées, dont les rameaux s'épanouissent en pleine lumière ; tous les sujets dont les cimes, subordonnées aux précédentes, végètent ou languissent en dessous d'elles, constituent l'étage dominé ou sous-étage.

Entre l'étage dominant et l'étage dominé, il s'établit une relation nécessaire quant à la densité ; le second, en effet, ne vit que de la lumière tamisée à travers le feuillage de l'étage supérieur; donc, plus celui-ci sera léger, plus la végétation basse pourra se développer.

On constate que les peuplements de même âge, quelles que soient leur origine et les essences qui les composent, ont toujours une tendance à se constituer en un étage unique, audessous duquel les longues tiges nues se profilent dans des espaces vides de feuillages. Cette tendance à l'uniformité est d'autant plus marquée que la station est plus fertile. Au contraire, plus les conditions sont mauvaises, moins les arbres affamés supportent l'état serré; au fur et à mesure que le massif s'éclaircit, le sol se couvre de générations nouvelles et l'uniformité originelle disparaît. C'est une indication dont il est utile de se souvenir, quand il s'agit de choisir le mode de traitement applicable à une forêt donnée. Quoiqu'il en soit, cette égalité absolue, qui, pendant longtemps, a été considérée comme le type idéal vers lequel devaient tendre tous les efforts des forestiers, doit autant que possible être prévenue et évitée. Car, sous son couvert insuffisant et qui va sans cesse en s'éclaircissant avec l'âge, le sol se tasse, se dessèche, les vents balayent les feuilles mortes et avec elles disparaissent les éléments qui le fécondent.

La présence de sous-étages, ne fussent-ils représentés que par les plus humbles sous-bois, prévient ces accidents. Aussi l'hygiène de la forêt commande-t-elle de conserver scrupuleu- 
sement cette végétation intermédiaire ou buissonnante, et, lorsqu'elle a disparu sous l'influence de pratiques mauvaises, telles que pâturage ou nettoiements inutiles, d'en provoquer la reconstitution par les moyens naturels, ou même artificiels. Telle est l'importance attribuée à ces sous-étages, que nous n'hésitons pas à considérer les sous-bois eux-mêmes comme

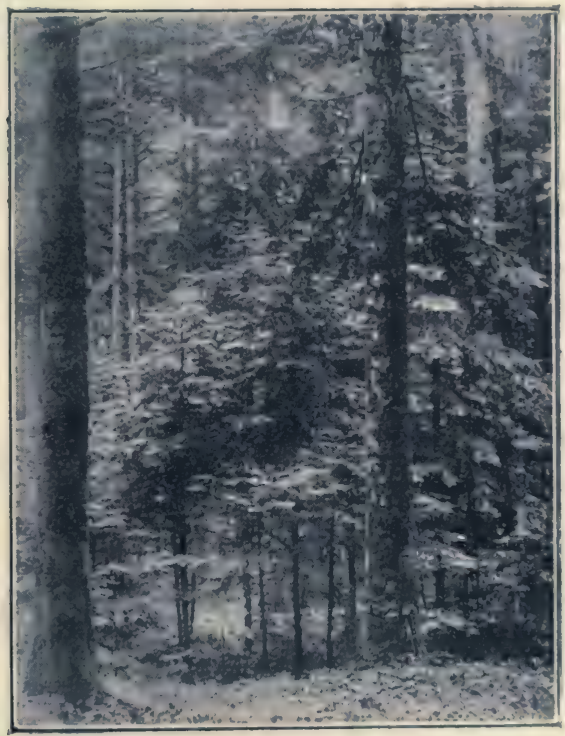

Fig. 31. - Essence d'ombre (sapin), se constituant à elle-même un sous-étage (forèt des Elieux, Meurthe-et-Moselle). (Photographie de M. A. Fron.)

faisant partie intégrante du peuplement, à la densité duquel ils concourent.

Il est évident que les essences à couvert épais peuvent mieux se passer de ces sous-étages bienfaisants que les espèces à couvert léger, et que le tempérament des essences d'ombre leur permet d'accepter le rôle de sous-bois (fig. 31), quand cette faculté est refusée aux espèces de lumière, qui périssent infailliblement dès qu'elles sont simplement surcimées.

Tous les avantages et les inconvénients qui accompagnent 
la présence ou l'absence des sous-étages ne sont plus à considérer quand il s'agit de peuplements d'âges multiples, qui présentent une masse de feuillage continue depuis le sol jusqu'aux plus hautes cimes et dans la verdure desquels l'œil ne perçoit aucun étage distinct.

Composition des peuplements. - Un peuplement peut être composé d'une seule essence ou de plusieurs espèces diversement associées. Dans le premier cas, on dit qu'il est pur, dans le second qu'il est mélangé.

Dans la nature, la différence des tempéraments des espèces facilite la dispersion des individus, augmente la zone des aires d'habitation, et favorise les mélanges. C'est un facteur nouveau, qui s'ajoute à la rapidité de la végétation pour créer les forêts mélangées. Si toutes les espèces exigeaient également la lumière, ou supportaient aussi mal l'ombrage, l'espace appartiendrait à celle qui végéterait le plus vite dans sa jeunesse (1).

Les peuplements purs ne seraient donc qu'une rare exception, si l'homme n'était intervenu pour propager, à l'exclusion des autres, l'espèce qui semble le mieux répondre aux besoins du moment. Dés chênaies, des sapinières, des taillis à écorce, sont ainsi entretenus à l'état pur, pour obtenir en plus grande quantité les produits spéciaux :qu'on leur demande. Mais, en dehors de ces causes voulues, des erreurs ou des fautes provoquent de semblables accidents : c'est ainsi, par exemple, qu'après des coupes à blanc, dans des futaies de chêne ou de hêtre, le sol peut se trouver envahi par des semis de charme pur; - que le bouleau ou des essences secondaires se substituent seules aux bonnes espèces dans un sol appauvri par des abus de jouissance, etc.

Des raisons culturales de premier ordre doivent, en toutes circonstances, faire préférer les peuplements mélangés aux peuplements purs. Les premiers, surtout lorsqu'ils sont composés d'espèce à assiette et à tempérament différents, résistent mieux à la violence des vents, au poids de la neige et du givre, aux dégâts des organismes nuisibles, insectes ou champignons. Les feuilles qui tombent sur le sol, ayant des consistances diverses, se décomposent plus facilement, la couche

(1) Vagener, Das Verhalten der Bäume im Kampfe um's Da ein. 
de terreau est aussi plus épaisse et mieux constituée : toutes causes qui, en augmentant la fertilité et l'ameublissement du sol, facilitent la régénération par la semence.

A la rigueur, les essences à couvert épais peuvent être cultivées à l'état pur, puisque leurs nombreux détritus enrichissent le sol. Cependant, on observe partout que, si les essences d'ombre sont mélangées entre elles, - le hêtre avec le sapin, par exemple, - la régénération est mieux assurée; c'est surtout vers les limites supérieures ou inférieures de l'aire forestière d'une espèce qu'une telle situation est désirable.

Mais, dès que les essences à couvert léger sont appelées au rôle prépondérant dans le peuplement, le mélange cesse d'être facultatif, et l'association d'espèces à couvert épais s'impose. On sait, en effet, que ces dernières peuvent, seules, remplir le rôle de sous-étage et donner au massif la densité indispensable.

En dehors de ces considérations, il faut ajouter que les peuplements mélangés ont des avantages économiques, puisque, par la variété de leurs produits, de tels massifs satisfont à un plus grand nombre de besoins, et, par cela même, augmentent l'utilité de la forêt.

Mélanges rationnels. - La question du mélange des essences est une des plus délicates qui se présentent en sylviculture. Il faut toutes les ressources de l'art du forestier pour obtenir, soit naturellement, soit artificiellement, la composition voulue. Chaque station demande, pour ainsi dire, un mélange différent. Ce qui est vrai, par exemple, en Lorraine, n'est pas forcément applicable aux mêmes essences en Normandie; pareillement sur un même point géographique, ce qui est bon au pied d'une montagne ne l'est peut-être pas à 1000 mètres plus haut; "vérité dans les Pyrénées, erreur dans les Alpes ", a dit Pascal. Mais en cherchant bien, surtout en interrogeant la forêt autour de soi, on trouvera toujours une solution satisfaisante, sans sortir des espèces spontanées.

Le hêtre. - A ce sujet, nous ne pouvons que recommander l'emploi du hêtre, qui se prète merveilleusement à toules les combinaisons. Il vit en plaine comme en montagne, son aire d'habitation, trés étendue, englobe celle de toutes lés 
espèces, auxquelles il peut être associé comme essence d'ombre. C'est, dès lors, l'espèce indiquée pour faciliter les transitions entre deux stations voisines, dans ces zones indécises, si délicates à manier, où une espèce va disparaître quand l'autre n'est pas encore bien installée: entre la région du chêne et la sapinière, par exemple, ou, à la limite supérieure de celle-ci, entre la forêt de rendement et le pâturage.

D'ailleurs, son tempérament plastique lui permet d'accepter tous les rôles; au gré du forestier, il sera, sur le même point, l'espèce précieuse atteignant les plus grandes formes ou le modeste buisson végétant en sous-bois. Nous connaissons trop de forêts qui ont été victimes d'une expulsion systématique du hêtre pour ne pas demeurer convaincus qu'on a tout à gagner en lui réservant la grande place qu'il mérite. Mais, c'est à la condition expresse de rester toujours son maître, sans jamais se laisser dominer par lui.

Conduite des peuplements mélangés. - Il ne suffit pas de créer le mélange, il faut encore savoir maintenir l'équilibre entre des voisins qui luttent à armes inégales. En effet, les espèces associées n'ont pas la même activité de végétation à leurs différents âges et l'on se fait souvent illusion sur l'avenir d'un mélange qui, au moment de sa formation, présente les plus belles promesses. On est donc conduit à intervenir d'une main adroite et patiente, pour donner à ces peuplements les soins culturaux qu'ils réclament pendant toute leur existence.

Il peut même se faire que le mélange intime - pied à pied - de deux espèces à tempéraments opposés soit irréalisable dans une région donnée. Quand, à l'espèce de lumière, on associe une essence d'ombre, dont les tendances envahissantes, par sa nature, sont encore exagérées par une meilleure adaptation au milieu, la première succombe toujours victime de la seconde. Il en est ainsi du chêne et du hêtre dans les forêts du Nord et:de l'Est de la France, où le hêtre, qui se trouve dans le centre de son aire, aura toujours des tendances à dominer le chêne et l'aura bientôt éliminé, si, par des éclaircies bien conduites, on ne vient pas sans cesse le cantonner dans le rôle secondaire qui lui est dévolu. Ainsi, encore, du hêtre et du sapin, aux altitudes un peu considérables. 
Aussi, quelles que soient les espèces et les stations, pour éviter ce danger, dont le caractère est permanent partout oủ il se présente, nous conseillons de préférer au mélange intime,

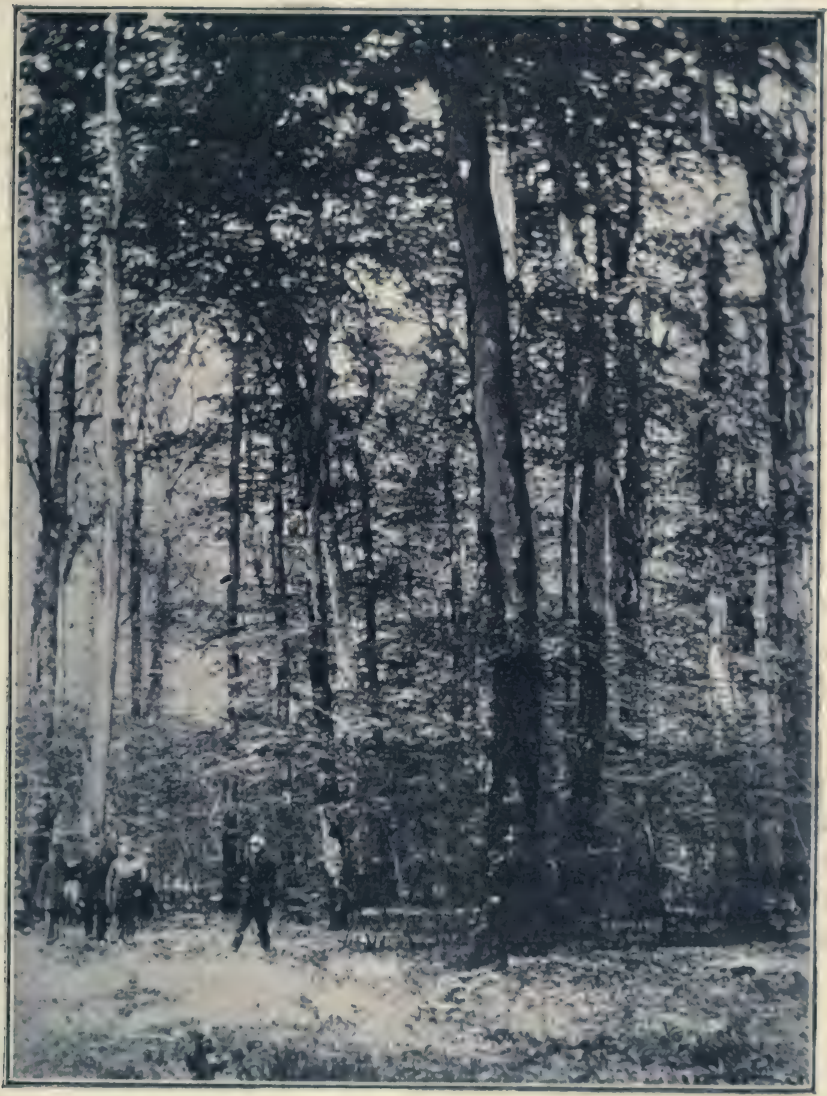

Fig. 32. - Essence de lumière (chêne rouvre) avec sous-étage d'essence d'ombre (hêtre), forêt de Bellême (Orne). (Photographie de M. Thiollier.)

le mélange par groupes, par places ou par compartiments, plus ou moins étendus, où chaque espèce sera traitée à l'état pur, suivant les besoins qui lui sont propres; de créer, en un mot, des peuplements de composition zébrée ou mouchelée. 
Il vaudra mieux encore obtenir des mélanges superposés, oủ l'essence d'ombre jouera un rôle subordonné, sous le couvert léger des espèces de lumière, dont les cimes s'étaleront librement au soleil (fig. 32). Voilà la forêt idéale!

C'est au prix de ces précautions, de ces soins éclairés, qu'on obtiendra des peuplements mélangés les résultats qu'il est permis d'en attendre. Mais ceux qui ne voudraient pas s'astreindre à de semblables exigences de la part de leur forêt, feront mieux, pour éviter tout mécompte, d'abandonner ce genre de culture intensive et de s'en tenir à des modes moins perfectionnés.

État de végétation des peuplements. - L'état de végétation d'un peuplement décide de son avenir, c'est-à-dire de sa durée probable ou de sa fin prochaine.

On dit qu'un peuplement est bien venant, quand la majorité des sujets dominants présente des jeunes pousses de longueur normale, des cimes à ramification complète et abondante, un feuillage à coloration saine et foncée, des écorces lisses. L'élasticité plus ou moins grande du sol et son bon état superficiel fournissent aussi des indications précieuses.

Tous les signes contraires, c'est-à-dire, pousses chétives, - cimes incomplètes et mal ramifiées, dans lesquelles la végétation abandonne la périphérie pour se rapprocher des centres, - feuillage pâle et étiolé, - écorces rugueuses et couvertes de végétations parasites, - sol dur et dégradé, caractérisent un peuplement mal venant ou sur le retour.

ARTICLE III.

\section{ACTION DES ARBRES SUR LE SOL}

Fertilité permanente des sols forestiers. - La couverture. - La couverture morte; son rôle physique. - Le terreau ; l'ameublissement du sol et la terre à bois. - Rôle chimique de la couverture morte ; l'acide phosphorique et la potasse; l'azote. - Les terreaux acides et tourbeux. - Ròle du sylviculteur dans la constitution de la couverture; dans celle du terreau. - La couverture vivante.

Fertilité permanente des sols forestiers. - En toute culture, qu'elle soit agricole, maraìchère ou fruitière, le 
labour et l'apport d'engrais sont indispensables. Or, en culture forestière, l'homme n'apporte pas d'engrais et ne laboure pas le sol. Pourquoi ce dernier conserve-t-il néanmoins sa fertilité?

C'est que, d'une part, - les analyses chimiques l'ont démontré, - le bois constitué de tout échantillon ayant dépassé les dimensions de branchettes ou de brindilles renferme en quantités très faibles les matériaux rares et précieux, comme l'azote, l'acide phosphorique et la potasse; si, donc, on se contente d'exporter de la forêt du bois ayant au moins le calibre de rondins, la production forestière sera beaucoup moins épuisante que les autres. Et, d'autre part, nous allons le voir, l'action du peuplement suffit pour entretenir le sol dans un état de fertilité satisfaisant et même pour l'améliorer.

La couverture. - L'instrument dont il se sert est la couverture, qui, par ce fait, est appelée à jouer, dans la vie de la forêt, un rôle capital, tant au point de vue physique qu'au point de vue chimique.

Comme son nom l'indique, la couverture est la couche superficielle qui recouvre immédiatement le sol minéral. On distingue la couverture vivante, généralement connue sous le nom de tapis végétal, composée par des plantes de petite taille, ligneuses ou herbacées, qui verdissent la surface du sol, sans jamais s'élever au point d'être confondues avec les sousbois, - et la couverture morte, véritable litière ou paillis de détritus de tous genres, tels que : feuilles, aiguilles, brindilles et branches mortes, lambeaux d'écorce, fruits, déjections et débris d'animaux, etc.

La couverture morte, son rôle physique. - D'après M. le professeur Henry, le poids de la couverture morte récoltée au mois de novembre dans des taillis sous futaie de vingt ans, aux environs de Nancy, varie suivant les sols, entre 4.600 et 5.500 kil. à l'hectare; et, dans les futaies de hêtre de la même région, il peut atteindre 7 à 8.000 kil. (1).

(1) E. Henry, Poids et composition de la couverture morte (Annales de la Science agronomique française et étrangère). 
M. le professeur Ébermayer (1), a résumé les principales propriétés physiques de la couverture de la manière suivante :

10 Elle offre de nombreux espaces capillaires, des sortes de canaux, qui la rendent comparable à une éponge et lui permettent de retenir une grande quantité d'eau par imbibition, quantité qui, parfois, peut atteindre deux fois et demi son poids;

$2^{\circ}$ Elle protège le sol contre l'accès direct de l'air et le met partiellement à l'abri des mouvements de l'atmosphère, empêchant ainsi une trop active évaporation;

$3^{\circ}$ Enfin, l'air renfermé dans ces canaux agit, comme dans le cas de la neige, en rendant la couverture peu conductrice pour la chaleur et diminue ainsi, tantôt le rayonnement du sol, tantôt la quantité de chaleur qu'il absorberait s'il était nu; la couverture empêche donc la couche superficielle du sol de s'échauffer ou de se refroidir trop rapidement.

Ajoutons enfin que la coůverture s'oppose au tassement du sol, en brisant le choc des gouttes qui viendraient le battre pendant les grandes pluies; qu'elle prévient le ruissellement des eaux et le ravinement des terres; qu'elle facilite enfin la pénétration des eaux athmosphériques dans les couches profondes.

\section{Le terreau, l'ameublissement du sol et la terre à bois.}

- Sous l'influence de ferments divers, agissant tans des milieux favorables, les éléments de la couverture se rẻsolvent en une matière pulvérulente, de couleur foncée, souvent complètement noire, dégageant une odeur de moisissure spéciale que tout le monde connaît. C'est l'humus ou terreau. Son importance est prépondérante, et nous devons, tout d'abord, enregistrer ses propriétés physiques, qui viennent s'ajouter aux précédentes pour le bénéfice du sol forestier.

Il absorbe et retient une quantité d'eau beaucoup plus considérable que tous les autres éléments terreux; mais s'il reste toujours frais, il n'est jamais mouilleux, car il est aussi filtrant qu'hygroscopique. Il semble, d'ailleurs, qu'en toutes choses, il se montre le pondérateur merveilleux des qualités physiques d'un. sol. Ainsi, il se comporte à la façon

(1) Ebermayer, Étude d'ensemble sur la couverture des forêts, Berlin, 1876; analyse de M. L. Grandeau, dans les Annales de la Science agronomique française êt étrangère, Nancy, Berger-Levrault, 1878. 
des argiles pour donner quelque cohésion aux sables les plus pulvérulents, sans que ses effets, dans ce sens, viennent s'ajouter à ceux de l'argile; bien au contraire, il divise les terres trop compactes. De même, il tempère les excès de chaleur et de froid et adoucit les propriétés mauvaises de certaines matières minérales.

D'autre part, le terreau est le principal agent d'un véritable ameublissement mécanique du sol, dont l'effet ne peut être comparé qu'à celui du labour. Darwin avait déjà appelé l'attention sur les procédés à l'aide desquels les vers de terre brassent les couches superficielles du terreau; mais, en ce qui concerne plus particulièrement les forêts, les travaux récents poursuivis en Danemark par M. le Docteur Müller(1) ont montré que, dans le terreau forestier, existent des pléiades d'organismes, de dimensions aussi variées que les embranchements auxquels ils appartiennent. Tous ces fouisseurs vivent dans le terreau et par le terreau; plantivores et carnassiers le perforent de leurs galeries et le transforment en une masse mouvante, sous l'effort constant des mangeurs et des mangés, qui le parcourent dans tous les sens à la poursuite les uns des autres. Mais bientôt, attirés par ce régal, les animaux d'un ordre plus élevé : musaraignes, taupes ou sangliers, achèvent de mélanger sol, sous-sol et terreau, comme ferait la pioche ou le soc de la charrue.

A ce propos, ajoutons que les arbres eux-mêmes contribuent, d'une manière directe, à donner au sol, sinon l'ameublissement, du moins la perméabilité, par leurs racines qui le pénètrent profondément.

Avec le temps, l'ensemble de ces actions concourt à transformer la terre sauvage en cette terre à bois, dont la consistance toute spéciale explique la sensation d'élasticité qu'on éprouve en marchant sur le sol d'un vieux peuplement.

Rôle chimique de la couverture morte, l'acide phosphorique et la potasse, l'azote. - Le rôle chimique de la couverture n'est pas moindre. En analysant les matériaux

(1) Dr P.-E. Müller, Studien über die natürlichen IIumusformen und deren Einwirkung auf Vegetation und Boden. Berlin, 1887. (Trad. par M. Henry Grandeau. Nancy, Berger-Levrault, 1889). 
qui constituent la couverture morte dans des forêts domaniales des environs de Nancy, traitées en taillis-sous-futaie, M. le Professeur Henry (1) a trouvé les chiffres suivants par hectare, dans un peuplement âgé de 20 ans.

Acide phosphorique.. $23 \mathrm{kil}$. en sol calcaire. $29 \mathrm{kil}$. en sol argileux. Potasse............ 16 33

Azote........ environ $43 \mathrm{kil}$. dans liun et l'autre sol.

Ce qui représente l'équivalent de 6.000 kilos de fumier de ferme, et, aux prix actuels des engrais chimiques, une valeur de 74 francs.

Si les feuilles rendent au sol ce qu'elles lui ont pris, où trouver la restitution des matériaux précieux exportés dans la récolte-bois et qui, malgré leur faible importance relative, ne constituent pas moins une perte sèche?

Cette restitution se fait par une sorte de mobilisation de la réserve du sol, sous l'influence de la vie du peuplement. “ On appelle réserve du sol, dit M. Henry, les éléments tels " que les silicates de potasse, de chaux, de magnésie, etc., qui, " insolubles pour le moment et inutilisables pour la végéta" tion, le deviennent peu à peu grâce à l'oxygène et surtout " à l'acide carbonique, dont l'air occlus dans les sols forestiers " contient toujours de notables quantitès. " Or, l'air pénètre dans les sols ameublis et fournit l'oxygène. D'autre part, l'acide carbonique en excès est dégagé par les microorganismes, lorsqu'ils réduisent 'la couverture en terreau. Ce dernier dissout les sels nutritifs, les digère pour ainsi dire et les prépare en aliments tout prêts à être livrés à la consommation des racines.

Mais d'où vient l'azote? Car, si les phosphates et la potasse restent fixés dans la terre tant que la végétation ne vient pas les lui prendre, on sait que les nitrates ne font que traverser les couches superficielles et se perdent avec les eaux de drainage.

On admet que les matières nitreuses en dissolution dans les eaux météoriques (pluies, neiges, rosées), lorsqu'elles traversent lentement les puissantes assises pénétrées par les racines

(1) E. Henry, Poids et composition de la couverture morte des Forêts (Comptes rendus Ac. des Sciences, 1896). 
des grands arbres, cèdent à ceux-ci plus d'azote qu'elles n'en peuvent fournir aux récoltes agricoles, dans leur court trajet à travers la mince couche arable qui les nourrit.

Quoiqu'il en soit, les chiffres empruntés à la statique chimique de la forêt dressée par M. L. Grandeau (I), démontrent que ces quantités sont insuffisantes pour rétablir l'équilibre entre les recettes et les dépenses de cette nature. Des recherches de $M$. Henry mettent en lumière ce fait nouveau que c'est encore la couverture qui vient combler le déficit. En effet, par l'intermédiaire d'infiniments petits, encore mal déterminés, les feuilles mortes ont la faculté de fixer directement l'azote de l'air, jouant, ainsi, pour les sols forestiers, le rôle des légumineuses en culture agricole. Dans les conditions où étaient installées ces expériences, il a été établi que le gain en azote dû à cette cause variait, par hectare et par an, de 13 kilogrammes, pour les feuilles de chêne, à 22 kilogrammes, pour celles de charme.

"Les feuilles " ajoute l'auteur du mémoire, "sont réellement des organes admirables..., on dirait qu'elles ont hâte, même mortes, de travailler pour l'arbre qui les a produites. Dès qu'arrivent les beaux jours, elles servent de pâture à des microorganismes, dont la présence et l'activité sont attestées par un fort dégagement d'acide carbonique, et dont un certain nombre a la faculté d'absorber, outre l'oxygène, l'azote de l'air, pour le faire entrer dans la constitution de leur protoplasma. "

Voilà donc le bilan des richesses que renferme dans son sein cette couverture morte dont tant de gens voudraient s'emparer gratuitement, comme d'une matière inerte, qui pourrit sans profit pour le propriétaire de la forêt. C'est comme si on voulait prendre au paysan le fumier de ses étables.

Les terreaux acides et tourbeux. - Mais le terreau ne se constitue pas toujours suivant la formule idéale dont nous venons d'esquisser les bienfaits.

Il suffit de regarder la couverture pour constater que

(1) L. Grandeau, Cours d'agriculture de l'École forestière. Nancy, Berger-Levrault, 1879. 
certaines feuilles, comme celles de hêtre ou les aiguilles d'épicéa, de consistance coriace, se décomposent très lentement; que d'autres, au contraire, comme les feuilles de charme, d'orme, de frêne, dont le bétail se montre très avide sous forme de feuillage vert, sont, en même temps, les plus recherchées, à l'état sec, par les plantivores du sol, notamment par les, lombrics. Cela explique pourquoi ces dernières ont disparu depuis longtemps, animalisées dans le terreau, quand les autres sont encore presque entières sur plusieurs lits superposés.

D'autre part, quand l'eau est en excès, l'action de l'oxygène et de la chaleur se trouve diminuée. La décomposition des débris organiques devient extrêmement lente et reste toujours incomplète. Les combinaisons acides se produisent abondantes et il en résulte un résidu analogue à la tourbe, dans laquelle, parmi nos grandes essences forestières, l'aune, le bouleau, le tremble, le pin de montagne peuvent seuls résister.

Inversement, quand il y a excès de sécheresse, le terreau se brûle et devient charbonneux, poudreux ou fibreux. Cette poussière brune ou noire, de décomposition ultérieure très difficile, est une véritable tourbe sèche, avec tous ses inconvénients. Elle se rencontre surtout dans les sables siliceux, auxquels elle se mélange pour donner les terres dites de bruyères.

Dans l'ouvrage déjà cité, M. le $\mathrm{D}^{\mathrm{r}}$ Müller insiste tout particulièrement pour caractériser ces différentes sortes dé terreau et il donne les moyens pratiques de distinguer facilement chacun d'eux. Dans l'humus, où l'acide carbonique est pour ainsi dire le seul produit acide, la vie animale abonde et, parmi ses colons, le grand lombric se montre très nombreux; le sol est gonflé par les taupinières, creusé par les vermillures des sangliers, et, au milieu des plantes qui lui sont spéciales, on trouve abondamment l'aspérule odorante, la mercuriale vivace, l'oxalis, l'anémone des bois, etc.

La tourbe, au contraire, constitue une sorte de feutrage, dont la résistance sous le pied est encore augmentée par l'enchevêtrement de racines superficielles et d'un mycélium spécial à cette formation. 
Tantôt elle est pulvérulente et imperméable, tantôt elle s'imbibe comme une éponge et retient l'eau en excès. Toujours riche en matières organiques acides, elle porte une végétation basse caractérisée par la canche flexueuse, par le triental et par les mousses, qui, à part quelques touffes de polytric, font défaut sur l'humus. Enfin, la faune, beaucoup moins riche, est surtout remarquable par l'absence presque complète de lombrics et de taupes. En un mot, sur ces terreaux déserts, la couverture échappe à l'action réductrice des milliers de tubes digestifs qui élaborent les engrais animaux, et attend, pour se décomposer, le fonctionnement des réactifs chimiques.

La tourbe est des plus communes dans les régions froides et brumeuses de l'Europe septentrionale. En France, où les saisons de végétation sont longues, où les sols calcaires sont abondants, elle est beaucoup plus rare. Elle se rencontre, cependant, sous la forme sèche, dans certaines forêts sablonneuses, - sous la forme humide, dans la montagne, et il importe d'y veiller.

\section{Rôle du sylviculteur dans la constitution de la couver-} ture morte; dans celle du terreau. - Sous ces réserves, voyons quelle est la part d'action du sylviculteur dans la confection des seuls engrais dont il puisse disposer. Sur la couverture, comme sur le terreau, il peut agir, autant par des soins culturaux que par des mesures répressives.

En maintenant les massifs complets, en respectant les sousétages, en évitant les découverts trop fréquents, il contribue à augmenter la quantité de détritus qui s'accumulent sur le sol. Mais il doit surtout s'opposer de la façon la plus énergique à l'enlèvement des feuilles mortes. Heureusement, ce fléau, qui sévit encore en Allemagne, est très localisé en France; car, partout, les expériences dirigées en vue de traduire par des chiffres l'influence de cette funeste pratique, lui ont fait imputer une perte de 50 p. 100 et plus sur la production ligneuse.

En ce qui concerne le terreau, on peut conseiller : de choisir des révolutions plutôt longues que courtes, afin de découvrir le sol le moins souvent possible:- de maintenir les 
sous bois; - de conserver, tout autour des enceintes, des arbres de lisières, qui tiennent le peuplement bien clos, à l'abri des coups de soleil et aussi des coups de vent; car ceux$\mathrm{ci}$, en bouleversant la couverture, rendent la vie difficile aux animaux qu'elle abrite; - enfin, de créer des forêts mélangées, où, la décomposition des feuilles se faisant beaucoup mieux, on évite la formation de ces litières de feuilles de hêtres, ou de ces feutrages d'aiguilles d'épicéa, parfois si gênants dans les forêts de la montagne.

Dans les sables grossiers, secs et brûlants : exagérer encore le principe du couvert bas et continu ; faire tous ses efforts pour maintenir les espèces à feuillage épais, qui ont trop de tendances à fuir ces régions où l'humidité leur fait défaut ; dans ce but, préférer les peuplements d'âges multiples aux peuplements d'un seul âge, ou choisir des révolutions très longues. Au contraire, dans les argiles froides et très humides : relever le couvert, pour faciliter l'accès de la chaleur; choisir des traitements qui découvrent périodiquement le sol; préférer le taillis sous futaie à la futaie pleine.

En résumé, dans toutes les circonstances et en tous lieux, maintenir le sol à l'état de saine fraîcheur que commande l'hygiène de la forêt, et qui convient aux lombrics et autres animaux, sur le rôle desquels on ne saurait trop insister.

La couverture vivante. - La couverture vivante, telle que nous l'avons définie, varie suivant la nature du sol; sa 。 présence est toujours l'indice d'une dégradation dans l'état du peuplement; en effet, elle absorbe à son profit les réserves alimentaires du terrain. Elle entrave la régénération naturelle en empêchant les graines d'arriver au contact de la terre; et les jeunes semis qui, malgré tout, viendraientà s'installer, ont à lutter contre les racines des plantes rivales, qui les enserrent et les affament.

Néanmoins, cette couverture vivante est encore préférable à un état de dénudation complet. Elle donne de l'assiette au sol, le protège contre les érosions des eaux et les ardeurs trop vives du soleil, empêche le tassement, et, de plus, par les débris morts qu'elle abandonne, fournit à la terre des éléments organiques, dont les grands arbres peuvent profiter. 
Aussi, en dehors du cas spécial où l'on cherche une régénération par la semence n'est-il pas indiqué de la faire disparaître par des travaux onéreux. Ce serait supprimer l'effet sans supprimer la cause; car l'évolution d'un tapis végétal est la conséquence nécessaire de l'arrivée au sol de rayons lumineux, tamisés à travers la frondaison insuffisante d'un peuplement incomplet ou mal composé. Le seul moyen d'en avoir raison, sans frais, est donc de laisser le peuplement se reconstituer normalement et de provoquer la réinstallation des sous bois.

ARTICLE IV

\section{ÉVOLUTION DU PEUPLEMENT}

Évolution du peuplement uniforme. - États de développements successifs des futaies régulières; - du taillis simple régulier. - Évolution des peuplements jardinés et furetés.

Évolution du peuplement uniforme. - Suivant son origine et sa forme, chaque peuplement évolue à sa manière et, à ce point de vue, il faut établir une différence fondamentale entre le peuplement uniforme et le peuplement d'âges mêlés.

Dans tout peuplement uniforme, les tiges d'avenir, celles que leur vitalité plus grande maintient dans l'étage dominant où rien n'arrête leur essor, s'élèvent en bloc, chaque année, d'une quantité à peu près égale pour toutes, jusqu'au moment oú elles ont atteint leur maximum de hauteur. Aussi, à tous les âges, le profil du massif est-il limité par une ligne régulière et parallèle au sol.

États de développements successifs de la futaie régulière; - du taillis simple régulier. - Dans cette suite non interrompue d'accroissement en hauteur, l'observation de phénomènes qui se reproduisent d'une manière constante a permis de distinguer certaines phases, certains états particuliers, qui, pour la futaie régulière, ont été appelés, dansl'ordre où ils se produisent: semis, fourrés, gaulis, bas et haut perchis, haute futaie et vieille futaie.

Chacun de ces états de développement peut ètre caractérisé 
dans les termes suivants, que nous empruntons à M. le Professeur Broilliard (1) :

... Tant que les jeunes semis se trouvent isolés l'un de l'autre sur le terrain découvert entr'eux, la végétation reste faible, et l'avenir de la forêt naissante est encore incertain; mais, quand le fourré s'est généralement constitué, s'élevant plus ou moins suivant les points, et alors même qu'il y reste quelques places vides, la forêt a pris possession du sql et se développe rapidement.

Les fourrés naturels offrent généralement des tiges de hauteurs régulières, et faisant un mélange confus de jeunes sujets de bonnes essences avec des espèces secondaires et des morts-bois, dont la présence hâte la formation du fourré et lui donne la densité désirable.

Le gaulis est formé de baguettes, ou gaules flexibles, ayant perdu les branches basses.... Le sol s'améliore rapidement par l'effet du couvert bas et complet et des détritus végétaux qui s'accumulent. Le nombre des petites cimes qui luttent entre elles en s'élevant pour prendre la place au soleil, diminue d'année en année, pour ainsi dire à vue d'oeil.

Une futaie se trouve à l'état de perchis, quand elle est principalement constituée par des perches, tiges de 1 décimètre de diamètre au moins... Dans les bas perchis, la production annuelle en bois arrive à son maximum; l'élagage naturel des branches basses s'opère encore avec rapidité et le nombre des tiges diminue de même.

On appelle haut-perchis ou demi-futaie le massif dont les fûts ont déjà pris une grande hauteur : hauteur qui correspond souvent à un diamètre minimum de 2 décimètres, à $1 \mathrm{~m}$. 30 du sol. Les cimes, dont les branches principales sont déjà fortes, occupent chacune une place assez large, les plus faibles résistent longtemps avant de périr sous l'étreinte de leurs voisines...

Quand les fûts sont entièrement constitués, le massif prcnd le nom de " futaie proprement dite " ou de " haute-futaie ". Les cimes élevées ont de fortes branches, qui persisteront à peu près indéfiniment, ou ne disparaîtront à la longue qu'en laissant au tronc des tares amenant la dégradation lente des arbres les plus faibles. Les trouées qui viennent à se produire se comblent dès lors difficilement, et, au-dessous d'elles, des semis se montrent en permanence sur le sol. La production ligneuse du massif est un peu plus faible que dans les perchis.

Chacun de ces états persiste un temps plus long que celui qui l'a précédé, le bas perchis plus longtemps que le gaulis, mais moins longtemps que le haut perchis et celui-ci moins encore que la haute futaie. Celle-ci prend le nom de Vieille futaie quand les arbres, devenus gros, approchent de la maturité. Les vieilles futaies ne sont pas toujours en massif uniforme par l'âge et la grosseur des tiges.

En résumé, dans l'évolution du peuplement uniforme, comme dans celle de tout être organisé, on peut distinguer trois grandes phases :

(1) Broilliard, Le traitement des bois en France, p. 238. 
$1^{\circ}$ Naissance et constitution du fourré ;

$2^{\circ}$ Jeunesse et croissance en hauteur, pendant les états de gaulis, bas et haut perchis.

$3^{\circ}$ Enfin âge mûr, pendant lequel les arbres adultes s'accroissent surtout en grosseur et fructifient abondamment.

Le taillis simple régulier ne se comporte pas tout à fait de même. Les rejets, à leur naissance, émergent de souches toujours plus ou moins éloignées les unes des autres, de sorte qu'il se passe un temps variable, mais toujours assez long, avant que les branches basses se rejoignent et s'entrelacent pour former le fourré. Pendant ce temps, les tiges principales dépassent les dimensions de gaulis et le peuplement devient un bas perchis, sans prendre l'aspect de gaulis. A partir de ce moment, il évolue comme la futaie pleine, dont il se différencie néanmoins par certaines tares qu'il doit à son origine: les tiges sont, en effet, plus ou moins déviées à la base, et leur section faite dans cette même région, au lieu d'être circulaire, est déprimée du côté de la souche dont elles sortent. Aussi, pour les distinguer de la futaie vraie, ajoute-t-on les mots sur souches, aux expressions qui caractérisent leur état de développement et l'on dit : bas et haut perchis sur souches, haute fulaie sur souches.

Évolution des peuplements jardinés et furetés - Bien différentes sont les allures des peuplements qu'un traitement systématique entretient dans les formes jardinées et furetées. Ici, les sujets mûrissent individuellement, et, sur chacun des points dénudés par la coupe qui les enlève, il en naîtra d'autres pour occuper leur place. Les bois de tous âges se développent irrégulièrement dans les espaces variables que leur ménagent les hasards des exploitations, leur croissance étant tantôt activée, tantôtralentie parla plus ou moins grande quantité de lumière qu'ils reçoivent à un instant donné. Pas plus que dans la masse de leur frondaison on ne distingue d'étages, on ne peut constater dans la durée de leur évolution aucune phase, aucun état particulier de développement. Si bien que ces peuplements, qui n'ont pour ainsi dire point d'âge, se perpétuent toujours semblables à eux-mêmes.

Pour mieux faire comprendre la situation, pour mieux faire 
ressortir la différence qui sépare ces deux types de peuplement, nous comparerons leur évolution à celle des parcs d'agrément, suivant qu'ils sont créés et entretenus d'après une des méthodes : française ou anglaise. Dans les parcs français, les arbres, souvent de même essence, .sont disposés, à la même époque, en longues bordures ou en avenues régulières, où, toujours solidaires les uns des autres, ils grandissent et vieillissent ensemble. Ils forment un tout homogène, et sous peine d'en rompre le caractère grandiose et l'harmonie, il est impossible de les rajeunir autrement que par un remplacement en masse. C'est l'analogue du peuplement uniforme.

Au contraire, dans le parc anglais, tous les éléments sont indépendants les uns des autres. Les arbres, plantés un peu au hasard, tout en suivant certaines règles de l'esthétique, peuvent être remplacés individuellement au fur et à mesure de la maturité de chacun, sans nuire au caractère de l'ensemble.

Comme les peuplements jardinés ou furetés, ces parcs conservent toujours le même aspect. En deux mots, l'évolution de l'un se fait à temps, tandis que celle de l'autre se prolonge à perpétuité.

A propos des traitements qui engendrent des formes aussi dissemblables, nous pouvons, dès maintenant, déduire les conséquences suivantes :

$1^{\circ}$ Chacune des unités de surface couverte par un peuplement d'âges multiples forme un tout complet qui, au point de vue du but final, évolue indépendamment des surfaces voisines. La vie de relation y existe à peine; c'est, en quelque sorte, la cellule unique des êtres inférieurs.

Comme l'organisme, le traitement restera simple.

$2^{\circ}$ Dans les peuplements uniformes, chacun des éléments est solidaire des autres, avec lesquels il marche d'ensemble vers le but commun. Pour assurer l'harmonie dans ce concert, un instrument nouveau est indispensable, dont le rôle n'est pas sans présenter une certaine analogie avec celui du système nerveux chez les êtres supérieurs. Le traitement de la forêt sera compliqué d'autant. 


\section{CHAPITRE IV}

\section{LES FORËTS}

Nous venons de constater les effets de l'intervention de l'homme sur la vie des peuplements. Mais, de celte étude, il ne faudrait pas conclure que la forêt se prête, sans protester, à tous les caprices de l'exploitant. Loin de là, l'expérience de tous les jours nous l'indique, chaque mode de traitement est cantonné dans un champ d'application dont les limites sont parfois très étroites et en dehors desquelles tous les accidents sont à craindre. Sans pousser les choses au pire, dès que la main de l'homme élimine l'une ou l'autre des espèces qui forment la forêt spontanée, la fertilité générale en souffre et s'amoindrit; cette modification dans le sens rétrograde peut avoir une marche assez lente pour ne pas être bien visible dans le cours d'une génération, mais elle n'en est pas moins fatale.

C'est dire que, toujours et partout, l'action de la nature est prépondérante. Dans chaque région, dans chaque station même, les agents sol et climat imposent à la forêt des allures particulières, lui façonnent une sorte de capacité forestière, qu'on ne saurait lui faire dépasser. Nous pensons en fournir la preuve dans les pages qui vont suivre.

\section{ARTICLE PREMIER}

\section{ACTION DU SOL}

Ròle du sol : généralités; classement. - Sols siliceux : proprićtís physiques; tapis végétal; allures des forêts; sobles à grains fins; alios; tourbières. - Sols argileux: propriétés physiques; tapis végétal ; allures des forêts; løhm. - Sols à base calcaire : propriétés physiques; lapis végétal; allures des forêts ; marne.

Rôle du sol. - Le rôle du sol, en même temps qu'il sert Bopre et Johyet. 
de support aux arbres, est de maintenir à leur disposition une quantité d'eau suffisante pour contre-balancer les effets de l'évaporation. D'autre part, nous avons dit que les végétaux ligneux et surtout les espèces sociales, qui forment la base de tous nos peuplements, sont très peu exigeants au point de vue de la richesse minérale dusol. Remarquons enfin, qu'il faut considérer comme faisant partie du sol forestier, non seulement la terre arable, mais encore toutes les zones sous-jacentes perméables aux racines; par conséquent la masse nourricière des forêts est beaucoup plus puissante que celle qu'utilisent les végétaux agricoles; cependant cette quantité n'est pas illimitée; car, en dehors des fissures qui, en raison de leur humidité constante, font pénétrer les racines jusqu'aux assises les plus profondes des rochers disloqués et des éboulis, il est rare que celles-ci fonctionnent au-delà de un ou deux mètres de profondeur.

Pour la forêt, les propriétés chimiques d'un sol importent donc beaucoup moins que ses qualités physiques; parmi lesquelles la profondeur et la perméabilité d'une part, et d'autre part l'aptitude, non seulement à absorber l'eau des pluies, des neiges, du givre et de la rosée, mais encore à la retenir, sont les plus importantes.

Dans la pratique, on a conservé l'habitude de classer les sols en trois groupes distincts: les sables siliceux, les argiles et les terrains à base calcaire. Sans doute, dans les milliers d'échantillons de terres qui se rencontrent à la surface du globe, la proportion des trois éléments : silice, argile et carbonate de chaux varie à l'infini ; mais il est rare que l'un d'eux ne prédomine pas suffisamment pour donner au milieu son caractère propre.

Sols siliceux. - Le sable siliceux pur, lorsqu'il contient peu de matière organique en mélange, forme un sol mẹble, sans liaison à l'état sec, n'ayant même qu'une faible cohésion sous l'influence de l'humidité.

J'eau, qui traverse facilement cet amas filtrant, entraine avec elle les particules fines dans les profondeurs; aussi la surface, n'offrant aucune résistance à l'érosion, est-elle facilement ravinée par les eaux ou déplacée par. les-vents. Sa 
faible ténacité n'offre aux grands arbres qu'une assiette insuflisante; du reste, aussi bien pour y chercher leur nourriture que pour trouver un point d'appui plus solide, les racines pénètrent fort avant dans ces sols et s'y étalent amplement.

Très pénétrable par l'air, le sable prend rapidement la température du milieu ambiant : il s'échauffe et se refroidit très

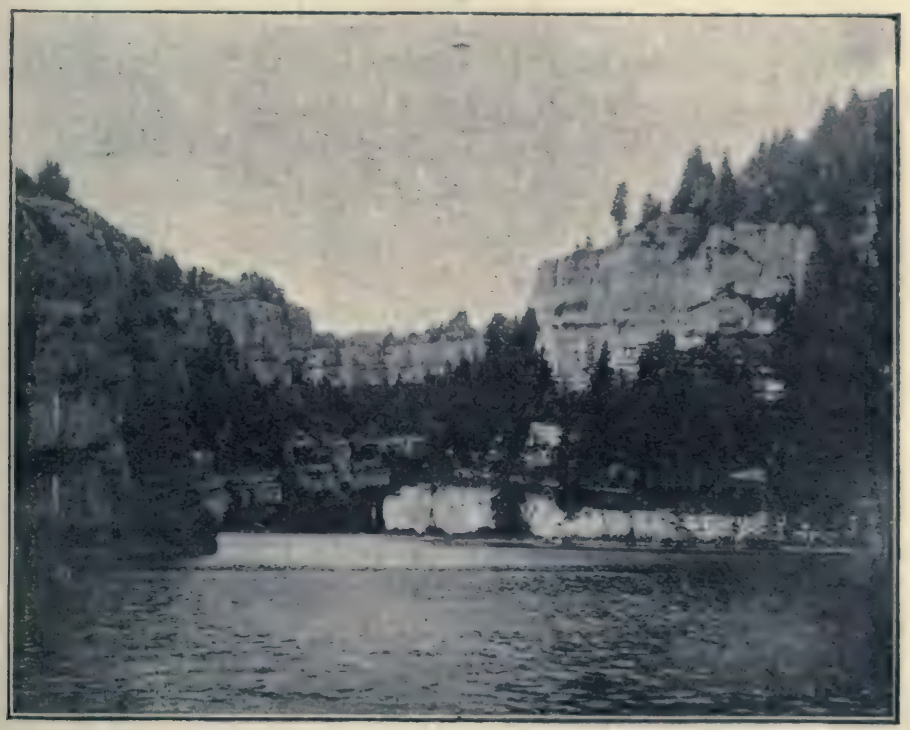

Figr. 33. - Escarpements calcaires des bassins du Doulss. (Photographie de M. Thiollier.)

vite; aucune terre n'est plus apte à se dessécher, à devenir aride dès qu'elle est ouverte à l'accès des vents et des rayons du soleil : toutes conditions mauvaises pour la bonne fabrication du terreau, qui prendra souvent la forme fibreuse. Les accidents de gelées, printanières ou autres, y sont aussi plus à craindre qu'ailleurs.

Le tapis végétal du sol siliceux est plus paurre en espèces que celui des terres plus fertiles; mais les plantes sociales, qui s accommodent de ces stations, se jettent à foison dans les vides et profitent de toutes les fautes pour en augmenter la gravité; 
car, une fois installées, elles ne se laissent plus exproprier, même par les grands arbres. Ces plantes sociales peuvent être herbacées, comme la grande fétuque bleue, mais elles sont plus souvent ligneuses : comme les genêts, les ajoncs, l'airelle myrtille, la callune et ces éternelles bruyères, qui, suivant les climats, feront la lande ou le maquis.

Malgré tout, le sol siliceux peut nourrir de belles et bonnes forêts, sous la condition d'être découvert le moins possible. Le semis y réussit très bien; par contre, les souches n'y rejettent que médiocrement : c'est dire que le traitement en futaie est préférable au traitement en taillis. Aussi les tentatives faites pour y perpétuer ce dernier mode ont-elles, le plus souvent, amené des désordres et des états d'épuisement tels, qu'il a fallu remplacer les espèces spontanées par d'autres plus frugales. Les trop nombreuses taches de résineux introduits, qui envahissent progressivement les régions autrefois peuplées de magnifiques forêts de hêtre et de chêne, n'ont pas d'autre origine.

Enfin, ces sols conviennent parfaitement à la culture en pépinière; car ils se travaillent sans effort et s'entretiennent à peu de frais en bon état de propreté. Essentiellement neutres, ils n'excluent aucune essence et acceptent sous forme d'engrais et d'amendement toutes les matières qu'on veut leur incorporer. D'ailleurs, à cause de leur état de division, les racines développent un chevelu abondant, qui facilite la reprise des sujets lors de la transplantation.

Ces propriétés moyennes peuvent être profondément modifiées en bien ou en mal par des causes diverses.

C'est ainsi que ces mêmes sols, quand les éléments en sont fins ou très fins, conviennent mieux que tous les autres à l'éducation des massifs de futaie; les sables les plus ténus, pourvu qu'ils soient imprégnés de terreau, jouent à s'y méprendre un rôle analogue à celui de l'argile et assurent une fraîcheur constante. Les arbres atteignent des hauteurs considérables, leurs fûts se rapprochent de la forme cylindrique et la régénération par la semence y est des plus faciles. Les argiles à silex, qui, malgré leur nom, rentrent dans la catégorie des sols siliceux, nourrissent les plus belles futaies 
de France: ainsi, celles de Villers-Cotterets, de Lyons, de Bellême, de Bercé, de Senonches, de Tronçais, pour ne citer que les plus remarquables par leur splendeur et leur élendue. On peut en dire autant des sapinières qui reposent sur les grès infraliasiques et les autres formations grèseuses, sous le climat humide des Basses-Vosges.

Souvent aussi, quelle que soit la grosseur des éléments et l'apparence filtrante de ces sables, on est surpris de les voir constamment mouilleux; c'est qu'alors il s'est formé, à une faible profondeur, celte couche d'alios si connue dans les Landes de Gascogne et dont la composition n'est autre que celle du sable lui-même, agglutiné en une sorte de roche compacte, imperméable, de couleur brune, par un ciment organique dans lequel les sels de fer ne manquent jamais. Partout où cet alios se rencontre, les effets ordinaires d'une végétation mauvaise sont encore aggravés par des accidents fréquents de gelées printanières et de gelées d'hiver. Quand les circonslances le permettent, et plus particulièrement lorsque le relief est peu accusé ou en forme de cuvelle, la présence de l'alios est pour ainsi dire fatale. Le seul moyen d'y remédier temporairement serait de perçer de loin en loin la couche imperméable par des trous de sonde, qui rempliraient l'office de puils perdus.

On peut encore faire rentrer les tourbières dans le groupe des terrains siliceux. Celles-ci, heureusement assez rares en France, n'y ont qu'une faible importance forestière. Souvent d'ailleurs, surtout dans la moyenne montagne, elles jouent, au point de vue de l'alimentation des cours d'eau pendant l'été, un rôle analogue à celui des glaciers. Aussi, sans prétendre les meltre en valeur par des assainissements coûteux, suffit-il de préserver par des fossés d'écoulement les cantons boisés qui les entourent, contre leur envahissement toujours à craindre.

Sols argileux. - Les terrains argileux sont froids, humides, et, quand ils se dessèchent sous l'action du vent ou du soleil, ils se transforment en une masse dure, crevassée par un retrait considérable et douée d'une odeur spéciale lorsqu'elle s'humecte. Les eaux séjournent à leur surface si la 
pente fait défaut; alors l'excès d'humidité, le manque de chaleur retardent la végétation et entravent la décomposition des débris organiques; le terreau y prend la forme acide et la pauvreté de sa faune ne lui permet d'amender que les couches les plus superficielles: Ces terrains sont, en général, profonds ; mais les racines des arbres s'enfonçent difficilement dans ces masses compactes, où elles ne fonctionnent qu'avec peine, faute d'oxygène.

Un couvert prolongé sera donc plus nuisible qu'utile aux sols argileux et l'accès modéré de l'air et du soleil ne peut que leur donner plus de fertilité; leur teneur suffisante en sels nutritifs, leur fraîcheur constante permettent de leur appliquer un genre de culture assez épuisant; toutes raisons qui justifient le traitement en taillis sous futaie ou même en taillis simple, qu'on applique généralement aux forêts feuillues de ces stations. Je pareils modes de traitement, quelque peu artificiels, conviennent à ces terrains, qui donnent de belles récoltes en bois, comme ils donneraient de belles récoltes en blé, à condition que l'homme se charge de veiller à la régénération de la forêt, aussi bien qu'il le fait pour la semaille des céréales. Par contre, ces terres, très difficiles à travailler, sont tout à fait impropres à la culture des plants en pépinière.

Le tapis végétal varie suivant le degré de compacité des argiles; souvent composé d'herbes denses et touffues : joncs, graminées, carex, il se réduit parfois, dans les cas extrêmes, à une couche de mousse peu épaisse, dans laquelle l'ensemencement semble assez bien réussir; mais où, bientôt, les jeunes plants disparaissent, faute de pouvoir enfoncer leurs radicelles dans la terre. Bien que la flore arbustive n'ait pas la variété de celle des terrains calcaires, on y trouve, outre la bourdaine, fréquente comme sur les sables, d'abondants fourrés d'épines noires et blanches. Parmi les grandes essences, les plus communes sont le chêne pédonculé, le frêne et surtout les bois tendres: tilleuls, aunes, saules, trembles, etc..., qui, d'ailleurs, y rejettent bien de souches.

Deux variétés principales se rencontrent dans les terrains de ce groupe : 
$1^{\circ}$ Le lehm ou le loss, que sa fertilité incomparable a converti depuis longtemps en nos meilleures terres agricoles, aussi n'en reste-t-il à la forêt que des lambeaux sur les rives submersibles des grandes rivières, comme l'Adour et la Saône.

$2^{\circ}$ Les terrains feldspathiques provenant de la décomposition de roches comme les granits ou les syénites, dont les éléments argileux sont divisés par de menus cristaux et qui, malgré leur peu de profondeur, sont très favorables à la végétation forestière; dans les régions montagneuses, où on les rencontre le plus souvent, ils sont couverts de fort belles sapinières.

Sols à base calcaire. - Dans la majorité des cas, les sols calcaires donnent une terre de ténacité moyenne, très avide d'humidité et se délayant en boue, mais qui se dessèche très vite, se fendille finement et tombe en poussière. Parfois aussi, les plateaux oolithiques ont leur ossature calcaire recouverte d'un manteau d'argile rouge ferrugineuse, terre excellente, d'une épaisseur toutefois trop variable. Dans l'un et l'autre cas, ces sols s'échauffent avec une grande facilité et, si l'humidité ne fait pas défaut, la couverture morte s'y décompose rapidement dans d'excellentes conditions, en même temps que les acides organiques s'y neutralisent. Toujours riches en principes nutritifs, ils conviennent tout particulièrement à la végétation ligneuse, bien qu'ils manquent, en général, de profondeur et que la nature fissurée des roches sous-jacentes les rende très perméables et les expose au dessèchement (fig. 34). Ces inconvénients sont, d'ailleurs, atténués ou aggråvés suivant l'orientement du système de fissures. Lorsque celles-ci sont verticales, les racines peuvent les suivre et 's'y nourrir jusqu'à de grandes profondeurs dans la terre qui les remplit. Si, au contraire, la direction en est horizontale, la roche se présente en la forme de dallages superposés, contre lesquels les racines se buttent en vain.

Dans ces conditions, le choix du mode de traitement est entièrement subordonné à la profondeur. Quand elle est suffisante, on peut tout demander et tout obtenir; partout où elle fait défaut, on s'efforcera d'éviter le dessèchement que causerait un découvert trop complet et trop souvent répété 
et, renonçant aux chênes et autres arbres pivotants, on ne cultivera que des espèces à racines traçantes. Dans les régions méridionales, les forêts en terrain calcaire sont exploitées surtout en vue de leur production en écorce, ou utilisées comme pâtures; chềne yeuse et chêne blanc, tráités en taillis simple, couvrent de grands espaces, dont l'étendue dépasse 100000 hectares. Assez souvent le pin d'Alep les accompagne.

Le calcaire nourrit une flore très variée; plantes herbacées, arbrisseaux, arbustes, grands arbres $\mathbf{y}$ abondent en espèces;

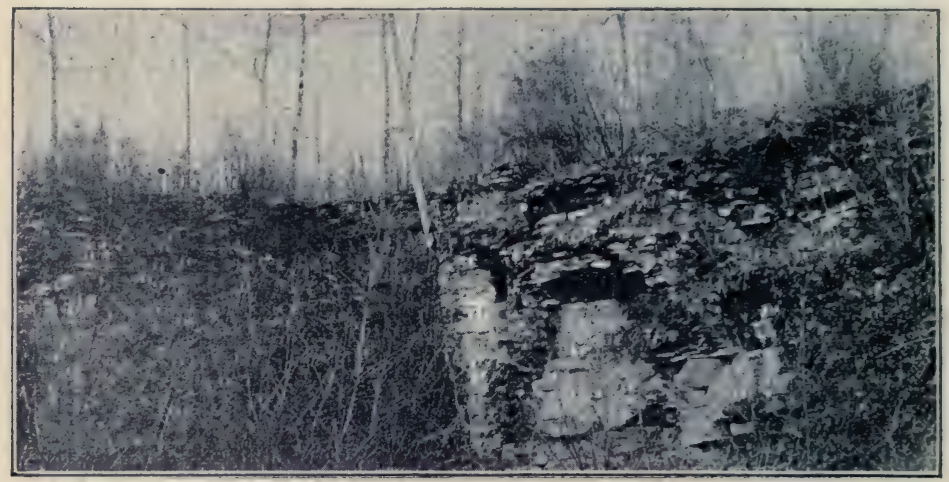

Fig. 34. - Taillis sous futaie sur roche calcaire fissurée (oolithe). Forêt de Chargey-lès-Port (Haute-Saône).

n'en sont exclues que les formes calcifuges déjà signalées. Les essences les mieux appropriées à ces terrains sont le hêtre et l'épicéa à cause de leur enracinement superficiel; puis les essences exigeantes : ormes de montagne, érables, fruitiers, à cause de la richesse du sol; enfin les espèces frileuses, comme le pin d'Alep et le chêne yeuse, qui y trouvent la chaleur dont elles ont besoin.

Étant donnée celte richesse de la flore ligneuse, partout où la sécheresse n'est pas absolue, il se rencontrera toujours quelques sujels assez accommodants pour se contenter des maigres condilions qui leur sont offertes par les calcaires les plus dégradés. Aussi le caractère véritable de ces stations se 
révèle-t-il par la hàte avec laquelle les surfaces dénudées se couvrent de broussailles. Quelque mal justifié que soit le traite-

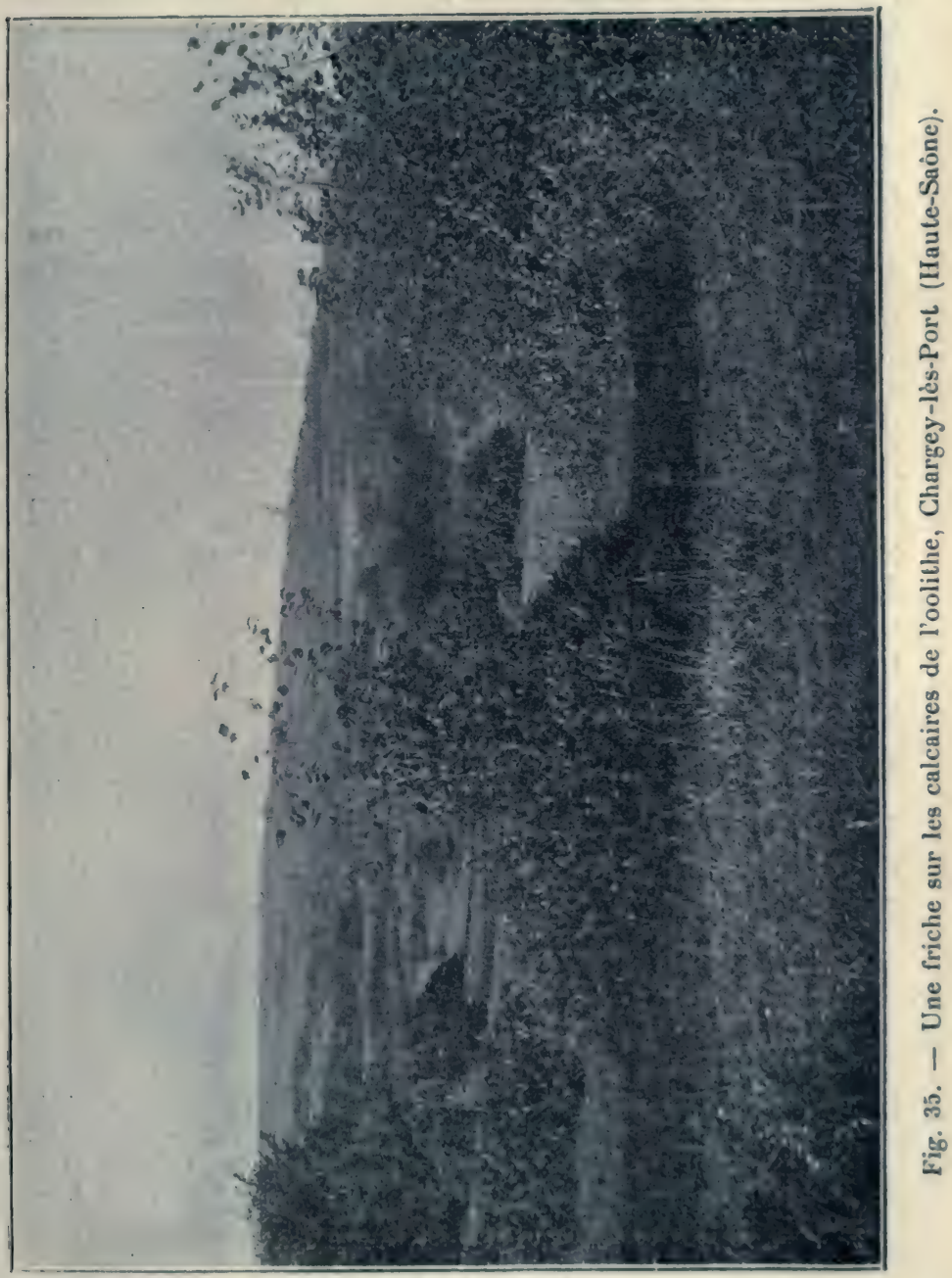

ment, quelqu'abusives que soient les exploitations, l'état boisé se maintient. Si les arbres disparaissent, arbrisseaux et arbustes seront toujours là, prêts à masquer les vides, à 
panser les blessures. Malgré leur vitalité, ils ne sont pas à redouter comme la tenace bruyère. En effet, tandis que celle-ci contribue à l'acidité du terreau, tandis qu'à l'instar de toutes les plantes sociales, elle couvre le sol d'un tapis continu et permanent, qui ne s'élève jamais assez pour jouer le rôle utile de sous-étage, les coudriers, les cornouillers et les différents arbustes des sols calcaires fournissent un excellent humus, et, si on a la patience de les laisser grandir, constituent un abri, sous lequel naissent abondants les semis de sapin ou des autres essences d'ombre. De même, au milieu de touffes de buis, de genévriers ou d'épines dans les friches ou les garriques, on voit poindre, suivant les altitudes, la flèche d'un épicéa ou d'un pin, la cime d'un charme ou d'un chêne (fig. 35). Et toute cette broussaille complaisante, après avoir abrité les grands arbres dans leúr jeunesse contre l'envahissement des plantes herbacées, contre les rayons du soleil ou la dent du bétail, se laisse dominer par eux et passe modestement à l'état de sous-bois.

Les pépinières établies en terrain calcaire se cultivent facilement, et, bien soignées, elles fournissent de bons plants ; mais, en raison de l'extrême diversité des plantes sauvages qui s'y développent, elles sont d'un entretien très coûteux.

Si la proportion du calcaire en mélange avec l'argile descend au-dessous de 30 p. 100 , la terre devient la marne, à laquelle les agriculteurs ne permettent plus aux forestiers de s'intéresser.

ARTICLE II

\section{ACTION DU CLIMAT}

I. Aptitude forestière. - II. Les climats de plaine : caractères généraux. - Division en zones. - Zone parisienne. - Zone girondine. - Zone provençale. - III. Les climats de montagne: caractères généraux. - Vosges. - Jura. - Alpes. - Plateau central. Pyrénées.

\section{I. - Aptitude forestière.}

En culture forestière, tout comme en agriculture, il s'agit de tirer parti de végétaux. Pour apprécier les aptitudes 
forestières d'un climat, on ajoute donc à la série des phénomènes atmosphériques que les cultivateurs apprécient ou

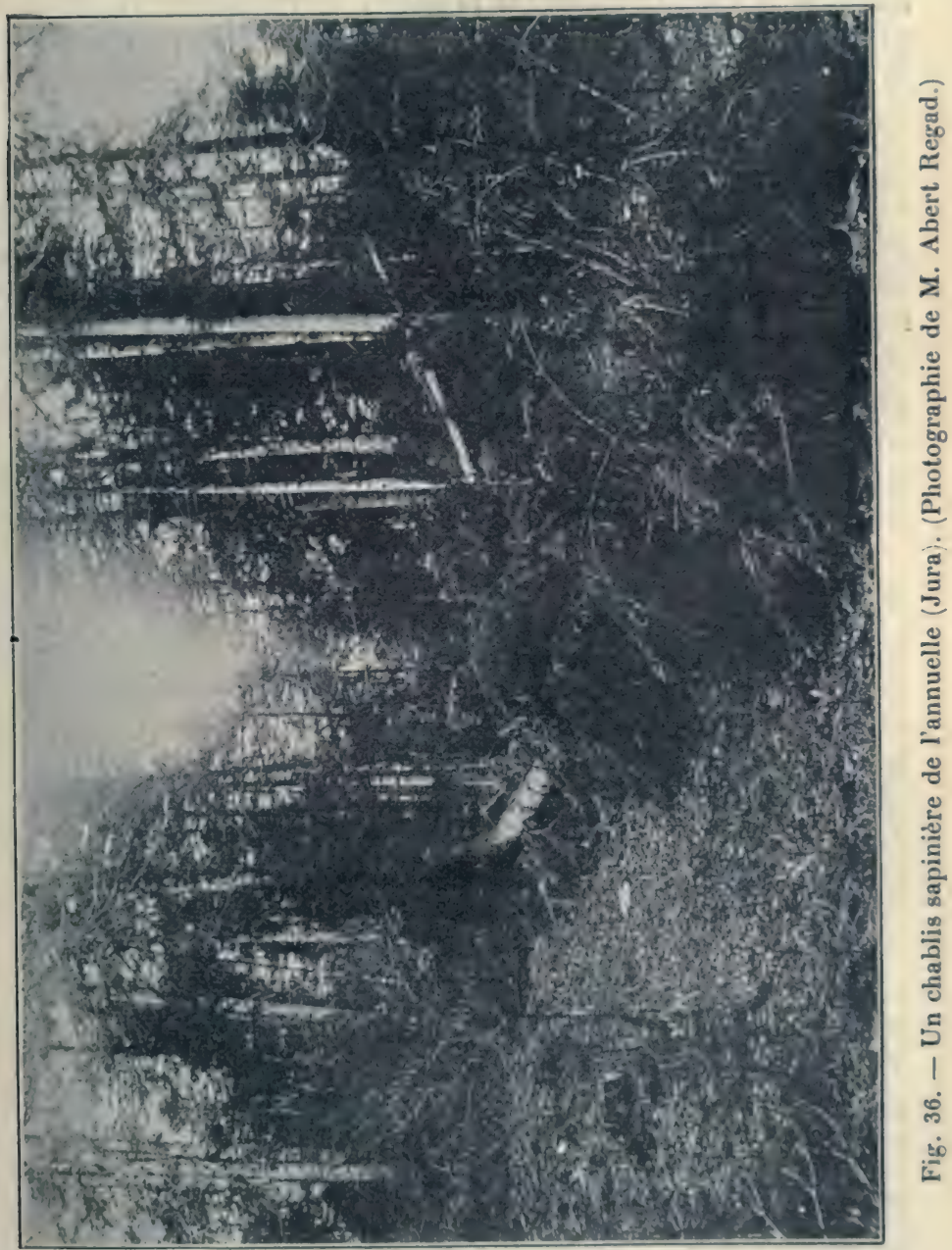

redoutent, certaines particularités, qui concernent plus étroitement le végétal arbre et, par suite, la forèt.

Pour maintenir un état de saturation constant dans sa 
masse ligneuse, qui doit renfermer toujours au moins 40 p. 100 de son poids total en eau, l'arbre a besoin d'une somme considérable d'humidité pendant la saison de végétation; il n'est pas surprenant, dès lors, que la forêt n'existe plus partout où le climat lui refuse le minimum d'eau nécessaire aux espèces les moins exigeantes à cet égard. La nudité relative des déserts, des steppes, des champagnes ou campines n'a pas d'autre cause. A ce point de vue, la culture forestière se rapproche beaucoup plus des cultures fourragères et pastorales que des autres; car elle a plus d'intérêt à favoriser le développement des axes et des organes verts que celui des graines ou des fruits mûrs. En un mot, régions forestières et régions pastorales se superposent, On peut donc dire que les contrées ou l'herbe reste verte toute l'année, grâce à leur climat humide, sont, en même temps, la patrie des belles et bonnes forêts.

Mais, plus que toutes les autres plantes cultivées, l'arbre craint la violence des vents; quand l'ouragan passe inoffensif sur les prairies et les champs, il est mécaniquement nuisible aux arbres des forêts, en déracinant les uns, brisant les autres, renversant parfois des cantons tout entiers. Les sujets brisés, arrachés par le vent se nomment chablis (fig. 36). Le danger que courent les massifs dépend de l'essence, de l'âge, de la saison, de l'altitude, de l'exposition et du mode de traitement.

Enfin des expériences aussi concluantes en France (1) qu'en

(1) Les expériences installées aux environs de Nancy, en 1867, par M. Mathieu, sous-directeur de l'École forestière, et continuées jusqu'en 1900 par les soins de la station de recherches, établissent les lois suivantes :

$1^{\circ}$ En forêt, pour cette région, la température moyenne annuelle est d'environ un demi-degré plus basse que dans les terres agricoles voisines ;

$2^{\circ}$ cet abaissement, très faible pendant les mois d'hiver, est surtout sensible en été;

$3^{\circ}$ la moyenne annuelle des minima est même relevée de près de un degré, tandis que celle des maxima est abaissée de deux environ, d'où une diminution dans l'écart entre les maxima et les minima atteignant presque trois degrés;

$4^{\circ}$ les hauteurs d'eau pluviale dans une clairière de forêt, - sur la lisière d'un massif, - et dans une région franchement agricole voisine, sont entre elles comme les nombres 100,95 et 77 ; 
Allemagne, concourent à faire admettre les faits généraux suivants comme chose jugée :

$1^{\circ}$ Les grands massifs forestiers abaissent quelque peu la température moyenne de l'année; mais, en même temps, ils régularisent les climats, en diminuant l'intensité des grands froids et des chaleurs extrêmes.

$2^{\circ}$ La forêt qui abaisse la température moyenne, facilite la condensation des vapeurs; d'autre part, la transpiration des feuilles augmente la quantité de vapeur d'eau contenue dans l'atmosphère; par conséquent, l'état boisé d'une contrée active la chute des pluies et le dépôt des rosées.

Ainsi, en modifiant l'état hygrométique d'un climat, la forêt le rend plus favorable à sa propre production. Elle exerce dans l'intérieur du continent un rôle analogue à celui de la mer sur les îles et les côtes. On peut donc dire que la forêt appelle la forêt et qu'il est toujours avantageux de la cultiver en grands massifs.

Telles sont les considérations qui nous ont servi de base pour diviser la surface de la France en deux groupes principaux de climats forestiers : les climats de plaine et les climats de montagne (1).

$5^{\circ}$ il pleut davantage sur la lisière Sud-Ouest d'un grand massif forestier que sur la lisière Est; mais c'est le centre du massif qui reçoit le plus d'eau;

$6^{\circ}$ le couvert des arbres feuillus intercepte, en été, environ 8 p. 100 de l'eau pluviale; mais comme la forêt reçoit 22 p. 100 d'eau en excès sur les champs voisins, c'est encore un bénéfice de $22-8=1$ \& p. 100 , en faveur du sol forestier, par rapport au sol agricole.

M. Mathieu, Météorologie agricole et forestière, Paris, 1878.

M. Fautrat, Id.

M. Bartet. Id. Bull. Ministère de l'agriculture, 1895.

M. Claudot, Id. Ann. Société d'émulation des Vosges, Épinal, 1897.

M. Hüffel, Influence des foréts sur le climat, Bull. Société forestière de Franche-Comté et Belfort, 1895.

(1) Nous ne pouvons ici que diviser la France en grands climats forestier's. Mais chaque département, chaque montagne, suivant l'exposition, suivant l'altitude, se subdivise, souvent, en plusieurs régions, caractérisées par leurs espèces forestières et que l'on peut reconnaitre, mème quand les grandes essences font défaut, à leur flore herbacée ou arbustive. On sait, par exemple, que l'on se trouve dans la zone du sapin, quand on voit a ses pieds Geranium sylvalicum. Prenanthes purpurea, Ranunculus aconitifolius, - dans celle du chène vert quand on rencontre Cistus monspeliensis, C. albidus, Lacandula lati- 


\section{II. - Les climats de plaine.}

Caractères généraux. - Un climat de plaine comprend, en général, celui des pays de collines et de coteaux. Il est caractérisé par des altitudes variables entre 0 et 600 mètres et des reliefs, qui, bien que parfois assez accusés, ne présentent nulle part les allures abruptes et tourmentées des pays franchement montagneux.

Dans chaque station la température est aussi chaude que leur latitude le comporte; les saisons sont bien marquées par les quatre grandes phases du mouvement de la terre qui les produisent. Le printemps, l'été, l'automne, et l'hiver se succèdent avec les variations annuelles qui leur sont propres : le printemps, plus ou moins régulier, avec ses alternatives de chaleur et de froid, qui activent ou suspendent la végétation, au point de la détruire quand surviennent les gelées dites printanières; - l'été, plus ou moins chaud; - l'automne, plus ou moins sec; - l'hiver plus ou moins froid. La suspension de la végétation par les froids de l'hiver dure quatre à cinq mois et la période d'activité se prolonge pendant sept ou huit. Mais, si la végétation est, en moyenne, plus rapide, la production annuelle est aussi plus variable; pendant telle année, un concours de circonstances atmosphériques favorables fait que la production ligneuse se trouve deux fois plus forte que pendant telle autre, où ces mêmes influences auront été mauvaises. Il en résulte que les bois formés dans les plaines présentent une assez grande irrégularité dans l'épaisseur de leurs couches annuelles et, par suite, peu d'homogénéité dans leur structure.

Division en zones. - En France, étant données la grande

folia, Thymus vulgaris, Genista scorpius, etc. M. Flahault, Professeur à l'Université de Montpellièr, a entrepris le travail considérable, mais d'un haut intérêt, qui consiste à établir des cartes forestières analogues, comme échelle et comme facture, aux feuilles de la carte géologique (Ch. Flahault, Projet de carte botanique, forestière et agricole de la France, Paris, Librairies-imprimeries réunies, 1895. Au sujet de la carte botanique, agricole et forestière de France, Annales de Géographie, 1896). 
étendue relative des régions dites de la plaine et la quantité variable de lumière, de chaleur et d'humidité qu'on y rencontre, il est nécessaire de les partager en un certain nombre de subdivisions. C'est encore la question d'humidité ou de répartition des pluies qui permet de distinguer les trois zones suivantes : $1^{\circ}$ zone du nord et de l'est ou pari-

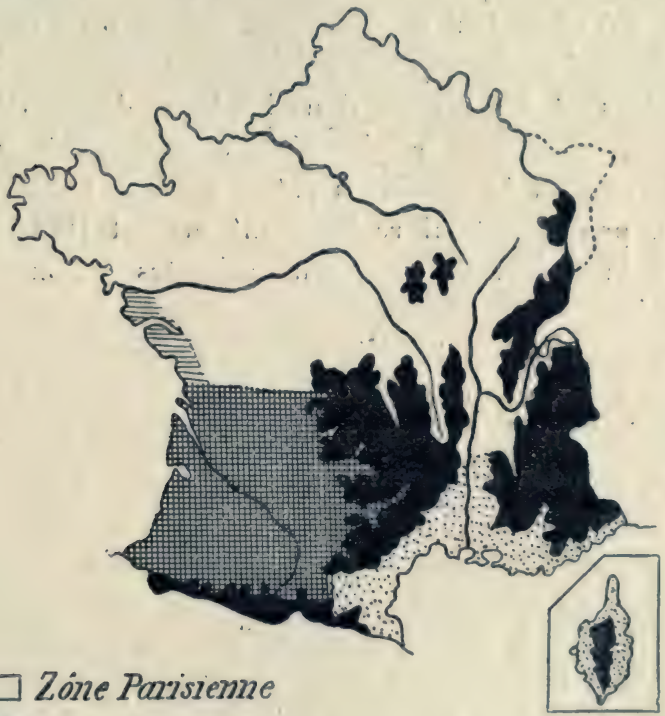

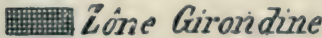

W. Zóne Proven cale

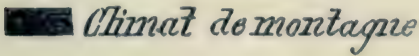

Fig. 37. - Carte des climats forestier's.

sienne; $2^{\circ}$ zone océanique ou girondine $3^{\circ}$ zone méditerranéenne ou provençale (fig. 37).

Zone parisienne. - Cette zone est la plus développée de toutes, elle embrasse plus de la moitié de la France, c'està-dire, toutes les contrées qui, à l'exception du Morvan, du Jura et des Vosges, s'étendent au nord d'une ligne orientée de l'Est à l'Ouest, de Valence à l'embouchure de la Gironde. Les forêts y sont, en totalité, peuplées d'essences feuillues? 
les bois résineux, pin sylvestre, pin maritime ou pin laricio d'Autriche, n'y apparaissent jamais qu'à l'état d'essences introduites par la culture. Le charme en est peut-être l'essence la plus caractéristique; le chêne rouvre et le chêne pédonculé sont plus nombreux là que partout ailleurs; le hêtre y abonde, sans appartenir en propre à cette région, puisqu'il s'élève dans la montagne. A côté des essences principales qui admeltent la culture en massif, on y rencontre, à l'état disséminé, des espèces précieuses, telles que: les frênes, les érables et les ormes, dont les bois, doués de qualités spéciales, apportent le plus utile contingent à la richesse forestière; c'est également la patrie des bois tendres : saules, tilleuls, peupliers, qui recherchent surtout les terrains fertiles et humides: de nombreux arbustes y constituent les sous-bois.

La quantité d'eau tombẻe atteint, en moyenne, 70 centimètres; mais, ce qui caractérise l'influence bienfaisante des pluies, c'est que la majeure partie se précipite en été, de sorte que les grandes sécheresses sont rarement à craindre. Les vents dominants sont ceux de l'Ouest, qui, chargés de nuages, activent peu l'évaporation et se condensent en brouillards et en pluies au moindre abaissement de température. Tout concourt donc à rendre le climat humide et essentiellement favorable à la végétation ligneuse. C'est, par excellence, la région qui produit les bons bois d'œuvre, région d'aspects très divers, toutefois, pour celui qui la parcourt de l'Ouest à l'Est.

A l'Ouest, sont les grands massifs forestiers bien limités, souvent isolés au milieu de vastes espaces livrés à la culture (fig. 38); beaucoup portent des noms historiques, presque tous sont d'anciennes forêts royales ou des biens d'apanage, dont les uns ont fait retour au Domaine, quand les autres sont devenus propriétés particulières. Si les étés sont moins chauds que dans l'est, du moins, les hivers y sont-ils plus doux: circonstances qui, jointes à la nature généralement siliceuse du sol, justifient le traitement en futaie, auquel nous devons les superbes forêts que nous avons déjà citées à l'article précédent. 
Par contre, le régime épuisant du taillis, la fréquence des gelées printanières et l'abus du pâturage aux siècles passés

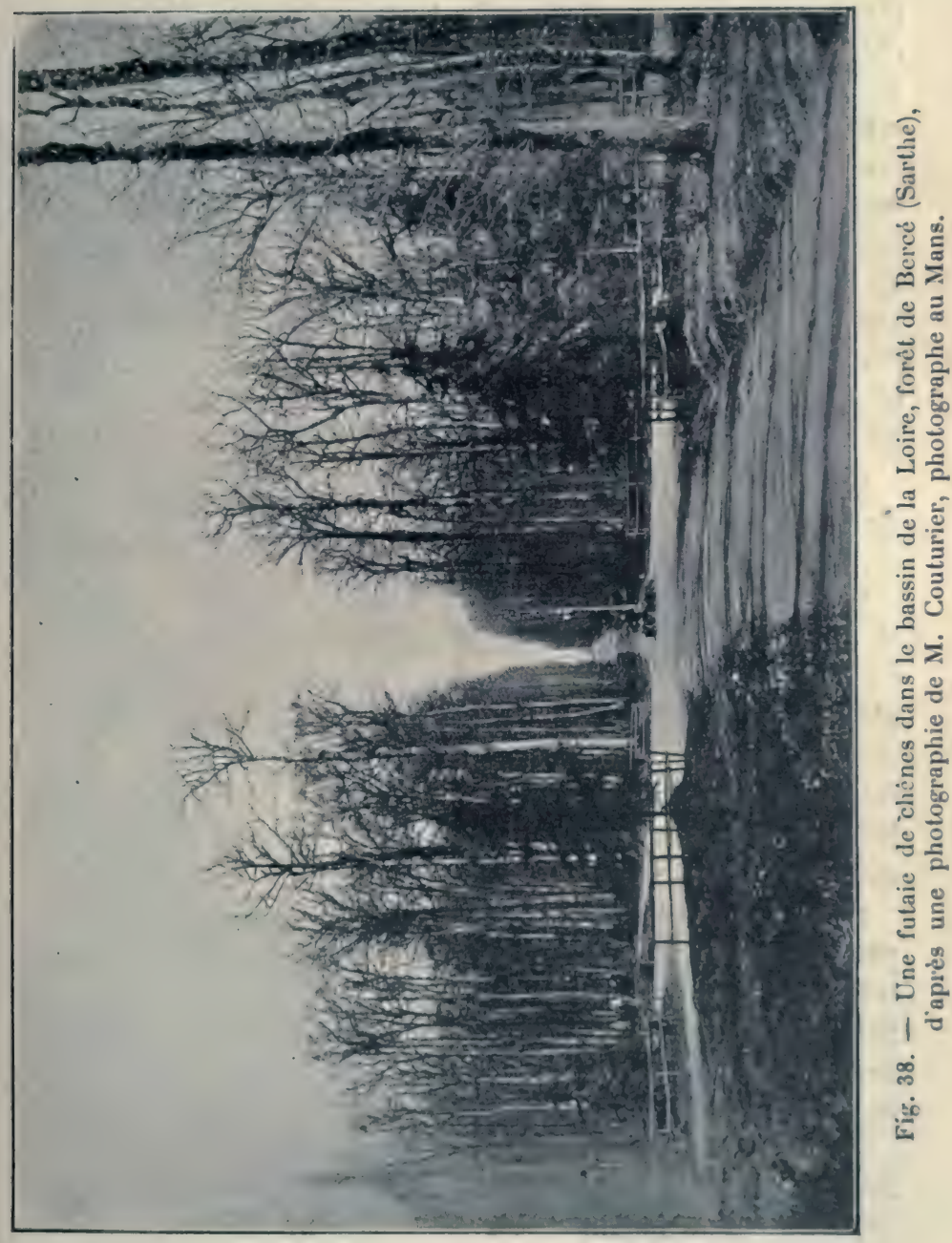

ont amené la ruine de nombreux massifs, dont les plus importants sont ceux de Fontainebleau, d'Orléans et de Montargis. 
A l'Est, le climat, plus excessif, prend un caractère continental, avec des étés plus chauds, des hivers plus froids. Les années de semence se font rares, et la nature généralement argileuse ou calcaire du terrain se prête mieux à la culture de l'arbre isolé ; par suite, le traitement en taillis sous futaie est commun et souvent très justifié. Suivant le relief et la fertilité des terrains, les forêts sont tantôt en massifs étendus, tantôt dispersées parmi les champs et les vignes, qui les entourent et les pénètrent. C'est le pays des gros chênes, à fût court, à boix nerveux, croissant au milieu de taillis, dont les produits, autrefois convertis en charbon, alimentaient les usines de la région. Le charme en est l'essence caractéristique; le hêtre abonde partout et sous toutes les formes : futaie pleine, réserve ou même cépée de taillis.

En fait, dans la zone parisienne, trois régions ou pays, bien caractérisés par les allures des forêts qu'on y rencontre:

$1^{\circ}$ les plaines de l'Ouest, ondulées par les collines du Perche et de la Bretagne, au climat doux et humide, sont le pays de l'herbe et de l'arbre, où les pommiers ombragent les pâtures, où le chêne et le hêtre s'alignent dans les haies du Bocage, en même temps qu'ils s'élancent dans les hautes futaies. Cette contrée se prolonge par les riches plaines du Nord, dont le sol, trop fertile pour elle, ne garde plus la forêt que sous la forme d'accident;

$2^{\circ}$ au Centre, la Sologne, pays siliceux, humide et déboisé par l'imprudence de l'homme, où, chaque jour, des travaux intelligents rétablissent la forêt indispensable à la vie; et, un peu plus au Nord, le grand îlot de la Champagne, dont le surnom de pouilleuse n'indique que trop l'antique pauvreté en arbres. Elle aussi se reboise;

$3^{\circ}$ enfin, à l'Est du ressaut de l'Argonne, dont la ligne de faîte se prolonge par le plateau de Langres jusqu'au Morvan pour fermer le bassin de Paris, s'étendent les plaines de la Lorraine, de la Franche-Comté et de la Bourgogne, avec leur taux de boisement qui dépasse 25 p. 100, avec leurs forêts feuillues, pour la plupart traitées en taillis-sous-futaie, où le 
hêtre, souvent expulsé par ce traitement, reparaît, en hâte, dès qu'on cesse de le maltraiter par des exploilations répétées à trop courts intervalles.

Zone girondine. - Elle suit les rivages de l'Océan, de Bayonne à l'embouchure de la Loire; c'est dans les Dunes et les Landes de Gascogne qu'elle est le mieux accusée. Ses caractères s'effacent et se fondent insensiblement avec ceux de la région tempérée, à mesure qu'on s'éloigne du littoral en s'avançant, dans la direction de l'Est, vers le plateau central. Le pin maritime (fig. 39 ), le chêne occidental et le chêne lauzin en sont les essences caractéristiques; ces deux dernières lui appartiennent en propre. On y remarque aussi le chêne pédonculé, particulièrement abondant dans les parties submersibles de la vallée de l'Adour, oủ il végète avec vigueur, atteint de remarquables dimensions et fournit ces excellents chênes de Bayonne, très recherchés dans les chantiers de constructions maritimes; - le chêne rouvre, qui, néanmoins, fait presque absolument défaut dans la région des Landes; le chêne yeuse, qui ne se trouve pas uniformément réparti et semble rechercher les terrains calcaires; aussi est-il rare dans les sables des Landes et n'a-t-il, en aucun point, la grande importance qu'il acquiert sur le littoral de la Méditerranée.

A ces végétaux essentiels des forêts se joignent d'assez nombreuses espèces arbustives de la région parisienne, auxquelles s'en ajoutent quelques autres de la région méditerranéenne.

Deux types de forêts distinguent cette région: les chênaies de l'Adour, et les massifs de pins maritimes réinstallés avec un plein succès sùr le sable des Landes. Franchement méridionale dans son aspect, avec le ton grisâtre de ses pins, ses touffes de daphne, de tamarix, de grandes bruyères, la Lande couvre de nombreux hectares, que limite la dune littorale du côté de la mer. C'est la terre classique de la lutte par la forêt contre l'envahissement des sables, contre l'insalubrité des eaux stagnantes. C'est aussi la seule contrée, en France, où l'on récolte la résine.

Le climat, moins chaud et moins sec que celui de la Pro- 
vence, convient parfaitement à l'agriculture. Il y tombe à peu près autant d'eau que dans la première zone; seulement, ici, ce sont les pluies d'automne qui sont les plus fréquentes, et les vents d'Ouest dominants y entretiennent une somme d'humidité suffisante pour les forêts, auxquelles la production des céréales, du vin et des fruits n'ont laissé qu'une bien

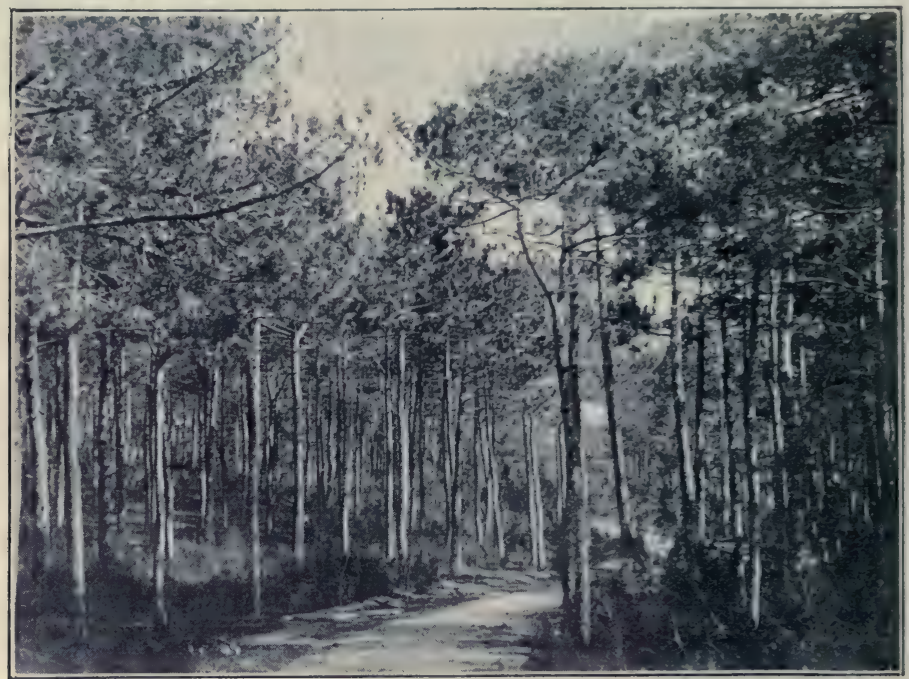

Fig. 39. - Forêt de pins maritimes à Arcachon, d'après une photographie achetée dans le commerce, auteur inconnu.

petite place, en dehors des départements des Landes et de la Gironde.

Zone provençale. - Région au climat sec et chaud, au sol en très grande partie calcaire, qui borde la Méditerranée de Nice à Port-Vendres; elle est resserrée à ses deux extrémités entre le littoral et le pied desAlpes-Maritimes à l'Est, celui des Pyrénées à l'Ouest, et s'élargit dans son parcours moyen; elle remonte le Rhône jusqu'aux environs de Valence, pénétrant, d'un côté, assez profondément dans les Alpes par les grandes vallées qui en débouchent, s’appuyant, de l'autre, sur les grands contreforts des Cévennes. Les forêts y 

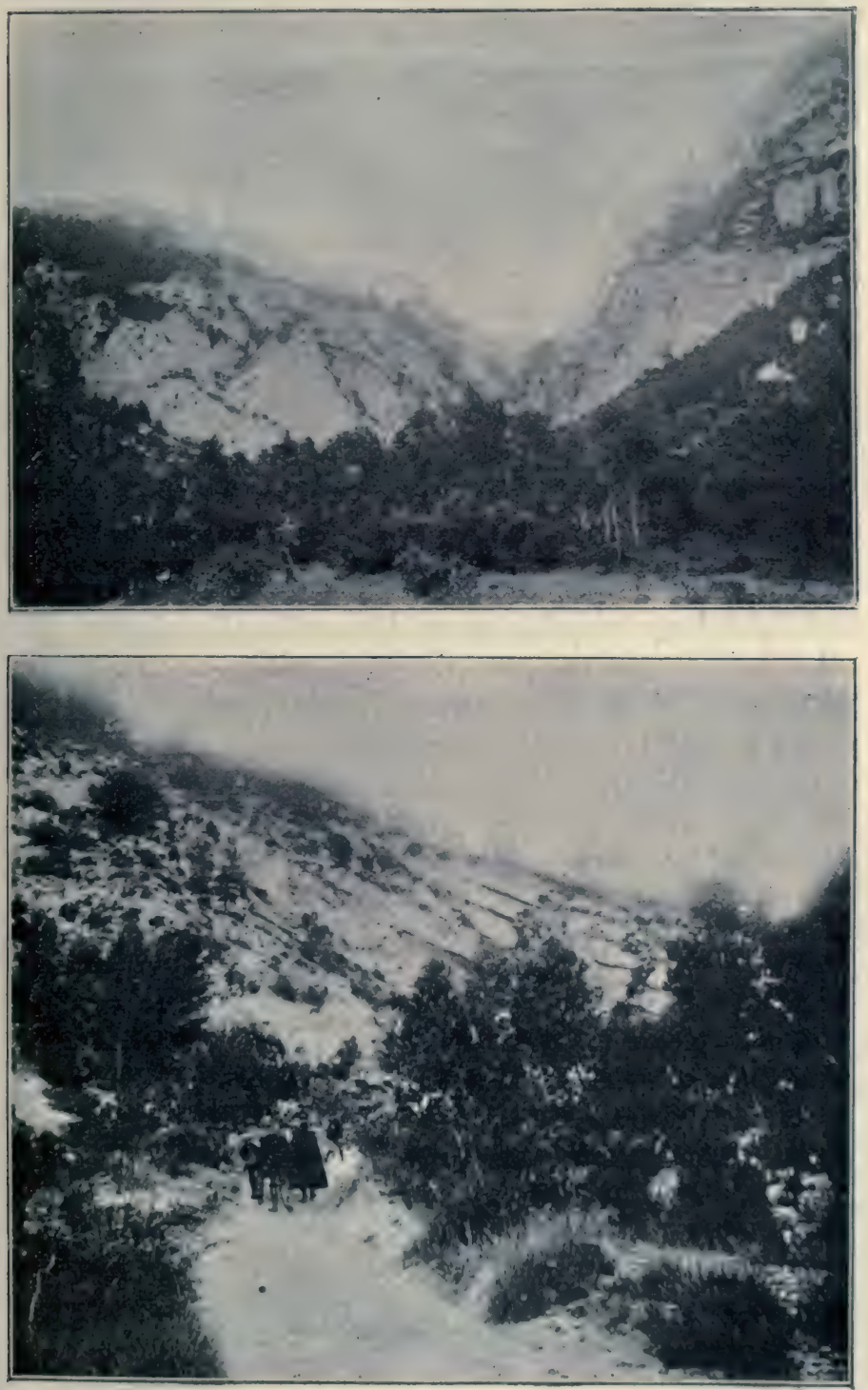

Fig. 40 et 41 . - Les gorges du Régalon et la Grande Combe dans la forêt de Mérindol (Vaucluse), pins d'Alep et chênes verts. (Photographie de M. J. George.) 
sont nombreuses, étendues; mais, malgré l'intérêt qu'elles offrent, elles ne présentent plus ces massifs frais et touffus, cette végétation vigoureuse, élancée des forêts des zones tempérées. Le chêne yeuse, le chêne blanc, et le pin d'Alep en sont les essences dominantes et caractéristiques (fig. 40 et 41); le pin maritime et le chêne-liège, qui, par ses produits accessoires, est appelé à devenir une des principales richesses du pays, n'apparaissent que sur les terrains siliceux des Maures et de l'Esterel. C'est, enfin, la région de l'olivier et du mûrier, qui n'appartiennent plus à la culture forestière. Les grandes espèces disséminées deviennent rares dans les forêts, oủ la végétation buissonnante prend un caractère spécial et une forme toute différente de celle des régions plus tempérées; les cistes, les térébinthes et les lentisques fournissent des sous-bois impénétrables, qui se dessèchent pendant l'été et sont l'une des principales causes d'incendies désastreux.

Les bois nourris sous ces climats chauds et bien ensoleillés jont, en général, durs, serrés, lourds, raides, sujets à se gercer et à se tourmenter; ils n'alteignent que rarement de forles dimensions, mais donnent des bois de feu appréciés, des charbons de première qualité et d'excellentes écorces à tan, dont la valeur va malheureusement en diminuant d'une façon inquiétante.

Malgré tout, même au point de vue forestier, il ne faut pas mépriser ces buissons d'yeuse qui s'arrondissent presque isolés sur des rochers brûlés par le soleil. L'état de misère dans lequel nous les voyons, ne fait que prouver leur résistance dans la lutte qu'ils soutiennent depuis des siècles contre la dent du mouton et la cognée du bûcheron piémontais. Loin de se montrer ingrats, ils favorisent la production de la truffe, dont, parfois, la récolte annuelle se loue plus de 500 francs par hectare. Signalons, enfin, le pin d'Alep: si l'on en juge par les massifs domaniaux qui ombragent le pied du Luberon, il peut fournir à l'industrie locale des produits fort intéressants.

La quantité annuelle de pluie tombée n'est pas de beaucoup inférieure à celle de la région parisienne; la distribution en est toutefois très différente. Plus de la moitié se pré- 
cipite en automne, l'autre moitié, en hiver et au printemps ; il en résulte, en été, des sécheresses persistantes; les averses d'automne élant très abondantes, on ne comple qu'un petit nombre de jours de pluie, circonstance qui, on le sait, est très défavorable à la forêt.

Le vent dominant est celui du Nord-Est; sous le nom de Mistral, il souffle avec violence dans la vallée du Rhòne.

\section{III. - Le elimat de montagne.}

Caractẻres gẻnéraux. - Les stations montagneuses sont caractérisées par un relief fortement accusé, des pentes raides, parfois abruptes, un sol souvent rocheux, mais, surtout, par des pluies et des neiges qui entretiennent une somme d'humidité à peu près constante, de telle sorte que les inconvénients de la sécheresse n'y sont à redouter dans aucune saison de l'année.

En montagne, l'hiver, au lieu de quatre à cinq mois, en dure sept à huit; il s'allonge ainsi au détriment du printemps et de l'automne. La neige, toujours abondante dans les hautes régions, fond brusquement et l'on passe, pour ainsi dire sans transition, de l'hiver à l'été. Il en est de même pour l'automne qui est abrégé par les chutes de neige prématurées. C'est ainsi que dans les Alpes, par exemple, aux altiludes de 1,800 à 2,000 mètres, la neige ne disparaît que vers le 15 juin et commence à tomber abondamment dès le 15 septembre; le printemps et l'automne, avec leurs influences utiles ou nuisibles, n'existent done plus que de nom dans ces stations élevées, où, au lieu de quatre, le nombre des saisons est réduit à deux : un hiver de huit mois sous la neige et un élé de quatre mois avec des quantités de lumière, de chaleur et d'humidité à peu près constantes chaque année.

Il en résulte qu'en montagne, les arbres ont une végétation beaucoup moins rapide ; mais que, croissant dans des conditions qui, d'une année à l'autre, restent toujours égales à elles-mêmes, leurs bois présentent des accroissements réguliers et homogènes. A ce point de vue, le climat des mon- 
tagnes présente une certaine analogie avec celui des régions les plus septentrionales du globe.

Il n'y a ici aucune subdivision à établir, car la conséquence de l'altitude se fait partout sentir sous la même forme, sans être sérieusement influencée par la latitude, dont le seul effet est de remonter ou d'abaisser le point où commence le climat montagneux. Il suffira donc d'indiquer sommairement les caractères spéciaux que présente chacun des cinq grands massifs naturels formés par les Vosges, le Jura, les Alpes, le Plateau central et les Pyrénées.

Vosges. - Le climat de montagne commence vers 350 mètres d'altitude dans les Vosges, qui forment une des contrées les mieux et les plus richement boisées de la France. Le sapin, le hêtre, auxquels se joint l'épicéa sur certains points, en sont les essences dominantes. Le chêne, le charme, n'en occupent que les contreforts et disparaissent complètement vers les sommets; les grands érables accompagnent le sapin jusqu'aux plus grandes altitudes. Le pin sylvestre, subspontané, est assez commun et provient, dans la plupart des cas, de travaux de reboisements entrepris sur une grande échelle, surtout depuis la création de l'Ecole des Eaux et Forêts.

La végétation forestière ne s'élève guère au-dessus de 1,250 mètres dans ces montagnes, où les cimes les plus élevées dépassent à peine 1,400 mètres.

Entre ces deux altitudes minima et maxima, cinq types de peuplements attirent surtout l'attention.

Si, placé sur la frontière de 1870 , on aborde le contrefort occidental de la chaîne au point où les collines de grès Vosgien s'éteignent dans la plaine Lorraine, on rencontre tout d'abord d'anciens taillis de charme, de chêne, de bouleau ruinés par ce mode d'exploitation que ne comportent, ni un sol trop pauvre, ni un climat déjà trop rude. Le sylviculteur avisé s'efforce d'y ramener le hêtre, partout oủ c'est encore possible; quand le mal est trop avancé, il a recours au pin sylvestre pour panser les blessures.

Plus haut, c'est la forêt des Basses-Vosges, où règne le noir sapin. Là, toute culture agricole devient impossible en dehors des parcelles irrigables, et la forêt couvre les ver- 
sants et les sommets; mais elle change d'aspect avec l'exposition; vigoureuse et touffue au Nord et à l'Est, elle s'éclaircit et se rapetisse à l'Ouest et au Sud, sous l'influence de la chaleur qui engendre la séchereśse. Les habitations sont agglomérées en villages et en hameaux au niveau des sources.

Au centre de la chaîne et dans sa partie méridionale, les terrains granitiques affleurent. L'eau, qui ne peut s'infiltrer dans les roches massives, abonde partout vers la surface. Aussi la sapinière est-elle souvent échancrée par des cultures eldes pâturages. Les habitations, dont chacune a sa fontaine, sont disséminées à toutes les altitudes et donnent à la région des Hautes Vosges un aspect tout particulier et qui n'est pas sans rappeler la Bretagne. La forêt, d'ailleurs, s'arrête vers les crêles, aux chaumes qui couronnent les ballons. Mais, avant de disparaître, elle se transforme en parcelles de protection, oú les arbres, à croissance lente, dégradés par la neige et couverts de lichens jouent, malgré leur faible rendement, un rôle des plus utiles, en abritant les massifs inférieurs contre les intempéries.

Sur la pente méridionale qui descend vers les plaines de la Franche-Comté, les schistes succèdent bientôt aux syénites et aux porphyres, et, brusquement, les sapins cèdent la place aux fouillies de chêne, exploitées en taillis simple en vue de la production des écorces. Autrefois, on y pratiquait le sartage conme dans les haies des Ardennes.

Signalons enfin, à la base occidentale, le petit pays de la Vosgge, îlot de grès bigarré, dont les sables à grains fins nourissent de fort belles futaies de chêne et de: hêtre. C'est de leurs assises que sourdent les sources minérales ayant donné naissance à la ceinture de stations balnéaires qui les entourent et dont Plombières est la plus ancienne.

Jura.- Les forêts du Jura sont moins élendues, en général, mais elles sont aussi riches, plus riches mème que celles des Vosges. Bien qu'assises en sol calcaire, tandis que celles de la région Vosgienne le sont en terrain siliceux, elles présentent avec ces dernières, dans leur peuplement et la nature des essences principales qui les forment, une similitude remarquable, avec un cachet spécial toutefois, à raison de la 
différence des latitudes, d'une part, et de l'autre parce que les derniers plateaux jurassiques appartiennent, par leur altitude, à la région alpestre, que les Vosges n'atteignent qu'exceptionnellement.

Les forêts du premier plateau, par leur végétation et leur composition, se rattachent bien plus à la région de la plaine qu'à celle de la montagne. Néanmoins, elles manifestent une tendance marquée à se laisser envahir par le sapin. D'ailleurs, il n'est pas facile d'établir si cette espèce descend vers la plaine, ou si elle ne fait que rentrer en possession des surfaces dont le traitement en taillis l'avait expulsée. Toujours est-il qu'aujourd'hui, partout où le sapin n'existe plus qu'à l'état de vieux arbres disséminés de loin en loin à travers les feuillus, des semis, longtemps cachés dans le sous-bois, pointent leurs flèches au-dessus des cépées et cherchent à se grouper en peuplements. Au prix actuel du bois de feu les propriétaires ne doivent pas regretter cette substitution.

Vers 500 ou 600 mètres d'altitude, sur les escarpements qui séparent le premier du second plateau, le sapin se montre, d'abord à l'état pur, pour se mélanger bientôt avec l'épicéa. Dans les sols riches et profonds qui appartiennent à l'assise oxfordienne de la formation jurassique, on rencontre les plus belles sapinières de France, celles de Levier et de la Joux, notamment. Ailleurs, les arbres sont moins élevés; mais les fûts se soutiennent encore bien, surtout quand ils sont poussés en hauteur par l'épicéa.

Celui-ci, moins difficile que le sapin sous le rapport de la profondeur du sol, manifeste surtout ses qualités d'essence rustique et productive dès qu'on atteint le troisième plateau, à 800 ou 900 mètres d'altitude. Plus haut, vers 1200 mètres, dans les forêts du Massacre et du Risoux, par exemple, on le voit se plier à tous les caprices d'un sol âpre et d'un climat rude. Il infléchit ses branches pour mieux résister au poids de la neige (fig. 8), et fait ramper ses racines traçantes jusqu'aux lésines pour y pénétrer. Il se régénère au besoin sur ses propres souches, comme nous l'avons dit plus haut. Souvent, après la mort brutale d'un peuplement renversé par le vent ou tué par les insectes, le sol, d'abord envahi par les sor- 
biers ou autres arbustes, se garnit avec le temps de semis qui végètent à leur ombre, jusqu'au jour oủ, solidement installés, ils referont la bonne forêt.

Au sapin, à l'épicéa et aux hêtres s'associe - quoique beaucoup plus rare - l'érable sycomore.Nous le mentionnons, car dans certaines forêts des contreforts du second plateau,

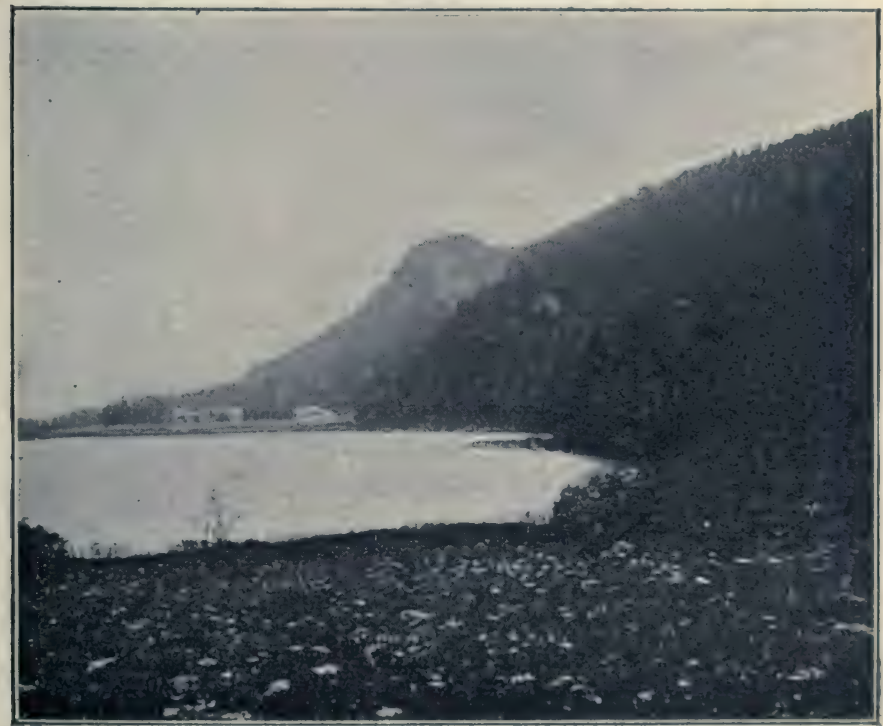

Fig. 42. - Chartreuse de Bonlieu, contreforts du deuxième plateau du Jura. (Photographie de M. J. George.)

en terrain argileux, il fournit, avec l'érable plane, une part notable des revenus de la forêt.

Toutefois, si le climat pluvieux (1) du Jura permet à la végétation forestière d'être luxuriante sur les calcaires les plus superficiels des grandes altitudes, il n'en est pas de même dans les régions plus chaudes, sur les pentes ensoleillées du

(1) Les plateaux du Jura, placés entre deux dépressions humides : la vallée de la Saône et la région des grands lacs, - étagés, de l'Ouest à l'Est, de façon à recueillir toute l'eau apportée de l'Ocian par les vents du Sud-Ouest, - reçoivent des quantités de pluie considérables, dépassant 1 mètre dans les hautes régions. 
Jura méridional notamment. Là exĩstent des taillis, qui sont d'un rapport plus que modeste. Il n'est que deux moyens d'en tirer parti - y cultiver le buis : expédient moins paradoxal qu'on peut le supposer, - ou leur substituer des peuplements de résineux.

Dans le Haut Jura, comme dans les Hautes Vosges, au-dessus de la forêt (fig. 43) s'étend une zone de pâturages, séparés

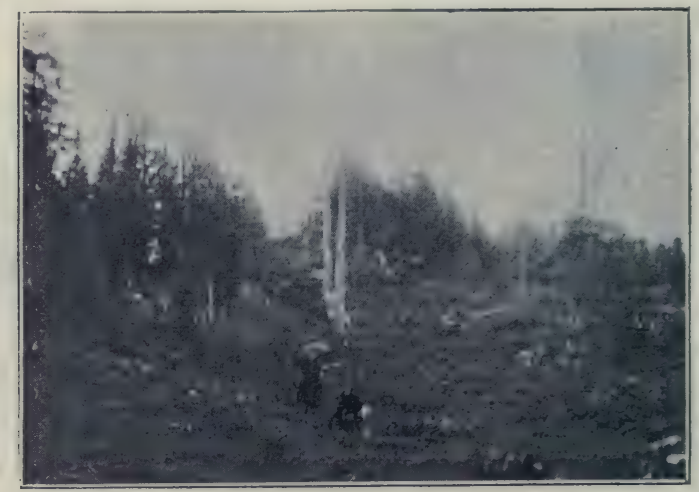

Fig. 43. - Limite de la végétation forestière, la Dôle (Jura).

(Photographie de M.J. George.)

des massifs inférieurs, tantôt par une lisière boisée, véritable forêt de protection, tantôt par cette culture mixte qui donne naissance aux prés-bois.

Alpes. - Le grand massif des Alpes, qui s'étend du lac de Genève à la Méditerrannée, présente, étagées les unes audessus des autres, toutes les zones de la végétation forestière française de la cote 0 à la limite supérieure de la végétation des espèces ligneuses.

La basse montagne et la chaînes des Alpines, jusqu'à 600 à 650 mètres d'altitude, bien qu'appartenant au massif par leur relief, jouissent encore des bénéfices du climat de la plaine provençale. On y trouve le chêne blanc, le chêne yeuse, le châtaignier, et toutes les espèces qui caractérisent cette station, - souvent aussi le pin sylvestre.

Plus haut, apparaissent les essences de montagne; d'abord, 
de vieilles connaissances : le pin sylvestre, le hêtre, le sapin, l'épicéa, puis trois nouveaux venus: le pin de montagne, le mélèze et le pin cembro.

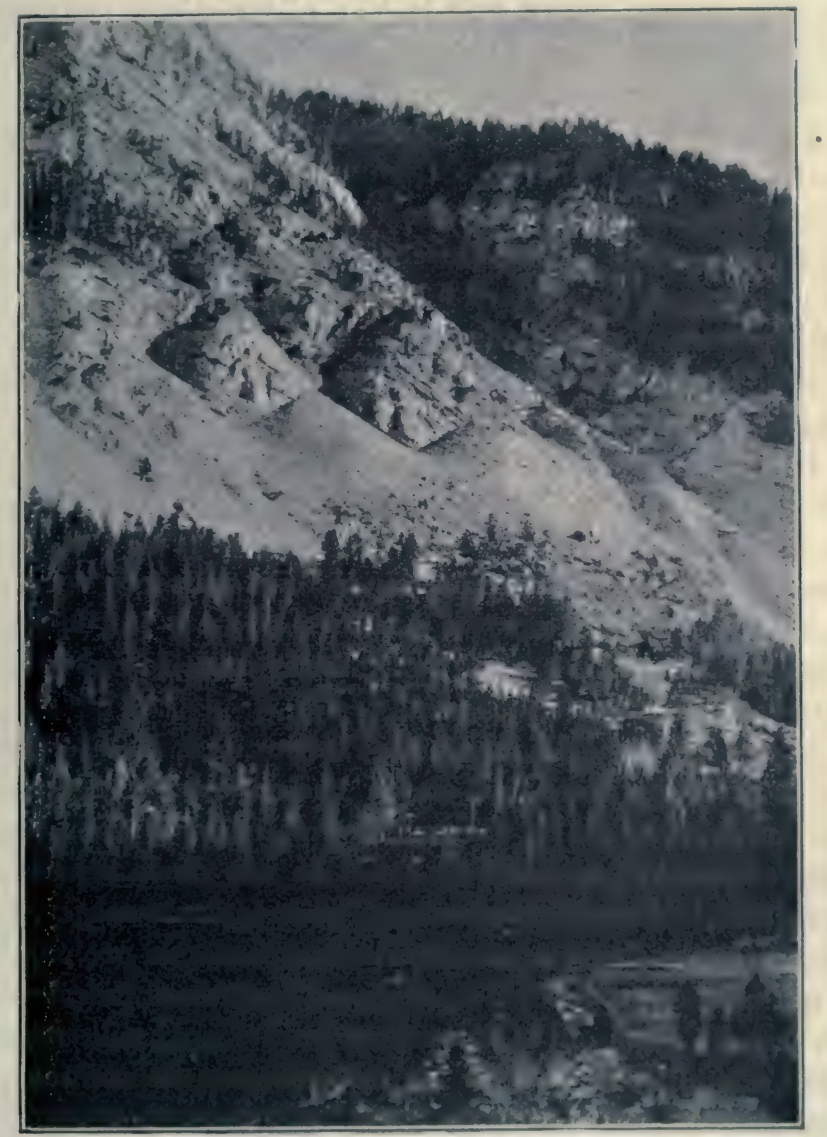

Fig. 44. - Forêt de montagne près de Saint-Étienne de Tinée (Alpes-Maritimes).

A l'exception du pin cembro, qui s'avance sous forme de sentinelle perdue, sur les confins des pâturages alpestres, toutes les autres espèces peuvent fournir de bons massifs, lambeaux plus ou moins étendus de la forêt continue qui 
couvrait les Alpes avant l'arrivée de l'homme. On peut citer encore: les hêtres du Vercors, les épicéas de la Tarentaise, les pins de montagne de l'Embrunois, les sapins de toutes les vallées fraîches, même dans le Var et les Alpes maritimes, et enfin les mélèzes du Briançonnais, du Queyras et du Comté de Nice.

Ce dernier fait la richesse forestière des Alpes. Tantôt, ce sont des massifs purs; tantôt, en mélange avec l'épicéa ou le pin de montagne, il s'accroche aux flancs les plus escarpés, - domine les crêtes les plus élevées et s'avance jusqu'aux éboulis et aux clappes, oú il a tout à souffrir des avalanches de neige et de pierres et surtout de la dent des chèvres.

Dans la partie française des Alpes, on peut distinguer deux régions forestières :

$1^{\circ}$ Les Alpes septentrionales, - les Alpes vertes, - du Mont Blanc au Pelvoux, dont les vallées fraîches, s'ouvrant au Nord, sont livrées à la culture pastorale et produisent assez pour nourrir leurs habitants, où la forêt préserve encore la montagne contre les ravages des torrents. Le hêtre, le sapin, l'épicéa forment les principaux massifs de cette Suisse française; les mélèzes y sont rares.

$2^{\circ}$ Les Alpes méridionales, du Pelvoux à la Méditerranée, où la direction Nord-Sud de la chaîne oriente les pentes du Dauphiné et de la Provence vers les expositions chaudes de l'Ouest et du Sud. Au point de vue de la répartition des pluies, le climat devient excessif ; à de violents orages, trop souvent mêlés de grêle, succèdent des sécheresses prolongées; le sol, calciné par le soleil du midi, est facilement entraîné par les paquets d'eau qui le délayent et le ravinent; ailleurs, des terres affouillables, que les assises rocheuses ne soutiennent pas, glissent, entraînant avec elles des pans de montagnes tout entiers. C'est la terre classique des torrents. Sans doute aux grandes altitudes, là où la roche donne de la solidité au terrain, il y a encore de belles et bonnes forêts (fig. 44). Sans doute, sur les versants Nord ou Nord-Est, sur les ubach, comme on les désigne dans certaines vallées, le sapin et l'épicéa forment des massifs pleins, ombreux et frais à quelques dizaines de kilomètres de la côte Niçoise; mais, souvent aussi, la forêt 
change d'aspect, elle est plus clairiérée et sa composition se modifie: l'épicéa se fait plus rare, il cède fréquemment la place au pin de montagne ou au pin sylvestre, suivant l'altitude; mais surtout, - et on ne peut que s'en louer - le mélèze acquiert une importance prépondérante. Comme feuillus, citons le hêtre, compagnon habituel des résineux, quand on ne l'a pas détruit systématiquement (1), - à titre de curiosité, le tremble, qui existe parfois en beaux exemplaires, en mélange avec les pins sylvestres, - enfin le châtaignier, doņt les massifs égayent le bas des versants et dont on ne saurait trop favoriser la propagation.

Mais dans l'une et l'autre de ces régions, faute de routes, ces arbres ne se vendent encore que des prix dérisoires. Souhaitons que l'initiative privée du commerce des bois introduise bientôt dans les vallées les mieux boisées des systèmes de transport perfectionnés, plans inclinés ou câbles aériens (2). Il est regrettable en outre que, dans ce pays, si bien doué par la nature pour la production du bois d'excellente qualité, les forêts aient en grande partie disparu par suite de l'abus du pâturage. L'administration forestière a reçu l'importante mission de reboiser les Alpes; mais c'est dans le but de consolider le sol que ces travaux sont entrepris, et il faudra que les forêts nouvellement créées aient accompli pendant bien longtemps ce rôle protecteur, pour qu'on puisse les considérer comme une véritable source de produits ligneux. En tout cas, jamais elles ne remplaceront les forêts de la plaine que certains économistes en chambre voudraient sacrifier à une ocuvre toute de restauration et d'entretien.

Plateau central. - Comme celles des Alpes, les forêts du Plateau central sont loin d'occuper la place qui leur serait assignée par l'exploitation rationnelle du sol; car, sous ce climat franchement montagneux, les bois sont susceptibles d'acquérir toutes les qualités désirables. La pauvreté actuelle des forêts résulte de la situation qui leur a été faite dans les temps passés, alors que, dans ce grand massif arrondi, les

(1) Sur quelques points, le hêtre fait naturellement défaut.

(2) E. Thiéry, Les transports par cables aériens. (Bull. Sociélé d'encouragement pour l'industrie nationale, Nancy, A. Nicolle, 1896.$)$ 
voies de pénétration étaient trop rares pour attirer le commerce des bois. Faute de débouchés pour les produits ligneux, les habitants n'ont estimé la valeur productive des forêts que sous forme de pâturage, et la dent des bestiaux a fait son cuvre de destruction. L'ouverture des grandes voies ferrées qui le traversent a sensiblement modifié la situation économique du pays; et sur ces terrains solides, où il suffit d'ensemencer le sol sans qu'il soit besoin de le soutenir, l'œuvre de reboisement, entreprise au milieu du siècle, marche rapidement avec plein succès. On y trouve déjà de beaux cantons en pleine production.

A ce groupe, peuvent se rattacher les montagnes du Morvan, où domine le hêtre, dont le hois de moule descend à Paris par les canaux et les rivières, - et celles des Cévennes connues par la forme de pin laricio qui porte son nom.

Pyrénées. - Au point de vue forestier, le massif des Pyrénées doit être séparé en deux régions distinctes; mais, ici, ce n'est plus comme dans les Alpes une simple affaire d'exposition, puisque dans tout son développement l'axe de la chaine orienté Est-Ouest, donne naissance à des vallées ouvertes du Sud au Nord; c'est une question d'influence climatérique plus complexe.

En effet, le climat et la flore des Pyrénées Orientales participent du climat provençal, tandis que le climat et la flore des Pyrénées Centrales et Occidentales sont influencés par le voisinage de l'Atlantique. La frontière entre ces deux stations peut être établie vers la ligne de séparation des eaux entre le bassin de l'Aude et celui de l'Ariège.

Dans la première, le climat se rapproche de celui des Alpes Maritimes, moins sec cependant. Les pluies torrentielles accompagnées de grêle y sont d'ailleurs moins fréquentes et moins redoutables; enfin le sol est plus solide. Aussi, bien que les inondations soient encore graves et dangereuses, ne prennent-elles pas le caractère désastreux des torrents des Alpes.

Les essences qui peuplent les forêts sont le sapin, le hêtre et le pin de montagne, qui prend le nom de pin à crochets. Les sapins forment dans le département de l'Aude, sur 
les derniers contre-forts de la Montagne Noire, de superbes massifs, dont les produits rivalisent en qualité avec ceux des Vosges et du Jura. Nulle part aussi, le pin de montagne ne donne des forêts plus denses et plus exclusivement pures. Il règne en maître au-dessus de l'aire du sapin, dans une région où épicéas et mélèzes, qui manquent complètement dans les Pyrénées, ne viennent plus lui disputer l'espace, comme ils le font dans les Alpes.

Les pâtures, médiocres, sont fortement dégradées par des abus de toute sorte et, plus particulièrement, par le pâturage immodéré des moutons et des chèvres.

La deuxième zone est beaucoup mieux partagée sous le rapport des pluies, que lui apportent régulièrement les vents d'Ouest. Sous leur action bienfaisante, les forêts et les pâturages s'améliorent. Les vallées, plus vertes et mieux boisées, prennent parfois l'aspect riant de la Suisse. Les bêtes à corne se substituent aux chèvres et aux moutons et les forêts, moins dégradées, se refont plus facilement.

Les essences sont à peu près les mêmes que dans la zone orientale; cependant, les forêts sont plus denses; le hêtre est en mélange plus nombreux avec les résineux; peu à peu le pin sylvestre se substitue au pin à crochets, qui devient d'autant plus rare qu'on se rapproche d'Irun et d'Hendaye. La montagne est ici franchement pastorale et forestière. Pourquoi faut-il que, malgré ses aptitudes, en dépit des lois et règlements, les habitants ne cessent de ruiner pâtures et forêts? Dans la partie centrale et notamment dans le département de l'Ariège, le mal a produit son maximum d'effet; car, sous la rubrique trompeuse de forêts domaniales, la statistique enregistre plus de 30.000 hectares de rochers nus et absolument improductifs. Jusqu’à ces derniers temps, la véritable situation forestière des Pyrénées était restée à peu près ignorée; en dehors des sapinières classiques de l'Aude, on parlait vaguement de surfaces couvertes de taillis de hêtre, agonisant sous le régime du fúretage; pour révéler, sinon les richesses, du moins les aptitudes forestières de la région, il a fallu que des forestiers fussent appelés par l'opinion publique pour préserver les stations balnéaires de Luchon

BOPPE et JOLYET. 
et de Barèges contre les inondations et mettre les sources thermales de Cauterets à l'abri des avalanches de pierres qui les mitraillaient du haut de la combe de Pégères. Mais ces sauvetages si vaillamment accomplis suffiront-ils pour convaincre les montagnards que la conservation des forêts est la meilleure prime d'assurance contre la ruine des pâturages? 


\title{
CHAPITRE $\mathrm{V}$
}

\section{LES MODES DE TRAITEMENT}

\author{
ARTICLE PREMIER
}

\section{LES OPÉRATIONS CULTURALES.}

La régénération. - Les dégagements de semis. - Les éclaircies; leu but cultural. - Leur but économique. - La manière de les conduire. - Leur importance.

La régénération. - Dans la culture forestière, l'intervention de l'homme se rapporte à deux ordres d'idées distincts : la régénération et les améliorations. De la régénération, nous ne dirons rien ici : il en a été parlé déjà à propos de la reproduction de l'arbre et, d'autre part, les procédés qui permettent de l'obtenir, variables avec les modes de traitement qu'ils caractérisent, seront étudiés au chapitre VI.

Les dégagements de semis. - Au contraire, les dégagements de semis et les éclaircies tendent toujours à un même but, procèdent toujours des mêmes principes et peuvent être, par suite, l'objet d'observations générales.

Les semis de nos grandes espèces ligneuses, les brins de dix, quinze et même yingt ans, ont une croissance lente, si on la compare à l'évolution rapide des morts bois qui se jettent au milieu d'eux et, surtout, des rejets de souche qui parfois les èntourent. Il peut aussi se faire, il arrive même presque toujours, que, dans un peuplement mélangé, une des essences se montre envahissante aux dépens d'une autre. L'opération qui consiste à retarder l'essor des espèces secondaires ou trop ambitieuses, tendant à entraver le développement normal du peuplement d'avenir, constitue le dégagement de semis.

Dans la première jeunesse, les brins de semence, dont 
les rameaux latéraux n'ont qu'une faible importance, vivent surtout par leur bourgeon terminal, et l'on favorise leur développement par la simple suppression des obstacles qu'ils

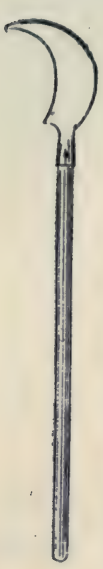

Fig. 45 . Croissant. peuvent rencontrer suivant la verticale : aussi, l'opération se fait-elle rapidement et à peu de frais. Sauf quand il s'agit de dégager de tout jeunes semis perdus sous un roncier qui les étouffe, point n'est besoin de couper rez terre : on se contente d'étêter les tiges nuisibles, en enlevant d'un coup de serpe les parties gênantes de leur cime. L'instrument le plus commode est le croissant, sorte de serpe, à manche long de 1 mètre à $1^{\mathrm{m}}, 50$, qui permet de faire l'opération très vite et sans se blesser (fig. 45).

En principe, un dégagement de semis n'est pas une guerre aux morts bois. L'objectif, dans une opération de ce genre, ne doit pas être la chose á détruire, mais le brin à dégager. Aussi écartonsnous le terme netloiement, qui est parfois employé comme synonyme de dégagement de semis. Chaque coup de serpe donné sans but précis est inulile et nuisible. Inutile, parce qu'il augmente sans profit les frais d'un travail toujours coûteux, - nuisible, parce qu'il est important de ne jamais détruire l'état de fourré, quelque négligeables que soient, au point de vue de leur valeur vénale, les espèces qui entrent dans sa composition. En isolant complètement les tiges d'avenir, on risque, en effet, de les voir se courber sous le poids de la neige, du givre ou de leur propre feuillage; de plus on découvre le sol : résultats fâcheux à tous les points de vue. Nous déconseillons donc absolument les expurgades trop souvent pratiquées.

Quand faut-il commencer les dégagements de semis? Quelle périodicité faut-il leur donner? Ces questions ne comportent pas de réponse catégorique. Un pareil dégagement n'est justifié que s'il est opportun : c'est au forestier de voir quand les semis ont besoin de son secours, et il doit le leur apporter aussitôt, et aussi souvent qu'il est nécessaire.

Nous prévoyons une objection : les dégagements de semis sont des opérations onéreuses... C'est vrai; mais les dépenses 
seront bien réduites si l'on évite des recépages inutiles. Quant à vouloir compenser les frais par la vente des produits, ce serait faire un mauvais calcul : on se laisserait aller à de véritables coupes, dont le préjudice dépasserait la mince rémunération.

Nous terminerons par deux conseils : le premier est de donner ses soins toujours aux mêmes individus. Il est regrettable, en effet, de prendre la peine de dégager une première fois un brin, puis de l'oublier pour s'occuper d'un autre qui, noyé jusque-là dans le fourré, a perdu toute vilalité. Le second est de faire exécuter, dans la mesure du possible, les dégagements par les gardes de la forêt. Un bon garde doit défendre les semis confiés à sa surveillance contre les morts bois, comme il les défend contre les délinquants. Des primes l'encourageant dans cette voie sont un argent mieux placé que des salaires donnés à des tâcherons ignorants des choses forestières et qui saccagent tout autour d'eux. Si même le propriétaire veut prêcher d'exemple et, quand il se promène dans son domaine, s'armer d'un croissant au lieu d'une canne, il sera tout étonné du nombre très respectable de jeunes chênes ou de jeunes épicéas qu'il " tirera d'affaire " dans un temps relativement court. Les dégagements les mieux faits sont ceux dont le prix ne dépasse pas la valeur de deux ou trois journées par hectare.

Les éclaircies; leur but cultural. - Pour l'éclaircie, au contraire, le bûcheron s'impose : nous travaillons au milieu de perches ou même d'arbres.

Bien que l'effet principal de l'éclaircie soit toujours de favoriser la croissance des sujets d'élite, on peut dire qu'elle poursuit un double but : au point de vue cultural, maintenir un peuplement dans les meilleures conditions de végétation, ou un mélange dans les proportions voulues; - au point de vue économique, augmenter le rendement.

Dans un peuplement, on constate à tous les âges la lutte pour la vie entre les sujets qui le composent, lutte qui se traduit par l'élimination d'un nombre considérable d'entre eux, surtout pendant la jeunesse.

D'après M. R. Hartig, le nombre de tiges à l'hectare dans 
les forêts de hêtre du Spessart, en Franconie, est environ le suivant (1):

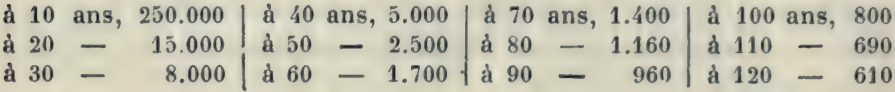

D'après M. Broilliard (2), la composition moyenne d'un peuplement régulier, bien complet, peut présenter en sapins des Vosges :

à $0^{\mathrm{m}}, 20$ de diamètre à hauteur d'homme :

à $0^{\mathrm{m}}, 30$

à $0^{\mathrm{m}}, 40$

$-$

à $0^{\mathrm{m}}, 50$

à $0^{\mathrm{m}}, 60$

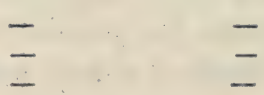

1.200 à 1.800 perches.

500 à 700 arbres.

300 à 400 -

200 มे 250

150 à 200

Or, pendant la durée des états de fourrés et de gaulis, les tiges les plus vigoureuses ont facilement raison des faibles : on peut laisser, sans inconvénients, la nature agir seule; on a même intérêt à le faire, car la sélection naturelle favorise les individus les mieux constitués. Au contraire, dès l'état de perchis, les éliminations portent sur des sujets plus gros, doués d'une plus grande vitalité et, par suite, présentant plus de résistance; les plus forts triomphent encore des plus faibles, mais les vainqueurs ne sortent pas indemnes de la bataille : il devient utile d'intervenir dans la lutte, pour pratiquer un véritable desserrement. Il arrive trop souvent que des arbres parviennent à se maintenir vivants en nombre excessif sur un espace donné; alors se constitue un peuplement dont les cimes étriquées, le feuillage insuffisant, attestent la situation précaire; non seulement la végétation se ralentit à l'extrême, mais ces peuplements sans vigueur sont à la merci de tous les accidents : ravages des insectes et des champignons, écrase. ment sous le poids de la neige, etc... Des éclaircies faites à temps et bien conduites auraient réagi contre cette tendance naturelle à s'uniformiser, toujours à craindre dans les peuplements d'un seul âge et constitués par une seule essence (3);

(1) Chiffres tirés du traité de M. Hüffel : Les arbres et les peuplements forestiers.

(2) Broilliard, Traitement des bois en France.

(3) Ce danger est particulièrement grand pour les peuplements d'origine artificielle. 
mais une fois les choses arrivées à ce point, le diamètre trop faible des tiges, hors de proportion avec leur hauteur, ne permet plus d'entr'ouvrir ces massifs, que le vent renverserait aussitôt.

D'autre part si, dans les peuplements mélangés, on ne continue pas, sous une forme quelconque, les dégagements commencés dans les fourrés et les gaulis, tous les sujets sauvés pendant le jeune âge seront bientôt irrémédiablement tués ou dégradés. D’où la nécessité de donner à l'éclaircie, dans certains cas tout au moins, le caractère d'éclaircie-dégage. ment.

But économique des éclaircies. - Nous avons, la chose est évidente, intérêt à activer le plus possible la croissance des arbres en hauteur et surtout en diamètre.

Beaucoup s'imaginent que plus les arbres sont serrés, plus leur croissance en hauteur est rapide, plus ils filent, suivant l'expression courante. C'est tout le contraire de la réalité. Sans doute un arbre isolé n'acquiert pas les hauteurs exagérées des arbres de massif, mais encore faut-il, pour qu'un arbre grandisse, qu'il dispose de l'espace nécessaire à la constitution de sa cime, et que sa frondaison reçoive une part suffisante de lumière. C'est dans l'atmosphère et par leurs feuilles que les arbres puisent la majeure partie de leur nourriture, et, s'ils sont mal nourris, ils ne s'allongent pas.

Il semble, qu'il soit bien inutile d'insister sur ce fait que des arbres trop serrés se développent mal, tant en hauteur qu'en diamètre... Varenne de Fenille, dans son premier mémoire (1790), signale les bons effets de l'éclaircie sur l'accroissement en hauteur de jeunes bois trop serrés. - Voici, littéralement traduit, ce qu'on lit, page 16 du volume de M. V. Baur, sur l'épicéa (1876): "C'est une erreur de croire qu'un état de massif trop serré favorise l'accroissement de la hauteur des peuplements; au contraire, la lutte trop longue et trop rude entrave le développement des hauteurs... "Le même auteur s'exprime d'une manière analogue au sujet du hêtre en 1881 (Die Rothbuche, page 115). Différents autres écrivains en 1885, 1888, etc... ont répété cette observation, devenue pour ainsi dire banale dans le monde forestier (1).

Le sapin se comporte de même, l'observation l'a démontré. Le fait que les éclaircies activent la croissance en diamétre

(1) G. Hüffel, Loc. cit. 
est trop connu pour qu'il y ait lieu d'en parler davantage. Il suffira de citer les chiffres suivants, tirés d'études de M. l'inspecteur Brenot sur les sapins du Jura (1):

\begin{tabular}{|c|c|c|}
\hline \multirow[b]{2}{*}{ Ages, } & \multicolumn{2}{|c|}{ Diamètre à hauteur d'lomme. } \\
\hline & $\begin{array}{l}\text { Sapins en massil } \\
\text { non éclairci. }\end{array}$ & $\begin{array}{l}\text { Sapins en massif } \\
\text { éclairci }\end{array}$ \\
\hline 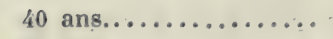 & $5^{\mathrm{cm}}, 5$ & $21 \mathrm{em}$ \\
\hline 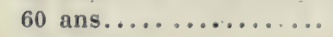 & $12 \mathrm{~cm}$ & $35^{\mathrm{cm}}$ \\
\hline 80 ans.. . . . . . . & $19^{\mathrm{cm}}$ & $48^{\mathrm{em}}$ \\
\hline 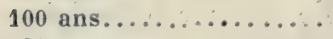 & $26^{\mathrm{cm}}, 5$ & $59^{\mathrm{cm}}, 5$ \\
\hline 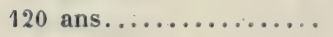 & $32^{\mathrm{cm}}$ & $69 \mathrm{~cm}$ \\
\hline 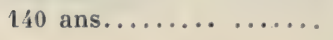 & $36 \mathrm{~cm}$ & $74 \mathrm{~cm}$ \\
\hline
\end{tabular}

De ce que le desserrement active la croissance en hauteur et en diamètre, il ne résulte pas qu'il soit toujours une cause d'augmentation de volume pour l'ensemble du peuplement; car celui-ci est appauvri de tous les mètres cubes réalisés par l'éclaircie. Mais, si l'on peut perdre en quantité, du moins est-on sûr de gagner - et beaucoup — en qualité : les tiges d'élite représentent les bons ouvriers, ccux qui fabriquent le bois de choix, le bois se vendant cher au mètre cube. On a donc tout intérêt à favoriser leur production, même au prix de sacrifices consentis au détriment de la masse, peu intéressante, des faibles ou des inhabiles.

Les éclaircies sont de véritables exploitations donnant des produits marchands: mais, ici encore, l'idée de récolte ne doit pas intervenir dans la conduite de l'opération, qui est d'ordre cultural avant tout. Toutefois, en même temps que l'éclaircie, peuvent se faire certaines réalisations, qui l'accompagnent sans en faire partie inhérente. Ainsi l'on profite de la présence du bûcheron pour débarrasser le peuplement des sujets tarés ou difformes, - de ceux qui sont envahis par les champignons et menacent de contaminer leurs voisins, - enfin de tous les bois morts ou mourants, qui attirent les délinquants, et ne jouent plus aucun rôle utile. De même encore, on fait coïncider avec une éclaircie l'exploitation des bois tendres, saules, trembles et autres essences sans longévité, qui dépériraient avant le passage de la coupe principale.

(1) Ces résultats sont traduits graphiquement, par M. Hüffel, loc. cit. 
Manière de conduire les éclaircies. - L'éclaircie consiste donc, à desserrer les sujets précieux, dans la région où leur cime manque d'espace, et cela progressivement. Les termes de cette définition demandent quelques explications.

Il s'agit de venir en aide aux sujets précieux; ceux là seuls seront notre objectif, et, agissant comme nous l'avons dit à propos des dégagements de semis, nous chercherons du re-

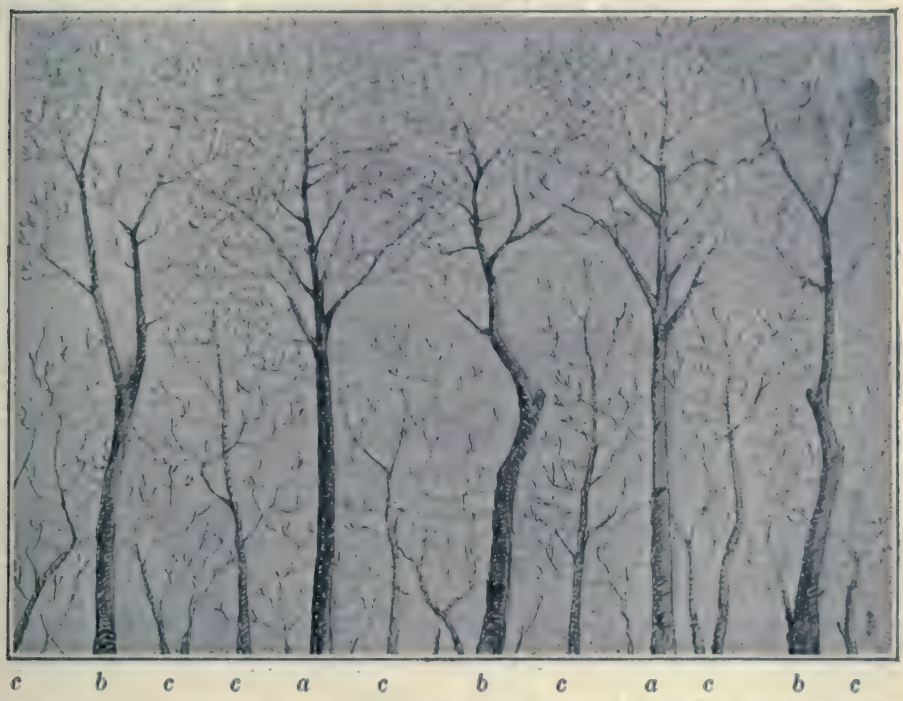

Fig. 46. - Schéma d'un peuplement à éclaircir. - aa, tiges d'élite; $b b$, sujets dominants mais qui nuisent aux tiges d'élite (à enlever); $c c$, sujets dominés (à conserver).

gard, non pas des tiges à abattre, mais des tiges à sauvegarder. Ces dernières sont celles qui, par leur espèce, par leur forme, et par la place qu'elles occupent, présentent le plus d'intérêt pour l'avenir. En général, dès les premières années, elles sont reconnaissables à leur aspect plus sain, à leur diamètre plus gros, à leur cime plus fournie (fig. 46). S'il en est autrement, ce qui arrive quelquefois, surtout dans les peuplements d'origine artificielle, la première éclaircie tend à rompre cette uniformité trop grande : on choisit au hasard, à des distances convenables, des perches à desserrer, auxquelles ce premier 
travail donne sur leurs voisins un avantage qu'il ne reste plus qu’à maintenir dans la suite.

L'éclaircie porte sur l'étage dominant, c'est-à-dire qu'elle enlève des perches, ou des arbres, dont le feuillage s'épanouit à peu près au même niveau que celui de l'individu dont on veut améliorer la situation. Au contraire, le respect scrupuleux de l'étage franchement dominé est la base fondamentale du système. Toutes les tiges qui, de guerre lasse, ont abandonné le combat pour la lumière, toutes celles qui sont réduites à végéter à un niveau inférieur à celui de rivales plus heureuses, tous les étages intermédiaires, tous les sousbois plus ou moins buissonnants, doivent échapper à la hache. Leur enlèvement serait inutile puisque leur feuillage ne peut plus faire concurrence à celui des sujets d'avenir. Il serait nuisible pour trois motifs: d'abord, leur maintien permet d'agir vigoureusement sur l'étage dominant sans crainte de découvrir le sol; en second lieu, ces bois dominés peuvent souvent reprendre leur essor et remplacer une tige d'élite détruile par un accident; enfin, celte végétation intermédiaire forme un écran, qui préserve les fûts contre la lumière directe du soleil, fait dépérir les branches basses, favorise en un mot l'élagage naturcl. Grâce à leur présence, on peut placer les sujets d'avenir dans cette situation excellente pour les arbres destinés à produire de bon bois d'œuvre : la cime au soleil, le fût à l'ombre, et les racines au frais.

Enfin l'éclaircie doit être progressive. Ceci nous amène à parler de l'époque à laquelle doit se faire la première éclaircie dans un massif, et de la périodicité avec laquelle les autres la suivront.

Dans les peuplements mélangés, les éclaircies-dégagements sont la continuation des soins donnés aux semis et leur succèdent sans qu'un intervalle trop long permette aux essences envahissantes de reprendre leur œuvre de dégradation. La grosseur des sujets et la réalisation de produits marchands fait changer l'étiquette de l'opération; son but reste le même.

Dans les peuplements purs, la première éclaircie se fait plus tôt quand il s'agit d'essences de lumière que quand il s'agit d'essences d'ombre. Il est important, dans tous les cas, 
d'opérer prudemment. En général, cette première éclaircie doit avoir lieu, dans les futaies, aussitôt les peuplements arrivés à l'état de gaulis ou, au plus tard, de bas perchis.

La périodicité varie surtout avec l'âge des peuplements. A chaque opération, on n'isole pas la cime des sujets d'avenir d'une manière complète, mais on se contente de dégager une ou deux de ses faces par l'abatage de quelques arbres gênants (1), avec l'idée d'opérer de même dans la suite aux autres orientations. Or, dans les peuplements jeunes, l'enlèvement d'une gaule ou d'une petite perche fait une lacune de peu d'importance bien vite refermée: les éclaircies devront donc se succéder à courts intervalles. Dans les peuplements âgés, au contraire, le vide créé par la disparition d'une grosse perche, ou d'un arbre, reste béant pendant plusieurs années : les éclaircies ne passeront que plus rarement.

La périodicité de 6 à 12 ans convient, dans les forêts traitées en fulaie, depuis l'état de gaulis jusqu'à la fin de celui de haut perchis, - et celle de douze à vingt ans, dans les hautes futaies constituées. Dans les sols fertiles, il faudra passer plus souvent que dans les sols médiocres et mauvais; plus, en effet, les conditions de végétation sont bonnes, plus les arbres tendent à prendre de l'ampleur, plus par suite leur nombre tend à diminuer à l'hectare; ainsi les forêts de pins sylvestres de l'Allemagne du Nord contiennent approximativement à l'hectare, les nombres de tiges suivants (2):

$\begin{array}{ccccc} & & \begin{array}{c}\text { Conditions } \\ \text { très bonnes. }\end{array} & \begin{array}{c}\text { Conditions } \\ \text { moyennes. }\end{array} & \begin{array}{c}\text { Conditions } \\ \text { mauvai ses. }\end{array} \\ \text { à } 40 \text { ans....... } & 1.750 & 3.050 & 5.600 \\ \text { à } 59 \text { ans....... } & 1.150 & 2.050 & 4.000 \\ \text { à } 60 \text { ans....... } & 800 & 1.500 & 2.800 \\ \text { à } 70 \text { ans } \ldots \ldots \ldots . & 650 & 1.100 & 2.000 \\ \text { à } 100 \text { ans....... } & 425 & 625 & 1.075\end{array}$

Dans la pratique, un bon criterium pour juger de l'opportunité d'une éclaircie est l'aspect même des arbres du peu-

(1) Ces arbres sont ceux dont la cime entrave le développement de celle de l'arbre d'élite. Ils peuvent fort bien ne pas être les tiges les plus voisines de ce dernier, mais s'en trouver séparés par quelques sujets dominés.

(2) G. Hüfrel, loc. cit. 
plement dominant. Si, par exemple, des chênes de futaie se couvrent de branches gourmandes, sans que leur âge très avancé ou toute autre cause accidentelle explique cette évolution anormale, c'est que leur cime manque de lumière dans les régions élevées; il en est ainsi encore des chênes dont la cime apparaît étriquée, souffreteuse, à frondaison chlorotique. De même, dans un perchis, un pin, un sapin, dépourvu de branches vivantes sur plus des deux tiers et surtout des trois quarts de la hauteur de sa tige, réclame d'urgence qu'on le desserre.

Nous avons dit qu'on rencontrait parfois des peuplements trop uniformes et trop serrés, dont les cimes réduites, usées par le frottement sous l'aclion du vent, accusaient l'état de langueur (fig. 47): on peut, à la rigueur, espérer les remettre en état par des éclaircies très prudentes, et répétées à intervalles fréquents, tous les trois ans par exemple. Dans les sapinières des Basses Vosges, des massifs compromis ont repris lentement leur essor, grâce à des opérations qui suivaient pour ainsi dire pas à pas la reconstitution du feuillage. Quoi qu'il en soit, il vaut mieux prévenir un pareil état de choses que que d'avoir à y remédier.

Dans les taillis sous futaies, on ne fait, en général, qu'une seule éclaircie, six à huit ans avant la coupe principale. Pourtant, sur certains sols, il peut y avoir intérêt à réaliser plus tôt, dans une première éclaircie, les bois tendres parvenus à maturité.

Importance des éclaircies. - L'éclaircie, telle que nous venons d'en exposer les principes, est appelée quelquefois éclaircie par le haut; elle est d'origine toute française. C'est elle que pratiquait le marquis de Rostaing, Réformateur général des eaux et forêts sous le roi Charles IX (1); c'est elle qui a fait l'objet des importants travaux de Varenne de Fenille en 1790 et 1791 ; enfin, mieux que toute autre, elle convient à notre tendance nationale qui nous invite à faire des individus, des beaux arbres, plutôt que des massifs d'une uniformité parfois monotone. Pendant quelque temps on a semblé

(1) G. Hüffel, loc. cit. 
l'oublier dans son pays natal, et il a fallu les travaux de MM. Bagneris et Broilliard, pour lui rendre la place qu'elle mérite dans la culture forestière.

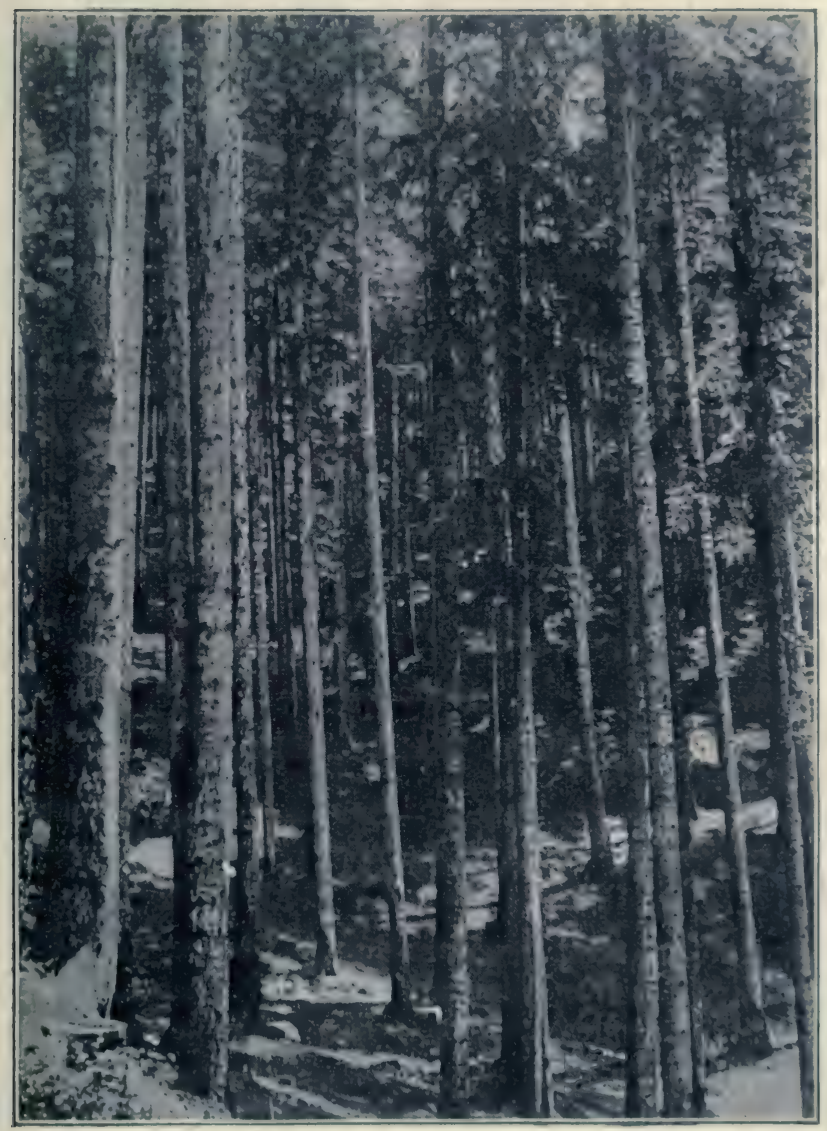

Fig. 47. - Perchis de sapins très serré, forét domaniale dés Élieux (Meurthe-et-Moselle). (Photographie de M. Juvanon du Vachat.)

Aujourd'hui, son rôle s'impose plus que jamais: on crée de tous côtés, par voie artificielle, des peuplements d'épicéa, de pin sylvestre, de pin noir, de mélèze, dans des stations 
souvent étrangères à ces essences. Si l'on ne se décide pas à entrer hardiment dans la voie des éclaircies, il ne faut pas espérer y produire autre chose que de la pâte à papier ou des étais de mine. Le maintien jusqu'à la haute futaie de pareilles forêts, toujours menacées de maladies cryptogamiques ou d'invasions d'insectes, n'est possible que si l'on y constitue des individus, en même temps que l'on hâte la formation d'un sous bois feuillu.

D'ailleurs, à une époque où il faut faire vite, il est bon de se rappeler que l'on a dans l'éclaircie le meilleur adjuvant à la rapidité de l'accroissement en grosseur. Ajoutons toutefois que, si l'éclaircie est toujours utile pour qui sait la manier, elle est d'une application délicate. Elle change de forme et d'intensité suivant les essences, suivant leur état de mélange, suivant le but poursuivi, suivant, enfin, les idées et le bon vouloir de celui qui la dirige. En un mot, c'est, pour les futaies pleines au moins, l'opération culturale par excellence, et celui qui n'en comprend pas le mécanisme fera mieux de s'abstenir.

ARTICLE II

\section{NOTIONS D'AMÉNAGEMENT}

Capital forestier. - Définitions. - Réalisation de la possibilité : par contenance, par volume, par pieds d'arbres. - Observations générales.

Capital forestier. - Un arbre ne donne des produits marchands qu'après de longues années d'existence. La récolte forestière est donc essentiellement périodique sur un point déterminé, et, pour la rendre annuelle comme l'exigent les besoins de l'homme, il est nécessaire de réaliser les produits, successivement, sur autant de points ou de surfaces différentes qu'il faut d'années aux arbres pour atteindre les dimensions requises. Pour satisfaire à cette condition, la forêt économiquement constituée, c'est-à-dire aménagée, doit donc présenter, sur des surfaces équivalentes, une suite non interrompue d'arbres ou de peuplements différant entre eux d'une année 
d'âge, depuis les sujets naissants jusqu'à ceux qui ont vécu le nombre d'années que ces mêmes dimensions comportent.

Ces derniers constituent seuls la récolte ou le revenu.

Le déplacement successif des surfaces occupées par les récoltes annuelles a fait naître l'idée de rotation, et on est convenu de nommer révolution le temps qui s'écoule entre deux récoltes successives sur le même point. C'est, en théorie, le temps laissé aux arbres pour atteindre les dimensions qu'on leur demande.

Les bois en croissance, c'est-à-dire ceux qui restent sur pied dans les surfaces autres que celles qui portent la récolte, font nécessairement partie du capital générateur, puisque, en dehors de leur présence, on ne peut concevoir de revenu annuel.

Exemples : Soit une forêt de 100 hectares couverte, par parties égales, d'une suite de peuplements âgés de un à vingt ans et dont les produits sont réputés réalisables à l'âge de vingt ans. La récolte sera fournie par les 5 hectares qui portent les bois de vingt ans, et les peuplements en croissance, répartis sur les dix-neuf autres surfaces, feront partie du capital d'exploitation.

De même, dans une forêt de 100 hectares peuplée de bois d'âges gradués de un à cent ans, le revenu sera fourni par le seul hectare où sont localisés les arbres de cent ans, et ceux qui sont âgés de un à quatre-vingt-dix-neuf ans et répartis sur les quatre-vingt-dix-neuf autres surfaces, seront comptés comme capital superficiel.

Il en résulte que la partie du capital générateur représentée par les bois en croissance est essentiellement variable. Son importance est entièrement subordonnée à la longueur de la révolution et va sans cesse en augmentant avec cette dernière : à des révolutions courtes correspond un capital superficiel restreint; les révolutions longues exigent un capital d'exploitation d'autant plus considérable qu'il renferme des arbres plus âgés et, par conséquent, ayant plus de valeur. - De plus, ce capital ligneux n'est pas seulement représenté par un cube de bois de dimensions quelconques, mais par ce même volume constitué d'une façon toute parti- 
culière au point de vue de la gradation des âges. C'est seulement lorsqu'il renferme la suite complète de ceux-ci, répartis sur autant de surfaces d'égale production, qu'il est normalement constitué : on dit alors que la forêt est normale.

Dès lors, le capital forestier se compose de deux éléments distincts, qui fonctionnent concurremment, mais chacun à sa manière, ce sont: d'une part le fonds de terre (1), dont la valeur, subordonnée à sa fertilité, est soumise aux mêmes fluctuations que celles des autres biens ruraux : il donne l'espace et fournit la quantité;-d'autre part, le capital superficiel, de valeur essentiellement variable, mais de forme définie : ce dernier se constitue par l'épargne et grandit avecle temps; il travaille uniquement à l'accroissement des produits en qualité.

Le caractère particulier du capital ainsi constitué est d'être toujours en partie mobilisable : ce qui expose à des confusions fâcheuses avec le revenu et, par suite, à des abus de jouissance. Par contre, toute fraction du revenu qu'on ne réalise pas, s'incorpore et fonctionne avec lui, naturellement et sans frais.

De la composition toute spéciale du capital forestier résultent les conséquences suivantes, qu'il suffit d'énoncer ici sous forme de propositions : $1^{\circ}$ toutes choses restant égales d'ailleurs, plus les révolutions sont longues, plus le revenu annuel est considérable ; $2^{\circ}$ le taux de placement suit une marche inverse, il est d'autant plus faible que les révolutions sont plus longues (2).

(1) Ce fonds de terre comprend le sol, avec son ensouchement ou les graines forestières prêtes à germer qu'il renferme, sa réserve d'humus, et aussi les travaux de l'homme qui facilitent l'exploitation de la forêt : bornages, chemins de vidanges, etc.

(2) Ce capital-superficie augmente avec la longueur des révolutions. Très faible dans un taillis simple aménagé à 15 ans, oủ il ne comprend que des taillis de 1 à 14 ans, presque sans valeur marchande, il devient très grand dans un taillis sous futaie riche en réserves, plus considérable encore dans les futaies pleines aménagées à 150 ou 200 ans. Dans la forêt domaniale de Bercé (Sarthe), certaines parcelles contiennent, à l'hectare, 700 à 800 mètres cubes de chêne, représentant une valeur de 30 à 40000 francs pour le capital-superficie. Dans la forêt de Levier (Doubs), plus d'un hectare, dont le sol nu vaudrait 100 francs à peine, porte 1000 mètres cubes de sapin d'une valeur de 25000 francs. Ces massifs sont peut-être les plus beaux de France; mais dans des forêts même beaucoup moins riches en matériel, le capital superficie est souvent assez considérable pour rendre 
Définitions. - Coupes (1). - On appelle coupe, toute étendue déterminée dans une forêt pour y abattre le bois, en totalité, ou avec réserve d'un certain nombre d'arbres. Le mot exploilation est souvent employé dans le sens de coupe ou de vente.

La désignation du lieu où doit se faire une coupe s'appelle l'assietle. Ainsi, asseoir une coupe ou faire l'assiette d'une coupe, c'est déferminer son emplacement.

Une coupe est en usance lorsqu'on l'exploite, elle est usée lorsqu'elle est exploitée et vidée.

Les coupes sont dites principales, lorsqu'elles ont pour conséquence la régénération des surfaces parcourues.

Quand, dans une coupe, on exploite tous les arbres, on dit que celte coupe est faite à blanc-étoc.

Accroissement. - L'accroissement de volume, ou simplement l'accroissement d'un arbre ou d'un peuplement est la quantité dont le volume de cet arbre ou de ce peuplement s'accroît au bout d'un temps déterminé.

L'accroissement annuel est celui que les bois prennent en un an; l'accroissement périodique est celui que l'on réalise au cours d'une période de plusieurs années. L'accroissement annuel moyen, ou l'accroissement moyen, est le quotient d'un accroissement périodique quelconque par le nombre d'années de la période considérée, laquelle peut correspondre à la durée totale de l'existence des sujets.

Rentes. - Taux de placement. - Le revenu brut d'une forêt est le montant de ses produits, sans déduction d'aucun frais. Si l'on déduit de ce revenu brut les frais de production, on a le revenu net ou la rente.

Le taux de placement est le rapport entre la rente et le capital qui la produit (2).

le capital fonds presque négligeable dans les estimations en fonds et superficie.

(1) Tassy, l'Aménagement des foréts, 3e édition, Paris, 1887.

(2) Le taux de placement est le rapport du revenu au capital générateur. Donc, si beau que puisse ètre le rendement d'une forèt comme celle de Bercé ou celle de Levier, elle fonctionnera toujours à un laux très réduit. Au contraire, les plus mauvaises " rapailles " de chène vert, avec un rendement infime fonctionneront à un taux très élevé, le capital engagé étant nul ou à peu près.

BOPpe et JOLYET. 
Aménaggement. - L'aménagement est un travail qui consiste à régler l'exploitation d'une forêt, de façon que celleci fournisse un revenu annuel aussi soutenu et aussi avantageux que possible.

Tout aménagement a pour but de créer l'état normal et de le perpẻtuer quand il est obtenu. Le moyen employé consiste à régler l'ordre et la nature des exploitations, après avoir fixé l'âge ou la dimension des arbres à couper et le volume des produits à réaliser annuellement.

Pour faire comprendre l'importance de ces questions, il est nécessaire d'indiquer sommairement ce qu'on entend par les termes exploitabilité et possibilité.

Exploitabilité. - Un arbre ou un peuplement est $e x$ ploitable, quand il réalise le mieux possible le genre d'utilité qu'on réclame de lui.

Sans entrer dans le détail des différents services qu'une propriété boisée est susceptible de rendre, nous constaterons les deux faits suivants :

$1^{\circ}$ Dans les forêts de protection, on doit, en principe, laisser les arbres sur pied jusqu'à leur dépérissement: les produits ligneux n'ont donc qu'une faible valeur marchande;

$2^{\circ}$ Dans un grand pays comme la France, l'industrie réclame des bois de fortes dimensions. Pour les obtenir, il faut exploiter à des âges très avancés ; cela conduit à entretenir sur pied un capital superficiel considérable, et abaisse le taux de placement à un chiffre trop peu rémunérateur pour les propriétaires particuliers:

Ces deux considérations justifient la possession par l'État d'un certain nombre de forêts. Seul, il peut logiquement accepter cette situation, en vue de l'intérêt général.

Possibilité. - Théoriquement, la possibilité est l'expression de la capacilé productive de toute surface boisée.

Il est évident que si, dans une forêt normale, on se contente tous les ans d'enlever à la forêt, sous forme de bois exploitables, un volume moyen équivalent à celui dont s'accroissent les peuplements répartis sur toute la surface, le revenu en matière sera constant et le capital générateur maintenu intact.

Toutefois, le propriétaire désire tirer de son bien un revenu 
constant, tandis que la quantité de bois fabriquée est évidemment variable suivant les années ; d'autre part, les forêts étant, en général, plus ou moins éloignées de l'état normal, en suite d'excès ou d'insuffisance du capital générateur, il peut y avoir nécessité de couper plus ou moins que la production, en vue de se rapprocher de cet état. Il en résulte que, dans les forêts aménagées, la récolte annuelle, ou taxe, diffère sensiblement de la possibilité vraie; néanmoins, et par extension, on est convenu de désigner la quotité qu'elle représente par ce même nom de possibilité. Dès lors, la possibilité devient l'expression pratique du revenu en matière, tel qu'il est fixé par l'aménagement en cours d'application (1).

Série d'exploitation. - La série d'exploitatıon ou simplement série, est l'unité de gestion de la propriété forestière, car chaque série est destinée à fournir, durant la révolution, une même suite de coupes principales annuelles, ayant chacune à peu près même valeur. Nous avons donné la définition de la série, page 106 , en note.

Parcelles. - Lorsque, dans une même station et sous l'influence d'un même traitement, le peuplement se constitue identique à lui-même dans toutes ses parties, la surface qu'il occupe représente une parcelle naturelle. Quand, dans un peuplement, il se rencontre des différences dues à des causes permanentes, étrangères au traitement (essences, sol, exposition), on a affaire à des parcelles distinctes. Ainsi constituće, la parcelle est l'unité culturale de la série.

Réalisation de la possibilité. - Your réaliser la possibilité, on peut: ou bien faire porter les exploitations sur des surfaces égales ; - ou bien abattre des arbres en nombre indéterminé jusqu'à concurrence d'un chiffre de mètres cubes fixé à l'avance ; - ou, enfin, couper chaque année un même nombre d'arbres, choisis parmi les tiges exploitables, sans se préoccuper autrement de leur volume. De là trois méthodes d'aménagement.

La méthode par contenance, la plus simple, suppose impl-1citement que les facteurs de la production sont partout les mêmes, et que les produits matériels sont entre eux comme

(1) A. Puton, Traité d'économie forestière. Paris. 1888. 
les surfaces. Elle est toujours appliquée aux forêts traitées en taillis, mais peut très bien convenir aux autres.

La méthode par volume nécessite la détermination de l'accroissement moyen en volume; quel que soit le systeme employé pour arriver à ce résultat, on taxe la forêt à un chiffre de mètres cubes que l'on réalise annuellement sans s'inquiéter du nombre de tiges nécessaires pour l'obtenir. Lne pareille manière de procéder n'est rigoureusement possible que dans les futaies régulières, où tous les arbres qui constituent un massif donné arrivent en même temps au terme de l'exploitabilité.

Quand on traite une forêt comprenant des peuplements d'âges multiples, et dont les individus arrivent un à un au terme de l'exploitabilité, on peut opérer autrement: on estime, par tradition ou par comparaison, la production éventuelle de cette forêt; on évalue d'autre part le volume de l'arbre réputé exploitable, et le quotient du premier nombre par le second donne le nombre des pieds d'arbres à réaliser. Les futaies jardinées, la réserve des taillis sous futaie, peuvent être aménagés de la sorte.

Observations générales. - Quelle que soit la méthode adoptée pour déterminer les possibilités par volume ou par pieds d'arbres, elle ne saurait être parfaite. La prudence exige donc que des inventaires fréquents - répétés tous les dix ans par exemple - établissent les oscillations du matériel sur pied. C'est le seul moyen d'éviter des abus de jouissance ou des épargnes exagérées. Si ces inventaires sont dressés de manière à faire ressortir l'accroissement, ils fourniront des renseignements intéressants sur l'effet cultural des opérations.

Dans le même ordre d'idées rentre la tenue de sommiers de contrôle, registres où sont inscrits, par parcelle et par année, la nature et l'importance des coupes, les prix de vente, les travaux d'amélioration exécutés, le but poursuivi, les débours, etc. Une colonne est réservée aux " faits divers » de la forêt: gelées printanières, invasions d'insectes, années de semence, etc.

Les anciennes pratiques forestières francaises, réglementées par les ordonnances remarquables qui ont servi de modèles à notre Code forestier, prévoyaient les exploitations par conte- 
nance. On ne concevait pas autrefois, dans notre pays, une forêt aménagée,sans compartiments délimités sur le terrain et affectés chacun aux exploitations de telle ou telle année. On a fait à ces méthodes le reproche d'être trop primitives, trop peu savantes. Peut-être les critiques oubliaient-ils qu'elles avaient été un objet d'études raisonnées pour des agents de la marine et des forestiers éminents, parmi lesquels on s'honore de compter Réaumur, Duhamel du Monceau, de Buffon, Varenne de Fenilles. Peut-être aussi ne songeaient-ils pas que l'ordre et la suite dans les opérations de gestion sont plus assurés quand on est lié par des divisions assises sur le terrain, que lorsqu'on a pour seul guide un nombre de mètres cubes d'un calcul souvent problématique.

D'ailleurs, rien n'empêche de concilier les deux choses; libre à chacun de faire intervenir les volumes dans son système d'aménagement; mais nous ne cesserons de recommander, avec M. Broilliard, la division de la forêt en compartiments limités par des chemins de vidange ou des tranchées bien tracées, d'une contenance de 5 à 10 hectares, vérilables unités de gestion, dont le développement sera suivi pas à pas.

Rappelons enfin, avant de commencer l'étude des divers modes de traitement, que si les auteurs forestiers ont dû, pour les besoins de leurs règlements ou de leurs traités didactiques, classer les systèmes employés, leur donner des noms et leur imposer des définitions, ils admettent, du moins, que les méthodes se fondent entre elles, et que chacune possède une élasticité suffisante pourse plier aux exigences des arbres.

Sous l'empire de cette idée, nous avons, dans les quatre premiers chapitres, attaché la plus grande importance à tout ce qui concerne la vie de nos essences ligneuses. Les données qui vont suivre, et surtout le sens forestier du sylviculteur, suffiront, dès lors, à lui tracer sa ligne de conduite.

Nous étudierons successivement :

$1^{\circ}$. Le régime de la futaie: futaie régulière, futaie jardinée.

$2^{\circ}$. Le régime du taillis simple : taillis simple régulier, taillis fureté.

$3^{\circ}$. Le régime du taillis sous futaie.

$4^{\circ}$. Les améliorations possibles en toutes situations. 


\title{
CHAPITRE VI
}

\section{LES DIFFÉRENTS MODES DE TRAITEMENT}

\author{
ARTICLE PREMIER
}

\section{LA FUTAIE RÉGULIĖRE}

Principe de la méthode. - Avantages et inconvénients. - La régénération. - Procédé par coupe unique. - Procédé par coupes successives. - Soins culturaux. - Application aux principales essences.

Principe de la méthode. - On se propose pour but :

$1^{\circ}$ d'assurer la régénération complète et naturelle de la forêt en essences appropriées au sol et au climat, et de créer une suite de peuplements uniformes, d'âges gradués et d'une composition déterminée à l'avance;

$2^{\circ}$ de profiter de cet état régulier pour améliorer la situation des peuplements en croissance.

Avantages et inconvénients. - L'irrégularité première qu'affectent, en général, les fourrés et les gaulis est favorable à leur végétation; car, en-dessous de l'étage dentelé, formé par les sujets les plus forts, se maintiennent des tiges retardataires et surcimées, dont le grand nombre augmente la densité du massif. Le sol, hermétiquement couvert, se maintient dans les meilleurs conditions de fertilité pendant toute la période de jeunesse qui précède la formation de la haute futaie.

L'état uniforme, le plus souvent acquis vers la dimension de bas perchis, permet d'intervenir efficacement pour donner aux sujets d'élite les soins culturaux convenables. En favorisant l'élagage naturel, il augmente aussi la production du bois d'œuvre: les arbres prennent une forme régulière et allongée, leur bois reste généralement sain. Au point de vue de la qualité, la longueur exagérée des fûts, qui-accompagne 
une faible épaisseur des couches ligneuses, est plus ou moins avantageuse suivant les espèces : excellente pour les sapins

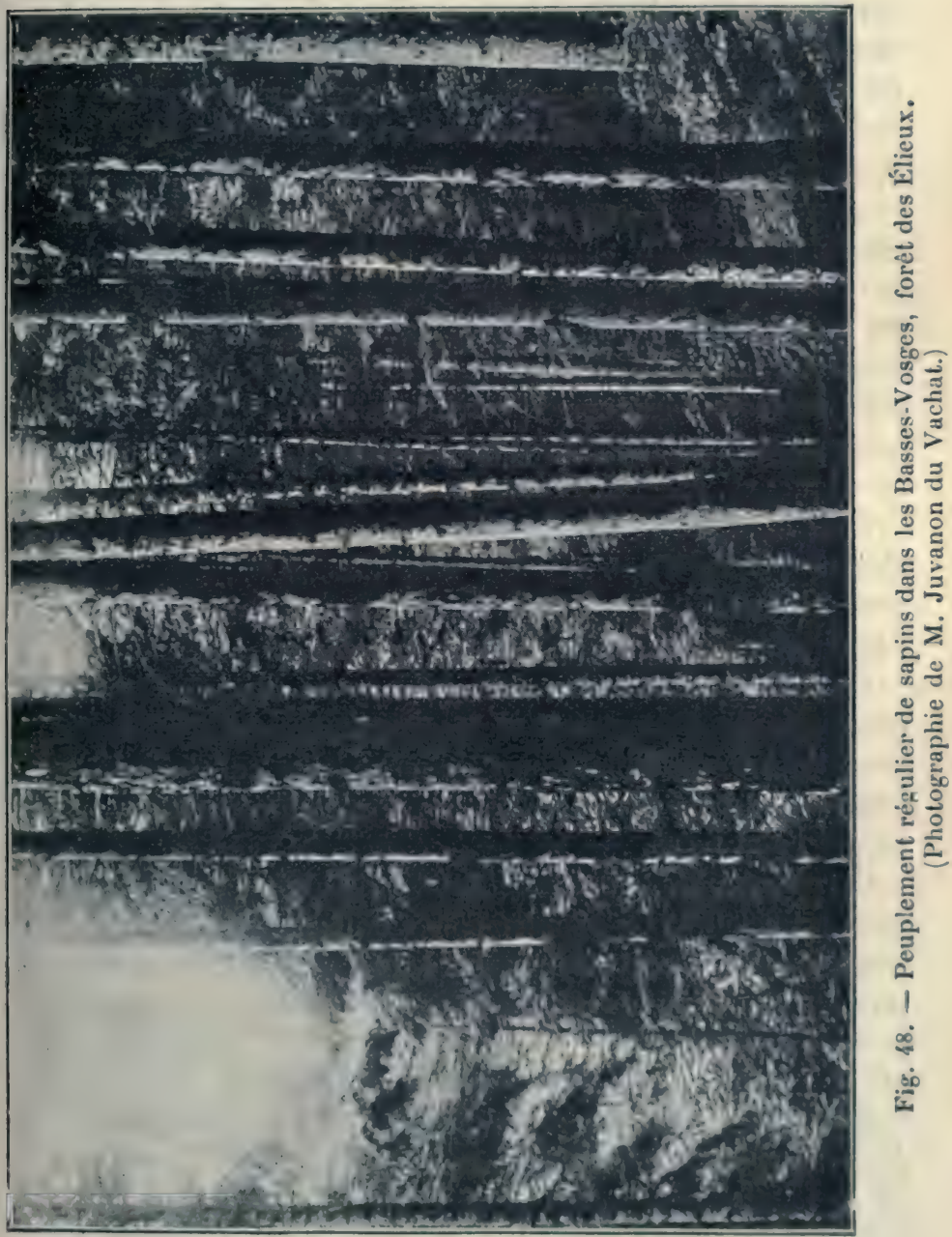

(lig. 48), les épicéas, les pins, - bonne pour les hêtres, parfois moins désirable pour les chênes.

Enfin, la futaie régulière assure la mise en ordre des forêts, 
le rapport soutenu, la facilité des exploitations et l'exactitude dans la complabilité et le contrôle.

Par contre, cette forme présente certains dangers, dontl'imporlance est atténuée ou aggravée par la composition des massifs. C'est ainsi que, dans les peuplements purs, la haute futaie, snuvent réduite à un seul étage, s'achemine plus ou moins vite, suivant le sol, le climat et les essences, vers l'état incomplet qui caraclérise la vieillesse. Le feuillage s'éclaircit, la couverture se dégrade, le sol se tasse, se durcit et là précisément où il va être appelé à recevoir les semences. De plus, ces massifs sont exposés à être renversés par les ouragans, ou encore écrasés par la neige, qui s'accumule en grande masse sur la surface horizontale de leurs cimes. Enfin, chaque sujet présentant les mêmes conditions d'âge et de végétation que ses voisins, ils offrent à l'invasion des insecles des milieux identiques et, partant, favorables à leur multiplication.

Dans les forêts mélangées, ces inconvénients sont en partie atténués; mais d'autres soins sont nécessaires, car ils exigent l'intervention continuelle du forestier pour maintenir l'équilibre entre des espèces de tempéraments différents.

En tout étal de choses, celle période de régénération, qui marque la fin d'un massif et le commencement d'un autre, conslitue un véritable temps de crise, perdant lequel une imprudence ou un accident de force majeure peut compromettre l'élat boisé.

En résumé, les allures des futaies régulières sont entièrement artificielles: les peuplements d'un seul âge ne se créent jamais spontanément que sur des espaces restreints, où la nature les a jelés au hasard, en un jour de colère; ils ne se perpétuent dans cette forme que grâce à l'intervention de l'homme et à l'aide de soins incessants. Aussi, bien qu'on doive toujours tenter de s'en rapprocher le plus possible, à cause des avantages incontestables qu'elle présente, sera-t-il prudent de ne la rechercher dans toute sa rigueur que si les agents naturels de la production peuvent se plier à toutes les exigences qu'elle comporte.

La régénération. - La régénération naturelle d'une futaie se réalise par trois sorles d'opérations consécutives : 
$1^{\circ}$ le desserrement des cimes, qui favorise la mise à fruits des porte-graines;

$2^{\circ}$ la destruction du sous bois et le relèvement du couvert, qui permettent au sol de recevoir la lumière et la chaleur nécessaires à la germination des graines et à l'évolution des semis;

$3^{\circ}$ la mise en état du sol, qui doit être assez meuble pour que les graines s'y enterrent légèrement et surtout que les racines des jeunes plants puissent y pénétrer.

Le desserrement des cimes est plus ou moins intense suivant les cas; il peut aller jusqu'à l'isolement complet.

La destruction des sous bois consiste dans l'enlèvement de toute la végétalion basse : sujets dominés ou morts bois. Le relèvement du couvert s'obtient par la coupe ou l'èbranchage des sujets dont le feuillage descend près du sol : les hêtres, les charmes surtout, sont le plus souvent dans ce cas.

La mise en élat du sol est inutile si le terreau et la couverture morte sont normalement constitués. Cette situation heureuse peut exister dans les forêts dont le pâturage est proscrit, quand on y a observé le respect des sous bois jusqu'à l'époque de la régénération. Mais, souvent aussi, le sol est trop tassé, ou bien une couverture vivante fait obstacle à l'installation des semis ; il faut alors intervenir.

Pour les semences lourdes, il suffit de remuer la couche superficielle du sol avec une charrue spéciale (1), ou simplement à la houe. L'opération se fait en automne, après la chute des glands et des faines. Ces " crochetages ", d'un usage courant dans les futaies de l'ouest de la France, où ils produisent les meilleurs effets, coûtent une dizaine de francs par hectare.

Quand il s'agit de graines légères, on détruit, avant leur chute, la couverture vivante (herbes, bruyères, myrtilles, mousses), ou bien on rompt la couverture morle trop épaisse (aiguilles d'épicéa, feuilles de hêtre) par les moyens les plus économiques dans la localité. Ces enlèvements se font tantôt sur toute la surface, tantôt sur des bandes alternes, de $0^{\mathrm{m}}, 60$ à

(1) Dubois, Travaux de reboisement exéculés à la charrue forestièré dans le Blésois. Blois, impr. Lecesne, 1862. 
$0^{\text {шi }}, 80$ de largeur, séparées par des intervalles incultes de 1 à 2 mètres. Mais, en toute circonstance, l'essentiel est de limiter les effets de la culture à quelques centimètres au-dessous de la surface, de façon à ne pas enfouir la couche de terreau en la mélangeant avec la terre minérale des zones plus profondes ; car c'est seulement lorsque les semences sont en contact immédiat avec l'humus, qu'elles germent et se développent assez promptement pour résister à la chaleur et à la sécheresse de l'été.

Parfois, dans les pentes exposées à l'ouest ou au midi, la superficie est dégradée à ce point que toute régénération naturelle ou artificielle y serait impossible. On peut améliorer la situation à peu de frais, en ouvrant une série de petils fossés disposés horizontalement en la forme de gradins. Les feuilles mortes s'accumulent au fond de ces rigoles, l'humidité s'y conserve, et il se forme une couche fertile, dans laquelle le semis naturel prend à la longue.

On peut aussi, dans certains cas, recommander l'exploitation par extraction de souches, qui ameublit le sol par places.

Enfin, dans les régions où l'habitude du panage s'est conservée, on a souvent recours à l'introduction des porcs. Mais cette pratique doit toujours être considérée comme un moyen de culture, et non comme un profit; car les produits de la glandée peuvent être entièrement dévorés par les hardes qu'on laisserait séjourner à jeun dans les coupes.

Nous ne saurions trop insister sur l'utilité de ces travaux de mise en état du sol. En les négligeant, on s'expose à attendre indéfiniment une régénération que quelques coups de pioche eussent suffi à provoquer.

Nous conseillerons d'ailleurs de ne pas hésiter à recourir à la régénération artificielle quand, logiquement, celle-ci s'impose, c'est-à-dire toutes les fois que les porte-graines sont en nombre insuffisant, toutes les fois que des gelées printanières répélées ou une cause accidentelle quelconque entravent manifestement l'intallation du semis. Il vaut mieux, en pareil cas, réaliser les bois exploitables avant qu'ils se dégradent, et reboiser par un des procédés que nous indiquerons dans le chapitre VIII. 
Enfin, la régénération par la semence et l'éducation des arbres en massifs uniformes pendant de longues années donnent inéluctablement l'a vantage aux essences les mieux appropriées au sol et au climat (1); à l'instar du hêtre, celles-ci éliminent toutes les autres. Si donc on préfère cultiver des espèces plus précieuses, mais moins bien armées pour la lutte, eu égard a la station, il faut adopter un autre mode de traitement: le taillis sous futaie, par exemple, où des recépages fréquents donnent plus de puissance à l'intervention du sylviculteur. II est illogique de vouloir marcher à l'encontre de cette loi naturelle. Nous avons vu quelquefois, avec regret, des régénérations en hêtre sur des terrains peu profonds de l'oolithe, qui ne demandaient qu'à prendre leur essor, et que l'on sacrifiait à l'espoir chimérique de leur substituer le chêne. Avec un pareil système, on dépense beaucoup d'argent, pour obtenir, en fin d'opération, des fourrés d'aubépine ou de cornouiller.

La régénération d'une futaie régulière peut se faire par coupe unique, ou par coupes successives.

Procédé par coupe unique. - Ce procédé consiste à exploiter systématiquement, en une seule fois, tout le matériel existant sur les surfaces à rajeunir, et à confier à la nature le soin de régénérer celles-ci par l'apport des graines provenant, soit des peuplements voisins, soit de quelques arbres réservés dans l'enceinte parcourue. En deux mots, la coupe unique est, pour la nature, une sorte de mise en demeure de procéder à la façon du malheureux qui ramasse un chiffon quelconque pour rapiécer son vêtement en lambeaux; elle prend une essence au hasard, la première venue, pour cacher la nudité du sol brutalement découvert. Ceci explique les bigarrures des vieilles futaies dont l'origine remonte à ce traitement. Ici, le hasard a favorisé le chêne; plus loin, le hêtre ou le charme sont à l'état pur; parfois encore, un champ de bruyères a succédé aux bois tendres et appelle la régénération artificielle en pins. L'ensemble constitue ces

(1) Exception peut être faite pour le sapin, qui, grâce à son abondante fructification et surtout à son tempérament d'ombre, est envahissant, même dans des stations oú il n'existe pas spontanément. 
"futaies irrégulières ", dont parlent Lorentz et Parade. Que l'exploitation soit faite à blanc étoc ou à tire et aire (1), la coupe unique ne peut être appliquée méthodiquement aux essences à graines lourdes; car, si le semis de ces espéces n'existe pas avant l'opération, il ne se formera plus aprés. En effet, le sol dénudé se dégrade rapidement et ne se couvre que d'cspèces à graines légères, de telle sorte que le jeune peuplement formé n'aura aucune ressemblance avec celui qui l'a précédé.

Même dans les circonstances les plus favorables, c'est-ìldire quand, au moment de son passage, le sol est garni de semis préexistants des essences à cultiver, la coupe rase n'est pas mieux justifiée :

$1^{\circ}$ parce qu'elle entraîne le développement de l'espèce unique dont le semis existe au moment oủ on vient découvrir le sol et donne ainsi naissance à des peuplements purs (généralement d'essences d'ombre);

$2^{\circ}$ parce que les semis préexistants disparaissent en grand nombre sous l'influence d'une trop brusque exposition aux agents atmosphériques (insolation, sécheresse, gelée);

$3^{\circ}$ enfin, parce que l'exploitation et l'enlèvement d'un matériel considérable sur des surfaces restreintes, fatigue le semis au point de compromettre son existence.

L'emploi de la coupe unique reste donc limité à la régénération des espèces à graines légères. Encore faut-il que celte condition soit accompagnée de certaines circonstances favorables: en montagne, par exemple aux grandes altitudes et sur les points oú la violence des vents empêche d'utiliser une méthode plus perfectionnée. En ces stations, le nombre des espèces est très restreint; les arbustes et les arbrisseaux faisant à peu près défaut, le sol dénudé ne se couvre que

(1) La coupe unique a été appliquée à toutes les futaies feuillues de l'Ile de France, de la Normandie et du Berry, en exécution de l'Ordonnance de 1669. Ces forêts étaient exploitées par contenance, de proche en proche et à tire et aire, avec réserve d'un certain nombre de porte-graines (20 par hectare); en même temps, on imposait l'obligation de ne jamais revenir èn arrière pour faire des coupes d'amélioration ou autres, et de répandre une certaine quantité de graines sur les parcelles récemment exploitées. 
d'herbes grêles et peu touffues, au milieu desquelles les graines ailées des mélèzes, des épicéas, des pins de montagne, provenant des massifs voisins, s'installent en plein découvert.

Pour faciliter leur régénération, ces coupes blanches sont disposées par bandes longues et étroites, et marchent à la rencontre des vents dominants. On cherche également à les établir dans la direction de la ligne de plus grande pente, plutôt que suivant l'horizontale; enfin, il est toujours prudent de réserver à la limite supérieure de la forêt une zone d'abri intacte. On doit d'ailleurs renoncer à cette méthode partout où les pentes sont très raides.

Quoiqu'il arrive, la régénération ne s'obtient qu'assez lentement et, souvent, on est obligé de la compléter artificiellement. Pour obvier à cet inconvénient, on 'a proposé de disposer les coupes par bandes alternées, dont les unes sont rasées et les autres conservées en massif plein; les bandes nues, mieux abritées et recevant la graine des deux côtés à la fois, auraient ainsi plus de chances de se régénérer. Nous connaissons dans les Alpes des couloirs d'avalanche qui simulent à merveille des coupes par bandes et qui se garnissent de semis. Mais, s'appuyer sur ces résultats accidentels pour ériger la chose en système de régénération, nous parait bien imprudent, d'autant plus que les bandes de massifs demeurés debout sont à la merci des ouragans.

Oủ la coupe unique donne, au contraire, des résultats à peu près certains, où elle semble d'une application logique et recommandable, c'est dans les stations chaudes des plaines girondines et provençales, lorsqu'on a affaire à des essences de lumière, et portant régulièrement de la graine, comme le pin maritime et le pin d'Alep. Il suffit d'exploiter le massif à blanc, en automne, avant la dissémination des graines; les cònes se détachent en ce moment et, si l'on prend la précaution d'activer l'exploitation et la vidange, de manière que le parterre soit débarrassé avant la germination des graines, on voit le sol se garnir d'une quantité de jeunes plants suffisante pour assurer la régénération. Ces jeunes sujets sont doués d'une végétation assez rapide pour s'élever en même temps que le fourré des morts bois qui les enserre de toute part. 
Dans les pignadars du Sud-Ouest, les cônes des arbres exploités jonchent le sol et le couvrent de graines qui germent bientôt; souvent, d'ailleurs, on laisse debout pendant deux ou trois ans quelques pins épars, analogues aux anciennes réserves prévues par l'ordonnance de 1669, et qui complètent l'ensemencement.

Procédé par coupes successives. - La méthode des coupes successives offre beaucoup moins d'aléa. Au lieu d'enlever en bloc tout le matériel sur pied (fig. 49) dans une parcelle donnée, on le réalise par fractions de telle sorle que le nouveau peuplement s'installe sous l'ombrage et se substitue graduellement à l'ancien. Ces opérations de régénération portent les noms de coupe d'ensemencement, coupes secondaires et coupe définitive.

Comme son nom l'indique, la première a pour but de favoriser l'ensemencement, en provoquant la production des graines et en mettant le sol en état de les recevoir avec utilité (fig. 50).

Si l'on tient compte de l'état superficiel du sol, de la fécondité locale des espèces et de la rigueur du climat, cette première coupe peut être faite tantôt sombre, tantôt espacée.

La coupe est sombre, dit M. Bagnéris (1), quand les branches latérales des cimes des réserves se touchent lorsqu'elles sont agitées par le vent.

Dans la coupe espacée, l'inlervalle entre les cimes peut aller de 2 à 5 et 6 mètres.

La coupe ombre est celle qu'on a le plus souvent l'occasion d'appliquer; elle est nécessaire toutes les fois que la semence est lourde et s'écarte peu du pied de l'arbre qui l'a produite, que le tempérament du jeune plant est délicat, que le sol est exposé à s'enherber fortement ou à se dessécher, qu'on opère sur les lisières des forêts ou dans les endroits exposés aux vents.

Quant aux porte-graines, il est évident qu'il faut les conserver parmi les pieds les plus vigoureux, ceux à fût élevé et dont la cime est largement développée. On doit, avant tout, s'attacher à une égale distribution du feuillage et non à la régularité de répartition des tiges.

Pour que la régénération soit suffisante à tous égards, il suffit que les espèces à cultiver soient représentées par quel-

(1) Bagnéris, Manuel de sylviculture, $2^{n}$ édition. Nancy, 1878. 
ques plants au mètre carré, pourvu qu'ils soient uniformé-

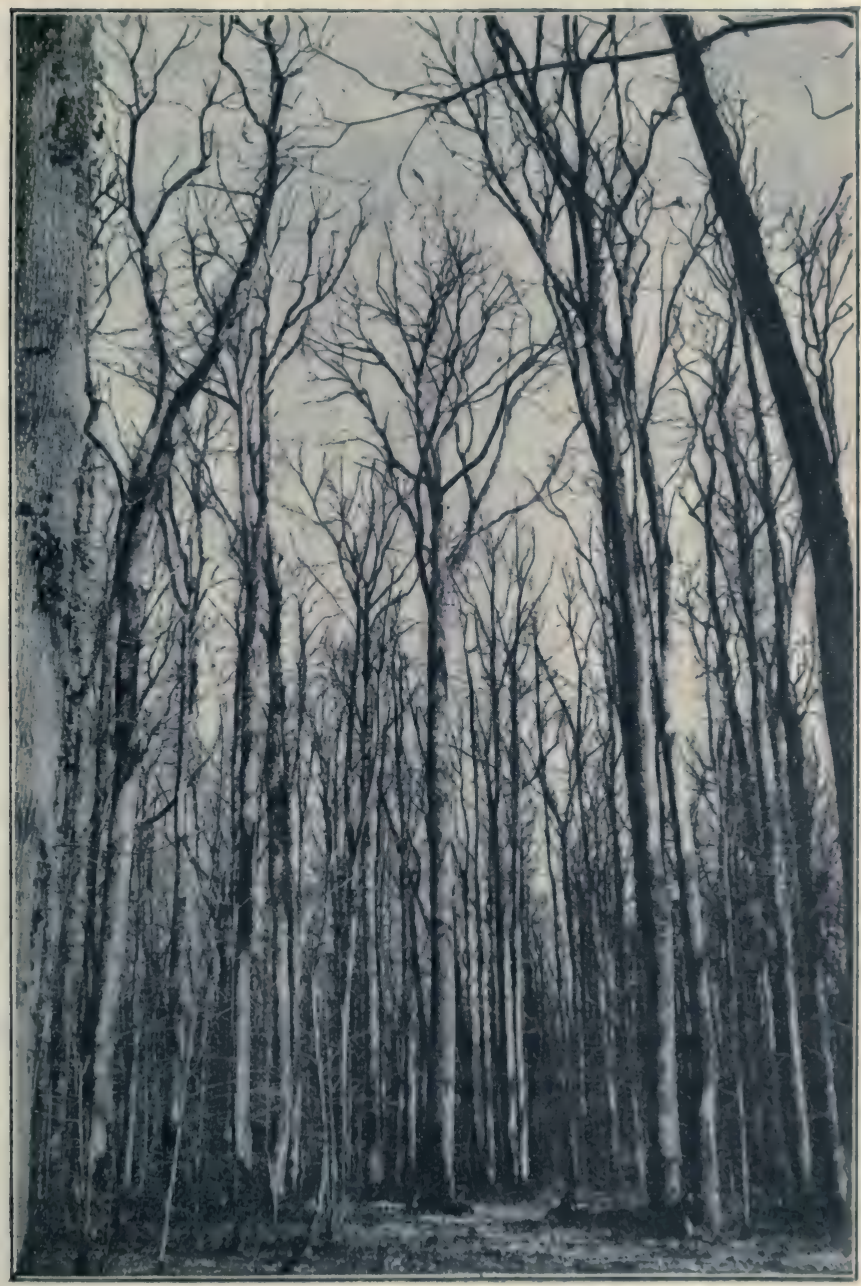

Fig. 49. - Vieille futaie de chênes, canton des Clos, dans la forét de Bercé (Sarthe). D'après une photographie de M. Couturier, photographe au Mans.

ment répartis. Ce résultat obtenu, il est inutile de refuser plus longtemps au.jeune semis la lumière dont il a besoin ; 
on juge d'ailleurs de son état de gêne à la pâleur de son feuillage et à la faible longueur de ses pousses.

Le moment est venu de faire les coupes secondaires. A cet effet,on désigne pour être abattus un certain nombre d'arbres, choisis parmi ceux qui recouvrent les semis les plus complets et les plus vigoureux: les plus gros sont généralement les plus nuisibles; on laisse mieux garnies et tout à fait intactes les places insuffisamment ensemencées ou celles qui sont peuplées de sujets trop jeunes (fig. 51), pour éviter à ceux-ci les insolations trop brusques, le desséchement du sol et les accidents de gelée (1).

Ces extractions se font avec une lenteur mesurée par la rigueur du climat, la fertilité du sol et le tempérament des essences.

Les circonstances fussent-elles aussi favorables que possible, on serait toujours amené à faire les coupes en plusieurs fois, afin d'épargner au jeune semis la fatigue considérable qui serait la conséquence de l'enlèvement d'une trop grande quantité de produits sur des espaces restreints.

Sous l'influence de ces coupes secondaires successives, le semis participe progressivement à la lumière ; il s'installe et grandit. En même temps s'opère le mélange naturel. Après les espèces sociales installées les premières, les formes disséminées, dont la graine légère vient des massifs environnants, apparaissent à leur heure, au fur et à mesure que le découvert fait naitre sur le sol les conditions favorables au tempérament de chacune d'elles. Quel que soit l'avenir réservé à ces régénérations de hasard, elles jouent, comme remplissage, un rôle des plus utiles et hâtent la formation du fourré.

On conduit ainsi le peuplement jusqu'au moment où, passant au gaulis, il n'a plus à redouter ni le plein soleil, ni la sécheresse, ni la gelée. Alors la coupe définitive lui donne un libre essor. Celle-ci fait disparaître les derniers représentants de l'ancienne futaie. A proprement parler, elle n'est que la dernière des coupes secondaires ; car elle n'enlève ni plus,

(1) Lès arbres d'abri : 10 diminuent l'intensité du rayonnement nocturne; $2^{\circ}$ empêchent un réchauffement trop brusque après la gelée. 
ni moins de matériel, elle ne donne ni plus, ni moins de lumière que ne le fait chacune de ces exploitations relativement à celle qui l'a précédée (1).

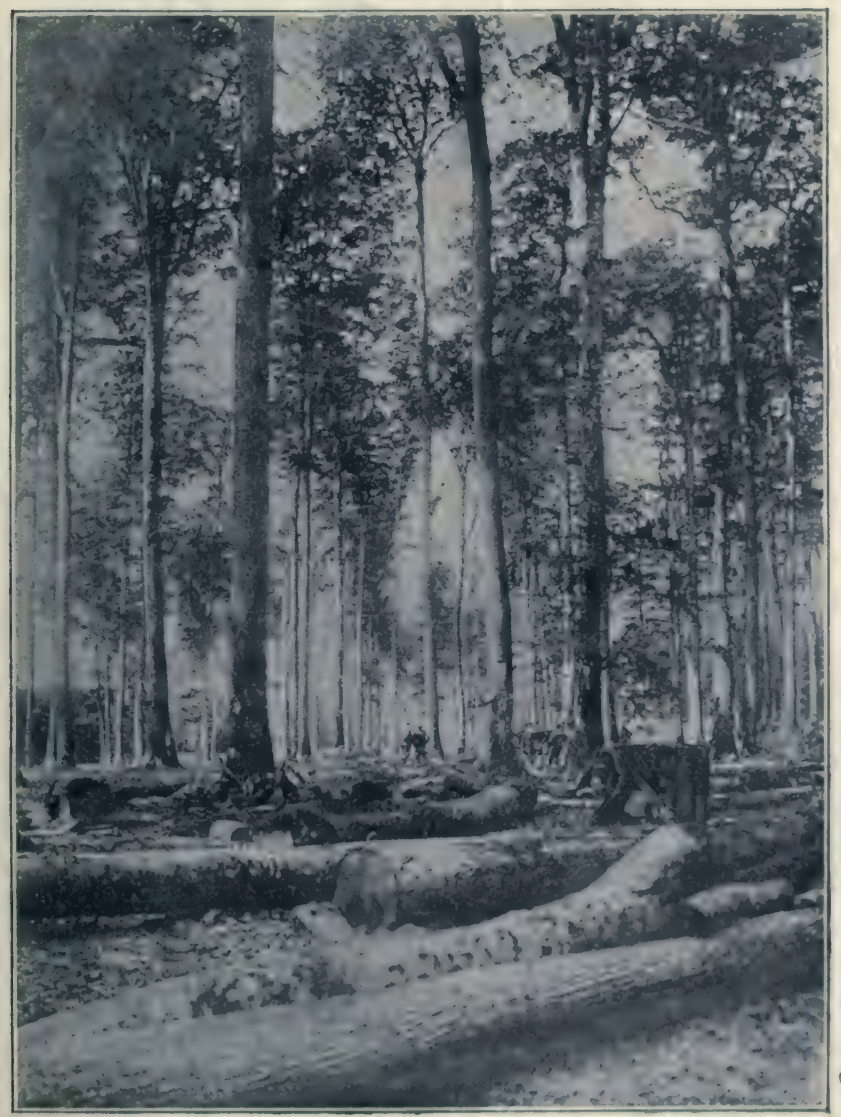

Fig. 50. - Coupe d'ensemencement dans une futaie de chênes, forêt de Bercé (Sarthe). (Photographie de M. P. Galland.)

Remarques générales $-1^{\circ}$ Suivant les circonstances, il faut compter qu'il s'écoulera de 10 à 25 ans entre la coupe

(1) Au point de vue économique, il serait souvent désirable de Iêisser sur: ipied, après le passage ${ }^{-}$de la coupe définitive, des chênes de végé - 
d'ensemencement et la coupe définitive. La durée de cette période sera plus longue chez les essences d'ombre (sapin, hêtre) que chez les essences de lumière (pin sylvestre, chêne); - plus longue dans les peuplements mélangés que dans les peuplements purs; - sous les climats rudes que sous les climats doux; - sur les points exposés aux gelées printanières que sur ceux où ces dangers ne sont pas à redouter.

$2^{\circ}$ Dans les sols médiocres, superficiels, dans les hautes stations, les coupes d'ensemencement devraient être faites moins sombres à cause de la rareté des années de semence, de la moindre fertilité des arbres et aussi de l'enchevêtrement des racines de la vieille futaie, qui dessèchent superficiellement le sol et ne laissent au semis aucune place disponible où il puisse s'installer. Mais ces coupes trop claires auraient l'inconvénient de dégrader davantage la surface; aussi est-il préférable de procéder par petites trouées, disposées de loin en loin, en choisissant les places où il existe déjà du semis. Le passage des coupes secondaires augmente l'étendue de ces découverts, au fur et à mesure que les jeunes brins naissent sur leurs bords; à la longue, les semis se rejoignant, la surface entière est régénérée. Ce moyen est également recommandable pour obtenir un mélange naturel.

$3^{\circ}$ La méthode par coupes successives donne naissance à des semis, qui se présentent sous forme de taches d'autant plus inégales en hauteur que la période de régénération aura été plus longue. Cet état, au lieu d'être nuisible, est plutôt avantageux puisque, tout en favorisant les mélanges, il donne plus de densité au peuplement et augmente sa force de résistance contre la neige, le givre ou le verglas. D'ailleurs, ces inégalités s'atténuent avec l'âge et disparaissent avant l'état de haut perchis.

Les soins culturaux. - Le plus souvent, il y a lieu de commencer les dégagenents de semis pendant la durée même

tation vigoureuse, qui acquerraient, da s la suite, des dimensions exceptionnelles. L'expérience a malhei.eusement montré que ces arbres se dégradent presque toujours ajissitôt après leur isolement, et quil faut les réaliser à bref délai. En tout cas, la question ne se pose que pour les essences de lumière à bois parfait distinct et de grande valeur, comme le chêne. 
de la période de régénération, dès les premières années qui suivent la coupe d'ensemencement. On les répète aussi souvent qu'il est nécessaire, jusqu'a ce que les tiges des espèces à cultiver soient généralement dominantes; il doit en être ainsi vers l'époque du passage de l'état de gaulis à celui de perchis.

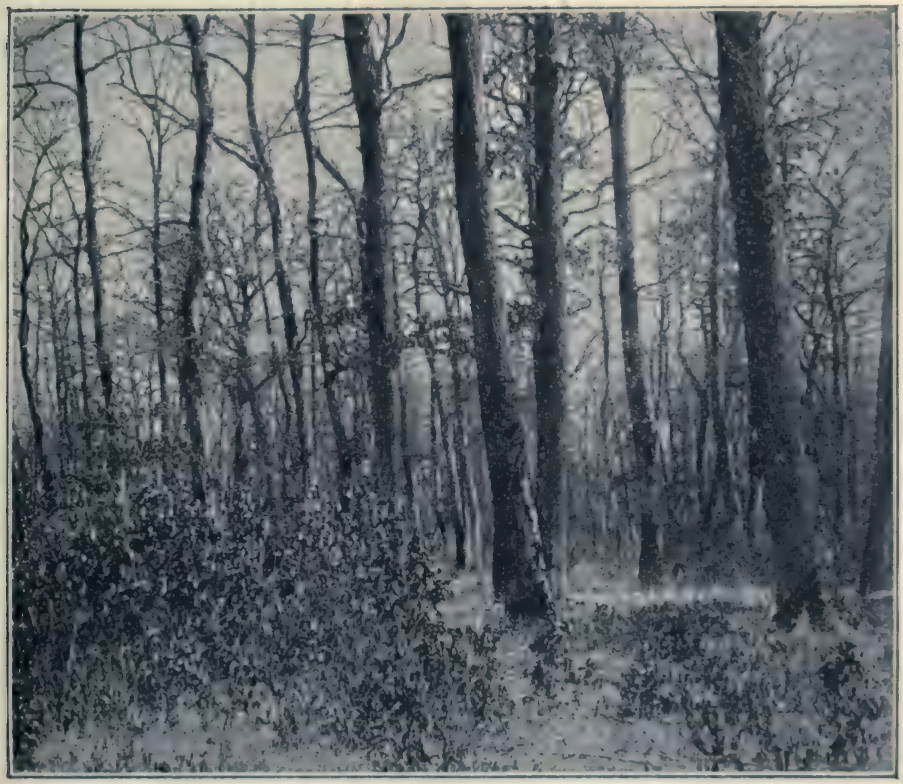

Fig. 51. - Coupe secondaire dans une futaie de chênes, forêt domaniale de Champenoux (Meurthe-et-Moselle). - A droite : espace occupé par un semis incomplet ou peu développé, au-dessus duquel on a maintenu des grands arbres. - A gauche : tache de semis bien développés, au-dess.ıs desquels on a fait un large découvert.

Alors la constitution générale du peuplement est acquise : il reste à l'améliorer dans sa composition, sa consistance et le choix des sujets d'avenix. C'est le rôle des éclaircies, étudiées dans le chapitre $\mathrm{V}$.

Application aux principales essences. - Nous donnons ci-après le résumé succinct des opérations applicables à celles de nos essences sociales 'qui sont susceptibles d'être 
traitées en futaie régulière, renvoyant aux Chapitres II (les essences) et III (les peuplements), pour tout ce qui concerne leur tempérament et leurs allures forestières.

\section{Hêtre.}

Régénération. - Fruits lourds : coupe d'ensemencement très sombre, formant un abri régulièrement réparti si la sta-

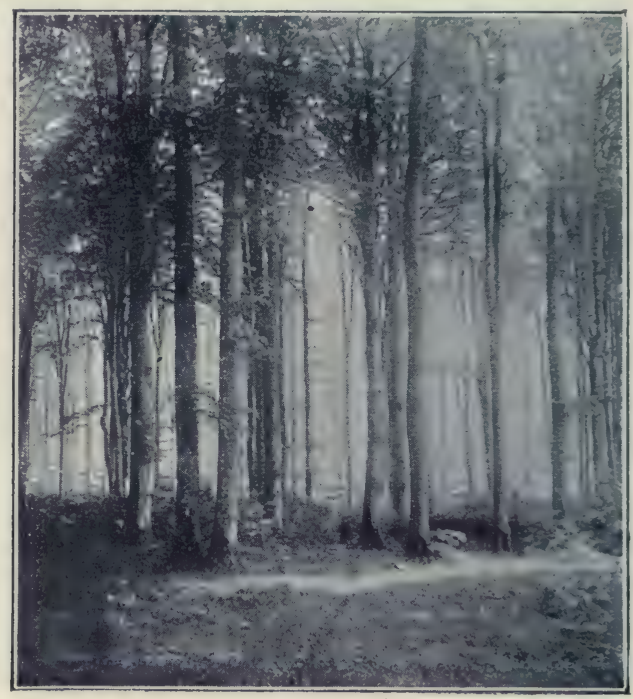

Fig. 52. - Coupe d'ensemencement et, au dernier plan, coupe secondaire dans une futaie de hêtres, forêt de Lyons-la-Forêt, canton de Pain d'Épices. (Photographie de M. J. George.)

tion est fertile (fig. 52), interrompu par de petites trouées sile sol est pauvre et superficiel.

Mise en état du sol par un ratissage des couches trop épaisses de feuilles mortes, ou par un crochetage des surfaces tassées et de la couverture vivante (mousses et plantes diverses). Profiter des semis préexistants (1) s'ils n'ont pas ététrop long-

(1) On appelle semis préexistants des semís, - appartenant presque toujours à des essences d'ombre, - qui s'installent sous un massif préalablement à toute coupe de régénération. 
temps dominés; attendre patiemment que les fainées partielles complètent les régénérations insuffisantes au début.

Coupes secondaires très prudentes, au nombre de quatre ou cinq, limitées chacune à l'enlèvement d'un arbre sur trois ou quatre avec retour tous les cinq à six ans. On sait, en effet, que les jeunes hêtres supportent aisément un couvert prolongé.

Dégagement de semis. - Le hêtre se défend contre les semis de toutes les autres grandes essences. Parfois, des dégagements peuvent s'imposer pour le protéger contre l'envahissement des rejets, des morts-bois et des ronces.

Eclaircies. - Le hêtre se plaît en massif très serré. La première éclaircie n'est nécessaire qu'une fois l'état de bas perchis bien affirmé. De ce moment, jusqu'à l'âge de cent ans : éclaircies tous les dix ans, à faire très prudentes, pour favoriser l'élagage naturel et éviter les ronces. A partir de cent ans, ne plus les répéter que tous les quinze à vingt ans.

Respect absolu de l'étage dominé; enlèvement systématique des sujets tarés ou branchus qui ne paient pas la place qu'ils occupent.

\section{Chềne pur.}

Régénération. - Fruits lourds : coupe d'ensemencement sombre, dont l'abri sera toujours régulièrement réparti.

Nettoiement radical du sol, excepté, pourtant, dans les régions où les années de semence sont rares ou très rares; on attendra, en pareil cas, pour faire l'opération, que la glandée soit cerlaine. Partout et toujours, crochetage au moment de la chute des glands.

Le semis général ne peut être que le produit d'une glandée complète, les résultats partiels disparaissant sous un couvert prolongé (1). Dès qu'on juge la régénération acquise, procéder aux coupes secondaires. Les faire d'autant plus intenses

(1) Souvent, les jeunes semis de chêne, dont la tigelle semble mort. conservent, dans les région. avoisinant le collet de la racine, une vitalité suffisante pour émettre des rejets qui s'élancent à nouveau dès que la lumière leur parvient. Aussi dit-on que les jeunes chènes se recèpent sous le couvert. 
que les gelées printanières sont moins à craindre; à chaque passage, prendre un arbre sur deux ou trois, en évitant d'abattre trop de matériel à la fois.

Dans la région girondine, une coupe secondaire, ou deux au plus, précèdent la coupe définitive; dans celle du Centre et de l'Ouest, deux ou trois sont nécessaires. Enfin, dans le Nord et l'Est de la France, on doit procéder plus lentement, pour éviter les accidents de gelée et pour permettre aux essences de remplissage de combler les lacunes d'une régénération trop souvent incomplète.

Dégagements de semis. - Ils sont presque toujours indispensables (sauf peut-être dans la région girondine). Leur nécessité s'impose partout où la rareté des années de semence permet aux morts bois et au hêtre de s'installer avant le chêne.

Éclaircies. - Jusqu'à l'état de bas perchis le chêne pur peut former des massifs assez denses. Mais, à partir de cette époque, les tiges demandent à être desserrées : dès qu'on les voit se couvrir de gourmands, on peut être certain qu'elles souffrent et qu'il faut intervenir. Alors, procéder hardiment en faveur des tiges d'avenir qui s'affirment; revenir tous les dix ans, et même plus souvent, jusqu'à l'état de haut perchis ; enlever les chênes dominés, qui sont voués à une mort inévitable, mais respecter tous les sous-bois de hêtre ou d'essences diverses qui peuvent exister. Il suffit de parcourir tous les vingt ans les hautes et les vieilles futaies.

\section{Chêne et hêtre mélangés.}

Régénération. - Opérer comme ci-dessus, mais en ayant soin, de toute nécessité, d'installer le chêne le premier; à cet effet, traiter les semis préexistants de hêtre comme des morts bois: les couper, ou mieux les arracher. Le chêne ayant pris possession du terrain après une bonne glandée, permettre seulement au hêtre de combler peu à peu les vides.

Donner à une même coupe secondaire une intensité variable suivant l'espèce à favoriser sur un point donné : la faire plus intense là oú l'on veut du chêne, plus timide sur les places résérvées au hêtre. 
Dégagements de semis. - Ces opérations sont toujours indispensables pour protéger le chêne contre le hêtre et les rejets envahissants.

Éclaircies. - Elles sont faites en forme d'éclaircies-dégagements, et répétées tous les quatre à cinq ans dans les gaulis et les bas perchis, sans jamais dépasser dix à douze ans, quel que soit l'âge du massif.

Ces soins culturaux sont bien simplifiés si l'on établit un mélange par compartiments. Il suffit alors de protéger le chêne sur les bords des placeaux où il vit à l'état pur, tout en bénéficiant de l'avantage d'une association avec le hêtre.

Une excellente pratique, indispensable même dans les futaies de chêne conduites au-delà de deux cents ans, est la création d'un sous-étage de hêtre sous les chênes arrivés à l'état de haut perchis.

\section{Sapin.}

Régénération. - Essence d'ombre, habitant, en outre, des stations exposées au vent: coupe d'ensemencement très sombre.

S'il y a lieu, détruire la couverture vivante (herbes, myrtilles, bruyères) et mettre le terreau à nu par bandes. Inutile de recéper les morts bois feuillus (sureaux, coudriers, etc.) qui envahissent fréquemment le parterre des coupes; car avec le temps, le sapin s'installera sous leur couvert devenu moins épais, dans l'excellent terreau formé par leurs détritus. Utiliser les semis préexistants dont l'avenir ne parait pas com. promis.

Commencer les coupes secondaires quand le sapineau est verticillé, c'est-à-dire âgé de six à huit ans. Les conduire avec lenteur. Dans la crainte des chablis, il est permis, aux grandes altitudes, où les insolations sont moins à craindre, de laisser la régénération s'installer solidement, puis, après dix à douze ans, de passer sans transition à la coupe définitive.

Quand la régénération se fait attendre, ou parait aléatoire, procéder par trouées prudentes, ou par bandes qui progressent du périmètre vers le centre du massif. 
Dégagements de semis. - Le jeune sapin arrive, en général, à percer, par ses seuls moyens, des fourrés épais de hêtres ou de morts bois. Néanmoins, afin d'éviter que les sujets dominés soient déshonorés par la perte de leur flèche, il est toujours utile d'intervenir par des dégagements de semis sobres et, par suite, peu coûteux.

Éclaircies. - Bien que, par son tempérament, le sapin accepte de vivre en massifs très denses, les éclaircies sont du plus haut intérểt. Très délicates à conduire dans des peuplements mal dirigés au début, elles deviennent faciles si l'on a toujours eu soin de respecter scrupuleusement les sujets dominés. Dans ces éclaircies, enlever avant tout les arbres champignonnés, chảudronnés ou mal conformés; adopter les mêmes périodicités que pour le hêtre.

Le sapin peut, à la rigueur, être traité à l'état pur dans les régions moyennes de son aire. Mais, sur les limites de celle-ci, un mélange rationnel avec les essences qui le précèdent ou qui le suivent est indispensable.

Aux altitudes basses, le hêtre s'impose. Aux altitudes élevées, cette essence donne des produits dont la valeur marchande est des plus médiocres; d'autre part, étant, en pareille station, bien plus souvent fertile que le sapin, elle devient envahissante; aussi, sans la proscrire systématiquement, semble-t-il indiqué de donner dans le mélange, - au besoin par voie artificielle, - une large part à l'épicéa, comme cela se présente spontanément dans le Jura et dans les Alpes. Dans l'un et l'autre cas, la conduite des exploitations sera modifiée pour tenir compte des exigences du nouveau venu.

\section{Épicéa:}

Régénération. - Semence ailée : coupe d'ensemencement assez claire; toutefois, les chablis éfant à redouter, il vaut mieux procéder par trouées éparses, en enlevant trois ou quatre arbres sur le même point.

Le ratissage par bandé de la couverture vivante, et surtout des couches épaisses d'aiguilles non décomposées, facilite l'installation du semis. 


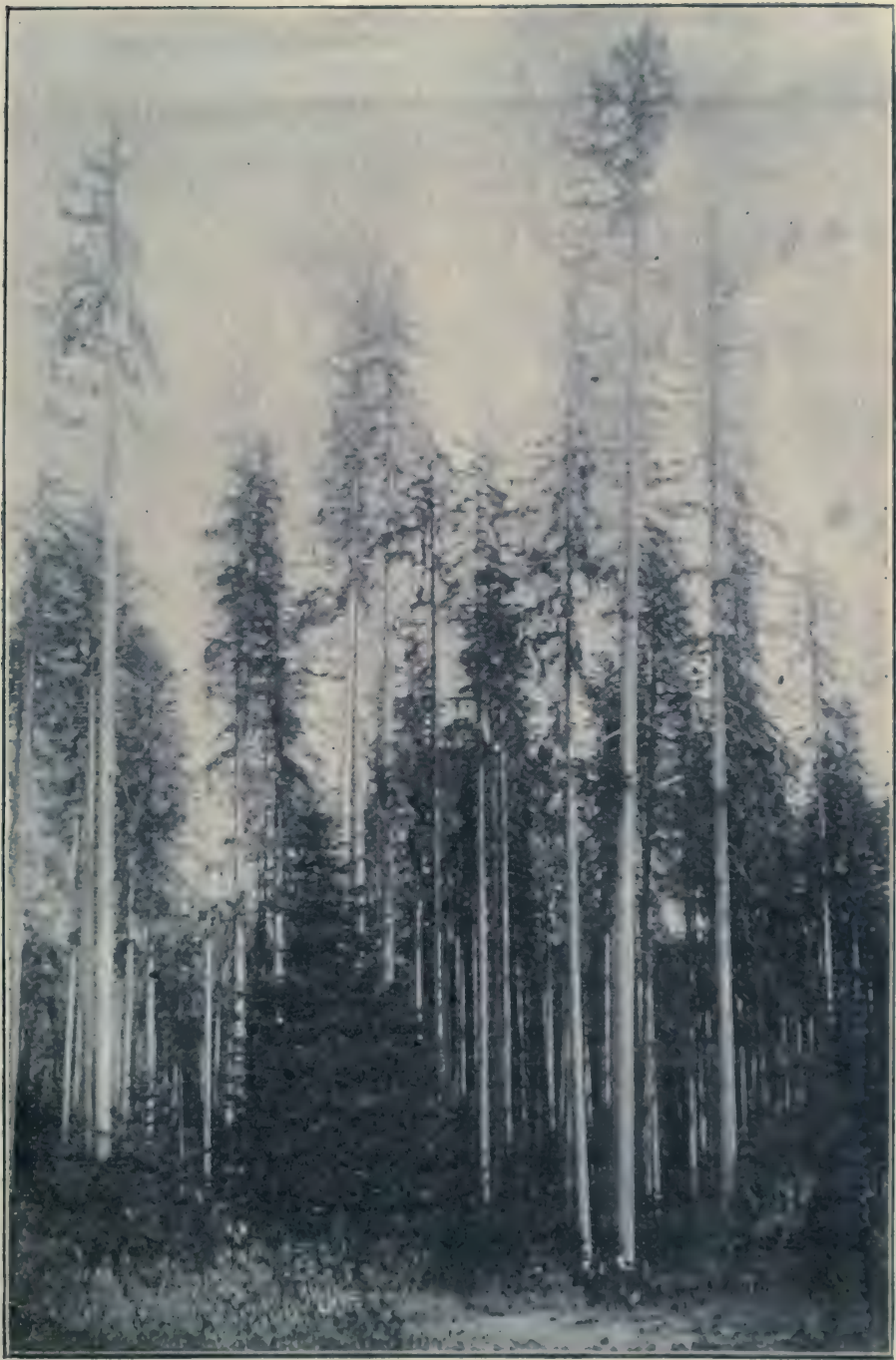

Fig. 53. - Épicéas de Gilley (Doubs), 1892. - Arbres alteignant $50 \mathrm{~m}$. de hauteur. (Photographie de M. Thiollier.) 
Dans la haute montagne, les jeunes épicéas supportent assez bien le couvert des grands hêtres, moins nuisible qu'en plaine par suite de la petitesse du limbe des feuilles; mais il faut extirper les semis préexistants de cette espèce, quand ils couvrent les parcelles à régénérer. Il n'en est pas de même des arbustes, dont la présence ou l'apparition n'ont rien d'inquiétant : " La régénération, dit M. l'Inspec" teur Guinier (1), est médiate et indirecte, quand elle a " lieu lentement et seulement avec l'intermédiaire de phases ") diverses de végétation, c'est-à-dire après l'occupation suc" cessive du sol par certaines plantes herbacées ou arbo" rescentes........ Il ne faut donc pas considérer l'avenir " comme compromis et la forêt comme ruinée parce que la " régénération immédiate et directe n'est pas obtenue et que " le sol est envahi par cette végétation appelée bien à tort " parasite. Au contraire, c'est une végélation auxilliaire... " A l'appui de cette théorie, rappelons certains cantons du Jura, où le massif, détruit par la tempête, a fait place à une végétation herbacée, puis frutescente, au milieu de laquelle sont nés, comme troisième stade dans la régénération de la forêt, de nombreux épicéas, qu'il n'y a plus maintenant qu'à dégager du fourré.

Une fois le semis installé, le découvrir rapidement, afin de le soustraire aux funestes effets de la coupe définitive brutale toujours subordonnée au caprice des ouragans. Si l'on a procédé par trouées, les coupes secondaires élargiront les premiers vides et en créeront de nouveaux.

Dégagements de semis.- La lente croissance de l'épicéa pendant sa première jeunesse rend ces opérations très utiles. A l'occasion, respecter les sorbiers, dont le couvert léger n'est point gênant, et dont les fruits attirent toute une population d'oiseaux, qui font la guerre aux insectes si dangereux dans de pareilles forêts.

Éclaircios. - L'épicéa ne prend les belles formes cylindriques et élancées qui font sa valeur (fig. 53), que si l'élagage naturel fonctionne régulièrement. Contrairement au sapin, il meurt

(1) E. Guinier, Trailement de l'épicéa dans les Alpes, Saint-Jean-deMaurienne, 1896. 
dès qu'il est dominé. Dès lors, faire de bonne heure des éclaircies très prudentes, mais souvent répétées. Profiter de leur passage pour enlever tous les arbres tarés ou dépérissants, qui favorisent la multiplication des insectes et deviennent des foyers d'invasion.

A tous ces points de vue, il est avantageux de mélanger l'épicéa avec le sapin et le hêtre (1), ou de lui constituer un sous bois de ces essences.

\section{Mélèze.}

Régénération. - Coupe d'ensemencement très claire, ou mieux par trouées éparses, après avoir rigoureusement fermé au pâturage les cantons à régénérer $(2)$.

Quand le sol est trop enherbé, donner une très légère culture, en procédant par bandes de $0^{\mathrm{m}}, 30$ de largeur, séparées par des intervalles de $1^{\mathrm{m}}, 50$ à 2 mètres.

Faire une seule coupe secondaire hardie, puis la coupe définitive, car on peut, sans crainte, laisser largement entrouverts les massifs de mélèze, dont l'enracinement est très puissant.

Dégagements de semis. - Les jeunes mélèzes croissant vite, et la végétation basse étant peu redoutable à ces hautes altitudes, les dégagements sont peu nécessaires. Il suffit, dans les semis très drus, d'opérer un dépressage.

Éclaircies. - Faire de bonne heure des éclaircies hardies. Pourtant, comme le mélèze a la cime peu étalée, ne pas exagérer leur intensité de façon à ne pas entraver le fonctionnement de l'élagage naturel.

A toutes les altitudes, il est bon de favoriser le mélange du mélèze avec d'autres espèces par crainte des maladies crypto-

(1) Dans ces mélanges, l'épicéa est toujours quelque peu dominant par rapport aux espèces associćes, ou, du moins, il les dépasse légèrement en hauteur. On ne peut que se féliciter d'un pareil état de choses.

(2) Les jeunes mélézes, dans les prés-bois alpins, arrivent pourtant, mieux que beaucoup d'autres conifères, à se défendre contre la dent du bétail : à la façon des genévriers ou des épines blanches, ils étalent leurs branches basses jusqu'à ce que les animaux ne puissent plus atteindre le centre de cette touffe, d'où s'élance une flèche vigoureuse. 
gamiques, - sous cette réserve que le mélèze demeure l'essence principale du peuplement.

\section{Pin sylvestre.}

Régénération. - Coupe d'ensemencement très claire, ou par trouées. Donner une culture au sol souvent durci, tassé et couvert de sous-abrisseaux de grande taille et envahissants (bruyères, genêts); au besoin, procéder par arrachis, suivant des bandes plutôt espacées et larges $\left(0^{\mathrm{m}}, 50\right.$ au minimum) que nombreuses et étroites. On évite cette dépense, en partie tout au moins, par l'extraction des souches des arbres exploités.

Dès que le semis est suffisant, on peutdécouvrir hardiment, brusquement même, les jeunes pins, qui ne craignent ni le soleil, ni les gelées. Éviter toutefois de fatiguer les régénérations par des coupes radicales.

Dans les mélanges de pin sylvestre avec le hêtre, installer d'abord celui-ci par une coupe d'ensemencement sombre; plus tard les pins se jetteront en abondance dans les vides, partout où les coupes secondaires apporteront de la lumière, et, grâce à leur végétation rapide, ils se raccorderont facilement avec ce qui les entoure.

Dégagements de semis. - S'ils ne sont que rarement nécessaires, du moins est-il important de pratiquer des dépressages toutes les fois que les semis, trop nombreux, se constituent en fourrés très deṇses, où l'évolution des champignons parasites est à craindre; ces dépressages s'imposent dans les pineraies de création artificielle.

Éclaircies. - L'éclaircie est la base du traitement des essences de pleine lumière, comme le pin sylvestre, dont la cime franchement desserrée dès le jeune âge, isolée même à partir de l'élat de haut perchis, doit alors occuper au moins le tiers de la hauteur totale du sujet. Sinon, la croissance est ralentie, l'arbre ne forme pas de bois de cœur, prend une forme étriquée, et devient la proie des insectes ou des champignons. Les gaulis et perchis serrés et uniformes sont fréquemment aussi écrasés par la neige.

Dans les peuplements artificiels, faire la première éclaircie 
dès l'âge de dix ans; dans les peuplements naturels, la retarder quelque peu. Répéter l'opération tous les quatre à six ans jusqu'à l'état de haut pérchis, puis tous les huit à douze ans.

Les pins dominés et les sujets mal conformés, doivent être enlevés systématiquement. Au contraire, le respect de toute la végétation de feuillus ou de mort-bois qui protège le sol, - la création même, sous les perchis de pins arrivés à l'âge de trente ou quarante ans, d'un sous bois de sapin, ou mieux encore de hêtre, - ne sauraient être trop recommandés.

\section{Pin maritime.}

Régénération. - Graines ailées, a bondantes ; régénération très facile quand le sable est nu et meuble; - quand il est tassé ou enherbé, une mise en état, par ouverture de bandes ou par extraction de souches, assure l'ensemencement.

Procéder très hardiment aux coupes de régénération, ou même faire une coupe unique avec une réserve de quelques porte-graines à l'hectare.

Dans les forêts résinées (1), on gemme à mort tous les arbres qui doivent être abattus et on les exploite, par extraction de souche, au fur et à mesure de leur épuisement.

Dégagements de semis. - La végétation rapide des jeunes pins maritimes rend ces opérations peu nécessaires.

Éclaircies. - Les pins destinés au gemmage réclament

(1) Pour gemmer un pin à vie, on ouvre au pied de l'arbre, vers le commencement de mars, une plaie ou carre large de 10 centimétres, dont la profondeur ne dépasse pas 1 centimètre. Pendant toute la période de végétation, le gemmier ravive cette carre, obstruée par des dépôts de résine solidifiée et l'élève progressivement, en lui conservant sa largeur et sa profondeur. A la fin de la premiére année, elle atteint une hauteur de 55 centimètres; à la fin de la cinquième année, elle arrive à $3 \mathrm{~m}$. 80 . On l'abandonne alors, pour en ouvrir une autre dans des conditions analogues.

Pour gemmer à mort, on ouvre à la fois un grand nombre de carres, autant que la grosseur du sujet en cornporte, de façon à soutirer rapidement la plus grande quantité possible de résine à l'arbre, qui meurt bientôt épuisé.

Dans l'un et l'autre cas, la résine est recueillie dans des pots en terre vernissée, fixés contre la carre au niveau de sa région active (système Hugues : page 97, flg. 28). 
un état plus clair encore que les pins sylvestres : leur cime doit être ensoleillée.

Une première éclaircie se fait à dix ou douze ans. On revient tous les cinq à six ans, hardiment, mais sans isoler complètement les perches avant l'âge de vingt ans, époque à laquelle il doit rester sur pied de six à sept cents tiges à l'hectare. De celles-ci, on gemme à mort deux cents, que l'éclaircie enlèvera, et ainsi de suite tous les quatre à cinq ans ; à trente ans, il ne reste sur pied que deux cent cinquante ou trois cents tiges; à soixante ou quatre-vingts ans : cent cinquante ou deux cents arbres.

On commence à gemmer à vie les perchis de trente ans ; il est bon, jusqu'à soixante ans, de laisser aux pins une année de repos après quatre ans de résinage. Ainsi ménagée, la pignadar peut durer cent vingt ans et plus.

Au passage des éclaircies, élaguer les branches basses jusqu'à la hauteur qu'atteindront les carres $\left(4^{\mathrm{m}}\right)$, sans plus.

\section{Pin d'Alep.}

Le pin d'Alep peut-être conduit à l'état pur, à la façon du sylvestre. La présence d'un sous bois de buis facilite les opérations en couvrant le sol et en permettant de donner au peuplement le degré d'éclaircissement qu'il exige.

Souvent aussi, cette essence est mélangée avec des chênes verts : ces derniers sont traités en taillis, et les pins, conservés sous forme de réserves, sont enlevés, en jardinant, lors du passage des coupes.

\section{Pin de montagne. Pins laricios.}

Nous avons vu que le tempérament de ces essences les rapproche beaucoup plus de l'épicéa que du pin sylvestre. Le traitement appliqué au premier leur convient donc à tous égards.

En ce qui concerne le pin noir d'Autriche, ajoutons que, dans le jeune âge tout au moins, il doit être tenu en massif ássez serré, sinon les arbres prennent une forme défectueuse, 
trapue, avec des branches qui acquièrent un développement démesuré en grosseur et en longueur. De préférence, enlever dans les éclaircies les pins fourchus, qui sont parfois très nombreux.

\section{ARTICLB II}

\section{LA FUTAIE JARDINÉE}

Principe de la méthode. - Avantages et inconvénients. - Cas oủ le jardinage doit être maintenu, - Pratique du jardinage. - Les rotations. - Les soins culturaux. - Application aux différentes essences.

Principe de la méthode. - Le jardinage, tel qu'il était appliqué à toutes les forêts résineuses, en suite de l'observation des anciens règlements généralisés par l'Ordonnance de 1669, était un mode de traitement parfaitement justifié, et qui, de nos jours encore, employé avec de légers correctifs, présente des avantages très sérieux.

Il consistait à parcourir annuellement toute l'étendue des forêts jardinées, en exploitant, par unité de surface, un nom. bre d'arbres fixé à l'avance, et constituant la possibilité. Ces arbres étaient enlevés çà et là, et choisis uniquement parmi ceux que leur dimension ou leur mauvais état de végétation rendaient exploitables.

Celte opération, qui constituait le véritable mode de jouissance de la forêt, était accompagnée de l'extraction des sujets morts. Tous ceux de ces bois morts, qui avaient la dimension d'arbre (1), élaient défalqués du nombre des tiges à réaliser. On ne tenait aucun compte des autres. En somme, tout en tirant parti des produits sains et exploitables, on procédait au curage de la forêt, comme il y a toujours lieu de le faire; mais il ne faudrait pas croire que le jardinage ne donnait que des bois en majorité tarés, loin de là.

Un semblable système a pour résultat de faire porter les exploitations annuelles, non plus sur des peuplements recouvrant des espaces continus, mais sur des arbres considérés individuellement, répartis au hasard dans toute l'étendue de

(1) Sujet ayant au minimum 1 mètre ${ }^{3}$ pieds de tour à hauteur d'homme. 
la forêt. La régénération se fait uniquement par points et non par surfaces, dans les endroits découverts, entourés de toutes parts par des arbres en croissance de dimensions variables, dont quelques-uns sont fertiles.

Dans ces conditions, la futaie jardinée revêt une forme toute spéciale et dont on ne rencontre l'analogue dans aucun autre mode de traitement. En effet, le peuplement jaräiné, considéré dans son ensemble, présente un mélange confus de tiges de toutes grosseurs sur chaque unité de surface (fig. 54). Il ne passe donc pas par les états de développement successifs d'un peuplement régulier.

D'ailleurs, l'aspect d'une forêt jardinée change avec le calibre choisi pour rendre l'arbre exploitable. Plus celui-ci est faible, moins il y a de tiges ayant dépassé l'âge de leur plus grand accroissement en hauteur: le nombre de celles qui ont des longueurs différentes sera maximum, et le profil du massif se dessinera suivant une ligne irrégulièrement brisée. Plus, au contraire, la grosseur sera forle, plus disparaîtra celle forme sinueuse; car les arbres qui, ayant dépassé le terme de leur accroissement en longueur, étalent leurs cimes dans une même zone de hauteur, seront alors plus abondants; ces sujets, grâce à leur nombre et à leurs grandes dimensions, forment la partie principale du peuplement et la catégorie la plus importante; comme arbres constitués, il sont les seuls qui frappent la vue et cela au point que certains massifs jardinés présententl'aspect de vieilles futaies régulières (1), à l'état plus ou moins clair.

Avantages et inconvẻnients. - Le profil sinueux des peuplements jardinés permet à la lumière de se projeter sur une vaste surface foliacée, et beaucoup de tiges, bien que dominées, sont maintenues vivantes sous l'influence de la lumière latérale. Tous les points du sol sont ainsi protégés par une végétation active, dont les étages se superposent en donnant d'abondants détritus. En fait, mieux que tout autre mode de traitement, le jardinage réglementé est à même d'assurer d'une manière permanente

(1) A distance, l'œil juge assez mal du calibre d'un arbre. 
Applications diverses. - Indépendamment des essences dont nous venons de parler, il peut être avantageux de cultiver en taillis simple d'autres espèces et, plus particulièrement, les suivantes :

$1^{\circ}$ dès l'âge de quinze ans, les taillis d'aune donnent de fort beaux rendements; mais ils peuvent, avec avantage, être conduits beaucoup plus loin. Les stations où le sol est frais et profond, les parties trop mouilleuses des régions de prairies, le bord des rivières conviennent à merveille à cette essence;

$2^{\circ}$ sous la dimension de faibles perches, le châtaignier présente déjà une bonne proportion de bois parfait, et, comme il pousse très vite, on a la coutume de le couper d'assez bonne heure, généralement vers douze à quinze ans. Dans les sols riches, en retardant l'exploitation et en pratiquant des éclaircies convenables, on obtient, dès l'âge de trente ans, des perches de vingt à vingt-cinq centimètres de diamètre, dont on tire d'excellents merrains. Le châtaignier, traité en taillis simple, ne prospère que dans les climats doux et sur les sols siliceux, légers et profonds. Les scories volcaniques du Plateau central lui conviennent particulièrement (1);

$3^{\circ}$ les taillis de robinier (vulgairement acacia), sont d'un bon rapport. Les plants doivent être espacés et maintenus à l'état pur, car ils ne supportent aucun mélange avec les essences indigènes. En général, on exploite le robinier rez terre tous les dix, douze ou quinze ans; mais, en le maintenant en massif clair, on peut le conduire avantageusement jusqu'à quarante et même cinquante ans. A quelqu'âge qu'on le coupe, il se reproduit très facilement par drageons. Le robinier croît vite; mais il craint le vent, l'état serré et le

(Ad. Chatin, De la truffe, de sa culture et de sa naturalisation, Bull. Société botanique de France, 1872.)

(1) En Alsace, le châtaignier fournit, à 4 ou 5 ans, des cercles de futaille, - à 15 ou 20 ans, d'excellents échalas pour la vigne. L'hectare de taillis de châtaignier, dont la création exigre une dépense d'environ 800 francs, sol et plantation compris, rapporte annuellement, dans la région du vignoble un revenu net de 100 francs. - Millischer, La culture du chataignier en Alsace. (Bull. Société forestière de Franche: Comté et Belfort, avril 1893.) 
couvert. On le plante trop souvent dans des sols sans profondeur ou compacts, sous le prétexte qu'il " reprend " presque toujours; c'est un tort, car il n'est d'une culture avantageuse et justifiée que dans des sols légers et profonds;

$4^{\circ}$ dans les terrains submersibles enrichis par le limon des cours d'eau débordés, les bois blancs, les saules surtout, prennent une végétation active et donnent de bons revenus. Les révolutions adoptées sont très courtes : elles dépassent rarement douze ans, et descendent parfois au-dessous de huit ans, pour former les menus taillis;

$5^{\circ}$ 'les perches ou barres de micocoulier, réduites au nombre de deux ou trois sur chaque souche, atteignent une dizaine de centimètres de diamètre à dix ou douze ans et valent un à deux francs pièce. Des taillis de cette essence peuvent être d'un rendement avantageux, mais ils exigent un sol divisé, bien ameubli et le climat méditerranéen.

Nous empruntons une bonne part des renseignements qui précèdentau Traitement des Bois en France de M. Broilliard. Nous ne pouvons que renvoyer à cet important ouvrage le lecteur désireux de plus amples détails sur la création et la conduite de ces divers types de taillis simples.

\section{ARTICLE IV}

\section{LE TAILLIS SIMPLE FURETÉ}

Forme des peuplements. - Circonstances dans lesquelles il se justifie. - Régénération. - Réglementation.

Forme des peuplements. - Pour fureter un taillis simple, on y revient à de courts intervalles, compris entre huit et quinze ans, en se bornant à couper les perches exploitables, c'est-à-dire, le plus généralement, celles qui ont de $0^{\mathrm{m}} 30$ à $0^{\mathrm{m}} 35$ de tour à hauteur d'homme. $\mathrm{Si}$, par exemple, on passe tous les neuf ans dans un taillis où il faut vingt-sept ans aux rejets pour atteindre cette grosseur, on trouvera, en théorie, sur chaque cépée, immédiatement avant la coupe, des rameaux de neuf, dix-huit et vingt-sept ans.

En fait, les choses se passent comme il suit: les exploita- 
tions, limitées sur chaque cépée à un petit nombre de perches choisies dans l'étage dominant, avec réserve de tous les rejets moins forts, occasionnent, çà et là sur la souche, des blessures qui tendent à se cicatriser; à la longue, la surface de cette souche présente donc des îlots de tissu cicatriciel (1), dans

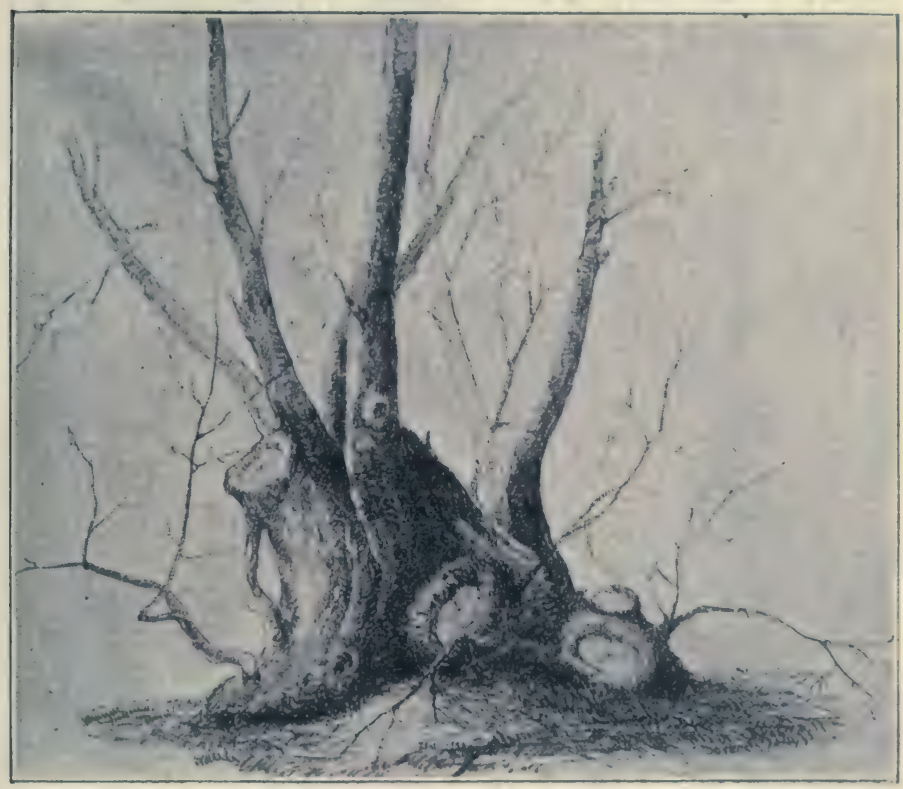

Fig. 57, - Souche de taillis fureté.

lequel s'organisent des bourgeons adventifs. De ces bourgeons, les uns restent à l'état dormant, d'autres s'allongent quelque peu en restant grêles et chétifs, mais tous sont prêts à se développer dès que le hasard d'une exploitation leur donnera l'espace et la lumière ; la question d'âge n'entre donc pour rien dans leur évolution successive: chacun peut devenir dominant bien avant son tour d'ancienneté.

(1) Un grand nombre de bourgeons adventifs naissent sur le tissu cicatriciel des blessures failes aux souches et aux racines par le trainage des produits et les autres accidents consécutifs à l'exploitation. 
Les éléments qui entrent dans la composition d'un taillis fureté représentent, par suite, une cépée en forme de buisson (fig. 57), dont les brins, émergeant tous d'une souche d'aspect tourmenté comme la cime d'un têtard, sont les uns plus ou moins dominants, les autres franchement chétifs et trâ̂nants: à vrai dire, au lieu de trois ou quatre classes d'âge, l'œil distingue à peine deux étages.

Dans son ensemble, le peuplement constitue un fourré perpétuel, ayant de 6 à 10 mètres de hauteur. Quand il est plein et parcouru par des exploitations modérées, il est souvent fort difficile d'y pénétrer: la présence des traînants, notamment, rend la circulation pénible pour peu que la pente soit accusée. Il n'y peut être non plus question de pâturage; et si, pour satisfaire aux exigences de propriétaires ou d'usagers, on coupe lesdits traînants, on perd tous les bénéfices de la forme furetée. Cette pratique funeste explique toutle mal que l'on a dit du furetage, procédé trop peu étudié et, par suite, souvent calomnié, véritable jardinage sur souches, qui partage avec le jardinage dans les futaies le grand mérite de tenir le sol toujours couvert et de bien le protéger contre l'érosion.

Circonstances dans lesquelles il se justifie. - Bien que des chênes, des châtaigniers, des fruitiers, puissent se jeter et se maintenir quelque temps dans un taillis fureté, ce mode de traitement ne concerne que le hêtre, et voici sa justification.

En général, les taillis simples réguliers de cette essence nese perpétuent facilement que dans lesstations tempérées, et sous la condition d'être exploités jeunes. Dès que les rejets naissants ne résistent plus, sans protection, à l'action d'un climat trop rigoureux par excès de chaleur ou de froid, dès que les produits, pour être utilisés, doivent atteindre une dimension qui dépasse celle où le hêtre repousse facilement de souches, il est nécessaire, si l'on veut rester dans le régime du taillis, de recourir au furetage. C'est, d'ailleurs, en de semblables circonstances, que ce mode de traitement est né dans le Morvan, dans les Pyrénées et, sans doute aussi, dans les Cévennes, en Savoie et en Suisse. 
Régénération. - La nécessité de ménager tous les rameaux d'avenir oblige à couper les perches exploitables à un certain niveau au-dessus du sol, de telle sorte que les bourgeons donnent des rejets qui ne s'affranchissent jamais; les souches furetées sont donc condamnées à mourir de vieillesse un jour ou l'autre. Pour les remplacer, il faut utiliser les faux drageons, les brins de semence qui naissent dans les intervalles, ou, à défaut, marcotter des traînants. Ces remplaçants sont recépés d'assez bonne heure pour provoquer la création de cépées, qui seront, à leur tour, mises à l'état fureté.

Réglementation. - On doit s'astreindre, non seulement à ne réaliser que des brins de calibre, mais encore à couper le plus grand nombre de ceux-ci. Sinon, les perches dominantes auraient des tendances à former un massif assez sercé, et arrêteraient le développement des rameaux intermédiaires et des bourgeons dormants; alors se constituerait un perchis plus ou moins régulier, qui ne serait plus un taillis fureté.

On n'a pas intérêt à multiplier le nombre des exploitations ; au contraire, en augmentant, dans une limite convenable, l'intervalle qui sépare les retours de la hache dans une même cépée, on rend plus nette la distinction des perches de calibre; le peuplement se trouve moins souvent exposé aux fatigues et aux abus d'une exploitation; enfin, on a l'avantage d'augmenter la surface des cantons où, les rejets étant plus âgés, le pâturage est moins nuisible. A tous les points de vue, les rotations de douze à quinze ans sont préférables à celles de huit à douze ans.

ARTICLE V.

\section{LE TAILLIS SOUS FUTAIE}

Constitution. - Solidarité entre la futaie et le taillis. - Avantages et inconvénients. - Régénération du taillis. - Constitution de la futaie. - Les dégagements de semis. - Les éclaircies.

Constitution. - Le taillis sous fulaie, ou taillis composé, est caractérisé, comme ce dernier nom l'indique, aussi bien par son mode de régénération, que par sa nature complexe. Il est formé, en effet, de deux éléments : un sous étage 
exploité à intervalles égaux, à la façon des taillis simples, et un étage composé d'arbres irrégulièrement disséminés, dont les cimes, qui dominent le taillis, se développent à l'état isolé jusqu'au terme de leur existence utile. Ces arbres constituent ce qu'on appelle la réserve ou la futaie.

Cette réserve, d'ailleurs, est constituée et s'exploite de la manière suivante: lors du passage des coupes, on réalise dans la futaie, individuellement et en jardinant, les arbres devenus exploitables. En même temps, on choisit, pour les conserver, un certain nombre de brins de l'âge du taillis, qui, à partir de cet isolement, sont acquis à la réserve et viennent la renforcer de façon à entretenir sa composition, sa consistance et sa production aussi constantes que possible.

Il résulte de cette manière d'opérer que les arbres de la futaie ont d'abord vécu avec le sous étage, dansle sein duquel ils ont été confondus pendant une révolution, et que, dans l'ensemble, la réserve se compose d'arbres appartenant à plusieurs classes, dont les âges différent entre eux d'un temps égal à la durée des révolutions de taillis.

Dans ces conditions, ces divers éléments peuvent se combiner de mille façons différentes; aussi la forme des taillis sous futaie est-elle essentiellement variable. Entre un taillis simple régulier, ombragé par quelques arbres épars, et un autre dominé par une réserve très nombreuse, il y a place pour tous les intermédiaires.

Solidaritẻ entre la futaie et le taillis. - D'ailleurs, entre les deux éléments du taillis sous futaie, il existe une solidarité complète.

D'une part, la consistance du taillis est en raison inverse de celle de la futaie, puisque ce taillis, qui forme l'étage dominé, est d'autant plus clair et chélif que la réserve, c'est-àdire l'étage dominant, est composée d'arbres plus nombreux et plus gros. Cette dernière considération engage donc à composer la réserve, autant que possible, avec des essences à feuillage léger, et dont la valeur acquise par le grossissement soit suffisante pour compenser la perte que leur couvert fait éprouver au sous bois.

D'autre part, la réserve agit sur la composition du peuple- 
ment, lequel est loin de conserver la stabilité relative que présentent les taillis simples. En effet, par son couvert, elle

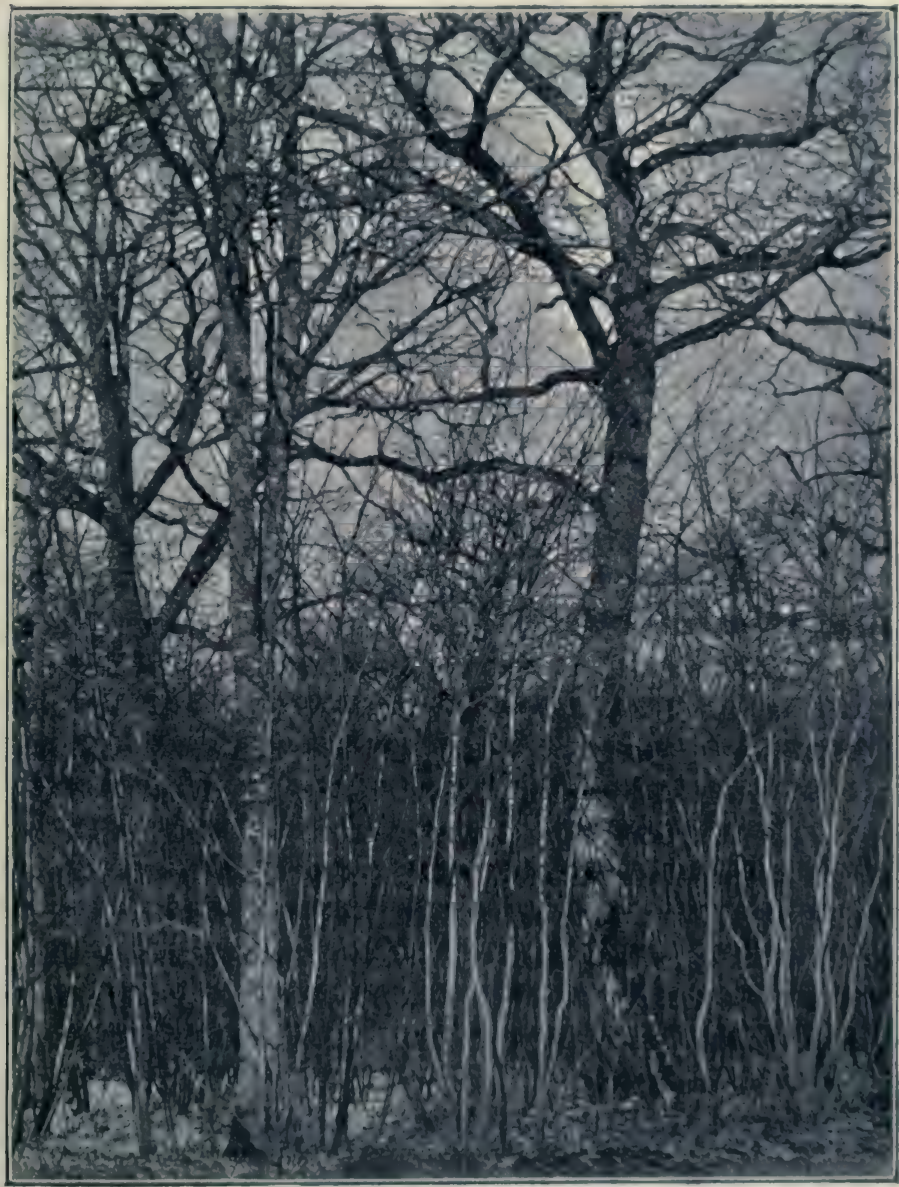

Fig. 58. - Un taillis sous futaie avant la coupe. Forêt de Champenoux. fait perdre à un certain nombre de souches la faculté de produire des rejets. D'après les recherches faites par M. d'Arbois de Jubainville dans les taillis de la Meuse (1), on peut admet-

(1) Recherches sur les Taillis sous Futaie, Grimblot. Nancy, 1860. 
tre que, dans les conditions moyennes, sous le couvert direct des chênes, 3 à 6 p. 100 des souches ne rejettent plus. Cette proportion augmente encore si, au lieu de chênes, la réserve est formée d'espèces à couvert épais, ou peu élevé, notamment de hêtres ou de charmies. De plus, la cépée dans laquelle on fait une réserve est, en général, perdue pour le sous étage, celle-ci ne devant être exploitée qu'à un âge où sa souche ne fournira plus de rejets durables.

Mais, ces dégradations sont compensées par l'apparition de brins de semence. Ceux-ci apparaissent dans les circonstances les plus diverses grâce à la disposition variée de la réserve, dont les arbres sont en grande partie fertiles. Ainsi les semis de bois blancs et des autres espèces à graines légères, telles que : frênes, ormes, érables, etc..., se jettent, aussilôt la coupe faite, sur le sol mis à découvert. Ces semis ont un sort variable : ceux de charmes, qui naissent nombreux, ne résistent à l'été que dans les sols assez frais pour ne pas se dessécher au delà de la zone peu profonde pénétrée par les racines superficielles de cette essence; ceux de chêne et de hêtre se produisent après chaque année de semence, mais ils ne rencontrent pas toujours des conditions favorables pour se maintenir : tant que le taillis est à l'état de fourré, à couvert très bas, leur réussite est impossible; s'ils se forment vers la fin de la révolution, les jeunes hêtres sont exposés à être tués parl'insolation trop brusque, consécutive à la coupe; quant aux semis de chênes, ils peuvent, sans doute, résister à la pleine lumière, mais, le plus souvent, ils sont dominés par des rejets de souches et disparaissent en presque totalité dans le cours de la révolution suivante, à moins qu'on ne vienne à leur aide.

Toutefois, profitant d'une série de circonstances propices, quelques brins de charme, de chêne, de hêtre, parviennent à entrer définitivement dans la composition du peuplement. On constate d'ailleurs que les semis se montrent d'autant plus nombreux que les révolutions sont plus longues, et la réserve plus riche en arbres fertiles. Aussi peut-on dire que, dans les taillis sous futaie bien tenus, l'action de la réserve tend à enrichir la composition du taillis en bonnes essences, plutôt qu'à l'appauvrir. 
Avantages et inconvénients. - Le taillis sous futaie emprunte au taillis simple son mode de régénération facile, sûr et gratuit; mais, mieux que lui, il utilise, en sol profond, toute l'épaisseur de la couche pénétrable aux racines. Le changement perpétuel dans la situation des réserves établit, dans cette zone, une sorte d'assolement entre les régions superficielles et les régions profondes, occupées alternativement par les racines des cépées, et par celles des arbres. Mieux que lui encore, il protège le sol et fournit à la couverture morte des détritus abondants; on ne remarque pas d'ailleurs qu'il exerce une action fâcheuse sur la fertilité des terrains de bonne qualité; toutefois, épuisant par nature, comme tout taillis, il peut être d'une application dangereuse dans les sols pauvres et secs, surtout si la révolution adoptée est courte.

Le sous étage, en tant que taillis simple, échappe à tous les dangers extérieurs. Les arbres de la futaie, eux-mêmes, grâce à l'enracinement solide qu'ils doivent à leur état isolé, offrent une grande résistance au vent; c'est seulement au cours des deux ou trois années qui suivent l'exploitation qu'ils risquent d'être renversés ; les anciens protègent, d'ailleurs, les modernes et surtout les baliveaux. Dans son ensemble, le peuplement, d'âges multiples et de composition variée, est peu exposé aux ravages des insectes. En toutes circonstances, et mieux que la futaie, le taillis sous futaie répare le lui-même les dégâts dont il a pu être victime; car quel que soit le sort de la réserve, l'ensouchement du taillis est toujours là, pour fournir une régénération par rejets et sauver l'état boisé.

Au point de vue économique, le taillis sous futaie se recommande par ses produits variés, de nature à satisfaire les besoins les plus multiples du commerce. 11 permel, en outre, d'obtenir, dans un temps relativement court, des chênes d'un fort diamètre, dont le bois est très nerveux, et, accessoirement, d'élever les grandes espèces disséminées que la futaie élimine presque toujours. Enfin, ce mode de traitement se prête aux combinaisons les plus diverses; il demeure applicable aux forêts de toute étendue, aux plus grandes comme aux plus 
petites; il est à la portée de toutes les bourses, de toutes les catégories de propriétaires.

Tels sont les avantages fondamentaux de ce mode de traitement; par contre, il exige, comme le taillis simple et pour les mêmes raisons, un climat doux, une longueur suffisante de la saison de végétation, un sol assez frais pour ne pas craindre des découverts souvents répétés, et, - en plus - un terrain dont la fertilité et la profondeur rendent possible la culture des essences précieuses, surtout du chêne.

Au point de vue économique, on peut reprocher au taillis sous futaie l'irrégularité dans l'épaisseur des anneaux ligneux fabriqués par les arbres, et l'excès de densité du bois de chêne : celui-ci, trop nerveux, se tourmente, et le travail en est difficile. D'ailleurs, les produits en bois d'œuvre sont moins sains que dans les futaies : les alternatives d'isolement et d'enclave au milieu d'un sous étage grandissant, disposent les arbres à contracler des tares, si bien que, même dans les circonstances les plus favorables, il est rare qu'ils fournissent de 40 à 50 p. 100 de leur volume total en bois d'œuvre; le reste n'est que du chauffage de médiocre qualité. - D'autre part, les peuplements s'éloignent des formes naturelles : ils sont le résultat de combinaisons entre des éléments antagonistes et trop variables pour qu'on puisse les modeler dans un moule unique ; chaque forêt, chaque climat, chaque sol, chaque propriétaire peut exiger, dans un sens ou dans l'autre, des modifications du type qu'on se serait imposé. - Enfin, si l'on n'y prend garde, la futaie partage, avec toutes les exploitations d'arbres considérés isolément, l'inconvénient d'être toujours mobilisable, et, par suite, exposée aux abus de jouissance.

Régẻnération 'du taillis. - Dans l'ensemble, le taillis assure l'ensouchement, c'est-à-dire l'état boisé, et constitue, en outre, une véritable pépinière, où se recrutent les brins destinés à remplacer les futaies qui tombent sous la hache. Il est, en un mot, l'agent fondamental de la perpétuation de la forêt.

La futaie, au contraire, est l'élément principal du revenu, c'est elle qui fait la richesse des peuplements.

La régénération du sous étage est identique à celle d'un 
taillis simple régulier en semblable condition : les mêmes précautions sont à prendre pour l'assurer (article V). Il n'y a pas, d'ailleurs, à faire fonds sur les rejets éventuels, et généralement sans avenir, que peuvent donner les souches des réserves exploitées.

Il est inutile, d'autre part, de trop s'attacher à la prédominance des bois durs dans le sous étage. Aujourd'hui bois durs et bois tendres, - les premiers moins recherchés qu'autrefois pour le chauffage, les seconds plus employés dans la fabrication des pâtes à papier, - se vendent aussi bien,... ou aussi mal l'un que l'autre. Le seul point essentiel est de veiller àu maintien, en nombre suffisant, des essences qui doivent assurer un bon recrutement de la réserve.

Constitution de la futaie. - Toutes les règles culturales propres au taillis sous futaie concernent donc la réserve, nous rechercherons à ce sujet : les essences qu'il convient de préférer ; - le choix à faire quant à l'origine de ces réserves; enfin la répartition la plus avantageuse à leur donner.

Les réserves à préférer sont, avant tout, les essences de lumière, puisque le besoin qu'elles ont d'espace pour étaler leur cime les dispose tout naturellement à croitre à l'état isclé, plutôt qu'en massif plein. D'autre part, c'est dans ce groupe que se rencontrent les espèces à feuillage léger, dont le couvert est le moins prèjudiciable au développement du taillis.

Parmi celles-ci, lęs chênes rouvre et pédonculé se rangent en première ligne; on peut même dire qu'en dehors de leur présence on n'a aucune raison sérieuse de faire du taillis sous futaie. Aux chênes, il est intéressant d'associer des essences disséminées, telles que : frènes, ormes, érables, fruitiers, qui, suivant les stations, fournissent chacun d'excellents produits. Les bois blancs, comme le tremble et le bouleau, dont le couvert est très léger, sont utilement conservés sur les points où ne se rencontrent pas de meilleures espèces. Ils poussent vite, et leur production se trouve pour ainsi dire, en supplément, car ils entravent peu la croissance des cépées qu'ils dominent. Au contraire, la conservation de l'aune et du 
tilleul, dont le couvert est assez épais, n'est à recommander que dans des circonstances exceptionnelles: l'aune sur les bords des ruisseaux, le tilleul dans les pierrailles amoncelées et les débris de carrières, où aucune autre essence ne prospère aussi bien que lui.

Le hêtre s'accommode assez mal de l'état isolé, et le régime du taillis composé ne lui convient pas en principe. Aussi disparaît-il bientôt des forêts en bon sol, où son absence n'est pas à regretter, puisqu'on peut obtenir mietxx à sa place. Au contraire, dans les sols médiocres, il persiste pendant assez longtemps, parce qu'il ne s'y trouve pas d'espèce plus vivace que lui pour l'éliminer.

Si le charme est l'arbre par excellence pour former les sous étages, il a moins sa raison d'être dans la futaie. Sa croissance est trop lente, son feuillage trop épais, son bois trop peu estimé pour le travail; en outre, à cause de son couvert bas, il tue le plus grand nombre de cépées qu'il couvre et ne permet à aucun semis de s'installer à son ombre. L'abus de la réserve charme est une cause de ruine pour les taillis; tout au plus est-il permis d'en conserver quelques sujets, auxquels on ne laissera pas dépasser la dimension de modernes; d'ailleurs, ceux-ci n'ont pas seuls la propriété d'enrichir en semis de l'espèce les taillis où elle serait insuffisamment représentée, car les perches fructifient de bonne heure et suffisent à tous les besoins.

En ce qui concerne la constitution de la réserve, nous devons citer les observations recueillies par. M. Mathey (1) dans le bassin de la Saône ; nous ne pouvons que résumer ce travail, où les taillis sous futaie de la région sont répartis en six groupes, définis par les qualités de leur sol, et caractérisés par leur flore ligneuse et herbacée. Les conclusions, souvent d'ordre très général, intéressent les propriétaires de taillis sous fulaie de toute la France.

Les trois premiers groupes comprennent les terres à chênes, celles où le taillis sous futaie donne son plein rende-

(1) Mathey, Élude sommaire des taillis sous futaie dans le bassin de la Saône. (Bulletin Société forestière de Franche-Comté et Belfort, scptembre, 1898.) 
ment. A tout seigneur, tout honneur..., la futaie est constituée en chêne. Pourtant, sur les colmatages, dont la fertilité est exceptionnelle, on lui associe des essences disséminées : frène, orme champêtre, sans toutefois donner une trop grande prépondérance à ces espèces, dont l'accroissement n'est supérieur à celui du chêne que pendant les deux premiers âges, dont la valeur marchande est variable, et qui sont très épuisantes. - Dans les sables argileux el siliceux fins, une petite place est faite au hêtre, auquel on ne laisse pas dépasser la dimension d'ancien de $1^{\mathrm{m}}, 50$ de tour. - Enfin, dans les marnes compactes, mais profondes et fertiles, les baliveaux et modernes de bouleau et de tremble, essences à couvert très léger et d'un bon rapport, sont utilement associés au chêne, quand celui-ci est insuffisant. On cède trop souvent sur de pareils sols à la fâcheuse habitude de réserver des charmes.

Le quatrième groupe englobe des argiles oxfordiennes ou autres, des conglomérats calcaires ou siliceux, terres de composition variée, mais toujours compactes, froides et acides. Le chène seul, dit M. Mathey, doit y constituer la futaie, mais, " au milieu des maigres taillis que décime la bruyère, " il végète mal. Aussi le forestier doit-il se préoccuper, avant " tout, de resserrer la trame ordinairement trop lâche et trop " uniforme du sous bois."

Quant aux deux derniers groupes, leur caractéristique est la profondeur de plus en plus faible du sol, et, comme corollaire, le rôle de plus en plus prépondérant du hêtre. Sur les calcaires marneux des pays de collines et de basse montagne, la terre est mélangée de plaquettes calcaires ou de rognons marneux; le chêne décline; il devient logique d'accepter largement le hêtre, qui est, en fait, l'essence la plus productive: " il ne " faut pas craindre de le multiplier en modernes, et d'en gar" der les beaux anciens, les plus longs; " quelques chênes, là où la profondeur sera suffisante, quelques alisiers torminaux, enrichiront la réserve et achalanderont les coupes. - Sur les arènes provenant de la décomposition des granits ou des porphyres, sur la terre rouge qui recouvre certains calcaires jurassiques, sols éminemment superficiels tous deux, reposant tous deux sur des roches dures, "la réserve du chêne 
" ne compense, à aucun âge, la perte du recrû qu'elle entraîne; " le hêtre, jusqu'aux dimensions d'ancien, est l'essence fonda" mentale et exclusivement rémunératrice de la futaie. "

Certes, nous le reconnaissons, l'introduction dans la réserve d'une essence d'ombre comme le hêtre, n'est pas conforme au principe du taillis sous futaie. Mais peu importe la théorie, si, ne pouvant faire mieux, nous constituons une forêt solide et d'un bon rapport. Or, il suffit de constater l'aspect chétif des chênes que l'on réserve, en semblable situation, pour se convaincre de leur faible production, c'est-à-dire de leur inutilité économique.

Pour entretenir en bon état la consistance du taillis, il y a lieu de ne pas choisir les baliveaux sur les grosses cépées, qui, donnant d'abondants rejets, forment sa véritable richesse. On leur préfère les brins de semis; à leur défaut, les rejets de jeunes souches, et les drageons; en dernier lieu, les brins isolés, détachés des cépées, comme il s'en rencontre presque toujours.

On admet que les brins de semence et les drageons ont le plus de vitalité. A ce point de vue, les rejets provenant du premier recépage d'un jeune sujet de franc pied ont à peu près même valeur que lui; en fait, ils fournissent même la majeure partie des bons baliveaux, car il est rare qu'un brin soit assez fort pour être isolé à la fin de la révolution au début de laquelle il est né : c'est seulement après avoir été recépé, qu'il s'élance avec assez de vigueur pour marcher comme le sous étage. Les rejets de vieille souche se carient d'assez bonne heure, et c'est se tromper soi-même que de conserver des arbres, sur l'avenir desquels on ne doit pas compter.

On choisit d'ailleurs les baliveaux parmi les tiges de forme régulière, droite et bien équilibrée, car les sujets fourchus risquent d'être déchirés par le vent, et, quand ils échappent à ce danger, leur bois est déprécié par la présence d'entreécorces.

Les arbres bons à être réservés ne se présentent pas tou- 
jours dans la station géométrique voulue pour obtenir un bon espacement. De plus, les réserves sont entremêlées de telle sorte que l'on peut voir avoisinées les espèces les plus différentes, appartenant à une catégorie quelconque de baliveaux, et parmi lesquelles il faut opter. Sans entrer dans plus de détails, ni discuter les problèmes que soulève la question, nous

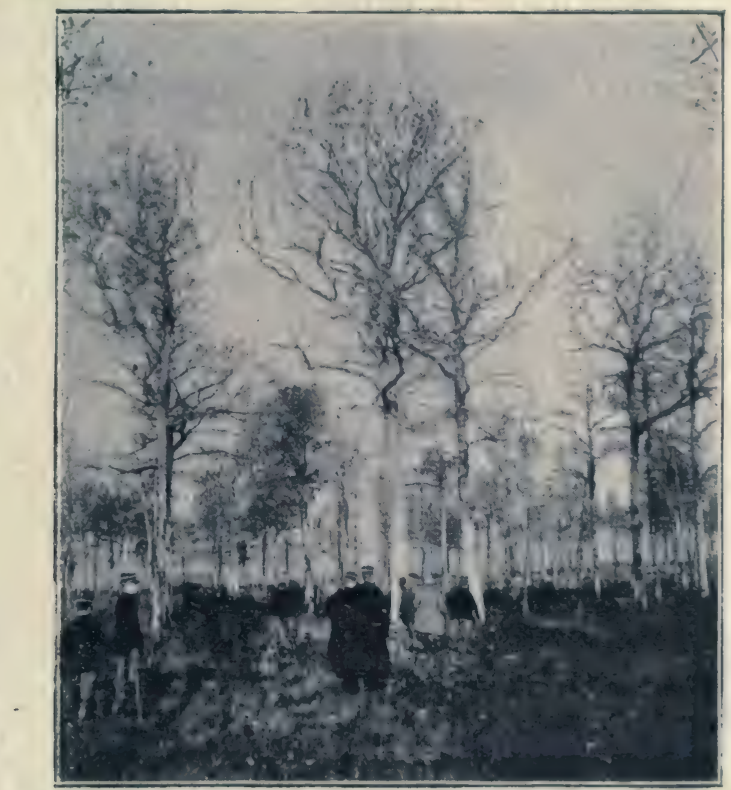

Fig. 59. - Un taillis sous futaie après la coupe. Forèt de Pont-àMousson (Meurthe-et-Moselle), terrain argileux. - Photographie de M. J. George.

engageons les opérateurs à se pénétrer de cette vérité que, sur chaque point, l'arbre le meilleur à conserver, est toujours celui qui, eu égard à l'espèce à laquelle il appartient, à sa vigueur, à son état sain, à ses dimensions, à sa valeur actuelle, travaille le plus utilement dans l'intérêt du propriétaire (1); ici nous marquerons un chêne ancien, là un hêtre moderne, ailleurs autre chose.

(1) Exemples :

Entre deux chênes d'ages différenta, s'il y a lieu de choisir, c'est, 
Dans la pratique, il est essentiel de porter d'abord toute son attention sur la grosse réserve. C'est seulement quand le choix de celle-ci est bien arrêté qu'on s'occupe des baliveaux, en les répartissant dans les espaces oủ les arbres manquent, en évitant surtout de les marquer trop près des modernes et des anciens, ou comme cela se fait trop souvent, sous leur projection. Ce serait, d'ailleurs, se faire illusion que d'en exagérer le nombre pour masquer l'indigence d'une réserve trop pauvre en arbres constitués; il faut être convaincu que les modernes et les anciens forment la véritable richesse de la forêt : c'est le capital indispensable à la fabrication des gros bois, les seuls qui se vendent cher au mètre cube.

Enfin, il est bon : $1^{\circ}$ de renforcer le balivage sur les lisières des forêts : on crée ainsi, sur 15 à 20 mètres de largeur, des rideaux de protection du plus utile effel; $-2^{\circ}$ de réserver, le long des tranchées ou des chemins de vidange, des cordons d'arbres de futaie, qui embellissent la forêt, rendent plus facile l'entretien des lignes et assurent une riche épargne, que l'on est heureux de trouver le cas échéant; cette excellente pratique, en honneur autrefois, s'est perdue de nos jours : à tous égards il serait avantageux d'y revenir.

Le balivage. - Tous les arbres qui entrent dans la réserve se nomment des baliveaux. Suivant leur catégorie, on les distingue en: baliveaux de l'âge, baliveaux modernes et baliveaux anciens. Au moment de la coupe, les premiers sont âgés d'une révolution, les seconds de deux, les autres de trois révolutions et plus. C'est ainsi que les ordonnances et le Code forestier les distinguent.

Mais, en fait, le terme de baliveau est exclusivement attribué aux brins de l'âge, aussitôt après leur isolement. On

en général, le plus gros qu'il faut conserver, à condition qu'il soit sain, bien entendu. C'est lui, en effet, qui fabrique le bois ayant le plus de valeur à l'unité de volume; c'est lui qui sera le plus tôt et le plus sûrement exploitable.

Entre plusieurs réserves d'essences autres que le chêne, c'est la plus vigoureuse qu'il faut conserver, quelle que soit sa grosseur, car ici le prix du mètre cube de bois fabriqué n'augmente pas sensiblement avec le diamètre. 
appelle modernes les arbres des deux âges, et anciens tous ceux de trois âges et au-dessus. Ces dénominations sont insuffisantes: il serait bon d'adopter des termes plus précis et permettant de distinguer chaque classe d'âge, par exemple :

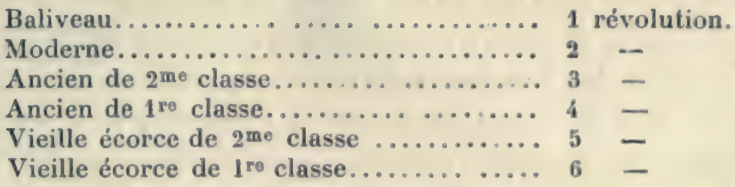

Il est d'ailleurs assez rare que le mème arbre puisse supporter six fois la crise de l'isolement, en restant assez sain pour ne pas être exploitable (1).

Leur désignation se fait par l'apposition de l'empreinte du marteau du propriétaire sur une flache, ou miroir, ouverte à la patte de l'arbre à réserver, savoir: une empreinte sur un miroir, pour les baliveaux de l'âge, - deux, chacune sur un miroir, pour les modernes, - trois, superposées sur le même miroir, pour les anciens. On ne saurait sans de graves inconvénients multiplier les miroirs et les coups de marteau, mais une excellente mesure consiste à tracer un numéro d'ordre sur l'écorce de chaque ancien, avec de la couleur ou avec une roanne; sur le calepin de balivage on inscrit, en regard de chaque numéro, le diamètre ou la circonférence de l'arbre. Ce procédé permet de reconnaître et de bien spécifier les exploitations frauduleuses; il permet aussi de suivre, dans l'avenir, la marche de l'accroissement de tous ces sujets de valeur.

Cette marque, dite en réserve, est évidemment le procédé donnant le plus de sécurité au propriétaire vis-à-vis de bûcherons insouciants ou mal intentionnés; mais ces plaies faites à la base des arbres sont une cause de pourritures. On peut leur substituer des indications à la couleur rouge ou de simples coups de griffes ou roannes, d'une imitation malheureusement bien facile. En tout cas, ce dernier procédé peut être employé

(1) Il est souvent difficile de déterminer l’àge d'une réserve, aussi se base-t-on sur la grosseur pour qualifier un arbre comme moderne ou ancien. On décide, par exemple, d'appeler anciens, les sujets de 35 centimètres de diamètre et au-dessus. 
sans inconvénient pour les baliveaux de l'âge, dont la valeur marchande est très faible; quant aux gros arbres, le garde marqueur prendra soin de faire le miroir le plus petit et le moins profond possible, et de le placer sur une grosse racine plutôt que sur le fût même du sujet. Pour éviter tous ces dangers, il serait préférable d'exploiter le taillis, après désignation des baliveaux de l'âge, et, seulement ensuite, de choisir dans la futaie les arbres à conserver et ceux à abandonner: ces derniers seraient alors marqués en délivrance par l'apposition d'une empreinte au corps et à la racine. Ce système, appliqué en Belgique, n'est pas encore entré en France dans le domaine de la pratique.

La base du traitement en taillis sous futaie repose sur ce fait que la régénération naturelle du sous-élage par rejet, et sa consistance en massif, doivent être assurés d'une manière permanente.

Ces deux conditions ne seront satisfaites que si l'on maintient toujours les réserves à l'état isolé. Par ce terme, il faut entendre qu'immédiatement après la coupe, les cimes des arbres voisins jouiront d'un espace tel qu'elles puissent se développer librement sans se rejoindre avant la fin de la révolution; c'est dire que jamais elles ne formeront massif. Cet espacement minimum, est d'ailleurs suffisant, et il n'y a pas d'espacement maximum : les arbres peuvent être aussi éloignés les uns des autres que le veut le bon plaisir du propriétaire.

Toutefois, si ce dernier dispose d'une marge considérable, encore ne doit-il pas marcher à l'aventure. En toutes circonstances, il est prudent d'établir, en même temps que la division en coupes, un plan de balivage, à l'esprit et à la lettre duquel l'opérateur devra se conformer.

Le plan de balivage fixe par hectare - approximativement tout au moins - le nombre de réserves de chaque essence et de chaque catégorie; il n'y a pas d'autre moyen de faire cesser l'état d'incertitude dans lequel flottent le capital d'exploitation et le revenu de la plupart des taillis sous futaie. Divers procédés ont été mis en avant pour l'établir. Le point de 
départ de tout calcul de ce genre est le choix d'un âge d'exploitabilité pour les réserves des différentes essences, et la détermination du nombre de sujets, parvenus à cet âge, que l'on entend réaliser au passage de la coupe. Ce nombre est subordonné à la longévilé des espèces, aux prix des marchandises sur les marchés, et au chiffre du capital que le propriétaire veut engager dans son exploitation; il est aussi fonction de la projection horizontale de la cime des réserves dans chaque catégorie: baliveaux, modernes, anciens et vieilles écorces.

"Dans une semblable question, dit M. le Conservateur Burel (1), le couvert des arbres est le facteur prépondérant, car il importe, par la distribution des futaies et leur abatage mesuré, de ménager à chaque catégorie la place indispensable pour se développer librement jusqu'au terme de l'exploitabilité. »

M. I'Inspecteur Galmiche (2), admet, en moyenne:

pour un arbre de 25 ans, un couvert

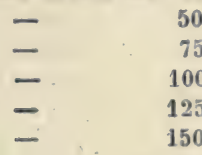

insignifiant;

de 22 mètres carris;

de 58

de 89

de 116

de 145

A chaque exploitation, on est dans la nécessité de réaliser, outre les arbres murs, un certain nombre de sujets appartenant à tous les âges de la réserve, mal venants ou mal choisis, tarés, brisés par le vent ou la neige; il en résulte un déchet, qui oscille entre le tiers et les deux tiers de l'ensemble, dit M. Galmiche dans le même travail, où nous lisons plus loin :

Si nous pouvions trouver en tous points, l'arbre qui nous convient: si cet arbre devait arriver sans encombre jusqu'au terme de l'expluitabilité, nous n'aurions qu'à diviser la surface de la coupe par la somme des couverts, pour obtenir le nombre des réserves de 1, 2, 3, \& révolutions à marquer; nous enlèverions exactement le nombre des arbres

(1) Burel, Élude sur les taillis composés. (Revue des Eaux et Foréts, février 18s5.)

(2) Galmiche, Élude sur les réserves des taillis sous futaie. (Bull. Société forestière de Franche-Comté et Belfort, aout 1893.) 
exploitables et désignerions un nombre égal de baliveaux pour les remplacer. Ce serait admirable !

Malheureusement, nous ne trouverons pas à la distance convenable l'arbire à réserver et, quelque désir que nous ayons de voir la coupe entièrement couverte par les cimes à l'expiration de la révolution qui va commencer, nous ne réaliserons jamais ce souhait : ce sera superbe si le couvert futur est des $3 / 4$ de la surface de la coupe; il y a plus de chance pour qu'il ne soit que des $2 / 3$; peut être même ne sera-t-il que de $1 / 2$. Nous réussirons moins encore à maintenir toutes les réserves sur pied jusqu'à une dimension uniforme.

Il est absolument chimérique, dès lors, de rêver la formule du plan de balivage intégral applicable en toutes circonstances. Il appartient à chaque propriétaire de calculer celle qui convient à sa fortune et à la situation de son bois, en tenant compte des circonstances de temps et de lieu.

A titre de spécimen, nous donnons, en forme de tableau, le résumé des opérationsà faire, sans d'ailleurs insister aucunement sur la valeur absolue ou relative des chiffres employés.

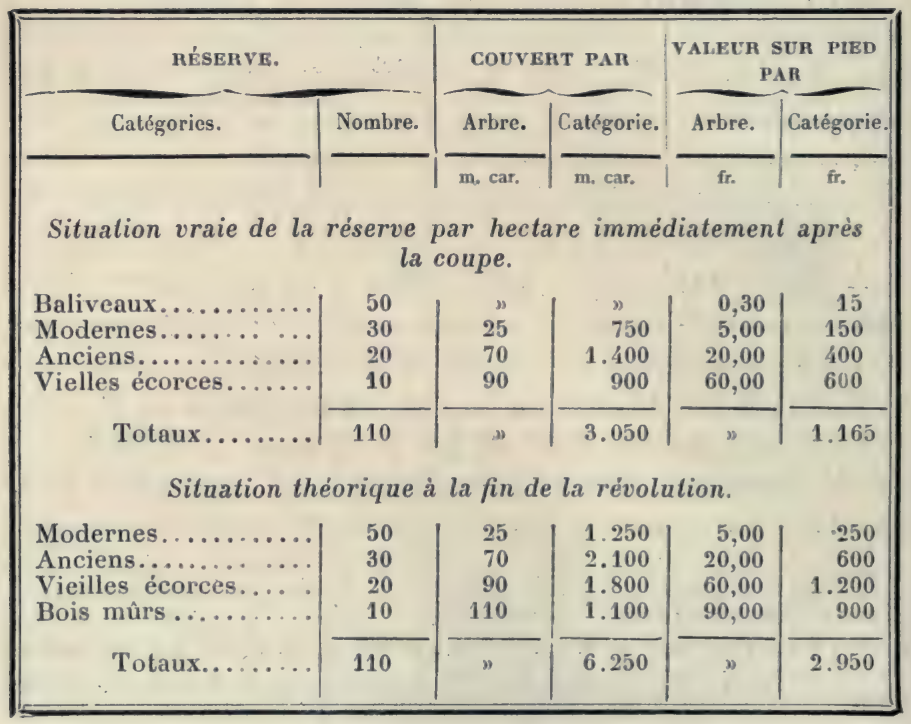

Si l'on veut ramener la réserve au taux initial et l'entretenir dans cette forme, il suffira, lors du passage de la coupe, d'exploiter 20 modernes, 10 anciens, 10 vieilles écorces et les 
10 arbres mûrs; soit 50 arbres, et d'ajouter 50 baliveaux choisis dans le sous bois. Au cas particulier, la valeur des réserves abattues, serait de $2950-1165=1785$ francs, somme qui représente la part de bénéfice résultant du fonctionne. ment de cet élément pendant le cours de la révolution. Pour compléter le bilan de l'entreprise, il rr'y aura plus qu'à tenir compte de la perte causée par le couvert des futaies à l'accroissement du sous étage.

Il est clair que le bénéfice produit par la réserve est surtout fonction de la valeur et du calibre des gros arbres, et qu'il varie avec le nombre de ceux-ci. La limite supérieure serait atteinte par cet état théorique d'un " sol couvert au moment de l'exploitation d'arbres isolés en croissance. "D'aucuns ont pris l'habitude d'appeler "futaies sur taillis" les peuplements dans lesquels le plan de balivage se rapproche de cet idéal; mais nous ne voyọns aucune nécessité d'adopter ce vocable, tant qu'on n'aura pas établi par des faits les limites qui séparent le taillis sous futaie vrai, de cette sorte de futaie sur taillis, et introduit dans la pratique une bonne définition de ces deux modalités.

Les dẻgagements de semis. - Les peuplements traités en taillis sous futaie comportent des soins de même nature que ceux que l'on applique aux futaies régulières; mais, ici, l'objectif constant doit être d'assurer le bon fonctionnement de la réserve. C'est dans ce but que seront pratiqués les dégagements de semis et les éclaircies.

Les dégagements de semis appellent les observations suivantes:

$1^{\circ}$ la végétation des rejets est trop rapide pendant les premières années pour justifier l'espoir de raccorder avec eux les brins de semence pendant la révolution en cours; on ne peut faire plus que les maintenir vivants;

$2^{\circ}$ la marche régulière du développement d'un taillis rend possible l'indication précise de l'époque à laquelle ces dégagements deviendront opportuns : le premier se fait, en général, quand le recrû à trois ans, - les autres suivent à trois, quatre ou cinq ans d'intervalle, jusque vers la quinzième année;

$3^{\circ}$ il est inutile de dégager tous les semis épars sur le par- 
terre d'une coupe; on concentre, au contraire, tous ses soins sur les petites taches de semis lorsqu'elles se présentent sur les points où leur présence est utile, nous voulons dire où il existe un vide dans la réserve; c'est, en tout cas, peine perdue que de faire le moindre travail sous la projection d'un gros arbre: une cinquantaine de placeaux bien choisis par hectare suffisent largement pour assurer l'avenir, si on ne les abandonne pas trop tôt à la concurrence des rejets; ce chiffre répond au nombre des baliveaux à conserver dans chaque coupe.

Les éclaircies. - L'éclaircie est conduite d'après les règles générales (Chapitre V), en se proposant pour objectif :

$1^{\circ}$ de continuer l'œuvre commencée par les dégagements de semis ;

$2^{\circ}$ d'assurer un espace convenable à la cime des perches qui se présentent dans les conditions voulues pour former un bon baliveau; en donnant ainsi de l'ampleur à leur cime, et, par suite, du diamètre à leur fût, on les prépare à subir la crise de l'isolement, on diminue donc le nombre de ceux qui risquent d'être courbés ou brisés par le poids de la neige ou de leur feuillage ; cette opération concerne tous les futurs baliveaux : brins de franc-pied, drageons ou rejets de souches;

$3^{\circ}$ de favoriser la naissance de semis naturels quelques années avant l'exploitation; l'opération doit être alors accompagnée de l'enlèvement des morts bois; mais ce n'est justifiable que quand ceux-ci sont assez dépérissants et le couvert du peuplement principal assez complet pour qu'ils ne donnent plus de rejets, c'est-à-dire quand le taillis a atteint ou dépassé l'âge de trente ans ;

$4^{\circ}$ de faire tomber les perches qui, pénétrant dans la cime des réserves, ou fouettant contre l'extrémité de leurs branches, menacent de les dégrader.

Si l'on excepte le cas où l'éclaircie continue les dégagements de semis, on conçoit qu'elle n'a aucune raison d'être - et même ne présente que des inconvénients, - quand la révolution est inférieure à trente ans. Passé ce terme, une seule éclaircie faite six ou dix ans avant la coupe, est suffisante.

On peut ranger à la suite des éclaircies la réalisation des bois tendres dans les sols très fertiles, réalisation qui se fait 
vers dix-huit à vingt ans, lors de la maturité de ces essences, en général peu longévives.

Trop souvent, sous le même nom d'éclaircie ou celui de nettoiement, on procède au recépage de toute la végétation basse d'un taillis sous futaie, morts bois et brins trainants, avec desserrement intérieur des cépées; une telle opération a pour conséquence immédiate de découvrir et de fatiguer le sol, c'est-àdire d'exagérer les inconvénients du régime. Nous n'ignorons pas que ces " détrappages " sont recommandés par nombre de praticiens, parmi lesquels on compte des forestiers distingués (1); sans nier l'efficacité relative de ces opérations sur les points où elles ont été étudiées et conduites avec le doigté et les soins minutieux qu'elles comportent, nous nous contenterons de faire à leur sujet les observations suivantes:

$\mathrm{Ou}$ bien les produits sont vendables, et il est à craindre que la serpe coupe plus que de raison pour augmenter le bénéfice du jour ;

Ou bien les produits, sans valeur, pourriront sur le sol; alors bien peu consentiront une avance de fonds dont la rentrée et le bénéfice, s'il y a lieu, n’interviendront qu'à longue échéance.

La raison et la prudence nous font donc un devoir de dire que, dans l'immense majorité des cas, il y a lieu de s'abstenir en laissant agir la nature (2).

ARTICLE VI.

\section{AMÉLIORATION DES FORÊTS TRAITÉES EN TAILLIS SIMPLE ET EN TAILLIS SOUS FUTAIE}

Transformations et conversions. - Leur peu de raison d'étre dans les forêts particulières. - But à poursuivre. - Améliorations proposées. - Allongement des révolutions. - Éducation d'arbres plus nombreux. - Substitutions d'essences.

\section{Transformations et conversions. - Quand la dégradation} d'une forêt a pour cause un mode de traitement appliqué à

(1) Du nelloiement des bois, par M. l'inspecteur Schoffer. (Bull. Société forestière de Franche-Comté et Belfort, juillet 1897.)

(2) Il importe de se mettre en garde contre une illusion d'optique 
tort dans une station ne le comportant pas, - ou quand, pour des raisons économiques, il faut produire des marchandises très différentes de celles dont on se contentait dans le passé, - il peut y avoir lieu de changer le mode de traitement, de faire une transformation ou une conversion, c'est-à-dire d'appliquer un de ces modes de traitements temporaires que nous avons définis plus haut (page 107).

En toutes circonstances, le procédé reste le même :

$1^{\circ}$ tracer sur le terrain et sur les registres le cadre du nouvel aménagement dans lequel les opérateurs devront se mouvoir;

$2^{\circ}$ exécuter, sur les points de la forêt qui paraissent le mieux se prêter à la transformation ou à la conversion immédiates, les coupes spéciales au nouveau mode de traitement: ainsi, pour transformer une futaie jardinée, ou pour convertir un taillis sous futaie en futaie régulière, pratiquer sur ces points les coupes successives de régénération par la semence;

$3^{\circ}$ conserver dans les autres cantons de la forêt, jusqu'à ce qu'ils arrivent à leur tour de transformation ou de conversion, l'ancien mode de traitement, que l'on modifie toutefois dans un sens qui rende plus facile, dans la suite, les véritables opérations de conversion.

La durée du traitement temporaire est, par nécessité, égale, en théorie du, moins, à celle de la révolution adoptée pour l'avenir; car, au moment où les derniers peuplements de l'ancienne forêt viennent d'être transformés ou convertis, il faut, pour qu'il n'y ait pas d'interruption dans les revenus, que les cantons attaqués et régénérés en premier lieu portent des bois exploitables. Bien plus, dans le cas de la conversion d'un taillis sous futaie en futaie régulière, l'obligation de laisser vieillir le taillis jusqu'à un âge tel que les rejets de souches n'évoluent pas en trop grand nombre dans les coupes affectées à la régénération conduit, préalablement à toute opération, à maintenir sur pied les peuplements pendant une " période d'attente" qui ne peut être inférieure à une trentaine d'années, mais

un peuplement que l'on a débarrassé de toutes les menues tiges, paraît, dans son ensemble, constitué par des sujets plus gros qu'un peuplement oú des tiges de tous calibres sont confusément mélangées. 
peut dépasser soixante ans. Inversement, quand il y a lieu de convertir en taillis simple ou en taillis sous futaie les lambeaux d'une futaie régulière disloquée, démembrée par un partage, on est souvent conduit à régénérer par la semence, une fois encore, les massifs trop vieux pour que l'on puisse compter sur une production suffisante de rejets après leur recépage.

Leur peu de raison d'être dans les forêts particulières. Ces traitements, dits temporaires, ont donc, en réalité, une durée très longue, si on la compare à celle de la vie humaine. Ils sont, de ce fait, incompatibles avec la situation économique des forêts particulières, exposées à des changements de main continuels.

D'autre part, ces opérations comportent des connaissances techniques que peuvent seuls posséder des professionnels expérimentés. Comme nous l'avons vu, il s'agit plutôt de combinaisons d'aménagement que de questions culturales proprement dites; car ces dernières sont, en général, peu différentes de celles qui se pratiquent dans les traitements permanents; mais leur distribution est autre.

D'ailleurs, pour passer d'un mode de traitement à capital restreint (taillis simple ou taillis sous futaie), à un autre mode à capital plus important (futaie pleine ou futaie jardinée), il faut toujours prélever sur les revenus des économies considérables, qui se traduisent par des diminutions, souvent même par des suspensions complètes de produits pendant un temps plus ou moins long.

Enfin, au cours d'une transformation ou d'une conversion, on est conduit tantôt à laisser des porte-graines se détériorer sur pied, tantôt à exploiter avant l'âge des arbres en pleine croissance, sacrifices qui, bien que justifiés par des nécessités culturales, n'en ont pas moins des conséquences économiques qu'on ne doit pas affronter sans les motifs les plus graves. En fait, l'État, et parfois les Communes, sont les seuls propriétaires qui puissent se permettre une pareille détermination.

But à poursuivre. - Par ces derniers mots, nous n'entendons nullement conseiller aux propriétaires particuliers un statu quo défectueux ; mais nous leur proposons d'améliorer 
graduellement leurs forêts : but qu'ils peuvent atteindre, avec quelque peine, sans doute avec du soin, de la patience et, certes, de l'économie, mais sans aller jusqu'à des suppressions totales de revenus incompatibles avec leur situation de fortune. Tout le monde a vu, dans l'industrie, des exploitations d'apparence modeste, qui, entre les mains d'un manufacturier éclairé, sachant profiter des découvertes de la science dans la mesure où ses capitaux le lui permettent, s'agrandissent, se transforment sans bruit, et rapportent finalement des bénéfices bien plus considérables que telle affaire lancée avec grand fracas de réclame et grand luxe de bâtisse. C'est un exemple à imiter.

Étant donnée la situation actuelle du marché des bois, le but à poursuivre est de faire des arbres plutôt que des taillis, et, d'une manière plus générale, d'augmenter le calibre, la grosseur des produits ligneux de nos forêts.

Les traitements en futaies régulière ou jardinée sont tout naturellement orientés vers ce but ; un choix judicieux de la révolution et des espèces à cultiver, l'opportunité, la bonne exécution des dégagements de semis et des éclaircies, conduisent, par la force des choses, au résultat souhaité. Il n'en est pas de même des taillis simples et des taillis sous futaie, que visent les améliorations exposées dans le présent article.

Améliorations proposées. - Nous ne nous occuperons pas ici des travaux d'entretien tels que : dégagements de semis, façons données au sol, plantations de brins destinés à faire les baliveaux de l'avenir ou à remplacer les souches épuisées, toutes choses inhérentes au traitement lui-même; ce que nous avons en vue, ce sont des mesures d'un ordre plus général, qui, sans changer le mode de jouissance, modifient le fonctionnement du capital-bois incorporé dans l'entreprise et augmentent la valeur de la récolte.

Les moyens sont:

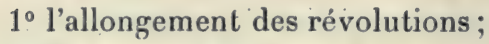

$2^{\circ}$ l'éducation d'arbres plus nombreux;

$3^{\circ}$ parfois même une transposition d'essences.

Allongement des révolutions. - Nous savons qu'au point de vue cultural, le terme supérieur des exploitations d'un taillis peut être fixé aux environs de quarante ans. D'autre 
part, la qualité des marchandises qu'il fournit augmente avec la durée de la révolution.

Ainsi M. Broilliard (1), faisant observer qu'il n'est pas rare de rencontrer des faillis qui, à l'âge de dix ans, ont à l'hectare un volume de 20 mètres cubes, établit le décompte suivant, très justifiable dans la pratique, et qui fait ressortir l'accroissement de plus en plus rapide du cube et surtout de la valeur, suivant que l'on exploite à dix, vingt ou trente ans :

Volume
Age. a l'hectare.

10 ans. 20 m.c.

$20-60$ -

30
Répartition en marchandises.

45 stères bois à charbon à $4 \mathrm{fr}$.

180

$\left.\begin{array}{ll}4 \mathrm{fr} & 180 \\ \text { aे } 8 \mathrm{fr} & 560\end{array}\right\} \quad 710$

70 - bois de moule à $8 \mathrm{fr} \ldots 560$ ?

45 - bois à charbon à $4 \mathrm{fr} . \ldots 180$

70 - bois de moule à $8 \mathrm{fr} . . .560\} 1.550$ n

$662 / 3$ - bois de perches à $12 \mathrm{fr} . . .800$

D'après M. Cardot (2), en 1897, les résultals de la vente des coupes de taillis sous futaie dans les forêts soumises au régime forestier du département de la Haute-Saône ont mis en évidence les résultats suivants :

$\begin{array}{ccc}\begin{array}{c}\text { Age } \\ \text { d'exploitatioz. }\end{array} & \begin{array}{c}\text { Prix de vente } \\ \text { à l'hectare. }\end{array} & \begin{array}{c}\text { La valeur de } 541 \mathrm{fr} \text {. an laux de } \\ 4 \mathrm{p} .100 \mathrm{est} \text { devenue : }\end{array} \\ 25 \text { ans. } & - & - \\ 30- & 541 & 65823 \\ 35- & 756 & 80179 \\ 40- & 1.025 & 1.01329\end{array}$

Dans la Haute-Marne, rapporte M. Devarenne (3), les forêts de Bourmont et de Forcey, comprenant chacune deux cantons contigus, situés dans des conditions semblables, mais exploités à des révolutions différentes, ont fourni à l'hectare les rendements moyens suivants, calculés sur toutes les coupes assises pendant une révolution entière :

(1) Loc. cil, page 90.

(2) Cardot, Allongement de la révolution dans les taillis. (Bull. Société forestière de Franche-Comté et Belfort.)

3) Devarenne, Mémoire sur le meilleur traitement des taillis sous futaie. (Bull. Société forestière de Franche-Comté et Belfort, juillet, 1899.) 
Bourmont : 24 ans, 10 st. rondin, 85 st. charbonnette, ensemble : $85 \mathrm{fr}$.

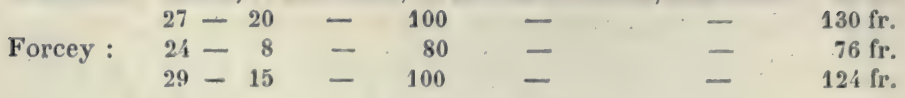

Les observations précédentes intéressent aussi bien les taillis simples que les taillis sous futaie. En ce qui concerne spécialement ces derniers, nous ajouterons :

$1^{\circ}$ que les longues révolutions seules, permettent de pratiquer les éclaircies et de réaliser toutes les mieux-values qui en sont la conséquence;

$2^{\circ}$ que les courtes révolutions multiplient dans un temps donné le passage des coupes, et, par suite, favorisent l'enlèvement des gros arbres: le propriétaire le mieux disposé saisira toujours quelque bon prélexte pour réaliser l'ancien auprès duquel il passe et dont la valeur le tente ;

$3^{\circ}$ que la hauteur de fût des réserves est subordonnée à celle du sous-étage, puisqu'elle est le résultat de l'élagage naturel; par suite, plus les révolutions seront longues, plus la production en bois d'œuvre sera considérable; à ce point de vue, il faut faire en sorte que le fût des réserves atteigne au moins 6 à 10 mètres de hauteur.

Il est bon, toutefois, d'appeler l'attention sur ce fait que l'on ne peut impunément allonger la révolution appliquée jusqu'alors à un taillis sous futaie sans prendre certaines précautions. En effet, quand le sous-étage est maintenu sur pied plus longtemps que par le passé, il continue à s'accroître en hauteur, et les cimes des réserves, englobées parmi ces perches qui les entourent, se dégradent. Aussi, dans la pratique, quand la réserve d'un pareil taillis est riche en arbres constitués, est-il prudent de répartir l'allongement cherché sur deux révolutions. Pour passer de vingt-cinq à trentecinq ans, par exemple, on se bornera, pendant la première révolution, à laisser pousser le taillis jusqu'à trente ans; à la révolution suivante, on fera le reste. Quand, au contraire, la grosse réserve est rare, ce sont les baliveaux ou modernes qui, pour le moment, sont l'élément principal : on peut alors, sans transition, prolonger la révolution du nombre d'années voulu, car les arbres jeunes peuvent encore allonger 
leur fût sans en souffrir, et les vieux arbres, qui seront prochainement exploités, n'auront pas le temps de se dégrader sérieusement.

Ajoutons que, si l'on prolonge une révolution, il est toujours prudent d'effectuer une éclaircie vers l'âge auquel l'exploitation se faisait antérieurement: on dégage alors la cime des réserves menacées d'enveloppement.

En résumé, nous recommandons, dans les taillis simples comme dans les taillis sous futaie, les révolutions de trente à quarante ans. Quand le sol sera frais, fertile, et les bois tendres abondants, on se rapprochera du chiffre de trente ans. Quand le sol sera peu profond, exposé au desséchement ou peu fertile, il faudra sans hésitation adopter une révolution de quarante ans.

Éducation d'arbres plus nombreux. - Sans en rechercher les causes, nous devons constater ici la pauvreté relative de notre littérature forestière à l'égard d'un mode de traitement aussi essentiellement Français que celui des taillis sous futaies. Quand les documents abondent pour les futaies, c'est à peine si nous possédons quelques données sur le rendement des 1200000 hectares de forêts domaniales et communales soumises à ce régime; mais il est surtout fâcheux que nous ne sachions rien de positif sur le traitement et sur la production des taillis sous fulaie appartenant aux propriétaires particuliers et dont la surface dépasse certainement $6000000 \mathrm{~d}$ 'hectares, sur les 9000000 qui composent la richesse forestière de la France.

Quoi qu'il en soit, l'expérience des faits nous permet de donner les indications suivantes sur le champ d'application du taillis sous futaie et des modalités qu'il comporte.

En principe, ce régime n'a sa raison d'être que dans les stations assez bien favorisées sous le rapport du sol et du climat, pour qu'on puisse y conduire, à l'état isolé et jusqu'à la dimension de gros arbres, les grandes espèces de lumière, telles que: frênes, ormes, érables et fruitiers et surtout les chênes.

Partout aù ces essences l'emportent en valeur sur les espèces d'ombre telles que le hêtre et le charme, on peut, sans sortir du régime, multiplier leur nombre jusqu'à couvrir le sol d'ar- 
bres isolés en croissance. Partout, au contraire, où les conditions inverses se présentent, partout notamment où le manque de profondeur du terrain ne permet pas l'éducation du chêne, il y a lieu de recourir à l'un des expédients suivants:

$1^{\circ}$ ou bien, renonçant à se payer de mots, abandonner le régime du taillis sous futaie, et se contenter du modeste taillis simple;

$2^{\circ}$ ou bien s'accommoder de la présence d'essences peu faites par leur nature pour entrer dans la réserve et modifier en conséquence l'application théorique du régime.

Examinons la première de ces deux hypothèses. Il est convenu que nous utiliserons le mieux possible le taillis simple en allongeant sa révolution; car, à défaut d'arbres, nous aurons ainsi du gros rondin de chauffage, c'est-à-dire du bois de luxe, le seul qui soit coté à un prix rémunérateur. Peutêtre y trouverons-nous même quelques autres marchandises: des perches pour étais de mines, du tremble pour la fabrication des pâtes à papier, etc.

$\mathrm{Si}$, pour une raison ou pour une autre, le propriétaire ne peut, ou ne veut, adopter cette mesure d'ensemble, - la seule logique à notre avis, - il pourra se contenter de n'en réaliser les heureux effets que sur un petit nombre d'individus, ou encore sur une essence seulement, en maintenant sur pied, pendant deux ou même trois de ces trop courtes révolutions, non pas des perches isolées, mais bien des cépées tout entières, choisies au nombre d'une cinquantaine par hectare. Celles-ci peuvent être éparses, et on les appelle des volières, - ou réunies en cordons de 2 à 3 mètres de largeur le long des sommières, des chemins, etc. MM. Broilliard, Mathey et beaucoup de sylviculteurs en recommandent l'usage. Il est à remarquer que le hêtre accepte cet état avec une complaisance très réelle, et c'est là un sérieux avantage de sa présence dans les taillis en sol superficiel.

Notre seconde hypothèse vise le taillis sous futaie et suppose l'admission du hêtre en quantité notable dans la réserve. Il s'agit, dès lors, d'une sorte de substitution d'essences, et quelques détails sont nécessaires à ce sujet.

Substitutions d'essences. - Sur les plateaux oolithiques 
de la Lorraine, de la Bourgogne, de la Franche-Comté, le foyard est souvent la seule essence qui fasse de beaux arbres. Ceux-ci fournissent déjà du bois de quartier recherché pour le chauffage; ils produiront du bois d'industrie en quantité notable le jour oú, en les maintenant à l'état plus serré, en augmentant les révolutions, on favorisera l'allongement des fûts. Pourquoi donc ne pas substituer peu à peu le hêtre au chêne? N'est-ce pas lui, d'ailleurs, qui a permis de remettre en état, sur les grés des Basses-Vosges, les taillis ruinés par des exploitations à courte révolution? le principe appliqué il y a quelque soixante ans par M. l'Inspecteur de Buffévent a été de marquer en réserve les hêtres bien venants et d'imposer le respect des semis de catte essence, de tout âge et de toute taille, que l'on rencontre noyés dans le taillis lors de l'exploitation de ce dernier; ses successeurs l'ont imité, et maintenant la forêt, en parfait état, s'achemine vers la futaie mélangée de chêne et hêtre.

Si nous accordons une importance toute spéciale à celle extension du hêtre, c'est qu'elle entraîne certaines modifications dans la conduite des balivages. Une réserve en chênes et essences disséminées suppose, en effet, des arbres isolés et maintenus sur pied le plus longlemps possible, afin d'en obtenir des produits de qualité supérieure. Il n'en est plus ainsi avec le hêtre, dont le prix au mètre cube n'augmente pas avec le diamètre à partir du moment où celui-ci est suffisant pour le débit en bois d'industrie: point n'est besoin, dès lors, de pousser les arbres au delà du diamètre de 50 centimètres qui les rend exploitables; plus tard, peut-être seraient-ils dépréciés par le défaut du " bois rouge ", ou d'autres tares plus graves encore. D'autre part, on sait que la valeur du foyard comme bois d'industrie, est liée à son état sain, à la rectitude et à la hauteur de son fût : la réunion des réserves de cette essence par pelits groupes ne pourra donc que contribuer à en améliorer la situation; de la sorte, sans prétendre à une production égale à celle des futaies régulières, du moins pourra-t-on s'en approcher; c'est done une disposition nouvelle à adopter dans la distrıbution des réserves.

Après celui du hètre, citons l'emploi des coniféres. 
En montagne, leur utilité saute aux yeux. Dans maintes circonstances, la substitution se fait d'elle-même, au profit du sapin; en général, le processus est le suivant : au milieu du taillis, on voit, épars, quelques sapins branchus, à fùt très court, arbres sans valeur marchande, mais tout à l'alentour leurs graines se répandent et les jeunes sapineaux percent les ronciers; la tache s'étend bien vite. On peut y aider par des plantations ou des répandages de graines sur les points où les semis naturels font défaut.

Dans la pratique des opérations, on évitera de dégrader les sapins d'avenir par les blessures du marteau : feuillus et résineux à abattre seront marqués en délivrance, et les sous bois seront recépés, avec réserve de tous les jeunes sapins et désignation, par griffage, d'un certain nombre de baliveaux feuillus.

Dans les régions de plaines ou de coteaux, les résineux peuvent encore fournir une aide des plus utiles. Quand des taillis sont clairiérés ou entrecoupés par des vides, quand ils manifestent des signes d'épuisement, l'introduction du pin noir si le sol est calcaire, du pin sylvestre sur le sable, est toute indiquée (1). Les aiguilles enrichissent le sol, reconstituent la couche d'humus détruite, et ces arbres, par leur croissance rapide ont vite compensé les frais de plantation. C'est ainsi que, dans les Basses-Vosges, quand le hêtre n'y suffit pas, dans les forêts d'Orléans, de Fontainebleau, de Montargis, etc., dans tous les terrains sablonneux en un mot, on sauve les forêts ruinées par le traitement en taillis $(2)$. On se demande quelquefois ce que deviendront, comment se régénéreront des taillis peuplés de résineux en plus ou moins grande abondance. Nous répondrons que tout d'abord l'introduction des résineux en pareille station est toujours suivie de la réapparition spontanée des feuillus, et notamment des chênes, dont les glands sont apportés par les oiseaux et les petits rongeurs, - et que, si l'avenir a de l'importance, le pre-

(1) Maire, loc. cit., Bulletin de la Socièté forestière de Franche-Comté et de Belfort.

(2) Le pin noir joue le même rôle dans les craies de la Champagne. 
l'entretien de la couverture morte et la fertilitć de la forêt. Les sujets les plus vigoureux, tout en continuant à appartenir au massif, se créent une certaine individualité. Par leur

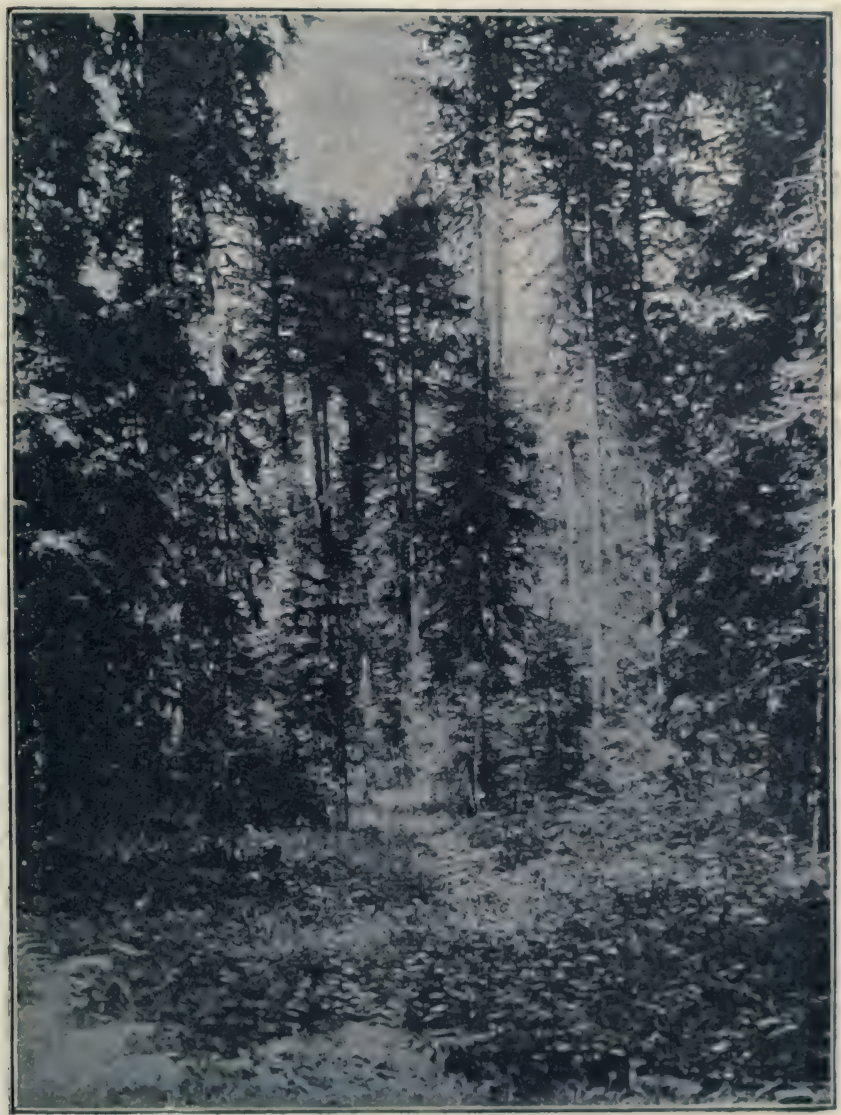

Fig. 54. - Une futaie jardinée dans le Jura.

(Photographie de M. A Fron.)

végétation meilleure, par leur enracinement plus profond, ils rendent la forêt plus solide et plus durable. Les massifs jardinés sont moins exposés que les peuplements réguliers aux dégals de la neige et du vent; ils ne présentent pas non 
plus un milieu aussi favorable au développement des organismes nuisibles.

I a forêt entière est sans cesse en voie de végétation, et ses peuplements, dont on ne saurait déterminer exactement l'âge moyen, se succèdent sans crise de transition, sans à coup, sans qu'on s'en doute, pour ainsi dire.

D'ailleurs, dans les massifs jardinés, l'intensité du couvert, variable dans le temps, diffère, par suite, à un moment donné, d'un point à un autre d'une même enceinte et favorise l'installation, ici d'une essence, là d'une autre. Les espèces forestières se succèdent donc et se mélangent avec une opportunité, un à-propos, auxquels ne peuvent prétendre les procédés factices du sylviculteur, dans le délai relativement court affecté à la régénération d'une parcelle de futaie régulière. Enfin le jardinage, dont l'organisme est simple, peut s'appliquer à toutes les forêts, aux plus petites comme aux plus grandes, et il est juste de constater qu'il a maintenu jusqu'à nos jours de nombreuses sapinières dans un état de prospérité remarquable.

Par contre, la qualité des produits laisse souvent à désirer. Les arbres ne fournissent pas toujours ces pièces élancées, de croissance régulièrement constante que produisent les peuplement suniformes.

Les exploitations portant sur de gros arbres englobés au milieu de tiges de tous âges occasionnent à ces dernières des dégâts importants. Leur dissémination sur de grandes étendues couvertes de bois en croissance rend la surveillance difficile et le transport des produits onéreux.

Les peuplements restent ce que la nature les a faits, leur composition, comme leur amélioration, échappent à l'action du forestier.

La possibilité, calculée sur des bases incertaines, ne permet pas de réaliser un rapport suffisamment soutenu, et les limites vagues dans lesquelles flotte le capital générateur rendent la confusion possible entre ce capital et le revenu; d'où des épargnes inutiles ou des abus de jouissance.

Cas oủ le jardinage doit-être maintenu. - Nous citerons en premier lieu, suivant les conseils de M. Broilliard (1), les

(1) Ch. Broilliard, Cours d'aménagement. Édition de 1878, p. 180. 
forêts de protection, c'est-à-dire celles où le maintien constant du massif est indispensable, soit à la sécurité publique pour prévenir les éboulements, les torrents, les avalanches, soit à la prospérité des foréts de rendement dont elles constituent la ceinture de défense; puis toutes les forôts où la régénération se fait avec lenteur. Tantôt c'est un escarpement, une pente très déclive, où la majorité des graines roulent entraînées par les eaux pluviales; tantôt c'est un éboulis rocheux, où les semis ne n'installent qu'à la longue dans les interstices des pierres, et à la seule condition que la mousse ne soit pas désséchée par un découvert irréfléchi; tantôt encore c'est le climat qui rend les arbres peu fertiles, le vent qui renverse les porte-graines isolés dans les coupes successives de régénération..... A quoi bon prendre la peine de fixer une révolution, d'établir des périodes, auxquelles correspondent sur le papier des affectations, si la nature bouleverse toutes les prévisions de l'aménagiste, si tel canton, qui doit se régénérer d'après le procès verbal en vingt-cinq ou en trente ans, ne se garnit qu'à la longue de semis se développant avec une lenteur désespérante? On se trouve conduit à prolonger la durée des périodes préalablement fixée, - ce qui est contraire au principe de la méthode, - et à conserver sur pied des sapins dépérissants, - ce qui, fait plus regrettable, entraîne des sacrifices de matériel importants.

A cette énumération, nous ajouterons les forêts de faible étendue, et toutes celles où l'on craint de ne pouvoir donner les soins culturaux, dégagements de semis et éclaircies, que comporte la futaie régulière.

D'ailleurs, il faut bien se dire que les inconvénients des futaies jardinées peuvent être atténués. L'irrégularité théorique de ces forêts est moindre dans la pratique qu'on ne pourrait le supposer. Quand un arbre est devenu dominant, il est rare qu'il perde la situation acquise, et, si le terme de l'exploitabilité est reculé, le peuplement prend cette forme d'apparence quasi régulière dont nous avons parlé plus haut: les produits qu'il fournit répondent très suffisamment aux exigences du commerce. 
La réalisation de la possibilité par volume, et surtout les comptages et les inventaires souvent répétés réduisent à peu de chose les dangers du système au point de vue économique; enfin, nous allons le voir, l'établissement de coupons remédie à la dissémination des produits, signalée comme préjudiciable au propriétaire de la forêt, aussi bien qu'à l'acquéreur des coupes.

Pratique $d u$ jardinage. - L'application du jardinage est d'une simplicité primitive, car la régénération est en grande parlie abandonnée à la nature. Néanmoins, si la régénération est incomplète ou se fait trop attendre sur un point, on lui vient en aide par la mise en état du sol, par des semis ou des plantations. De même, un martelage bien dirigé fait tomber de préférence les arbres âgés dont le couvert étouffe des placeaux de jeunes semis; ailleurs, sur les points où la lumière arrive au sol en quantité insuffisante, il rompt le massif pour faciliter l'ensemencement. Tout en laissant faire la nature, il n'est pas défendu de conduire intelligemment la cognée.

Le principal soin nécessaire est de bien choisir les arbres à exploiter. On prend de préférence, parmi les gros, ceux qui couvrent la jeunesse et qui sont les moins bien venants. On évite de faire de larges trouées, de dégarnir les lisières, d'isoler les arbres pauvres en branches et, par suite, en racines. On se garde bien d'enlever les perches dominées qui seront un jour ou l'autre des sujets de remp!acement. Quand le hêtre se trouve mélangé aux résineux, on coupe les gros hêtres, qui s'étalent, de préférence aux sapins et aux épicéas, à moins que les hêtres ne soient rares ou placés sur les bords du massif, auquel ils font alors comme un manteau protecteur. (1)

Les rotations. - Les séries jardinées seront de faible étendue, de façon à égaliser autant que possible les facteurs de la production. Cette précaution prise, dans le but de réglementer les exploitations, de faciliter le choix des arbres, et de garantir la bonne exécution des opérations, on a imaginé de restreindre encore la surface annuellement parcourue, en partageant la série en un certain nombre de divisions, dans chacune desquelles la coupe de l'année se trouve concentrée. Il s'établit ainsi une véritable rotation.

(1) Ch. Broilliard, Traitement des bois en France, p. 311. 
Le nombre des divisions ou coupons, qui fixe la périodicité du retour du jardinage sur le même point, règle en mème temps l'intensité de la coupe sur chaque unilé de surface. En effet, étant donnée une série de cent hectares, avec une possibilité d'un arbre par hectare et par an, si la surface est divisée en dix coupons, dans chacun de ceux-ci successivement on réalise cent arbres, en une seule fois. Si le nombre des coupons est réduit à cinq, la surface de chacun se trouve doublée, et, comme le nombre des arbres à prendre reste toujours de cent, lintensité de la coupe est réduite de moilié.

Ce procédé est donc un palliatif ingénieux, mais l'importance donnée aux exploitations constitue une dérogation au principe de la méthode. Si on augmente outre mesure le nombre des coupons, si l'on en fait vingt, par exemple, on découvre le sol vingt fois plus qu'on ne le doit théoriquement et l'on s'expose à perdre les avantages culturaux du jardinage.

Pour concilier ces derniers avec les avantages économiques du coupon, on est conduit, en tenant compte de la situation, du climat et des essences, à faire varier la durée des rotations entre cinq et quinze ans. En général, il faut revenir plus souvent chez les essences d'ombre que chez celles de lumière, plus fréquemment dans les sols fertiles que dans les sols pauvres, dans les climats doux que dans les climats rudes. Parfois même, dans les régions alpestres où la végétation est très lente, on se croit autorisé à dépasser le chiffre de quinze ans. Cela permet de réglementer le pâturage, de cantonner le bétail dans les coupons où les semis, âgés de huit à dix ans déjà, souffriront moins de sa présence.

Les rotations un peu longues créent, par suite des vides qui, relativement grands au début, vont sans cesse en se réduisant et permettent aux diverses essences de trouver l'une aprés l'autre les conditions propres à leur régénération; elles facilitent donc le mélange.

Les coupons sont, bien entendu, établis une fois pour toutes sur le terrain et délimités par des tranchées ou des chemins de vidange. En outre, quelle que soit la méthode adoptée pour le recrutement de la possibilité (volume ou 
pieds d'arbres), la division en tour d'exploitation doit être entièrement parcourue par la coupe; il est facile d'obtenir ce résultat si la taxe de chaque coupon a été individuellement calculée, comme si ce dernier constituait une série à part.

Soins culturaux. - En principe, la futaie jardinée ne comporte pas de soins culturaux. D'une part, en effet, la régénération est supposée obtenue par les seules forces nalurelles, ce qui supprime les dégagements de semis. D'autre part, l'impossibilité où l'on est de distinguer les individus appelés à jouer les rôles principaux dans le peuplement, de ceux qui seront éternellement réduits à l'état dominé, ne permet pas de faire des éclaircies, opérations destinées par définition à l'amélioration des sujets d'avenir.

Cependant, quand de jeunes sapineaux luttent péniblement pour se dégager d'un roncier ou d'un fourré de hêtres, quelques coups de serpe donnés à propos peuvent être fort utiles. De même, au passage de la hache, on coupera les perches mal conformées qui gênent un sujet voisin de belle venue. La différence entre ces soins culturaux et ceux qui sont en usage dans les futaies régulières, est que, dans celle-ci, ils constituent une ou plusieurs suites d'opérations syslématiques spéciales et indépendantes des coupes principales. Dans le jardinage, au contraire, tout se fait à la fois dans la même enceinte : en même temps que l'on réalise les "vieux ", on améliore la situation des " jeunes ". Le passage fréquent de cette coupe à tout faire assure un travail durable et suivi, pourvu que les exploitations soient strictement limitées aux nécessités culturales.

Application aux différentes essences. - Le jardinage étant surtout à sa place dans les régions montagneuses, ne trouvera son application que dans des forêts généralement peuplées de conifères.

D'autre part, le jardinage vrai, ne convient qu'aux essences d'ombre, et, en fait, c'est dans la sapinière qu'il a pris naissance, c'est à elle seule qu'il peut s'appliquer sans réserves; d'ailleurs, la présence du hêtre ne s'oppose en rien à l'application d'une méthode grâce à laquelle le mélange se maintient en d'excellentes proportions. 
Dans le Jura, le sapin file à travers les hêtres, et il est facile de le sauver. Dans les Vosges, il exige plus de précaution et de soins. Mais partout il se tire d'afraire dans la futaie jardinée, car, à l'état dominé comme à l'état dominant, il prime en hauteur les hêtres voisins, et prend ainsi la meilleure place (1).

Le propriétaire ne peut pas rêver mieux.

Quant au mélèze, bien que comportant peu l'élat jardiné, il se rencontre à de hautes altitudes, dans la zone alpine où la plus grande prudence s'impose, quel que soit le nom du traitement choisi. Or, le jardinage se prête à toutes les modalités, et permet au sylviculteur d'agir au mieux des intérêts de la forêt. On peut, en effet, parcourir les cantons en enlevant des arbres, toujours cà et là, mais, de préférence, en créant de petites trouées oú les semis naîtront par bouquets : le mélange des âges s'établira non plus par pieds isolés, mais par petits groupes plus ou moins homogènes. Les arbres qui constituent ces forêts ont d'ailleurs assez de résistance individuelle aux intempéries et aux coups de vent pour qu'il n'y ait pas trop de danger à entrouvrir les massifs. Sous ses réserves, on peut jardiner le mélèze dans les Alpes, comme le pin de montagne et même le pin sylvestre dans les Pyrénées et dans toute situation analogue (fig. 55). Il suffira, par exemple, d'exploiter de ci et de là quelques arbres sur un même point.

Pour l'épicéa, la question est plus discutable. En dehors de la zone alpine, où le jardinage peut lui être appliqué comme au pin et au mélèze, on sait: $1^{\circ}$ que son semis a besoin de lumière pour s'installer; - $2^{\circ}$ que plus tard, au contraire, si l'on v̀eut en obtenir du bois de bonne qualité, cette essence doit former des peuplements très pleins; or, ces considérations semblent incompatibles avec le jardinage.

Nous ne pensons pas cependant devoir en proscrire systématiquement l'application. Tout d'abord, il favorise d'une manière générale le mélange des essences et ne peut être, en particulier, nuisible à l'association sapin et épicéa. L̇n second lieu, dans les forêts d'épicéa pur à de grandes altitudes, les arbres, dont les cimes se rapprochent plus ou moins de la

(1) Ch. Broilliard, loc. cit., p. 310. 
forme columnaire, ne constituent, malgré leur grand nombre, que des massifs peu serrés; on pourra donc, sans trop se préoc-

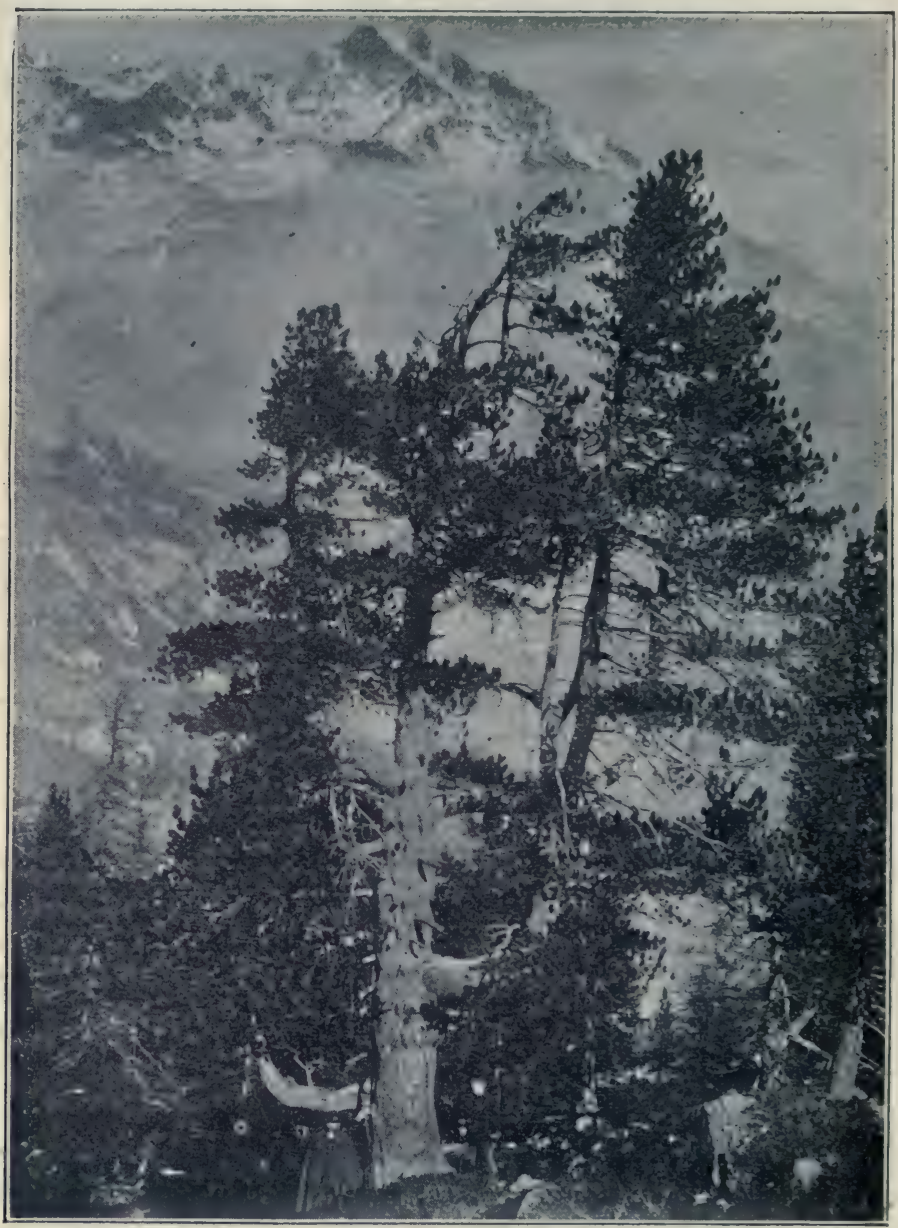

Fig. 55. - Mélèzes et pins cembros à 2000 mètres d'altitude, forêt de Villarodin Bourges, près de Modane. (Photographie de M. Thiollier.)

cuper d'une régénération qui, avec le temps, se produira toujours, tantôt sur les vieilles souches, tantôt dans les clairières, 
réaliser, en jardinant, les gros bois, de préférence les arbres sur le retour ou menacés par les bostriches. La production du sol atteignant à peine deux mètres cubes, par hectare et par an, il faut ménager un matériel dont la croissance est lente, mais la qualité exceptionnelle.

On peut, à la rigueur, ranger dans les forêts jardinées les peuplements de chêne-liège des Maures et de l'Esterel. Ces chênes forment des massifs très clairs, ou même sont à l'état d'arbres isolés au-dessus d'épais maquis de bruyères, de cistes et d'autres arbustes méridionaux; le plus souvent, ils poussent en mélange avec les pins maritimes. Le seul produit de valeur étant le liège, on exploitera - en jardinant - les arbres où la levée de l'écorce sera devenue, avec l'âge, peu rémunératrice. Pour propager l'essence précieuse, il est indispensable d'en dégager radicalement tous les semis et de découvrir, en temps opportun, les jeunes tiges par des extractions de pins en forme jardinatoire. L'incendie, qui détruit les résineux, n'atteint pas la vitalité des souches de chêne : celles-ci fournissent d'abondants rejets après le passage du feu ; .... mais ce n'est pas là un procédé de culture à recommander!

ARTICLE III

\section{LE TAILLIS SIMPLE RÉGULIER}

Principe de la méthode. - Avantages et inconvénients. - Régénération. - Soins culturaux. - Application dans les régions tempérées. - Application dans les régions méridionales. - Applications diverses.

Principe de la méthode. - Quand on coupe systématiquement à blanc étoc, et sans y faire aucune réserve, une surface continue, peuplée de bois feuillus susceptibles de rejeler de souches, les peuplements se constituent en taillis simple régulier. La régénération est la conséquence nécessaire de l'exploitation. Dès le printemps qui suit la coupe, le recrû, formé de rejets et de drageons, naît sur toute la surface à la même époque, et son ensemble représente le type le plus parfait de peuplement uniforme. 
La densité d'un taillis, eu égard au nombre des cépées, est fonction de la révolution. Comme chacune de celles-ci se développe avec les années, sa projection occupe d'autant plus d'espace qu'on la laisse davantage vieillir. Par conséquent, le nombre des centres de reproduction est d'autant plus faible, et par suite le fourré véritable, - abstraction faite des morts bois, - s'établit d'autant plus tard, que les révolutions sont plus longues.

Le mode de régénération par rejets entraîne une liaison intime entre le peuplement à venir, et celui qui va tomber sous la hache. En fait, il n'y a pas de nouvelles individualités créées, puisque les anciens sujets continuent leur existence sous la forme des rejets émergeant de leurs souches mutilées. Il y a rajeunissement plutôt que naissance d'une génération nouvelle; aussi, théoriquement, en dehors de la question d'âge, ne devrait-il y avoir aucune différence entre le peuplement ancien et le peuplement nouveau, oú les mêmes essences se retrouvent dans la même situation.

Les seules causes de perturbation dont il y ait lieu de tenir compte sont : la mort naturelle d'un certain nombre de souches, - l'évolution possible de drageons, - enfin l'apparition de quelques brins de franc pied, provenant de semences apportées par les vents ou par les animaux.

Avantages et inconvénients. - L'exploitation en taillis simple régulier est facile et commode. Elle fournit des produits constants. Le capital engagé reste toujours très faible et fonctionne à un taux de placement élevé.

Les peuplements de cette forme sont peu exposés aux dégâts des champignons et des insectes (1), non plus qu'aux bris de vent ou de neige; d'ailleurs, en cas d'accident, on ne perd que la récolte et les bois en croissance : l'ensouchement reste toujours intact.

Enfin, le taillis, dont les tiges flexibles résistent à l'arrachement, dont les racines nombreuses s'entrelacent dans la partie la plus superficielle du sol, est tout indiqué pour prévenir et corriger les ravinements.

Par contre, les taillis simples, du moins dans leur jeunesse,

(1) Un Coræebus est pourtant nuisible dans les forêts de chêne vert. 
sont très exposés à l'atteinle des gelées. La première année surtout, les rejets peuvent être arrêtés dans leur lignification par les gelées d'automne el disparaître victimes de l'hiver, qui les trouve mal " aoutés ». C'est, alors, une année de végétation à peu près perdue, et si l'accident se répète plusieurs années de suite, les souches meurent en grand nombre. Les gelées printanières, quand elles sévissent fréquemment dans un canton, entravent aussi la croissance des taillis, qui prennent un aspect chétif et rabougri caractéristique; on dirait d'un abroutissement par le bétail. Le régime du taillis exige donc un climat doux, une grande somme de chaleur et un temps de végétation suffisamment long: ce qui rend son aire d'application beaucoup plus restreinte que celle de la futaie.

En outre, le traitement en taillis, qui dénude complètement les surfaces à des intervalles rapprochés et ne produit que des bois jeunes, fatigue le sol, auquel il n'apporte que des restitutions insuffisantes. D'ailleurs, les effets de l'épuisement sont d'autant plus rapides que le terrain est d'une nature plus sèche et la révolution plus courte.

L'influence des taillis sur les phénomènes météoriques est aussi bien moindre que celle des futaies. Enfin les taillis ne fournissent que du bois de chauffage, du charbon, des écorces, ou du menu bois d'industrie; encore la quantité de ces derniers est-elle subordonnée à la longueur des révolutions.

Régénération. - Toute la méthode de régénération des taillis simples réguliers repose sur le fait même de l'exploitation. Les points essentiels à considérer, pour assurer la production permanente des rejets, sont relatifs à l'âge de l'exploitation des peuplements, à la saison la plus favorable pour faire la coupe et au mode d'abatage des produits, toutes questions étudiées au chapitre $\mathrm{I}^{\text {er }}$.

Soins culturaux. - Les soins culturaux se bornent, en général, à quelques plantations sobrement faites, pour rétablir l'état de massif continu en bonnes essences, sur les points clairiérés par la mort accidentelle d'une ou de plusieurs souches.

Ces plants doivent être dégagés périodiquement en la forme prescrite, afin qu'ils ne soient pas étouffés par la poussée des 
essences inférieures. Le plus souvent, les plantations seront rendues inutiles, sil'on prend soin de sauver les semis naturels qui se rencontrent accidentellement disséminés, et qui proviennent de semences fournies par le taillis lui-même (1), ou par des arbres constitués, fructifiant dans les environs.

Dans les taillis simples réguliers, systématiquement exploités à des révolutions courtes, souvent inférieures à vingt-cinq ans, tous les sujets ont même avenir et sont appelés à une même fin prochaine. Les cépées existent aux distances que comporte la révolution; elles s'étalent sans se gêner l'une l'autre. Dès lors, si la lutte s'engage, ce n'est pas de cépée à cépée, mais de rejet à rejet dans une même cépée. Cette lutte est d'assez courte durée pour qu'il ne soit pas nécessaire d'intervenir; car, partout où l'on n'a aucun intérêt à créer des individualités, l'éclaircie ne se justifie pas.

Il n'en est plus de même si les révolutions atteignent ou dépassent 30 ans. Alors une éclaircie s'impose, dans le même esprit que celle des peuplements de futaie. Dans chaque cépée, traitée comme une unité à part, on enlèvera, au profit des plus belles tiges, les perches surabondantes ou tarées de l'étage dominant, en respectant scrupuleusement tous les brins dominés, rabougris ou traînants, qui composent le sous étage et lui donnent sa densité. Une telle opération est aussi délicate à diriger qu'à exécuter; car il n'est pas loujours facile au bûcheron de lancer sa hache au milieu d'une cépée pour y abattre, sans froisser les autres, le ou les seuls brins à faire tomber.

Exceptionnellement, en sol fertile, dans un mélange de bois tendres et de bois durs, quand les premiers sont exploitables avant les seconds, on peut les réaliser en temps opportun, avant la coupe principale. Mais alors ces éclaircies, qui sont de véritables exploitations anticipées, perdent en partic leur caractère cultural...

Application dans les régions tempérées. - En France, on trouve les taillis simples dans les régions méridionales

(1) Les cépées de chêne rouvre et surtout de chêne pédonculé donnent des quantités assez considérables de glands. Mais beaucoup de ceux-ci, quoique de belle apparence, demeurent stériles. 
comme dans les régions septentrionales. Malgré leur importance - ils couvrent plusieurs millions d'hectares - et leur variété, nous ne ferons que passer rapidement en revue les principaux types.

Dans les climats tempérés, ce mode de traitement, très justifié alors que diverses industries (salines, verreries, forges, etc...) consommaient des quantités considerables de bois de petits calibre, répond de moins en moins à la situation économique de notre pays, à une époque oủ le charbon et les bois à brûler perdent de jour en jour de leur valeur. Les écorces à tan, d'un produit autrefois très rémunérateur, souffrent aussi d'une baisse de prix considérable. Il est donc urgent de songer à transformer les taillis simples en vue de la production de marchandịses d'un plus fort diamètre.

Le rôle et la composition de, ces taillis différent sensiblement d'une région à l'autre. Dans l'ouest, ce sont des taillis de chêne tauzin; en Sologne, on trouve le chêne rouvre el le chêne pédonculé; dans les Ardennes, dans les Vosges méridionales, le chêne rouvre domine. Un peu partout, aux chênes se mélangent : le bouleau, sur les sables pauvres, - les bois blancs dans les stations fraîches, - le charme, l'érable cham. pêtre, le coudrier dans des sols secs et moins profonds. Partout, des améliorations importantes pourraient être apportées à ce genre d'exploitation et en atténuer les mauvais effets; la principale serait l'allongement des révolutions, précédant la conversion en taillis sous futaie.

En ce qui concerne les taillis de chêne écorcés (1), nous avons vu au Chapitre $1^{\text {er }}$ que l'exploitation pouvait, sans inconvénients sérieux pour l'évolution des rejets, être reculée jusqu'en mai, époque de la levée des écorces. D'autre part nous pensons que l'écorçage sur pied est peu préjudiciable, à condition que l'exploitation des perches soit précédée d'une incision annulaire ouverte au dessus du niveau du sol, et que la coupe ait lieu ensuite rez-terre; et cela malgré l'opinion des sylviculteurs autorisés qui considèrent cette incision comme inutile et même nuisible (2).

(1) Bouvart. De l'écorģage du chène. (Revue des Eanx et Foréts, 1866. )

(2) Société centrale forestière de Belgique, avril 1899. 
Nous restons convaincus que le plus grand inconvénient de l'écorçage sur pied consiste dans le va-et-vient de nombreux ouvriers dans les coupes oú commencent à évoluer les rejets; et, pour ce motif, nous croyons bon de tenir la main, - afin de délivrer le plus vite possible la forêt de toute cette population qui la fatigue, - à l'abatage des perches écorcées dans le plus court délai possible, alors même qu'il serait démontré que le maintien sur pied de ces perches, pendant une quinzaine de jours, n'est pas nuisible à la vitalité de leurs propres souches.

Il va sans dire que les propriétaires de forêts ne peuvent que souhaiter l'extension des procédés d'écorçage à la vapeur. - après exploitation, - inventés par MM. Maitre et de Nomaison (1).

Disons enfin un mot de la pratique du sartage, ne fût-ce que pour en signaler le danger.

Dans certaines régions, notamment dans les Ardennes et les Cévennes, les taillis de chêne sont encore soumis à cette pratique. Le sartage consiste, après l'exploitation d'un taillis simple, à brûler les rémanants répandus sur le sol, de façon à le faire profiter, sans frais, des matières fertilisantes immédiatement assimilables et à permettre, grâce à cet engrais, d'intercaler une culture agricole, du seigle généralement, entre deux exploitations forestières (2).

Mais le mince bénéfice qu'on en tire s'obtient au détriment de la forêt : $1^{\circ}$ la mise à feu se faisant vers le mois d'août, toute la pousse de l'année est perdue, et un certain nombre de souches, les unes fatiguées par cette pratique qui dérange le mouvement de la sève, les autres directement atteintes par le feu, cessent de repousser et meurent; le sarteur, qui ne cherche que le chêne, aide à la destruction de toutes les autres essences : charmes, bouleaux, fruitiers, qui disparaissent et sont remplacés par des morts bois et surtout par le genêt, dont la multiplication est favorisée par la grande quantité de potasse contenue dans les cendres; $-2^{\circ}$ sur les versants à

(1) Bull. Société des Agriculteurs de France, mai 1873.

(2) Cornebois. Notice sur le sartage dans l'arrondissement de Rocroy, Paris, J. Tremblay, 1882. 
pente rapide, la terre végétale est brûlée et perd, pendant quatre ou cinq ans au moins, la cohésion nécessaire pour résister à l'action des pluies; le retour de chaque exploitation en enlève une bonne part, et les flancs des montagnes montrent de plus en plus les rochers stériles qui en forment les

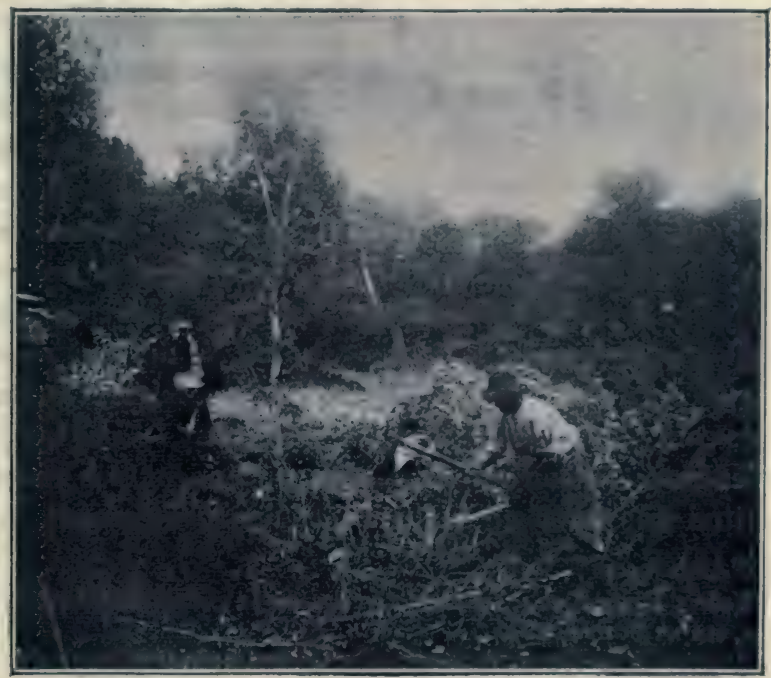

Fig. 56. - Un taillis de chêne vert; exploitation par łe saut du piquet. Forêt de Mérindol (Vaucluse). (Photographie de M. J. George.)

assises; $-3^{\circ}$ enfin le sartage met obstacle à l'allongement des révolutions et à la conversion en taillis sous futaie.

Du reste, tous les prétendus avantages attribués au sartage au point de vue forestier, ne supportent pas la discussion. On entend dire, en effet, que les taillis se sontmaintenus grâce à lui; or, il est facile de se rendre compte de ce fait que, pour sarter, il faut couper soigneusement les taillis rez-terre; c'est donc grâce à leur bonne exploitation que ces taillis se sont perpétués quoique, - et non parce que, - sartés. Le sartage perd d'ailleurs du terrain dès que l'aisance pénètre dans les pauvres contrées qui en vivaient.

Applications dans les régions méridionales. - Les taillis 
de chêne yeuse, pur ou mélangé de chêne blanc (fig. $5 \ell$ ), donnent la seule forêt qu'on puisse demander aux terrains calcaires brûlés par le soleil de la Provence, sur lesquels ils sont localisés $(\mathbf{1})$.

Pour ceux-ci, comme pour tous les précédents, l'allongement des révolutions s'impose. Leur durée actuelle, qui varie de huit à quinze ans, peut, sans inconvénients, être portée à vingt ou vingt-cinq ans. Sans rien perdre au point de vue de la production des écorces, on auraitl'avantage d'augmenter la valeur des produits ligneux et de faciliter l'exercice du pâturage (2).

Heureux le propriétaire de pareils taillis qui, à défaut de bois, peut y récolter des truffes (3). Peut-être, comme le conseille M. Broilliard, des éclaircies bien dirigées augmenteraient-elles la production de cet intéressant cryptogame. Il semble nécessaire que le taillis ait huit à dix ans pour que les truffes y soient abondantes; elles disparaissent quand les cépées sont plus âgées, et, malheureusement, — sur les pentes du Ventoux tout au moins, - ne reviennent pas quand on substitue une forêt nouvelle à l'ancien peuplement devenu stérile (4).

(1) Voir : Regimbeau, Le chêne yeuse ou le chêne vert dans le Gard, Nimes, impr. Jouve, 1879.

A. Rousset, Recherches expérimentales sur les écorces du chêne yeuse, Paris, imprim. Nationale, 1887.

E. Rouis, Note sur le développement et la gestion des forêts communales dans le déparlement du Gard, Avignon, Séguin, 1896.

V. de Larminat, Les forêts de chêne vert, Troyes, L. Lacroix, 1893.

F. Tessier, Le versant méridional du Ventoux. (Revue des Eaux el Forêts, janvier, février, mars 1900.)

(2) Voir chapitre VII.

(3) En 1892, la commune de Bédouin (Vaucluse) a tiré un revenu de plus de 55,000 francs d'une forêt truffière, créée par M. l'Inspecteur général Bédel, alors qu'il était chef de service à Avignon en 1865.

(4) Nous n'avons pas besoin d'ajouter que le chêne "truffier " est une pure chimère en tant que race ou variété. Des glands nés de chênes verts ou de chênes blancs quelconques, peuvent donner des cépées truffières, si, d'ailleurs, les conditions nécessaires à la vie du champignon sont remplies. Peut-être, cependant, de la terre ou des feuilles, prises dans une forêt où vivent des truffes, en apporteraient-elles des spores.

Pour créer une truffière artificielle, on défriche le terrain, on cultive des bandes de quatre à six mètres de largeur et on y sème des glands de chêne yeuse autant que possible. Dans l'intervalle, et en attendant que les truffes apparaissent, ou peut pratiquer une culture agricole 
sent en a tout autant, sinon plus. D'ailleurs, une forêt riche en matériel, avec un sol en bon état, est toujours une forêt d'avenir. A chaque âge suffit sa peine.

Nous irons plus loin : dans les mauvais taillis, même si la forêt ne renferme pas de vides, nous serions tentés de recourir aux résineux pour faire une forêt plus riche, d'un plus grand rapport.

Dans ce but, on peut procéder radicalement: faire une coupe rase du taillis et planter des pins noirs sur les calcaires, des pins sylvestres sur les sables. L'opération est, en général, fructueuse, mais c'est un véritable reboisement, dont les frais sont majorés du coût des dégagements rendus nécessaires par l'évolution des rejets. Aussi, est-il indiqué de chercher à réduire la dépense en mettant à profit tout ce qui est utilisable dans l'ancien peuplement, et en n'introduisant les résineux que çà et là, sur des points choisis à cet effet. Plus tard, on augmentera, si on le juge à propos, la surface qui leur est attribuée.

Sans doule, ici encore, il faut lutter contre la poussée des essences feuillues qui ne se laissent pas exproprier sans résistance; mais les sujets à défendre sont peu nombreux, et l'utilisation du sapin et de l'épicéa, qui souffrent moins que les pins, du voisinage des buissons, diminue le travail (1). Entre ces deux espèces laquelle choisir? Cela dépend des cas et des convenances; sous la réserve expresse que le sapin, tout en acceptant de vivre assez loin des montagnes où il est spontané, exige, néanmoins, des stations suffisamment fraîches, accidentées ou maritimes, rappelons que l'épicéa se recommande par la facilité de sa reprise, le sapin par son aptitude à se réensemencer naturellement au milieu des taillis: le plus sage sera, le plus souvent, de planter des épicéas en majorité, avec une faible proportion de sapins destinés à servir plus tard de porte-graines. M. l'Inspecteur des forêts Runacher (2), paraît

(1) Ces dégagements de semis sont facilités par le feuillage vert des résineux, qui tranche, en hiver, sur la coloration uniforme des taillis.

(2) Runacher, Utilité de l'introduction du sapin et de l'épicéa dans les taillis médiocres de la région jurassique. (Bull. de la Sociélé forestière de Franche-Comté et de Belfort, octubre 1899.)

Boppe et Jolyet. 
vouloir généraliser l'emploi du sapin et de l'épicéa dans cette œuvre de restauration, et certes, sur bien des points, on ne pourrait mieux faire que de suivre ses conseils. Cependant, pin Weymouth et mélèze méritent aussi qu'on leur réserve quelques belles places.

En somme, il est certain que les résineux sont appelés à jouer dans les forêts de la plaine un rôle plus important que celui qui leur avait été réservé jusqu'alors. Et, pendant qu'on en sera à restaurer les peuplements dégradés à coup de bêche et à coup de pioche, il ne paraîtra pas imprudent de risquer la plantation de quelques pieds isolés d'essences exotiques en voie d'introduction, le chêne rouge d'Amérique, par exemple, qui se multiplie dans certains taillis sous futaie des Vosges et plusieurs espéces dont nous parlerons au chapitre VIII. Nous croyons avoir suffisamment recommandé le principe de chaque essence dans sa station, pour pouvoir nous permettre ces exceptions; mais, une fois de plus, nous signalons les dangers des cultures d'espèces étrangères plus exposées que les indigènes à toutes les maladies cryptogamiques ou autres. Aussi, avant d'avoir recours aux formes exotiques, fera-t-on bien de consulter la flore forestière locale; souvent, avec ses seules ressources, on pourra composer des mélanges de feuillus et de résineux d'une résistance assurée; témoin les excellents résultats que donne, au point de vue économique, la multiplication du pin d'Alep dans les taillis méridionaux, tout comme celle du sapin dans les taillis du Bas-Jura. 


\section{EXPLOITATION ET PROTECTION DES FORÊTS}

\section{ARTICLE PREMIER}

\section{GÉNÉRALITÉS}

Tandis que la nature arme suffisamment la forêt sauvage pour sa défense contre les ennemis qu'elle lui suscite, la forêt domestiquée, celle dont nous venons d'étudier les conditions d'existence, modifiées de mille façons en vue de satisfaire à nos besoins les plus divers, exige, en échange, de notre part, modération et protection. Ses forces amoindries par la culture sont, en effet, devenues impuissantes à conjurer les périls dont celle-ci est la cause ou l'occasion.

Exploitation et production se rattachent ainsi directement à la sylviculture.

Les détails que comporte la pratique de la coupe et de l'enlèvement des produits appartiennent à un autre sujet (1): nous nous bornons donc, ici, à prémunir la gestion contre les dommages éventuels qui sont une conséquence inévitable de l'introduction en forêt de la hache du propriétaire, et, comme s'il s'agissait de tout autre dégât imputable à l'homme, d'indiquer les moyens d'en atténuer les effets.

Sous celte réserve, toutes les misères qui sont de nature à entraver les énergies productrices de la forêt et que nous avons intérêt à écarter de son chemin, lui peuvent venir de l'une des causes suivantes :

$1^{\circ}$ Dommages causés par l'homme;

$2^{\circ}$ Dégâts des animaux;

$3^{\circ}$ Dégâts des végétaux;

$4^{\circ}$ Dégâts des météores.

(1) L. Boppe, Cours de lechnologie forestière, Nancy, BergerLevrault, 1878. 
ARTICLE II

\section{DOMMAGES GAUSÉS PAR L'HOMME}

Fait du propriétaire : à l'occasion des exploitations ; - des élagages ; des émondages; - des assainissements. - Fait des délinquants: les causes; - la répression. - Les concessions de menus produits : tolérances nécessaires; - menus produits végétaux; - menus produits minéraux. - Le páturage : la situation actuelle; - nocuité du pâturage suivant les régions; - influence de la nature du bétail introduit; - de l'état des peuplements; - conclusions. - Les incendies : leurs causes et leurs conséquences; - régions montagneuses; - zone parisienne; - régions méridionales.

\section{Fait du propriétaire.}

Les exploitations. - Celles-ci comprennent l'abatage des bois, le façonnage et le transport des produits.

Pour l'abatage, la saison d'hiver est la plus avantageuse dans les coupes principales des forêts feuillues. Toutefois, ces travaux doivent être suspendus par les froids rigoureux; car, le bois a perdu alors toute élasticité, il brise les outils et fatigue les bûcherons; les arbres gelés risquent aussi beaucoup plus de se rompre dans leur chule. Le moment le plus favorable est la fin de l'hiver et le commencement du printemps avant l'ouverture des bourgeons.

Les essences résineuses doivent, de préférence, être exploitées en temps de sève, leur bois est alors plus léger el conserve un plus bel aspect lors du débit; il est aussi plus facile de les écorcer pour éviter les piqûres des insectes. Dans le cours de cette saison, il suffit de suspendre les exploitations pendant les deux mois qui suivent la montée de la sève, époque où les jeunes pousses sont extrêmement fragiles et pendant laquelle les tiges elles-mêmes guérissent beaucoup plus difficilement leurs blessures (1).

Quand les arbres feuillus ou résineux sont exploités par

(1) On remarque néanmoins, que souvent, les bois résineux exploités en été, surtout quand ils sont flottés, sont tachés par le mycélıum de divers champignons dont ils ont pris le germe en forêt. Le redoutable Merulius lacrymans, qui détruit le solivage et les lambris de toute une maison, peut y être importé de la sorte. 
extraclion de souches suivant la forme dile à culée noire, leur chute est facilitée et rendue moins dommageable par l'emploi du brocque en usage dans certaines forêts de l'Ouest et du

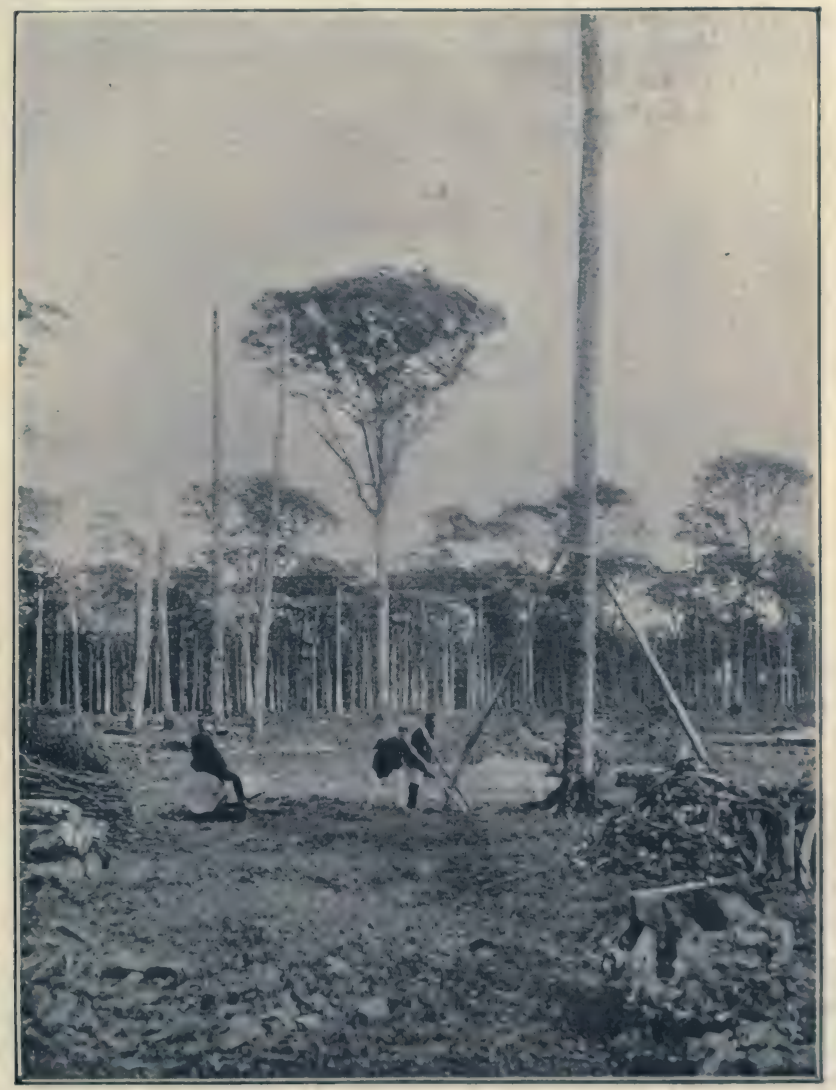

Fig. 60. - Abatage à culće noire, à l'aide de brocque's, forêt de Senonches (Eure-et-Loir). (Photographie de M. d'Alverny.)

Centre, notamment, dans celle de Senonches (fig. 60).

L'ébranchage ou bollage des arbres à abattre sera prescrit partout où il sera jugré nécessaire dans l'intérêt des régénérations acquises ou des arbres réservés. Bien qu'il soit plus commode et moins dangereux pour le bûcheron de 
couper les branches de haut en has, l'opération est meilleure lorsqu'on la pratique de bas en haut, parce que, dans le premier cas, les branches coupées, en tombant sur les branches inférieures encore attachées à l'arbre, les brisent, les arrachent et occasionnent des déchirures qui déprécient la tige.

Dans les belles futaies de l'Ouest, pour éviter les dégradations et les brisures qui pourraient atteindre les longues

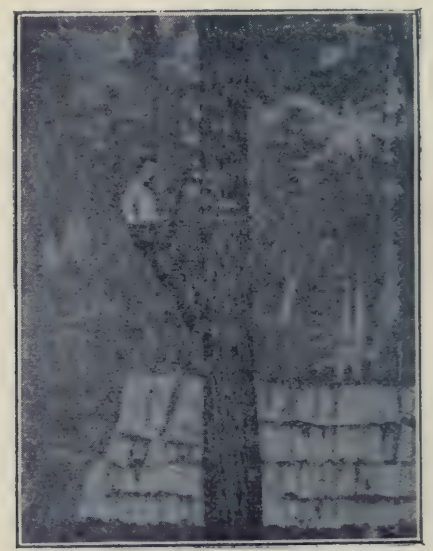

Fig. 61. - Bûcheron appuyć sur ses crampons, soutenu par une ceinture de corde, et tronçonnant un chêne. (D'après une photographie de M. Takasima, Élève Japonais à l'École forestière.)

pièces dans leur chule et en déprécier le débit comme bois de fente et de sciage, on a pris l'habitude de les tronçonner sur pied en billes de 4 à 6 mètres de longueur (fig. 61 et 62).

Les coupes d'éclaircies peuvent être exploitées indifféremment en toute saison, et même, si l'on a intérêt à éviter la production des rejets, il vaudra mieux faire l'abalage en été. Dans les bois feuillus, pourtant, on préfère la saison d'hiver, parce qu'alors les bûcherons travaillent à meilleur marché, et aussi parce que les menus bois coupés avec leurs feuilles se façonnent moins bien et moins proprement que ceux qui en sont dépourvus; c'est ce qui fait que les fagots feuillés 
passent, avec raison, pour avoir été coupés en délit, et les honnêtes gens ne les achètent pas.

Aussitôt après leur chute, les arbres sont découpés suivant le genre du débit qu'ils comportent. Les divers produits ainsi obtenus sont ensuite rassemblés et disposés de façon à encombrer le moins possible le parterre des coupes, en attendant qu'on vienne les enlever.

Dans l'enceinte des ventes, le débit des bois ne doit pas

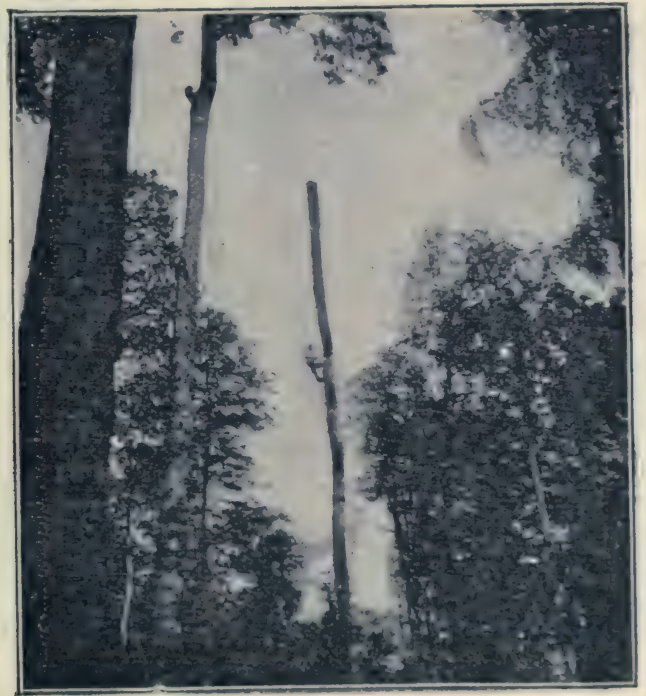

Fig. 62. - Tronçonnement d'un arbre sur pied, forêt de Bellême (Orne). (Photographie de M. J. George.)

être poussé au delà de la forme d'un premier façonnage brut, indispensable pour qu'on puisse en faire l'enlèvement et pour en réduire les poids par la dessication. Il ne faut jamais y tolérer l'installation de chantiers destinés à transformer la matière première en produits fabriqués tels que : sabots, merrains, échalas, sciage, etc... Cette coutume ramène chaque année, en forêt, toute une population ouvrière qui s'installe pendant plusieurs mois sur les points qui demanderaient à être le mieux garantis; non seulement les places d'atelier sont tassées par la fréquentation des ouvriers et de 
leur famille, mais le piétinement exerce partout son influence fâcheuse et les régénérations les mieux, assurées ne lui survivent pas. C'est à de pareils abus qu'on doit, dans une certaine mesure, attribuer la destruction de belles futaies de chêne, où de maigres régénérations artificielles en pin sylvestre succèdent aux peuplements les plus riches.

Quoi qu'il en coûte, il faut faire cesser un tel état de choses. Rien n'est plus simple que de déterminer, dans

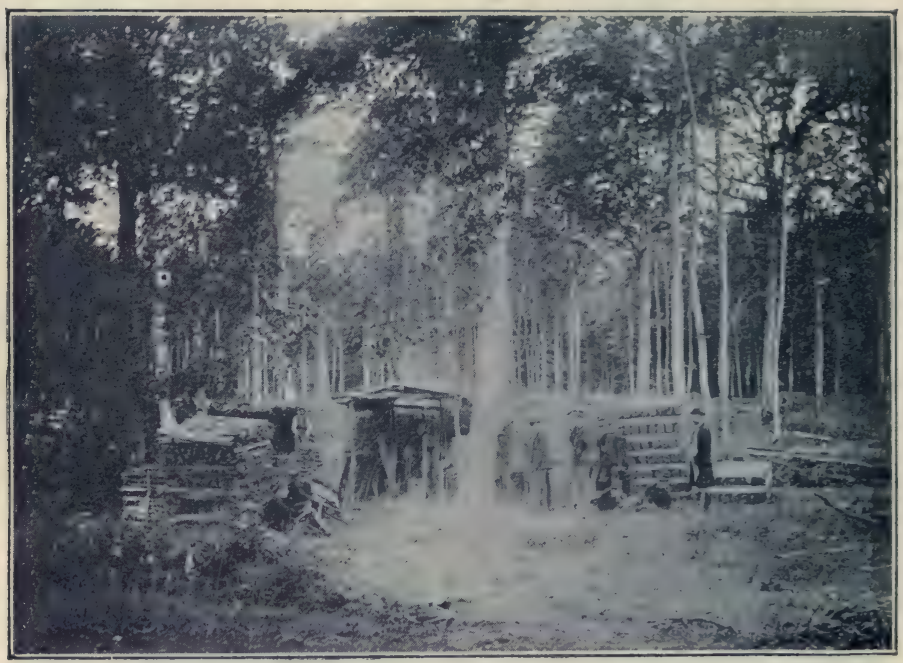

Fig. 63. - Atelier de fente dans la forêt de Senonches.

(Photographie de M. Juvanon du Vachat.)

chaque série, un certain nombre de places bien choisies qui seront consacrées, d'une manière permanente, à l'installation des chantiers de débit. Le propriétaire de la forêt pourrait ainsi faire la dépense de baraquements loués aux adjudicataires; cette première mise de fonds serait bientôt couverte au grand profit de tout le monde. L'emploi, pour le débardage, de petits chemins de fer ou porteurs Decauville, faciliterait considérablement les améliorations à introduire dans des habitudes funestes, enracinées depuis des siècles.

Le transport des produits hors des coupes se fait à dos 
d'homme ou à la brouette, pour les bois de chauffage et les pièces de faibles dimensions; au moyen de chariots, d'avanttrains ou de traîneaux pour les billes trop grosses pour être maniées à bras d'homme. Tous ces procédés peuvent être mis avantageusement en pratique suivant les cas, et les inconvénients qu'ils présentent sont proportionnés à l'intelligence, au bon vouloir et à l'adresse des charretiers; on doit aussi

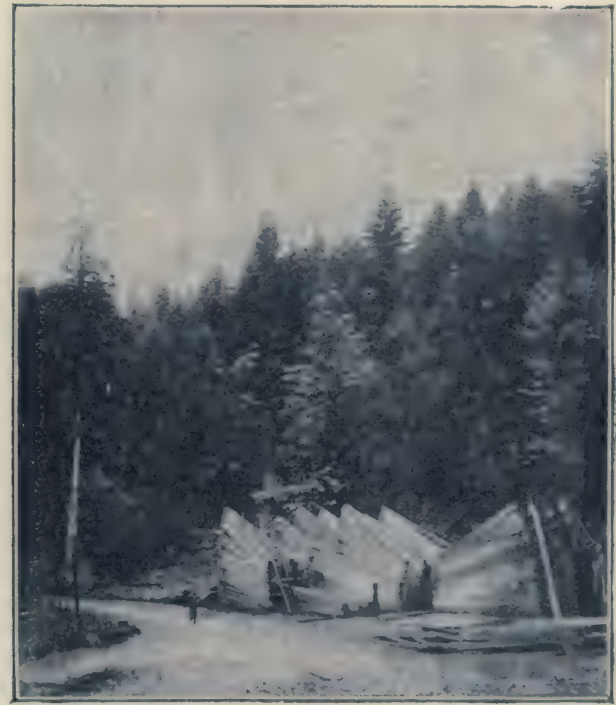

Fig. 6i. - Eimpilage de planches de sapin, scierie du Grand-Roué (Vosges). (Photographie de M. J. George.)

tenir compte des dommages causés au jeune recrû, qui augmentent avec la longueur sous laquelle les pièces de service sont maintenues.

Le traînage ou glissage direct des tronces sur le sol, qui déplace la couverture et commence les ravinements, doit être proscrit, autant que possible, des coupes principales. Nous en dirons autant du lançage, fût-il amélioré par les couloirs creusés en cuvetle en vue de diriger les billes dans leur chute. C'est seulement dans la grande montagne que ces procédés primitifs seront tolérés et cela ne sera jamais 
sans dommages; car on sait combien de billes bondissent hors dulançoir, et, dans leur chute désordonnée, mutilent ou brisent tout ce qu'elles rencontrent (fig. 68, b). On devra toujours leur préférer le transport par traîneaux sur les chemins de bois dits chemins de schlitte (fig. 65), ou, mieux encore, les câbles aériens (1). La dépense sera grandement récupérée

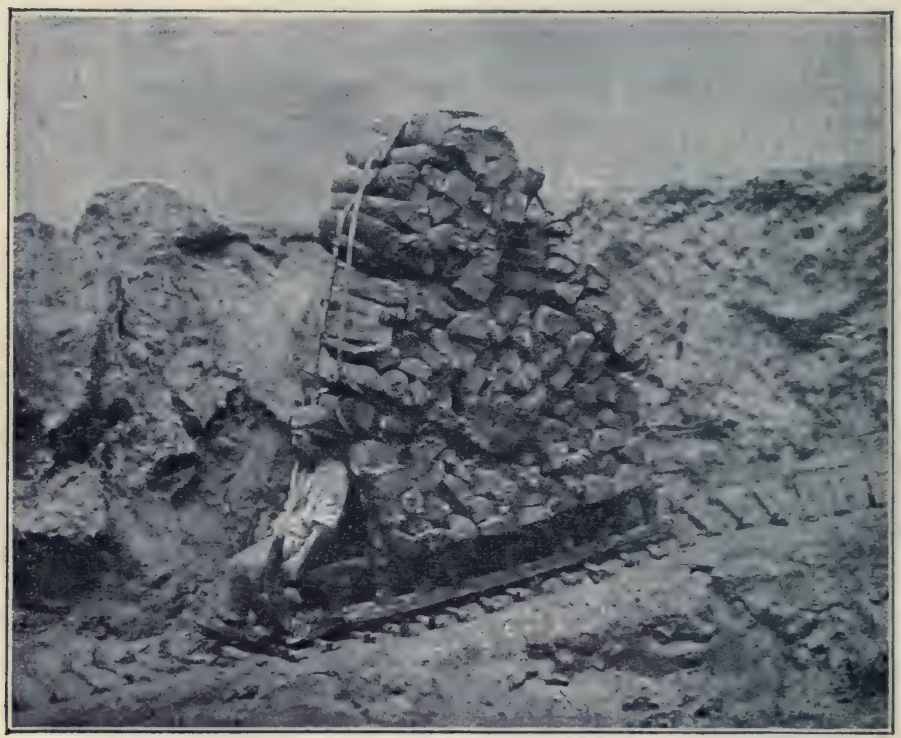

Fig. 65. - Le schlittage dans les Vosges.

par la plus-value des marchandises et la meilleure tenue des peuplements.

Dans les futaies feuillues, le passage des voitures ou des avant-trains ne fait pas de dommages aussi considérables qu'on pourrait le supposer : les brins de semis se courbent sous les pieds des chevaux ou sous les roues des voitures et se relèvent bientôt sans paraître trop souffrir des lésions qui leur sont faites. Il suffit d'éviter de faire passer plusieurs voi-

(1) E. Thiéry. Étude sur les petits chemins de fer forestiers. Nancy. Berger-Levrault. 1893. - E. Thiéry et Demonet. Les transports par câbles aériens, Nancy, Nicole, 1896. 
tures de suite dans le même sillon, ce qui crée un vérilable chemin battu dans lequel tout est écrasé. D'ailleurs, si cela est nécessaire, on peut avoir recours au recepage. Quand des soins convenables ont été pris, deux ou trois ans après une coupe, le fourré sera complètement rétabli sans conserver de traces bien sensibles de la dernière exploitation. Dans les forêts résineuses, plus de précautions sont nécessaires, car les jeunes plants écrasés se relèvent difficilement et

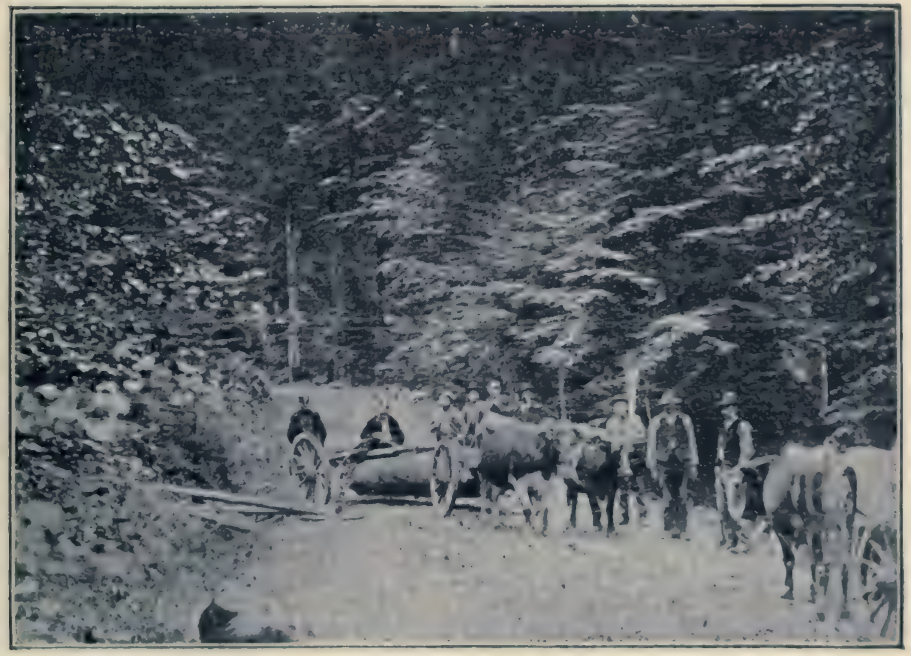

Fig. 66. - Enlèvement diune tronce de sapin, vallée de Ravines (Vosges.) (Photographie de M. Fron).

n'ont pas, comme les feuillus, la faculté d'émettre des rejets.

D'une manière générale, on peut dire que le dommage causé au peuplement par le transport des arbres à travers bois est proportionnel à la longueur des billes plutôt quà leur poids. C'est ainsi que, dans les régions où l'on fabrique de la planche marchande, les plus grands sapins ou épicéas sont débités sur place en tronces de 4 mètres, dont le transport se fait facilement et d'une façon inoffensive (fig. 66). Tandis qu'il faut avoir vu, dans le Jura, les douze à quinze paires de 
bœufs attelées à une de ces énormes poutres dont la longueur dépasse 30 mètres et destinées aux chantiers de constructions navales de la Méditerrannée, pour comprendre les ravages que de tels convois font subir à la régénération; sur une largeur de 4 à 5 mètres, le long de leur parcours, toute la jeunesse est écrasée. De plus, les ingénieurs forestiers doivent calculer les courbes de leurs routes avec un rayon suffisant pour que les prolonges puissent parcourir les tournants sans venir butter contre les talus en déblai.

Quelles que soient les essences, il est d'un intérêt majeur d'enlever les produits aussi rapidement que possible. Moins longtemps le parterre des coupes sera fréquenté, mieux s'en trouveront les peuplements; car, pour réparer les fatigues de toute sorte qui sont la conséquence nécessaire d'une exploitation, la forêt demande à être au plus vite rendue à ellemême (1).

Dans les coupes de taillis, la vidange doit être conduite plus rapidement encore que dans les futaies. Leur fréquentation par les hommes et les animaux pendant la saison d'été qui suit l'abatage, devient une cause de dommages considérables pour la régénération; car un grand nombre de jeunes rejets sont détruits alors qu'ils sont cassants et mal allachés. Certainement, il en repoussera de nouveaux au printemps suivant, mais les rejets de la première année sont les plus abondants et les plus vigoureux; de plus, il y a toujours un certain nombre de souches ainsi fatiguées qui ne repoussent plus. C'est là une des principales causes de l'appauvrissement des taillis en bonnes essences. Pour se rendre compte de ce fait, il suffit de comparer deux coupes voisines, en tout semblables et exploitées en même temps; dans l'une, la vidange aura été terminée avant le printemps qui suit l'exploitation, tandis que, dans l'autre, cette opération se sera

(1) Avec les délais de vidange admis dans la pratique de la vente des coupes sur pied, l'adjudicataire se sert pendant plus d'une année du parterre de la vente comme d'un lieu de dépôt dans lequel il peut, au fur et à mesure de ses livraisons, prendre les produits pour les conduire directement au bûcher de l'acheteur. Il gagne ainsi les frais de chargement et de déchargement qu'il subirait en les accumulant dans ses chantiers. 
prolongée pendant tout l'été suivant: la différence entre le développement des deux régénérations est saisissante en faveur de la première.

Les produits des éclaircies sont portés à dos d'homme sur les chemins existants. A ce point de vue, il est avantageux de multiplier, dans les limites raisonnables, le nombre de ceux qui traversent les massifs, et de rendre praticables aux voitures

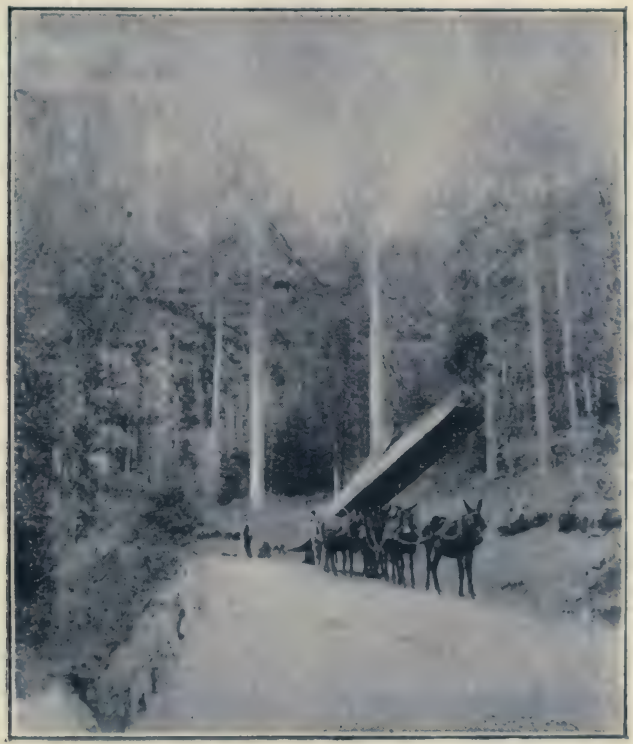

Fig. 67. - Enlèvement de pièces de pin laricio, forêt d'Aitone (Corse). (Photographie de M. J. Bregeault.)

toutes les lignes d'assiette de l'aménagement. Ces chemins intérieurs, établis sur une largeur de 2 à 3 mètres lors de l'exploitation des coupes principales, peuvent être maintenus pendant toute la vie du peuplement sans nuire sensiblement à la production totale.

Toutes les précautions qui précìdent sont du ressort de la gestion. La seule prescription qui concerne les aménagistes est contenue dans le formulaire des Régles d'assielle. La deuxième de ces règles dit : 
Les coupes doivent être disposées de manière que les bois d'une coupe en exploitation ne soient pas dans le cas d'être transportés à travers d'autres coupes récemment exploitées.

Pour s'y conformer, il suffit que chaque coupe soit indépendante des autres et qu'elle aboutisse directement, soit sur une route ou un chemin, soit sur un ruisseau.où le bois puisse se flotter, soit enfin sur les terres riveraines (1).

Ce qui vient d'être dit, suffit, en général, pour concilier les exigences de la perception des revenus avec celles de la régénération. Sans doute, dans la pratique, quelques légères modifications y seront apportées en vue de mieux les adapter aux habitudes ou circonstances locales; mais il est superflu d'imposer aux adjudicataires des charges souvent onéreuses que la routine éternise dans les documents administratifs sans aucun profit pour la forêt. Il en est ainsi, par exemple, de l'arrachage des épines. A quoi sert, en effet, d'arracher les épines? Ce travail, lorsqu'on l'exige, est toujours mal fait; de plus, la plupart des morts-bois et arbustes dits nuisibles, notamment les ronces, les épines noires et blanches drageonnent facilement, et l'enlèvement de la souche, qui ne peut nécéssairement s'étendre jusqu'à l'extrémité des racines, provoque leur expansion sur une grande surface.

Les élagages. - A l'occasion des méfaits commis par la hache ou la serpe du propriétaire, nous devons parler des élagages. Mais, pour ceux-ci du moins, on à la faculté de laisser les outils se reposer et, dans la majorité des cas, aussi bien dans l'intérêt de ses arbres que dans ceux de sa bourse, le mieux sera de s'abstenir.

L'élagage est une sorte de taille appliquée aux arbres, en vue de leur donner, en une seule fois, une forme plus avantageuse au moyen d'amputations totales ou partielles des rameaux réputés inutiles ou nuisibles.

Séduit par l'aspect extérieur des arbres élagués, et faute de s'être rendu un compte exact de la conséquence des opérations, on a, pendant longtemps, érigé en un véritable systême d'exploitation forestière cette pratique d'ailleurs excel-

(1) Lorentz et Parade, Culture des bois. 
lente pour les arbres de parc ou d'alignement(1). Mais si la taille est réellement profitable à celui qui - chaque jour - intervient pour diriger un bourgeon bien placé, en vue de lui faire C

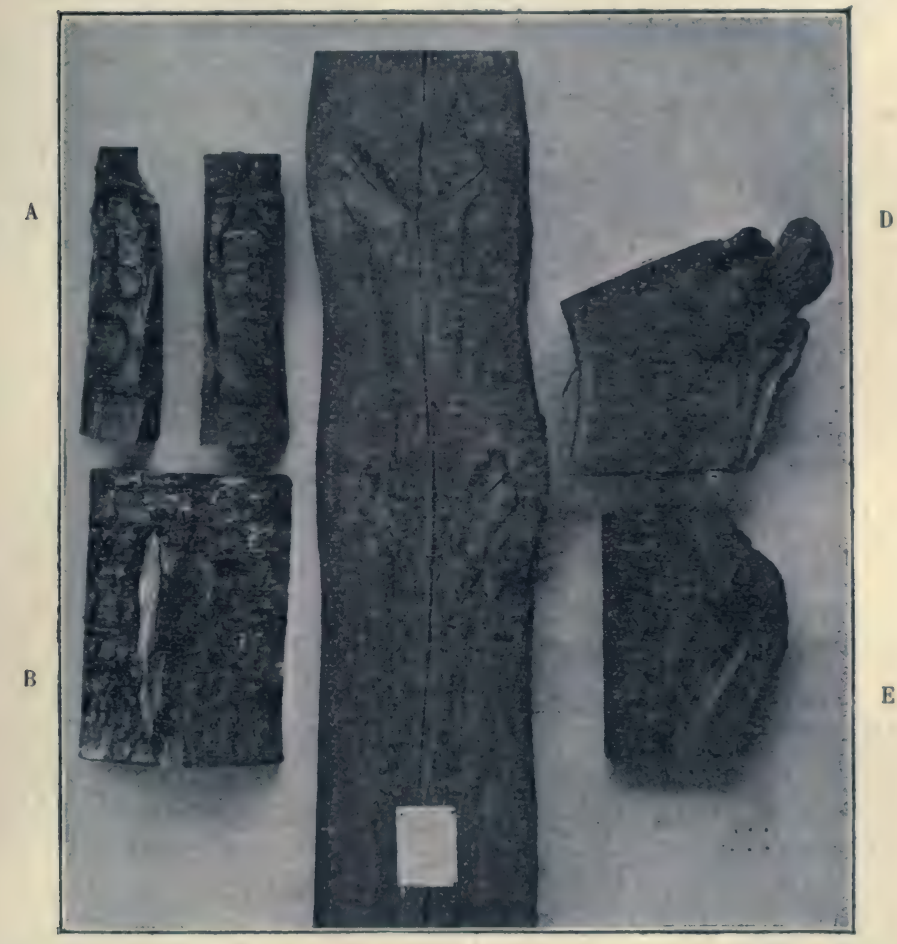

Fig. 68. - a, Tares indélébiles consécutives à une inscription faite sur l'écorce. - $b$, Plaie contuse occasionnée par le choc d'une tronce lancée sur un versant de montagne. $-c$, Élagage rez-tronc bien fait : solution de continuité dans les tissus. $-d$, Elagage rez-tronc suivi de pourriture. $-e$, Élagage en chicot suivi de pourriture.

produire, à son gré, des fruits, des fleurs ou des ombrages, en forêt, oủ l'on cherche - avant tout - à fabriquer du bois sain, elle ne saurait être qu'une fallacieuse mutilation. Il faut se souvenir que l'enlèvement de toute branche, de tout rameau, se traduit par une perte de substance et une

(1) L'élagage des arbres, par le comte des Cars, Paris, Rothschild, 1867. 
plaie à recouvrir. Pour permettre à l'arbre qui en est victime de réparer ces dommages, il lui faut une nourriture supplémentaire qu'un arboriculteur avisé ne lui ménage pas. Mais, en forêt, qui apportera à l'opéré les médicaments et fortifiants nécessaires à sa guérison?

Quoiqu'il en soit, il est certain que l'élagage rez-tronc, à section verticale, présente certains avantages sur les procédés en chicot ou à section oblique; mais il n'en produit pas moins des blessures incurables, avec tout le cortège de maladies qu'elles engendrent, en dépit des pansements les mieux appliqués. Le recouvrement (fig. 68 , a) cache la surface de section sans la cicatriser dans le sens physiologique du mot; et, avant qu'il soit complet, le bois mis à nu a tout le temps de mourir, de se gerçer et l'on sait que toute crevasse est la porte ouverte aux germes des champignons destructeurs (1).

Aujourd'hui, l'expérience a fait justice de toutes ces chimères et il n'est pas trop tôt d'arrêter les mutilations infligées, de parti pris, à la plupart des chênes réservés dans les taillis composés. Il suffit, en effet, d'ouvrir le tronc d'un de ces arbres pour constater toute l'étendue du mal (1) (fig. 68, $c, d, e$ ).

"En fait, dit, M. Broilliard (2), tout arbre constitué ñe saurait être amputé d'une ou plusieurs grosses branches sans qu'il en résulte un ralentissement dans la végétation, un trouble marqué dans les fonctions vitales, et une plaie nuisible par elle-même ét souvent désastreuse par les vices qu'elle occasionne dans le corps de l'arbre ".

L'élagage doit donc être proscrit des forêts, en tant que procédé méthodique de traitement, mais on doit se demander dans quelles limites il est permis d'en user, à titre d'opération chirurgicale, suivant qu'il s'agit de branches vivantes, de branches mortes ou de branches gourmandes.

L'élagage des branches vivantes ne peut être toléré que dans les cas suivants :

(1) Martinet, Garde général des forêts, Considérations et recherches sur l'élagage des essences forestières, Paris, librairie agricole, 1876.

D'Arbois de Jubainville, sous-inspecteur des forêts, Observations sur le système d'élagage de Courval et des Cars, Paris, Rothschild, 1869.

(2) Broilliard, Cours d'aménagement des forêts, Nancy, Berger-Levrault et Cie, 1878, p. 258. 
$1^{\circ}$ sur les plants de haute tige élevés en pépinière et qu'on peut soumettre à une taille raisonnée dans le but d'améliorer une forme défectueuse ;

$2^{\circ}$ sur les branches basses appartenant à la cime des baliveaux de l'âge et des jeunes modernes. La suppression de ces branches basses, dont le calibre ne dépasse pas 5 à 8 centimètres de diamètre à la base, peut se faire sans graves inconvénients, car d'aussi petites plaies se recouvrent bientôt et les solutions de continuité qui en résultent sont placées près du centre de l'arbre et n'en déprécient pas sensiblement le débit; quand il s'agit de chênes, le bois mis ànu étant encore entièrement à l'état d'aubier, celui-ci se recouvre d'un enduit gommeux qui le préserve du contact immédiat de l'air et le met à l'abri des champignons. D'ailleurs, ces branches basses sont condamnées à disparaître sous l'influence de l'élagage naturel: en les supprimant on ne fait que devancer la nature;

$3^{\circ}$ il est également permis de couper, si on le juge nécessaire pour favoriser un ensemencement, les branches appartenant à des sujets destinés à être exploités à brève échéance.

On ne saurait trop se garder de toucher à une branche, quelque petite qu'elle soit, sur les conifères ; car, toute plaie faite à un arbre de ce groupe provoque des écoulements de résine et des désordres plus graves encore que chez les arbres feuillus.

Les branches mortes doivent être traitées comme les branches vives, puisque, pour obtenir le recouvrement, il faut reculer le point de section jusque dans le bois vif (1). Il vaut donc mieux laisser les branches mortes continuer, lentement et au grand jour, leur œuvre de destruction, en surveillant le mal pour couper l'arbre avant qu'il soit plus dégradé.

Cette observation s'applique surtout aux grosses branches mortes insérées directement sur le fût des arbres. Certes, pour retarder les pourritures, il serait utile de remplacer par une section nette la cassure esquilleuse qui les termine le

(1) L. Boppe, Cours de technologie forestiere. Nancy, Berger-Levrault et Cie, 1887. 
plus souvent; mais l'opération est trop onéreuse et l'on évite de faire des sacrifices pour des sujets qui sont affectés de tare compromettant leur avenir. Du reste, on ne pourrait pratiquer ces amputations qu'en montant sur les arbres à l'aide de crampons, dont l'usage est peut être encore plus nuisible que l'élagage lui-même.

Quant aux branches mortes dans la cime, branches appartenant aux ramifications secondaires, il n'y a pas lieu d'y toucher. Les essences de lumière, notamment les chênes réservés dans les taillis composés, présentent toujours de pareilles branches dans la couronne, quand ils arrivent à un âge avancé; ces cornes sont la conséquence de leur tempérament et des crises qu'ils ont à supporter après chaque exploitation. Tant que l'arbre se maintient d'ailleurs vigoureux, elles disparaissent assez vite sous l'effort du vent et, avant longtemps du moins, leur rupture n'occasionnera aucun désordre sérieux. Au contraire, chez les essences d'ombre, chez les hêtres principalement, les branches mortes dans la cime sont toujours un indice fâcheux, et il n'est jamais prudent de conserver les individus qui portent ainsi les signes d'un dépérissement prochain.

M. Mer conseille d'enlever, sur les arbres résineux, les branches mortes en dessous de la cime, de façon qu'elles ne soient pas englobées, - véritables chevilles, - dans le fût qui grossit autour d'elles. Leur amputation se fait à la scie, à quelques millimètres au-dessus du bourrelet de base, pour ne pas en entamer les tissus vivants.

Les émondages. - On admet que l'évolution des branches gourmandes ou des gourmands sur le fût des réserves est la conséquence du changement d'état que leur impose chaque exploitation; les gourmands, abandonnés à euxmêmes, disparaissent pendant le cours de la révolution. On accuse leurs évolutions successives d'engendrer des $e x$ croissances, des nodosités, des broussins, dont le tissu làche et madré, est de qualité médiocre.

L'opération qui consiste à supprimer ces rameaux gourmands, porte le nom d'ébourgeonnement ou d'émondage. Nombre de forestiers altachent à la pratique des émondages 
une importance capitale; d'autres, n'ont dans leur efficacité qu'une confiance très limitée; un observateur de premier ordre, M. le Conservateur d'Arbois de Jubainville les signale même comme nuisibles à l'égal des élagages. Nos recherches personnelles nous font partager cette dernière opinion; aussi, sans proscrire systématiquement les émondages, recommandons-nous de ne pas en généraliser l'application et de les restreindre à un petit nombre de cas particuliers, que tout praticien saura discerner : il y a, pour les propriétaires qui en abusent par tradition, de sérieuses économies à réaliser de ce côté.

Quoiqu'il en soit, partout où l'opération sera jugée indispensable, on devra se conformer aux indications suivantes:

$1^{\circ}$ procéder à l'opération au milieu de l'été, ou mieux, en automne, de façon à ne pas exposer les ouvriers aux démangeaisons avec accès fébriles qu'occasionnent les poils de la chenille processionnaire très commune sur les chênes;

$2^{\circ}$ limiter strictement la coupe aux gourmands dont le diamètre à la base ne dépasse pas 1 centimètre, en employant des instruments spéciaux, bien tranchants et maniés avec assez d'habileté pour ne pas entamer les parties vivantes de l'écorce. En effet, dès que les branchettes ont dépassé ce calibre, elles laissent des plaies étendues, dont la marqueindélébile déprécie, lors du débit, les échantillons qui les portent : émondage devient élagage.

Les assainissements. - Mais, dans la trousse du chirurgien forestier, la hache n'est pas seule à torturer les peuplements. Pendant qu'elle ampute les arbres soi-disant pour les redresser, la bêche et la pioche, sous prétexte d'hygiène, épuisent le sol par d'inutiles saignées.

L'eau n'est réellement nuisible aux arbres que si elle resle stagnante à la surface du sol pendant la saison de végétation; ces parties marécageuses se présentent, dans la plupart des cas, sous forme de tourbières, de mares ou de fonds de cuveltes sans écoulement. En semblables conditions, on confond, trop souvent, la cause avec l'effet; car, si l'eau reste à la surface, c'est que le sol est imperméable, 
aussi bien aux racines qu'à l'eau, et cet inconvénient est encore plus accentué après qu'avant le drainage.

Quant aux fossés d'assainissement ouverts dans les stations humides ou aquatiques dont l'eau se renouvelle, ils ont pour effet de ralentir la végétation des arbres déjà développés. Ils modifient en mal la qualité du bois des chênes, des ormes, des frênes et compromettent, enfin, leur mélange avec les bois tendres. On ne bouleverse pas impunément les conditions dans lesquelles les arbres ont vécu; car les peuplements forestiers se constituent en fonction du sol et de état permanent de ses propriétés physiques. Quand celles-ci sont modifiées, l'ancien peuplement dépérit, pour faire place à un autre dont le tempérament sera mieux en harmonie avec la situation nouvelle.

Peu importe d'ailleurs l'état submergé d'un terrain en hiver, quand l'excès d'eau disparaît au moment de la végétation. On constate que les cantons soumis à ces inondations périodiques fournissent les meilleurs chênes de France, aussi bien dans le Nord que dans le Centre et dans le Midi.

L'assainissement ne se justifie donc que dans les terrains improductifs par excès d'eau stagnante; dans ce cas, l'opération présente un caractère purement local et ne doit être exécutée que si les bénéfices à prétendre sont de nature à compenser la dépense ; partout ailleurs, l'assainissement érigé en système, outre qu'il augmente les crues des rivières, est inutile, onéreux et nuisible au point de vue strictement forestier.

Au surplus, par la transpiration des feuilles, par la pénétration des racines et surtout par les propriétés hygrométriques de la couverture, la végétation forestière exerce sur tous les sols une action asséchante des plus marquées; aussi, dans l'immense majorité des cas, l'excès de sécheresse est-il plus à craindre que l'excès d'humidité.

A l'appui de ce fait, citons un exemple bon à noter.

Partout, en montagne, il est facile de constater les effets dus à l'ouverture d'un chemin qui coupe la pente d'un versant quelque peu rapide. Les eaux superficielles d'amont sont captées par la tranchée des talus de déblai et conduites dans les 
fossés bordiers jusqu'au prochain caniveau, par où elles s'écoulent en flots inutiles et parfois nuisibles par suite des ravinements qu'elles produisent; dès lors, les parcelles d'aval, privées des eaux superficielles qui les alimentaient, souffrent de la soif et leur végétation se ralentit. Pour atténuer l'intensité du dommage, le seul moyen est de multiplier les caniveaux pour rendre aux eaux leur cours naturel d'infiltration à travers toutes les surfaces : irriguer, aprés avoir asséché.

Lors donc qu'il s'agit de remettre en valeur des terrains sans déclivité que la nature du sous-sol et leur état de nudité rendent marécageux, il peut être nécessaire de faire des assainissements préalables, en vue d'y rétablir l'ancien état boisé; mais, une fois les peuplements reconstitués grâce à un travail d'ensemble qui englobe toute une région, la forêt fera le reste ; c'est du moins ainsi que cela s'est passé dans certaines contrées autrefois infectées par les fièvres paludéennes et en partie assainies par la forêt, comme : les Landes de Gascogne (1), la Sologne (2) et les Dombes.

Enfin les intéressantes recherches de M. Chevandier au sujet de l'influence des irrigations sur la végétation des forêts permeltent deconclure que de telles améliorations ne peuvent être généralisées dans la pratique, mais qu'il est utile de mettre à profit toutes les circonstances dans lesquelles la nature les fournit sans frais; - qu'il n'est donc pas logique, - par exemple, d'assainir d'une façon exagérée les tourbières dans la haute montagne où elles jouent un rôle analogue à celui des glaciers et fournissent, en été, l'eau nécessaire pour irriguer les versants et alimenter les sources de la plaine.

\section{Fait des délinquants.}

Les causes. - De tous temps, les forêts ont eu à souffrir du voisinage des populations riveraines. Cela tient à ce fai

(1) Chambrelent, Mise en valeur des landes de Gascogne, Bordeaux, 1862 .

(2) A. Brongniart, Rapports sur les reboisements de la Sologne (Annales forestières, t. XI, 1865.) 
que la forêt, avant d'être reconnue, en droit, comme une propriété privée, était regardée comme un bien banal, où chacun pouvait prendre ce qui se rencontrait d'utile à l'entretien du ménage.

De telles traditions à réprimer justifient les rigueurs du Code forestier. L'enlèvement frauduleux de tout produit du sol y constitue un délit; le łois mort, les herbes, la litière, les pierres, etc..., aussi bien que le bois sur pied, rien ne peut être exporté sans autorisation; certains faits sont même punissables, alors qu'ils dénotent la simple intention de commettre un délit. De ce côté, tout est prévu, le propriétaire est suffisamment armé; à lui de veiller à l'exécution de la loi, en faisant bonne garde.

On prévient aussi les délits, dans une certaine mesure, en donnant satisfaction aux besoins les plus impérieux des habitants pauvres, au moyen de concessions sagement réglementées. Ces délivrances, limitées comme il sera dit plus loin, font aimer et respecter la forêt:

La répression. - En ce qui concerne la répression directe, on remarque que les délits augmentent bientôt lorsque la police forestière n'est pas assurée d'une manière convenable ; aussi les propriétaires de forêts ne doivent-ils pas regretter les dépenses que leur occasionne un personnel de surveillance actif et suffisant; les économies mal entendues sur les frais de garde coûtent souvent bien cher. Au surplus, les fonctions du vrai garde forestier ne doivent pas être limitées à la répression des délits; il peut exécuter une foule de menus travaux, dont l'ensemble représente une somme d'améliorations notables pour la forêt : il est, en un mot, l'auxiliaire naturel du propriétaire dans tous les actes relatifs à la gestion de son domaine. Mais, avant tout, il faut qu'un garde ait une moralité et une tenue en rapport avec la dignité d'officier de police judiciaire dont il est investi; on doit donc le mettre, par un traitement convenable, dans une situation pécuniaire supérieure à celle des bûcherons et des manœuvres qu'il dirige.

Bien que, chez nous, le garde forestier ne puisse pas donner à la chasse tout le temps que comporte une source aussi 
importante de revenus pour les forêts, il ne devra jamais s'en désintéresser. Mieux que personne, il est à même de connaître les allures des braconniers, et jamais il ne manquera l'occasion de les prendre en flagrant délit. Que si, dans ces recherches aussi minutieuses que dangereuses, il n'a pas toujours le temps d'opérer lui-même, du moins ses indications seront-elles des plus précieuses pour les gardes particuliers spécialement chargés de ce service. L'entente et la bonne harmonie régneront entre eux.

D’ailleurs, qu'il s'agisse de délits de bois ou de chasse, la surveillance sera singulièrement facilitée par la création de chemins de ronde, dissimulés sous bois à quelques mètres des bordures; en les suivant, matin et soir, pour se rendre à son travail, le garde qui a quelque sentiment du devoir saura bien reconnaître sur le sol la trace d'une piste douteuse. De plus, en pays de montagne tout au moins, l'ouverture de sentiers de surveillance pénétrant au milieu des massifs a donné les meilleurs résultats dans les forêts soumises au régime forestier; leur réseau s'étend tous les jours. D'un parcours plus aisé que les lignes d'aménagement souvent dirigées suivant la plus grande pente, ils permettent l'accès de tous les cantons au garde et à l'agent; ils font aussi le bonheur du touriste.

On recommande, enfin, l'emploi de cabanes rustiques campées sous bois, çà et là; celui qu'elles abritent peut, à loisir, dévisager le délinquant qui, dans ses randonnées et ses feintes, passera par mégarde à leur portée. "La vue permanente, d'ailleurs, de ces sombres sentinelles, engendre, chez le braconnier, une crainte salutaire et le décide, souvent, à aller en d'autres lieux exercer ses talents " (1).

\section{Les concessions de menus produits.}

Tolérances nécessaires. - Ia forêt renferme dans son sol et fournit spontanément un grand nombre de substances uti-

(1) De Brus, Les chasses aux braconniers, Paris, E. Dentu, 1885. 
lisables, d'origine soit végétale, soit minérale, auxquelles on donne le nom de menus produits. Ce sont, parmi les produits végétaux : les bois morts, les souches mortes, les morts-bois, la litière, les feuilles mortes, les herbes, les fruits, les champignons, les plantes herbacées diverses, pharmaceutiques ou autres, etc...; et, parmi les produits minéraux: les pierres roulantes, les pierres en place, les minerais, castines, sables, la terre de bruyère, la tourbe, l'eau, etc.

Le plus souvent, ces menus produits sont concédés aux populations riveraines, moyennant des redevances évaluées, soit en journées de travail, soit en argent. On les considère comme inutiles à la forêt, et les bénéfices procurés par leur enlèvement, quelque modestes qu'ils soient, semblent de l'argent trouvé. Il y a lieu de réagir contre cette opinion trop répandue, en France, parmi le public et même dans le monde forestier; car ces concessions donnent lieu à bien des abus.

D'autre part, il faut reconnaître que certains de ces menus produits satisfont à un besoin réel pour les populations riveraines des forêts. Il y a lieu, toutefois, de les considérer. non plus comme la source d'un revenu éventuel, mais, à la façon du parcours, comme un mal nécessaire. Il est clair que de tels enlèvements, lorsqu'ils sont modérés, ne produisent jamais de préjudices bien sérieux; et ce serait folie que de vouloir les supprimer d'une façon radicale; ce qui est à craindre, ce sont les abus auxquels on se laisse trop facilement entraîner, lorsqu'on ne se rend pas un compte bien exact des faits, et c'est mal comprendre l'hygiène de la forêt que de ne pas attacher à cette question l'importance qu'elle comporte. En forêt, comme ailleurs, les plus petites causes peuvent avoir des effets désastreux : par exemple, le parcours ou l'enlèvement des feuilles mortes, en relentissant la végétation, engendrent les invasions d'insectes. On sait aussi que la forêt demande la tranquillité ; or, la fréquentation des massifs et l'enlèvement d'une fraction de la couverture morte ou vivante ont pour conséquences nécessaires de diminuer la production de l'humus, d'amoindrir la densité 
des peuplements, d'augmenter enfin le degré de tassement et de désséchement du sol: toutes causes qui sont de nature à changer bientôt en perte sèche pour le propriétaire, les prétendus bénéfices réalisés sous cette forme.

Il convient donc, parmi ces concessions, de pouvoir discerner celles qui pourraient devenir dangereuses, afin de les supprimer en temps utile, ou tout au moins de les réglementer.

Menus produits végétaux. - L'enlèvement des bois morts sur pied donne lieu à de nombreux abus : aussi celte jouissance du bois mort doit-elle être limitée au menu bois gisant, sans jamais permettre la coupe ou la rupture des branches sèches encore attachées aux arbres.

Les souches morles, en se décomposant dans la terre, se transforment en humus; elles augmentent ainsi la fertilité du sol et sa porosité. L'extraction n'en est utile que dans des cas déterminés, par exemple: pour faciliter la régénération naturelle dans les futaies, les plantations dans les taillis sous futaie, ou encore pour empêcher le développement exagéré des insectes dans certaines forêts résineuses.

Les morts-bois contribuent, dans les peuplements jeunes, à couvrir le sol et à hâter la formation de l'état de fourré. Ils ne peuvent disparaître sans danger, même quand les grandes essences se sont constituées en massif au-dessus d'eux. Si, momentanément et sur certains points, ils deviennent gênants, c'est sous forme d'opération culturale et non sous forme de concession qu'il faut s'en débarrasser. En dehors des contrées oủ les incendies sont à craindre, le débroussaillement radical, ou soutrage, n'est donc jamais justifié.

La récolte des herbes, pour fourrage ou pour litière, ne doit être tolérée, à la faux ou à la faucille, que sur les chemins et les sentiers oú les arbres manquent absolument; partout ailleurs, même dans les vides et clairières, elle ne doit être permise que par arrachage à la main; autrement l'étendue de ces clairières va sans cesse augmentant et jamais on ne les verra se repeupler. Dans ces conditions, le prix de la cueillette ne permet pas à la grande et à la moyenne culture d'en tirer profit. Il y a même lieu de l'interdire d'une 
façon absolue dans les parcelles en régénération; car ces herbes protègent les jeunes plants contre les ardeurs trop vives du soleil, et, quand, par leur présence, elles s'opposent à l'installation du semis, ce n'est pas en les fauchant qu'on améliorera la situation : il faut les arracher, en donnant au sol une légère culture, vers l'époque de la chute naturelle des graines.

Dans le voisinage des grandes villes, l'enlèvement des mousses peut aussi prendre des proportions telles qu'il en résulte un véritable danger. C'est alors par wagons que la mousse est ramassée; le sol sans cesse piétiné, tassé, pelé, s'appauvrit graduellement, au point de ne plus pouvoir nourrir que de maigres broussailles. L'état déplorable dans lequel se trouvent certaines forêts des environs de Paris n'a pas d'autres causes, et le temps n'est pas loin, où il faudra chercher un mode de traitement compatible avec des déprédations qu'on sera de plus en plus impuissant à empêcher.

Les graines forestières telles que : glands, faînes, doivent être ramassées à la main, sur le sol, dans les cantons où l'on ne désire aucune régénération par la semence. Le ramassage au moyen de râteaux, balais, est nuisible, en ce qu'il déplace ou détruit la couverture; en aucun cas, si ce n'est pour son usage particulier, le propriétaire ne devra permettre la récolte des graines sur les arbres.

La cueillette des baies comestibles tels que : fraises, framboises, myrtilles, etc., occasionne des piétinements et des ruptures de tiges parfois très préjudiciables danș les jeunes peuplements; il est bon de soumettre ces tolérances à des restrictions, dans les cantons où elles ne pourraient être exercées sans dommage.

L'enlèvement des champignons comestibles ne présente d'autres dangers que ceux auxquels sont exposées les personnes qui les récoltent, lorsqu'elles commettent des erreurs sur les espèces. Nous avons vu que l'un d'entre eux, la truffe, acquiert même, dans certaines régions méridionales, une importance assez considérable pour engager les propriétaires à disposer les peuplements dans les conditions les plus favora- 
bles à sa production, sans tenir trop grand compte des produits ligneux.

Quant aux plantes herbacées ramassées pour leurs vertus pharmaceutiques ou extraites en mottes pour une transplantation, il est clair que leur disparition est toujours limitée à un trop petit nombre de sujets pour que la fertilité du sol puisse en subir un dommage appréciable. Néanmoins, on entend les botanistes se plaindre, avec raison, de ce fait que, dans le voisinage des villes, les belles plantes sauvages disparaissent sans autre profit que celui qui est réalisé par les maraudeurs qui en font le commerce; en effet, ces plantes, organisées pour vivre dans les conditions de sol et de couvert que leur ménage la forêt, périssent infailliblement dès qu'elles sont transplantées, en plein air, dans les jardins : si l'on a intérêt à cultiver certaines d'entre elles, c'est par la semence qu'il faut tenter de le faire.

Nous ne saurions trop répéter que l'enlèvement des feuilles mortes, qu'elles soient destinées à faire de la litière ou à tout autre usage, ne doit être toléré nulle part.

Ajoutons enfin que ces petites industries de la forêt ne sont, en général, pratiquées que par des infirmes, des paresseux ou des insoumis, à qui, trop souvent, elles servent de prétexte pour se livrer au braconnage.

Menus produits minéraux. - $\mathrm{Si}$, des matières végétales, on passe aux matières minérales, les inconvénients des extractions, pour être d'une nature différente n'en sont pas moins réels. Sur les pentes rapides, l'enlèvement des pierres roulantes occasionne des éboulements de débris qui causent des plaies dangereuses au pied des arbres. On constate qu'en semblable situation, si, dans les éclaircies, on ménage les petites perches dominées mais encore vivantes, celles-ci arrêtent les pierres et, en diminuant leur vitesse de chute, préservent les arbres d'avenir.

La délivrance de blocs dans les moraines et les murgers, des montagnes granitiques, détruit les conditions d'équilibre de la masse et occasionne des éboulements; de plus on sait que là, plus qu'ailleurs, tout déplacement de la surface empêche le reboisement naturel de ces parties rocheuses et en éternise la stérilité. 
Quand les matériaux sont extraits decarrières, il en résulte des défrichements qui diminuent la surface en production tant que dure l'occupation; et, dans la suite, de longues années s'écoulent avant que le terrain puisse rentrer dans le cadre du traitement appliqué aux parcelles environnantes. En outre, ces dernières ont à souffrir du voisinage des ouvriers, et, sur tout le périmètre des parties occupées, il se crée des lisières, avec toutes les conséquences fâcheuses que l'on connaît.

Les exploitations en galerie, sous le soldes forêts, amènent l'ouverture de crevasses, des effondrements, des chutes d'arbres, des troubles nombreux dans la jouissance et des pertes sensibles pour le propriétaire de la surface. Il est nécessaire de tenir compte de tous ces faits lors du règlement des indemnités qui lui sont dues, soit par l'exploitant quand les marchés ont été consentis amiablement, soit par les industriels à qui certaines législations spéciales confèrent des droits considérables.

Enfin, s'il s'agit de concessions d'eaux, il sera bon de les limiter aux eaux courantes qui sortent de la forêt, en interdisant les travaux de captage qui pourraient assécher une partie plus ou moins étendue de la surface.

\section{Le pâturage.}

La situation actuelle. - Le pâturage, même modéré, appauvrit la forêt; l'abus est sa ruine.

On désigne sous le nom général de parcours, le fait d'introduire des animaux domestiques en forêt pour les y faire pâturer; le plus souvent, on distingue le pâturage qui s'applique aux bêtes aumailles, - du pacage, qui se rapporte particulièrement au menu bétail : chèvres et moutons, et du panage, qui ne concerne que les porcs.

Mettre une forêt en défends, c'est en interdire l'entrée aux bestiaux, parce que les jeunes bois n'y sont pas assez élevés pour échapper à l'abroutissement. On appelle forêt défensable celle qu'on peut ouvrir au parcours parce qu'elle n'a plus rien à craindre de la dent du bétail. 
Tout propriétaire, en vertu de son droit d'user de la chose qui lui appartient, peut exercer le parcours dans ses forêts; à lui d'apprécier le dommage qu'il en subira. Depuis les premiers temps du moyen âge, des droits de cette nature ont été aussi concédés, sous forme de servitude d'usage, aux populations riveraines, par les seigneurs propriétaires; on a tout bénéfice à s'affranchir de ces lourdes charges par le rachat à prix d'argent, dans les conditions prévues par la loi. Le parcours peut enfin être exercé en délit, sans le consentement du propriétaire; il constitue alors un fait punissable. C'est affaire de surveillance.

Nocuité du pâturage suivant les rẻgions. - Actuellement, dans les pays de coteaux et de basses montagnes des zones parisienne et girondine, où le climat est assez humide et la terre assez fertile pour permettre la culture et le fauchage des prairies naturelles et artificielles, le parcours n'a plus sa raison d'être. A vrai dire, la forêt ne porte d'herbes nutritives que dans les très jeunes bois et dans les vides; on tourne, dès lors, dans un cercle vicieux ; car, si la forêt produit d'autant plus d'herbes qu'elle est en situation plus précaire, c'est alors qu'elle a le plus besoin de repos et qu'il faut en éloigner le bétail; aussi, les cultivateurs avisés se rendent-ils parfaitement compte du bénéfice illusoire qu'on tire de cette pratique lorsque, comme de raison, elle est limitée aux seuls cantons défensables; ils savent que, dans toute forêt en état moyen de production ligneuse, l'étendue de ceux-ci va du tiers à la moitié de la surface totale et qu'on n'y rencontre pas plus d'un dixième de vides. Dans ces conditions, on a calculé que le bénéfice annuel oscille entre 60 centimes et $1 \mathrm{fr} .50$ par hectare pour l'ensemble de la forêt et que le nombre des bêtes à admettre ne doit pas être supérieur à une tête par 2 à 4 hectares de cantons défensables; entre ces limites, les chiffres varient avec la nature du sol, avec la fertilité du climat, avec la composition des peuplements et leur traitement, suivant enfin les ressources qu'ils présentent en herbe : dès qu'elle sont dépassées, on peut être assuré que le bétail, faute d'herbe, attaquera le 
bois (1); un si mince profit est loin de compenser la perte totale des engrais et le dommage éventuel causé aux arbres. Il faut des années exceptionnellement sèches, comme l'été de 1893, pour que, dans ces régions, on ait intérêt à lâcher le bétail dans les bois; ceux-ci rendent alors un service signalé à l'agriculture, en lui permettant, non pas de tirer une rente du bétail admis au parcours, mais simplement de le maintenir vivant jusqu'à la prochaine récolte. Et à quel prix! La crise passée, la forêt cache ses blessures sans les guérir, et ce n'est que plus tard, alors que viendront en tour d'exploitation les peuplements pâturés à l'état de jeune bois, qu'on pourra évaluer l'énormité du dommage; nos enfants paieront nos méfaits.

Il n'en est pas de même dans les hautes montagnes, et dans les régions provençales où, en été, toute trace de verdure ayant disparu en sol découvert, on ne rencontre plus de rares brins d'herbe que sous l'abri des buissons et des arbres. Dans l'un et l'autre cas, pâturage et pacage deviennent une nécessité, et il faut s'incliner devant la formule du primo vivendi. Toutefois, si l'on va au fond des choses, on constate que, souvent, ces besoins sont plus artificiels que réels. Dans la haute montagne, la question du pâturage en forêt est entièrement liée à celle du pâturage en général, question vitale et pleine d'actualité, dont les pouvoirs publics sont en ce moment saisis, mais qui demande trop de développements pour être traitée ici avec tous les détails qu'elle comporte.

Quant aux forêts méridionales de la plaine et de la basse montagne, taillis de chêne yeuse et de chêne blanc, elles sont toutes plus ou moins ruinées, l'antique capital bois ayant été progressivement dévoré par les moutons; dans l'état de dégradation où elles se trouvent, beaucoup ont été transformées depuis longlemps en pâtures ligneuses n'ayant plus de forêt que le nom. Il n'est pas surprenant, dès lors, que certaines rapportent en viande et en laine beaucoup plus qu'en bois (2).

(1) A. Mathey, Le pâturage en forêt, Besançon, Paul Jacquin, 1900.

(2) V. de Larminat, Inspecteur-adjoint, Les forêts de chẻne vert, Troyes, Lacroix, 1893. 
Voilà l'argument fondamental invoqué en faveur du parcours.

Encore serait-il juste, du moins en ce qui concerne les forêts communales, que chacun des habitants, qui réprésente une fraction du propriétaire, fût appelé à prendre sa part de profit dans cette mobilisation du patrimoine commun. Or, il n'en est pas ainsi, et la manière dont s'exerce ce parcours ruineux est aussi injuste qu'anti-démocratique.

Citons un exemple entre mille. D'après un document récent (1), la situation des forêts sur le versant méridional du mont Ventoux a été établie de la façon suivante, au point de vue du parcours : " $6,1 \%$ seulement du tolal des che $/ s$ de famille usent de cette part de la propriété commune à tous les habitants, et parmi ces $6,1 \%, 2,6 \%$, les plus riches, possédent des troupeaux nombreux et accaparent la totalité des produits. "

Il faut avoir habité la montagne, ajoute l'auteur, pour se faire une idée de l'âpreté avec laquelle les gros propriétaires luttent contre le reboisement.

Ce sont ces égoïstes obstinés qui peuplent les conseils municipaux. Pour mettre fin à leurs exigences insatiables, les agents forestiers préposés à la défense du patrimoine commun sont unanimes pour demander, comme seul remède pratique, d'affouager les pâturages : ce qui revient à donner à chaque feu sa légitime part des produits des pâturages, comme cela se fait pour ceux des forêts (2).

Quoi qu'il en soit, puisque le pâturage est un mal nécessaire, il importe de connaitre ses conséquences afin de mieux le réglémenter. On constate que l'intensité du dommage varie d'une part, suivant l'espèce de bétail admis au parcours, d'autre part, suivant la nature du sol, suivant l'àge et l'état des peuplements qui le subissent.

Influence de l'espèce de bétail. - La chévre est celui des animaux domestiques qui cause le plus de mal à la forêt.

(1) F. Tessier, Inspecteur-adjoint, Les foréts du versant méridional du mont Ventoux. (Revue des eaux et forêts, nos de janvier et février, 1900.)

(2) Ch. Guyot, Le régime pastoral (Revue des eaux et foréts, août 1899.) 
On la laisse divaguer en toute saison : ce qui fait qu'elle se nourrit presqu'exclusivement des jeunes plantes ligneuses qu'elle peut atteindre jusqu'à une assez grande hauteur en se dressant sur les pattes de derrière. Pour la chèvre, il n'y a pas de cantons défensables; de plus elle s'aventure, par instinct, dans les endroits les moins accessibles où la végétation forestière ne s'installe que sous l'influence d'un repos absolu.

Bien qu'à nombre égal, ils soient moins nuisibles que les chèvres, les moutons sont aussi des hôtes très dangereux, surtout si on les laisse cheminer à leur gré, ou séjourner longtemps sur le même point ; car, alors, ceux qui marchent en tête du troupeau n'avancent qu'après avoir brouté le meilleur de l'herbe. Ceux qui suivent tondent les touffes de plus près et il ne reste aux derniers que les racines, si bien que, à défaut de celles-ci, ils doivent attaquer le bois pour ne pas mourir de faim. Quand, par force majeure, la forêt doit leur être ouverte, on peut user des précautions suivantes pour atténuer le dommage et retarder la ruine : donner la préférence aux petites races, - composer le troupeau en brebis plutôt qu'en moutons. Dans ce cas, le berger qui les dirige devient un véritable administrateur: il doit être intelligent, vigilant et habile à donner tous ses soins à l'élevage (1); il offre donc, à tous égards, beaucoup plus de garantie que le chemineau quelconque à qui l'on confie les troupeaux nomades.

Les bêtes à cornes pâturent les jeunes plants forestiers en même temps que les herbes; elles en arrachent un grand nombre, surtout par les temps pluvieux. Les chevaux et les ânes feraient moins de mal, s'ils se contentaient des jeunes pousses que leurs dents coupent net sans les arracher; mais, trop souvent, en rongeant les écorces, ils font de graves blessures dans la partie du tronc qui a le plus de valeur. Toutes ces bêtes aumailles tassent le sol et arrêtent le fonctionnement des réactions qui entretiennent sa fertilité.

De tous les animaux domestiques, les porcs sont cerlaine-

(1) V. de Larminat, loc. cit. 
ment les moins nuisibles à la forêt. Mais il ne faudrait pas abuser de leur séjour permanent dans des espaces restreints. Témoins, tous ces cantons qui nous sont parvenus sous le nom de clairs-chênes et qui, tant qu'ils ont été fréquentés par les hardes, n'étaient peuplés que de vieux " glandiers " disposés à la façon d'une Normandie, où les chênes, comme des pommiers, laissaient tomber leurs branches jusqu'à terre, sur un sol nu, sans cesse tourmenté par les souilles, où nulle végétation n'avait le temps de s'installer; le repos en a fait d'excellents massifs où le chêne abonde.

A ces dégradations, il faut encore ajouter celles qui sont le fait des pâtres, souvent des enfants, dont les jeux, taxés d'innocents, se traduisent par de véritables actes de destruction : l'un étêtera un jeune brin d'avenir; un autre détachera un lambeau d'écorce sur le cerisier, le bouleau ou le sapin le plus lisse de la forêt, ou gravera son nom sur le fût d'un hêtre (fig. 68 a); un troisième, allumera du feu dans un vieux tronc, y laissera couver un tison d'où naîtra l'incendie ; tous s'amuseront à faire rouler sur les pentes des pierres qui, en rebondissant, iront frapper les arbres et leur ouvrir de larges plaies. Ces petits méfaits, que le jeune âge excuse, finissent, lorsqu'ils sont répétés tous les jours, par coûter à la forêt plus cher que le salaire d'un pâtre sérieux et intelligent.

Influence de l'état des peuplements. - Les feuillus ont plus à souffrir que les résineux, les essences de lumière plus que les essences d'ombre. Les peuplements jeunes sont les plus malmenés par le bétail; plus aussi, on s'approche du moment de la régénération, plus le pâturage est nuisible; pour bien faire, tout bétail doit être exclu des cantons en voie de régénération dix ans au moins avant l'ensemencement et douze ou quinze ans après la naissance des semis ou des rejets; il en résulte que les bois d'âge moyen peuvent seuls être déclarés défensables; or, ceux-ci, étant les plus serrés, renferment le moins d'herbe. Quant aux forêts jardinées ou furetées, qui toujours sont mélangées de jeunes bois, le bétail ne devrait jamais y pénétrer en principe. Telle est la source de l'éternelle querelle entre usagers et propriétaires, entre cultivateurs et forestiers. 
Quand le sol est en pente rapide, qu'il soit d'une nature terreuse ou couvert de pierrailles, le parcours y augmente les chances de ravinement; dans le dernier cas, les bestiaux détachent des pierres roulantes qui occasionnent, au pied des arbres atteints, des tares, bientôt suivies de pourritures; sur les plateaux terreux ou mouilleux le plus grand dommage vient du tassement.

Conclusions. - Tels sont les faits contre lesquels on doit se prémunir. On n’y parviendra qu'à l'aide des précautions suivantes :

$1^{\circ}$ s'assurer contre les délits par une surveillance constante sur les points les plus exposés ;

$2^{\circ}$ établir une forte et solide clôture entre le sol boisé et les pâturages voisins, faire de même le long des pistes fréquentées par les troupeaux. Cette clôture sera, suivantles cas, un fossé difficile à franchir, avec les terres disposées en rempart à l'intérieur du bois, - un mur en pierres sèches, - des haies vives, - un landrage en bois brut disposé à la Suédoise, un cordon en fil de fer nu ou façonné en ronce artificielle;

$3^{\circ}$ enfin, soumettre le droit de parcours aux restrictions sévères dont on trouvera le détail aux articles 66 à 85 du Code forestier.

Constatons, en terminant, que dans toute l'Europe centrale, le $\mathrm{xIx}^{\mathrm{e}}$ siècle aura eu pour mission de réparer, dans les forêts, les désordres imputables au pâturage pendant les temps passés. Veillons !!

\section{Les incendies.}

Leurs causes et leurs conséquences. - Les incendies dans les forêts sont occasionnés, le plus souvent, par des imprudences ou par la malveillance; ils proviennent très rarement des effels de la foudre. Presque toujours, le feu, allumé sur le sol, est alimenté par des matériaux inflammables qui s'y accumulent pendant la sécheresse; il se propage parfois sur de très grandes surfaces.

Du niveau du sol, il peut s'élever jusqu'au sommet des arbres résineux, dont les aiguilles gorgées de résine sont in- 
flammables à l'état vert, et alors, de proche en proche, des massifs considérables peuvent être entièrement dévastés. Rarement il gagne la cime des arbres feuillus.

Par un temps sec, si la saison est favorable, il suffit d'un fragment d'allumette ou d'amadou encore en combustion, d'une étincelle échappée du fourneau d'une pipe ou d'un cigare, d'une bourre enflammée par la décharge d'un fusil, d'un charbon tombé du cendrier d'une locomotive pour déterminer l'embrasement presque subit d'un espace trop étendu pour qu'un homme seul puisse l'éteindre.

D'ailleurs, le nombre des incendies et la gravité de leurs conséquences varient suivant les régions que l'on considère.

Régions montagneuses. - Sous le climat humide de la haute montagne, la couverture morte est toujours mouillée et le feu ne s'y propage pas facilement; aussi, malgré la constitution des forêts en massifs résineux, les incendies sont-ils peu à craindre. Toutefois, il ne faut pas abuser de cette apparente sécurité pour commettre des imprudences: les feux qu'on allume volontairement en forêt doivent être surveillés, en montagne comme partout ailleurs; il faut éviter de les placer sous le feuillage des arbres résineux, dont les branches trop basses pourraient être atteintes par la flamme, et on ne doit jamais abandonner un brasier sans l'avoir éteint complètement.

Zone parisienne. - En plaine, dans la zone parisienne, oú les forêts sont en majeure partie peuplées d'espèces feuillues, l'incendie ne quitte guère le sol. C'est au printemps, quand les feuilles mortes sont desséchées par le vent du Nord-Est (vulgairement appelé le hâle de mars), que le feu prend; le danger existe pendant quelques semaines au plus, car il suffit que les herbes entrent en végétation pour l'écarter.

L'incendie se propage en détruisant la couverture, il endommage les parties inférieures des tiges et les portions de racines qui sont à découvert. Poussé par le vent, il marche dans la même direction que lui, et s'avance tant qu'il trouve des aliments et aussi loin que le massif se prolonge, à moins qu'une pluie abondante ou les secours l'arrêtent.

Les gros arbres en souffrent généralement peu; par contre les jeunes tiges sont presque toujours mortellement atteintes: 
il est d'ailleurs, facile, de se rendre compte de la situation en examinant les couches cambiales mises à nu par de légères incisions pratiquées au couteau sur quelques tiges de moyenne grosseur, choisies dans les cépées de toutes les essences. Dès que cette couche apparaît, teintée en noir, même légèrement, il faut prescrire le recépage immédiat; sinon, la pousse encore possible des feuilles masque la situation: il arrive que les bourgeons, grâce à l'eau qui monte dans les tissus ligneux, parviennent encore à s'ouvrir; mais la sève élaborée ne pouvant plus descendre et se diffuser par la couche cambiale nécrosée, les racines insuffisamment nourries, si elles ne sont pas complètement mortes, au printemps suivant, ne donneront plus, du moins, que des rejets chétifs et sans avenir. Dans le doute, le mieux est encore de se résoudre au sacrifice immédiat; car, si un grand nombre de tiges partiellement atteintes continuent encore à vivre tant bien que mal, on voit apparaître à leur pied, sur les zones brûlées, des plaies chancreuses qui ralentissent leur croissance et déprécient la marchandise.

Il faut veiller avec soin à la préservation des bois résineux d'origine artificielle, pour qui le danger est permanent surtout pendant les années sèches, comme l'été 1893, où l'on a vu les incendies prendre le caractère de désastres, aussi bien en France que dans la Campine Belge. Aussi, dès qu'une de ces forêts est plus particulièrement exposée par la proximité d'habitations ou d'une ligne de chemin de fer, y a-t-il lieu de procéder au nettoiement du sol et, dès l'état de gaulis, d'élaguer les branches basses jusqu'à 50 ou 60 centimètres au-dessus de terre; mais, sous cette réserve expresse que les brindilles provenant de ce travail, au lieu d'être abandonnées sur le sol, comme cela se fait trop souvent, seront emportées au loin, hors des enceintes parcourues; autrement c'est enfermer le loup dans la bergerie. En semblable: situation, il sera prudent, de la part des compagnies dé chemin de fer, dont la responsabilité est si sérieusement engagée, de s'entendre avec les propriétaires riverains de la voie et de leur fournir les subventions nécessaires-pour procéder à ces travaux de préservation, en tenant 
la main à leur exécution. La division des surfaces par de larges tranchées garde-feu, comme nous recommandons plus loin de le faire, serait encore d'un grand secours.

En général, le feu n'est pas allumé par la malveillance; personne, dans cette région, n'ayant intérêt à détruire l'état boisé.

Dès qu'un incendie est signalé, les populations riveraines doivent, au besoin, être mises en demeure d'accourir pour l'éteindre; il faut reconnaître que leur bonne volonté ne fait jamais défaut, et que, le plus souvent; leur concours est spontané. Les hommes arrivent munis de pelles, de pioches et de râteaux; si l'incendie ne présente qu'un foyer peu actif et peu étendu, on éteint le feu en le piétinant, en le couvrant de jets de terre, ou en le frappant avec des branchages; toutefois ce dernier moyen n'est pas trop à recommander, car, en lançant des flammèches dans toutes les directions, on peut allumer de nouveaux foyers en arrière des travailleurs, On attaque le feu de préférence par ses flancs et dans le sens de sa marche, en cherchant à rétrécir de plus en plus la largeur du front jusqu'à fermeture complète.

Quand le foyer est trop ardent pour qu'on puisse l'approcher et, pour ne pas exposer ses hommes à des accidents, le chef des travaux se transporte avec une bonne équipe en avant du feu et dans sa direction; là, les travailleurs, avec des râteaux, débarrassent une bande de terrain de tous les matériaux combustibles; celte bande est tracée perpendiculairement à la direction du feu et à une distance suflisante pour qu'on ait le temps d'achever le travail avant son arrivée ; il s'éteint, alors, faute d'aliments. Si, néanmoins, la flamme franchit cet obstacle, elle a, du moins, perdu sa violence, et on l'étouffe. Ordinairement il suffit de donner à la tranchée une largeur de 2 à 3 mètres.

L'incendie réprimé, on doit veiller sur le théâtre du feu jusqu'au moment où il n'y a plus à craindre de le voir se ranimer. Il faut remarquer qu'un tison peut couver longtemps encore dans les arbres creux, dans les troncs pourris, dans les racines et qu'il faut l'éteindre parfois, soit en l'inondant, soit en l'étouffant sous des jets de terre. 
Régions méridionales. - Dans les climats méridionaux, et, plus particulièrement dans l'Esterel et dans les landes de Gascogne, les incendies sont le fléau des forêts résineuses.

La fécondité du climat, la nature siliceuse du terrain font naître à profusion, sur le sol des forêts, des plantes socialse qui se dessèchent en été et couvrent la terre de matières inflammables. Les forêts en terrain calcaire avec leur flore plus variée et surtout plus riche en arbustes à feuilles persistantes, sont, à ce point de vue, beaucoup moins exposées que les précédentes.

Dans les forêts des Maures, dit M. Faré (1), sous le couvert des pins maritimes et des chênes-liège qui, avec le chataignier, forment la grande masse des peuplements, la végétation arbustive se développe șur le sol en buissons impénétrables, les cystes et la bruyère arborescente sont, de beaucoup, les espèces les plus dangereuses. Pendant la saison chaude, ce sous-étage, à l'état naturel dans tous les massifs de la contrée, parvient à un tel degré de dessiccation, qu'il suffit d'une étincelle pour produire la combustion. Lorsque le feu s'est déclaré, il se propage avec une rapidité plus ou moins grande suivant la violence du vent, et on est disposé a admettre que, si le mistral souffle avec force, tous les moyens employés pour combattre l'incendie sont, le plus souvent, inefficaces et dangereux pour les travailleurs. Dans ces circonstances, les cônes de pins seraient un agent de propagation des plus dangereux; de nombreux témoins occulaires affirment, en effet, que, sous l'influence de la chaleur de l'incendie, ces cônes encore verts éclatent et que leurs débris enflammés peuvent allumer de nouveaux foyers à plusieurs centaines de mètres de leur point de départ; les flammèches et les fragments des écorces de résineux, qui pétillent sous l'action du feu, concourent au même résultat, de sorte qu'il n'est pas rare de voir de nouveaux incendies se multiplier en dehors de la ligne oủ les travailleurs combattent la marche du feu. Ces conditions font de l'arrêt de l'incendie une opération toujours délicate. Le plus souvent, les secours, quelques dévoués qu'ils soient, restent impuissants contre les forces aveugles du fléau et le feu ne s'arrête que lorsqu'il n'y a plus rien à dévorer.

Ces incendies proviennent, pourla plupart, de l'imprudence des fumeurs, des chasseurs, des ouvriers charbonniers ou de ceux qui pratiquent les écobuages. On dit même que le feu peut être allumé et communiqué à distance par les dégagements d'huiles essentielles odorantes, produits, pendant les grandes chaleurs, par certaines plantes de la famille des la-

(1) Faré, Enquête sur les causes des incendies dans la région des Maures et de l'Esterel, Imprimerie nationale, 1869. 
biées : lavande, thym, serpolet etc...; cette assertion aurait besoin d'être confirmée. On a malheureusement trop de preuves qu'ils doivent aussi être attribués à la malvẹllance.

Il est rare que le foyer conserve la forme du feu courant sur le sol; presque toujours, il monte dans les cimes des pins, entraînant la mort de tous les arbres atteints, quand même il ne s'attaquerait qu'aux brindilles sèches et aux feuilles ou aiguilles; le plus souvent, d'ailleurs, les arbres ne sont consumés en entier que lorsqu'ils sont creux. Les cantons ainsi parcourus doivent être exploités, el, dans les massifs trop jeunes pour donner de la semence, les espèces feuillues reparaissent seules sous forme de rejets ou de drageons.

Le seul moyen efficace de diminuer les chances de sinistres est le débroussaillement. L'opération est malheureusement trop coûteuse pour qu'on puisse l'imposer à tous les propriétaires; cette dépense est évaluée en moyenne à 80 ou 100 francs par hectare, pour la première opération; les débroussaillements ultérieurs, qui doivent être répétés à des intervalles de 5 à 10 ans, coûtent, suivant les difficultés locales, de 5 à 10 francs par hectare. Dans l'impossibilité de détruire la cause des incendies, on a eu recours à une loi spéciale promulguée les 6 juillet-3 août 1870, pour en limiter les effets. Cette loi interdit l'usage du feu, même pour les exploitations forestières ou agricoles usitées sous la dénomination d'écobuage, taillards, issards et petit feu, en dehors des époques déterminées par des arrêtés préfectoraux. Elle prescrit, en outre, l'ouverture et l'entretien de tranchées garde-feu, soigneusement défrichées et d'une largeur de 20 à 50 mètres entre deux propriétés contiguës et non débroussaillées. Si cette loi était sévèrement appliquée, on pourrait en attendre de sérieux résultats.

Dans les landes de Gascogne (1), les forêts sont de création récente; elles sont en presque totalité peuplées de pins maritimes que l'on résine. Comme dans les Maures, les incendies se propagent sous l'influence de la végélation buissonnante extrêmement touffue et formée d'ajoncs, de bruyères, de fou-

(1) Rapport de M. Faré, Enquéte sur les incendies dans la région des landes de Gascogne. Imprimerie nationale 1873. 
gères et de diverses plantes herbacées parmi lesquelles il faut citer la Canche en gazon (Aira cespitosa).

Les causes des incendies sont les mêmes que partout ailleurs, mais ici il faut attribuer une beaucoup plus large part aux accidents causés par la circulation des locomotives et, aussi, à la malveillance. On a constaté, en effet, que, trop souvent, surtout dans les forêts plantées en exécution de la loi de 1857 , les bergers allument le feu, volontairement, dans le but de détruire un état boisé qui entrave la jouissance des terrains autrefois livrés au parcours. Les meilleures précautions à prendre sont encore le débroussaillement et l'ouverture de tranchées garde-feu peuplées d'essences feuillues et plus spécialement de chêne pédonculé et de chênes américains : Q. tinctoria, Q. phellos, Q. palustris...

Quoiqu'il en soit, dans ces deux régions, le feu a déjà causé des pertes immenses. Comme on est sans cesse exposé à de nouveaux ravages, il faut savoir comment s'y prendre pour arrêter la marche de l'incendie.

Au premier signal, dit M. Faré (1), il faut réunir sur le lieu du sinistre des travailleurs en grand nombre, placés sous une direction éclairée et munis des instruments nécessaires. Mais, comme la population est peu dense et les chemins aussi peu nombreux, il y a dordinaire bien du temps perdu avant que les secours soient organisés. Les habitants d'une commune une fois réunis devraient être placés sous la conduite d'un homme compétent qui imprimerait à tous les efforts une direction unique. Il arrive souvent, en effet, que, par suite du défaut de commandement, les secours se divisent au grand détriment du résultat à obtenir, et la vie des travailleurs se trouve même parfois menacée. Le manque d'instruments de travail paralyse parfois le dévouement, et, à ce propos, on a fait remarquer la convenance de placer dans les cantons habités un dépôt d'outils appropriés à ce genre de travail auquel peut participer toute la population. Les mesures à prendre consistent d'ordinaire à disposer les travailleurs sur une route ou sur une ligne de pare-feu parfaitement débarrassée de matières combustibles. Chacun d'eux est muni d'une perche-garnie de ses feuilles vertes et c'est en frappant les parties embrasées soit sur le périmètre de la ligne, soit en arrière, lorsqu'un nouveau foyer produit par des flammèches portées au loin vient à éclater, qu'on arrête l'incendie. Cette opération suppose l'existence d'une route ou d'un parefeu ; mais, dans tous les autres cas, c'est en plein massif qu'il faut agir et alors il convient de se donner une base d'opération. On commence

(1) Rapport ci-dessuş mentionné. 
done par alattre les bois sur une largeur déterminée de manière å former une ligne déblayée d'arbres et de morts-bois; on établit ensuite un fossé dont on rejette les terres du côté du foyer et c'est sur cette ligne qu'on combat la marche du feu. Dans certaines circonstances, quand l'incendie est attisé par un vent violent, il.y aurait témérité à attendre le feu sur une ligne étroite, telle qu'on peut l'établir pendant la marche du feu; on recourt, alors, au contre-feu, pratique dangereuse dans ses applications, et qui exige de la part de ceux qui l'emploient beaucoup de tact et de mesure. Le contre-feu peut-être considéré comme une arme à deux tranchants; c'est parfois le seul remède efficace à employer, mais s'il est appliqué mal à propos, il peuf provoquer un nouvel incendie.

Il faut aussi se préoccuper de l'alimentation à fournir sur place aux travailleurs qui, entendant sonner le tocsin, quittent tout et s'empressent de se rendre à l'appel. Cette question, secondaire en apparence, est signalée comme ayant eu une importance capitale sur des points éloignés de tout centre habité où les travailleurs, à bout de force et manquant de nourriture, étaient contraints de suspendre la lutte.

\section{ARTICLE III}

\section{DÉGATS DES ANIMAUX}

Les mammifères. - Les oiseaux. - Les insectes.

La forêt abrite et nourrit nombre d'animaux : mammifères, oiseaux et insectes, parmi lesquels, pour quelques espèces utiles, on en compte une foule de nuisibles. Une revue rapide de tout ce grand et petit monde nous permettra de distinguer les amis des ennemis, et de traiter chacun comme il le mérite pour le plus grand bien des massifs.

Les mammifères. - Les carnassiers ne font jamais de mal aux arbres et, si l'on ne tenait pas compte des pièces de gibier ou des animaux domestiques qu'ils dérobent, loups et renards pourraient être considérés plutôt comme utiles que comme nuisibles, car l'un et l'autre détruisent une grande quantité de campagnols. Les renards se nourrissent aussi d'insectes et notamment de hannetons à l'état de larves ou d'insectes parfaits.

Les sangliers rendent quelques services par les insectes et 
les souris qu'ils détruisent, et par l'ameublissement qu'ils donnent au sol en le fouillant de leurs vermillures. Ils sont néanmoins nuisibles, en ce sens qu'ils mangent une grande quantité de semences, ravagent les pépinières et bouleversent les semis et les plantations. Ils fouillent de préférence les potets ou les rigoles fraîchement travaillés; ce n'est pas, comme on le suppose généralement, pour mâcher les racines des jeunes plants, mais pour chercher les vers et les autres animaux qu'ils trouvent en plus grande abondance dans le sol ameubli. Mais c'est, surtout par les dégâts qu'ils causent dans les cultures riveraines des forêts que la préșence des sangliers est redoutable : du mois de mars au mois de novembre, quand toutes les graines forestières ont germé ou sont pourries, ils ne trouvent plus à se nourrir sous bois et forcément ils vont dévaster les récoltes. L'action en responsabilité de ces dommages est, d'ailleurs, assez délicate à intenter, car le sanglier est un animal essentiellement nomade.

Les bêtes fauves: cerfs, daims et chevreuil, lorsqu'elles fraient leur tête pendant le refaît des bois, occasionnent sur les perches des blessures, auxquelles, en langage de chasse, on donne le nom de frayoirs (fig. 69 b). Au printemps, ces mêmes animaux broutent les jeunes bourgeons et attaquent l'écorce de certaines essences, notamment du charme, qu'ils arrachent en longues lanières. On dit que cette nourriture les enivre. En hiver, quand la neige les empêche de pâturer, ils attaquent brindilles, écorces et bourgeons. A cet égard les daims font moins de mal que les chevreuils et les cerfs, qui absorbent de plus une grande quantité de glands.

Quand une épaisse couche de neige recouvre le sol, les lièvres rongent, à la base, l'écorce des jeunes arbres, surtout des fruitiers; dans les pépinières, ils sont aussi très friands des jeunes pousses des espèces légumineuses, telles que : robinier, cystise, genêt, etc.

Les lapins sont certainement les hôtes les plus dangereux de la forêt; ils recherchent les terrains sablonneux meubles qui s'égouttent rapidement, et affectionnent les cantons traités en taillis sous futaie, les pineraies claires où le sol est toujours sec. Ils évitent les grands massifs de hêtre sous 
le couvert continu desquels règne une humidité constante. Partout où ils s'installent, les lapins fouillent le sol, rongent les écorces jusqu'au bois, broutent les jeunes semis, déracinent les plants : rien de ce qui est vivant n'échappe à

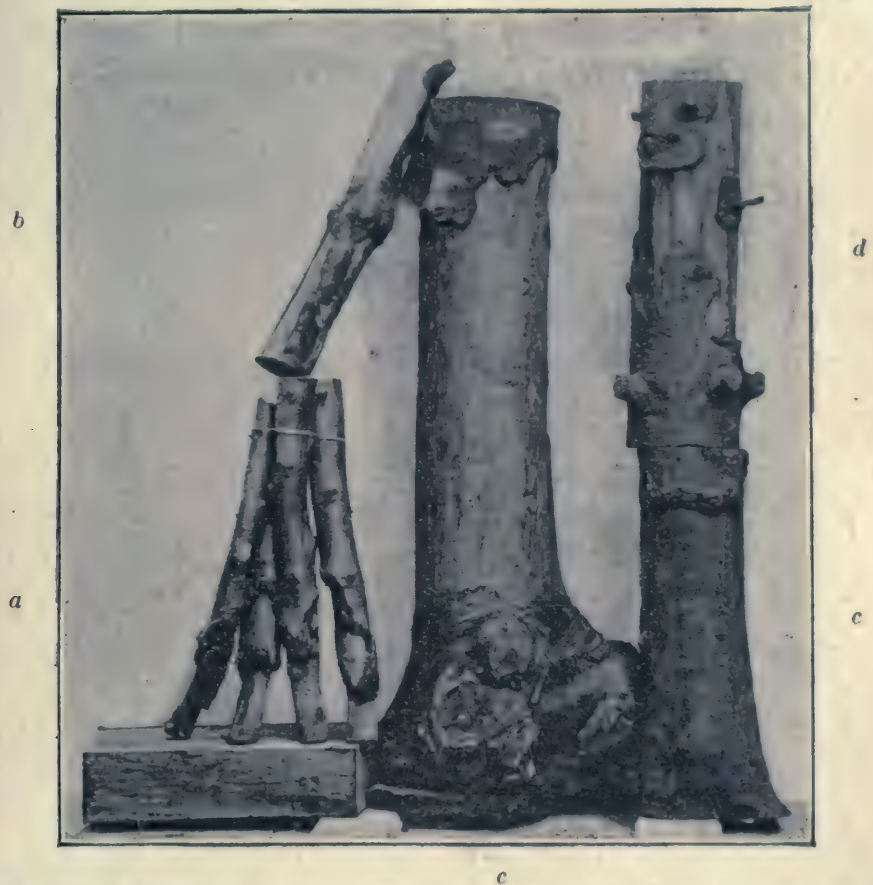

Fig. 69. - Dégâts des mammifères : $a$, brins de charme rongés à leur base par les campagnols: $b$, frayoirs de bète fauve sur une perche de hêtre; $c, c$, tiges de frêne et de hêtre attaquées par les lapins; $d$, cime d'épicéa décortiquée par les écureuils.

la voracité et à l'instinct de destruction de ces rongeurs; en cas de famine, ils s'attaquent au bois vif et même au bois sec (fig. $69 c . c$.). De plus ils se multiplient, en peu de temps, d'une façon effroyable, et foisonnent pour ainsi dire à la manière des insectes : aussi le lapin doit-il être exterminé par tous les moyens possibles et banni de toute forêt bien tenue. Le moyen le plus économique d'empêcher leur propagation serait de s'opposer à la destruction des renards, fouines, 
belettes..., etc., ses ennemis naturels, à qui la nature a dévolu le rôle d'en arrêter la multiplication exagérée. Il est vrai que toute mesure de ce genre sera incompatible avec une exploitation luxueuse de la chasse.

Toute réserve étant faite à l'égard du lapin, on peut dire que le gibier n'occasionne à la forêt qu'un mal-insignifiant. D'ailleurs, dans le cas où l'une ou l'autre espèce viendrait à se multiplier d'une manière inquiétante, on possède dans la chasse un moyen efficace d'en diminuer le nombre. De tels dangers sont trop rarement à craindre en France; il est même à désirer qu'une législation mieux entendue permette un jour de mettre à profit les facilités naturelles que la forêt présente à la production du gibier, pour augmenter son rendement et procurer à l'alimentation publique des ressources qu'on emprunte aujourd'hui à l'étranger.

En dehors du gibier proprement dit, les espèces les plus dangereuses appartiennent au groupe des rongeurs.

Les écureuils coupent un grand nombre de cônes et de jeunes bourgeons; faute de mieux, ils rongent jusqu'au bois la jeune écorce des sapins, des épicéas et des hêtres; à ce point de vue, il est bon de ne pas les laisser trop se multiplier (fig. $69 d$ ).

Les souris détruisent une énorme quantité de semences, non seulement pour leur nourriture, mais encore pour obéir à un instinct de prévoyance outréc, qui les porte à faire des provisions considérables, - un décalitre et plus, - auxquelles souvent elles ne touchent même pas. En hiver, quand les fruits leur font défaut, ces animaux, et particulièrement les campagnols, s'attaquent à l'écorce des jeunes brins, surtout des charmes et des coudriers, qu'ils rongent sur une hauteur de plusieurs centimètres. Les tiges ainsi attaquées sont perdues et il est nécessaire de les recéper (fig. 69 a). Ces petits rongeurs apparaissent parfois subitement, en très grand nombre, sous forme d'une véritable invasion; leurs dégâts sont alors considérables; on a des exemples de superficies de 500 hectares, et plus, dévastées pendant un seul hiver; la nature heureusement pourvoit elle-même à leur destruction, et il est rare qu'ils se montrent en nombre exagéré 
pendant plusieurs années de suite. D'ailleurs, pour les combattre, on ne possède aucun moyen pratique applicable sur de grandes surfaces comme celles des forêts; le mieux serait de ne pas détruire, par préjugés ou par plaisir mal entendu, les animaux qui leur font la guerre : le hibou, la buse, le milan, le renard et le chat; mais on se trouve, ici encore, en présence d'un intérêt contraire à celui des chasseurs.

Les oiseaux. - Parmi les espèces dites forestières, les rapaces diurnes et surtout les rapaces nocturnes sont utiles en détruisant une grande quantité de rongeurs.

Les passereaux granivores sont parfois nuisibles à cause des fruits qu'ils mangent et des dégâts qu'ils commettent dans les pépinières au moment du semis et de la germination des graines résineuses: les jeunes plantules ont alors un attrait irrésistible pour les pinsons. A leur décharge rappelons qu'à l'époque des nichées tous les oiseaux détruisent des insectes.

Les insectivores passent, à juste titre, pour utiles. Sans doute, il ne faut pas compter sur eux pour arrêter une invasion, et, parmi les insectes mêmes, les Ichneumonides les Tachinines et bien d'autres, qui vivent en parasites, sont d'une action plus efficace; néanmoins l'oiseau contribue à maintenir dans la multiplication de tous ces ennemis des forêts et des vergers un équilibre qu'il est imprudent de détruire. La raquetle, qui brise les pattes des rouges-gorges et des fauvettes, est d'une cruauté révoltante; le collet, qui étrangle les grives et les merles, la pipée, qui fait des hécatombes de mésanges, sont également néfastes. Toutes ces petites chasses s'exercent de préférence dans les boqueteaux et sur les lisières des massifs; aussi, tout en dépeuplant leurs ombrages, privent-elles les parcs, les vergers, les jardins et les champs de leurs plus aimables auxilaires.

Nous appelons du fond du cour la convention, jusqu'alors trop platonique, qui assurera la protection internationale de la gent ailée qui nous aide et nous égaye.

Les pics s'attaquent toujours au bois vermoulu ou piqué pour rechercher les larves dont ils se nourrissent; mais le

(1) H. de Blanchère, Les oiseaux utiles et les oiseaux nuisibles, 5e édition. Paris, 1889. 
$D^{\text {r }}$ Altum (1), les accuse de faire sur l'écorce des tilleuls des séries de piqûres mathématiquement alignées en forme de couronne autour du tronc : cette fois ce n'est plus pour chercher les insectes, mais pour percer les petites ampoules cachées sous l'écorce et boire une goulte de la sève sucrée qu'elles renferment. Les pics fréquentent de préférence les forêts mal tenues, où l'on rencontre une grande quantité de bois morts sur pied, car c'est là qu'ils trouvent le plus facilement leur nourriture. A défaut d'arbres dépérissants, ils émigrent, plutôt que de peiner à jeun contre le bois sain.

Les insectes. - Les forêts ont à supporter de la part des insectes des dommages considérables, et qui, dans certains cas, peuvent atteindre la proportion de véritables calamités publiques (2). Dans le grand nombre des espèces nuisibles, chacune des parties vivantes de l'arbre trouve son ennemi spécial : les uns dévorent les feuilles ou les aiguilles, les autres rongent la couche d'accroissement, ou creusent leurs galeries dans le bois ; un certain nombre mangent les racines, les bourgeons, les fleurs ou les fruits.

Quelle que soit la nature de ces ravages, les résineux ont beaucoup plus à souffrir que les feuillus. Ces derniers, pourvus de bourgeons proventifs, réparent plus facilement leurs frondaisons détruites; d'ailleurs, ils cicatrisent mieux leurs plaies, aussi succombent-ils rarement et les dégâts les plus importants se traduisent-ils par un ralentissement sensible dans la végétation et des pertes de substances assez notables (3). Les résineux, au contraire, périssent en grand nombre quand leurs aiguilles sont dévorées; les plaies amènent des écoulements de résine qui épuisent les arbres; enfin, la destruction du bourgeon terminal les déshonore et diminue leur valeur industrielle.

(1) U'nsre Spechte und ihre forstliche Bedeuting. Berlin, 1878.

(2) En 1791-93, une invasion du bombice du pin a détruit 23,000 hectares de forêts dans une seule province d'Allemagne.

(3) On peut facilement constater sur la section transversale d'un chêne la trace de ces dégâts : les couches ligneuses correspondant aux années où la frondaison a été détruite par les hannetons, sont réduites à une épaisseur souvent insignifiante. 
En général, chaque espèce d'insecte vit sur un arbre déterminé. Aussi, quand, sous l'influence de circonstances favorables, l'une ou l'autre se multiplie en nombre exagéré, les cantons peuplés de l'essence qui la nourrit sont-ils très compromis. Dans les peuplements purs, il n'est pas rare que des surfaces considérables soient dévastées : on est alors obligé d'abattre le massif entier pour tirer parti des bois dépérissants ou morts et pour empêcher la propagation du mal; on a même été contraint parfois d'avoir recours à l'incendie.

La principale cause des invasions tient à la grande fécondité des insectes, qui permet à leur nombre de s'augmenter en progression géométrique, quand l'influence des milieux est favorable à leur développement. Parmi les circonstances qui activent cette multiplication, nous citerons :

$1^{\circ}$ l'abondance de nourriture convenable;

$2^{\circ}$ l'absence d'ennemis;

$3^{\circ}$ la température.

Les forestiers conservent, dans certaines limites, une action sur les deux premières circonstances; ils sont absolument impuissants en présence de la troisième.

Pour justifier les moyens préservatifs à employer contre ces redoutables fléaux, il est nécessaire de diviser les insectes nuisibles en deux groupes: les insectes lignivores et les phyllophages.

Les insectes lignivores sont ceux qui perforent le bois constitué comme : Bostrichus lineatus, Sirex gigas, dans les résineux ; Cossus ligniperda, Zeuzera osculi, dans les bois feuillus; - ceux qui creusent leurs galeries dans le liber et l'écorce comme : Bostrichus typographus, si redoutable dans les forêts d'épicéa, Itylobius abietis dans les forêts de pin sylvestre, Scolytus ulmi chez les ormes; - ceux qui détruisent les jeunes pousses et les bourgeons, comme : Iyylesinus piniperda, Tortrix buoliana, T. turionana; - ceux enfin qui, vivant dans le sol, rongent le chevelu des racines, tels sont: la larve du hanneton commun (Melolontha vulgaris) et la courtilière (Grillotalpa vulgaris) dans toutes les phases de son développement. IEt nous n'arons prétendu nommer ici que les types 
de familles dans lesquelles les espèces nuisibles sont légion.

Les plus redoutables parmi les phyllophages, c'est-à-dire ceux qui détruisent les feuilles et les autres parties vertes des végétaux, sont: dans les forêts résineuses, les chenilles de Lasiocampa pini, Liparis monacha, Fidonia piniaria, Lophyrus pini; et, dans les forêts feuillues, celles de Orgya pudibunda, Bombyx neustria, Bombyx processionnea, de Pyrales et de Tinéides. En de certaines régions, le hanneton commun à l'état parfait exerce périodiquement ses ravages.

A chacun de ces groupes correspondent des moyens préventifs spéciaux.

$1^{\circ}$ Presque tous les insectes lignivores ont besoin, pour se développer en grand nombre, de bois morts, dépérissants ou atteints de maladies. L'abondance de ces matériaux dans une forêt crée un foyer de contagion oú les insectes se multiplient en telle quantité que les arbres qui leur ont servi de berceau deviennent bientôt insuffisants; les bois en bon état de végétation sont attaqués, à leur tour, et menacés d'une perte imminente. Pour empêcher la formation de ces foyers, il faut : entretenir les peuplements à l'état sain par des soins appropriés, - cultiver les essences adaptées au sol et au climat et leur appliquer un traitement rationnel, - donner la préférence aux peuplements mélangés et surtout au mélange de feuillus et résineux, - exploiter radicalement et annuellement les bois morts ou dépérissants, - écorcer totalement les arbres résineux dès qu'ils sont abattus, et, pour rendre l'opération possible, les couper de préférence en temps de sève, - enlever les produits aussitôt après le façonnage et ne pas laisser de bois gisants en forêt.

$2^{\circ}$ Sauf les cas d'invasions, les insectes qui mangent les feuilles ou les aiguilles trouvent toujours la nourriture qui leur convient; il n'est pas possible, comme pour les lignivores, d'augmenter ou de diminuer l'élément principal de leur multiplication. On reste donc à peu près désarmé contre eux; cela est d'autant plus regrettable que ce groupe renferme les espèces les plus à craindre pour les bois résineux comme pour les bois feuillus. 
De plus, lorsqu'ils ne font pas périr complètement les massifs, ils les affaiblissent au point de préparer les invasions des xylophages ; ceux-ci achèvent fatalement l'œuvre de destruction.

Les seuls moyens à recommander sont : l'éducation de peuplements mélangés adaptés au climat et au sol ; - la conservation de la couverture morte dans les forêts et la protection des animaux insectivores utiles.

Mais ces moyens restent souvent inefficaces et, quel que soit le groupe auquel il appartiendra, la meilleure sauvegarde contre un insecte sera la connaissance complète de ses mours. Il est rare qu'une invasion se produise soudainement, - le cas se présente néanmoins - : en général, elle marche progressivement et celui-là seul qui possède une connaissance exacte des faits peut la prévenir et la combattre. Le plus sûr sera donc d'avoir immédiatement recours à un homme compétent à qui l'on adressera, avec les exemplaires de l'insecte en cause, un échantillon des dégâts dont on l'accuse et des fragments de toutes les parties dégradées ou suspectes de l'arbre; car, bien souvent, les dégâts d'un insecte ne sont que le corollaire de l'attaque préalable du sujet par une autre espèce ou par un champignon. Ce spécialiste pourra, en même temps que sa consultation, renvoyer aux ouvrages et articles d'entomologie qui traitent la matière.

\section{ARTICLE IV}

\section{DOMMAGES CAUSES PAR LES VÉGETAUX.}

Les plantes sarmenteuses. - Les plantes parasites. - Le gui. Les champignons. - Les bactéries.

Pas plus que nous l'avons fait pour les animaux, nous ne pouvons, au sujet des végétaux nuisibles, entrer dans tous les détails que comporte une branche aussi importante de l'histoire naturelle appliquée aux forêts. Le simple aperçu qui va suivre n'a d'autre but que de signaler les principaux dangers, et d'engager les intéressés à recourir, en cas de besoin,' aux botanistes autorisés:

Boppe et JoLyet. 
Sans nous occuper du tapis végétal, nous ne parlerons que des espèces directement nuisibles aux arbres. Celles-ci sont toujours ou sarmenteuses ou parasites; et, parmi ces dernières, plus on étudie la forêt, plus on est frappé de l'importance énorme que prennent les champignons.

Sous le climat de la France, les plantes sarmenteuses nuisibles appartiennent aux classes supérieures du monde végétal : le lierre, les clématites et le chèvre-feuille des bois sont ligneux; les crampons et les circonvolutions de leurs tiges fatiguent et dégradent les sujets qui leur servent de support; il y a lieu de les extirper partout où on les rencontre quels que soient l'âge et la forme des peuplements (fig. $70 \mathrm{c}$ ).

Le gui vit en parasite sur un grand nombre d'essences feuillues ou résineuses. Bien qu'il soit très rare sur le chêne, on l'y rencontre néanmoins; c'est, sans doute, en raison de cette rareté extrême que les Druides l'avaient choisi comme plante sacrée. Mais Vellèda, notre patronne, avait dû révéler à ses prêtresses les procédés d'en multiplier les buissons nécessaires à l'entretien du culte.

En général, le gui fait peu de mal aux bois feuillus, parce que, installé sur leur cime, ses racines ne dégradent que les régions destinées à être débitées en bois à brûler. Sur les résineux, sur les sapins surtout, ses racines traçantes, partent des branches sur lesquelles la touffe a pris naissance et peuvent atteindre le tronc, où elles laissent des traces profondes et causent de sérieux dommages (fig. $70 \mathrm{~b}$ ). Amputer les branches envahies par le gui, c'est évidemment empêcher sa propagation par les oiseaux qui se nourissent de ses baies; mais le moyen est peu pratique; le vrai reméde serait, au passage des éclaircies, d'enlever de préférence les sapins qui portent de ces buissons.

Etant donnés les ravages qu'ils engendrent, les champignons peuvent être comparés aux insectes les plus nuisibles. De même que ces redoutables ennemis de la culture forestière, les champignons s'attaquent aux arbres, qu'ils endommagent ou font mourir; - aux massifs, qu'ils éclaircissent ou détruisent en tuant les sujets qui les constituent; - au bois, qu'ils rendent impropre à tous usages. Quelques exemples. 
permettent de se rendre compte de ces faits; citons: OEcidium elatinum, qui fait le balai de sorcières et, sans tuer le sapin, provoque le grave défaut connu sous le nom de chau-

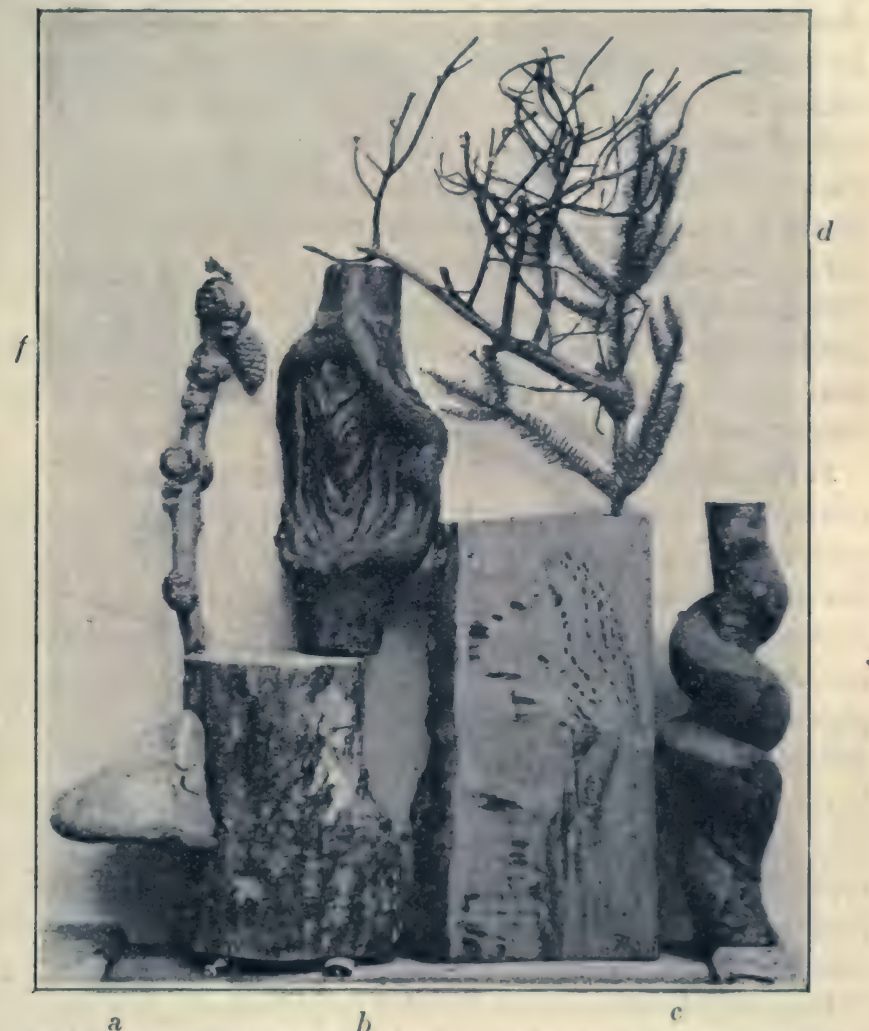

Fig. 70. - Dégâts des végétaux : a, fructification en épaulette d'un polypore qui ravage une tige de sapin $; b$, plateau de sapin portant les traces des racines du gui; $c$, tige de hêtre déformée par les circonvolutions du chèvrefeuille des bois; $d$, balai de sorcière sur une tige de sapin ; au point d'insertion, on peut voir le renflement qui donnera un chaudron; $\boldsymbol{e}$, tige de charne déformée par le chancre (Nectria ditissima); $f$, nodosités causées par la bactérie du pin d'Alep.

dron (fig. $70 \mathrm{~d}$ ) ; C'coma pinıtorquum, qui déforme simplement les tiges; Peridermium pini (var. corticola), Agaricus melleus, Trametes radiciperda, qui tuent un grand nombre 
d'arbres dans les pineraies; Rhizoclonia quercina, qui s'attaque aux forêts de chêne; Peziza calycina, qui atteint le mélèze et fait périr des arbres en pleine vigueur. On peut remarquer ce fait que les conifères sont plus exposés aux attaques des champignons que les feuillus; sous ce rapport l'analogie se continue entre la nocuité de ceux-ci et celle des insectes. Quant à l'action funeste exercée sur le bois, elle n'est pas moins redoutable pour les feuillus que pour les conifères: c'est ainsi que Hydnum diversidens, Telephora perdix, Polyporus sulfureus, Polyporus igniarius, détruisent le bois de chêne, - comme le font, des bois de sapin, de pins et d'épicéas : Polyporus fulvus, Polyporus vaporarius, - tous produisant chez les uns et les autres les vices connus depuis longtemps sous les noms de : rouge, grisette, etc..., sans qu'on les eût rapportés à leur véritable cause.

Si l'on commence à connaître assez bien bon nombre de champignons nuisibles aux forêts à des titres divers, non seulement dans leur structure, mais encore dans leur mode de développement et dans l'action qu'ils exercent sur le végétal ligneux dont ils sont l'hôte, on n'est pas toujours à même de mettre les forêts à l'abri de leurs dégâts. Les spores de champignons sont d'une extrême ténuité qui facilite singulièrement leur transport par les vents; elles sont aussi très nombreuses; enfin, assez fréquemment, elles germent sur un végétal très différent de celui sur lequel s'est constitué le corps reproducteur qui leur a donné naissance, et ce végétal est parfois ignoré : c'est le cas, par exemple, pour Ecidium elatinum.

Cependant, même dans cet état imparfait de la science, il est certaines mesures de protection dont l'efficacité n'est pas à dédaigner. D'une façon générale, il sera bon de supprimer tous sujets sur lesquels se sont développés les corps reproducteurs; quand il s'agit des espèces dont les spores ne germent que sur le bois, - souvent même, que sur le bois de cœur, on devra éviter, avec le plus grand soin, de le mettre à nu, proscrire, par conséquent, tous les élagages sur les essences comme le chêne et le sapin, très sujettes à l'altération de leurs tissus ligneux. Enfin, pour quelques espèces, il y aura d'autres 
mesures spéciales à prendre, tel, l'Agaricus melleus, qui produit dans les pineraies la maladie dite $d u$ rond, parce qu'elle s'étend en cercle à partir des premiers arbres attaqués et qui se propage sous terre au moyen d'une forme spéciale de mycélium, connu sous le nom de rhizomorphe; on le combat avec efficacité par la suppression, non seulement des sujets atteints, mais encore de ceux qui les entourent immédiate. ment, et par une culture du sol après extraction de toutes les racines, - ou, tout au moins par l'ouverture d'un fossé d'isolement.

Ce serait sortir de notre cadre que parler des bactéries et autres infiniment petits, dont, paraît-il, nous avons autant à craindre pour nos forêts que pour nos personnes. Témoin, ces nodosités dues au travail de formes spéciales, parmi lesquelles on peut excuser les espèces qui agrémentent cerfains rameaux d'enjolivures variées, au grand profit des fabricants de cannes ou de manches de parapluie. Mais les autres?...

En résumé, si tout forestier ne peut pas être initié aux secrels les plus intîmes de la mycologie, chacun peut, du moins, diagnostiquer la présence des champignons, soit par leurs fructifications en forme de chapeau, d'épaulettes ou de toufles, soit par les lames de mycélium dont les filaments, à la façon de radicelles, serpentent sous l'écorce ou dans les tissus du bois. Dès que ces signes apparaissent, il faut, sans hésiter, abattre et enlever l'arbre qui les porte; car, désormais, on ne peut rien attendre de lui que pourriture progressive, et, de plus, il devient un bouillon de culture, d'où s'échappent des milliards de spores avides de nouvelles victimes (fig. 70 a).

Disons, en passant, que les champignons ne s'attaquent pas seulement aux arbres sur pied, ils détruisent aussi le bois après son emploi. Il appartient à la technologie forestière et à l'art du constructeur d'indiquer les moyens à employer pour meltre le bois en ceuvre à l'abri de ces dangereux parasites. 
ARTICLE V

\section{DÉGATS CAUSÉS PAR LES MÉTÉORES.}

Généralités. - Le vent : troisième règle d'assiette et massifs de protection. - Le froid et les tares qu'il engendre. - Les coups de soleil. - La foudre. - La neige et les avalanches. - La grêle, le givre et le verglas.

Généralités. - Nous avons établi que, sur chaque point, la forêt spontanée est la résultante de tous les phénomènes météoriques d'une suite en quelque sorte indéfinie d'années; par leur longévilé, les arbres ou les peuplements ont traversé les saisons les plus excessives: les hivers les plus rigoureux, comme les étés les plus secs. Une telle forêt n'a donc rien à craindre des effets normaux du climat dont elle est fonction; et, même, les accidents sporadiques, dont les actions mauvaises dépassent la moyenne, n'y atteignent que les individus sans compromettre l'existence de l'organisme en tant que terrain boisé : ce ne sont qu'épisodes dans son processus vers les fins de la nature.

Mais, ce qui est vrai pour la forêt en soi, ne s'applique plus à nos sépies aménagées. Celles-ci, en effet, sont composées d'arbres ou de peuplements savamment distribués en raison de leur âge et de leurs dimensions; toute action qui, à un moment donné, change l'état d'un seul des éléments dans un tout si bien organisé, en détruit l'harmonie générale.

En cela, la culture forestière est encore bien différente de la culture agricole. Quand un accident météorique supprime la récolte pendante de céréales ou autres plantes annuelles, là se borne la perte ; l'instrument de production, la terre, reste indemne, et, dès l'année suivante, les choses reprenant leur cours normal, le cultivateur continue à toucher son revenu moyen. En forêt, au contraire, le météore brutal frappe au hasard jeunes et vieux : les bois en croissance, comme les bois mûrs ; derrière lui, le revenu gâché est compromis pour un temps dont la durée dépasse parfois l'existence du propriétaire. 
DÉGats CAUSÉs PAR LES MÉTíores.

Quand bien même la tourmente épargne les plus vieux cantons, toute avarie dans le capital générateur amoindrit son

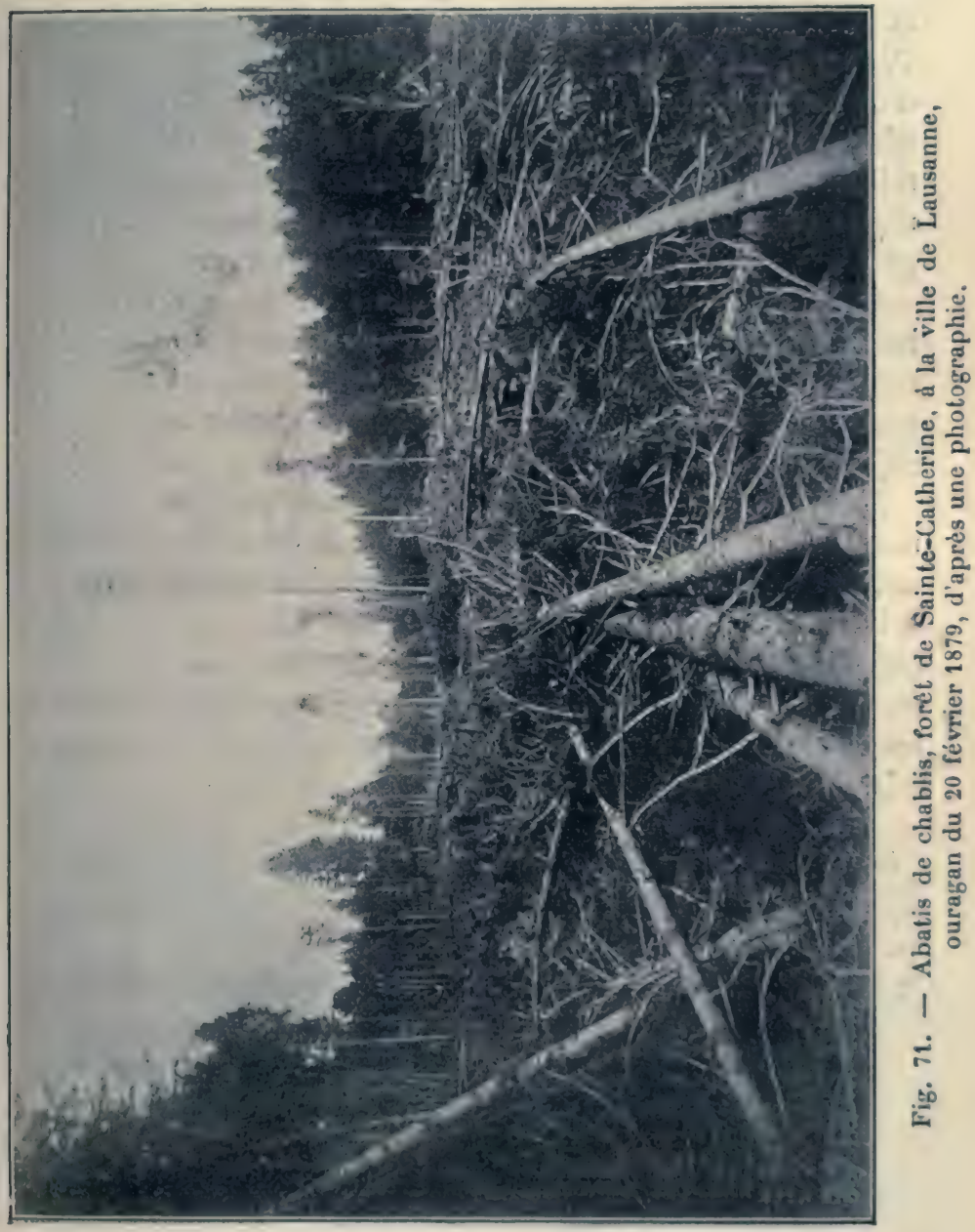

activité productive et marque un temps d'arrêt dans la marche de l'ensemble vers le but commun. Car, sur ces champs de bataille, il faut compter, non seulement avec les cadavres 
gisants ou en estant, mais encore avec les blessés, qui, pour muets qu'ils soient, n'en souffrent pas moins d'infirmités incurables; la matière bois va se dégrader progressivement dans la forêt, qui en est l'entrepôt en même temps que la fabrique. Par suite, si l'on tient compte de la durée plus que séculaire d'un massif de futaie ou d'une réserve de taillis, il faut bien admettre que tout arbre ou peuplement aura, au moins une fois en sa vie, à subir, de l'un ou l'autre des agents météoriques, une des atteintes néfastes que les hommes d'une génération peuvent ne connaître que par ouï-dire.

Le devoir du sylviculteur est donc de prévoir l'improbable comme le possible, et de garer la forêt des méchants coups, même les plus rares, et d'où qu'ils viennent : froid, vent, foudre, neige, grêle, etc... Voyons quelles sont à ce sujet les limites de ses pouvoirs.

Le vent. - Maintes fois déjà nous avons parlé du vent el -noté, à leur place, les influences, bonnes ou mauvaises, du sol, de l'exposition, de la forme des peuplements, etc...

Il suffit à un profane de visiter un abatis de chablis (fig. 71) pour être saisi d'une épouvante salutaire. Mais les forestiers, comme les marins, s'habituent au danger de tous les jours, et quelques avis sont nécessaires pour les ramener à la réalité des faits (1).

(1) On peut dire que, en montagne surtuut, ces accidents sont devenus la règle. Il ne faut pas remonter bien haut dans l'histoire forestière d'une région pour trouver trace de nombreuses catastrophes qui ont bouleversé les plus sages prévisions. Rien que dans les forêts de l'Est, les dates suivantes sont enregistrées comme particulièrement néfastes :

novembre 1868 : Jura et Suisse; désastre suivi d'une redoutable invasion de bostriches.

28 octobre 1870 et 11 novembre 1875 : plaines et collines de la Lorraine.

20 février 1879 : Suisse.

22 août 1889 : Basses-Vosges.

septembre 1890: Vosges, Jura et Suisse.

29 mars 1891 : Vosges.

janvier 1895: Jura et Suisse.

Trop souvent, la plaine n'est pas épargnée: témoin les ouragans qui, pendant l'hiver 1899-1900, ont saccagé les forêts de l'Ouest, entre autres les beaux massifs de chênes de Bercé. 
Tout d'abord, une étude approfondie de la météorologie du lieu et de son passé forestier permettra de délimiter exactement toutes les zones dangereuses. Chacune de celles-ci sera soumise au traitement que comportent les formes d'âges multiples, les plus résistantes entre toutes: le jardinage notamment. Partout ailleurs, et quelque rassurantes que puissent être les allures du climat, les tempêtes, toujours à craindre, commandent d'user pour chaque série de cerlaines précautions intérieures et extérieures.

A l'intérieur, le formulaire des règles d'assiette trouve encore son application. Citons le texte de la troisième règle, emprunté au manuel de sylviculture de M. le professeur Bagnéris.

Dans toute forêt ou série d'exploitation, les coupes devront être assises, de manière à toujours marcher à l'encontre des vents les plus dangereux régnant dans la contrée, généralement, en France, en allant du Nord-Est au Sud-Ouest.

Le principal objet de celte troisième règle est de protéger, contre les dégâts du vent, non seulement les arbres réservés dans les coupes, mais encore les peuplements en croissancc.

Par malheur, - on le constate tous les jours, - si les jeunes peuplements, quelle que soit d'ailleurs leur forme, ont peu à craindre de ce côté, il n'en est pas de même des vieux bois. Parmi ces derniers, les plus exposés sont, d'une part, les plus âgés et ceux qui se rapprochent le plus de l'état uniforme à un seul étage: d'autre part, ceux qui sont plus ou moins interrompus par le fait des exploitations; tel est le cas des arbres d'abris conservés dans les coupes successives de régénération et des réserves dans les taillis sous futaie (1).

(1) Les observations faites dans la forêt de Haye, cantonnement de Nancy-École, à la suite des ouragans des 28 octobre 1870 et 11 novembre 1875 , nous ont permis de constater les faits suivants :

$1^{0}$ dans les parcelles de futaie en régénération, le volume des chablis a été vingt-trois fois plus grand que dans les cantons non entrouverts ou traités en taillis sous futaie, bien que la surface des premières fut huit fois moindre que celle des autres;

$2^{\circ}$ dans l'ensemble des parcelles traitées en taillis sous futaie, les chablis étaient localisés, pour les deux tiers de leur volume, dans les coupes en exploitation ou exploitées depuis un à trois ans.

D'autre part, le relevé des produits mis en vente dans l'ensemble 
Si les points d'attaque sont du côté opposé à la direction des vents à redouter, ceux-ci, maintenus à la hauteur des cimes par les massifs intacts, passeront avec moins de dangers sur ceux que des exploitations récentes rendent moins résistants; dans les forêts de plaine, le mieux sera de faire marcher les coupes en allant du centre vers la circonférence. - Dans les taillis sous futaie, on constate également que les coupes riches en grosses réserves sont moins endommagées que celles où la jeunesse domine: les anciens soutiennent et protègent les modernes et les baliveaux; en toute situation, on devra renforcer les martelages le long des tranchées et sur les rives de la forêt.

A l'extérieur, il sera prudent de conserver, sur tous les périmètres des grandes forêts, des rideaux d'abri maintenus à l'état de massif, par le jardinage, ou soumis à un traitement indépendant de celui des suites de coupes contiguës.

Dans les futaies résineuses, en montagne, la stricte observation de la troisième règle d'assiette doit avoir une portée plus étendue encore. Elle doit être appliquée, non seulement de coupe à coupe, mais de canton à canton et de série à série. Il serait même désirable de faire accepter entre propriétaires voisins le principe des zones de défense tel qu'il existe dans certaines provinces de l'Allemagne centrale, où de larges bandes de forêts sont, au point de vue du traitement, grevées de véritables servitudes de voisinage. En tout état de choses, il est indispensable, dans les régions montagneuses, d'établir, vers les limites supérieures de la végétation, un rideau protecteur qui sépare la forêt régulièrement traitée des parties réservées au pâturage; ce rideau sera plus ou moins large, suivant l'altitude, la violence des vents et la configuration du terrain; il ne subira d'ailleurs que des exploitations modérées et toujours faites en jardinant, car il est extrêmement difficile, pour ne pas dire impossible, de régénérer par les mé-

des sapinières des Vosges, pendant la période quinquennale écoulée de 1879 à 1884, fait ressortir que, dans un total de 668.053 mètres cubes, les chablis entrent pour 204.533 mètres cubes, soit dans l'énorme proportion de 43 p. 100, par rapport au volume des coupes vendues sur pied. 
thodes perfectionnées les massifs aboutissant à la crête des hautes cimes ou voisins des alpages.

Il est inutile d'insister sur ce fait que la troisième règle d'assiette n'est efficacement applicable que dans les peuplements d'un seul âge chacun ; elle perd sa raison d'être dans les cantons jardinés ou furetés.

Le froid. - Le froid, qui trace les frontières septentrionales des essences spontanées, occasionne aussi, dans les limites de ces zones et surtout vers leurs confins Nord, les troubles les plus graves: tantôt les gelées tardives et précoces

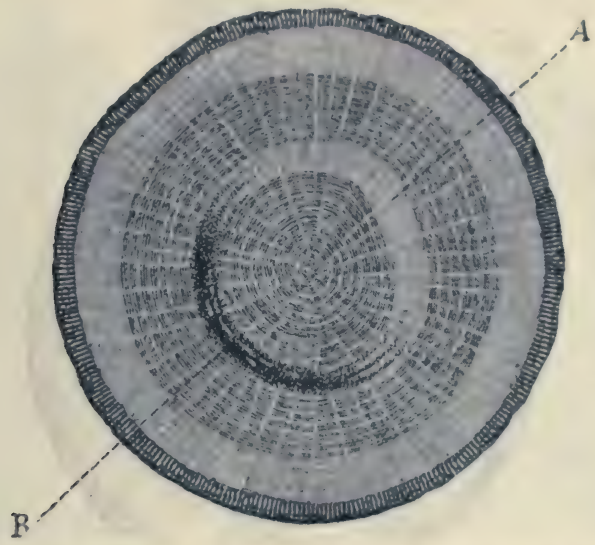

Fig. 72. - Rondelle de chêne présentant, dans sa région centrale, une lunure, dont la portion de gauche est déjà attaquée par la pourriture.

détruisent les organes verts au printemps et à l'automne; tantôt les gelées d'hiver tuent la zone cambiale, produisant des nécroses d'abord superficielles, mais qui deviennent de plus en plus profondes, et qu'on nomme, suivant les cas : gélures, morsures de gelée, friasses, elc... ; ou bien encore, pénétrant dans le corps de l'arbre, elles provoquent dans le bois toutes ces tares que, d'aprés leur aspect, les praticiens ont appelées : lunure ou double aubier, quand les couches les plus jeunes atteintes dans leur vitalité, ne peuvent plus se transformer en bois parfait; - gélivure ou roulure, quand les dislocations, rayonnantes ou circulaires, crevassent la masse toute entière. 
Nous en donnons (fig. 72 et 73) les principaux spécimens.

Pour en préserver les arbres, nos moyens se bornent à cette précaution générale qui consiste, connaissant le tempérament de nos essences, à maintenir chacune à la place où ces dangers seront le moins à craindre pour elle. On remarque, tou$d$

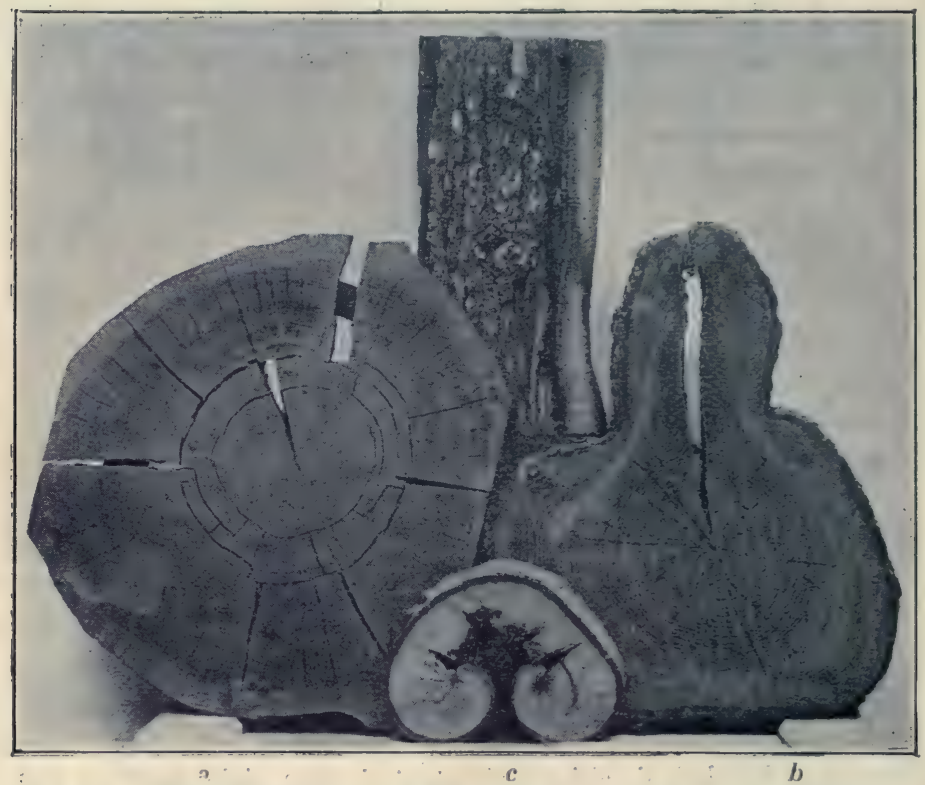

F.g. 73. - Dégàts causés par les météores : $a$, roulure ; $b$, gélivures ; $c$, nécrose due à un coup de soleil avec bourrelets de recouvrement; $d$, nécrose de gelée.

tefois : $1^{\circ}$ que les accidents morbides qui accompagnent les abaissements de température tiennent bien moins au nombre de degrés accusé par le thermomètre au dessous du zéro centigrade, qu'aux circonstances qui accompagnent ou qui suivent le refroidissement; c'est ainsi que les passages brusques du froid au chaud sont bien plus dangereux, toutes choses restant égales d'ailleurs, que les transitions lentes; il en résulte qu'une nuit très froide, suivie d'un jour clair, pourra occasionner des gélures sur les points directement frappés par les 
rayons du soleil, quand, à tous les autres aspects, le sujet restera indemne $;-2^{\circ}$ que dans les sols siliceux, meubles, qui se laissent plus profondément pénétrer par les froids vifs,. les accidents de lunures, de gélivures, de roulures sont plus fréquents que dans les sols argileux, argilo-calcaires et surtout tourbeux; - $3^{\circ}$ que les arbres à écorce lisse, et vivante dans toute son épaisseur, sont plus souvent nécrosés que ceux dont la couche cambiale est protégée par un rhytidome épais.

De ces faits, on peut tirer les conséquences suivantes :

éviter de faire des repeuplements artificiels à l'aide d'essences introduites au nord de leur station d'origine; - placer les plantes à feuilles persistantes de préférence aux expositions froides, pour leur épargner les passages trop brusques du froid au chaud; - dans les dépressions plus particulièrement exposées aux gelées printanières, planter les espèces à frondaison "tardive ou peu sensible; - craindre la transition rapide, de l'état du massif à celui d'isolement pour les essences à écorce mince et privée de rhytidome; - et tant d'autres qu'un sens des choses de la forêt suggère à tout forestier, qui sait, par expérience, que, quoi qu'il fasse, il a toujours des chances à courir, bonnes ou mauvaises.

Les coups de soleil. - L'excès de chaleur, comme l'excès de froid, peut occasionner des nécroses. Ce sont encore les espèces à écorce lisse, les jeunes sujets, ceux qui sont nouvellement plantés et dans le corps ligneux desquels la sève circule mal, qui ont le plus à souffrir. On atténue le danger en prenant partout les mèmes précautions que contre le froid, et, en ce qui concerne les hautes tiges, en les orientant, lors de la mise en place, aux mêmes aspects que lorsqu'ils élaient en pépinière; - en les arrosant abondamment pendant la sécheresse; - en habillant leurs tiges d'un fort manchon de paille: toutes choses qui intéressent plus le verger que la forêt.

La foudre. - La foudre produit sur les arbres les désordres les plus variés, depuis la décortication partielle ou le simple sillon qui marque la trace de son passage, jusqu'à la rupture la plus complète (fig. 74), qui pulvérise un arbre en mille éclats; 
souvent même, à coté de ces effets mécaniques, les arbres voisins de celui qui est foudroyé, sont tués, sans lésion apparente, par ce qu'on appelait autrefois le choc en retour.

On a beaucoup discuté sur les causes qui font tomber la

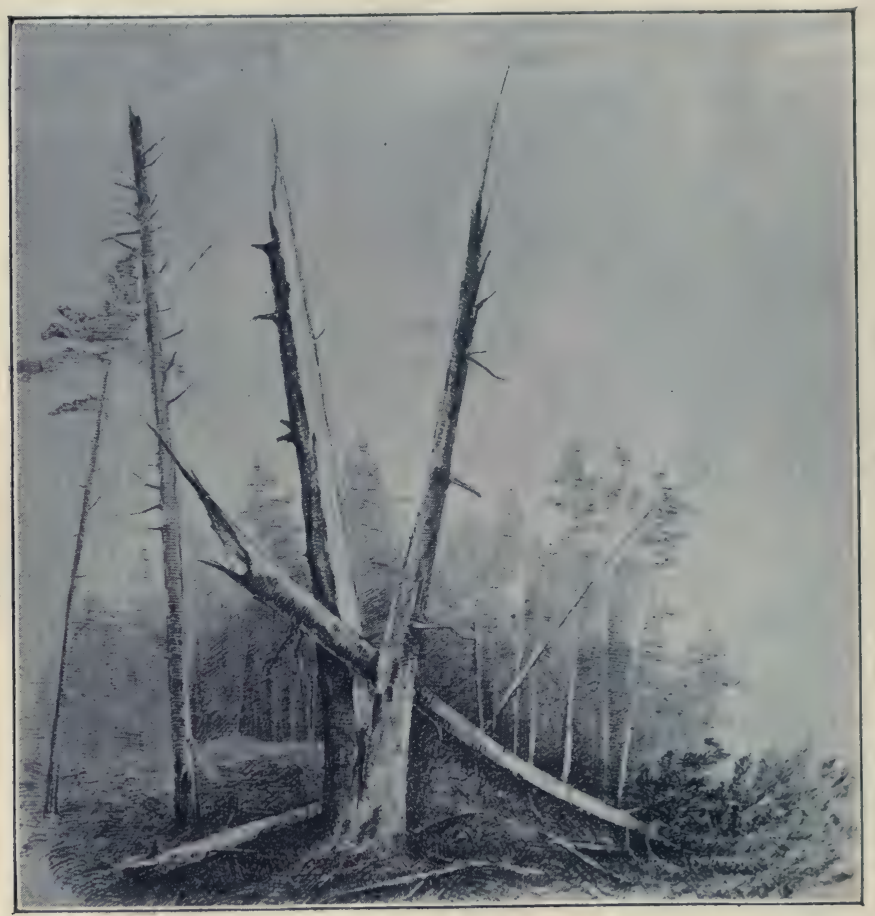

Fig. 74. - Sapin brisé par la foudre dans les Hautes-Vosges.

foudre sur tel arbre plutôt que sur tel autre. Les uns attribuent cette disposition fâcheuse à une forme plus ou moins aiguë de la cîme, d'autres à l'épaisseur du feuillage, d'autres encore à la proximité des eaux stagnantes ou courantes; des observations récentes semblent en avoir trouvé la véritable cause dans la conductibilité variable du bois suivant les essences et son état vert ou sec. Malgré notre incompétence sur cette question de physique, il nous semble, en tout cas, 
assez naturel qu'un chêne, dont les racines pivotantes atteignent les couches toujours humides du terrain, soit frappé plus souvent qu'un hêtre dont l'enracinement reste superficiel.

La seule conclusion pratique à en tirer pour le forestier est que, surpris par un orage, il fera bien de ne jamais s'abriter sous un gros arbre, pas plus sous un hêtre que sous un chêne, et, surtout, sous un fruitier isolé en rase campagne.

La neige et les avalanches. - On cite de nombreux exemples de peuplements dégradés ou écrasés par la neige, lorsqu'elle tombe prématurément ou tardivement sur des arbres couverts de leur feuilles; mais, il faut reconnaître qu'on a rarement à déplorer, dans les forêts d'essences feuillues, des désastres aussi impossibles à prévoir qu'à conjurer, tels que celui qui a dévasté la forêt du Sihlwald, appartenant à la ville de Zurich, le 28 septembre 1885 (fig. 75.). Au contraire, les forêts résineuses de la basse montagne ont à souffrir annuellement de dégâts analogues, mais dont on peut atténuer les effets par des éclaircies faites à propos et judicieusement conduites.

Dans la haute montagne, il faut prévoir et combattre les avalanches, dont tout le monde connaît les elfets désastreux. On sait qu'une fois la masse en mouvement, rien ne l'arrête plus dans sa chute vertigineuse : arbres, chalets, ponts, ouvrages d'art, tout est écrasé sur son passage (fig. 76.). Mais, on sait aussi que jamais l'avalanche ne prend naissance dans les terrains boisés; c'est donc à la forêt qu'il faut demander aide et protection. Dans une étude magistrale publiée en 1881, M. Coaz, inspecteur général des forêts à Berne (1), donne les moyens suivants de combattre le fléau.

Après avoir repéré exactement la ligne de première cassure, par des recherches personnelles, et sans trop s'en rapporter au dire des gens du pays, on corrige le sol nu et glissant des couloirs d'origine à l'aide d'obstacles artificiels : fossés, pieux, murs en pierre sèche ou en maçonnerie. En

(1) Voir dans la Revue des eaux et foréts du 1er avril 1900, les fragments traduits par M. l'Inspecteur-Adjoint Mathey.

Voir également : P. Mougin, La correction des avalanches en France. Revue des eaux et Forêts, mai 1900. 


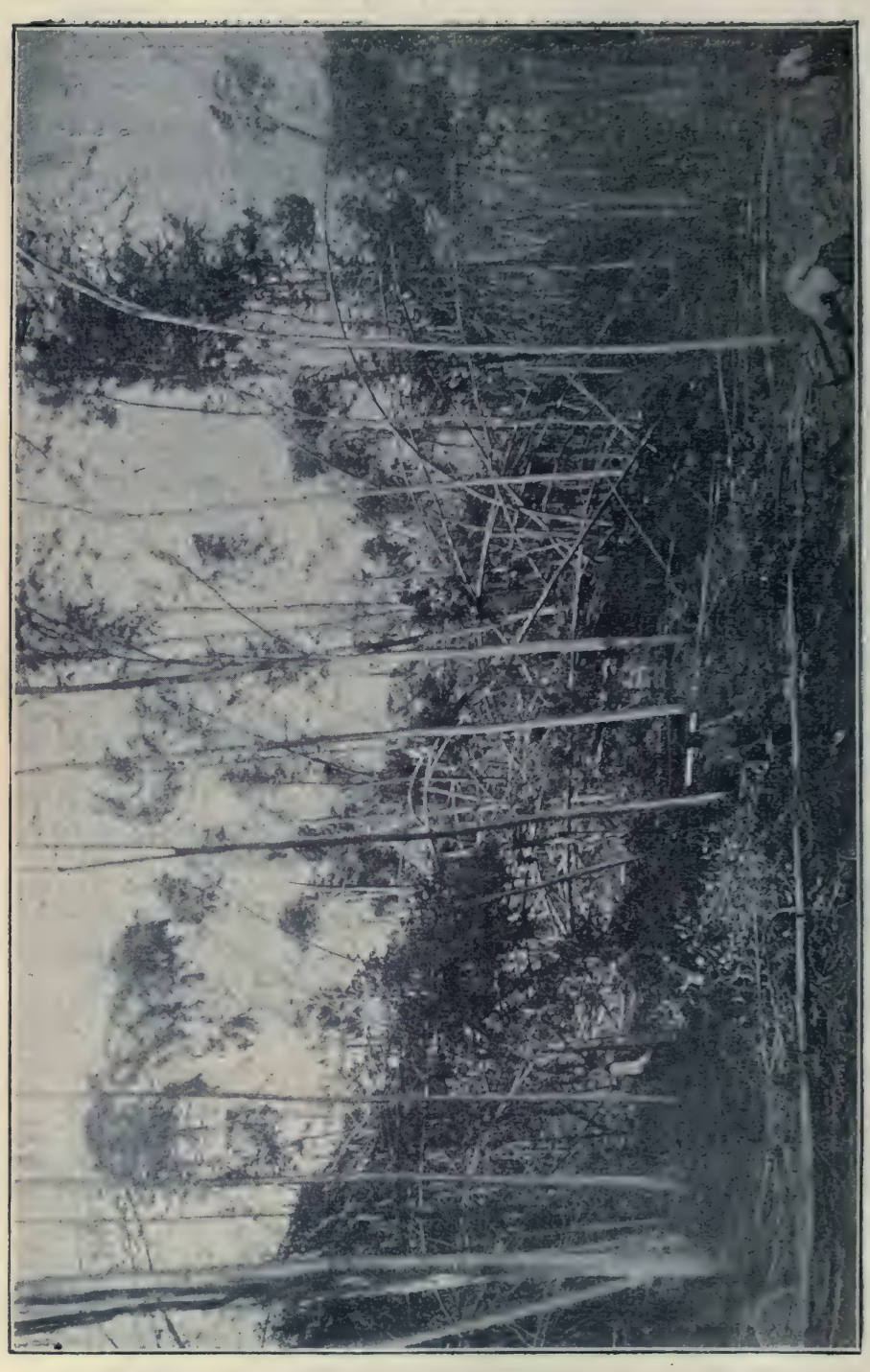


DÉGATS CAUSÉS PAR LES MÉTÉORES.

aval de ces abris morts, exposés aux injures du temps, on plante des arbres qui seront seuls d'un secours définitifs; la

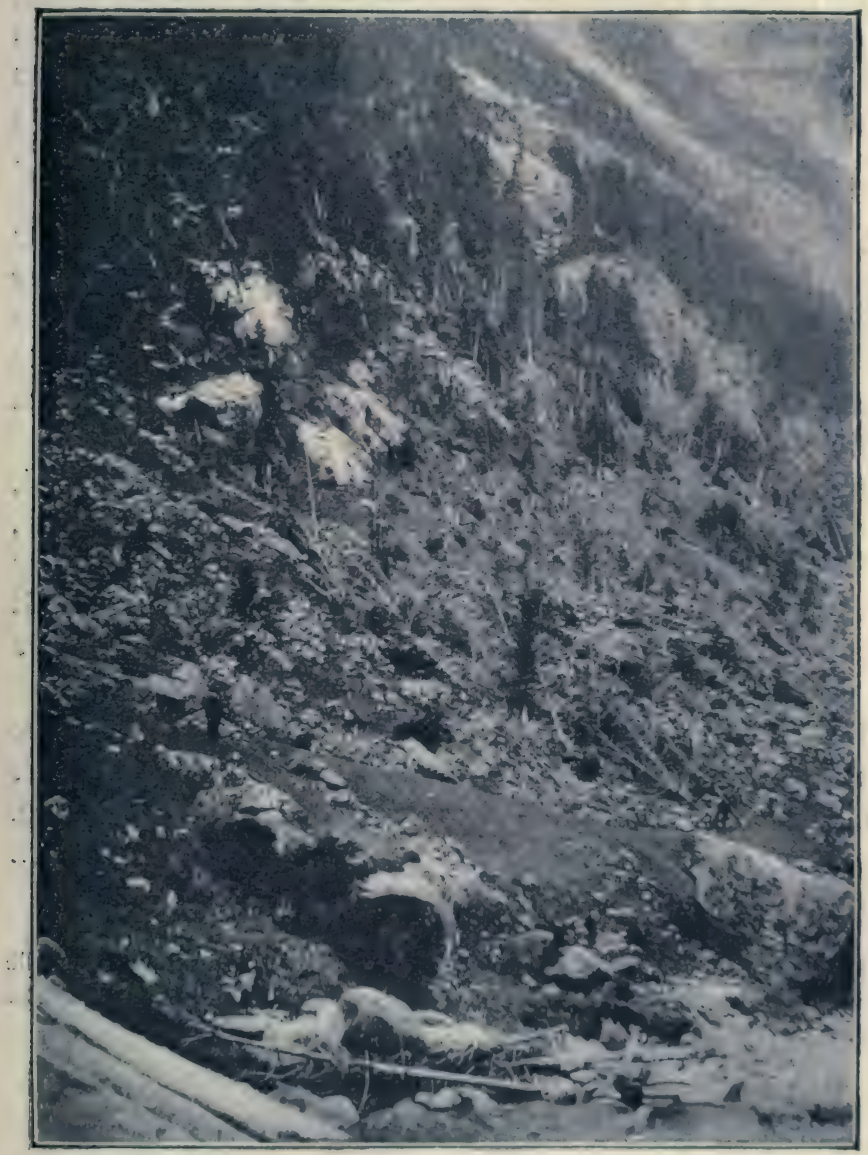

Fig. 76. - Arbres renversés par le déplacement de l'air au passage d'une avalanche sur les pentes de la Jungfrau, le 1er mai 1879 , d'après une photographie.

meilleure essence à employer est le pin cembro, l'arolle des montagnards.

Partout où la forêt n'a pas encore été détruite, dans les Boppe et Jolyet. 
zones dangereuses, elle devra être ménagée avec le plus grand soin, afin de prévenir les accidents à naître. Dans ces contrées, il ne faut pas risquer les régénérations trop systématiques; et si, contraint par la nécessité, on est conduit à réaliser un arbre mort ou vivant, il est sage de le couper à 1 mètre ou $1^{\mathrm{m}}, 50 \mathrm{au}$-dessus du sol, de façon que le chicot attenant à la souche continue à jouer longtemps le rôle de soutien dévolu à l'arbre sur pied.

La grêle, le givre, le verglas. - Bien que les forêts passent pour écarter les nuages à grêle, on pourrait citer nombre d'exemples d'arbres portant dans leur sein des traces de blessures causées par les grêlons, il y a vingt, cinquante ans et plus, et recouvertes par un nombre égal de couches ligneuses.

A cela nous ne pouvons rien! On prétend, aujourd'hui, que des salves d'artillerie empêchent, dans un certain rayon, la formation de la grêle; mais une longue expérience nous fait craindre que, de tous les biens de la terre, la forêt soit encore la dernière en faveur de qui on fera parler la poudre de protection.

Enfin, nous signalerons les graves accidents dus au givre et au verglas, dont le poids couche les jeunes brins et brise les plus grosses branches des vieux arbres. Souhaitons au lecteur de n'avoir jamais à les constater dans ses forêts ;... faire plus, nous est impossible (1).

(1) Dans la forêt de Fontainebleau, on a évalué à 150000 stères le volume des débris, - arbres et branches - cassés par le verglas pendant les journées des 22 et 23 janvier 1879. 


\title{
CHAPITRE VIII \\ LES PEUPLEMENTS ARTIFICIELS
}

\author{
ARTICLE PREMIER
}

\section{OBSERVATIONS GÉNÉRALES}

But à poursuivre. - Définitions. - Les différents procédés de boisement.

But à poursuivre. - Si nous portons nos regards en arrière, nous constatons que la sylviculture, telle que nous avons cherché à la présenter dans son application, nous enseigne à exploiter les forêts surtout à l'aide des moyens naturels, sans avoir recours à des capitaux étrangers, si ce n'est pour subvenir aux dépenses de mise en valeur.

Dans les pays de vieille civilisation, où l'on doit poursuivre, partout et toujours, l'exploitation rationnelle du sol, la forêt a sa principale raison d'être quand elle fonctionne à l'aide du temps dans les terrains non réclamés par d'autres emplois. Sa culture est donc extensive au premier chef.

Quel que soit le point de vue auquel on se place, ce caractère s'impose. En effet, s'il s'agit de la forêt de protection, on est largement payé du service indirect qu'elle rend par sa seule présence, pour qu'il ne soit pas nécessaire d'engager des capitaux en vue d'augmenter une production en bois qui n'est ici que l'accessoire. S'il s'agit de forêts de rendement, le bois, premier objectif de leur culture, n'est réellement utile que s'il est aliondant et à bon marché ; dès qu'il devient cher, la consommation l'abandonne pour le remplacer par des succédanés, souvent de qualité moindre, mais qu'on obtient à meilleur compte. Aussi, tout progrès doit-il être recherché dans un choix judicieux des modes de traitement les mieux adaptés aux conditions locales : car le surcroît de production 
obtenu à l'aide de procédés dispendieux a pour effet d'augmenter le prix de revient de la matière ligneuse dans des limites qui dépassent l'intérêt normal des sommes engagées : c'est la conséquence nécessaire du fonctionnement des capitaux forestiers à intérêts composés.

Si, de la forêt naturelle nous passons á la forêt artificielle, nous constatons que les mêmes causes doivent amener les mêmes effets. Ici encore, nous devons demander à la sylviculture les moyens de réduire les dépenses nécessitées par le boisement à leur strict minimum, de façon que le résultat financier de l'entreprise soit accessible à toutes les catégories de propriétaires et au plus grand nombre des bourses.

Définition. - Nous appelons boisement toute formation nonvelle de peuplements forestiers dans les sols nus.

Le plus souvent, en France du moins, l'homme ne fait que rendre à la forêt les terrains dont ses ancêtres l'avaient dépossédée ; cependant, le fait n'est pas assez général pour justifier, dans tous les cas, le terme de reboisement. Quoi qu'il en soit, nous nous proposons d'étudier les procédés, dits artificiels, mis en pratique pour créer, de main d'homme, la forêt dans les espaces où elle n'existe pas. A cette occasion, nous parlerons des travaux de même nature qui s'exécutent daus les peuplements acquis dont on veut améliorer la situation.

Les différents procédés de boisement. - Les plus usités sont le semis et la plantation. La bouture et la marcotte, très employées par les arboriculteurs, ne trouvent leur application en sylviculture que dans certains cas particuliers dont nous indiquerons les principaux.

Mais faut-il planter, faut-il semer? Telle est la première question qui se pose. Les circonstances si diverses de climat, de sol et de situation dans lesquelles on est appelé à opérer, la variété même des essences à employer, ne permettent pas de répondre d'une manière catégorique. Toutefois, d'après l'ensemble des faits acquis, on peut résumer la situation comme suit :

Il vaut mieux planter que semer :

$1^{\circ}$ sur les terrains mouilleux, sur ceux que les gelées sou- 
lèvent avec facilité, ou qui sont couverts d'herbes, et dans les stations élevées où le climat est rude;

$2^{\circ}$ dans les endroits où les graines sont exposées à être dévorées par les animaux ;

$3^{\circ}$ dans les régions chaudes, oú les racines doivent être de suite assez profondément enfoncées pour résister à une vive insolation et à des sécheresses prolongées.

En outre, avec la plantation, on peut, mieux qu'avec le semis, régler à son gré la consistance des peuplements et le mélange des espèces. Enfin, on s'aperçoit plus tôt des insuccès et il est plus facile de les réparer.

La plantation sera donc la règle, le semis se trouvant localisé :

$1^{\circ}$ dans certains terrains pierreux où la plantation serait très difficile;

$2^{\circ}$ dans les régions où l'on peut obtenir de la graine à très bas prix, et quand le terrain, pour être ensemencé, ne demande pas une préparation particulière; car, dans tous les autres cas, il coûte plus cher que la plantation.

Un des principaux inconvénients du semis est, en cas de réussite, de donner sur un même point des plants trop nombreux, qu'il faut largement desserrer de bonne heure, surtout lorsqu'il s’agit de résineux. Ce travail occasionne un surcroît de dépense dont il faut tenir compte.

- Depuis que l'art de planter, trop longtemps négligé en France, a rapidement progressé sous l'impulsion des grands travaux de reboisement, la pratique de la plantation a établi sa supériorité sur le semis : le fail est incontestable. Mais, en matière de boisement, pas plus qu'en matière de traitement, il ne faut s'en tenir à la théorie immuable; car rien ne peut se résoudre en dehors des questions de temps et de lieu. 'Tous les moyens sont bons quand on sait les employer à propos. 


\section{ARTICLE II}

\section{LE BOISEMENT PAR SEMIS}

Qualité des semences. - Leur récolte. - Leur conservation. Préparation du sol avant le semis. - Les différents modes de semis : en plein ou à la volée; - par places; - par bandes continues ou brisées; - par trous ou potets; - en terrain non préparé ; - semis expéditifs. - Répandage de la semence. Quantité de semence à employer. - Saison favorable pour faire les semis. - Application aux essences sociales. - Soins à donner aux semis.

Qualité des semences. - La réussite du semis dépend, en premier lieu, de la qualité de la semence; on doit toujours s'en rendre compte avant l'emploi.

A la simple inspection, on peut juger si la graine est bien pleine, si elle a une odeur et une saveur fraîches, si l'amande présente la couleur normale de l'espèce à laquelle elle appartient; on peut aussi, en plongeant les semences dans l'eau, considérer comme bonnes celles qui tombent au fond et comme vaines, celles qui surnagent; ou bien encore, en les mettant sur une plaque de fer rougie au feu, on regarde celles qui éclatent en sautillant comme ayant conservé leur faculté germinative, tandis que celles qui se consument lentement sont mauvaises. Mais tous ces procédés ne donnent que de simples présomptions; pour avoir des renseignements à peu près certains, - et la question en vaut la peine, - il faut soumettre toutes les graines à l'épreuve du germoir artificiel : c'est également le seul moyen de constater la fraude qui consiste à livrer certaines espèces, au lieu d'autres, dont les fruits sont plus rares. A défaut d'appareils spéciaux, on peut toujours placer dans les conditions convenables à la germination un certain nombre de graines comptées à l'avance, et établir la proportion de celles qui germent et de celles qui ne germent pas; il suffit, par exemple, de les semer dans des terrines que l'on place sous vitraux sur couche chaude; ou bien de les disposer, dans une serre ou dans une chambre à température élevée, entre deux 
doubles de flanelle dont les extrémités plongent dans un récipient plein d'eau, etc.

D'une manière générale, plus les semences sont légères plus grande est la proportion des stériles. A l'exception des glands et des faînes, dont la bonne qualité est toujours facile à constater, les semences des autres espèces feuillues sont peu employées sous forme de semis direct; elles s'obtiennent du reste à des prix assez bas pour qu'il n'y ait pas grand intérêt à les ménager. La qualité des graines résineuses est très variable suivant les espèces; ainsi, quand les semences de sapin pectiné, d'épicéa, de pin sylvestre, pour être considérées comme bonnes, ne doivent pas renfermer plus de 10 p. 100 de graines vaines, on en accepte 30 p. 100 pour celles de mélèze ( 1 ).

Il faut aussi tenir compte de ce fait qu'à l'air libre, la germination naturelle donne toujours un résultat inférieur à celui des épreuves; outre les accidents météorologiques à redouter, les mulots, les oiseaux et les insectes occasionnent, en effet, un déchet sérieux. Dans tous les cas, si l'on achète des graines dans le commerce, c'est une économie mal placée que de s'adresser à des maisons n'inspirant pas toute confiance, ou d'utiliser des "fonds de sac " des années précédentes. Le mieux, quand cela est possible, est de ramasser soi-même en forêt les semences dont on a besoin. Il faut alors donner tous ses soins à leur récolte et à leur conservation; les indications suivantes serviront de guide dans cette double opération.

Récolte des semences. - A moins de circonstances exceptionnelles, on ne doit récolter que des graines provenant d'arbres sains, exempts de tares héréditaires (fig. 77), vigoureux et croissant dans leur station. La récolte ne se fait que quand les graines sont complètement mûres. Les graines lourdes se ramassent sur le sol après leur chute naturelle; les graines

(1) Les cônes de résineux, dans leurs portions terminale et basilaire, ne renferment souvent que des graines stériles. Des cônes très courts, comme ceux du mélèze, fournissent done une proportion de bonne graine bien inférieure à celle que l'on trouve chez des espéces à cóne allongé, comme l'épicéa. 
légères et les cônes des résineux doivent être cueillis directement, en montant sur les arbres.

Les glands des chênes mûrissent et se disséminent vers la fin de l'automne. Parmi ceux qui tombent les premiers, il s'en

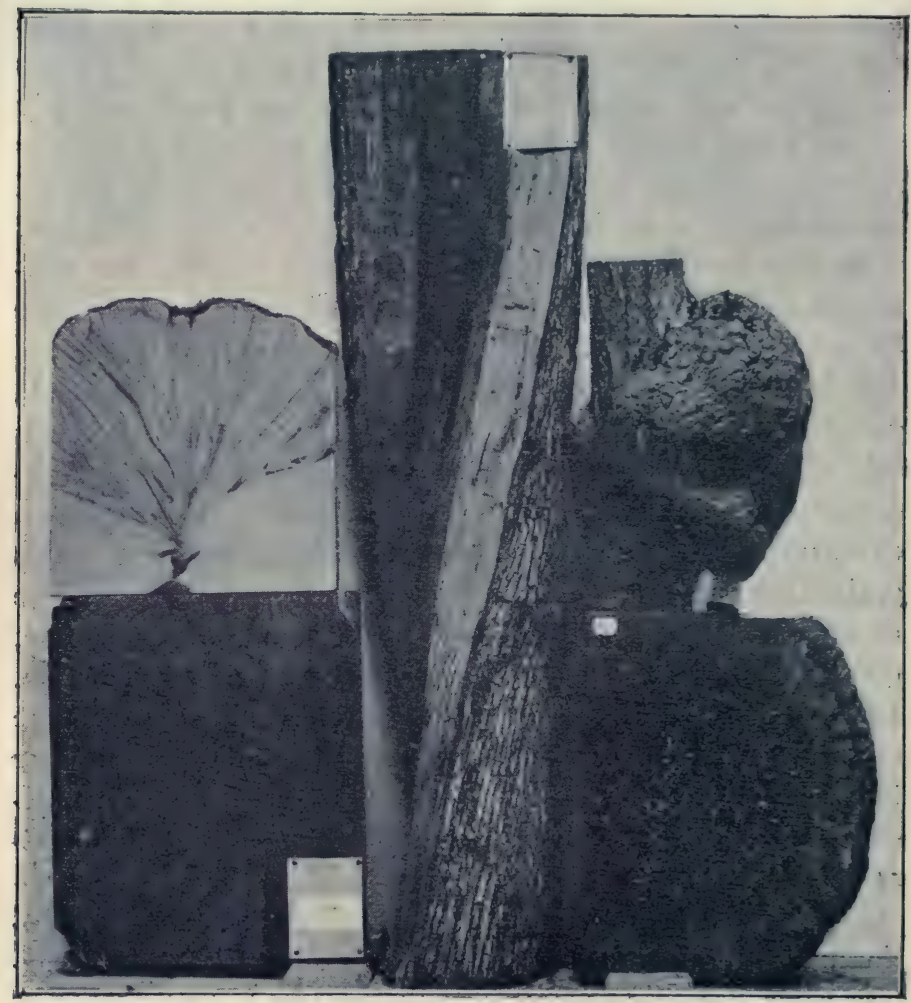

Fig. 77. - Anomalies de structure : fibre-torse (défaut héréditaire); loupe (défaut purement local) et sections faites dans les loupes.

trouve beaucoup de véreux ; pour les ramasser, il faut donc attendre le moment où la chute se fait abondamment; on choisit alors ceux qui sont fraîchement tombés et les plus gros, l'expérience ayant indiqué que ces derniers donnent les plants les plus forts. En faisant cette récolte, il est extrêmement important de bien distinguer les 
glands du chêne rouvre et ceux du chêne pédonculé; les deux espéces ont, on effet, des exigences bien différentes, dont il faut tenir compte, sous peine de mésaventures difficilement réparables.

La faine mûrit également à la fin d'automne; on la recueille par terre, à la main ou au balai ; quelquefois, on la fait tomber sur des draps étendus au pied des arbres, dont on frappe les rameaux avec de longues perches.

Les glands de charme se récoltent à la main vers la fin d'automne, après la chute des feuilles, ou bien on gaule les arbres, comme cela vient d'être dit pour les faînes. La samare des érables et celle du frêne, mûrissent aussi en automne; on les récolte à la main, sur l'arbre, après la chute des feuilles; chez l'érable sycomore et le frêne, ces semences restent suspendues jusqu'à la fin de l'hiver, celles de l'érable plane se disséminẹnt plus tôt.

La samare des ormes mûrit vers la fin de mai, ou au commencement de juin; elle s'envole bientôt. On la ramasse surtout le long des routes et dans les allées des promenades publiques où ces arbres sont abondants. Il importe alors de savoir distinguer l'orme champêtre de l'orme de montagne, car ces deux espèces, dont les bois ont deș qualités bien différentes, sont souvent mélangées comme arbres d'agrément. Les semences d'orme germent de suite après leur dissémination; beaucoup sont vaines.

Les cônes du bouleau mûrissent en septembre; ils se désarticulent bientôt après, et la graine tombe en même temps que les écailles. Ceux qui mûrissent les premiers, de même que ceux qui restent sur l'arbre jusqu'en novembre, ne contiennent généralement que des semences infertiles.

Les cônes des aunes blancs et glutineux ne se désarticulent pas; ils s'entr'ouvrent et laissent échapper leurs potites graines, depuis le commencement de décembre jusqu'au printemps. On ramasse ces cônes, en automne, dès que les écailles en deviennent brunes; on les conserve dans un lieu sec, et la semence se sépare tout naturellement.

Les graines de sapin pectiné mûrissent en septembre et tombent des cônes avec les écailles, surtout après les gelées, 
dans l'espace d'une quinzaine de jours. Les cônes sont dressés sur les branches du sommet de l'arbre; il faut se hâter de les y cueillir au moment précis de la maturité.

Les cônes d'épicéa mûrissent au mois d'octobre, ils ne s'entr'ouvrent pas de suite et on les cueille pendant l'hiver. Lorsque la température est douce, les graines commencent, dès cette saison, à s'échapper des cônes et à s'envoler; le plus souvent la dissémination ne se fait qu'au printemps.

La récolte des graines de méléze s'opère dans les mêmes conditions que celles de l'épicéa, en évitant de ramasser les cônes vides, qui restent plus d'une année attachés à l'arbre après la dissémination. On peut aussi la récolter sur la neige durcie, dans les dépressions où le vent l'a balayée.

Les cônes de pin sylvestre mûrissent dix-huit mois après la floraison, pendant l'automne de l'année suivante; les semences se disséminent au printemps. On cueille les cônes pendant l'hiver. Il en est de même des pin maritime, pin de montagne, pin laricio, pin cembro. Les graines de pin d'Alep se récoltent au printemps de la troisième année qui suit la floraison; les cônes vides restent indéfiniment attachés aux rameaux.

La désarticulation des cônes du sapin se fait facilement et par simple torsion dans les mains. Tous les autres cônes s'ouvrent sous l'influence de la chaleur et de la sécheresse.

La récolte et la préparation des graines, - des graines résineuses surtout, - sont l'objet d'une industrie très importante. Le plus souvent, le commerce les façonne dans des établissements spéciaux, auxquels on donne le nom de $s e ́-$ cheries; à défaut de séchoir artificiel, on soumet les cônes à la chaleur solaire, en des endroits exposés au midi et bien abrités contre les vents. Les graines ainsi obtenues sont munies de leurs ailes; si elles doivent être conservées un certain temps, il est préférable de les laisser intactes, mais, avant l'emploi, il convient de les désailer. Celte opération se fait en humectant légèrement la graine; on la met ensuite dans des sacs remplis au quart environ, et qui sont secoués par 
des ouvriers tenant chacun deux des quatre coins. Le nettoyage s'obtient au moyen de vans (1).

Conservation des semences. - Les fruits lourds, comme la châtaigne, le gland et la faîne, moisissent facilement; il est impossible de les conserver pendant plus d'un hiver, et encore est-il nécessaire de prendre certaines précautions assez délicates, car il faut les garantir en même temps contre la germination (2), la moisissure, le froid, le desséchement et la voracité des animaux. C'est dans des silos qu'on obtient les meilleurs résultats; on peut aussi renfermer les glands dans des tonneaux percés de trous et qu'on immerge dans de l'eau courante. Si l'on préfère conserver ces semences dans un grenier, - ce qui est également bon, - on doit, aussitôt après la récolte et le nettoyage, les étendre au soleil, en couches minces; on les brasse deux ou trois fois par jour, si elles sont très humides. Après complet desséchement, on les place par couches de 3 à 5 centimètres d'épaisseur sur le plancher d'un grenier, où il faut les remuer tous les jours pendant le premier mois, - ensuite, une fois par semaine. Une forte aération du grenier est nécessaire.

La graine de sapin est aussi très délicate, à peine se conserve-t-elle pendant un hiver.

Celles des pin d'Alep, pin pinier, pin cembro, qui renrenferment une assez grosse amande, rancissent très vite et se conservent difficilement au-delà de six mois. De même les semences légères et sèches de plusieurs bois feuillus, tels que l'orme, le bouleau, l'aune, perdent en quelques mois leurs facultés germinatives.

Les glands de charme, les samares de frêne, quelquefois aussi les samares d'érable, restent une année en terre avant de germer; on les stratifie alors, durant un an, au fond de simples rigoles ouvertes dans un terrain sain et frais, en les recouvrant de 10 à 15 centimètres de terre. La graine d'orme doit être semée au printemps, aussitôt après dissémination;

(1) Voir les articles de M. Thil sur la " Récolte et préparation des graines résincuses " (Revue des eaux et foréts, t. XXIII, 1884).

(2) Une température de $+4^{0}$ suffit à la germination des glands. 
le même procédé permet de retarder sa germination jusqu'au printemps suivant.

Toutes les autres graines se conservent plus ou moins longtemps dans des greniers bien aérés, où l'on a la précaution de les remuer pour éviter l'échauffement. Mais la conservation d'une semence a toujours pour effet d'en altérer peu à peu la vitalité, jusqu'au moment où toute faculté germinative est perdue. En général, plus on s'éloigne, en semant, de l'époque de la récolte, plus la germination est irrégulière, lente à se produire, et moins aussi les plants obtenus ont de vigueur. Le tableau suivant contient le résultat des essais de graines de différents âges, poursuivis par M. l'Inspecteur Pierret à l'École forestière des Barres.

\begin{tabular}{|c|c|c|c|c|c|c|c|c|}
\hline \multirow[b]{2}{*}{ ESSENCES. } & \multirow{2}{*}{\multicolumn{2}{|c|}{ 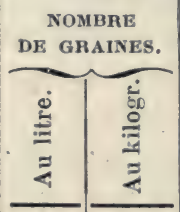 }} & \multicolumn{5}{|c|}{$\begin{array}{c}\text { FACULTÉs } \\
\text { GERMINATIVES (Tau p. 100). }\end{array}$} & \multirow[b]{2}{*}{ OBSERTITIOXS. } \\
\hline & & & 遶 & 离 & 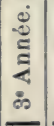 & 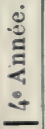 & 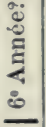 & \\
\hline & 66.300 & 136.000 & $\%$ & $\%$ & $0 \%$ & $0 \%$ & 0,0 & \\
\hline Pin sylvestre .... & $\begin{array}{l}\text { à } \\
71.800 \\
61.500\end{array}$ & $\begin{array}{l}150.000 \\
165.000 \\
124.000\end{array}$ & 74 & 49. & 28 & ” & 5 & Graines désailées. \\
\hline Pin de montagne... & $\begin{array}{c}\mathrm{a} \\
79.150\end{array}$ & $\begin{array}{c}a \\
150.300\end{array}$ & 72 & 57 & 50 & 42 & 25 & id. \\
\hline Pin laricio (noir).... & 28.045 & 53.086 & 76 & 50 & $n$ & $n$ & 5 & id. \\
\hline - (de Corse). & 33.684 & 67.684 & 77 & 50 & $"$ & ” & 5 & id. \\
\hline - (des Cévennes). & $\begin{array}{r}31.649 \\
9.771\end{array}$ & $\begin{array}{l}66.600 \\
17.142\end{array}$ & 74 & 50 & w & ") & 5 & id. \\
\hline Pin maritime. & $\begin{array}{c}\mathrm{a} \\
11.363\end{array}$ & $\begin{array}{c}\text { à } \\
19.176\end{array}$ & 74 & 68 & 70 & 74 & 66 & $10^{\circ} \mathrm{an}, 540 / 0$, id. \\
\hline Pin d'Alep........ & 27.055 & 52.763 & 74 & 68 & 65 & $"$ & $"$ & Graines desailée. \\
\hline Pin Weymouth.... & 24.427 & 50.365 & 66 & 33 & 10 & ) & ) & id. \\
\hline Pin cembro........ & 1.775 & 3.412 & $25-52$ & 3 & 0 & $\mathbf{0}$ & 0 & id. \\
\hline Mélèze............ & 81.761 & 169.539 & $39-44$ & $16-18$ & $5-8$ & 0 & 0 & id. \\
\hline Epicéa............ & 65.312 & 122.386 & 83 & 53 & 26 & 7 & $n$ & id. \\
\hline Sapin..................... & 5.941 & 22.013 & $?$ & $?$ & $?$ & $?$ & $?$ & Graines ailees. \\
\hline
\end{tabular}

On remarque que les graines des essences qui vivent dans les stations élevées (mélèze, pin cembro) n'ont qu'une très faible faculté germinative et la conservent moins longtemps 
que celles des espèces poussant dans les stations chaudes (pin maritime, pin d'Alep). M. Pierret attribue ce fait à une maturation insuffisante. Néanmoins le pin de montagne ferait exception.

Il semblerait aussi résulter d'expériences récentes, entreprises dans les pépinières par M. de Vilmorin, que la macération dans la pepsine donne un regain de vitalité aux vieilles graines.

Pour préserver les graines de toutes essences contre la voracité des animaux rongeurs et des oiseaux, aussi bien dans les réserves que lors de la mise en terre, il est bon de les enduire de minium rouge. A cet effet, on saupoudre les tas avec une quantité suffisante de minium, et le mélange intime s'obtient par les brassages successifs destinés à prévenir la fermentation.

Préparation du sol. - Quand le boisement par semis direct est justifié, il est nécessaire, le plus souvent, de faire subir une certaine préparation au sol; car les jeunes plants naissants risquent d'être étouffés par la végétation sauvage qui les environne, et dont certaines espèces se propagent avec une telle rapidité, qu'elles ont vite raison des semis les mieux réussis. En terrain calcaire, ces espèces nuisibles sont très nombreuses; en sol siliceux, pour être moins variées, elles n'en sont pas moins dangereuses, et l'une d'elles, la fétuque bleue (Molinia cœrulea), constitue, en certaines régions, un des principaux obstacles à vaincre pour les travaux de reboisement. Il faut donc des conditions tout à fait spéciales pour justifier le semis en terrain naturel.

La manière de préparer le sol diffère avec son état superficiel, les conditions de couvert et la composition minéralogique; mais la règle est partout, et toujours, de débarrasser les parties à semer de la végétation herbacée gênante, et d'accompagner cette opération indispensable d'une culture, d'une façon, qui revient à défoncer le terrain sur une profondeur variable. Ce défoncement est surtout utile quand le climat est sec, quand le sol, par sa nature et son exposition, est sujet à se dessécher. La culture, en effet, a les résultats suivants : 
$1^{\circ}$ elle augmente les facultés d'imbibition du sol à l'égard des eaux atmosphériques;

$2^{\circ}$ la couche superficielle ameublie joue, par rapport aux régions plus profondes du terrain, avec lesquelles ses liens sont rompus, le rôle d'une couverture morte : elle diminue l'évaporation;

$3^{\circ}$ elle permet aux jeunes plants de développer rapidement leur appareil radicellaire, qui pénètre ainsi dans des régions où la sécheresse ne se fait plus trop sentir;

$4^{\circ}$ la destruction de la végétation herbacée, dont elle est le corollaire, entraîne la disparition de tout un monde de plantes qui feraient aux jeunes semis une trop rude concurrence dans la lutte pour l'eau.

Par contre, dans les stations où les accidents de gel et de dégel sont à craindre, la culture augmente les chances du déchaussement.

En tóutes circonstances, il faudra veiller à ce que la graine se trouve en contact avec la partie de la terre la plus riche en terreau, parce qu'elle est, en même temps, la plus hygrométrique et la plus perméable; on évitera donc le brassage complet de la terre, en ayant soin de laisser en place la couche superficielle, sans ramener à la surface la terre minérale pauvre des parties plus profondes; le contraire a lieu quand il s'agit de plantations. Pour profiter de l'humidité si nécessaire à la germination, il est bon de ne faire cette culture qu'au moment de répandre la graine.

Les différents modes de semis. - Dans l'exécution des semis, on distingue plusieurs méthodes, dont les principales sont les suivantes : semis en plein, - par places, - par bandes alternes continues ou brisées, - par potets, - sur terrain non préparé, - et semis expéditifs.

Semis en plein ou à la volée. - Le semis en plein consiste à ensemencer régulièrement le terrain après en avoir nettoyé avec soin toute la superficie. Il se justifie quand le sol a déjà subi une préparation en vue d'une culture agricole, ou lorsque le travail ne coûte rien (extraction de souches ou de bruyères; d'ailleurs l'emploi de la charrue, mode qui, au point de vue économique, est le plus avantageux; permet, 
en même temps qu'on fait le semis de graines forestières, d'obtenir une récolte de céréales. Le semis en plein n'est applicable que dans les terrains en plateau ou en pente légère ; en montagne, la terre ne saurait être ameublie en plein, sans dangers de ravinements. Il faut aussi que la graine soit à très bas prix; car, sous cette forme, le semis en absorbe une grande quantité.

Semis par places. Ce n'est autre chose qu'un semis en plein localisé sur de petits espaces de forme et de contenance variables, sur des places, en un mot, choisies à cet effet. Cette méthode économise les soins et le travail; mais l'ensemble reste parfois irrégulier, surtout si quelques places voisines l'une de l'autre viennent à manquer; de plus l'envahissement par les herbes est toujours à craindre.

Elle trouve son application dans les trois cas suivants :

$1^{\circ}$ en forêt, pour compléter une régénération naturelle insuffisante;

$2^{\circ}$ en forêt, encore, pour créer des placeaux d'une essence précieuse au milieu des espèces spontanées ;

$3^{\circ}$ enfin dans les terrains nus oú la roche formant sous sol, est à une profondeur notable sur certains points, tandis qu'elle affleure tout à côté. On ensemence alors les bons endroits : les autres se regarniront dans la suite par voie naturelle ou par plantation, quand les îlots de verdure obtenus leur auront assuré un peu d'ombre et de fraîcheur.

Dans de pareilles conditions, l'emploi de la charrue est impossible, et c'est la pioche ou la bêche qui préparent le terrain.

Semis par bandes continues ou brisées. - On divise la surface à reboiser en bandes parallèles, plus ou moins larges et plus ou moins éloignées entre elles, dont les unes sont cultivées et ensemencées, quand les autres restent en friche. Ces semis exigent moins de graines que les précédents, ils donnentaússi plus de facilité pour soigner les jeunes plants ; ils joignent à cela l'avantage de permettre le maintien, dans les intervalles incultes, de la végétation naturelle, dont l'abri sera d'un grand secours contre les effets du soleil, des vents et des eaux pluviales et dont la présence active la condensation des rosées. 
La largeur dès bandes cultivées est comprise entre $0^{\mathrm{m}}, 50$ et 1 mètre; plus le sol a de tendances à s'enherber, plus les bandes doivent être larges; on les diminue, au contraire, a mesure que la pente augmente. L'espace inculte varie de 1 à 3 mètres, suivant les essences employées, en tenant compte de ce fait que plus les bandes cultivées sont écartées, plus tard aussi se forme le fourré.

Chaque fois que cela sera possible, les bandes seront ouvertes à la charrue; mais, quand le sol est pierreux, peu profond, encombré de racines, ou si les pentes sont accusées, cette préparation se fait à bras d'homme. Si la charrue est recommandable à tous égards dans les terres abandonnées par la culture, le versoir présente, dans les friches, l'inconvénient de ramener à la surface la terre sauvage; alors nous préférons la houe.

Quand le terrain est plat ou peu incliné, il faut toujours ouvrir les bandes dans la direction Est-Ouest; la végétation des parties incultes, aussi bien que le bourrelet formé par les terres provenant des cultures, servent d'écran du côtẻ du Midi. Dès que les pentes atteignent 7 ou 8 p. 100, il faut nécessairement tracer des bandes horizontales, c'est-à-dire suivant la direction perpendiculaire à la ligne de plus grande pente; le fond cultivé en est réglé et nivelé avec un léger dévers du côté d'amont. Jamais elles ne sont ouvertes dans le sens des pentes, car il se produit alors des ravinements et les eaux ruisselantes entraînent les graines.

En montagne, il est impossible d'obtenir une horizontalité parfaite, à moins de frais supplémentaires et très superflus; d'autre part, s'il existe la moindre pente, les eaux s'amassent dans les parties basses et provoquent un commencement de ravinement. Dans ces conditions, il est préférable de briser les bandes, c'est-à-dire de ne les ouvrir que par troncons de 5 à 6 mètres de longueur, séparés entre eux, dans le sens de leur direction, par des intervalles incultes de $1^{\mathrm{m}}, 50$ à 3 mètres; ceux-ci sont disposés de telle sorte que le milieu de la partie cultivée, dans la ligne supérieure, corresponde avec le milieu de l'espace inculte dans la ligne inférieure.

Semis par trous ou potets. - Ce genre de semis se fait 
en plaçant les semences une à une, ou en faible quantité, dans des trous plus ou moins profonds préparés à cet effet et symétriquement disposés. Le procédé est économique; mais il expose à des insuccès nombreux; car, si les jeunes semis courent peu de risques de la part des animaux fouisseurs, ct surtout des vers blancs qui éprouvent de la difficulté à forer leurs galeries d'un potet à l'autre, ils ont, par contre, à souffrir des herbes qui les envahissent par quatre côtés à la fois.

Semis sur terrain non préparé. - Pour que cette méthode offre des chances réelles de succès, il faut tout à la fois:

$1^{\circ}$ que le sol soit naturellement meuble;

$2^{\circ}$ que la graine coûte très bon marché;

$3^{\circ}$ que les semis de l'essence employée aient une végétation rapide qui leur permette de lutter contre les plantes sauvages.

Ces trois conditions se trouvent réalisées dans la dune blanche des côtes de l'Océan, où le pin maritime, semé à la volée sur le sable nu, fait merveille, sous réserve de couvertures qui empêchent le décapage par le vent; mais, si le sable est envahi par une végétation basse qui lui donne de la cohésion, la pioche doit intervenir.

Parfois, dans les landes montagneuses du Plateau central, afin d'éviter les frais d'arrachage de la bruyère, les semeurs répandent les graines de pin sylvestre sans aucun travail préalable, et se font suivre par un troupeau de moutons; ceux-ci secouent la bruyère et font tomber les graines sur le sol où leur piétinement les recouvre suffisamment. Mais il faut conslater que les mécomptes sont aussi fréquents que les résultats acceptables.

Ce genre de semis est plus justifié dans les terrains rocailleux de la haute montagne, au pied des escarpements, sur les cônes d'éboulis désignés dans les Alpes sous le nom de clappes, où toute culture est impossible; là, d'ailleurs, si le sol n'est pas précisément meuble, dı moins est-il toujours frais, et la graine trouve des abris dans les interstices des pierres; d'autre part, si la végétation des jeunes semis est très lente, du moins n'a-t-elle pas de concurrents sérieux dans les toulfes de gazon, les mousses et les lichens qui ont

Boppe et Jolyet. 
àssez à fairé de lutter contre la rigueur du climat. On sème alors soit sur la neige, soit sur terrain nu, sans aucun travail préalable.

Le semis sur terrain nu se fait toujours en plein. Dans les pentes rocheuses, clappes ou autres, il faut avoir soin de lancer fortement la semence par un mouvement de bas en haut, de façon que les graines, pénétrant sous les pierres aussi avant et en aussi grand nombre que possible, soient préservées contre la sécheresse et l'entraînement par les eaux.

Le semis sur la neige se pratique en répandant la graine, à la volée et en notable quantité, quand la surface est encore recouverte de névés assez résistants pour que le parcours n'en soit pas trop pénible. On choisit un temps calme, par une belle journée de printemps, afin que les graines répandues dans la matinée puissent pénétrer de quelques millimètres dans la neige fondue par les rayons du soleil; cela suffit pour qu'elles ne soient plus balayées par le vent. Pour qu'on puisse compter sur un succès, il est indispensable que le terrain couvert de neige soit susceptible d'être reboisé par semis direct, c'est-à-dire qu'il ne soit pas exposé au soulèvement après la fonte des neiges. Il est évident que, partout où le déchaussement est à craindre, le semis ne réussira pas mieux sur la neige que sur le même sol nu; c'est certainement pour ne pas s'être rendu compte de ce fait que bien des insuccès ont été reprochés, à tort, à ce procédé qui peut rendre de très utiles services, car il permet, en même temps, d'opérer à une époque oủ tout autre travail est impossible, et d'utiliser tous les instants d'une saison de végétation très courte: les graines ainsi répandues arrivent, en effet, sur le sol dans des conditions de macération éminemment favorables à leur prompte germination.

Semis expéditifs. - Ces méthodes varient suivant la nature du terrain, les outils dont on dispose, et la grosseur des semences, lesquelles ne sont pas répandues à la volée sur un sol non préparé; mais le travail trés sommaire, consiste à obtenir, en un tour de main, tout à la fois l'enfouissement d'une pincée de graines de distance en distance, à la profondeur 
voulue, - et une légère façon, toute locale, dont bénéficiera le jeune semis.

Ainsi, pour repiquer des glands ou des faines, il suffit de soulever une motte de gazon avec le tranchant d'une houe; on jette deux ou trois semences dans cette ouverture, et, avec le pied, on presse légèrement la terre.

Quand il s'agit de graines légères, on peut, d'un seul coup de pioche, rompre la couverture vivante; puis, sur la terre émiettée, on répand une pincée de semences qu'on recouvre légèrement à la main de façon à les cacher à la vue. Souvent encore, avec un sarcloir, avec une petite herse spéciale, ou même, simplement, avec le fer d'un piolet ou l'extrémité d'un bâton ferré, on gratte la surface du sol pour faire un placeau où l'on jette la graine.

Ces modes de semis, économiques avant tout, se recommandent pour compléter les régénérations naturelles dans les terrains boisés. Ils trouvent encore leur application dans les friches, lorsque l'état d'ameublissement du sol et l'humidité constante du climat les rapprochent des conditions précédentes; ainsi, dans les grandes altitudes, ou dans une région froide quelconque, quand le sol n'est couvert que d'un gazon court et qui reste vert toute l'année. Ils s'imposent même dans les terres mobiles, croulantes, en partie dépourvues de végétation, ou toute culture quelque peu étendue serait dangereuse; on cherche alors, pour les ensemencer, les points les plus stables, soutenus par une touffe d'herbe ou abrités par un bloc, et on mélange avec la graine forestière la semence d'une plante herbacée rustique, à végétation rapide, qui consolide temporairement le terrain.

Parmi les différents outils en usage, nous devons une mention spéciale aux semoirs de M. l'Inspecteur Prouvé, qui consistent en un tube de métal de la longueur d'un manche de bèche, du diamètre d'une pièce de cinq francs, dont l'extrémité inférieure est terminée par une houlette permettant de creuser le sol ou d'en ameublir la surface. Le tube du semoir conduit naturellement dans cette sorte de potet les deux ou trois glands qu'on y laisse glisser sans avoir la peine de se baisser; parfois même, il constitue un véritable réservoir 
contenant une provision de menues graines, dont un déclanchement très simple fait tomber, à la volonté de l'ouvrier, là quantité suffisante.

Hâtons-nous d'ajouter que l'emploi d'un outil, quelque perfectionné qu'il soit, ne remplace jamais une bonne préparation du sol.

Répandage de la semence. - On n'a aucun intérêt à se servir des semoirs mécaniques : la main suffit. Dans le semis en plein, on jette la graine à la volée, après l'avoir intimement mélangée, s'il y a lieu, avec une demi-semence de céréales; dans les semis par bandes ou par potets, on répand la graine en ayant soin de la laisser tomber assez près de la terre pour qu'elle ne soit pas emportée par le vent dans les parties incultes. Partout, on facilite la répartition des graines légères en les mélangeant au préalable avec plusieurs fois leur volume de sable bien sec. Pour bien régler le répandage, il est prudent, dès le début, de répartir la semence et le terrain en une même quantité de fractions égales.

Quantité de semences à employer. - La quantité de semences à employer varie avec le sol, avec la pente et le climat local; elle augmente avec les dangers qui menacent les jeunes plants pendant les premières années : sécheresses, gelées, ravages des animaux, etc.. ; elle dépend, enfin, de la grosseur de la semence, du mode de culture donné au sol, de la qualité même de cette graine et de la saison des semis.

Nous résumons ci-après un tableau publié par l'Agenda du Forestier (1), et dressé d'après les travaux de MM. Bouquet de la Grye, Caquet, Mathieu, Fliche, Pierret et autres :

(1) Agenda du Forestier, année 1900. Édité par Paul Jacquin, à Besançon, sous les auspices de la Société forestière de Franche-Comté et Belfort. 


\begin{tabular}{|c|c|c|c|c|c|c|c|}
\hline \multirow[b]{2}{*}{ ESSENCES. } & \multirow[b]{2}{*}{$\begin{array}{l}\text { MARCHE ET DURÉE } \\
\text { DL LA } \\
\text { GERMIXATION. }\end{array}$} & \multirow{2}{*}{ 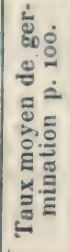 } & \multirow{2}{*}{ 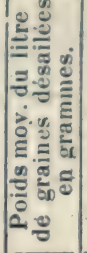 } & \multicolumn{4}{|c|}{ 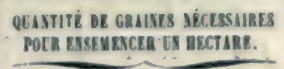 } \\
\hline & & & & Semi & 㱠 & 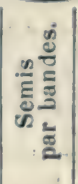 & 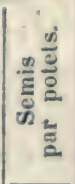 \\
\hline Aune.......... & 3-6 semaines. & 30 & 325 & 0,6 & $15-20$ & $15 \mathrm{~kg}$ & $15 \mathrm{~kg}$ \\
\hline Bouleau ....... & $4-5 \quad-$ & .45 & 90 & 4 & $30-40$ & 30 & 30 \\
\hline Charme....... & Souv. $2^{e}$ année. & 50 & 415 & 6 & $60-70$ & $4 \mathrm{hl}$ & $2^{\mathrm{hl}}$ \\
\hline Châtaignier.... & 3-6 semaines. & D & 500 & 8 & $3-500 \mid$ & 5 & 3 \\
\hline $\begin{array}{r}\text { Chênes, rouvre } \\
\text { et pédouc..... }\end{array}$ & $4-6=-$ & 70 & 575 & $8-12$ & $4-600$ & $5-7$ & $3-5 h^{\prime}$ \\
\hline Epicéa......... & $4-6 \quad-$ & 70 & 530 & 0,17 & 9 & $6 \mathrm{~kg}$ & $4 \mathrm{~kg}$ \\
\hline Erable symoc.. & $4-6$ & $n$ & 130 & & $40-45$ & $2 \mathrm{hl}$ & $1,5 \mathrm{hl}$ \\
\hline Frêne..... & $4-6$ & 60 & 170 & $2,5-3$ & $40-50$ & 2 & 1,5 \\
\hline IIêtre..... & $3-4$ & 60 & 415 & 6,8 & $230-300$ & 4 & 2 \\
\hline Mélèze..... & $3-5$ & 50 & 480 & 0,6 & 30 & $20 \mathrm{~kg}$ & $5-12$ \\
\hline Orme (ailé)..... & $2-3$ & . & 45 & 6 & 20 & 15 & D \\
\hline Pin sylvestre... & $3-6 \quad-$ & 75 & 500 & 0,16 & $8 \cdot 10$ & 5 & $3 \mathrm{~kg}$ \\
\hline Pin à crochet... & Levée as. rapide. & 70 & 480 & 0,17 & 8 & 5 & 3,5 \\
\hline Pin laricio (Corse). & id. & 77 & 500 & 0,20 & 10 & 6 & 4 \\
\hline $\begin{array}{c}\text { Pin noir d'Au- } \\
\text { triche........ }\end{array}$ & Levée rap., $15 \mathrm{j}$. & 75 & 520 & & & 8 & 4 \\
\hline Pin maritime... & $\begin{array}{c}\text { id. } \\
\text { id. }\end{array}$ & 75 & 590 & $\begin{array}{l}0,20 \\
0,20\end{array}$ & 12 & 8 & 5 \\
\hline Pin Alep........ & id. & 75 & 510 & 0,20 & 10 & 6 & 4 \\
\hline Pin Cembro.... & Germ. lente, irr. & 55 & 520 & " & $"$ & $n$ & D \\
\hline Robinier,f.acac. & $2-4$ semaines. & 55 & 150 & " & $20-22$ & 15 & 12 \\
\hline Sapin (ailé'...... & $3-6 \quad-$ & 60 & 250 & $3-4$ & $75-80$ & 60 & 50 \\
\hline Tilleul......... & Souv. $2^{\circ}$ année. & 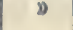 & $m$ & $x$ & a. & $x$ & $n_{1}$ \\
\hline
\end{tabular}

Ces chiffres peuvent être doublés, triplés même, si les conditions sont particulièrement mauvaises. C'est ainsi, par exemple, qu'il n'est pas exagéré d'employer jusqu'à 40 kilogrammes de graine désailée de mélèze, d'épicéa ou de pin de montagne pour les semis à la volée sur la neige.

Saison favorable pour faire les semis. - Le temps le plus propice pour faire les semis serait celui où la graine mûre se dissémine naturellement. Mais l'opération n'est pas toujours possible à cette époque pour les motifs suivants : la préparation de la graine récoltée, surtout quand il s'agit de cônes résineux, exige un certain temps; - à cause des variations dans les prix de main-d'oeuvre, c'est une question d'économie qui fera préférer le printemps ou l'automne; - enfin 
la mise en état du sol rend souvent désirable l'ajournement des semis.

En général, pour les semences qui mûrissent vers la fin de l'été, en automne et en hiver, le mieux est de les conserver jusqu'au printemps et de les semer alors seulement. Employées en automne, la plupart des graines sont exposées à la voracité des animaux et les jeunes plants, qui apparaissent de bonne heure au printemps sur une surface manquant d'abri, sont souvent atteints par les gelées tardives.

On peut admettre le semis d'automne pour les graines qui se gardent difficilement, celles du sapin pectiné et des pins à grosses amandes, les glands et les faînes; cependant, si on a de bons moyens de les conserver, le semis de printemps sera préférable; cette dernière saison devra être adoptée pour toutes les autres essences. En un mot, la saison des semis doit être choisie de telle sorte que la germination s'opère le plus vite possible, afin que la graine, puis les jeunes plants, traversent rapidement la phase où de nombreux dangers les menacent; c'est-à-dire assez tôt pour qu'ils profitent de l'humidité du sol au sortir de l'hiver, pas trop tôt cependant avant les premières chaleurs du printemps.

Application aux essences sociales. - Les résultats du boisement par semis direct se font longtemps attendre, car la végétation des plants forestiers est souvent très lente dans les premières années. Il faut s'armer de patience et se garder de toute comparaison avec les régénérations naturelles en forêt; autrement, les semis faits de main d'homme paraitront toujours trop rares. Dès qu'on est assuré de la réussite d'un plant par mètre carré, on a lieu d'être satisfait; en vouloir davantage, serait exagérer inutilement la dépense. Ce résultat obtenu, on doit savoir oublier les boisements, - en les surveillant, - et, quelques années plus tard, on sera fort surpris de trouver le fourré formé, là où l'on croyait ne rencontrer que. des brins trop espacés.

Les semis des essences disséminées, qui ne comportent pas l'état de massif pur, nè se font généralement qu'en pépinière. On ne considérera donc ici que ceux des espéces sociales.

Le chêne, essence de lumière, peut être semé en plein dé- 
couvert; le semis est alors préféré à la plantation à cause de la longueur de pivot qui rend cette dernière opération onéreuse et difficile. Dans les terres suffisamment profondes, l'emploi de la charrue est tout indiqué; après un premier labour en plein, qui ameublit le sol et que l'on peut faire à l'automne, on dispose les glands dans des sillons parallèles que le versoir de l'instrument recouvre à un second passage; tantôt, on laisse inculte les espaces de $1^{\mathrm{m}}, 50 \mathrm{à} 2$ mètres qui séparent deux lignes de glands; - tantôt on donne une culture complète au terrain sur lequel on fait une demi-emblavure de céréales; - tantôt, ce qui vaut mieux encore, on occupe les interlignes avec des pommes de terre plantées aussi à la charrue, et qui, plus que l'avoine, le seigle ou le blé, et jusque dans les premiers jours de septembre, entretiennent le sol dans un état de propreté et de fraîcheur dont profitent les chênes; - tantôt on abrite les rigoles par des plantations de bouleaux ou des boutures de saule marceau. Dans les friches, on peut procéder par bandes ou par potets. Pour les glands surtout, le semis de printemps est préférable à celui d'automne, à cause des dégâts auxquels ils sont exposés en hiver. Les glands devant être recouverts de 4 à 6 centimètres de terre seulement, le fer de la charrue sera réglé en conséquence. Le semis expéditif, en forme de repiquage, est le plus économique et donne de bons résultats avec une dépense par hectare de 3 à 4 hectolitres de glands et 4 à 5 francs de main d'œuvre.

En pays de plaines, de collines ou de basses montagnes, le semis de hêtre ne doit être tenté qu'en forêt. On repique alors les faînes à la houe, comme les glands. Ces faînes, qui se conservent difficilement, peuvent être semées en automne. Quand on a su les garder jusqu'au printemps, il est extrêmement. important de les mettre en place toutes fraiches et aussitôt après la sortie des réserves oủ elles sont stratifiées; car, si les semences ont déjà germé, il suffit, en cet état, qu'elles soient exposées à l'air quelques instants, pour être perdues; c'est seulement pour les semis à faire en pépinière, lorsque la distance entre les réserves et les bandes est assez faible pour que le transport se fasse it la minute, qu'on peut avoir 
intérêt à les faire germer avant la mise en place. Sur les sols granitiques et schisteux des Cévennes, aux altitudes de 1,200 à 1,500 mètres, on est parvenu à installer, en terrain nu et par voie de semis, de jeunes massifs du meilleur aspect sur des points où la violence du vent s'opposait à la bonne venue des espèces résineuses les plus rustiques; on procéde de la manière suivante : on ouvre une rigole orientée, si la pente le permét, suivant la direction Est-Ouest, et à laquelle on donne 15 centimètres de profondeur et 10 centimètres de largeur; on forme avec le déblai une sorte de butte sur le bord Sud de cette rigole; après avoir bien ameubli la terre du fond, on y sème les faînes, à raison de un litre par 5 mètres courants, et on les recouvre de 8 centimètres de terre. Dès la levée, on comble progressivement la rigole avec la terre de déblai jusqu'à la hauteur des feuilles cotylédonaires d'abord, des feuilles vraies ensuite, et cela jusqu'à épuisement de la terre en réserve. On entretient ainsi toujours fraîche la zone où se trouvent les racines des jeunes plants, qui peuvent, dês lors, résister à la sécheresse du premier été. Le même procédé peut être employé dans les pépinières, en réduisant l'espacement entre les bandes à la place strictement nécessaire pour installer les buttes, c'est d'ailleurs celui qui est indiqué par Cotta et rappelé par MM. Lorentz et Parade. (Cours de culture des bois, $\S 720$ ). Mais la réussite ne tient pas, comme le croyaient lés auteurs, à une sensibilité extrême de la tigelle aux rayons lumineux, mais à ce fait que la terre rapportée protège les racines contre le dessèchement superficiel. - Les semis artificiels de cette espèce semblent plus exposés que les régénérations naturelles aux dégáts d'un champignon: Peronospora infestans.

Le semis de charme peut se faire à la volée, en forêt, dans les sols frais et sous l'abri interrompu d'une coupe secondaire. En plein découvert et dans les sols exposés à se dessécher, cette essence aurait peu de chances de réussir à cause de la faiblesse de son enracinement; un peu de fraîcher ou l'abri de quelques broussailles sont nécessaires; il est d'ailleurs indispensable, dans ces sols nus, toujours tassés, d'enfouir la semence de charme à 1 ou 2 centimètres de profondeur. 
Le semis de sàpin pectiné ne réussit bien qu'en forêt, à l'abri d'un peuplement déjà formé ; alors, il peut se faire par bandes ou par places. Un moyen économique de l'installer consiste à racler la surface du sol, sur des placeaux de 25 centimètres de côté, en donnant un léger ameublissement superficiel; dans chacun de ces placeaux, on désarticule un cône frais cueilli. Il faut à la graine de sapin un sol riche en terreau et un recouvrement d'un demi centimètre de terre. On sait que, pour elle, le sèmis d'automne est préférable à celui du printemps.

Les semis d'épicéa se font au printemps, par bandes ou par potets; ils demandent un sol bien préparé et surtout débarrassé des mauvaises herbes. On recouvre la graine légèrement de manière que, par une pluie, même assez forte, elle ne puisse pas être mise au jour ; on obtient ce résultat en remuant la graine répandue sur le sol avec un râteau à dents courtes, ou en passant, en forme de hersage, un fagot d'épines sur les parties ensemencées. Les jeunes plants faiblement enracinés, dont la croissance est lente, sont exposés à être soulevés par la gelée d'hiver; par contre, les gelées printanières ne leur font que peu ou point de mal. Dans les stations basses, les épicéas demandent un ombrage modéré; ils réussissent néanmoins en plein découvert, si le climat n'est ni trop chaud, ni trop sec.

De toutes les essences feuillues ou résineuses, le pin sylvestre est, avec le chêne et les pins méridionaux, celle qui s'installe le mieux par semis direct. En toutes stations, il accepte le plein découvert; il préfère les terrains siliceux, mais réussit généralement dans tous les sols, pourvu qu'ils soient graveleux. Tous les modes de semis lui sont applicables: le semis en plein avec une demi semence de céréales, tout aussi bien que le semis par bandes ou par potets. C'est avec le pin sylvestre seul qu'ont parfois réussi les semis sur bruyères, en terrain non préparé. A côté de ces avantages, il est regrettable que, dans leur première jeunesse, les semis de pin sylvestre soient sujets à la maladie cryptogamique dite du rouge, connue en Allemagne sous le nom de schälte, et dont les dégâts prennent' la proportion d'un véritable fléau. Si, à la 
rigueur, un traitement convenable peut atténuer le mal dans les pépinières,il n'y a aucun remède pratique à recommander pour les semis en place ; la plantation de sujets sains et assez âgés pour avoir dépassé l'époque de la crise sera la seule bonne solution. Les semis naturels échappent heureusement en grande partie à ce danger.

En sol calcaire, le pin noir d'Autriche se comporte mieux que le pin sylvestre: il est d'ailleurs aussi rustique, et peut être employé de la même manière; toutefois, comme sa graine est toujours d'un prix assez élevé, il vaut mieux, en général, procéder par plantation.

A cause de la rapidité de sa croissance, de la valeur des produits divers qu'on en retire et surtout de son extrême frugalité, le pin maritime est l'essence la plus précieuse pour boiser les terrains siliceux, pauvres, des climats maritimes; il est tout indiqué pour la mise en valeur des landes et la fixation des dunes du littoral. C'est sous forme de semis direct qu'il est le plus employé, et les procédés spéciaux qui servent à l'installer seront indiqués plus loin.

Dans les régions calcaires de la Provence, le pin d'Alep se présente avec les précieuses qualités qu'on vient de constater chez le pin maritime. Mieux que ce dernier encore, il accepte les conditions de sol les plus mauvaises et les chaleurs les plus excessives; on le voit s'installer naturellement entre les fissures des rochers stériles où la terre végétale fait presque entièrement défaut. On le sème par potets; le semis d'automne serait préférable à celui de printemps, si de nombreux ennemis ne venaient pas, en hiver, détruire soit les graines, soit les jeunes plants naissants; en général, on opère dans les deux saisons, en complétant, au printemps, les semis d'automne insuffisants. Telle est la résistance du pin d'Alep à la sécheresse, qu'on est parvenu, grâce à son aide, à boiser le rocher nu du mont Faron, où, sur bien des points, il a fallu ouvrir à la barre à mine des trous qu'on remplissait ensuite de terré rapportée.

Le semis direct du méléze ne se pratique guère hors de sa station, sur les hautes montagnes. On l'y sème comme l'épicéa, en prenant toutefois la précaution de faire macérer la 
graine dans l'eau, pendant 15 ou 20 jours, avant de l'employer; elle doit être mise en terre, humide et sans qu'on lui laisse le temps de se ressuyer. Un peut semer le mélèze sur la neige, c'est même à l'aide de ce procédé qu'on a obtenu les meilleurs résultats, sur les gazons continus comme sur les éboulis rocheux.

Les renseignements qui précèdent s'appliquent également aux semis d'essences mélangées; quand les graines ont mêmes exigences et même grosseur, il suffit de préparer le mélange en proportions voulues avant le répandage; si elles sont de grosseurs différentes, il faudra d'abord semer les graines lourdes, qui demandent à être plus profondément enfoncées, et, seulement ensuite, les graines légères.

Soins à donner aux semis. - Les semences forestières de bonne qualité, semées avec soin et en bonne saison, germent presque toujours; il est assez rare, en effet, qu'elles restent inertes dans le sol, et qu'elles y fondent, suivant le terme employé en horticulture. Mais il faut tenir compte des dégâts trop fréquents des animaux; nous renvoyons à l'article III (pépinières) pour tout ce qui concerne les moyens de lutter contre ces déprédateurs petits et grands.

C'est donc surtout après la levée que surviennent les véritables dangers auxquels les semis directs sont exposés. La sécheresse du premier été et le déchaussement à la fin du premier hiver sont à craindre tout d'abord et, quelles que soient les précautions prises, il y a toujours de ce côté certaines chances défavorables à courir. Malgré tout, quand les circonstances de sol et de climat autorisent le boisement par par semis direct, un premier échec ne doit pas décourager et, en cas d'insuccès marqué, il faut recommencer le travail, en comptant sur des temps meilleurs.

Quand la réussite, suffisante en général, est incomplète sur des taches disséminées, on regarnit les vides par de nouveaux semis en potets, ou, mieux encore, par des plantations de jeunes sujets extraits dans le semis même, sur les points oủ ils se rencontrent trop serrés.

Pour les semis en plein, avec ou sans préparation du sol, il est à peu près impossible de décider, dès la fin de la première 
année, si la réussite est bonne : il faut attendre deux et même trois ans avant de pouvoir se prononcer. Au contraire, quand les semis sont faits par bandes ou par potels, l'inventaire des plants d'avenir est relativement facile; c'est une raison de plus pour donner la préférence à ces derniers modes.

Lorsque le jeune semis a résisté aux épreuves de la première année, il n'a plus que rarement à souffrir des accidents météoriques, mais il risque d'être étouffé par les plantes sauvages qui l'envahissent. Il est toujours utile, et parfois même indispensable, de procéder à leur enlèvement, soit à la main, soit à la faucille; ces travaux s'exécutent vers la fin du printemps, en tout cas, avant la floraison des végétaux nuisibles; ils doivent être répétés annuellement jusqu'à ce que les jeunes plants soient assez forts pour n'avoir plus rien à redouter de ce côté.

Enfin, quand les semis d'essences de lumière, notamment ceux de pin sylvestre et de mélèze, naissent trop serrés, il faut procéder à leur dépressage. En effet, les jeunes brins s'affament mutuellement, s'entravent dans la poussée de leurs branches latérales et forment des paquets oủ la lumière ne peut pénétrer : les plus chétifs sont exposés les premiers à l'invasion des insectes et des champignons; les autres seront ensuite attaqués, car, on le sait, les champignons recherchent les endroits abrités contre le vent, et un jeune peuplement très serré leur crée ce milieu favorable; il semblerait, dès lors, que la nature ait chargé ces organismes de maintenir à l'état clair les espèces qui, par tempérament, demandent semblable situation; mais, si on les laisse seuls faire la besogne, le plus souvent ils dépasseront le but. Aussi faut-il, dès que les semis ont atteint l'âge de quatre à cinq ans, procéder soi-même à l'opération, qui sera d'autant plus facile qu'on la commencera plus tôt. On opère par section, au ciseau ou au sécateur, plutôt que par l'arrachage qui ébranle, en même temps, les racines des sujets d'avenir. Plus tard, quand les semis non dépressés sont devenus des gaules trop longues pour leur faible diamètre, ce sont les dégâts de la neige qui les menacent, comme dans les plantations, et nous savons qu'il faut les éclaircir. 
ARTICI.E III

\section{LE BOISEMENT PAR PLANTATIONS}

\section{Les plants.}

Qualité des plants. - Leur origine. - Leur âge et leur dimension.

Qualité des plants. - De même que la réussite des semis demande l'emploi de bonnes graines, de même, pour assurer l'avenir d'une plantation, il faut avoir à sa disposition de bons plants.

Au moment de leur mise en terre, les plants, quelle que soit leur force, doivent présenter, dans la mesure du possible :

$1^{\circ}$ des racines complètes, touffues, régulières et surtout un chevelu développé;

$2^{\circ}$ une tige droite, régulière, une cime bien ramifiée et annonçant la vigueur; des branches latérales proportionnées à l'âge du plant;

$3^{\circ}$ le feuillage ou les bourgeons complets et bien constitués;

$4^{\circ}$ un aspect sain; la tige et les racines sans blessure aucune, ni cicatrices suspectes.

Mais toutes ces qualités, fussent-elles développées autant qu'on pourrait le désirer, deviennent inefficaces si le plant n'est pas frais, c'est-à-dire s'il n'est pas bien vivant dans son ensemble, et si les parties les plus grêles, les plus délicates du chevelu sont restées trop longlemps exposées à l'action desséchante du vent et du soleil.

Origine des plants. - Il y a deux moyens de se procurer les plants forestiers. On peut les prendre directement en forêt parmi des semis naturels, ou les élever en pépinière.

Les plants extraits des peuplements naturels, ayant vécu au milieu d'un massif, sur un sol non ameubli, ont le défaut de manquer de chevelu, d'offrir des tiges grèles, sans ramifications suffisantes. On ne saurait trop réagir contre cette opinion erronnée qu'ils sont préférables aux sujets élevés en pépinière, trop bien soignés, dit-on, dans leur jeunesse pour accep- 
ter des conditions d'existence médiocres ou mauvaises. C'est tout le contraire : nés sous l'ombrage, ils souffrent, lorsqu'ils sont exposés subitement à toutes les influences atmosphériques; dailleurs la transplantation est une crise, et chez les arbres, comme chez les hommes, un individu chétif et mal nourri ne résiste pas aussi bien que si les conditions inverses étaient remplies. Enfin, les sujets arrachés en forêt sont extraits sans soin, et la nécessité de les ramasser un à un empêche de les traiter convenablement; exceptons cependant: les bouleaux que l'on trouve parfois en véritable gazon, sur des terrains meubles, - les hêtres et les sapins, quand on a l'occasion de les enlever en mottes.

En résumé, il faut des circonstances exceptionnelles pour justifier l'utilisation des plants sauvages.

Les sujets élevés en pépinière sont de beaucoup les meilleurs; la culture et les soins constants dont ils sont l'objet permettent de leur donner toutes les qualités requises ; l'extraction en est facile, et peut se faire sans qu'il soit besoin de briser ou de déchirer leurs racines.

Age et dimension des plants. - On distingue les plants en: bassestiges, demi-tiges et hautes tiges.Les premiers sont des sujets toujours très jeunes, ayant en hauteur depuis quelques centimètres jusqu'à 1 mètre; les demi-tiges ont de 1 à 2 mètres, et les hautes tiges dépassent 2 mètres.

Les plantations réussissent d'autant mieux qu'elles sont faites avec des plants plus jeunes, le fait est bien reconnu aujourd'hui. Aussi, les demi-tiges, et à plus forte raison les hautes tiges, ne sont-elles utilisées que dans des cas spéciaux, pour boiser, par exemple, des terrains couverts de grandes herbes ou livrés au parcours du bélail. L'emploi de ces sujets sur de grandes étendues coûterait d'ailleurs trop cher: ce sont de véritables plantations de luxe, qui demandent des précautions dont les détails ne peuvent trouver leur place que dans un traité d'arboriculture.

Il est donc, en général, convenable de planter des basses tiges et même de très jeunes sujets sans descendre, toutefois, au-dessous d'une limite raisonnable, car les plants trop exigus sont d'une manutention délicate; leur enracinement ne 
dépasse pas les couches les plus superficielles du sol qui se dessèchent infailliblement en été ; enfin, pour eux, le phénomène du déchaussement est plus à redouter. Une hauteur de 15 à 20 centimètres à partir du collet de la racine est une bonne moyenne.

Quant à l'âge, il varie suivant les conditions dans lesquelles on opère, suivant aussi la rapidité avec laquelle les semis des différentes espèces forestières développent leur appareil aérien et surtout radicellaire. Les pins des climats chauds (pin maritime, pin d'Alep, pin pinier) se plantent à un an, deux ans au plus; le pin sylvestre, le pin d'Autriche, le mélèze à deux ou trois ans,l'épicéa à trois ou quatre ans, le sapin à quatre ou cinq ans. Les feuillus (chêne, hêtre, érable, bouleau, frêne, charme), peuvent se planter à deux ou trois ans; les robiniers et les aunes à un ou deux ans.

$\mathrm{Si}$, comme nous le dirons plus loin, on peut souvent éviter l'opération coûteuse du repiquage pour les sujets de deux et même trois ans que l'on élève soi-même en pépinière, du moins ne faut-il pas, sil'on achéle les plants dont on a besoin, reculer devant un supplément de prix de quelques francs par mille pour se les procurer repiqués : leur reprise est toujours beaucoup plus certaine.

\section{Les pépinières.}

I. installation d'Une pépiniène centrale

Capital d'exploitation. - Choix de l'emplacement. - Division du terrain. - Préparation du sol. - Les engrais. - Le terreau. - Aménagement de la pépinière. - Son étendue. - Les outils. - Les clotures. - Les dangers à combattre.

Capital d'exploitation. - Une pépinière centrale est une véritable exploitation horticole. Son installation demande une forte dépense de premier établissement; il faut, en outre, un fonds de roulement assez important. Aussi, en dehors du commerce, un propriétaire ne doit-il se résoudre à une pareille entreprise que s'il veut boiser des terrains d'une étendue considérable.

Choix de l'emplacement. - L'emplacement d'une pépi- 
nière permanente doit répondre aux conditions suivantes (1):

$1^{\circ}$ se trouver dans une position aussi centrale que possible par rapport aux différents cantons à reboiser et qu'elle devra approvisionner, en tout ou en partie;

$2^{\circ}$ offrir un accès facile pour le transport des plants ainsi que des engrais et des amendements à y employer ;

$3^{\circ}$ être à la portée de la résidence de la personne chargée d'en diriger l'exploitation, ou mieux, être attenante à une maison où le chef de culture sera logé;

$4^{0}$ présenter une surface aussi homogène et un périmètre aussi régulier que possible, permettant une bonne division et facilitant les clôtures ;

$5^{\circ}$ ne pas occuper des fonds bas et humides où les gelées et le déchaussement sont le plus à redouter, mais, au contraire, des versants en pentes très douces, exposés, de préférence, à l'Est et au Nord-Est, afin d'éviter une trop grande précocité, au printemps, et un trop grand prolongement de la végétation, en automne ;

$6^{\circ}$ être susceptible d'irrigation à l'eau courante, surtout dans les régions à climat sec;

$7^{\circ}$ posséder un sol d'une fertilité moyenne ou meilleure encore, car elle doit produire des plants vigoureux et bien équilibrés, très aptes à une reprise certaine et prompte.

Quand on connaîtra d'une façon bien précise la destination à donner aux plants, on choisira un terrain qui, par sa constitution et sa base minéralogique, présente une certaine analogie avec les cantons à boiser; car les plants d'une même espèce se constituent un enracinement variable suivant la nature du sol dans lequel ils végètent; les racines produites en sol humide ne conviennent pas pour une plantation à faire en sol sec, toutes n'y fonctionneraient pas; de même, des plants nés en terrain siliceux, léger, seraient mauvais pour boiser des sols argileux ou compacts. Sous ces réserves, on constate que les terrains siliceux, meubles, sont les plus avantageux pour l'installation d'une pépinière permanente: les travaux de culture y sont moins coûteux qu'en

(1) Landolt, La forêt; manière de la rajeunir et de la soigner, 3 e édition. Traduction par X. Amuat, Porrentruy, 1880. 
terrain argileux; ceux de sarclage plus faciles qu'en terrain calcaire; les jeunes plants s'y forment, d'ailleurs, un chevelu trés abondant; le déchaussement y est aussi moins à craindre; enfin, un terrain neutre est toujours préférable, en ce sens qu'aucune espèce n'en est exclue : il suffit, pour les unes ou pour les autres, d'ajouter, sous forme d'engrais ou d'amendement, les éléments nutritifs qui, à un moment donné, pourraient faire défaut; on crée de la sorte des stations artificielles pour l'éducation d'espèces étrangères à la flore locale. D'ailleurs, si les terres légères conviennent mieux pour la réussite des semis, une terre un peu forte sera préférable pour l'éducation des plants repiqués à élever en moyennes ou hautes tiges.

Contrairement à ce préjugé que, pour établir une pépinière, il faut éviter les terres trop fertiles, on doit être assuré qu'un terrain de très bonne qualité formera des plants plus vigoureux, mieux pourvus de racines et d'une reprise plus certaine que celle des sujets chétifs et mal nourris, élevés en un sol trop maigre; il en est de ceux-ci comme des plants sauvages.

Si, dans les espaces dont on dispose, il ne se rencontre aucune parcelle présentant les conditions convenables, il vaudra donc mieux s'abstenir, ou se résoudre à chercher ailleurs un terrain favorable, qui sera loué, ou mieux, acheté.

Division du terrain. - L'emplacement déterminé, on calcule l'étendue à donner à la pépinière, en s'appuyant sur les données qui seront indiquées plus loin.

Le premier travail consiste à délimiter un certain nombre de grandes divisions par des chemins de $2^{\mathrm{m}}, 50$ à 3 mètres de largeur, permettant aux charrettes de circuler en tous sens. On partage chacune de ces sections en carrés ou en rectangles, d'une surface de 10 à 15 ares, séparés entre eux par des petits chemins de 1 mètre de largeur et accessibles à la circulation des brouettes.

On procède, ensuite, au nivellement des chemins grands et petits et on les combine de façon à rendre, d'une part, inoffensif l'écoulement des eaux pluviales et à permettre, d'autre part, l'irrigation des carrés. A ce point de vue, il est avan-

Bopre et JoLyet. 
tageux, chaque fois que la chose est possible sans trop grands frais, de donner une horizontalité parfaite à chacune de ces surfaces : les eaux de pluie ou d'arrosage pénètrent plus régulièrement dans le sol et tout danger de ravinement est écarté. Un usage général consiste à disposer la superficie des carrés en saillie de 15 à 20 centimètres au-dessus du niveau des chemins; dans les terrains meubles et filtrants, dans les climats chauds et secs, une disposition inverse serait certainement préférable : les chemins tracés en relief au lieu de drainer le sol serviraient de barrage pour empêcher la perte des eaux quelle qu'en soit la provenance; mais, pour que cette disposition puisse être utilement adoptée, il est nécessaire que la surface du carreau soit parfaitement horizontale.

On fera bien, en quelque endroit retiré de la pépinière, tout en préparant les fosses à fumier et les pourrissoirs, de réserver, sur les points les plus humides, l'emplacement nécessaire pour l'installation d'une oseraie. Les meilleures espèces de saules à choisir pour cet usage sont les suivantes : Salix fragilis, S. viminalis, S. pentandra, S. Lambertiana. De même, il est utile de planter autour des bassins d'irrigation quelques touffes de jonc (Juncus glaucus) destinées à fournir des liens; dans les endroits secs des climats méridionaux, cette espèce peut être remplacée par le sparte (Ligeum spartum).

Préparation du sol. - En tout état de choses, les carrés seront défoncés à une profondeur d'environ $\mathbf{4 0}$ centimètres (2 fers de bêche). Cette opération est indispensable, et de sa bonne exécution, dépend l'avenir de la pépinière. Le défoncement a pour but d'ameublir la terre, de la rendre plus perméable aux racines, à l'air, aux pluies, - plus productive enfin. On ne saurait trop insister sur ce fait que la culture des plants forestiers en pépinière, comme l'aboriculture fruitière, ont beaucoup de points communs avec la culture maraîchère : partout et toujours, le sol doit être préparé de la même façon, c'est-à-dire, être profondément défoncé et largement fumé.

Le défoncement se fait, soit à la bêche, soit à la houe ou 
à la pioche; quand cela est possible, l'emploi de la charrue sous-sol est plus économique. L'ouvrier qui travaille à la houe ou à la bêche ouvre d'abord une jauge de 1 mètre de largeur sur la profondeur voulue, et, pour "se donner du champ ", il conserve toujours cet espace libre entre la ligne de défrichement et le jet des terres; celles-ci sont disposées, par couches, en talus à 45 degrés : les mottes sont ainsi mieux divisées, et celles qui échappent tombent au fond de la jauge, où il est facile de les briser. On répand ensuite les engrais bien également sur tout le talus des surfaces remaniées, en ayant soin de ne pas en laisser tomber dans le fond de la jauge, où ils seraient enterrés à une trop grande profondeur, et l'on continue, de proche on proche, par tranches de 20 à 25 centimètres de largeur, jusqu'à l'extrémité du carré que l'on nivelle avec la terre provenant de la première jauge (1). Les couches superficielles élant ainsi mélangées avec les plus profondes, la masse présente une compacité et une fertilité moyennes, en même temps qu'une égale richesse en engrais.

Tout en procédant au défoncement, on débarrasse le sol des plus grosses pierres ou pierrailles (tous les fragments qui dépassent la grosseur d'une noix devront être enlevés). Ces matériaux serviront pour assainir et empierrer les chemins et sentiers.

Il est nécessaire de laisser à la terre le temps de se tasser avant de lui confier les graines; pour cela, on termine le travail à la fin de l'automne dans les terrains à ensemencer au printemps suivant, et à la fin du printemps pour ceux qui recevront les semis d'automne. Il suffira de donner un léger labour quand le moment sera venu de semer ou de repiquer.

Les engrais. -- La question des engrais est tout à fait capitale quand il s'agit de pépinières permanentes. Car, si une pépinière qu'on installe dans un sol forestier nouvellement défriché peut, à la rigueur, fournir deux ou trois récoltes, sans engrais, elle se refusera le plus souvent, à en produire une quatrième; le sol des pépinières est, en effet,

(1) Gressent, Le potager moderne, 40 édition, p. 209, Paris, Goin, 1875. 
exposé sans protection aux influences atmosphériques; de plus, aucune matière fertilisante ne vient l'entretenir comme celui des massifs forestiers. "Quelles que soient leur nature minéralogique et leur fertilité première, dit M. Grandeau (1), les terrains consacrés pendant de longues années à des semis feuillus ou résineux, qu'on exploite tous les deux ou trois ans (tiges et racines), s'épuisent comme les sols livrés à la culture agricole: les jeunes plants d'arbres nécessiteront des quantités de matières minérales bien supérieures annuellement à celle que les arbres eux-mêmes réclament plus tard. " Ainsi, de toute nécessité, il faut fumer les pépinières à la façon des terres arables.

D'autre part, les analyses de terreaux de feuilles ont permis de constater que ces engrais, d'ailleurs suffisamment riches en potasse, sont très pauvres en azote (ils en renferment à peine 0,40 p. 1000 ) et en acide phosphorique. Terreau ou humus forestier, employés purs et tels qu'il est possible de se les procurer gratuitement dans la forêt, sont donc insuffisants.

Le fumier de ferme, par sa porosité, entretient l'état meuble et l'aération dans une terre qui, couverte de la même récolte pendant 2 ou 3 années consécutives, ne reçoit pendant cet intervalle que des façons superficielles et insignifiantes. Toutefois, il présente l'inconvénient d'être de composition mal titrée; aussi, tout en lui donnant la préférence chaque fois qu'il sera possible de se procurer à bon compte du fumier de qualité moyenne, tel qu'il est mis en œuvre par des cultivateurs sérieux, on devra s'assurer de sa teneur en matières fertilisantes et l'additionner d'engrais chimiques, de façon à le transformer en engrais complet, eu égard à l'état du sol et à la nature des récoltes qu'on veut obtenir. En général, le fumier de cheval convient mieux dans les terres froides et compactes, celui de vache dans les terres meubles et sèches,

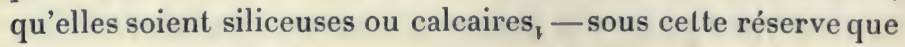
le fumier de vache expose plus que celui de cheval à la propagation des larves dangereuses.

(1) Grandeau, Annales de la station agronomique de l'Est, tome I, p. 400 . 
A défaut de fumier de ferme, pour rendre au sol la matière organique dont il ne peut se passer, on a recours à des composts faits de déchets de jardin, de feuilles ramassées en forêt, d'herbes, de pourrissoirs, d'ajoncs, de genêts, etc... préparés dans les conditions qui seront indiquées plus loin. Nous venons de constater la pauvreté de ces composts en sels nutritifs; aussi, pour rétablir l'équilibre rationnel entre l'importation et l'exportation, est-il toujours indispensable de leur mélanger des engrais organiques ou minéraux; ceux-ci sont incorporés dans la masse des composts au moment de leur préparation, et dans des proportions variables suivant la qualité du sol naturel et son degré d'épuisement en telle ou telle substance.

Il existe dans le commerce toutes sorles de poudres ou mélanges dont les vendeurs disent merveille pour leur emploi en pépinière. Il y a toujours lieu de se méfier des falsifications, et le plus sage sera de se procurer des engrais titrés en s'adressant aux syndicals et aux stations agronomiques. En toutes circonstances, et pour éviter des analyses longues et coûteuses, on peut, sans crainte, choisir entre l'une des formules ci-après, qui se recommandent par la notoriété de leurs auteurs :

"Pour les vergers, dit M: Grandeau, on se trouve bien, à l'hectare, des fumures suivantes :

2000 kilog. de scories de déphosphoration;

ou 600 - de kaïnite;

ou 200 - de chlorure de potassium.

" On peut aussi employer le mélange indiqué par P. Wagner, savoir :

200 kilos superphosphate double;

ou 500 - de superphosphate à $16 \mathrm{p} .100$
et 160 de chlorure de potassium;
ou 230 - de phosphate de potasse
et 40 de chlorure de potassium.

" Ces fumures de tête doivent être incorporíes dans les "fumiers composts avec lesquels ils sont répandus avant la " façon; après le labour et l'épandage des graines au prin- 
" temps, on sème, à la volée, 200 kilos de nitrate de soude à "l'hectare (1). "

Les pépinières de l'École forestière d'Eberswalde (Prusse) en sol siliceux très pauvre, ont été entretenues dans un parfait état de rendement par l'emploi, à l'hectare, sous forme de mélange dans les composts, de :

50 kilos de poudre d'os azotée

$$
\begin{aligned}
50 \text { - de scories de déphosphoration } \\
100 \text { - de sang desséché } \\
50 \text { - de sulfate d'ammoniaque } \\
150 \text { - de carnallite (2). }
\end{aligned}
$$

Ces quantités suffiront pour une et, au plus, pour deux récoltes; il est facile, d'ailleurs, d'apprécier l'époque du renouvellement par l'aspect des cultures.

Fumiers et composts nourris sont, en général, employés dans la proportion de 8 à 10 mètres cubes à l'hectare.

Enfin, comme source gratuite d'azote, on peut semer dans les jachères du lupin ou toute autre plante légumineuse. Nous nous sommes très bien trouvés de ces cultures dérobées, qui, en même temps qu'elles sont utilisées comme engrais verts, étouffent les mauvaises herbes et dispensent des sarclages.

En ce qui concerne l'emploi du fumier, on remarque que les racines des jeunes plants, mises en contact immédiat avec des matières fraîches, sont exposées à la moisissure. Pour éviter cet inconvénient, il est bon de faire précéder le semis d'une culture de plantes sarclées, ou mieux, de stratifier les fumiers pendant une année dans des fosses abritées contre les pluies et le soleil et où ils seront nourris.

Le terreau. - Quant au terreau utilisé en couverture, lors de l'exécution des semis, on peut le préparer économiquement de la manière suivante :

En dehors de la pépinière, mais aussi près que possible et en donnant la préférence aux endroits bien abrités, on creuse

(1) L. Grandeau, La fumure des champs et des jardins, 6'édition, p. 126, Paris, Librairie agricole, 1897.

(2) Schwappach, Ueber Mineraldüngung in Forstgärten (Zeitschrift für Forst-und-Jagdwesen, t. VII, juillet 1891). 
une tranchée de 60 à 80 centimètres de profondeur et d'une largeur de 3 à $\mathbf{4}$ mètres; on en règle le fond avec une inclinaison convenable vers des fossés d'écoulement, de telle sorte que l'eau n'y séjourne jamais. Dans ce pourrissoir, on accumule des feuilles sèches ramassées à l'automne, au moment de leur chute nalurelle et, de préférence, par un temps humide; on les tasse d'ailleurs convenablement; on y ajoute tous les débris de la pépinière : ce sont, des herbes, des brindilles non lignifiées, des mousses, des pailles, des gazons, des cendres (1); mais il faut avoir le plus grand soin, et cela est d'une importance capitale, de ne jamais jeter au pourrissoir que des plantes qui n'ont pas fleuri. Dès qu'une mauvaise herbe est montée en graine, elle doit être brûlée, autrement on la resème avec les composts.

Pour activer sa décomposition, la masse doit être remaniée deux fois par an, le plus sonvent en mai et en septembre, époques où l'ouvrage ne presse pas dans les pépinières; on profite de ces moments pour faire les additions convenables d'engrais chimiques. Il faut, en moyenne, trois ans pour obtenir l'état de terreau.

La longueur à donner à la fosse sera proportionnée à la quantité de terreau qu'on veut produire annuellement, en tenant compte de ces faits, que cette losse doit toujour's présenter : $1^{\circ}$ une place vide pour recevoir la provision de feuilles à ramasser à l'entrée de l'hiver; $2^{\circ}$ un premier tas de feuilles stratifiées depuis un an; $3^{\circ}$ un second de celles de deux ans; $4^{\circ}$ le terreau de trois ans prêt à être employé. Il faut, de plus, avoir soin que ces dépôts soient séparés entre eux d'un espace suffisant pour qu'on puisse les remuer en les déplaçant par un simple jet de pelle.

Par les temps secs, il sera bon d'arroser le pourrissoir de temps à autre. Ces arrosages seront rendus plus efficaces quand ils seront additionnés de purin, des eaux de vidange, de lessive, de savon ou de cuisine, ou enfin de guano dissous. Tous les terreaux, quelle que soit leur provenance, doivent être passés à la claie avant leur emploi.

(1) En forêt, on peut s'en procurer de grandes quantitís, et à très bon compte, en les ramassant dans les baraques des bùcherons. 
Aménagement de la pépinière. - Une pépinière destinée à fournir des plants d'espèces et de dimensions variées, doit être divisée en grandes sections dans lesquelles on cantonne:

$1^{\circ}$ les semis feuillus ou résineux à planter à demeure, dès l'âge de deux ans, sans repiquage ;

$2^{\circ}$ les semis destinés à être repiqués à un an ;

$3^{\circ}$ les planches à repiquer, ou bâtardières, qui se divisent en compartiments séparés pour les bases tiges, demi-tiges et hautes tiges.

Afin de simplifier les travaux de toute nature, il est bon qu'un compartiment ne soit occupé que par des plants à extraire au même âge; sous cette réserve, on peut admettre plusieurs essences dans le même carré.

Quand les plants sont extraits au printemps pour être mis en place, la saison est, le plus souvent, trop avancée pour qu'on puisse immédiatement les remplacer par de nouveaux semis. Le carreau d'où ils sortent reste donc improductif pendant une saison; on devra néanmoins le sarcler avec autant de soins que s'il était occupé ; aussitôt après l'extraction des plants, on répandra sur leur emplacement la quantité d'engrais nécessaire, puis on donnera un bon labour; dans cette terre, ainsi fumée et bien préparée, on cultivera une récolte de plantes agricoles sarclées : betteraves, carottes, pommes de terre, choux, légumineuses, fourrages, etc., dont la valeur paiera, en grande partie, les frais de fumure et le travail; au printemps suivant, il suffira d'une légère façon pour que le carreau se trouve en parfait état pour recevoir les semis. Il faut donc, de toute manière, consacrer trois carreaux à la production de plants de deux ans et deux à celle de plant de un an.

Une disposition semblable sera adoptée pour les semis extraits à un an pour repiquages; on ne donnera, toutefois, à la terre livrée à la culture agricole qu'une fumure proportionnée à la durée de la rotation.

Cette alternance de cultures agricoles et forestières établit, dans l'ensemble de la pépinière, une sorte d'assolement régulier dont l'effet ne peut qu'être utile à la bonne venue des plants, et les mettre à l'abri des germes infectieux, dont la 
vitalité persiste plusieurs années sous terre. Dans la rotation ainsi établie, il sera bon de faire alterner les semis de feuillus avec les semis de résineux, de façon à ne pas demander au même terrain deux récoltes consécutives de la même essence.

Étendue de la pépiniẻre. - Comme les plants de deux ans ne s'obtiennent qu'à raison d'une récolte sur trois carreaux, il faut multiplier par trois la surface occupée par les plants bons à être mis en place.

En général, on peut compter sur une production moyenne de 400 plants par mètre carré : soit 40000 plants par are de semis de deux ans, et un tiers, ou 13333, par are de pépinière. En tenant compte des déchets, des accidents et des non-valeurs, ce chiffre doit être abaissé à 10000 ; il justifie donc la donnée empirique généralement adoptée pour les pépinières qui approvisionnent les chantiers des boisements obligatoires, laquelle consiste à fixer pour l'ensemble des pépinières une étendue représentée, en ares, par le chiffre des surfaces à reboiser annuellement, exprimé en hectares. Si l'on veut employer des plants repiqués, il faudra tripler, quintupler même les surfaces cultivées, et la dépense sera augmentée dans la même proportion.

Les outils. - Les outils à employer dans les pépinières n'ont rien de spécial ; ce sont ceux dont se sert généralement la culture maraîchère dans la région où l'on se trouve. D'ailleurs, en ce qui concerne l'exécution des semis, l'important n'est pas d'aller vite, mais de bien faire. Il faut donc se méfier des outils soi-disant perfectionnés dans le seul but d'activer le travail. Le semis est toujours une opération minutieuse, et le temps que l'on croit gagner dans une exécution plus rapide se paie souvent fort cher par une réussite incomplète.

Les clôtures. - Toutes les pépinières permanentes ou volantes doivent être défendues par une clôture contre les dégâts des animaux domestiques ou sauvages et contre les maraudeurs. Suivant les cas, cette clôture sera formée de murs, de haies vives, de palissades ou de treillages.

Pour les pépinières volantes, on choisit de préférence un mode économique de clôture mobile : treillage à larges 
mailles, fils de fer, cordon simple ou double de ronce artificielle, piquets et longrines provenant d'exploitations quelconques, etc....

Les dangers à combattre. - Outre les accidents provenant des causes atmosphériques et dont on trouvera plus loin des moyens de prévenir les fâcheux effets, les pépinières sont exposées à de nombreux dangers de la part des animaux : sangliers, lapins, taupes, mulols, oiseaux, larves de hannetons, courtilières, etc. Elles sont aussi attaquées par des organismes inférieurs de la classe des champignons.

On se préserve contre les sangliers qui viennent dévaster les semis de glands ou de faînes, soit avec de solides clôtures, soit en piquant dans les carreaux ensemencés des baguettes à l'extrémité supérieure desquelles sont suspendus des chiffons imbibés de pétrole qu'on renouvelle quand il est évaporé. - Contre lièvres et lapins les entreillagements sont nécessaires, bien qu'on ne doive pas toujours compter sur leur efficacité. - Les taupes se prennent aux pièges. Quand les mulots sont très abondants, il est fort difficile pour ne pas dire impossible de s'en débarrasser, même avec le poison, dont l'emploi, toujours dangereux, n'est pas à recommander.

Malgré la protection que nous réclamons en faveur des petits oiseaux, nous ne pouvons qu'engager ici à se défendre énergiquement contre ceux d'entre eux qui se montrent très friands de certaines graines et surtout des semences résineuses; le danger existe de leur côté depuis le moment où la graine est mise en terre jusqu'au moment oủ la jeune tigelle est débarrassée de l'enveloppe ou chapeau qu'elle pousse hors de terre. Pour les éloigner, on fait circuler autour des carrés un enfant muni d'un fouet, ou un garde armé d'un pistolet qu'il tire à blanc de temps en temps. Quand on peut se procurer à bon marché de vieux filets de chasse ou de pêche, on les utilise en les étendant à 30 ou 40 centimètres au-dessus des planches, mais en ayant soin de les tenir bien fixés contre terre sur tout leur pourtour et de fermer soigneusement tous les trous. Ces différentes précautions sont les seules efficaces, 
car, en général, les épouvantails produisent peu d'effet. On dit cependant qu'il y en a de bons (1) et il n'en coûte guère de les essayer; on fera bien, dans ce cas, d'en changer souvent la forme et l'emplacement.

Parmi les insectes, la larve du hanneton, connue sous le nom de mans ou ver blanc, est un des plus redoutables, surtout dans les terrains meubles. Un moyen pratique, sinon pour la détruire, du moins pour atténuer ses dégâts, est de la chercher en terre, soit à la main, soit avec un outil, partout où le dépérissement rapide des plants signale sa présence. On facilite d'ailleurs ces destructions en plantant, comme le font les jardiniers, des bordures-pièges formées de plantes dont ces larves sont très friandes, tels que des fraisiers et des laitues; les recherches sont alors localisées sur ces bordures oủ les dégâts sont aisés à constater ou à suivre. Dans une étude, publiée dans la Revue des eaux et forêts (2), M. l'Inspecteur Croizette-Desnoyers recommande, comme très efficace, l'emploi de benzine répandue dans le sol, à raison de 30 kilogrammes par hectare, au moyen du pal-injecteur Gonin.

La chenille d'un lépidoptère, Agrotis segetum, connue sous le nom de ver gris, commet des dégâts en tout semblables à ceux de la larve du hanneton, notamment sur les jcunes semis de chêne, dont le chevelu et le pivot sont complètement rongés; on la détruit par des procédés analogues.

Les courtilières, à tous les âges, rongent les racines des jeunes semis; quand elles sont nombreuses, elles ravagent en peu de temps des carreaux entiers, dont elles parcourent toutes les rigoles. Les procédés les plus usuels pour les combattre sont les suivants :

$1^{\circ}$ on fait la perquisition des nids renfermant les œufs et on les détruit. Les courtilières fréquentent de préférence les

(1) Citons les petits miroirs de la grosseur d'une pièce de 5 francs que l'on suspend par de longues ficelles à cité des planches de semis : les rayons lumineux qu'ils lancent de cotés et d'autres inquiètent les oiseaux.

(2) Aout 1888. 
lieux humides, les abords des fossés d'irrigation; c'est là qu'on trouve leur trace sur les points où gisent les débris des plantes dévorées ; ces nids, parfois assez profondément enfoncés dans le sol, se présentent sous la forme d'une boule de terre de la grosseur du poing, dans laquelle les œufs sont renfermés. La recherche des nids doit se faire en mai et en juin ;

$2^{\circ}$ on prend des pots à fleurs de 20 centimètres de diamètre environ, on bouche avec un liège l'ouverture inférieure et on les enfonce aux endroits des plates-bandes oú de petits sillons annoncent le travail de l'insecte; le rebord supérieur du vase doit être à quelques centimètres au-dessous du niveau du sol : la courtilière, en suivant son sentier habituel, tombe dans le vase d'où elle ne peut sortir. Le mieux est d'entourer les planches attaquées, ou celles qu'on veut préserver, avec des voliges fixées verticalement et faisant saillie de 10 à 15 centimètres sur le sol; les pots sont disposés, comme il vient d'être dit, à l'intérieur et à l'extérieur et tout contre ces planches, qui guident l'insecte vers les pièges lorsqu'il veut entrer dans l'enceinte ou en sortir;

$3^{\circ}$ on recommande de préparer, en septembre, dans les pépinières contaminées, des tas de fumier de cheval mesurant de 1 à 2 mètres cubes. En décembre ou en janvier, si on rompt ces tas, on y trouvera en quantité les courtilières engourdies (1). Le fumier doit être frais;

$4^{\circ}$ au printemps, on étend sur le sol nouvellement arrosé, des paillassons, sous lesquels les courtilières viennent s'abriter contre la chaleur du jour ;

$5^{\circ}$ on peut aussi leur faire la chasse en introduisant, par l'ouverture des galeries, des huiles grasses ou minérales, qui font immédiatement sortir les insectes ou les tuent dans leurs réduits.

Mais tous ces procédés ne sont que des palliatifs; ils atténuent le mal, sans le supprimer, surtout quand on a eu la mauvaise fortune d'installer une pépinière dans un milieu favorable à la multiplication de ces hôtes dangereux.

(1) Séante de la Société pomologique, avril 1880. 
Un remède enfin contre les limaces, qui détruisent les feuilles cotylédonaires des semis de hêtre aussi bien en pépinière qu'en forêt: on enduit de graisse ou de beurre,- - qui peut être rance, -- des petites planchettes qu'on dispose dans les semis, la face grasse tournée vers le sol; en visitant ces pièges de bonne heure le matin, on détruit quantité de cette gluante vermine.

Les champignons engendrent sur les plantes tout un cortège de maladies. Tous les jours on en découvre de nouveaux, et il n'est pas surprenant que les cultures artificielles, qui leur fournissent en grande masse la nourriture préférée, soient plus exposées encore à leur ravage que les peuplements spontanés. Ne pouvant entrer ici dans tous les détails que comporte la question, nous ne citerons que les maladies les plus connues et les plus graves.

Il est rare, par exemple, que dans une pépinière déjà ancienne, les semis de pins sylvestre, comme ceux de toutes les races de laricios ne soient pas atteints par le Rouge, maladie provoquée par le mycelium de Leptostroma pinastri. Pour le combattre, M. l'Inspecteur Bartet (1) a eu l'idée d'user de bassinages à la bouillie bordelaise dans les conditions employées contre le mildew de la vigne (2); à la pépinière de Bellefontaine, dans les bandes ainsi traitées, le plus grand nombre des plants ont été sauvés, tandis que, dans les bandes témoins, intercalées au milieu des autres et laissées sans traitement, tout était perdu. Le procédé peut donc être recommandé; il est même probable qu'il trouverait son application dans d'autres circonstances.

Phylophtora fagi, est commun à toutes les essences et plus particulièrement au hêtre, chez lequel il provoque la maladie de l'embryon. Dès la germination, les plants sont atteints et, si le temps est humide, la pourriture s'étend bientôt de proche en proche: les taches contaminées semblent roussies par le feu. La sécheresse arrête le mal.

Rosellinia quercinia attaque les jeunes semis de chêne en

(1) Bartet et Vuillemin, Note présentée à l'Académie des sciences, séance du 27 février 1888.

(2) Société pomologique, loc. cit. 
pépinière lorsqu'ils sont âgés de un à trois ans. Le mycelium se propage sur les racines et tue les plants.

Les moyens pratiques pour combattre ces germes infectieux sont à peu près les mêmes pour toutes les espèces; et nous résumons ceux que recommande le savant Professeur Robert Hartig dans son traité sur les maladies des arbres forestiers (1):

ne pas installer les pépinières de résineux au milieu des forêts de pins; les placer, autant que possible, sur le périmètre Ouest, de façon que les vents dominants qui viennent de cette direction aient passé sur des champs plutôt que sur des peuplements forestiers, d'où ils arrivent chargés de germes morbides ;

orienter les bandes de semis dans la direction Nord-Sud en élevant à l'Ouest un petit bourrele̊t de terre qui arrêtera ces mêmes germes ;

ne semer ni pin, ni hêtre dans les planches où des maladies se seraient manifestées sur des espèces similaires depuis moins de quatre ans; on peut cependant, en ce qui concerne le hêtre, y repiquer des jeunes plants de deux ou trois ans, les champignons à craindre ne s'attaquant qu'aux sujets naissants ;

éviter tout ce qui peut entretenir l'humidité, c'est-à-dire les abris, les couvertures, les paillis; en un mot aérer, et activer l'évaporation dès que le mal apparaît ;

extraire avec soin tous les plants malades, et les placer de suite dans un récipient fermé d'où ils ne sortiront que pour être brûlés ;

éviter de passer dans les planches saines en sortant des planches malades, car les spores s'attachent aux vêlements, aux chaussures, et on les transporte avec soi. C'est d'ailleurs à cause de la propagation par les hommes et par les animaux qu'on voit surtout le mal se développer le long des chemins et sentiers ;

quand il s'agit de maladies souterraines qui s'attaquent

(1) R. Hartig, Wichtige Krankheiten der Waldbaüme, Berlin 1874. Traduction de MM. Gerschel et Henry, Nancy, Berger-Levrault 1891. 
aux racines, creuser des fossés profonds autour des parties contaminées, de façon à arrêter la marche du mycelium dans le sol.

Ajoutons qu'une excellente mesure consiste, dès qu'un compartiment de pépinière est attaqué par un champignon, à isoler les parties indemnes des points contaminés par un rideau de branches hautes de 2 mètres environ et feuillées jusqu'au bas: ce n'est là sans doute qu'un palliatif, mais il suffit à rendre les progrès de la maladie assez lents pour qu'on ait le temps de prendre des mesures plus énergiques. Enfin, quand au cours d'une saison de végétation, on a constaté sur les semis d'espèces à feuilles caduques les signes d'une invasion cryptogamique, la prudence commande de ramasser et de brûler, dès les premiers jours de l'automne, les feuilles qui se détachent, car, bien souvent, c'est dans les feuilles mortes traînant sur le sol, que les spores du champignon arrivent à maturité: cette dernière remarque concerne spécialement le mélèze.

\section{2. - exploitation de la pépinière.}

Exécution des semis : en plein; - en rigoles; - en coffres ; - procédés spéciaux; - quantité de graine à employer; - saison des semis. - Soins d̀ donner aux semis : pralinage des graines; - abris contre la sécheresse; - abı is d'hiver; - abris contre les gelées printanières; - abris permanents ; - arrosages et irrigations ; sarclages; - binages. - Travaux divers : repiquage des plants ; leur extraction; - leur taille et leur rhabillage; - transport et emballage.

Exécution des semis. - Pour les semis de toutes essences, on prépare le sol en planches parallèles, ayant une largeur de $1^{\mathrm{m}}, 20$ et séparées entre elles par de petits sentiers. S'il a été possible d'orienter les côtés des grands carreaux dans les directions, les uns Nord-Sud, les autres Est-Ouest, on tracera les planches parallèlement à ces derniers; on verra plus loin que cette disposition est avantageuse lorsqu'il s'agit de placer les abris.

Semis en plein. - Dans chaque planche, on peut semer en plein, comme cela se fait pour les carottes dans un jardin; 
mais ce mode, qui distribue inégalement la graine sur toute la surface, en exagère la quantité; de plus, les sarclages et les binages à l'aide d'outils sont impossibles, tout doit se faire à la main, ce qui est bien plus coûteux; les maladies cryptogamiques s'y propagent aussi plus rapidement; enfin l'extraction des plants y est rendue difficile par l'enchevêtrement général des racines: un grand nombre de brins, plus ou moins meurtris, doivent être rebutés. Aussi ce mode n'est-il à recommander que lorsqu'on destine les plants à être extraits en gazons pour les plantations à faire en motles.

Semis en rigoles. - Il est préférable de généraliser le système des rigoles ou sillons, dirigés, soit longitudinalement, soit transversalement et espacés entre eux de 10 à 30 centimètres, suivant l'âge auquel on se propose de conduire les plants. Pour les résineux non repiqués, qu'on plante à demeure à deux ans, l'espacement de 15 à 18 centimètres est le plus convenable, en ce sens qu'il permet le passage du fer des outils à sarcler, à biner et à rechausser.

On peut tracer les rigoles à la binette, à la latte, ou à la planche bavaroise. Dans les deux premiers cas, ces rigoles sont ouvertes suivant le sens de la longueur, dans le dernier, suivant le sens de la largeur des planches.

Quand on opère à la binette, on tend un cordeau dans toute la longueur de la planche et à 10 centimètres de l'un des bords; avec la pointe de l'outil, on trace, le long du cordeau, un sillon de 2 ou 3 centimètres de profondeur; on sème à la main et on recouvre la graine en nivelant le sol au râteau, puis on replace le cordeau à la distance convenable, et on ouvre un nouveau sillon; ainsi de suite pour toute la planche.

A la latte, on fait de même, en employant des lattes de 3 à 4 mètres de longueur, qu'on dispose le long du cordeau et qu'on frappe à la masse, de façon à tracer dans le sol un sillon de profondeur égale à leur épaisseur, c'est-à-dire 2 centimètres environ. Les lattes pourvues d'une double gorge semblable à celle de la planche bavaroise présentent un sérieux avantage sur la latte simple.

La planche bavaroise se compose d'un madrier en bois dur 
(chêne, orme ou frêne) de 3 centimètres d'épaisseur, d'une longueur égale à la largeur des bandes (fig. 78 et $81, b$.), soit $1^{m}, 20$ et large de deux fois l'espacement adopté entre les rigoles. Sur la face inférieure de cetle planche sont disposées deux paires de baguettes triangulaires espacées entre elles de la largeur entre les sillons, soit 18 centimètres, et distantes des bords de la planche de moitié de cette largeur; chacune des baguettes juxtaposées a 3 centimètres de base sur 2 cen-

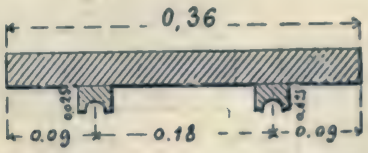

Fig. 78. - Coupe de la planche bavaroise. timètres 1/2 de hauteur. En appuyant la planche ainsi disposée sur un sol meuble et en la damant, on imprime deux doubles sillons bien espacés et d'une profondeur uniforme; la graine, qui tombe sur la crête de la double gorge, glisse à droite et à gauche et se répartit en deux rangs espacés de 3 centimètres.

Chaque chantier est muni de trois ou quatre planches semblables que l'on juxtapose successivement, et une à une, suivant toute la longueur de la bande. Ces petits appareils, qui nécessitent une terre meuble et saine, facilitent la besogne, économisent beaucoup de temps et donnent d'excellents résultats.

Quel que soit le mode employé, au fur et à mesure que l'on trace les sillons, on y répand la semence, aussi uniformément que possible, en faisant varier la quantité suivant la grosseur et les espèces. Pour les résineux à petites graines, conıme : le pin sylvestre, le pin de montagne, l'épicéa, le sapin, le mélèze, il suffit qu'il y ait, au fond de chaque rigole double du système bavarois, une seule série de semences se touchant dans leur travers.

Pour assurer une bonne répartition de la graine et se mettre à l'abri des nervosités toujours possibles dans les doigts du semeur, on a imaginé des instruments réglés à l'avance pour répandre automatiquement les quantités voulues. Certaines de ces machines sont bien compliquées et ne fonctionnent bien quà la condition d'ètre parfaitement entretenues: mais nous recommandons tout 
particulièrement la régle semoir imaginée par l'Inspecteur Cardot et qui a fait ses preuves dans les pépinières communales de l'arrondissement de Pontarlier. Cet outil très simple, et que tout le monde peut fabriquer, consiste en une règle de la lon-

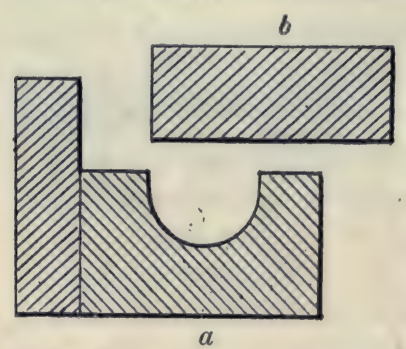

Fig. 79. - Coupe de la règle semoir Cardot. gueur de la planche bavaroise dont elle est en quelque sorte l'annexe. La section transversale a en est disposée comme l'indique la figure 79 , c'est-à-dire que sa face supérieure est creusée d'une gorge dont laprofondeur est calculée de telle sorte qu'elle renferme, pour chaque unité delongueur, la quantité de graine à semer. Il suffit de remplir la gorge de semence et de faire circuler la réglette mobile $b$ le long de l'arrête $c$ pour faire tomber le trop plein ; puis, posant la règle chargée le long de la rigole, d'y verser son contenu. Il va sans dire qu'on assortit tout un jeu de règles aux différentes semences et aux différents états de densités que l'on cherche à obtenir pour le semis d'une même essence.

Quand on veut produire des plants résineux et bons à être mis en place, non repiqués, à l'âge de deux ans, il suffit de 3 à 5 kilos de pin sylvestre, pin d'Autriche, épicea et mélèze par are, - si d'ailleurs les semences sont de bonne qualité. Il faudrait semer

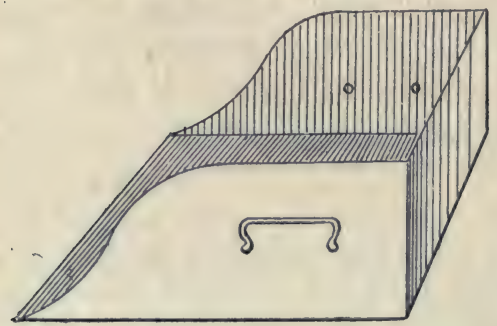

Fig. 80. - Coffre à terreau utilisé à la pépinière de Bellefontaine. plus dru, si les graines étaient de qualité moindre ou si l'on ne voulait faire que des plants d'un an.

Derrière le semeur, marche immédiatement un autre ouvrier, qui, muni d'une sorte de coffre en bois ou en tôle (fig. 80), remplit les sillons d'un compost formé de moitié terreau et de moitié sable ou encore de sciure de bois. Les expériences poursuivies depuis quelques années à la pépinière de 
Bellefontaine ont permis de constater que l'emploi de la sciure pure, provenant de bois de toutes essences, sans excepter le chêne, donne d'excellents résultats ; la levée s'est môme montrée plus complète et plus hàtive que dans le compost de terreau; la seule condition essentielle à remplir, c'est que la sciure soit bien saturée d'eau lors de l'emploi.

Il suffit de rabattre la substance employée en couverture jusqu'au niveau du sol, au moyen du revers d'une pelle, pour que l'opération soit terminée. D'ailleurs, la profondeur des sillons indique la quantité de terre qui doit recouvrir les semences; cette quantité reste fixée à 2 ou 3 centimètres pour toutes les graines de résineux les plus usuelles de petite et moyenne grosseur.

Semis en coffres. - Comme le terrain d'une pépinière coûte toujours cher d'achat ou de mise en valeur et d'entretien, il faut que chaque unité de surface produise son maximum de rendement en plants forestiers. Aussi ne saurait-on donner trop de soin à chaque opération. En ce qui concerne le semis, nous recommandons d'entourer les plates-bandes d'un cadre de planches brutes dressées en la forme du coffre employé par tous les horticulteurs ou maraîchers. Cette disposition présente une foule d'avantages : elle permet de renouveler sans grands frais la terre épuisée par la culture, ou d'y doser plus facilement les amendements et engrais, sans risque de déchets dans les zones d'entourage; de créer les milieux artificiels pour cultures spéciales; enfin elle facilite singulièrement la mise en place des différents appareils destinés à protéger les semis contre tous les dangers auxquels ils sont exposés.

Le semis dans les colfres se fait d'ailleurs par les procédés ci-dessus détaillés; il convient, surtout, pour les plants destinés à être repiqués à un an, qu'on peut semer très dru sans crainte de déchets, et pour économiser les graines dont le prix est élevé.

Procédés spéciaux. - Pour les semis de grosses yraines, qui demandent à être plus profondément enterrées que les précédentes, on trace, à la binette, des raies de 1 à 6 centimètres de profondeur et de largeur assez grande pour que 
deux ou trois graines puissent y entrer, placées de front.

Souvent, lorsqu'on sème les glands en automne, et pour éviter les accidents causés par la gelée, on les enterre jusqu'à 10 ou 15 centimètres de profondeur, sauf à décaper la surface au printemps. Ce procédé n'est pas à recommander, parce que, malgré les précautions prises, les graines se trouvent enfouies à des profondeurs inégales et la germination ne se produit plus uniformément : on voit des chênes lever jusqu'en juillet et même seulement au printemps suivant, ce qui nuit à la régularité des bandes. Aussi, vaut-il toujours mieux semer à la profondeur normale, et, dans les régions où la gelée est à craindre, couvrir les semis de paillassons ou de feuilles mortes qu'on enlève au premier printemps.

Quelle que soit la saison, une sage précaution à prendre immédiatement avant la mise des glands en terre, sera de les mouiller légèrement et de les agiter dans des sacs renfermant quelques poignées de minium rouge. Cette poussière minérale forme autour des graines un enduit qui les préserve de la voracité des animaux.

L'enracinement du jeune chêne est constitué par un pivot simple, dont la longueur, dans un terrain bien défoncé, peut atteindre 50 centimètres, dès la première année. Semblable conformation rend délicates et onéreuses les manipulations, extractions et mise en place de ces plants; depuis longtemps, les forestiers ont cherché à supprimer le pivot ou du moins à limiter son développement. Duhamel du Monceau signale la pratique, dite de Brelagne, qui consiste à établir au fond des rigoles un dallage qui arrête le pivot à la profondeur voulue. Dans le même ordre d'idées, M. le conservateur Levret a proposé de semer les glands sur un lit de pierres cassées, comme celles qui servent à l'empierrement des routes, et de les recouvrir d'une couche de bon terreau de $0^{\mathrm{m}} 10$ d'épaisseur. Nous avons expérimentéces deux procédés qui ne nous ont rien donné de satisfaisant, et il ne pouvait guère en être autrement; car, d'une part, le dallage étanche entretient une humidité exagérée qui pourrit les racines; d'autre part, le macadam produit un drainage qui assèche les plants. 
LE BoIsemeNT PAR PLANTATIONS.

Quelques praticiens font germer les glands et en cassent le pivot avant de faire le semis. Une telle mutilation a pour effet principal de ralentir la végétation; il n'est pas surprenant, dès

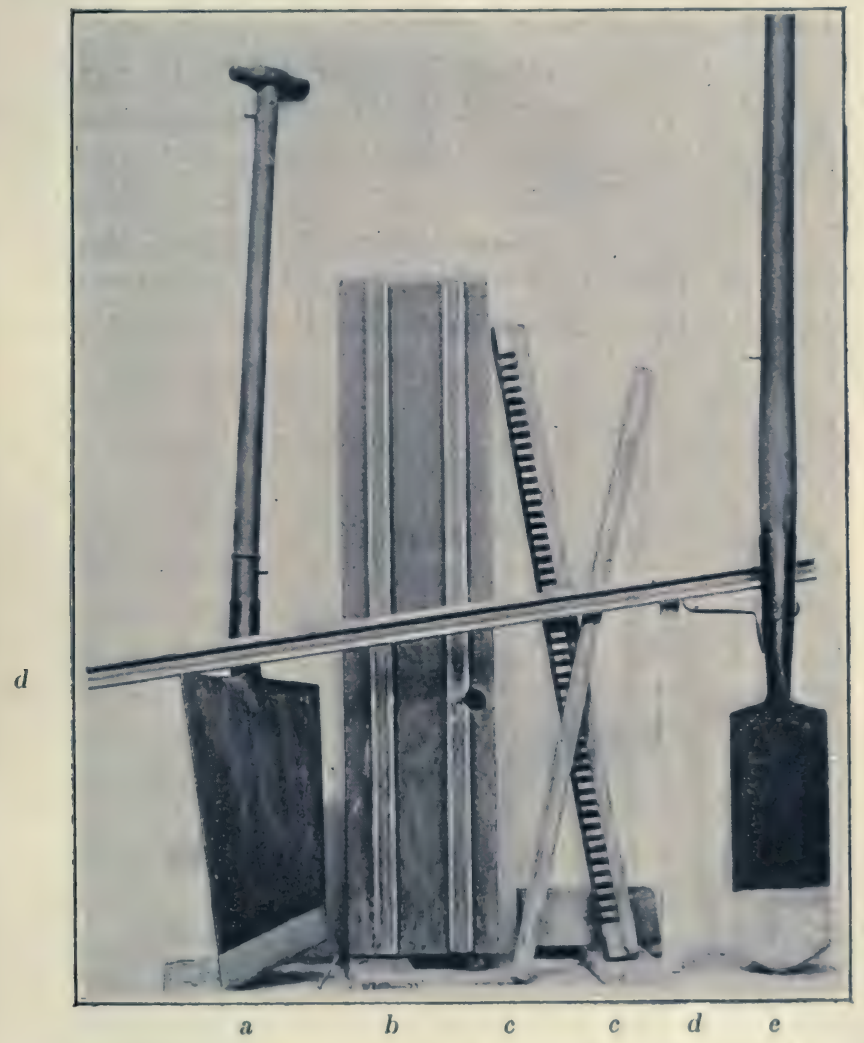

Fig. 81. - Quelques outils.

$a$, bêche coupe-pivot; $b$, planche bavaroise; $c, c$, règle à repiquer; $d, d$, règle à semer; e, bêche Prouvé à étrier pour extraction d'un semis de chêne.

lors, que des sujets chélifs aient un pivot moins développé que celui des brins provenant des semences intactes.

On a beaucoup parlé aussi de la bêche coupe-picot (fig. 81 a) importée d'Allemagne, dont le fer, en biseau, bien tranchant, 
est introduit obliquement entre les rigoles et va couper les pivots à la profondeur voulue. Nous pouvons dire par expérience que ce vieil instrument, dont l'emploi est limité aux sols meubles et absolument dépouvus de pierrailles, ne fait partout que d'assez mauvaise besogne.

Tout compte fait, il est préférable de laisser les semis de chêne se développer librement, de les arracher à un an, et de les mettre en place en se servant des outils de M. Prouvé partout où les circonstances locales permettent de les utiliser; - ou, mieux encore, surtout quand on veut des tiges moyennes ou hautes, de repiquer les plants d'un an en pépinière après résection du pivot, comme il sera dit plus loin, pages 384 et suivantes.

Le semis de faînes demande des soins tout particuliers et le procédé employé par M. l'Inspecteur Croizette Desnoyers dans les pépinières de Fontainebleau se recommande à tous égards. Au commencement de mars, on stratifie dans du sable, sur une aire bien saine, les faines conservées jusqu'alors dans un grenier, c'est-à-dire qu'on les dispose par lits alternes de 2 centimètres d'épaisseur de faînes et de 5 centimètres de sable. On prend, autant que possible, du sable grenu, bien lavé; en général, le sable ordinaire de rivière suffit. Les tas, pour ne pas être trop volumineux, ne dépassent pas la capacité d'un demi mètre cube; on leur donne soit la forme d'un cône, soit celle qui est en usage pour les matériaux d'empierrement sur les routes.

Le but étant de faire germer les faînes au premier printemps, on entretient dans ces tas une humidité constante, par des arrosages, si la sécheresse l'exige. Généralement, suivant la température de l'air, trois semaines ou un mois après la stratification, les germes apparaissent.

Quand ils sont généralement sortis, sans attendre toutefois que leur longueur dépasse 2 à 3 centimètres, on sème dans des rigoles peu profondes, espacées entre elles de 20 à 30 centimètres pour faciliter les binages. Le semis se fait assez clair et ne demande pas plus de 60 litres par are de terrain disposé en rigoles de $0^{\mathrm{m}}, 18$ d'écartement; on comble les rigoles avec le compost de couverture. L'important, dans cette mani- 
pulation, est de ne pas donner aux germes le temps de se flétrir: les faînes mélangées de sable passent, directement et immédiatement, du grermoir à la planche de semis.

Contrairement à la pratique consacrée par l'usage, l'inventeur du procédé recommande tout particulièrement de ne pas rechausser les tigelles des plants; car cette opération conduit à trop recouvrir les graines qui ne sont pas levées, ce qui retarde encore leur développement. On constate, en effet, que la germination des faînes, comme celle des glands, n'ayant jamais lieu en même temps sur un même point, il s'écoule souvent 15 jours ou trois semaines entre l'apparition des premiers plants et la levée complète ; ces retards font que les derniers levés n'ont pas un enracinement assez profond lorsque surviennent les sécheresses de l'été et qu'ils périssent en grand nombre dans les terrains qu'on n'irrigue pas. A partir de la levée, des sarclages et des binages répétés entretiendront les surfaces toujours meubles et bien propres.

Cet excellent procédé est applicable aux chênes dans les mêmes conditions.

Il existe, pour le semis de hêtre, bon nombre d'autres recettes, accompagnées de tour de main, dans le détail desquels nous ne pouvons entrer.

On recouvre toutes ces graines lourdes de 3 à 6 centimètres de terreau, la couche étant d'autant plus épaisse que la terre est plus légère.

Les semis de semences légéres, aune et bouleau, se font en plein, et la graine n'a pour ainsi dire pas besoin d'être enterrée; il suffit de répandre à la surface une mince couche de terreau et de tasser légèrement avec un revers de pelle. L'aune doit être placé dans des conditions telles qu'il puisse être constamment irrigué; les bords humides d'un fossé conviennent très bien à ce genre de semis.

La graine de bouleau germe difficilement dans les terres dont la surface n'est pas parfaitement ameublie. A défaut de terrain graveleux, on peut semer cette essence sur les anciennes places à charbon. Le procédé suivant est égale. ment à recommander pour le répandage de la semence, quand il est possible de disposer de porte-graines dans les forêts 
voisines de la pépinière : avant la maturité complète des cônes, on coupe sur les arbres des branches de faible grosseur et bien pourvues de semences; ces branches sont plantées sur le terrain à ensemencer; dans cette situation, les cônes achèvent de mûrir et la dissémination des graines se fait natu rellement: il suffit d'une pluie, même peu abondante, pour les coller sur la terre nue et, sans autre préparation, elles germent au printemps suivant. Ce même procédé peut être avantageusement employé pour le semis direct de cette essence. On réussit encore le semis de bouleau en répandant sa graine mẻlangée avec la proportion de sciure suffisante pour former sur le sol une mince couverture continue. Dans aucun cas, il ne faut craindre d'exagérer la quantité de cette semence, qui présente toujours une forte proportion de graines vaines.

Comme toutes les graines légères, celle du bouleau germe et se maintient infiniment mieux quand la semence est abritée par un léger paillis de suite après l'épandage. Lcs aiguilles de pin font, à ce point de vue, une excellente couverture; une couche très mince suffit ; mais il faut craindre les dégâts des limaces, qui se réfugient en grand nombre dans ces milieux frais et humides. Nous préférons le procédé cité plus haut et employé par M. l'Inspecteur Bartet, qui consiste à semer la graine en mélange avec une quantité égale ou supérieure de sciure de bois.

Les autres espèces feuillues se sèment rarement en pépinière, ou, du moins, en petite quantité. Les semences sont à bon marché et on ne les ménage pas, d'autant plus que les plants sont presque toujours extraits à un an pour être repiqués en vue de la production des moyennes ou hautes tiges. Rappelons seulement, au sujet des charmes, frènes et érables, que la semence ne germe régulièrement qu'après une année de stratification en rigoles, comme cela a été dit à propos de la conservation des graines. La semence mise en place ne doit pas être couverte de plus de 2 à 4 centimètres de terreau.

Les essences résineuses de lumière sont celles qui sont le plus souvent cultivées dans le terrain découvert des pépinières. Les procédés indiqués plus haut leur sont applicables 
en tous points et sans autres précautions spéciales. Il n'en est pas tout à fait de même du sapin, essence d'ombre, beaucoup plus délicate à manier; à ce point que, même parmi les forestiers, certains prétendent qu'il n'est pas possible de l'obtenir de semis en pépinière. Rien n'est plus simple cependant : il suffit d'avoir un terrain tel qu'il y trouvera l'abri nécessaire à son tempérament. Dans ce but, on choisit sous bois, dans une parcelle mise à l'élat d'ensemencement, une ou plusieurs surfaces, suivant la quantité de plants à produire, dont la forme sera quelconque entre les tiges des porte-graines; on nettoie le terrain, on le défonce en le débarrassant de toutes les racines vivantes et, dans la terre à bois ainsi préparée, on sème, comme s'il s'agissait du pin sylvestre ou de l’épicea. - Il va sans dire que cette petite pépinière annexe sera traitée, au point de vue des clôtures et des soins divers, comme l'établissement principal. Les abris d'hiver et d'été lui étant seuls fournis par le peuplement qui les protège, il faudra recourir aux engrais si l'on demande plusieurs récoltes consécutives à la même surface.

Quantité de graines à employer. - Cette quantité varie, non seulement avec l'espèce, mais, pour une même espèce, avec la nature des plants à obtenir. Il faut semer plus dru pour utiliser les plants à un an que pour les employer à deux ans, et, d'une manière générale, d'autant plus clair que les plants resteront plus longtemps en place. Cette considération est très importante, car un semis fait pour être extrait à un age déterminé ne peut demeurer en rigoles, au delà de cet âge, sans subir une perte considérable. Chez les résineux, par exemple, qui végétent rapidement entre la deuxième et la troisième année, le déchet, par suite de manque d'espace, réduit d'environ moitié le nombre de plants récoltés à trois ans, alors qu'ils étaient semés pour l'être à deux ans. Aussi, malgré la dépense qu'entraîne le repiquage, il faut, si des circonstances indépendantes de la volonté empêchent d'utiliser les plants à l'âge voulu, les extraire et les rigoler en pépinière. Si l'on n'a ni le temps, ni le terrain préparé nécessaire pour cette opération, ne pas 
hésiter à faire le dépressage, mais, en se gardant bien de procéder par arrachage à la main ou avec un outil quelconque. La coupe au ciseau, par petites bandes alternes dans le travers de la rigole, est beaucoup plus expéditive, en ce sens qu'elle limite à la proportion voulue l'enlèvement des sujets surabondants, sans nuire à ceux qui restent en place.

Pour les semis faits en rigoles et destinés à ètre extraits après leur deuxième année, dans les conditions moyennes de sol et de climat, il suffit d'employer, par 1,000 mètres courants de rigoles, les quantités suivantes:

6 à 7 kil.d'épicéa désailé.

5 à 6 - de pin sylvestre (id.)

7 à 8 - de mélèze (id.)

8 à 9 -depin noir d'Autriche(id.)

30 à 35 kil. de sapin désailé.

30 - de frêne.

30 - d'érable.

12 - d'orme.

Si les semis doivent être extraits après la première année, ces quantités pourront être augmentées d'un tiers.

On compte, par are, de 600 à 700 mètres courants de rigoles espacées entre elles de 15 à 18 centimètres, déduction faite des sentiers de 33 centimètres réservés entre chaque planche de $1^{\mathrm{m}}, 20$ de largeur.

Saison des semis. - Quelles que soient les essences et les régions, le printemps paraît la saison la plus convenable. Suivant que le climat sera plus ou moins sec, on commencera plus ou moins tôt, pour profiter de l'humidité accumulée dans le sol pendant l'hiver. A partir du 15 mars, dès que la terre est assez ressuyée à la surface, on peut profiter de tous les beaux jours pour semer, et il n'y a pas de temps à perdre, car, à ce moment, les travaux à faire pressent de tous côtés dans une pépinière comme ailleurs.

Soins à donner aux semis : pralinage des graines. Dès qu'elles sont mises en rigoles, avant même la germination, les graines sont exposées à la voracité d'animaux de toute sorte. On atténue les dégâts par des procédés analogues à ceux qui sont employés pour les plantes agricoles (1).

(1) M. Neuville, professeur à l'École pratique de Neubourg recommande le pralinage au pétrole par le procédé qu'il décrit en ces termes:

On prend 200 grammes de pétrole et 6 litres d'eau bouillante; on verse d'abord l'eau bouillante sur 100 grammes de goudron de gaz 
Dès leur levée, les semis réclament d'autres soinsjusqu'au moment de la récolte.

Abris contre la sécheresse. - Ce sont, d'abord, les abris qui les maintiendront ombragés contre la chaleur.

De ces abris, les uns sont formés par des tiges vivantes et cultivées, à cet effet, le long des bandes; on plantera, parexemple, sur la ligne du côté sud, soit des boutures de saule ou de peuplier, soit des feuillus de demi-tiges de manière à former une série d'écrans; on peut également semer sur cette même ligne du seigle, des fèves de marais, ou toute autre plante annuelle à tige droite et rigide, pouvant s'élever jusqu'à 60 ou 70 centimètres et dont on obtiendra une récolte utilisable.

Mais, au lieu de ces cultures dont la réussite est incertaine, qui fatiguent le sol et qui sont à demeure jusqu'à la récolte, il vaut mieux employer des abris morts qu'on peut placer ou enlever à volonté.

En forêt, les plus économiques sont des branchages de bois résineux ou de bois de feuillus coupés au moment où les feuilles sont déjà développées, que l'on fixe en terre le long des bandes et de façon à les abriter du côté du midi; l'orientation des bandes dans la direction Est-Ouest facilite beaucoup cette opération. Les branchages peuvent être simplement plantés sur une ligne ou, mieux encore, sur les deux bords de la bande et entrelacés au-dessus d'elle en forme de berceau; cette disposition fournit des abris d'une solidité à toute épreuve, mais elle augmente sérieusement la difficulté des sarclages. On peut aussi installer le long des bandes deux lignes de lattes parallèles supportées par des crochets de 63 à 70 centimètres de hauteur et sur lesquelles on étend les branchages. A défaut de rames feuillées, on se

épuré, en agitant, puis on ajoute 100 grammes de pétrole en remuant le mélange; on remet de nouveau du goudron puis du pétrole, en même quantité, jusqu'à épuisement des provisions, qui sont dosées dạns la proportion de :

$100 \mathrm{gr}$. de goudron de gaz

$100 \mathrm{gr}$. de pétrole épuré

1 litre 1/2 d'eau bouillante,

proportion qui suffit pour 50 litres de semence. Celle-ci est trempée dans le mélange refroidi et employée ressuyée. 
sert, suivant les cas, de toiles, de claies, de paillassons, en donnant la préférence au meilleur marché.

Quelquefois, on garnit les intervalles des rigoles avec de la mousse. On attribue à cette couverture l'avantage: $1^{\circ}$ de garantir le sol contre le durcissement superficiel et de diminuer le nombre de binages que les semis néces. sitent pendant l'été; $2^{\circ}$ d'empêcher, dans une large mesure, la levée des mauvaises plantes et d'économiser ainsi une partie des sarclages $; 3^{\circ}$ de garantir les plants contre les fortes pluies et surtout contre la sécheresse, en conservant au terrain une partie de sa fraîcheur et diminuant ainsi, ou même supprimant, les arrosages. Par contre, la mousse présente l'inconvénient de faire obstacle à l'influence bienfaisante des rosées et de servir de repaire à une foule d'animaux nuisibles.

Abris d'hiver. - Les plants forestiers indigènes n'ont pas beaucoup à craindre du froid; mais, dans certains sols, comme les marnes, on perd beaucoup de plants par l'effet du déchaussement. Bien que ces accidents soient surtout à craindre pour les semis de l'année, ils produisent parfois des effets tels que des semis de tous âges et même des plants de haute tige sont complètement arrachés (1).

On prévient ces accidents, en grande partie du moins, en recouvrant les semis, dès l'automne, d'un paillis grossier ou de feuilles séches fixées par des branchages. Pour être efficace, la couverture doit être maintenue jusqu'au moment où les plants entrent en végétation; car c'est au mois de mars que le déchaussement est le plus à craindre; quand il se produit, malgré les précautions prises, on relève avec soin les plants renversés et on en rhabille les racines et les jeunes tigelles au moyen de terreau qu'on répand sur le sol à travers un tamis; jamais on ne doit les brutaliser, en les renfonçant

(1) On peut citer des exemples, notamment dans les environs de Barcelonnette, de poteaux télégraphiques soulevés par l'effet du déchaussement. En semblable circonstance, il ne faut songer, ni à créer des pépinières permanentes ou volantes, ni à faire des semis en place; on ne peut que planter, et encore faut-il avoir recours à la plantation oblique, de telle sorte que le soulèvement par la gelée déplace en même temps tout l'appareil souterrain sans le tirer en dehors. 
avec les doigts, comme cela se fait trop souvent. On peut aussi butter la terre autour des lignes de semis, soit à la houe, soit avec des outils spéciaux.

Ajoutons que les paillis, qui empêchent le réchauffement rapide de la surface dès le premier printemps, retardent l'évolution des bourgeons assez pour les garantir contre les gelées printanières.

Abris contre les gelées printanières. - Ces accidents sont tellement fréquents en toute région, aussi bien au Midi qu'au Nord, qu'il est prudent, en règle générale, d'abriter tous les plants et plus particulièrement les espèces délicates et à végétation hâtive, comme le sapin, le hêtre, le frêne, etc...

A cet effet, on se sert de paillassons qu'on enlève pendant le jour, ou, plus simplement, de grandes toiles maintenues par des piquets à 1 mètre au-dessus du sol et qu'on peut installer ainsi d'une façon permanente, tant que cela est jugé utile.

Le gardien de la pépinière doit aussi être pourvu du matériel nécessaire pour la création de nuages artificiels.

Abris permanents. - Pour éviter toutes ces dépenses d'appareils et de main d'veuvre, on a été conduit à créer des abris permanents contre tous les effets météoriques: vents, chaleur, sécheresse, froid, etc... Le plus souvent ce sont des haies vives d'essences feuillues ou résineuses : charme, épicéa, thuia, qu'on taille suivant une épaisseur réduite autant que possible, en les maintenant à une hauteur de 2 mètres environ. Si leur disposition varie dans le détail, du moins sont-elles toujours orientées suivant la direction EstOuest. Certainement ces abris en-tous-cas ont une efficacité réelle, mais nous ne pouvons passer sous silence leurs inconvénients : ils occupent une place beaucoup plus grande que leur faible projection semble l'indiquer ; car les racines s'étalent à droite et à gauche et interdisent toute culture profitable à une dislance de 1 mètre à $1^{\mathrm{m}}, 50$ de chaque côté de leur pied; - les haies doivent être entretenues par une taille sévère et soigneuse qui absorbe du temps; - leur présence diminue considérablement les dépôts de rosée.

Quoiquil en soit, nous pouvons citer comme exemple la 
disposition adoptée dans les pépinières de la forêt domaniale de Lyons-la-Forêt et dont les forestiers locaux disent le plus grand bien. Les haies sont en charme, dont les feuilles marcessantes continuent, en toute saison, les effets qu'on demande à l'abri. La figure 82 donne le plan de leur installation.

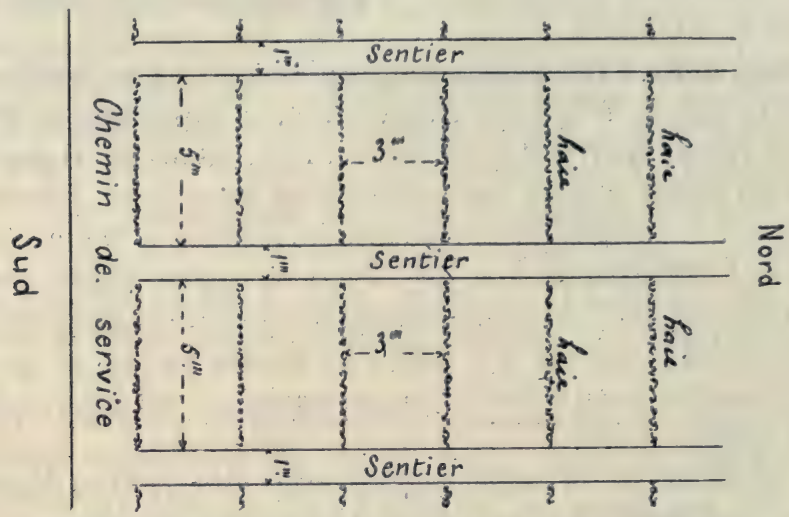

Fig. 82. - Haies en charmille servant d'abri permanent eontre les gelées printanières.

Nous ne terminerons pas cette question si importante des abris sans rappeler combien l'emploi des coffres simplifie tous les travaux, surtout en ce qui concerne les semis d'un an qui occupent la plus grande place dans les pépinières de résineux. Rien de plus facile, notamment, que de construire des cadres tendus de grillage galvanisé ou de claies en menues lattes ayant la largeur du coffre et protégeant les plants contre les oiseaux ou le soleil.

Arrosages, irrigations. - Les arrosages, tel qu'on les pratique le plus souvent, ne sont pas à recommander. L'eau • qu'on jelte sur la terre, en grande masse à la fois, n'y pénètre que difficilement et s'écoule inutilement à la surface pour peu que le terrain présente une pente, même légère. Un arrosage, pour être efficace, ne doit laisser arriver l'eau sur la terre qu'en quantité égale à celle qui peut être immédiatement absorbée, et l'opération doit se prolonger tant que la terre n'est pas saturée jusqu'à la profondeur pénétrée par les racines. 
Avec les ustensiles ordinaires, une telle opération serait trop onéreuse pour être appliquée à de grandes étendues; au lieu de produire un effet utile, les arrosages incomplets encroûtent la surface du sol et, pour ne pas devenir nuisibles, ils doivent être suivis d'un binage. Les seuls arrosages convenables seraient faits à la lance fixe qui projelte l'eau en goutelettes fines et qu'on déplace à volonté; mais ces appareils coûtent cher, et on ne dispose pas toujours de la pression convenable.

Aussi, au lieu d'arroser, vaut-il mieux irriguer. La dépense de première installation nécessitée par l'établissement d'un système complet d'irrigation sera largement compensée par les économies qui en résulteront plus tard et par la meilleure qualité des plants obtenus. Quand l'eau courante peut être amenée jusqu'au bord des planches, un ouvrier creuse, le long de chacune d'elles, un sillon où il dirige une petite quantité d'eau qu'il y laisse assez longtemps pour que la bande entière soit imbibée entre deux terres, par voie de filtration, sans que l'eau puisse couler à la surface : ce qui serait nuisible et parfois dangereux. Pendant les années ordinaires, deux irrigations, pratiquées à des époques bien choisies, sont suffisantes pour entretenir en bon état de fraîcheur un carré de semis. Dans les régions plus sèches, le mode d'irrigation par submersion, dans des compartiments bien nivelés et entourés d'un bourrelet de terre, donne d'excellents résultats.

Sarclage. - Le sarclage consiste dans l'enlèvement de la végétation herbacée qui se présente plus ou moins abondante dans toutes les cultures. On doit sarcler la pépinière aussitôt que les mauvaises herbes commencent à poindre et avant qu'elles aient développé de fortes racines; quand ces herbes sont encore jeunes, on les arrache facilement à la main, surtout aprés une petite pluie; si on les a laissées grandir, il vaut mieux les couper: l'arrachis ne pourrait plus se faire sans danger pour les jeunes plants. Le point essentiel est de ne jamais laisser les mautaises herbes monter en semence; de telles négligences sont impardonnables, car les graines mûres se répandent à profusion sur le sol et le terrain en est littéralement empoisonné pour plusieurs années. A ce point de vue, 
il est important que les carrés vides, les chemins et les abords des pépinières soient sarclés comme les bandes de semis ellesmêmes. Quand l'espacement entre les lignes de semis est suffisant pour qu'on puisse y passer le fer d'un racloir, on fera facilement et économiquement le sarclage en même temps qu'un binage.

Binage. - Les jardiniers prétendent que binage vaut arrosage. L'opération consiste à donner au sol une légère culture pour en ameublir la surface. Elle a pour effet, en augmentant la perméabilité du sol, de faciliter l'absorption de l'air et des eaux de pluie, de fixer les vapeurs et les rosées, de ralentir l'évaporation, en un mot, de rompre la liaison entre la couche supérieure et celle sous-jacente: ce qui fait remplir à la première le rôle de couverture.

Travaux divers : repiquage. - Le repiquage ou le rigolage est toujours une pratique très coûteuse, non seulement à cause de la dépense qu'entraîne l'opération elle-même, mais encore, et surtout, à cause de la grande étendue du terrain qu'elle nécessite et dont l'entretien en bon état de production est très onéreux. C'est en faisant les semis d'une manière convenable et dans des carreaux bien fumés qu'on se dispensera de ces repiquages ; car le plus souvent, si les plants résineux de 2 ans ne sont pas assez forts pour être plantés à demeure, c'est parce que l'engrais ou les soins nécessaires leur auront manqué. Aussi, malgé la supériorité incontestable des plants qu'il procure, le repiquage doit-il être l'exception pour les plantations de résineux en basses tige; il est, au contraire, la règle pour les essences feuillues; ajoutons enfin que nombre de pépiniéristes sont partisans du repiquage quand même; il importe dès lors d'en connaitre les effets et la pratique.

Prenons, par exemple, deux plants de pin sylvestre de 2 ans provenant du même semis. L'un (fig. 83 a) a été extrait directement dans le sillon où il a été semé; l'autra (fig. 83 b.), enlevé de la bande de semis et repiqué à sa première année, ayant par conséquent une année de repiquage. Le premier est élancé, grêle; ses aiguilles pâles et rares, son enracinement représenté par de longs filets à peine ramifiés forment un ensemble de médiocre aspect. Le second est trapu; ses aiguilles 
longues et de couleur foncée, ses racines nombreuses et bien pourvues de chevelu témoignent de sa vigueur.

A cause de la faiblesse de leur enracinement, les épicéas

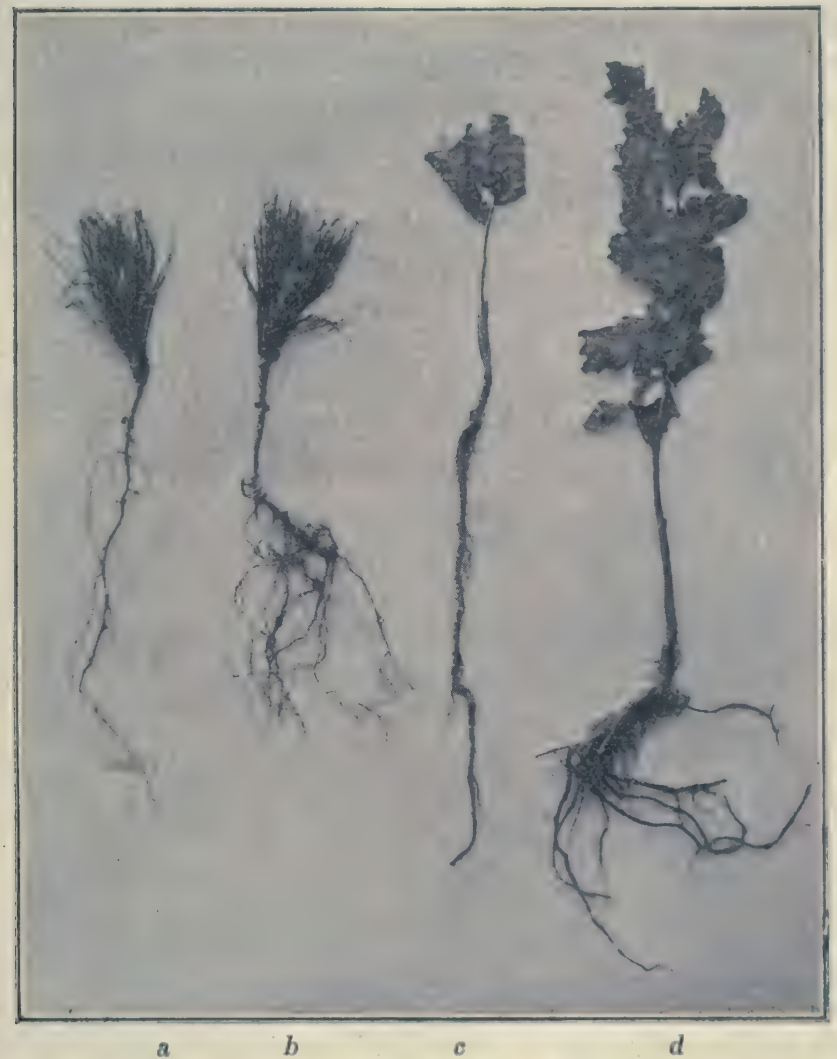

Fig. 83. - Effets du repiquage. - a, Pin sylvestre de 2 ans, non repiqué; $b$, pin sylvestre repiqué; $c$, Chêne pédonculé de 2 ans, non repiqué; chêne pédonculé de 2 ans repiqué après résection du pivot.

doivent presque toujours être repiqués avant emploi. Toutes les essences exigrent cette pratique quand on veut les conduire jusqu'aux dimensions de moyennes et de hautes tiges; alors plusieurs opérations successives sont nécessaires, en 
augmentant les espacements en proportion de la taille qu'elles atteindront.

Plus particulièrement en ce qui concerne les chênes, les types $c$ et $d$ de la figure 83 , nous montrent les effets du repiquage. Le premier représente un plant de 2 ans, tel qu'il a été extrait de la rigole avec toutes les précautions voulues pour qu'il soit complet : cime chétive, tige grêle, pivot simple d'une longueur exagérée. Le second, repiqué à un an, après résection du pivot, s'est formé un appareil radiculaire multiple, bien pourvu de radicelles en même temps qu'il étoffait sa région aérienne. Inutile d'insister pour faire ressortir lequel des deux sera d'une reprise plus certaine.

Mais venons à la pratique du repiquage. Il se fait en pépinière dans des compartiments spéciaux, qu'on nommait autrefois des batardières.

Qu'il s'agisse d'espèces résineuses ou feuillues, on emploie, pour être repiqués en pépinière, des jeunes plants âgés d'un ou deux ans au plus. On les arrache avec les précautions nécessaires pour ne pas endommager les racines; on en fait le triage et l'on écarte les sujets trop grêles ou trop chétifs. Les bons sont abrités avec soin contre le soleil et le vent, pour les préserver du desséchement.

La mise en terre de ces plants se fait d'après l'une des trois méthodes suivantes :

$1^{\circ}$ au moyen d'un plantoir à main, dont la grosseur et la longueur sont proportionnées à la dimension des jeunes plants, on fait une série de trous alignés au cordeau, et régulièrement espacés; dans chacun de ces trous, on enferme un plant de manière que le collet de la racine se trouve au niveau du sol; puis on les rebouche au moyen d'une simple pression qu'on exerce avec le plantoir enfoncé à une faible distance de la première ouverture. Ce procédé s'applique surtout aux résineux d'un an;

$2^{\circ}$ avec une bêche, on ouvre, le long d'un cordeau, une simple fente qu'on élargit à sa partie supérieure en appuyant successivement à gauche et à droite sur le manche de l'outil; dans cette fente, on glisse les plans un à un, à la distance voulue, et l'on remplit avec du terreau; on les fixe 
ensuite, en pressant la terre, soit avec le pied, soit avec la bêche ;

$3^{\circ}$ un ouvrier creuse à la houe une rigole alignée au cordeau ; un autre, le plus généralement une femme, pose les plants, un à un, en les appuyant contre l'une des parois de la rigole que l'on a eu soin de maintenir à peu près verticale : pendant qu'il les soutient en bonne position avec la main gauche, il fixe les racines en refoulant, de la main droite, la terre meuble qu'on vient de retirer de la rigole. Il suffit, quand une planche est entièrement remplie, de donner un coup de râteau entre les lignes pour terminer la besogne. Cette troisième méthode, de beaucoup préférable aux deux autres, s'applique aux plants de toutes dimensions.

D’ailleurs, la rapidité du travail et la régularité parfaite dans l'emplacement des plants peuvent être assurés par l'emploi de la règle à repiquer (fig. 81 c. c.) dont M. l'Inspecteur Cardot a fait le pendant de sa règle à semer (1). Sensiblement de même forme et de même dimension que celle-ci, elle porte sur une de ses faces une suite d'encoches équidistantes, dont chacune est destinée à recevoir un plant, qui y est posé de telle façon que le collet de la racine

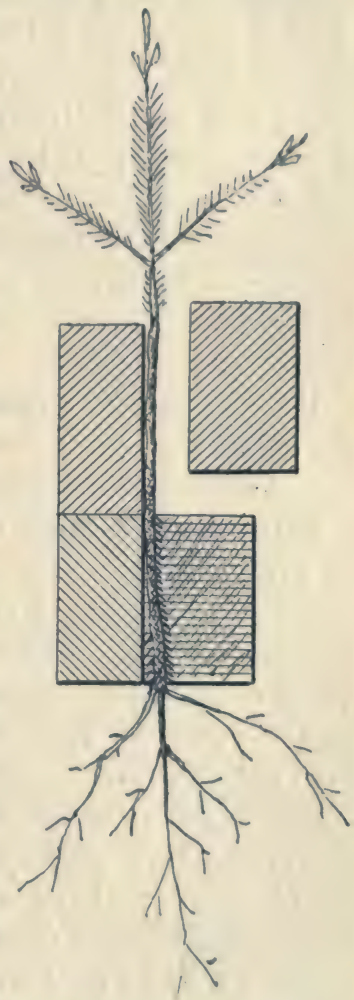

Fig. 8i:- Coupe de la règlé à repiquer. soit au niveau de la face inférieure de la rìgle. Quand toutes les encoches sont garnies, on maintient la série en appuyant la réglette mobile dans l'angle supérieur de magasin et l'on place le tout sur le bord de la rigole; il suffit de rechausser

(1) Le principe est celui de l'antique règle d̀ repiquer, outil en fer, mal commóde, et dont per'sonne n'avait jamais songé à se servir. 
les racines en procédant comme ci-dessus, après quoi on enlève l'appareil.

La règle peut servir à des espacements différents en garnissant à volonté une encoche sur $2,-3$, - etc.

L'espacement à donner aux plants varie avec l'emploi auquel on les destine. Sil'on ne veut que des basses tiges, l'écartement entre chaque ligne pourra varier, suivant les essences, de 15 à 25 centimètres et la distance dans les lignes de 4 à 5 centimètres; pour les moyennes tiges, on devra augmenter ces dimensions et donner 10 à 15 centimètres à l'espacement des plants dans les lignes; enfin, pour les hautes tiges, il conviendra de donner aux lignes au moins 50 centimètres d'écartement et aux plants un espacement minimum de 10 centimètres.

Il est entendu qu'au point de vue de la fumure, des sarclages, des binages et même des irrigations, les planches repiquées seront entretenues comme les planches semées.

Extraction des plants. - Lors de l'extraction des plants, on évitera de blesser les racines et les petites tiges; aussi l'arrachage à la main doit-il être absolument proscrit. Le meilleur procédé, celui qui est surtout facilité par le semis ou le repiquage en lignes, consiste à ouvrir, sur le bord des sentiers et tout contre la première rangée, une jauge assez profonde pour atteindre l'extrémité inférieure des racines; puis, au moyen d'une bêche, ou mieux encore d'une fourche à dents plates, on exerce une pression du côté opposé de façon à renverser les plants dans la jauge, sans que la terre se détache des racines; celles-ci restent absolument intactes quand il s'agit de jeunes plants résineux d'un ou de deux ans; dès lors, il n'y a jamais besoin de les tailler ou d'en retrancher une portion quelconque. L'extraction est toujours suivie d'un triage, qui permet, tout en comptant les plants, de rebuter tous ceux qui ne paraitraient pas de bonne qualité. Cette opération doit être faite sous un hangar fermé ou, tout au moins, à l'abri de paillassons, pour éviter le contact d'un air trop agité ou trop chaud et, par suite, le désséchement.

Les feuillus d'espèces pivotantes, les demi ou moyennes tiges s'arrachent beaucoup plus difficilement; il faut faire des 
trous profonds et, malgré cette précaution, il est rare qu'on puisse extraire les racines entieres. Alors, la taille de ces racines est justifiée; mais l'opération se limite à pratiquer une section bien nette, faite obliquement avec une serpette tranchante, immédiatement au-dessus des cassures ou des portions trop fortement lésées; en aucun cas, il ne faut rien retrancher aux parties saines, car les filaments les plus tenus sont les plus utiles.

Quand il s'agit de plants précieux ou délicats, le mieux est de les extraire et de les transplanter en motle; le surcroit de dépense que l’opération entraîne est largement compensé par une reprise plus certaine et une végétation meilleure. D'ailleurs, quand on veut assurer la transplantation en motte d'un sujet, quelles que soient ses dimensions, on procède de la manière suivante : dès le printemps qui précède la saison oú se fera le déplacement, on creuse, tout autour de la tige et à une distance variable entre $\mathbf{4 0}$ centimètres et 1 mètre, suivant la force du sujet et la nature de son enracinement, une jauge circulaire de la largeur d'un fer de bêche et de 50 ou 60 centimètres de profondeur. Cette jauge, taillée à parois verticales, au moyen d'une bêche avivée, est ensuite remplie avec de la bonne terre mélangée de terreau. Si l'on a soin de l'arroser plusieurs fois dans la saison de végétation, il se formera, dans cette terre neuve, un chevelu abondant, et, quand le moment sera venu, on extraira la motte sans occasionner de nouvelles lésions aux racines (1).

Taille ou rhabillage des plants. - Quand les plants de basses tiges ont été convenablement arrachées, les racines sont suffisamment intactes pour qu'il ne soit pas nécessaire de les parer, à moins qu'il ne s'agisse de rabattre le pivot sur des sujets destinés à être rigolés en pépinière.

Quant aux branches des feuillus ou des résineux, il ne faut jamais y toucher. En effet, la règle est de ne mettre en place que des plants bien conformés; ceux-ci n'ont pas besoin qu'on enlève quoi que ce soit à leurs tiges ou à leurs rameaux. La taille ne devient nécessaire que quand les sujels sont

(1) A. Chargerand. Traité des plantalions dialignement et d'ornement. Paris. Rothschild, 1896. 
mal équilibrés : tige trop grêle ou fourchue, rameaux insuffisants ou trop développés. Dans ce cas, il vaudra mieux l'opérer dans la pépinière, rigoler à nouveau les plants et n'en disposer qu'une année ou deux plus tard lorsqu'ils seront suffisamment refaits; c'est ainsi qu'on est souvent conduit à recéper les individus mal conformés, soit au moment de la mise en terre, soit en pépinière, ce qui vaut mieux.

Transports et emballage des plants. - Il faut se persuader que de la bonne conservation des plants dépend, en grande partie, la réussite des plantations et ne jamais perdre de vue cet axiome important : moins longtemps les racines restent à découvert, mieux elles se conservent.

A partir de l'extraction jusqu'au moment de la plantation à demeure, les sujets, surtout les résineux, dont les racines sont très délicates, doivent être garantis des accidents de nature à dessécher le chevelu ou à leur occasionner des lésions. S'ils ne doivent pas être transportés au loin, et si l'on juge inutile de les emballer avec soin, on les dispose, par bottes, dans un panier ou dans une brouette en les entourant de mousse humide, de ramilles ou de terre meuble. On recommande souvent de plonger les racines dans une bouillie liquide de terre grasse et, après les avoir entourées de mousse, de les emballer dans des paniers ou dans des caisses, mais ce pralinage a le grave inconvénient d'agglutiner les racines en pinceau et d'en provoquer la mise en terre dans des conditions tout à fait anormales.

Pour les espèces à feuilles persistantes, il est prudent d'entre-croiser les bottes de telle façon que les feuilles de l'une soient en contact avec les racines de l'autre, parce que les parties vertes pressées et accumulées en grande masse fermentent facilement. Il faut éviter aussi de faire voyager les plants par un froid trop rigoureux ou une chaleur excessive, sous peine de s'exposer aux accidents très fréquents de gelée ou d'échauffement.

Si les sujets ne peuvent être expédiés ou plantés à demeure aussitôt après l'extraction, on creuse une fosse dans laquelle on les dresse et on couvre leurs racines avec de la terre fraîche ; cela s'appelle mettre les plants en jauge. De même, 
dès leur arrivée à destination, les plants doivent être immédiatement déballés et mis en jaugre. En hiver, pendant les grandes gelées, les espèces à feuilles persistantes seront utilement recouvertes d'un paillasson ou de branchages.

\section{3. - LES pépinières volantes ou Locales.}

Avantages. - Installation.

Avantages. - Les pépinières volantes suffisent largement à tous les besoins des repeuplements à faire, sous forme d'amélioration, dans les terrains déjà boisés ; souvent même, bien qu'il s'agisse de terrains nus, les pépinières ne peuvent être établies que dans cette forme. C'est le cas, par exemple, pour les reboisements à exécuter sur le sommet des montagnes, en des climats rudes, dont les saisons de végétation ne correspondent pas avec celles des stations plus tempérées où les pépinières permanentes sont en général établies.

Installation. - Les pépinières volantes se font en pleine forêt, soit dans une petite clairière, soit à l'abri de grands arbres. Après avoir choisi l'emplacement convenable, on défriche le terrain et l'on défonce, comme s'il s'agissait d'une pépinière permanente, sans toutefois apporter d'engrais. On y fait des semis ou des repiquages, en vue d'obtenir les plants dont on peut avoir besoin dans les environs immédiats, et, quand la terre est fatiguée, on abandonne la place, après y avoir laissé autant de plants qu'il est nécessaire pour en assurer le boisement.

Ces petites pépinières intelligemment disposées, présentent les avantages suivants : $1^{\circ}$ elles ne doivent fournir qu'un nombre de récoltes assez faible pour qu'on puisse se passer d'engrais; $2^{\circ}$ elles offrent la possibilité d'élever, pour ainsi dire sur place, et dans les conditions climatériques où ils doivent végéter, les sujets destinés à être plantés; $3^{\circ}$ elles suppriment les nambreux inconvénients des emballages et des transports à grandes distances.

En montagne, les espaces de petite étendue, ayant la dimension de pépinières volantes, peuvent, par exception, être 
traités à la façon des pépinières permanentes à cause de la rareté des emplacements qui conviendraient à ces dernières. On est souvent obligé de les maintenir dans cette forme; car, de toute nécessité, les plants doivent être produits sous un climat identique à celui des espaces où ils seront plantés à demeure. Dans ces conditions, il faut créer autant de centres de production qu'il y a de zones d'altitude, c'est-à-dire de climats différents.

\section{Exécution des plantations.}

Préparation du sol. - Disposition des plants. - Confection et dimension des trous. - Manipulation des plants. - Plantation à racines nues; - par touffes; - en butte; - en corbeilles. - Plantation en terrain non préparé. - Saison favorable à la plantation. - Application aux principales essences. - Soins à donner aux plantations.

Préparation du sol. - Ce que nous avons dit, à l'article précédent, de l'heureuse influence d'une culture du sol sur la réussite des semis, nous dispense d'entrer dans de nouveaux détails, et notre conclusion est tout aussi absolue dans un cas que dans l'autre : à la rigueur, on peut se dispenser de cultiver le sol dans les terres naturellement très meubles, tels que les sables sans consistance; - dans les forêts constituées où la terre à bois est déjà préparée de longue date; - enfin, dans les terrains enherbés où la présence d'un tapis continu de plantes basses, se maintenant en végétation pendant toute la saison d'été, témoigne d'une constante fraîcheur. En toutes les autres circonstances, la culture doit être considérée, le plus souvent, comme indispensable, - toujours, comme avantageuse.

On procede aux façons de la terre par les moyens les plus économiques dans chaque région, en cherchant par le brassage des couches, à donner à l'ensemble une composition moyenne; contrairement à ce qui a été recommandé pour les semis, il sera préférable de localiser la meilleure terre dans les zones profondes, oú les racines vont être appelées à fonctionner immédiatement.

La culture en plein n'est jamais nécessaire, elle présente 
même certains inconvénients; en effet, dans les pentes rapides, un sol trop ameubli peut être entrainć par les eaux; de plus, l'expérience indique que les dégâts du ver blanc sont bien plus à redouter dans un terrain cultivé que dans ceux qui ne l'ont pas été. L'ouverture de bandes continues n'est avantageuse que pour des plantations de hautes tiges dont la mise en terre demande de larges défoncements; elle est inutile lorsqu'il s'agit de basses tiges; les dégâts de ver blanc y sont d'ailleurs à craindre,comme dans les cultures en plein. Reste la préparation dusol par trous ou potets à laquelle nous donnerons la préférence, comme permettant de défoncer profondément le sol sans entraîner une grande dépense : elle réunit donc tous les avantages culturaux et économiques.

Disposition des plants. - Ces trous seront disposés suivant le tracé de figures géométriques: triangles, carrés ou quinconces, de manière que les plants puissent être retrouvés facilement, soit pour les soigner, soit pour remplacer les manquants; un bon ouvrier prend toujours la précaution de jalonner sur le terrain les lignes équidistantes suivant lesquelles seront disposés les plants : une baguette coupée à la longueur voulue, ou bien un cordeau portant des nouds en ficelle de couleur, donnent leur espacement dans les lignes.

Quel écartement convient-il de donner aux plants? Cela dépend des circonstances. Tout d'abord, voyons quelle est l'influence de cet écartement sur la végétation.

Des expériences récentes entreprises par M. l'Inspecteur Bartet à la station de recherches de l'École Nationale des Eaux et Forêts semblent établir les faits suivants, sous réserve de vérification dans d'autres milieux :

$1^{\circ}$ à tous égards, l'espacement de 2 mètres, plus que les espacement moindres, convient à l'épicéa; on peut en trouver l'explication dans le besoin d'espace latéral réclamé par l'enracinement superficiel de cette essence;

$2^{\circ}$ pour tous les résineux, le même espacement, plus que les espacements moindres, donne aux plants un riche appareil foliacé, et favorise leur croissance en diamètre;

$3^{\prime \prime}$ pour tous les résineux autres que l'épicéa, on ne peut 
encore rien avancer de certain quant à la croissance en hauteur; il semble toutefois, contrairement à l'opinion qu'on pourrait se faire à priori, que les grandes distances sont peu favorables à l'allongement des espéces de lumière : celles-ci, en effet, ont souvent une tendance à buissonner qu'il est bon de combattre en hâtant la formation du fourré; M. le Professeur Fliche nous a souvent entretenus de la forme trapue et par suite défectueuse que prennent les jeunes pins noirs d'Autriche quand ils constituent des peuplements trop clairs. Un article que nous avons publié sur ce sujet dans le $\mathrm{Bul}$ letin du Ministère de l'Agriculture (1), est résumé par les lignes suivantes : “ à notre avis, la croissance en hauteur n'a pas une importance capitale pendant les premières années consécutives à la plantation. La vigueur et la bonne santé de l'arbre, accusées par l'abondance du feuillage et l'accroissement en diamètre, en ont au contraire beaucoup. Si donc, laissant de côté les massifs résineux destinés à produire à brève échéance telle ou telle marchandise spéciale (perches à houblon ou étais de mine), nous songeons à créer une forêt solide et définitive, nous constatons une fois de plus avec combien de raison M. Broilliard, dans le Traitement des bois, recommande de ne pas surcharger les plantations d'un nombre inconsidéré de sujets, trop souvent plantés à l'économie. ")

Nous rejetons les plantations faites avec des écartements inférieurs à 1 mètre $(2)$ : ou bien elles sont exécutées sans soin, avec des plants à bon marché et conduisent à un insuccès total, - ou bien les sujets viennent trop serrés et l'on se trouve dans la nécessité d'en sacrifier la moitié par dépres. sage. Nous considérons aussi comme imprudentes, au moins jusqu'à preuve du contraire, les distances égales ou supérieures à 3 mètres. Pour qui veut un chiffre, nous recom-

(1) Année 1899.

(2) Dans certaines forèts de la Bavière, on fait les plantations excessivement serrées; nous avons vu dans le Spessart des plants disposés à $0^{\mathrm{m}}, 30$ les uns des autres, dans des lignes espacées entre elles de 1 mètre; cela représente 30000 plants à l'hectare. La mise en place coûte 55 francs, quand je prix de la journée ne dépasse pas 2 fr. 50 pour les hommes, 1 fr. 75 pour les femmes. Même à ce prix, il y a exagération et les forestiers enseignants réagissent contre le gaspillage des pratiquants. 
mandons pour toutes les essences feuillues et résineuses, les écartements de $1^{\mathrm{m}}, 50$, dans les lignes espacées de 2 mètres, - ou tout au moins de $1^{\text {in }}, 50$ dans tous les sens.

Le tableau suivant donne le nombre de plants à l'hectare, dans les limites les plus ordinaires des écartements entre les lignes et entre les plants d'une même ligne.

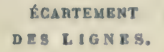

$1^{\mathrm{m}}, 20$
$1^{\mathrm{m}}, 20$
$1^{\mathrm{m}}, 40$
$1^{\mathrm{m}}, 50$
$1^{\mathrm{m}}, 80$
$2^{\mathrm{m}}, 00$
$1^{\mathrm{m}}, 50$
$1^{\mathrm{m}}, 80$
$2^{\mathrm{m}}, 00$
$1^{\mathrm{m}}, 80$
$2^{\mathrm{m}}, 00$

Rearteuent
E S PL A N
dans chaque li
$0^{\mathrm{m}, 80}$
$0^{\mathrm{m}}, 90$
$1^{\mathrm{m}}, 00$
$1^{\mathrm{m}}, 00$
$1^{\mathrm{m}}, 00$
$1^{\mathrm{m}}, 00$
$1^{\mathrm{m}}, 20$
$1^{\mathrm{m}}, 20$
$1^{\mathrm{m}}, 20$
$1^{\mathrm{m}}, 50$
$1^{\mathrm{m}}, 50$

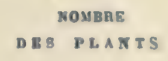

10,417

9,260

7,143

6,666

5,555

5,000

5,555

4,630

4,166

3,636

3, 333

Confection et dimension des trous. - La dimension des trous est subordonnée à celle des plants : il suffit que les racines puissent y être étalées dans leur position naturelle.

Pour les basses tiges de résineux, des potets de $0^{\mathrm{m}}, 25 \times 0^{\mathrm{m}}, 25$ d'ouverture, et de $0^{\mathrm{m}}, 25$ de profondeur sont, en général, très satisfaisants. On fera, d'ailleurs, en sorte de leur donner la même largeur au fond qu'à l'ouverture, en évitant la forme en entonnoir; le fond en sera toujours ameubli.

L'outil le plus commode pour les ouvrir est la pioche, si le sol est d'un travail moyennement facile. Quand il est caillouteux ou trés dur et compact, on peut recourir au pic de terrassier pour le rompre, tandis que la pioche sert à extraire les déblais. Enfin, quand nous avons eu à ouvrir des potets de dimensions excédant la normale, pour des plantations de demi-tiges, par exemple, nous nous sommes bien trouvés de l'outil dont se servent les vignerons, pour la confection des fosses.

En extrayant la terre au fur et à mesure du défoncement, on la disposera en lots séparés, suivant ses différentes qualités. Les gazons seront mis à droite, par exemple; la terre fine qu'ils recouvrent immédiatement, ì gauche; la plus mau- 
vaise terre du fond, en avant; on aura ainsi sous la main, au moment de la plantation, les matériaux tout prêts pour en disposer au mieux de l'opération. Les grosses pierres seront également conservées près des trous pour être utilisées comme il sera dit plus loin; dans les terrains en pente rapide, elles pourront être amoncelées, à la main, en forme de talus ou de mur de sontènement du côté d'aval. En plaine, ces petits amas se feront de préférence du côté du Midi.

Quand le terrain comporte l'emploi de la charrue, on peut ouvrir à l'aide de cet instrument des sillons distants de $1^{\mathrm{m}}, 50$ et se coupant à angle droit; à chaque point d'intersection, on ouvre un potet. Mais ce procédé n'est qu'une manière de tracer l'ouvrage et de rendre plus facile le travail de la pioche, qui seule peut creuser des trous convenables. Le système expéditif qui consiste à disposer des plants dans une raie ouverte à la charrue et à les recouvrir par un second passage du versoir expose à des échecs fréquents.

Dans les terrains meubles, secs, filtrants, les trous peuvent être ouverts au moment de la plantation; dans les sols plus ou moins compacts, ils seront faits quelques mois à l'avance pour profiter de l'ameublissement naturel que donnent la gelée ou la sécheresse.

Manipulation des plants. - Tous les ouvriers planteurs se munissent de paniers dans lesquels les racines sont soigneusement abritées; jamais celles-ci ne doivent rester exposées à l'air et il ne suffit pas, comme cela se fait trop souvent, de placer les plants dans la poche d'un tablier. Le maintien du chevelu à l'état frais est la condition la plus importante pour la réussite; on ne saurait trop insister sur ce point. Tous les plants seront donc mis en jauge, en attendant leur emploi; et, dans l'organisation des chantiers, pour éviter les pertes de temps, il sera avantageux d'adjoindre aux planteurs un certain nombre d'enfants, chargés d'aller prendre, dans des paniers de rechange, les provisions nécessaires pour remplacer les paniers vides.

Les racines sont préservées du contact de l'air par du terreau frais, ou de la mousse humide ; quand les ouvriers sont inhabiles ou peu soigneux, il peut $y$ avoir avantage à rem- 
placer ces matériaux trop faciles à disperser par un morceau d'une vieille couverture de laine lâchement cousue au panier par deux ou trois de ses côtés et assez long pour abriter les plants.

Plantation à racines nues. - C'est le mode le plus communément employé. Les autres procédés ne trouvent leur application que dans des cas spéciaux.

Chaque trou est garni de la manière suivante: l'ouvrier s'assure que le fond du potet est bien ameubli, sinon, il y jette quelques poignées de terre fine, puis il prend un plant dans le panier, - en ayant soin de ne pas découvrir les autres, - et, de la main gauche, il le soutient contre la paroi du trou qui lui fait face, à une hauteur telle que le collet de la racine se trouve, soit au niveau du sol en place, soit à 2 ou 3 centimètres en dessous; de la main droite, il étale les racines en position normale dans toute la largeur du trou, puis les fixe au moyen de la terre meuble qu'il trouve à sa gauche et qu'il emploie ainsi jusqu'à épuisement. Enfin, il achève de combler avec la terre la plus grossière amoncelée en avant et qu'il tasse légèrement avec le pied. Pour tenir compte du foisonnement, on donne à la surface la forme d'une butte élevée de quelques centimètres au-dessus du sol en place; sans cette précaution, le sol s'affaisse et met à nu quelques racines.

Il existe un inconvénient réel à enfouir les racines des plants à une profondeur exagérée, comme à damer avec plus ou moins de brutalité la terre qui sert à les recouvrir. Privées d'air, elles fonctionnent mal, ne peuvent s'allonger ni se ramifier : le plant languit, tant qu'un nouvel enracinement n'a pu se substituer à l'ancien. comme on peut s'en rendre compte par la figure 85 . Sans doute, nous restons convaincus que l'ennemi du planteur est toujours la sécheresse, et nous préférons exposer nos pins ou nos épicéas à sou//rir pendant deux ans, faute d'oxygène, plutôt qu'à mourir en six mois, faute d'humidité; quoi qu'il en soit, la question peut se résumer ainsi : - dans les terrains sablonneux très meubles, qui sont tout à la fois très perméables à l'air, ef très exposés à se dessécher, planter prolond: - dans les argiles 
très compactes, oủ l'asphyxie menace toujours les plants, ne pas enfouir ceux-ci plus profondément que le collet de la racine, au risque d'avoir un insuccès si le hasard veut que l'année soit très sèche; - enfin, dans les sols calcaires, dans les terrains granitiques, ne pas exagérer la profondeur, mais utiliser, pour préserver les plants des chaleurs estivales, les pierres qui ne font jamais défaut sur de pareils terrains.

Il est avéré, en effet, que toutes les fois que l'on couvre le sol, au pied du sujet mis en terre, de grosses pierres plates, on entretient d'une façon remarquable la fraîcheur du terrain, et l'on assure la réussite. Les dalles calcaires, si fréquentes sur certains sols de l'oolithe, ont sauvé bien des plantations et se prêtent à merveille à cet emploi ; mais tous autres quartiers de roche peuvent être utilisés de même.

Comme moyens préservatifs contre la sécheresse, on a recours encore à des talus de 1 à 3 décimètres de hauteur élevés au midi de chaque polet et qui assurent un peu d'ombre au plant: tantôt l'abri est une butte de terre; tantôt un petit mur de pierres sèches; souvent on se contente d'amonceler les gazons découpés pour l'ouverture du potet, et que l'on dispose, les racines en l'air.

Sur les pentes trés déclives, on modifie la disposition du plant dans son potet: le sujet doit être maintenu, pendant que l'on comble le trou, sur la paroi aval. Sinon les eaux courantes, en entraînant la terre fraîchementremuée, déchausseraient la tigelle.

Plantation en mottes ou en gazons. - La plantation en mottes ou en gazons consiste à découper, dans une planche de semis, des mottes de 8 à 10 centimètres de côté qui sont enlevées avec la terre et disposées en cette forme dans des trous préparés à l'avance. Ce mode, qui a l'avantage de conserver le chevelu dans un état de fraîcheur parfait, a l'inconvénient d'employer inutilement un grand nombre de sujets, d'augmenter beaucoup les frais de transport, et d'appauvrir le sol des pépinières; aussi n'est-il employé que pour les plants extraits directement en forêt parmi les régénérations naturelles ou parmi ceux qui surabondent dans des régénérations artificielles. 


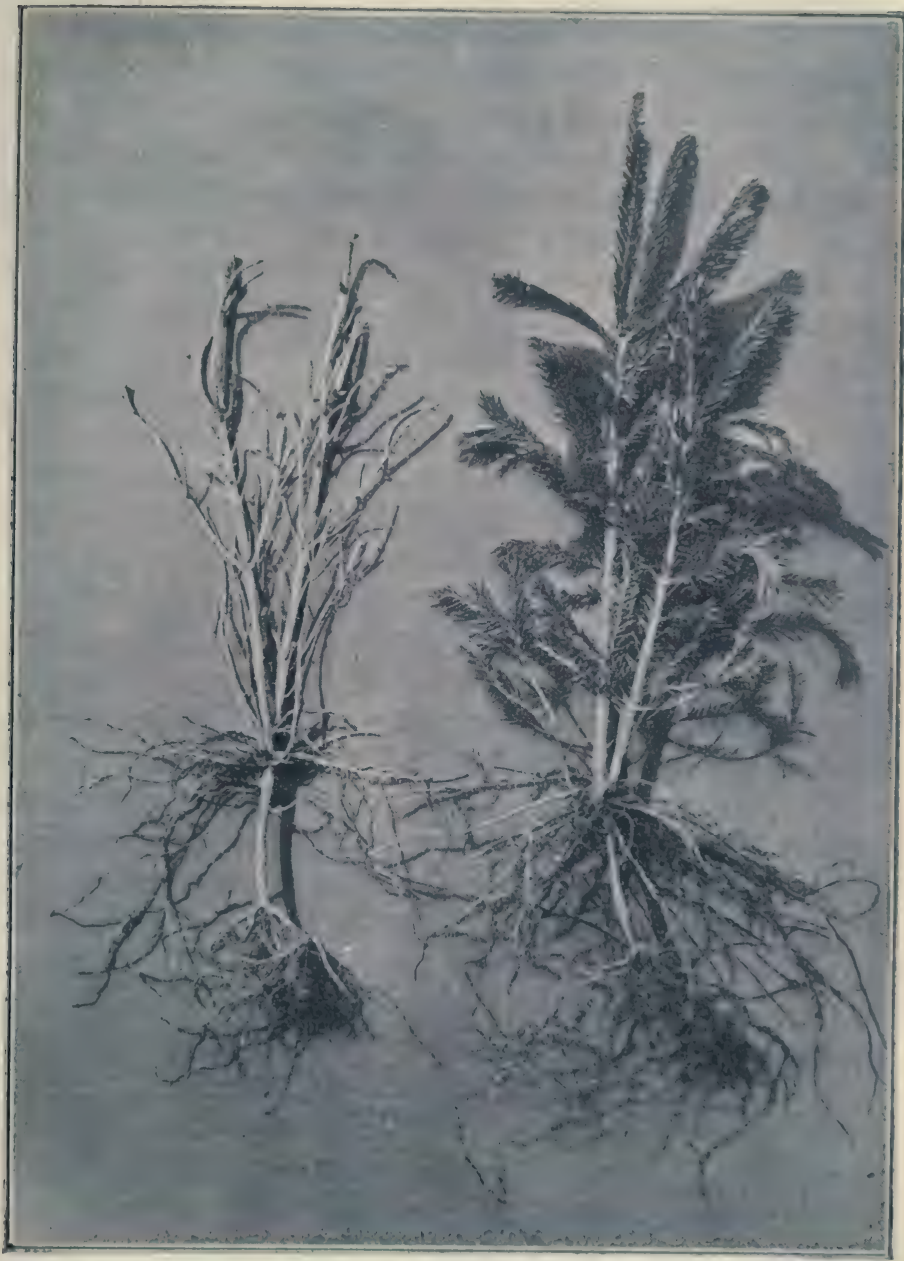

Fig. 85. - Épicéas plantés à une profondeur telle qu'un nouvel enracinement a dù se substituer à l'ancien I'.

(1) La plantation d'oú ces types ont été extraits, faite au début d'une année sèche, a donné d’ailleurs des résultats satisfaisants. 
Ainsi, dans les sapinières et les hêtraies, on rencontre souvent des semis assez nombreux pour former par place de véritables brosses aussi fournies qu'un carreau de pépinière; - ainsi encore quand un semis par bandes de pin ou d'épicéa a bien réussi, rien n'est plus facile que de prélever dans ces lignes des mottes d'une reprise certaine.

Malgré le surcroît de dépense considérable qu'entraîne ce procédé, on est obligé d'y avoir recours dans les situations difficiles: notamment dans les très mauvais sols, dars les rocailles où les plants à racines nues périraient nécessairement. S'il existe des jeunes semis ou des plantations récentes dans les environs, il est naturel d'y puiser: on a réussi par ce procédé le boisement de stations très ingrates comme des éboulis rocheux ou des marnes exposées au déchaussement (1).Il est indispensable, toutefois, que le terrain où sont découpées les mottes présente une certaine cohésion, sans quoi la terre est trop meuble pour supporter le transport et tout se désagrège.

Plantation, par touffes ou par bouquets. - M. Demontzey recommande, au lieu de planter par pieds isolés, de réunir dans un même trou une touffe de trois à quatre plants. Suivant cet habile praticien, ce mode présente le double avantage d'être le plus économique et le plus sûr de tous les moyens employés.

“ D'une part, en effet, il augmente considérablement les chances de succès d'une plantation et épargne ainsi de nombreux regarnissages, non seulement coûteux et souvent difficiles, mais encore dangereux pour les plants qui ont bien végété, à cause du passage des ouvriers sur des pentes souvent très fortes.

“ D'autre part, il permet d'espacer davantage les plants, et par suite de diminuer la dépense à l'hectare. Car ces touffes forment au début une sorte de petit massif dans lequel les plants se soutiennent mutuellement, ce qui leur permet d'attendre plus longtemps et sans danger le moment où le massif général se constituera.

(1) A l'aide de ce procédé, M. le conservateur Carrière a obtenu les plus beaux succès dans le périmètre de Seyne (Basses-Alpes). 
"On a fait à ce mode l'objection que si les plants réunis ainsi en touffes venaient à pousser tous également, ils ne tarderaient pas à s'affamer mutuellement et à produire des arbres grêles, incapables d'une longue durée.

" A cette objection, on peut répondre d'abord que la touffe n'implique pas nécessairement un grand nombre de plants, et que dans les limites que nous posons, c'est-ì-dire de deux à quatre plants par touffe, ce danger devient illusoire.

"Mais, bien plus, une longue expérience a démontré l'inanité de ces craintes. Il est rare, en effet, que deux, trois ou quatre plants poussent, dès le début, avec une vigueur identique; le plus souvent, l'un d'eux prend le dessus et quand le massif général se produit, il demeure seul. D'autre part, bien souvent, un seul plant, deux au plus, reprennent avec vigueur, les autres viennent à mourir, soit immédiatement après la plantation, soit un an après, de sorte qu'en dernière analyse, on se trouve dans les conditions de la plantation par brins isolés, mais avec des chances de réussite bien plus complètes. Enfin, il serait toujours facile d'obvier à peu de frais à l'inconvénient d'une égale pousse, s'il venait à se manifester (1). "

Il. semble, à première vue, que cinq ou six plants identiques, placés dans les mêmes conditions, doivent tous reprendre ou tous mourir, le plus souvent du moins. En fait, les choses ne se passent pas ainsi, et les sujets d'une même touffe se prêtent, sans doute, un mutuel appui, car leur résistance à la sécheresse est supérieure à celles des plants isolés.

Ce procédé ne nous semble pourtant pas devoir être généralisé. Son application reste confinée dans la montagne, quand on opère dans des conditions d'une difficulté toute spéciale, - ou bien en plaine, quand on veut créer le plus vite possible une barrière de verdure délimitant une plantation et l'isolant des champs ou des pâturages voisins.

lin effet, il occasionne une consommation de plants exagé-

(1) Demontzey, Éudes sur les trataux de reboisement des montagnes, p. 227.

Bopps et Jolytit. 
rée, et, de plus, les touffes constituent bientôt des milieux qui sont très propres au développement des maladies contagieuses si l'on ne se hâte de procéder à un dépressage. Les faits ne manquent pas à l'appui de cette observation : ainsi, au printemps 1886 , les dégâts de Peridermium pini, ont été constatés dans le périmètre de Barèges (Hautes-Pyrénées) sur des pins de montagne qu'on avait omis de desserrer en temps utile; des plantations voisines, faites par pieds isolés, étaient à peu près indemnes. Semblables accidents ont été signalés depuis sur bien d'autres points, notamment dans les Basses-Alpes.

Pour le même motif, la plantation par touffes est à déconseiller d'une manière absolue avec le mélèze; dans la pratique, elle n'est guère utilisée qu'avec les pins.

Plantation en butte. - Ce procédé, imaginé par M. le baron de Manteuffel (1), consiste à créer de toutes pièces des buttes ou monticules de terreau sur lesquels on plante. Il a l'inconvénient de coûter très cher de main d'œuvre; c'est une véritable plantation de luxe. D'ailleurs il n'est applicable qu'en forêt, ou, du moins, à proximité de terrains déjà boisés et pouvant fournir, à un prix abordable, la quantité énorme de terreau nécessaire, quantité qui peut être évaluée à 4 mètres cubes par mille plants, soit au minimum 12 à 15 mètres cubes par hectare. Le procédé Manteuffel n’a du reste été imaginé que pour boiser les terrains tourbeux dans lesquels on ne pouvait planter directement; la butte forme ainsi un sol d'apport tout artificiel dans lequel s'enracine le jeune plant.

Dans des conditions analogues, on peut employer la méthode de Biermann, qui conduit à peu près au même résultat d'une manière infiniment plus économique. On trace à la charrue, à 70 ou 80 centimètres de distance, deux sillons ouverts en sens opposé, de telle sorte que la terre en soit versée sur l'intervalle inculte entre les deux sillons; on forme ainsi une série d'à-dos régulièrement alignés, espacés 'entre eux de deux mètres, par exemple, et sur les-

(1) L'art de planter, par M. le baron de Mauteuffel, traduit par S. P. Stumper. Paris, Rothsehild, 1868. 
quels on plante, à distance convenable, dans une terre assainie, ameublie par l'insolation et enrichie par les débris de la végétation herbacée.Cette préparation doit être faite quelques mois avant la plantation, de façon à laisser au gazon renfermé dans les buttes le temps de se décomposer.

Plantation en corbeilles. - Le procédé consiste à creuser un trou en forme de tronc de cône renversé, ayant de 60 centimètres à 1 mètre d'ouverture et de 25 à 35 centimètres de profondeur; tout autour des parois de ce trou, on place une série de basses ou moyennes tiges (généralement des feuillus), espacées entre elles de 10 à 15 centimètres et disposées à la façon des baguettes qui forment la carcasse d'un gabion; on remplit ensuite le trou avec de la terre meuble, mélangée de terreau si cela est possible. Dans les sols exposés au soulèvement par la gelée, on couvre la terre rapportée avec de grosses pierres; celles-ci conservent l'humidité en même temps qu'elles fixent la surface.

La reprise des plants est assurée et, en espaçant ces trous de plusieurs mètres entre eux, on crée une série de petits îlots de verdure; si l'on a pris soin de planter des espèces susceptibles de se propager par le marcottage, la végétation s'étale rapidement en zones concentriques et s'avance en raison du carré des diamètres de chacun de ces cercles mobiles : bientôt tous les intervalles se trouvent boisés. Ce mode, imaginé par M. le conservateur Carrière, alors qu'il était chef du service du reboisement dans les Basses-Alpes, a été employé par lui dans les atterrissements du fond du lit des torrents et des ravins, où, complété par le marcottage, il a produit des résultats parfaits dans des sols qui, au delà de la couche la plus superficielle, étaient encore réfractaires à la végétation ligneuse. Il serait applicable dans maintes circonstances.

Plantation en terrain non préparé. - La plantation en terrain non préparé consiste à ouvrir dans le sol un simple trou ou une fente de dimension proportionnée à celle des racines des sujets à planter; dans ce trou, dirigé soit verticalement, soit obliquement (1), suivant la profondeur du terrain,

(1) M. l'inspectcur Prouvé recommande "d'ineliner les plants vers 
on glisse les racines du plant, en ayant soin de les étaler aussi régulièrement que possible sans qu'elles restent jamais en pinceau ; puis, on ferme la fente en pressant la terre avec le pied, ou bien avec l'outil.

Si ce mode primitif a l'avantage d'être très économique, ses inconvénients sont nombreux; car les racines des sujets se trouvent disposées dans un même plan et en situation toute différente de celle qu'elles devraient occuper normalement; enfouies dans un sol vierge et pauvre, trop loin de la couche d'humus superficielle où les jeunes plants naturels étalent de préférence leur chevelu, ces racines sont en outre bien souvent pressées avec exagération par des mouvements dont il n'est pas facile de mesurer le degré d'intensité, et, par suite, dans les sols argileux compacts, privées de l'air nécessaire à leur respiration. Sans doute, on a peu à craindre les dégâts du ver blanc; sans doute, on évite le dessèchement; mais la végétation reste extrêmement lente; si les plants ne meurent pas, ils boudent, comme disent les pépiniéristes, et, dans cet état languissant, ils risquent d'être étouffés dansles herbes. D'une manière générale, avec ce système, les résultats sont d'autant meilleurs que l'on emploie des plants plus jeunes et, partout où l'envahissement des herbes n'est pas à craindre, on se servira avec avantage de sujets feuillus âgés d'un an et de résineux âgés de 1 à 2 ans, suivant leur force.

Pour activer le travail, quand on plante en terrain non préparé, on a imaginé, aussi bien en France qu'en Allemagne, une foule d'instruments spéciaux : bêches, plantoirs, haches, fourreaux destinés à protéger les racines du plant quand on les glisse dans la fente qui doit les recevoir. Les outils construits sur les indications de M. l'Inspecteur Prouvé (1), se recommandent parmi tous les similaires. Avec ceux-ci, un des meilleurs est certainement le plantoir triangulaire à nervure,

le Midi, afin de diminuer l'effet funeste des rayons solaires sur la tige au moment de la transplantation "n.

(1) Revue des eaux et forêts, t. XVI, p. 305 ; XVII, p. 433 ; XIX, p. 273 et Prouvé, Régénération par plantation des coupes de futaies, Paris, P. Mouillot, 1899 (Bull. Société des Agriculteurs de France.) 
en usage dans le Palatinat qui, tout en donnantle moyen d'ameublir le sol par un mouvement circulaire, permet d'ouvrir des fentes assez larges dans les sols forestiers encombrés de racines.

Sans rejeter de parti pris ces instruments qui, dans des circonstances données, peuvent présenter des avantages réels, il est nécessaire, à leur sujet, de faire certaines restrictions : c'est que, d'abord, un outil, quelque perfectionné qu'il soit, ne compensera jamais l'absence de préparation du terrain ; et qu'ensuite les plantations se font seulement dans un laps de temps très court, deux mois au plus dans l'année ( 3 ou 4 semaines au printemps et autant en automne); or, l'ouvrier, aussi bien le laboureur que le bûcheron, ne travaille adroitement qu'avec l'outil qu'il a l'habitude de manier, l'outil du pays, celui avec lequel son père et ses ancêtres ont nourri leur famille; chaque fois qu'on lui change cet outil, il y a pour lui un apprentissage à faire, et l'on risque ainsi de perdre tout le bénéfice qu'on attendait de l'instrument perfectionné.

Saison favorable à la plantation. - En principe, il est imprudent de transplanter un végétal quelconque pendant le plein de la saison de végétation si l'on ne peut l'arroser et lui donner tous les soins que comporte cette période de crise. De plus, on ne peut pas planter quand la terre est durcie par la gelée: ce n'est qu'exceptionnellement, sous les climats très doux, que les travaux se prolongent sans interruption pendant tout l'hiver. Restent donc, dans la majorité des cas, l'automne et le printemps. La question est de savoir à laquelle de ces deux saisons donner la préférence; nous recommandons l'automne pour les motifs suivants :

$1^{\circ}$ les grandes pluies du solstice et les neiges de l'hiver, survenant après la mise en place des plants, font couler les particules de terre entre les racines; elles tassent d'une façon progressive et des plus heureuses le sol fraîchement remué;

$2^{\circ}$ chez les sujets mis en terre dès l'automne, il se produit pendant l'hiver un travail végétatif dont les effets semblent localisés dans les racines : le chevelu se reconstitue, s'allonge et facilite l'évolution normale au premier printemps;

$3^{\circ}$ on évite le danger des hâles de mars, trop souvent mor- 
tels aux jeunes plants que l'on manipule et dont on expose plus ou moins les racines au grand air, - nuisibles à la reprise quand, immédiatement après la plantation, ils soufflent pendant de longues semaines, avec une constance désespérante.

M. Duparchy (1) pense qu'on peut planter les résineux dès la seconde quinzaine de septembre; dans les environs de Saint-Claude (Jura), il s'est bien trouvé des travaux faits dans une saison que d'autres trouveront prématurée. Quant à nous, nous conseillons d'attendre cette époque de l'année difficile à définir, mais que savent reconnaitre tous ceux qui ont habité la campagne, où l'on sent que l'on n'est plus en été : c'est le moment des premiers brouillards, celui où mûrissent les raisins et les alises, où les grives commencent à passer; en un mot, vers les premiers jours d'octobre. On plante d'abord les résineux; quant aux feuillus, qui peuvent être encore en pleine frondaison, on a la ressource d'enlever les feuilles, ou mieux encore d'attendre leur chute naturelle.

Dans quelques cas, néanmoins, la plantation de printemps doit être préférée ; ainsi :

$1^{\circ}$ dans les climats montagneux, où la brusque transition des saisons réduit à quelques jours le temps propre aux plantations; là, l'automne n'existe pour ainsi dire pas, et c'est au printemps, dès la fonte des neiges, qu'il faut planter ;

$2^{\circ}$ dans les terrains exposés au déchaussement par la gelée.

Signalons ce fait, bien connu en arboriculture, que les espèces délicates à feuilles persistantes supportent la transplantation en temps de sève, même à une époque tardive, tandis que leurs racines mutilées, placées au début de l'hiver dans un sol bientôt durci par la gelée, ne peuvent assurer l'approvisionnement en eau nécessaire pour contrebalancer les pertes consécutives à la transpiration. Mais, en général, les essences utilisées dans les grands travaux de boisement sont assez rustiques pour ne rien craindre de ce chef, à moins

(1) Duparchy, Mémoire sur le déboisement et le reboisement dans la région de Saint-Claude. (Bull. Société forestière de Franche-Comté et Belfort, septembre 1898). 
que l'on ait la malchance de subir un hiver exceplionnel par l'intensité du froid et l'absence de neige.

Quelle que soit la saison adoptée, il ne faut jamais planter dans une terre trop détrempée, qui se prenne en boue collante, sans qu'on puisse la disposer convenablement autour des racines.

Application aux principales essences. - Dans les sols nus, assez profonds pour que l'on puisse y cultiver le chêne, cette essence est généralement introduite par voie de semis. C'est elle, au contraire, qu'on a le plus souvent l'occasion de planter en forêt. Les plants d'un ou deux ans non repiqués sont utilisés dans les régénérations de futaies; accidentellement, on les plante dans les taillis simples exploités pour leurs écorces; ces plants n'ont, en général, pour toute racine, qu'un long pivot presque nu et l'absence de chevelu rend leur reprise assez chanceuse; néanmoins, dans les sols où l'on peut creuser des trous proportionnés à la longueur du pivot, divers outils spéciaux permettent de les utiliser; partout ailleurs il vaudra mieux n'employer que des plants repiqués après amputation du pivot et mis en place dans des potets défoncés. Dans les taillis sous futaie, on plantera des demi tiges ou, tout au moins, des basses tiges vigoureuses, repiquées une ou deux fois; le sol sera cultivé avec soin pour activer, autant que possible, la croissance. Si les pépinières sont mal approvisionnées, il vaudra mieux s'abstenir, plutôt que de mettre en terre des plants trop vieux et mal constitués, comme cela se fait trop souvent.

Le jeune hêtre s'utilise surtout en forêt, soit sous forme de peuplement principal, soit, comme sous étage, sous des essences de lumière. Dans ces conditions, les plants de deux ans repiqués réussissent très bien. A défaut de ceux-ci, on a recours au mode de plantation en mottes ou en gazons : ce procédé permet de prolonger le travail assez avant dansle printemps, alors même que les semis ont déjà poussé leurs premières feuilles.

La plantation du charme est facile; pourtant cetle essence boude pendant plusieurs années, quand le sol n'est pas frais 
et fertile. Vu le faible enracinement, de l'espèce, employer de préférence des plants ayant au minimum trois au quatre ans de pépinière.

Les frênes, les érables, les ormes, disséminés au milieu d'essences sociales dans les travaux de boisement des terrains nus, sont, en pareil cas, plantés à l'âge de deux ans, après repiquage : les semis sauvages sont d'une reprise trés incertaine. Sous forme d'amélioration, ces espèces sont introduites dans les coupes de taillis sous futaie, concurremment avecles chênes : alors comme ces derniers, on les emploie à l'état de demi et de hautes tiges.

L'aune est d'une plantation facile. On utilise soit des plants de pépinière de un ou deux ans, soit des semis naturels de deux ans.

Le bouleau est également facile à transplanter dans sa première jeunesse; il l'est moins dans un âge plus avancé et quand son écorce commence à blanchir. Lâge le plus convenable pour la plantation est de deux à quatre ans; bien que les plants de pépinière soient préférables, on peut, nous l'avons vu, se procurer de bons plants sauvages en forêt.

Les saules et les peupliers sont plus souvent introduits sous forme de boutures que sous forme de plantations.

La plantation du sapin se fait dans les mêmes conditions que celle du hêtre. La seule différence est que, si l'on plante à racines nues des sujets isolés, il est préférable de n'utiliser que des sujets de quatre ans repiqués; plus jeune, le sapineau, à peine ramifié, est trop délicat. La plantation en mottes donne aussi d'excellents résultats.

L'épicéa à trois ou quatre ans, le méléze, le pin sylvestre et le pin noir à deux ou trois ans sont les espèces résineuses les plus couramment employées dans les plantations en terrain nu. Ce choix est justifié par la facilité de leur reprise et de leur frugalité.

Nous devons une mention toute spéciale à l'épicéa. Si le pin noir réussit dans des sols plus superficiels, si le pin sylvestre accepte des sables plus grossiers, c'est l'épicéa, par la facilité avec laquelle on se le procure dans le commerce, par la complaisance avec laquelle il reprend presque en toute station et en tout terrain, qui a décidé les habitants de nos 
campagnes à créer dans les mauvais sols ce qu'ils appellent des "bois de sapins ". Il ne faut point abuser pourtant de sa souplesse pour l'employer en tous lieux ou le planter sans soins, car, s'il ne meurt pas, il restera chétif et mal venant.

Ajoutons qu'en tout état de choses, sa végétation est très lente pendant les toutes premières années qui suivent la plantation : ce n'est que quand ses branches couvrent bien le sol, quand il a tué l'herbe à son pied, qu'il commence à s'élancer. Mais, alors, il a bientôt rattrapé le temps perdu!

Pendant toute cette période d'installation, le feuillage jaunit, l'arbre prend une forme buissonnante et son aspect reste languissant; dans les terrains susceptibles de s'enherber fortement, des sarclages feront le meilleur effet. Cette première crise passée, on remarque que, sur un même terrain, planté dans des conditions identiques, avec des sujets de même origine, un certain nombre d'individus conservent leur feuillage jaunâtre, quand leurs voisins ont revêtu définitivement la couleur d'un vert bleu qui caractérise l'espèce : les plants jaunes entrent en végétation longtemps avant les autres, ils sont dès lors beaucoup plus exposés aux gelées printanières; leur croissance est plus lente, et dans certaines terres, ils meurent après avoir langui plus ou moins longtemps. Ne serait-on pas en présence de ces deux variétés signalées par M. Brenot aussi bien dans le Jura que dans les Alpes et qu'il a décrites sous les noms d'épicéa à cônes verts et d'épicéa à cônes rouges (1)? Le tempérament de ce dernier n'est pas sans une certaine analogie avec celui des sujets à feuillage jaune. Il n'y aurait d'ailleurs rien que de naturel, puisque les graines du commerce sont récoltées dans les stations où les deux variétés se rencontrent en mélange.

Le pin d'Alep réussit, par voie de plantation comme par voie de semis, dans les conditions de sécheresse qui seraient fatales à toutes les autres essences; il lui suffit de quelques pouces carrés de terre pour qu'il puisse s'installer. Sa végétation est assez rapide pour qu'on mette en place des plants d'un an.

(1) Brenot, Remaryues sur les deux variélés de l'épicéa commun. Paris, Imprimeric nationale, 1878. (Revuedes eaux et forêts, juillet 1870.) 
On ne plante guère le pin maritime que pour regarnir les semis directs dont la réussite est incomplète. On prend alors les jeunes sujets à replanter sur les points voisins les mieux fournis : l'opération n'offre d'ailleurs aucune difficulté si l'on n'emploie que des plants âgés d'un an ou de deux ans au plus.

Soins à donner aux plantations. - La réussite des plants mis en terre n'est pas toujours assurée, même après une saison de bonne végétation; plus les sujets sont forts, plus il faut de temps pour qu'on puisse répondre de leur avenir, et la période de crise qui accompagne tout déplacement d'un sujet, dure, en moyenne, deux ans pour les basses tiges et trois ans, au moins, pour les hautes. Quelles que soient donc les précautions prises, on est exposé à voir disparaître un certain nombre de plants pendant les premières années et l'on doit considérer la réussite comme bonne, si ce déchet n'atteint pas 10 p. 100 ; pour éviter les clairières, il faut remplacer les manquants dès l'automne qui suit l'époque de la plantation. On facilite le travail, si, avant de mettre les ouvriers en chantier, on parcourt avec soin toutes les surfaces, pour y compter les pieds manquants et en marquer la place par des fiches apparentes. En général, il est bon d'employer des sujets de même essence et de même taille que ceux qu'il s'agit de remplacer; cependant, si l'on a tardé plusieurs années avant de procéder à cette opération, le mieux sera de profiter de l'abri existant pour introduire dans les places les plus claires quelques pieds d'essences d'ombre.

Dans les stations fraîches et humides, les basses tiges des espèces à croissance lente, celles d'épicéa notamment, sont exposées à être étouffées dans les herbes; il est indispensable de nettoyer ces plantations et l'on procède alors d'une manière analogue à celle qui a été indiquée plus haut pour les semis. Si, néanmoins, au lieu d'arracher les herbes à la main ou de les couper à la faucille, on les enlève à la houe, en donnant une légère culture au sol, on assure la reprise des sujets ainsi protégés, en activant leur végétation d'une façon tout à fait exceptionnelle.

La pioche sert encore à butter, au printemps, les tiges 
déchaussées par les gelées de l'hiver. Ce travail est précisément facile dans les terres abandonnées par l'agriculture, où l'insuffisance du tapis herbacé rend plus fréquent ce genre de dommage. D'ailleurs, l'emploi des pierres en couverture prévient le déchaussement et l'envahissement des herbes.

Le recépage des plants, au moment de la plantation, est une mutilation que rien ne justifie, lorsqu'on opère sur des sujets bien conformés et bien équilibrés; les brins de deux à trois ans sortant de pépinière ne doivent jamais avoir besoin d'être recépés. En effet, le plant déplacé subit une forte crise ; il n'a pas trop de toutes les ressources qu'il renferme pour l'aider à la supporter; à quoi bon, dès lors, le priver de la réserve alimentaire contenue dans sa tige? Pourtant, quand il faut utiliser des plants sauvages, mal équilibrés, pourvus d'un mauvais enracinement, il est souvent utile de les rabattre plus ou moins; de même encore, dans des stations très sèches où l'essentiel est de diminuer l'évaporation, on peut être conduit à restreindre la surface de l'appareil foliacé en sectionnant la tige ou les rameaux; mais ce ne sont là que des cas particuliers, et cette pratique ne doit pas être érigée en principe.

Au contraire, le recépage d'essences feuillues plantées depuis quelques années, dont la reprise est bien certaine, mais qui restent languissantes, qui ne poussent pas de tête, donne les meilleurs résultats. L'opération est trop souvent négligée et c'est ainsi que l'on voit des chênes ou des charmes bouder indéfiniment, se recoqueviller sur eux-mêmes, à la façon des végétaux gelés ou abroutis par le bétail : un coup de serpe ou de sécateur remplace ce mauvais buisson par des rejets vigoureux. Toutefois, il ne faut jamais recéper les sujets plantés sous un couvert, quelque léger soit-il.

Enfin, on évite tout recépage si, aux chênes et aux charmes, on a eu soin de mélanger des pins, des mélèzes, des épicéas ou des bouleaux, qui forcent leurs compagnons à s'élancer s'ils ne veulent être dominés.

La taille des essences feuillues pendant les années qui suivent la plantation n'est pas à recommander en principe; elle 
ne doit être pratiquée que très sobrement et avoir, pour chaque individu, un objectif précis : supprimer, par exemple, une fourche naissante, couper une branche horizontale qui s'allonge démesurément ou une autre qui se redresse pour former une double cime. Il ne faut pas poursuivre autre chose; c'est en vain qu'on demanderait à la taille de faire filer les sujets qui lui sont soumis; l'opération ralentit plutôt la croissance. A plus forte raison ne faut-il jamais élaguer les conifères, en dehors des cas suivants: quand, au moment où un jeune plant résineux prend son essor, il lui pousse deux ou trois flèches, on fait choix de la plus belle de ces pousses et on rabat les autres; de même, quand les pins noirs d'Autriche se bifurquent à 2 ou 3 mètres au-dessus du sol, il est bon d'enlever, de suite, une des deux flèches avec un échenilloir; plus tard, la section ferait une plaie de mauvaise nature; dans ces deux cas, au lieu de couper le rameau rez-tronc, il vaut mieux étêter la pousse de l'année, ou simplement casser le bourgeon terminal ; - on voit quelquefois la tige des jeunes sapins transplantés se couvrir, au niveau d'un ou de plusieurs entrenouds, de véritables branches gourmandes, qui montent parallèlement à l'axe principal en s'insinuant entre les branches plus âgées: les briser à la main ou les couper;-M. Mer (1) indique la possibilité d'activer l'allongement des jeunes sapins par l'amputation ou le raccourcissement des branches basses, qui s'étalent parfois d'une façon exagérée; nous n’avons jamais procédé à ces amputations complètes, mais très souvent nous avons coupé l'extrémité de ces longs rameaux s'étalant à quelques centimètres au-dessus du sol et qui semblent concentrer toute l'énergie vitale d'un sapincau: toujours nous avons obtenu d'excellents résultats; - enfin, quand un accident a rompu ou froissé la flèche d'un jeune résineux, il ne faut pas hésiter à faire une section en dessous la blessure, avec l'espoir de voir une nouvelle pousse se substituer à l'ancierine.

Certes, tous ces soins sont bien minuticux et leur applica-

(1) Mer, Moyen d'activer l'allongement des jeunes sapins. (Revue des eaux et forêts, 1890 . 
tion dans des reboisements de grande étendue serait d'autant plus onéreuse qu'on ne peut en confier l'exécution qu'à des ouvriers très soigneux. Si donc nous en avons parlé, c'est qu'ils peuvent intéresser le garde qui, au cours de ses tournées, trouve du plaisir à rendre meilleure la situation de quelques plants, ou le propriétaire qui ne craint pas d'utiliser ses loisirs en veillant sur ses épicéas comme un amateur taille ses espaliers. Mais, nous le répétons, dans des boisements importants, le plus simple sera de laisser agir la nature, et de faire tomber, dans les dégagements de semis et les éclaircies, les sujets défectueux.

Les dépressages sont moins urgents, sans doute, dans une plantation que dans un semis bien réussi, - du moins, si l'on a adopté entre les plants un espacement au moins égal à un mètre. Mais, quand le cas se présente, il ne faut pas hésiter à procéder à l'opération.

ARTICLE IV

\section{PROCÉDÉS SPÉGIAUX DE BOISEMENT}

Les boutures. - Les plançons. - Les marcottes.

Les boutures. - La multiplication par bouture ne concerne qu'un petit nombre d'essences indigènes appartenant toutes à la catégorie des bois tendres, notamment: les saules et les peupliers.

Comme boutures, on choisit des branches lisses, longues de 75 centimètres à 1 mètre, âgées de un à trois ans, dont on retranche les rameaux latéraux en ayant soin de ne pas endommager l'écorce; la section inférieure de la bouture est taillée en biseau, à quelques centimètres au-dessous d'un bourgeon. Les rameaux ainsi préparés sont enfoncés en terre jusqu'aux deux tiers de leur longueur, de telle façon qu'il reste en dehors trois ou quatre yeux bien formés. Plus le sol est sec, plus les boutures doivent être placées profondément, et c'est en leur donnant une direction oblique qu'on a le plus de chance de réussite. Les boutures reprennent d'autant mieux qu'elles sont mises en terre plus 
vite après avoir été coupées; cependant, comme, le plus souvent, elles doivent être préparées à l'avance pour être transportées au lieu d'emploi, il faut les préserver contre la sécheresse en les conservant dans l'eau, ou, mieux encore, en les couvrant de terre dans un lieu frais et exposé au nord, de façon à retarder autant que possible leur végétation; car, dès que les bourgeons évoluent, il est trop tard pour les mettre en place.

Quel que soit l'état d'ameublissement de la terre, on ne doit jamais enfoncer directement les boutures dans le sol; afin de ne pas détacher leur écorce, il faut les introduire dans un trou ouvert à l'avance soit avec un pic, soit avec une barre à mine; elles sont ensuite consolidées par un tassement convenable. Dans les terrains très exposés au déchaussement par la gelée, il est prudent de disposer les boutures très obliquement, presque horizontalement, pour qu'elles ne soient pas rejetées hors de terre.

Les plançons. - Les plançons ne sont autre chose que de fortes boutures de $1^{\mathrm{m}}, 50$ à 2 mètres de longueur et pouvant avoir jusqu'à 5 à 6 centimètres de diamètre. Ils sont préparés et mis en terre de la même façon que celles-ci et, quelle que soit leur grosseur, il ne faut jamais les frapper pour les faire entrer de force à la profondeur voulue. Les trous auront un calibre suffisant pour que le plançon puisse y glisser librement et, pour assurer la reprise, il est utile, après la pose du plançon, de remplir le vide avec de la terre meuble et de bonne qualité.

Le mode de repeuplement par bouture est de beaucoup le plus économique; il ne peut malheureusement être utilisé que dans les terrains frais et humides et convient surtout pour fixer les berges des torrents; dans ces conditions, les plançons, employés comme piquets de clayonnages, prennent racine et constituent des barrières vivantes des plus utiles pour la solidité et la durée des ouvrages.

Les marcottes. - Le marcottage des branches basses d'un arbre ou des brins les plus extérieurs d'une cépée permet de faire rapidement gagner du terrain à un centre de végétation existant. On peut encore, de la manière suivante, obtenir 
des sujets susceptibles de transplantation : 1l suffit de recouvrir le pied d'une jeune cépée d'une butte de terre de 15 à 25 centimètres de hauteur et dans laquelle les jeunes rejets en croissance prennent racine au-dessus de la souche; deux ou trois années après, en rompant la butte, on trouvera autant de tiges enracinées qu'il y avait de rejets; détachés au niveau de la souche, ils pourront être mis en terre à la façon des brins de semence. Si l'on prend la précaution de faire une légère entaille au pied des rejets dans la région enterrée, on facilitera l'évolution des racines.

On sait d'ailleurs que la marcotte fournit un très petit nombre de sujets relativement à celui des pieds étalons. En forêt, elle n'est employée que pour remplacer les souches usées dans les taillis furetés; elle est au contraire très en faveur dans les travaux de boisement pour diminuer, dans la mesure du possible, les frais de plantation, dont le prix est d'autant plus élevé que les conditions de reprise sont plus difficiles.

ARTICLE $v$

\section{LES ESSENCES DE BOISEMENT}

1. Généralités.

Choix des essences: - Caractères de la forêt artificielle.

Choix des essences. - Quelles essences employer dans les boisements? La question est des plus complexes, tant est grande la variété des circonstances de temps et de lieux, la diversité des services demandés à l'entreprise.

Aussi, quel que soit le but poursuivi : forêt de rendement ou de protection, boisement temporaire ou définitif, avant de rien décider, faut-il se rendre compte des faits.

Caractères de la forêt artificielle. - Quiconque veut boiser doit tout d'abord se mettre moralement au point : c'està-dire dépouiller le "vieil homme ", dont l'esprit est hanté par l'évolution rapide des cultures usuelles : semer aujourd'hui, récolter demain. Car la forêt est un au-delà, dont l'avenir 
lointain correspond à une période d'enfantement d'autant plus longue que les surfaces à meltre en valeur sont ellesmêmes plus pauvres ou dans un état de dégradation plus avancé. En toute situation, il faut compter. sur le temps, qui travaille gratuitement, afin d'épargner l'argent, qui fonctionne à intérêts composés.

D'autre part, quels que soient les régions et les procédés employés, on constate que tout peuplement créé de main d'homme ne conserve pas la composition simple et l'aspect uniforme qu'il doit à son origine artificielle. Bientôt, la nature intervient pour reprendre ses droits : un véritable travail de reconstitution s'opère (1), la flore et la faune se modifient en même temps que le sol prend la consistance de terreà bois.

On voit ainsi les massifs se modifier lentement pour se rapprocher sans cesse des types spontanés; alors seulement que cette forme dernière sera bien acquise, on pourra considérer le sol comme rendu à la production forestière; car, on ne saurait trop le répéter, la forêt naturelle est seule durable: adaptée au sol et au climat, elle porte en elle les remèdes contre les maux qui peuvent l'atteindre; elle s'est installée en dépit de ses ennemis et sait au besoin les combattre et les vaincre. Qu'il s'agisse de boisements, de brousaillements, d'enherbements, la loi reste la même; elle domine toutes les situations : méconnaitre ses effets serait tenter une aventure.

Si cette métamorphose contrarie parfois le but économique poursuivi, du moins a-t-elle, au point de vue forestier, un effet doublement heureux :

$1^{\circ}$ elle remplace le peuplement pur et uniforme, constitué le plus souvent à l'aide d'espèces plus ou moins dépaysées, par des individus dès longtemps accoutumés à la station, c'est-àdire plus résistants à tous égards ;

$2^{\circ}$ elle transforme lentement un sol quelconque en cette chose toute spéciale et complexe qui constitue la terre à bois.

Ajoutons enfin que, si l'on fait une culture ligneuse temporaire et sans s'inquiéter de l'avenir, on peut, à la rigueur, sacrifier quelques principes, tandis que la création d'une forêt perma-

(1) P. Fliche, Un reboisement (Annales de la science agronomique française et étrangère 1888). 
nente est toujours subordonnée à la stricte observation des règles culturales: faire une forêt solide, se régénérant par la voie naturelle, et cela avec le moins de frais possible, tel est le but à atteindre.

Ceci étant admis, nous allons passer en revue les principales essences indigènes, en indiquant sommairement le parti qu'on peut tirer de chacune d'elles, tant à l'état pur qu'à l'état mélangé; nous renvoyons d'ailleurs au Chapitre II pour tout ce qui concerne leur tempérament et leurs allures forestières. Puis, nous donnerons quelques détails sur les espèces exotiques introduites jusqu'à ce jour, en appréciant les services qu'elles sont appelées à rendre.

\section{LES ESSENCES INDIGÈNES.}

Essences résineuses. - Essences feuillues. - Les mélanges.

Essences résineuses. - Les essences résineuses se recommandent tout d'abord par leur frugalité. Elles prennent vite possession du terrain et fournissent à courte échéance des produits marchands : échalas, perches à houblon, étais de mine, poteaux télégraphiques, bois de raperie ou de boulangerie. Les espèces de lumière : pin sylvestre, pin maritime, pin d'Alep, mélèze, épicéa, - ajoutons même le pin laricio noir, originaire d'Autriche, et qui se comporte chez nous à la façon des espèces spontanées, - sont toutes à notre disposition. Nous pouvons choisir. Souvent même, alors que les ressources locales seront insuffisantes, nous nous adresserons à des formes étrangères à la contrée; mais l'expérience de tous les jours tend à restreindre le nombre de ces auxiliaires. Sachant que le dernier mot reste à la nature pour arrêter la situation définitive, on ne fait usage que des espèces les plus communes, les plus rustiques, de celles enfin, qui, douées d'une faculté d'accommodation suffisante, permettent de réaliser au plus tôt, et au meilleur marché, le premier état boisé que l'on cherche, C'est ainsi, que, sur tout le territoire de la France, le pin sylvestre s'installe facilement dans les sols arénacés de la

Boppe et Jowrit. 
plaine et de la basse montagne, - le pin noir dans les sols calcaires, secs, peu profonds de ces mêmes stations, - le mélêze dans les lieux bien éclairés et bien aérés, - l'épicéa en toutes contrées froides et fraîches, - le pin maritime sur les sables siliceux du littoral, - le pin d'Alep sur les rochers calcaires de la Provence, - le pin de montagne dans les tourbières alpestres, etc...

Ajoutons à ces grandes lignes quelques remarques d'ordre plus spécial :

Sous le climat parisien, le pin sylvestre pousse encore assez vigoureusement dans les argiles, pourvu qu'elles ne soient pas trop compactes; et, dans les terrains calcaires eux-mêmes, sa croissance, pendant la jeunesse, est plus rapide que celle du pin noir; cependant, nous lui préférons ce dernier, dont le couvert plus épais et les détritus plus abondants améliorent rapidement la fertilité du sol.

Épicéa et mélèze acceptent toutes les terres, bien qu'ils préfèrent naturellement les meilleures. On doit, néanmoins, donner la plus grande place à l'épicéa qui est beaucoup plus facile à manier : sans doute, le bois du mélèze se recommande entre tous, même dans les stations basses; mais, employée en masse, sous un climat trop chaud pour elle, cette essence est sujette à des maladies qui rendent sa vie précaire.

Dans les régions méridionales, concurremment avec le pin sylvestre et le pin noir, se présentent le pin maritime et le pin d'Alep. Si ces deux essences acceptent des sols ingrats, elles sont sensibles au froid; il est donc toujours dangereux d'en tenter l'introduction au nord de leur aire d'habitation. Les dégâts de l'hiver 1879-80 ont été une dure leçon pour les propriétaires des pignadars de la Sologne et de la Normandie.

Sous les climats franchement montagneux, dont on connaît le caractère, on utilisera, suivant les cas : le mélèze, l'épicéa, le pin de montagne et le pin cembro.

Essences feuillues. - En général, la mauvaise qualité des terres incultes et sauvages que l'on rend à la forêt, aussi bien que le plein découvert ou l'on opère, limitent à un petit nombre d'espèces le choix à faire parmi les feuillus, Le bouleau, l'aune et le saule marceau crnviendraient à peu près 
sculs pour constituer la masse d'un boisement; encore, ce dernier ne pourrait-il être utilisé, concurremment avec de nombreux arbustes et arbrisseaux, que pour servir de premier abri dans les cas difficiles; - l'aune s'emploie dans les stations fraîches et même mouilleuses; - le bouleau un peu partout, à cause de la facilité de sa reprise el du bon marché des plants; mais ce n'est pas une raison pour en trop généraliser l'emploi, car les résineux en même situation prennent plus de valeur que lui.

En sol frais et profond, le choix est moins limité; car toutes les essences de lumière qui prospèrent dans les forêts voisines peuvent être employées. Parmi celles-ci, le chêne se recommande entre toutes, non seulement par les qualités de son bois, mais encore par le bon marché avec lequel il est facile de l'introduire par voie de semis dans les terres qui le comportent et plus particulièrement dans les champs abandonnés par l'agriculture. Les autres ne peuvent guère être employées sur de grandes surfaces qu'en forme de mélange et nous allons voir dans quelles conditions.

Les mélanges. - Le mélange des essences dont nous avons constaté les bons offices dans les vielles forêts en état de rendement (page 113), retrouve son utilité, pour des motifs semblables, dans la forêt naissante (1).

Bien que, - nous venons de le voir, - la composition initiale de la forêt artificielle se modifie sans cesse, il est certain qu'en jetant, dès les premiers jours, sur un sol nu, des éléments variés, nous donnons à la nature un plus grand choix de matériaux, qu'elle façonnera à son gré et, le plus souvent, au mieux de nos intérêts (fig. 86).

A un fond d'une espèce donnée, choisie comme la plus avantageuse économiquement, nous en associerons d'autres; nous adjoindrons, par exemple, des pins et des mélèzes à une majorité d'épicéas ou inversement. Mais il sera surtout excellent de mélanger feuillus et résineux. Les essences de lumière, les seules dont il ait été question jusqu’à présent, nous oflrent

(1) Mélanges naturels et artificiels, par R. B. - Traduit du Journal suisse d'Économie forestière, par P. Poirot, élìve de l'ḱcole forestière. Revue des Eanx et Foréts, mars 1900). 
déjà les éléments d'un grand nombre de formules: rien n'empêche, si le sol est profond, de jeter quelques glands dans une plantation résineuse, de disséminer des charmes dans les parties fraîches, des bouleaux dans les interlignes des sols les plus pauvres, etc.; toutefois, en ce qui concerne le bouleau, on doit se méfier des dommages qu'il cause aux résineux, quand, par les grands vents, ses rameaux grêles viennent fouetter les cimes de leurs voisins et en briser les bourgeons.

Puis, dans la solution du problème des mélanges, les essences disséminées et les essences d'ombre sont appelées, à leur tour, à nous venir en aide. Dans l'immensité des surfaces à reboiser, il y a place pour tout le monde : c'est précisément le talent du metteur en ceuvre d'y loger chacun chez soi.

Se rencontre-t-il, par exemple, quelques poches de terre, plus profondes et plus fertiles que l'ensemble, les frênes, les ormes sont tout désignés pour les occuper. Dans quelles proportions? c'est affaire de fantaisie et de convenance de la part du propriétaire; mais il conviendra rarement de dépasser le chiffre du dixième. Partout, - et dans la même mesure, des places seront réservées, çà et là, aux grands érables, principalement au sycomore.

Un rôle différent est dévolu aux essences d'ombre. On sait qu'elles ont, en général, le couvert épais; on connaît enfin leur tendance envahissante et leur faculté de résister sous le couvert : toutes qualités dont on doit tirer parti au profit de la forêt à venir. Leur introduction est chose peu malaisée quand on y met de la patience, vertu dont il faut toujours être armé dans une entreprise d'aussi longue haleine que la création d'une forêt. Ainsi, dès le début, et partout où le climat le comporte, on profite de tous les abris qui se rencontrent sur les surfaces à travailler : touffes de genévriers, buissons d'épines, haies, rochers, murs, versants rapides sont une occasion de planter, à leur ombre, au Nord, quelques pieds de hêtre ou de sapin ; les grosses pierres, les quartiers rocheux sont surtout précieux, car ils entretiennent un ameublissement et une humidité dont profitent les racines des plantes les plus voisines; si, comme cela se présente dans certaines régions, notamment en Franche-Comté, les friches sont entrecoupées 
par ces longues bandes de buissons appelées des lisiéres, on profitera de leur abri du côté Nord pour faire une ou deux

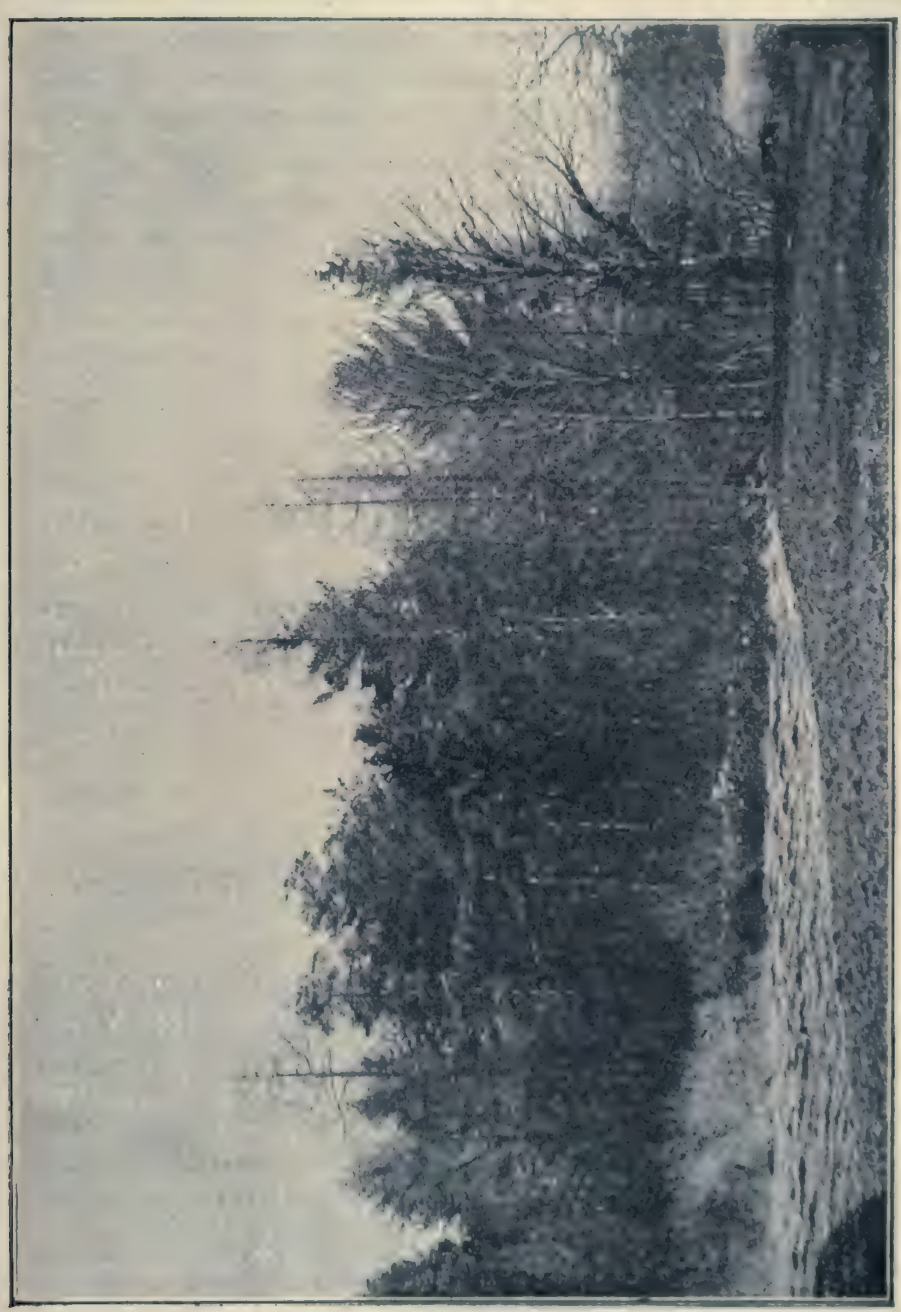

อ

lignes d'espèces d'ombre, la plus voisine de l'abri, en sapin, la seconde, en hêtre; ailleurs, ce sera autre chose, le principe 
restant le même. Plus tard, quand le moment est venu de combler les vides, on remplace les manquants de la première heure par des sujets d'ombre. Plus tard encore, une fois le massif constitué à l'état de perchis, on peut, sous son couvert, créer un sous-bois en essences d'ombre. Tout cela est facile, il suffit d'y penser et de vouloir; car, les jeunes plants de hêtre et de sapin élevés en pépinière et bien constitués sont plus accommodants qu'on le suppose généralement; et, pour peu que l'attitude et le climat s'y prêtent, ils ne sont pas réfractaires aux terrains nus; il en est ainsi dans toute la zone parisienne à partir de 300 mètres d'altitude, dès que la moindre broussaille donne un peu d'abri; à plus forte raison en montagne, dans les genêts et les fougères. Sous les climats plus méridionaux, il faut renoncer à ces deux espèces et le châtaignier peut avantageusement les remplacer; en sol sableux, il croît à merveille sous l'ombrage des pins, dont il abrite le pied, en les préservant des invasions d'insectes.

A chaque pays sa forêt. Cherchez vos auxiliaires et vous les trouverez; et si, ce dont nous doutons fort, la flore indigène ne vous donne pas pleine satisfaction, la flore exotique sera pour vous une suprême ressource.

\section{LES ESSENCES EXOTIQUES.}

Généralités. - Le choix à faire. - Les essences les plus connues. Conclusions.

Gẻnéralitẻs. - La flore forestière de France peut certes suffire à tous les besoins, sans qu'il soit indispensable d'avoir recours aux espèces exotiques. Telle était, du moins, l'opinion de notre savant maître M. Mathieu (1).

Avec de semblables richesses en mains, dit-il, il n'est d'autre difficulté que celle de choisir, et le choix peut-être fait sûrement, sans rien laisser au hasard, puisqu'il s'agit de végétaux spontanés, bien connus, dont les exigences se traduisent par les faits de l'association la plus simple : sol, exposition, altitude.

Négliger d'aussi vieux amis, dont les services sont certains, pour des nconnus, des végétaux exotiques, serait peu sage assurément, et per-

(1) A. Mathieu, Le reboisement et le regazonnement des Alpes, p. 25. Paris, Imprimerie Nationale, 1875. 
sonne n'y songe, sans doute. Il peut n'ètre pas hors de propos, néanmoins, d'examiner en quelques mots ce que l'on peut attendre des essais de naturalisation et d'acclimatation en matière forestière. Ce sera la justification de la réserve avec laquelle cette voie doit être suivie dans les travaux de reboisement.

Pour qu'un végétal mérite d'être placé au rang des essences forestières, il faut qu'il soit complètement naturalisé, c'est-à-dire qu'il présente " les caractères des plantes spontanées indigènes, croissant et se multipliant sans le secours de l'homme, ayant traversé des séries d'années pendant lesquelles le climat a offert des circonstances exceptionnelles. " (De Candolle, Géog. bot., p. 608). Mais, ce n'est pas tout, il doit encore pouvoir atteindre les dimensions, les qualités qui ont engagé à en tenter l'introduction, en un mot, conserver dans sa nouvelle patrie la marche de végétation, la longévité qui lui étaient propres dans la contrée dont il est originaire; enfin, il est indispensable qu'il soit social, propre à croître en massif, aptitude refusée à beaucoup d'arbres, qui recherchent l'isolement et l'état de dissémination. C'est seulement alors que le végétal aura subi ces longues épreuves, réalisé toutes ces conditions, que le problème sera résolu, qu'on sera fondé à en proclamer la conquête.

Alors que M. Mathieu écrivait ces lignes remarquables, il était appelé à donner son avis, avec toute l'autorité attachée à son nom, sur une œuvre gigantesque d'un caractère social, où il n'y avait aucune faute à commettre. La sagesse, comme la raison, imposaient à celui aux lumières de qui l'État avait recours, de signaler tous les dangers, de prévenir toutes les aventures.

Nous partageons les idées de notre Maître vénéré, dans ce qu'elles ont de local et de particulier; mais l'étude des essences exotiques a fait des progrès depuis vingt-cinq ans, et l'emploi judicieux de certaines d'entre elles, dans les situations moins graves qu'un reboisement obligatoire, peut rendre des services, ne fût-ce que pour multiplier dans tous les milieux la série des expériences en dehors desquelles on n'atteindrait jamais la certitude.

La question d'ailleurs a déjà donné lieu à bien des controverses.

"Les opinions moyennes, fait remarquer M. Hickel (1), sont " rares : les uns repoussent, presque avec horreur, toute ad" dition à notre flore ligneuse indigène; les autres, moins

(1) Hickel, Un essai d'acclimatation d'essences exotiques à Veinheim. Orléans, G. Michau et Cle. 
" nombreux ne rêvent qu'exotiques, et peupleraient volon" tiers nos forêts d'essences aussi bizarrement choisies que " variées ». Il y a donc une mesure à garder.

La principale objection que l'on puisse faire à leur emploi est celle-ci : moins bien adaptées à notre climat que les espèces indigènes, elles seront éliminées par ces dernières. Mais la chose n'est pas certaine, et l'on manque d'observations pour décider de ce qui se passera.

En effet, comme l'a établi M. le Professeur Fliche (1), les données paléontologiques, de plus en plus abondantes, attestent d'une façon indubitable que beaucoup des espèces que l'on cherche à réinstaller dans notre pays y étaient spontanées à une époque parfois peu lointaine de l'histoire géologique de notre globe.

Citons, au hasard; les genres Salisburya et Taxodium; s'ils ont disparu, c'est que les milieux se sont modifiés dans un sens qui leur fut défavorable. Mais, à côtéde ces formes qu'on pourrait appeler archaïques, dont certaines n'existent même plus nulle part à l'état sauvage, il y a des espèces appartenant à des genres modernes; elles ont même, le plus souvent, des similaires dans notre pays, similaires qui, vivant sous un autre climat, n'ont pas évolué d'une façon absolument identique. Qui sait, si, parmi elles, quelques unes ne se trouveraient pas, par hasard, appropriées à notre région? qui sait même si, ayant vécu sous un ciel plus rude, elles ne seraient pas plus résistantes aux intempéries? Il est certain, par exemple, que le Chêne rouge d'Amérique s'est mieux comporté en France que notre rouvre pendant l'hiver néfaste de 1879-80. D'ailleurs, même en admettant qu'abandonnées à leurs seuls moyens de défense elles risquent d'être éliminées de nos peuplements, n'arriverait-on pas à les y maintenir à l'aide de sacrifices moindres que ceux qu'entraîne la protection du rouvre ou du pédonculé contre l'envahissement du hêtre?

Plus grave est la crainte de voir une espèce dépaysée disparaître chez nous, victime d'une maladie jusqu'alors inconnue ou peu dangereuse 'dans son pays d'origine.

(1) Fliche, Communication faite à la réunion biologique de Nancy, décembre, 1897. 
Toutes ces questions, dit M. l'Inspecteur-adjoint Pardé (1), appellent l'expérience. $\mathrm{Ni}$ nos lois successoriales, ni nos mœurs françaises ne favorisent la transmission héréditaire de grands domaines pendant plusieurs générations, et l'veuvre d'expérimentation forestière chez les particuliers sera forcément rare; les personnes mêmes qui pensent, en principe, que l'initiative privée doit s'exercer partout où la logique ne lui impose pas des limites, reconnaitront que, dans cet ordre d'idées, l'État, en France, peut et doit faire beaucoup plus que les particuliers. M. Pardé conclut en émettant le vœu que les résultats obtenus, aussi bien les mauvais que les bons, soient portés à la connaissance du public et que les Sociétés forestières de tous les pays veuillent bien leur réserver une place de choix dans leurs bulletins.

Nous nous rallions pleinement au vœu de M. Pardé; mais, en attendant, nous pensons qu'il sera prudent, d'ici longtemps encore, de confiner les essences exotiques dans un rôle accessoire, accidentel, en les employant toujours sous forme de mélanges. Dans ces limites, voyons les ressources qu'elles nous offrent actuellement, et quelle peut être leur utilité.

Le choix à faire. - Dès que l'on compare la flore ligneuse étrangère à celle de la France, on constate combien cette dernière, malgré sa richesse apparente, est relativement pauvre, On sait, en effet, que les végétaux, comme les animaux et les hommes, ont leurs mouvements de migration, leurs marches d'invasion. Dans notre vieux monde la loi des transports a toujours tracé les routes d'exode de l'Est à l'Ouest, d'Asie en Europe. Mais, au fur et à mesure qu'une famille, qu'un genre s'étendait dans ce sens, il laissait en route des retardataires : les membres de cette famille, de ce genre, sont donc moins nombreux à la dernière étape qu'à la première : la France est précisément cette dernière étape, dont le point d'arrêt est fixé par le littoral de l'Atlantique. Pour reconsti. tuer la liste des retardataires, il nous faudrait remonter le courant vers ses sources, - vers l'Asie centrale, - ou nous

(1) Pardé, Communication au Congrès international de sylviculture, juin 1900. - Rapport de M. Maurice de Vilmorin. 
trouverions cette flore curieuse que les découvertes récentes des explorateurs et les remarquables travaux de M. Franchet commençaient à nous faire connaître. Ce serait, sans doute, la mine à exploiter pour en faire bénéficier nos forêts.

Dans la pratique et jusqu'à ces temps derniers, on s'est adressé surtout au Continent américain plus accessible dans toutes ses parties. Sa flore forestière, mieux étudiée, nous présente, rien que pour l'Amérique du Nord, dit M. Hickel, plus de 30 espèces de pins, tandis que l'Europe n'en compte que $8 ;-50$ espèces de chênes habitant les régions tempérées, tandis que nous n'en avons pas plus de 8... Quels mobiles déterminants nous feront-ils adopter telle de ces espèces plutôt que telle autre?

Après la question de rusticité qui domine toutes les situations, se présente celle de l'originalité. L'espèce adoptée doit, en effet, posséder des qualités spéciales qui ne se retrouvent dans aucune des espèces indigènes. A quoi bon cultiver le hêtre ferrugineux d'Amérique, s'il faut la loupe du botaniste pour distinguer les caractères scientifiques qui le séparent du hêtre commun? A quoi bon, si ce n'est pour le snobisme du catalogue, cultiver le Sapin de Fraser, le Sapin baumier ou la Sapinette blanche, qui ne sont que des moules réduits du sapin pectiné ou de l'épicéa, dont ils n'ont ni les dimensions, ni les qualités, même dans leur pays d'origine?

Au contraire, on aurait tout profit à multiplier : le Hinoki des Japonais, dont le bois satiné fait de si belle menuiserie, Abies grandis, Abies nobilis, Abies cilicica, Pseudotsuga Douglasii, qui atteignent des dimensions inconnues chez nos sapins, - Abies Nordmanniana, Fraxinus alba, que leur végétation tardive meitent à l'abri des gelées printanières, et d'autres encore... Car nous ne pouvons passer en revue toutes les formes actuellement à l'étude. Nous recommandons aux sylviculteurs que la question intéresse, la lecture des ouvrages et articles publiés à ce sujet (1).

(1) Michaux, Historre des arbres forestiers de l'Amérique septentrionale. Paris, 1810-1812; - Sargent, The silva of North America. Boston et New-York, 1893 et suiv.; - J. Houba, Les chênes de l'Amérique septentrionale en Belgique. Hasselt, 1887; - De Kirwan, Les 
Les espèces les plus connues. - 'T'outefois, à ceux qui seraient effrayés par le nombre et l'importance de ces documents, nous pouvons, à titre de simple indication, citer les espèces les mieux connues, celles qui sont d'une utilisation assez ancienne et assez courante pour qu'on puisse, sans grand danger, les admettre dans nos cultures.

Les plus rustiques sont, parmi les feuillus:

Le Chêne rouge d'Amérique (Quercus rubra), très employé en Belgique et dans bon nombre de forêts françaises, où il se régénère naturellement et montre même des tendances envahissantes. Il se recommande par sa croissance plus rapide que celle du rouvre, sa forme plus régulière, sa plus grande résistance au froid, sa fructification plus fréquente et plus abondante, son couvert plus épais ; on dit que, dans son pays d'origine, son bois grossier est beaucoup moins estimé que celui de nos chênes. Chez nous, dans la plaine des Vosges du moins, il ne semble pas inférieur à ces derniers ; cela tient, sans doute, à ce que, jouissant, en France, d'un temps de végétation plus long que dans son pays d'origine, il fabrique plus de bois d'été et, par suite, acquiert plus de dureté et de densité. Il a contribué, dans une large mesure, au boisement du sol ingrat des Campines belges. Il a toutefois les allures d'une plante calcifuge et nous ne le conseillons pas en dehors des terrains siliceux.

Dans la Campine encore, on utilise avec succès le Cerisier tardif (Prunus serotina), espèce américaine très voisine de notre Cerisier à grappes, très peu exigeante au point

forêts du Japon. Bruxelles, 1887 ; - L. Ussèle, A travers le Japon, Paris, 1891 ; E. Dupont, Les essences forestières du Japon. Paris, BergerLevrault. 1880. - Meyr, Monographie der Abietineen des japanischen Reiches. Munich, 1890; - Hickel, Les jardins hotaniques des bords du Rhin. Feuille des jeunes naturalistes, janvier 1900 ; - Dr. Schwappach, Denkschrift betreffend die Ergebnisse der in den Jahren 1881-1890 in den Preussischen Staatsforsten ausgeführten Anbauversuche mit fremdlän. dischen Holzarten, Zeitschrift für Forst und Sagdwesen, 1891; - Catalogue des végétaux ligneux indigènes et exotiques, existant sur ledomaine des Barres. Paris, imprimerie Nationale, 1878; - De Kirwan, Les conifères indigènes et exotiques. Paris, 1867-1868;-Cannon, Le propriétaire planteur. Orléans, 1887. - De nombreux articles dans le Bulletin de la Société centrale forestière de Belgique et dans la Revue des Eaux el Forêts, etc... 
de vue de la fertilité du sol, et dont les dimensions sont suffisantes pour fournir du bois d'ébénisterie. Ce cerisier ne paraît pas craindre le calcaire et, comme sa transplantation est des plus faciles, il semblerait tout désigné pour accompagner le pin Noir dans toutes les terres pauvres des formations oolithiques. Malheureusement, les plants en sont assez rares en France, où les pépiniéristes le confondent avec le Cerisier de Virginie, de forme bien différente et de qualité beaucoup moindre; il est cependant facile de les distinguer : le cerisier tardif a les feuilles aussi vertes sur la face inférieure que sur la face supérieure, le calice de la fleur persiste même après la chute des fruits (1).

Le robinier faux acacia que tout le monde connaît et que nous aurions pu comprendre dans la monographie de nos essences, tant est grande son expansion sur tous les points de notre territoire. Rappelons seulement que c'est une espèce assez exigeante et qui n'atteint toutes ses qualités que dans les bons sols.

On en pourrait dire autant des platanes, dont le bois analogue à celui du hêtre, a sur celui-ci l'avantage de présenter, quelqu'en soit le débit, de très belles maillures qui justifient son emploi en menuiserie apparente.

Parmi les Conifères, on peut admettre, aux mêmes titres que les précédents :

Le pin du Lord ou pin Weymouth (Pinus strobus), d'un usage courant dans les reboisements en Allemagne. Il y est aussi apprécié qu'on l'estime peu en France, où sa mauvaise réputation tient, sans doute, à ce fait que, grâce à sa croissance très rapide, on le coupe très jeune, alors que son bois est encore à l'état d'aubier dans toute son épaisseur. Mais, si on le laisse vieillir, il fabrique un bois de cœur de teinte rosée et dont les qualités sont au moins égales sinon supérieures, à celles des bois d'épicéa ou de sapin dont les anneaux ligneux auraient même largeur que les siens; les voliges, même débitées dans l'aubier pur, peuvent être utilement employées, à l'égal de celles de peuplier, pour les emballages,

(1) Le cerisier sauvage d'Amérique, à fruits noirs (Cerasus serotina). Notice de M. Berger, Annales des travaux publics de Belgique, t. Ier. 
et, à l'usage, elles ne s'arrachent pas en esquilles comme celles du sapin. Le pin Weymouth accepte tousles sols, même ceux qui sont mouilleux; il est donc tout indiqué, pour mettre en valeur les terrains de cette nature.

Le Sapin de Douglas (Pseudotsuga Douglasii), couvre déjà de grandes surfaces en Allemagne et en Écosse. Il forme des massifs d'une densité extrême et dont l'obscurité dépasse celle de nos sous bois les plus noirs; il atteint des dimensions superbes et son bois de cœur, rouge et d'excellente qualité, se prête aux meilleurs emplois. Il est de reprise facile, ne boude pas comme l'épicea, mais s'élance rapidement à la façon du mélèze. Sans être exclusif, il préfère les sols siliceux. Par contre, son aubier est toujours très épais, comme chez les laricios, et un ennemi déjà très connu, du genre Botrytis, engage à ne pas l'utiliser à l'état pur.

Bien que moins étudiés, en forêt surtout, semblent recommandables encore :

le noyer noir (Juglans nigra), qui résiste mieux au froid que notre noyer commun ( $J$, regia) et dont le bois est très recherché; de même les Carya alba, C. porcina, C. amara;

le tulipier (Liriodendron tulipifera), dont le bois très léger, serré et tendre répond à des besoins spéciaux; il est recherché par l'industrie du tranchage ;

le cédrèle, Cedrela sinensis, rustique, croissance assez rapide, bois dense, élastique, résistant;

les planères, Zelkowa crenata, rustique, à croissance rapide, dont le bois vaut au moins celui de l'orme, et Zelhoura acuminata, également rustique, à bois très souple se rapprochant de celui du frêne

Dans les carrefours, sur les points que l'on voudrait garnir de plantes ornementales et un peu partout, ¿̀ titıe d'expérience, on peut placer avec des chances sérieuses de réussite les belles espèces suivantes:

Un sapin : Abies concolor; - deux cupressinées américaines: le cyprès de Lawson (Cupressus Lawsoniana) et le Thuia géant (Thuia Lobbii) et deux japonaises : le Hinoki (Chamœeyparis obtusa), très rustique sous nos climats, dont nous avons déjà parlé, et le beau Thuiopsis dolabrala. 
Nous serions moins affirmatifs pour le libocèdre (Libocedrus decurrens) à végétation très vigoureuse, mais qui souffre des hivers rigoureux sous le climat de Paris.

Dans des circonstances particulières, on peut aussi utiliser : - si le terrain est mouilleux, submergé même, à côté du Pin Weymouth: Thuiopsis borealis et Chamocyparis Nutkaensis - sur les landes maritimes, les chênes américains: Q.palustris, Q. Phellos ou encore Cupressus Lambertiana, et, si le climat est trop rude pour le pin maritime, le pin raide, Pinus rigida, qui n'est bon qu'à fixer les surfaces les plus rebelles à la végétation. - Dans la basse montagne méridionale, au-dessous de la station du sapin pectiné, on peut essayer le cédre de l'Atlas (Cedrus atiantica) comme l'Administration forestière l'a tenté sur les versants du Mont Ventoux et le sapin algérien, Abies baborensis vel numidica, qu'il ne faut pas confondre avec Abies pinsapo originaire d'Espagne. - Enfin si, dans les terrains siliceux, on veut installer quelque refuge pour le gibier, on pourra créer des remises avec le petit chêne de Banister, véritable buisson, dont la fructification est des plus abondantes et qui fournit des glands recherchés par les animaux de chasse.

A titre d'expérience ou d'essai, on peut encore jeter, çà et là, quelques pieds des érables américains ou japonais, - de bouleau merisier, bonne espèce sur laquelle nous appelons spécialement l'attention et que recommandent les qualités spéciales de son bois, - de mélèze du Japon (Larix leptolepis), - de divers pins et épicéas : Picea Alcockiana, Picea orientalis, Picea alba, Picea Menziesii, Pinus ponderosa, Pinus Jeffreyi. Ces quatre dernières espèces sont d'origine américaine; habituées au climat excessif du versant de l'Atlantique, elles ont plus de chance de réussir chez nous que les espèces californiennes qui, à l'instar des formes japonaises, jouissent d'un climat marin d'une douceur exceptionnelle, dont on ne trouve l'analogue en France que sur les confins du littoral réchauffé par le Gulf-Stream.

Mais n'oublions jamais qu'au-delà de cette zone, le climat de nos plaines, d'allures trop continentales, est souvent meurtrier pour les plantes à feuilles persistantes. 
Conclusions. - Ce court résumé n'a d'autre but que de bien faire comprendre au lecteur l'importance que nous attachons à l'étude des essences exotiques, en l'engageant à recourir aux ouvrages spéciaux; de bien lui faire voir que nous admettons toutes les tentatives, toutes les expériences d'abord en petit - en matière de boisement; que, loin d'adopter la formule des feuillus quand même, nous sommes partisans de l'introduction raisonnée des résineux dans certaines forêts où tant de vides, tant de clairières s'éternisent dans leur stérilité première, quand depuis longtemps le frugal conifère les aurait comblés et utilisés.

En un mot, mettre en œuvre les facultés du sol pour obtenir sur chaque unité de surface le maximum de rendement en bois, telle est la limite dans laquelle nous comprenons la culture forestière intensive.

ARTICLE VI.

\section{LA MISE EN VALEUR PAR LE BOISEMENT}

1. GÉNÉRALITÉs.

Les boisements facultatifs. - Les boisements obligatoires. Règle commune à tous deux.

Les boisements facultatifs. - Le boisement est facultatif quand il a pour but de satisfaire d'une façon directe et immédiate à un intérêt privé; l'intérêt public, lorsqu'il existe, ne passe qu'en seconde ligne.

Au propriètaire du fonds : Département, Commune, Établissement public ou Particulier, appartient exclusivement l'initiative de l'opération. L'État peut encourager ce propriétaire, l'aider, le subventionner même (1); mais nul ne peut exercer contre lui aucun moyen de coercition.

Pareille opération doit être fructueuse et le problème financier se pose dans toute sa rigueur (2). Les données pour le

(1) Lois du 21 juillet 1860 et du 8 juin 1864 .

(2) Arthur Noël, Essai sur les repeuplements artificiels, chap. xu et xIII. Paris, Berger-Levrault et Clo, 1882. 
résoudre présentent un certain degré d'incertitude, en ce sens que, si l'on peut se rendre un compte assez exact de la valeur du fonds, des frais occasionnés par le boisement et de la durée probable de l'engagement du capital avant d'en tirer profit, du moins ignore-t-on quelle sera la valeur de la marchandise au terme de cette échéance. Néanmoins, on est à peu près certain de faire une entreprise avantageuse chaque fois que, s'adressant à des terres d'une faible valeur vénale, on procède avec une connaissance suffisante des faits pour réussir sans engager dans l'opération matérielle du boisement un capital supérieur à 100 francs par hectare, y compris l'achat des graines ou des plants.

Les boisements facultatifs se rattachent à l'une des catégories suivantes :

a. les améliorations en forêt;

b. la mise en valeur des terres arables abandonnées par l'agriculture;

c. celle des terres vagues et incultes en pays de plaines et de coteaux;

d. celle des terres stables en montagne.

Les boisements obligatoires. - Chaque fois que l'intérêt public commande la constitution de l'état boisé comme unique moyen de défense contre l'action destructive des éléments, le boisement est obliqatoire.

Il en est ainsi principalement lorsqu'il s'agit :

a. de la restauration des montagnes;

b. de la fixation des dunes.

En semblables conditions. l'État, investi par la loi des pouvoirs nécessaires (1), a la mission de poursuivre l'entreprise pour la terminer avec succès.

Règle commune à tous deux. - Dés qu'un terrain est destiné à être boisé, il importe, avant toutes choses, d'interdire l'introduction du gros et du menu bétail de la façon la

(1) Loi du 28 juillet 1860 sur le reboisement des montagnes; - Loi du 8 juin 1864 sur le gazonnement des montagnes; - Décret du 10 novembre 1864 pour l'exécution de ces deux lois; - Loi du 4 arril 1882, relative à la restauration et à la conservation des terrains en montagne ; - Décret du 11 juillet 1882 pour l'exćcution de cette lní 
plus scrupuleuse. Il y a incompatibilité absolue entre la jouissance pastorale et la forêt naissante.

\section{LES BOISEMENTS FACULTATIFS.}

a. Les améliorations en forêt : considérations générales. - Applications dans les futaies; - dans les taillis.

Considérations générales. - Bien que la régénération naturelle soit la base de tout traitement rationnel des forêts, il faut lui venir en aide dans bien des cas. En effet, quelle que soit la méthode appliquée, des accidents de force majeure, des situations particulièrement difficiles, des abus de jouissance, des opérations mal raisonnées peuvent dégrader les peuplements et en faire disparaître les bonnes essences : ce qui conduit à les reconstituer par des procédés artificiels.

Mais, avant d'avoir recours à ces moyens extrêmes, toujours très coûteux et dont les résultats sont souvent incertains, on doit, sous peine de travailler en pure perte, se rendre un compte exact des faits qui ont amené la dégradation, pour en faire cesser immédiatement les causes. C'est ainsi que, suivant les cas, il faut : changer le régime appliqué; réglementer le pâturage ou même le supprimer complètement; rajeunir les parties sans avenir; ou encore, augmenter la durée des révolutions trop courtes et réformer les balivages défectueux. Quand, ces remèdes étant inefficaces, des repeuplements artificiels s'imposent, il y a toujours lieu de les exécuter avec la plus stricte économie.

Lorsqu'il s'agit de réintroduire une espèce précieuse dans un peuplement d'ailleurs complet, on doit utiliser toutes les ressources que présente le massif à améliorer; en général, il suffira de disposer convenablement une faible minorité de sujets dans la masse des tiges naturelles. L'important est alors de mettre les nouveaux venus dans des conditions telles qu'ils se raccordent le plus tôt possible avec leur entourage. Il est clair que, pour qu'il en soit ainsi, le moment le plus favorable coïncide toujours avec la naissance du peuplement à soigner : c'est-à-dire avec l'époque où l'on y provoque une régénération, soit par la semence, soit par rejets.

BOPre el Jolyet. 
Exceptionnellement, lorsqu'on veut créer par voie artifificielle des sous étages dans des massifs réguliers d'essences de lumière, il faut procéder quand, ce peuplement étant à l'état de perchis, on vient d'y pratiquer une éclaircie avec extraction de toutes les tiges surcimées, - qu'on peut faire disparaître sans arrière pensée, puisque leur tempérament les condamne à une mort prochaine et qu'on va les remplacer par des essences d'ombre. Le sol estalors en bon état de conservation et, quel que soit le moyen employé, - semis ou plantation, - les sujets introduits réussiront à merveille sous le couvert du peuplement principal.

Enfin, quand il s'agit de vides ou de clairières, il est d'une bonne administration de les repeupler immédiatement, afin de remettre aussitôt que possible le sol en état de production.

Applications dans les futaies. - Si, la semence étant fournie en quantité suffisante par des porte-graines convenablement répartis, le sol dégradé n'est pas en état d'assurer l'installation du semis naturel, toute la dépense se borne à la préparation du sol par des crochetages; nous avons insisté dans le chapitre VI, pages 185 et suivantes, sur l'urgence et les bons résultats de cette pratique.

$\mathrm{Si}$, au contraire, les porte-graines font défaut ou que, pour tout autre motif, la régénération naturelle soit incertaine ou impossible, il faut semer ou planter. Dans un peuplement mis à l'état d'ensemencement, le procédé par voie de semis est tout indiqué, puisque toutes les conditions favorables sont réunies pour assurer la germination des graines, - qu'elles soient apportées du dehors ou disséminées naturellement. Il suffit de déterminer les espaces affectés à l'espèce à introduire et de disposer les semences de façon à obtenir, soit le mélange intime, soit le mélange par places ou par compartiments plus ou moins étendus. La plantation par mottes peut d'ailleurs être assimilée au semis quant à l'âge, ou, pour mieux dire, à la situation du peuplement dans lequel on opère.

Certaines circonstances locales s'opposent à l'emploi du semis. Il en est ainsi quand la semence de l'espèce à introduire fait défaut, ou bien quand ces semences sont exposées à la voracité des animaux: sangliers, mulots, pigeons ramiers. 
pour les glands et les faines, - oiseaux granivores, pour les espèces résineuses : il est toujours imprudent, par exemple, de semer en forêt des glands ou des faines apportés du dehors pendant le cours des années oủ les chênes et les hêtres n'ont pas fructifié dans la région. Il faut alors planter des sujets aussi jeunes que possible, afin de réduire les dépenses à leur minimum.

De même, on est obligé d'avoir recours à la plantation pour terminer des régénérations acquises dans l'ensemble, mais que la nature a laissées incomplètes; en effet, le plus souvent, on ne constate ces insuffisances que quand le sol est déjà plus ou moins dégradé sur les espaces à regarnir. Il est alors trop tard pour semer, et l'on plantera des sujets dont la force sera proportionnée à celle des semis naturels qui les entourent. Il faut tenir compte de ce fait que le. semis réussit mieux dans les sols médiocres que dans les terrains fertiles, où les jeunes sujets naissants sont exposés à être envahis et étouffés par une abondante végétation herbacée.

Les espaces à repeupler artificiellement dans les futaies jardinées se présentent, le plus souvent, sous forme de vides ou de clairières; comme tels, ils seront remis en état le plus promptement possible, par les procédés généraux les plus économiques adaptés aux exigences de la station.

Applications dans les taillis. - Dans les taillis composés, les repeuplements artificiels doivent être exécutés dans le même esprit que les dégagements de semis, c'est-à-dire localisés sur les points où l'état de la réserve demande une amélioration. Aussi, dans la majorité des cas, faut-il planter avec grand soin, dans un sol bien préparé, des sujets assez forts pour résister à l'étreinte des rejets. F'aire peu, mais ne rien négliger pour bien faire, tel est le principe dont on ne doit jamais se départir. Pour tenir compte du déchet, il est toujours prudent de ne pas planter des brins isolés, mais de les réunir par groupes de 4 à 8 individus, convenablement espacés. Puisque l'objectif est ici de créer des ressources pour les balivages futurs sur les points où la réserve présente des lacunes, ce sont toujours des espèces de lumière el notamment des chênes qu'il s'agit d'introduire; suivant les cas, ormes. 
frênes, érables, peuvent être associés ou substitués aux chênes. Les plantations seront donc faites en plein découvert, immédiatement après l'expiration des délais fixés pour l'enlèvement des produits. C'est d'ailleurs à cette époque qu'on peut le mieux juger, en constatant l'état de la réserve, de la nature et de l'imporlance des travaux à exécuter. Un procédé simple et économique consiste à faire ces plantations sur les emplacements des souches concédées sous forme de menus produits; les places de loges ou d'ateliers, les places à charbon, les chemins temporaires peuvent également êtrc repeuplés à peu de frais. Ces menus travaux suffisent, dans la plupart des forêts situées en bon sol, pour entretenir la richesse du taillis en essences précieuses et pour assurer le recrutement de la réserve.

Mais les plantations ne sont profitables que si l'on vient périodiquement les dégager dans la même forme que les semis naturels ; les deux opérations sont solidaires l'une de l'autre. Et si, en faisant ces dégagements, on prend la précaution de donner une légère culture au sol autour des brins plantés, on leur assure une vigueur telle que l'on pourra souvent les réserver comme baliveaux à la fin de la révolution au début de laquelle ils auront été introduits.

Nécessairement ces dégagements sont onéreux; aussi, tout compté, est-il préférable de planter des moyennes ou des hautes tiges. Quelques sujets bien soignés coûteront, en définitive, moins cher que les milliers de basses tiges jetées sans soin et au hasard, et dont l'entretien sous forme de dégagements nécessite, en même temps que des frais énormes, une perte de production considérable. Il ne manque pas d'exemple de ces plantations négligées dont le profita été nul pour la forêt.

On suit des procédés analogues dans les taillis simples réguliers, soit pour y combler les vides, soit pour y ramener les bonnes essences, - notamment le chêne dans les haies à écorces.

Dans les taillis simples furetés, indépendamment des semis et plantations, on a recours à la marcotte pour remplacer les cépées mortes de vétusté. 
b. La mise en valeur des terres arables abandonnées par l'agriculture : conditions du déclassement. - Les procédés de boisement. Boisements spéciaux. - Arboriculture fruitière. - Les têtards; les arbres d'émonde et les ramilles-fourrage.

Conditions du déclassement. - Les motifs qui engagent à restituer à la forèt les terres autrefois livrées à l'agriculture se rattachent à des questions économiques; telles sont : l'abondance de la terre arable, le prix élevé de la main d'œuvre, la rareté des fermiers, etc... Ces causes sont d'ailleurs purement locales, souvent même passagères. Ainsi, dans une province riche en terres fertiles, franchement agricole, ou la main d'œuvre est chère, où les bois sont rares, on sera conduit économiquement à abandonner comme trop mauvaise, telle terre, qui, dans une autre région moins bien partagée, serait encore considérée comme d'une culture avantageuse.

Quoi qu'il en soit, avec la culture intensive que l'on poursuit à l'aide des engrais minéraux, l'étendue des surfaces emblavées diminue tous les jours et la contenance des terres disponibles augmente d'autant. D'ailleurs, on a pu dire avec raison que " même sur un sol fertile, la forêt peut lutter, parfois avec avantage, contre le champ cultivé " (1). M. Hüffel cite l'exemple de la ferme de Dombasle, connue par sa bonne exploitation, qui donne à son propriétaire un revenu net de 40 francs par hectare, tandis que la forêt communale de Pontà-Mousson (Meurthe-et-Moselle), qui lui est contiguë et traitée en taillis sous futaie, rapporte 60 francs par hectare et par an.

Les procédés de boisement. - L'ancienne culture agricole est toujours une excellente préparation pour le reboisement. Elle a d'ailleurs des conséquences pratiques qui influent sur le choix des méthodes à employer :

$1^{\circ}$ le sol est débarrassé de la végétation sauvage et meuble, ce qui rend possible le boisement par semis direct;

$2^{\circ}$ il est facile à travailler et suffisamment profond pour permettre l'emploi de la charrue avec cultures en plein ;

(1) G. Hüffel, Sols forestiers et sols agricoles. Nancy, imprimerie centrale, 1894. 
$3^{\circ}$ souvent il conserve encore assez de la fertilité due aux anciennes avances pour qu'on puisse associer une plante agricole aux essences forestières : une plante sarclée avec le chêne, - une céréale de printemps avec les résineux. La récolte en grains ou en pomme de terre paie une bonne part des frais de semis.

M. Henry (1) cite, à titre d'exemple, le décompte suivant des dépenses et recettes d'une pareille exploitation dans une région calcaire de la Meuse :

\section{A. Dépenses.}

Labour d'automne à quatre chevaux, suivi d'une légère façon au printemps................. $70 \mathrm{fr}$.

Avoine de semaille, 125 kilogr. ............... 20

Graine de pin, 10 kilogr.................. 50

Répandage à la volée de l'avoine et de la graine de pin.............................. \&

Hersage et roulage...................... 16

Frais de récolte de l'avoine................ 30

$\overline{190} \mathrm{fr}$.

B. Recettes.

14 hectolitres d'avoine................. $112 \mathrm{fr}$.

1.000 kilogr. de paille..................... 30

$142 \mathrm{fr}$,

Excédent des dépenses : $190-142=48$ francs par hectare (2).

Dans les terres profondes, froides, argileuses sans trop de compacité, on donne la préférence au chêne. On le sème en rigoles et, entre les lignes, si l'on ne plante pas des pommes de terre, on lui associe des espèces à croissance rapide telles que saules bouturés, ou aunes plantés. Les grandes espèces disséminées formeront aussi un utile mélange.

Dès que, la profondeur faisant défaut, la sécheresse est à redouter, on choisira parmi les résineux l'espèce la plus convenable à la station. Ces derniers sont, tantôt plantés par potets, tantôt semés en plein ou en bandes. Les essences

(1) Henry, Un reboisement à bon marché (Revue des Eaux et Foréts, 1 er juin 1900).

(2) Voir aussi, pour le reboisement " à bon marché " : l'article de M. Dufay, dans le Bulletin de la Société forestière de Franche-Comté et Belfort, de juillet 1899. 
feuillues disséminées, et les résineux autres que les espéces courantes : épicéa, pin sylvestre, etc.., sont toujours plantées.

\section{Ajoutons deux observations:}

1. dans les régions infestées de lapins, les plants sont infailliblement détruits. Les semis, au contraire, ont beaucoup plus de chances de passer inaperçus; encore faut-il que le semis soit fait en plein, les bandes indiquant à ce désagréable rongeur qu'il y a des dégâts à commettre ;

2. le déchaussement est plus à craindre dans les anciennes terres agricoles que partout ailleurs. Ainsi, au mois de mars dernier, une de nos plantations a été très endommagée de ce fait, et cela dans une région où nous n'avions jamais souffert de pareil accident: mais il s'agissait d'un champ cultivé en pommes de terre l'année précédente, et dont la terre friable était dépourvue de toute végétation. Il sera donc prudent, dans les conditions analogues, non seulement de renoncer au semis, mais encore de planter des sujets assez forts, âgés de trois ou quatre ans par exemple; d'ailleurs, la facilité avec laquelle se creusent les potets permet de leur donner, sans augmentation de dépense, des dimensions un peu supérieures à la normale.

Boisements spéciaux. - L'état morcelé des terres arables est le principal motif qui détourne le propriétaire d'y créer une fôrêt. Aussi, quand une parcelle a moins de 5 hectares d'un seul tenant, peut-on, tout en la reboisant, orienter sa mise en valeur vers un but spécial.

Quelle qu'en soit la variété, ces cultures ligneuses ont un caractère commun : les bois y sont réalisés à court terme et en bloc.

C'est ainsi que cela se pratique dans certaines contrées du nord de l'Europe, oú l'on fait entrer une récolte forestière dans la rotation d'un assolement agricole. A cet effet, on boise le terrain appauvri par une culture vampire en mélangeant la graine forestière à une demi semence de céréales. On donne les soins convenables au peuplement que l'on coupera à blanc étoc, entre vingt et cinquante ans. Après le défrichement du terrain, on profite de son humus pour en obtenir 
deux ou trois récoltes agricoles. Puis on reboise à nouveau et ainsi de suite.

En France, on peut utiliser de la sorte le pin sylvestre, le pin noir et le pin maritime. Nous déconseillons le mélèze, qui, même dans sa jeunesse, ne comporte pas l'état serré, et le pin Weymouth, bien que ce dernier soit souvent employé à cet usage dans d'autres pays.

Ailleurs, on supprime l'alternance des céréales, et l'on fait une culture ligneuse permanente. C'est le cas des taillis de coudriers, de robiniers et de châtaigniers, qui donnent des cercles de futaille ou des échalas dans les vignobles, des taillis de tilleul dans l'Aube et dans toutes les régions où les fibres libériennes de cette essence servent comme matière textile, - des oseraies, - des taillis de micocoulier, etc. Nous avons dit un mot déjà de ces différentes exploitations (page 225), renvoyant au Traitement des bois en France, par M. Broilliard, pour les détails qui concernent leur création et leur exploitation.

Nous mentionnerons toutefois d'une façon plus spéciale les taillis de charme créés dans certains pays où la propriété est morcelée, - sur les coteaux calcaires de Franche-Comté notamment, - par des cultivateurs qui utilisent ainsi des pièces de terre de qualité médiocre. La mévente des bois taillis ne les inquiète pas, car ils consomment des quantités considérables de bois pour la cuisson des aliments destinés aux bêtes de boucherie. Au besoin, on brûle le bois de ses " buissons " et l'on vend sa part d'affouage. C'est une culture très rationnelle.

De même se recommandent: le bouleau dans les champs sablonneux; sa présence est peu gênante pour les cultures agricoles voisines, - l'aune sur les parcelles de prairies trop mouilleuses ou trop exposées aux inondations, - et bien d'autres encore.

C'est là, direz-vous, de l'arboriculture industrielle, plutôt que de la sylviculture. Peu importe, pourvu que le sol rende. Nous irons même plus loin dans ce sens.

Arboriculture fruitière. - Alors que les céréales ont baissé de prix, deux productions restent rémunératrices: 
la viande et les fruits. Toutes deux peuvent s'obtenir de pair : il suffit de créer des pâtures-vergers, en utilisant trois choses qui ne manquent pas en France : la terre, l'eau et le soleil.

"L'arboriculture fruitière, dit M. Baltet, est entrée dans une voie nouvelle de grande culture et de grande production. De simple délassement d'amateur, elle est devenue une branche importante de la richesse nationale en approvisionnant nos marchés de fruits frais, ou transformés par l'industrie, et en ajoutant une source de revenus à l'exploitation agricole (1)".

D'ailleurs entre les cerisiers ou les pommiers l'herbe pousse abondante, et le kirsch, le cidre, le lait, le beurre se vendent toujours bien. On a dit, avec raison, que la France était le "verger de l'Europe "; c'est ici l'occasion de le rappeler.

Les têtards; les arbres d'émonde et les ramilles-fourrage. - Dans les prairies humides, sur le bord des rivières ou des ruisseaux, partout oú les gelées printanières rendent impossible la culture des arbres fruitiers, partout encore où, bien que donnant la plus large part à la production des herbages, on veut néanmoins récolter les produits ligneux nécessaires au chauffage domestique, les essences forestières réapparaissent, mais cultivées sous la forme de têtards ou d'arlres d'émonde.

Quand on tronçonne la tige d'un arbre feuillu, naturellement disposé à fournir des rejets, on voit évoluer des rameaux nombreux dans la zone qui avoisine la section; ces rameaux se développent à la façon des rejets de souche. Exploités euxmêmes systématiquement à de courtes révolutions, ils forment bientôt, au sommet de la tige mutilée, un renflement caractéristique, qui a fait donner le nom de têlards aux arbres ainsi traités.

Le têtard n'est donc autre chose qu'une cépée aérienne, se reproduisant à l'abri des atteintes du bétail, et dont l'élévation au-dessus du sol est généralement suffisante pour permettre la circulation des hommes et des animaux sous des

(1) Ch. Baltet, Traité de l'arboriculture fruilière, commerciale et bourgeoise. Paris, Masson, 1884. 
rameaux qui ombragent les pâtures sans les étouffer. Les saules sont les plus cultivés sous cette forme; indépendamment du menu bois qu'ils fournissent, ils contribuent par leur enracinement à protéger les berges des cours d'eau contre les érosions et les éboulements. Cependant toutes les espèces feuillues peuvent accepter ce traitement; dans certaines plaines, notamment dans celle des Laumes, on voit des frênes, des ormes, des charmes traités en têtards.

Les têtards ont un fût trop court et qui se carie de trop bonne heure pour qu'on puisse en tirer aucun parti. Si donc on veut combiner une exploitation de rejets sur tige avec la production du bois d'œuvre, il faut traiter les sujets en arbres d'émonde. Pour cela, laissant la tige entière, on élague périodiquement tous les rameaux latéraux, à l'exception d'un faible bouquet qui reste intact au sommet. Sur celte tige, chaque branche amputée forme un centre de production, une façon de cépée, d'où sortent de nouveaux rameaux après chaque exploitation et dont l'ensemble constitue un véritable taillis sur futaie. L'arbre se couvre de broussins, mais ne se dégrade pas aussi vite que le tétard : son bois madré, à fibre tourmentée, souvent interrompue par des traces de cicatrices, reste longtemps sain; si, à cause de sa structure irrégulière, il ne peut donner ni sciage, ni bois de fente, du moins fournit-il à la construction des pièces d'une grande résistance.

Les peupliers d'Italie, communs autrefois le long de toutes nos grandes routes, sont le meilleur type d'arbres d'émonde. Les chênes pédonculés existent encore sous cette forme au milieu des haies de la Normandie et du centre de la France. M. Marcel Vacher, - dans un éloquent plaidoyer en faveur des haies vives, qui fournissent d'excellentes clôtures, et un abri pour les oiseaux insectivores, - expose les revenus vraiment merveilleux que l'on peut se créer de la sorte avec quelque peu de soin et de patience (1). Dans certaines régions même, dans le Quercy par exemple, l'émonde semble méthodiquement adoptée à un traitement régulier de la forêt : car,

(1) Marcel Vacher, Les haies: utilité, plantation, taille. (Revue des Eaux et Forêts, 1er décembre 1899). 
dans bon nombre de taillis sous futaie, toutes les réserves sont émondées, et l'on voit les sous étages en chêne pur, se développer, à la façon d'un taillis simple, sous ces futaies, dont la projection est pour ainsi dire nulle.

Nous n'avons envisagé jusqu'ici l'arbre d'émonde que comme producteur de bois d'œuvre et de chauffage. Il peut encore fournir du fourrage et un fourrage apprécié, ainsi qu'en témoignent les expériences faites à ce sujet de différents côtés (1).

M. Grandeau (2) à la suite de nombreuses analyses a constaté que les feuilles et les ramilles d'arbres sont beaucoup plus riches en matières protéiques au printemps qu'en été; mais, comme de tous les organes d'un végétal, ce sont toujours les feuilles qui ont le plus de valeur alimentaire, et comme, d'autre part, c'est en été que ces feuilles enţrent en majeure proportion dans la masse d'un rameau, il en résulte que l'on récolte presque autant de substance azotée en cueillant les branches en août et même en septembre qu'en le faisant aux débuts de la végétation. D'ailleurs, ces branches feuillées sont un fourrage d'une réelle valeur : " les foins les plus riches en matière azotée, tel que le foin des Alpes, dit M. Grandeau, atteignent seuls la teneur en cette précieuse substance des ramilles de printemps bien préparées, et sont très peu supérieurs aux ramilles d'été. Quant aux pailles, elles se montrent infiniment plus pauvres que les ramilles de bonne composition ".

Toutefois, cette ramille alimentaire est d'autant plus nutritive que les parties de l'arbre qui la composent sont plus jeunes. Elle devra donc être exclusivement constituée " avec des pousses de l'année (axes et feuilles) et des jeunes branchettes dont le diamètre n'excèdera pas 5 à 6 millimètres ".

(1) Cormouls Houlès, Utilisation des ramilles d'arbres ensilées pour l'alimentation du bétail. Mazamet, Carazol, 1893.

Circulaire ministérielle de juillet 1893.

Poessler, Uber Futterwerth und Gerbstoffgehalt des Laubes, der Triebe und der schwachslen Zueige der Eiche, 1891.

Dr Ramann et Iena, Holtzfütterung und Reisigfütterung. Berlin, 1890.

(2) L. Grandeau, La Forét el la disetle de fourrage. Paris, C. Pariset, 1893. 
Il y a longtemps d'ailleurs que les Romains avaient inventé l'arbre d'émonde pour suppléer aux prairies fauchées rares dans leur pays, et pour se procurer le fourrage d'hiver nécessaire au bétail pendant les courts moments qu'il passait à l'étable. Cette pratique s'est conservée jusqu'à nos jours telle qu'elle était au temps de Virgile, dans la plupart des vallées de nos basses montagnes, surtout dans la France méridionale. Nous ne pouvons qu'en souhaiter le maintien et l'expansion, comme un des meilleurs moyens de détourner des forêts le fléau du pâturage de printemps.

c. La mise en valeur des terres incultes en pays de plaines et de coteaux : les landes. - Les friches. - Les garrigues.

Les landes. - La lande est caractérisée par la nature siliceuse du terrain. Nous savons que sur de pareils sols le boisement par semis direct donne les meilleurs résultats, pour certaines espèces, du moins.

On emploiera suivant les cas : pin sylvestre, pin maritime, chêne, bouleau, en y ajoutant par plantation, si on le juge à propos, les essences exotiques appropriées à la station, notamment le cerisier tardif.

Les landes les plus étendues sont celles de Sologne et de Gascogne. En Sologne, on fera bien de s'en tenir au pin sylvestre et au chêne comme essences principales de reboisement. Malgré les désastres que l'hiver de 1879-1880 a fait éprouver aux nouvelles forêts de pin maritime, bon nombre de propriétaires reviennent encore à cette espèce à cause de la rapidité de sa croissance pendant la première jeunesse; avant de s'engager dans cette voie, on fera bien de considérer que le pin maritime est loin de présenter tous les avantages qu'on lui suppose trop généralement (1).

On sème le chêne et le pin sylvestre après trois cultures agricoles, dont la dernière, faite par petit billons, avéc emblavure de sarrasin, rend le sol meuble et propre. La récolte faite, on répand à la volée, sur chaque hectare, cinq hecto-

(1) E. Girard, Traité de sylviculture pratique en Sologne. Romorantin, Joubert éditeur, 1881. 
litres de glands, en automne, ou huit kilogrammes de graine de pin sylvestre, au printemps suivant. La dépense est en partie payée par la récolte du sarrasin.

Si le terrain n'est pas susceptible d'être ensemencé en céréales, on procède par bandes; la dépense ne dépasse pas 100 à 120 fr. par hectare. Le semis par potets est surtout employé pour regarnir les vides dans les parties traitées par les procédés précédents; de même encore, dans les forêts récemment exploitées, où la présence des souches empêche l'emploi de la charrue. La dimension des bandes et potets est variable, suivant l'état superficiel du sol : on donne jusqu'à 1 mètre et $1^{\mathrm{m}}, 33$ de largeur aux unes et de côté aux autres dans les terrains fortement envahis par l'ajonc et la fétuque bleue; dans ceux où l'on ne rencontre que de la bruyère courte, cette dimension est réduite à 40 centimètres et même 25 centimètres; la dépense, dans ces dernières conditions, ne dépasse pas 70 à 90 francs par hectare. Malgré les bons résultats obtenus par le semis, bon nombre de praticiens préfèrent la plantation bien faite, surtout pour le pin sylvestre (1).

La région autrefois désolée et malsaine de la lande de Gascogne s'est transformée rapidement en riches domaines forestiers, grâce au système d'assainissement appliqué vers 1850 par M. l'ingénieur en chef Chambrelent. Sur ce grand triangle dont la surface dépasse 8,000 kilomètres carrés, le sol est formé d'un sable siliceux à peu près pur. $A$ une profondeur moyenne de 30 à $\mathbf{4 0}$ centimètres règne une couche continue imperméable à laquelle on a donné le nom d'alios, laquelle est formée du même sable agglutiné par un ciment de sels de fer et de matières organiques. La présence de ce sous-sol étanche et le peu de relief du terrain entretenaient dans la région une humidité insalubre, et s'opposaient en même temps à la végétation ligneuse. Une étude approfondie a permis à M. Chambrelent d'y découvrir un système de pentes naturelles et, par suite, un moyen de l'assainir. De grands canaux ont été

(1) David Cannon, le Propriélaire planteur. Orléans, 1887. 
creusés qui déversent les eaux au Sud, vers l'Adour, et au Nord, vers la Garonne ou les grands étangs qui longent le littoral : Arcachon, Cazau, Biscarosse, Aureilhan, etc. A ce système général se rattache tout un lacis de fossés, formant une sorte de drainage à ciel ouvert, et qui vont partout chercher l'eau en excès. Chaque hectare assaini présente environ $\mathbf{4 0 0}$ mètres courants de ces fossés, dont le prix actuel ne dépasse pas 15 centimes par mètre (1).

La lande devient ainsi un bon sol forestier, dans lequel le chêne pédonculé et surtout le pin maritine s'installent sans aucune difficulté. On connaît d'ailleurs la facilité avec laquelle le pin maritime se multiplie dans cette région. Il suffit pour obtenir un semis complet de peler la terre soit par bandes, soit par trous et d'y répandre 10 à 12 kilogrammes de graines par hectare; le prix de revient de l'hectare semé est d'environ 50 à 60 francs. Le chêne pédonculé, encore beaucoup trop rare jusqu'à présent, y prend aussi de superbes accroissements. Il serait intéressant, à titre d'essai, d'y tenter l'acclimatation de quelques chênes d'Amérique à végélation très rapide, tels que: Quercus rubra, Q. tinctoria, Q. palustris et Q. Phellos.

Les pouvoirs publics devaient donner leur appui à l'œuvre de M. Chambrelent : la loi du 19 juin 1857 règle la question de la mise en valeur par l'assainissement et le boisement, d'environ 400.000 hectares de terrains communaux, en même temps qu'elle renférme des dispositions relatives à l'ouverture de routes agricoles.

Les friches. - La friche est dans les terrains calcaires ce qu'est la lande dans les sols siliceux. Son caractère est l'irrégularité: irrégularité dans la profondeur du sol, le plus souvent assez faible, mais qui peut être nulle quand la roche affleure, ou au contraire assez considérable dans les poches ou les crevasses, - irrégularité dans l'aspect de la superficie, tantôt gazonnée, tantôt recouverte de blocs ou de pierrailles éparses, de murgers, de débris de carrière ou de vestiges de clôtures abandonnées ; - irrégularité enfin dans la végé-

(1) Croisette-Desnoyer, Notice forestière sur les landes de Gascogne Glermont (Oise), Daix, 1874, 
tation spontanée: ici, c'est un gazon court et londu par les moutons, là, un mélange de plantes aussi variées dans leur taille que dans leurs espèces, mais souvent ligneuses et buissonnantes : coudriers, troènes, trembles, charmes, épines, genévriers, etc. (fig. 35, p. 137).

Le sylviculteur avisé saura, comme nous l'avons dit, se plier à cette diversité, et, utilisant chaque chose à sa place, attendre patiemment, après avoir mis en valeur les meilleures parties, que la nature se charge de repeupler les mauvaises.

Il convient donc de respecter soigneusement toutes les broussailles existantes, - il est même bon de receper celles qui sont trop fatiguées par la dent du bétail, - puis on ouvre des potets partout où il y a de la terre. Avec un peu d'habitude, on reconnaît vite les bonnes places, que l'on marque par des jalons; mais le propriétaire devra se charger lui-même de cette besogne ou la faire exécuter par des hommes de confiance. En général, où poussent des sureaux hyèbles ou des épines noires, le sol est profond; de même, quand on remarque sur la surface du terrain des sortes de ressauts, d'escaliers, il y a des chances pour qu'au bas de chaque contremarche existe une crevasse, une fracture du soussol; au contraire, la petite terrasse qui forme la marche est le plus souvent constituée par une roche à fleur de terre. On vérifie d'ailleurs très vite ce qu'il en est en sondant le terrain avec une barre à mine.

Tout ce que nous avons dit à propos des essences indique suffisamment à chacun ce qu'il peut planter et comment il doit s'y prendre, en utilisant tous les abris naturels et toutes les ressources locales. Dans les friches, plus que partout ailleurs, il faut faire preuve d'opportunisme et songer aux petites recettes.

C'est ainsi qu'il peut être permis d'avoir recours au semis direct, en terrain non préparé dont M. l'Inspecteur des forêts Pierre Leddet signale les excellents résultats (1): dans les friches de Reuilly et Saint-Georges (Indre), on a jeté à la volée $10 \mathrm{kilog}$. de semence $(8 \mathrm{kilog}$. de pin noir

(1) Bulletin de la Sociélé des Amis des arbres, no 25, 1899. 
et 2 kilog. de pin sylvestre) par hectare; avec un double hersage à tour croisé suffisant pour recouvrir la semence, les frais n'ont pas dépassé 58 francs par hectare, y compris l'achat de la graine. Le résultat a été parfait et les massifs de plus de 30 ans sont très beaux. Il s'agit d'un terrrain appartenant au groupe corallien de l'oolithe moyenne, aride au point d'être appelé Champagne; la nature filtrante dủ sous-sol, fissuré en tous sens, draîne les surfaces sans permettre à la capillarité d'y faire rencontrer les eaux profondes; la mince couche de terre ne conserve quelque fraîcheur qu'en raison de son état de tassement : tout ameublissement, toute culture ne feraient qu'en augmenter l'extrême sécheresse. En semblable situation, on peut procéder de même; mais pour être certain de réussir, il faut comparer la flore sauvage des deux stations : car rien ne fait mieux ressortir l'analogie des milieux que la présence simultanée des mêmes plantes : arbustes, herbes, mousses ou lichens.

A titre d'exemple, nous citerons encore l'intéressante étude de M. Duparchy (1), oùr l'auteur décrit le procédé qu'il a mis en usage pour boiser des versants chauds pauvres en humus des environs de Saint-Claude, dans le Jura:

... On doit tout d'abord renoncer à la satisfaction de faire des plantations bien alignées, contenant le nombre réglementaire de plants à l'hectare... il faut se borner à mettre ceux-ci au hasard des places qui semblent propices, de préférence tout contre un buisson, si petit soit-il, et le plus possible du côté nord. J'ai acquis la conviction que là où végète un buis de médiocre vigueur, là aussi peut vivre et prospérer un épicéa, à toute exposition, sauf peut-être le plein midi; mais à la condition que le plant sera placé de manière que ses racines soient en contact immédiat avec les racines du buis et, par conséquent avec l'humus qui entoure celles-ci... Comme il s'agit ici d'un sol pierreux et pauvre en terre végétale, on devra s'abstenir de faire des trous à l'avance et à la pioche, ce serait s'exposer à perdre le peu d'humus qu'on aurait déplacé... Il est préférable de sè servir d'une forte barre de fer ou sorte d'épieu terminé, à une de ses extrémités, par une pointe aciérée, et à l'autre, par une tête arrondie. Avec la pointe on sonde le sol, et, quand on a trouvé un fond suffisant, en agitant la barre, on élargit le trou assez pour pouvoir y introduire le plant. Quelques coups donnés avec le pommeau serrent la terre contre les racines.

(1) Loc. cit. page 406. 
A chacun de s'ingénier!

Les garrigues. - La garrigue, c'est la friche sous le soleil du midi. On peut la boiser, grâce au pin d'Alep et au chêne vert, qui réussissent partout oủ le vent n'est pas trop violent.

On peut encore y introduire des plantes ligneuses diverses, arbrisseaux ou sous-arbrisseaux, tels que le sumac fustet, l'épine-vinetle, qui ont des emplois industriels, et surtout le buis, si recherché des tourneurs, mais qui se fait rare dans le midi comme dans le Jura. La bruyère en arbre, l'arbousier sont dans le même cas.

Enfin, les garrigues pourraient être transformées en pâtures ligneuses; diverses espèces de genêts, de saules, de peupliers, le noisetier, les variétés de robinier sans épines, etc., présenteraient des ressources sous ce rapport. " Je suis convaincu, dit M. Lecoq (1), que la plupart des terrains secs, des pacages, par exemple, donneraient d'abon dantes feuillées, bien supérieures en quantité aux maigres plantes herbacées qui les couvrent ou que l'on peut y semer, si on y plantait des arbres à feuillage, dont les souches, taillées chaque année, donneraient en abondance des jeunes pousses qu'une sécheresse prolongée ne saurait détruire, comme elle arrête la végétation des prairies. "

M. Grandeau (2) cite dans une brochure signée M. A. P. et imprimée à Bourg, les lignes suivantes, qui peuvent trouver place ici, bien qu'intéressant une autre région de la France :

Dans quelques parties du haut Máconnais, on coupe, au mois de septembre, les taillis de l'âge de six ans pour fourrage, et on les vend, à cet age, aussi cher que si on les vendait à dix pour le bois seulement... On coupe les branches pour feuillées, avant les brouillards, autant que possible, par un beau jour d'automne, et on fagote le len: demain, alors que la feuille s'est un peu fanée au soleil...

L'utilité de ces fourrages ligneux étant ainsi bien démontrée, nous ferons seulement à leur emploi deux objections :

1. Il ne faut employer qu'avec prudence, dans l'alimentation du bétail, les ramilles ou les jeunes pousses dont l'usage n'est

(1) Traité des plantes fourragères. Paris, Libralrie agricole de la maison rustique, 1862.

(2) Loc. cit.

Boppe et Jor.yet. 
pas courant: des indispositions, ou, tout au moins, des symptômes suspects se sont produits déjà à différentes reprises; ainsi, les hématuries qui sont attribuées à l'abus des papilionacées. De plus, quelles que soient les essences, les feuilles vertes récoltées au premier printemps amènent des désordres analogues à ceux qu'on appelle le mal du brou ou la folie des chevreuils, chez les bêtes fauves.

2. Un terrain quelconque, - mais surtout un terrain pauvre et superficiel, sous un climat chaud, - consentira-t-il à produire des récoltes indéfinies et quasi annuelles de fourrage ligneux? Il s'épuisera sans aucun doute, car on n'a pas la prétention de fumer de pareilles cultures comme on le fait pour les oseraies, - mode d'exploitation analogue et dont on connaît les exigences. - Nous avons aussi souvenance de certaines landes des environs du Mans où, à force de couper la bruyère, on est arrivé à un état d'appauvrissement tel qu'elle-même s'est refusé d'y croître. Pourtant, s'il est un végétal frugal, c'est bien la bruyère!

Quoi 'qu'il en soit, nous concluons qu'au lieu de mener le bétail pâturer en forêt, ce qui entraîne le gaspillage, le tassement du sol, la perte des engrais, le mieux serait, au point de vue agricole comme au point de vue forestier, de récolter les feuilles vertes, en cas de pénurie de fourrage, ou de couper au sécateur les jeunes ramilles feuillues, de préférence en juin, juillet et août, dans les coupes destinées à être exploitées l'hiver suivant.

\section{d. La mise en valeur des terrains stables en montagne: les procédés} de boisement. - Les prés-bois.

Les procédés de boisement. - Dans les massifs montagneux qui, par suite de leur âge géologique, de leur nature rocheuse, de la végétation herbacée ou ligneuse protégeant les surfaces, semblent avoir acquis des conditions définitives d'équilibre, la dénudation se présente sous forme d'accidents purement locaux : les torrents dangereux n'existent pas. Les reboisements conservent alors leur caractère de mise en valeur et d'utilité privée : ils restent facultatifs. A cette caté- 
gorie appartiennent les Ardennes, les Vosges, le Jura, le Morvan, le Plateau central, les collines de Bretagne et les montagnes basses des Maures et de l'Esterel.

En toutes ces régions, on peut procéder à l'aide des seuls moyens déjà cités : rien de spécial ne doit précéder ou accompagner l'opération. Il suffit de choisir les essences les mieux appropriées au sol et au climat, en tenant compte de l'altitude, et de suivre les procédés les plus économiques suivant les ressources locales. S'il se présentait quelque difficulté, elle serait facilement résolue grâce aux indications données plus loin, à propos des boisements obligatoires en montagne.

Les prés-bois. - Mais ici encore, plus qu'ailleurs peutêtre, il y a lieu pour un propriétaire de bien peser si son intérêt est de boiser partout et à tout propos, s'il ne convien. drait pas, au contraire, de réserver une place à la production des herbages ou, mieux encore, d'associer sur certains espaces l'exploitation forestière et l'exploitation pastorale.

Cette association existe dans la forme excellente du pré-bois. Dans le Jura, ces sont de bouquets d'arbres disséminés en îlots parmi les pâtures et constitués par des essences diverses, dont les plus importantes sont : le hêtre, le coudrier, l'épicéa et le sapin. Ces deux dernières se trouvent quelquefois à l'état de sujets isolés et prennent alors une forme spéciale et pittoresque, comme on se plaît à se représenter les anciens cèdres des montagnes du Liban. Dans les Alpes, l'espèce habituelle est le mélèze qui, par tempérament, accepte mieux la forme de " clairs-bois ", dont le sol couvert d'herbe est pâturé ou lauché (fig. 87). Dans les Pyrénées, le pin de montagne, le sapin et le hêtre se prêtent à cette double culture.

Nous prendrons pour type les prés-bois du Jura,qui passent, à juste titre, pour les modèles du genre (fig. 88). Les vallées de la Suisse, celles de la Savoie en possèdent aussi de très beaux exemples, et l'administration des Laux et Forêts emploie toug ses efforts à en généraliser l'usage aussi bien dans les Alpes que dans les Pyrénées et dans le Plateau central (1).

(1) Gebhart, Pâturages et foréts. Paris, Berger-Levrault, 1889. 
Un pré-bois, dit M. Broilliard, est un terrain, mi-partie en prairies ou en pâturages à peu près dépourvus d'arbres, et mi-partie en bois à l'état de bouquets ayant une certaine consistance; les arbres isolés n'y sont qu'exceptionnels. Les parties déprimées, doucement ondulées, fraîches, riches en terre végétale, sont laissées à l'herbe, qui s'y développe abondante, grâce aux rosées et à l'abri des arbres voisins. Ceux-ci, occupant les hauteurs, les pentes raides, les parties pauvres ou rocheuses, forment des massifs, des bosquets, des lisières, défendus sur les bords par des buissons et des arbres de tailles diverses qui en font comme une masse de feuillage (1).

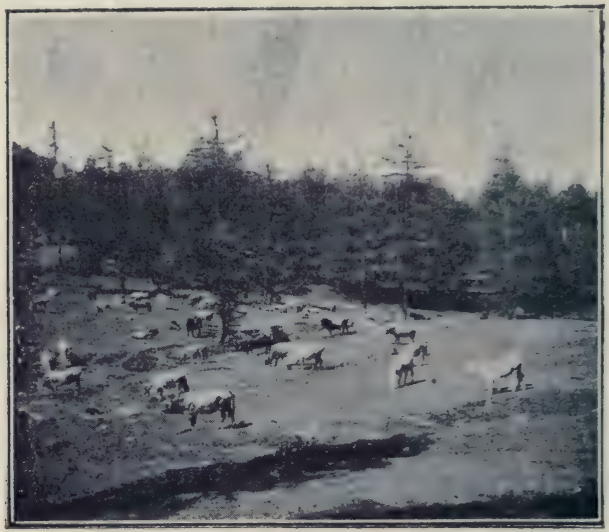

Fig. 87. - Clairs bois de mélèzes au Lautaret. (Photographie de M. S. George.)

Rien de frais et d'agréable à l'œil comme ces véritables parcs, sortes de jardins anglais qu'animent les magnifiques troupeaux jurassiens. Rien aussi de mieux compris au point de vue utilitaire.

Au bénéfice cultural que le voisinage des arbres entretient la fraîcheur dans ces prairies sur sol naturellement sec et superficiel, s'ajoute le profit que l'on tire du bois. Les boqueteaux fournissent les perches nécessaires à l'édification des clôtures, le chauffage indispensable pour la fabrication du fromage et des épicéas, des sapins, qui, s'ils n'ont pas comme bois de sciage et comme bois de fente toutes les qualités de leurs congénères des futaies voisines, trouvent pourtant acquéreur dans des conditions très acceptables; enfin ils abritent le bétail

(1) Broilliard, loc. cit., édition de 1894, p. 359. 
contre les ardeurs du soleil, contre la pluie et la tempête.

Mais, si l'on n'y prend garde, un des associés absorbe l'autre et la faillite générale est fatale. En fait, c'est toujours l'arbre qui succombe viclime de l'herbage; cherchons le motif et le remède :

D'abord, là comme en forêt, l'arbre est un capital, dont la réalisation tente toujnurs le proprićlaire. De plus, sur les

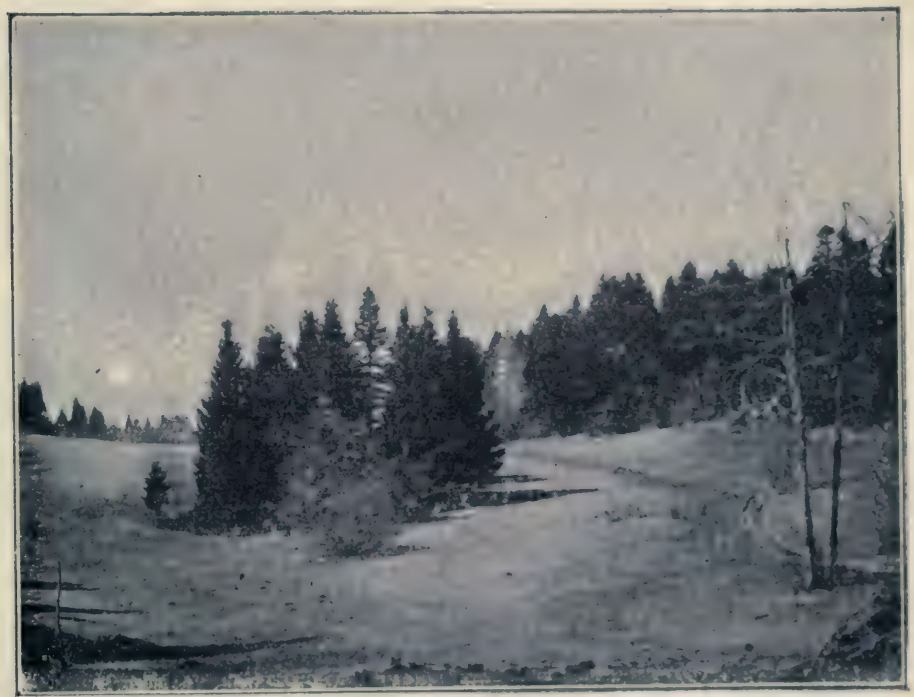

Fig. 88. - Un pré-bois près de Levıers (Doubs), (Photographie de M. Juvanon du Vachat).

surfaces en prés-bois, on ne peut pas indiquer par deschiffres les étendues relatives des pâtures et des boisés : le rapport varie à l'infini suivant l'état des lieux; l'essentiel est de mettre chacune des deux exploitations aux points que lui assigne la nature, en affectant aux arbres les endroits rocheux et les pentes rapides, car, quelles que soient les précautions prises, tout versant un peu incliné se dégrade quand il est livré au parcours.

Une fois reconnue la place faite au bois, pour y maintenir ou pour y installer les essences ligneuses, il suffit de mettre 
à profit un fait d'observation: l'union intime qui existe entre l'arbre et le buisson. Partout, en effet, nous voyons les bouquets de hêtres ou d'épicéas maintenus à l'abri de la dent du bétail par une ceinture d'épines, de genévriers, véritable rempart plus ou moins abrouti, mais qui supporte tous les mauvais coups. La chose est plus frappante encore, quand on assiste à la formation naturelle d'un pré-bois : comme dans toute forêt naissante, les arbrisseaux s'installent les premiers, - arbrisseaux épineux pour la plupart - et, quand ils ont pris possession du sol, les semis des grands arbres se jettent dans le fourré.

Utilisons donc tous ces auxiliaires dévoués et, quand ils font défaut, remplaçons-les par des obstacles inertes. Ainsi, dans le Jura, on entoure de fagots d'épines les jeunes plants d'épicéa. Aux environs d'Ischl, dans le Salzrammergut, on nous a montré des plantations faites au milieu de pâtures et que protégeaient efficacement des planchettes de 4 à 5 centimètres de largeur dépassant le sol de 20 à 30 centimètres, et fichées près de chaque sujet ; ce modeste obstacle suffisait à empêcher les vaches de poser le pied sur le jeune épicéa ou de brouter dans son voisinage immédiat. A notre avis, le meilleur est encore la clôture continue en ronce artificielle, en fil de fer galvanisé ou en palissade "à la suédoise ». Ces défenses, utiles partout, sont indispensables autour des parties nouvellement boisées ; elles n'empêchent pas d'ailleurs le bétail de venir chercher près des arbres l'ombre et la fraîcheur.

Les herbages réclament aussi nos soins. Le plus efficace est un bon aménagement, laissant à chaque pâture, à tour de rôle, un repos de deux ou trois années, pendant lequel l'herbe fatiguée " se refait "; les vides disparaissent, les bonnes espèces fructifient. Cette jachère, revenant tous les huit à dix ans, remplace l'engrais que l'on donne aux prairies de la plaine. D'ailleurs, le propriétaire qui comprend son intérêt profite de ce moment pour épierrer le pâturage, pour arracher la gentiane, le véraire, le genêt sagitté, les aconits, les linaigrettes, les euphorbes et autres mauvaises plantes, pour défricher aussi les genévriers et les épines, là où l'on ne 
veut pas que le bois s'installe, - pour étendre les fourmilières et les taupinières - pour semer un peu de bonnes graines dans les vides, etc., etc., - toutes précautions que nous avons entendu recommander si justement aux éleveurs jurassiens par M. I'Inspecteur Cardot. Il en est de ceci comme des dégagements de semis: un bon pâtre, aussi bien qu'un bon garde, peut, le plus souvent, suffire lui-même à la besogne. L'un et l'autre méritent d'en être bien récompensés.

Mais, qu'il s'agisse du Jura, des Alpes, des Pyrénées ou du Plateau Central, tous ces soins sont perdus si chèvres et moutons sont admis au pré-bois. Seules les bêtes bovines en méritent les honneurs.

\section{LES BOISEMENTS OBLIGATOIRES.}

a. La restauration des montagnes: les causes de la dénudation. - La méthode de travail suivie. - Les travaux de soutien. - Les boisements proprement dits; - traitement des parties stables; - des parties mouvantes; - des terres noires. - Les enherbements. Le gazonnement. - Conclusion.

\section{Les causes de la dénudation.}

Deux forces antagonistes se trouvent en présence dans les Alpes, dit M. Mathieu (1), et de la préminence de l'une ou de l'autre, dépend la ruine ou la prospérité du pays. La première est la force de dénudation qui démolit les crêtes, ravine les versants, comble les vallées, porte partout la dévastation. La seconde est celle de la végétation, victorieuse autrefois, vaincue aujourdhui par l'aveuglement de l'homme, qui a tout fait pour l'amoindrir et a causé la disparition du tapis de verdure, auquel il devait aisance et sécurité; toujours prête cependant à cicatriser les plaies, à réparer les désastres.

Les phénoménes de dénudation sont de deux ordres et, suivant la cause qui les détermine, doivent être subis ou peuvent être prívenus.

Parmiles premiers, se rangent les éboulements qui se produisent au pied des hauts escarpements calcaires, les chutes de rochers, le's glissements lents ou subits de terrains parfois étendus, qui descendent à des niveaux inférieurs, avec maisons, forêts et paturages. Les exemples de ce genre ne sont pas rares... Ce sont là des conséquences inévitables de la constitution grologique des Alpes; soulevées à des époques relativement récentes et formées le plus souvent de terrains sédimentaires ou métamorphiques, alternativement délayables et résis-

(1) Mathieu, Reboisement des Alpes, page 8. 
tants, elles n'ont point encore pris leur assiette et la doivent prendre; nulle force humaine ne saurait s'y opposer.

Mais à côté des faits de ce genre, locaux et accidentels en définitive, il en est d'autres d'un caractère plus général, auxquels revient la plus large part des ruines dont les Alpes sont couvertes. Ceux-là peuvent être prévenus : reboisement et regazonnement en fournissent les moyens certains.

Ces moyens d'action sont exposés avec détail par M. l'Inspecteur général Demontzey (1). Les procédés mis en œuvre sous son habile direction dans les Alpes françaises sont applicables à toutes les autres régions montagneuses, car cette contrée, la terre classique des torrents, présente les plus grands exemples de la dévastation et les difficultés les plus sérieuses. C'est à l'ouvrage de M. Demontzey et au rapport de M. Mathieu que sont empruntés la plupart des renseignements qui vont suivre.

D'ailleurs, en dehors des grands cataclysmes qui ont pétri la surface du globe pendant la suite des âges géologiques, nous voyons autour de nous la nature tout former et tout détruire à l'aide des infiniments petits: la goutte d'eau et la cellule végétale jouent le rôle fondamental dans tous ces phénomènes.

Spécialement en ce qui concerne l'érosion des montagnes, l'observation des faits a démontré que les torrents ont pour cause première le déboisement et l'abus du pâturage. C'est par la reconstitution des forêts et des pâtures que nous parviendrons à les éteindre.

La méthode de travail suivie. - Dans l'ensemble de la région dévastée, on a procédé à la reconnaissance des torrents en activité, et délimité sommairement, pour chacun d'eux, le bassin de réception des eaux dangereuses. En présence de l'immensité du travail à accomplir, la méthode commandait de concentrer tous les efforts, toutes les ressources disponibles, sur un petit nombre de points choisis parmi ceux où le danger était le plus imminent. Au début surtout, il fallait aboutir, et c'est en fractionnant la tâche qu'on a réussi.

(1) Demontzey, Étude sur les Iravaux de reboisement et de regazonnement des montagnes. Paris, imprimerie Nationale, 1878. 
On peut envisager leversant d'une montagne déboisée comme constitué par une série d'ì-dos, dont la ligne médiane serait dirigée suivant la pente et séparés les uns des autres par des ravins plus ou moins profonds, parcourus ou non, en temps ordinaire, par un ruisseau, mais où se précipitent, lors des grandes pluies ou des grandes fontes de neige, des masses énormes d'eau chargées de boue et roulant des blocs de rochers.

Il faut donc :

$1^{\circ}$ enrayer la vitesse des eaux au fond des ravins; c'est la

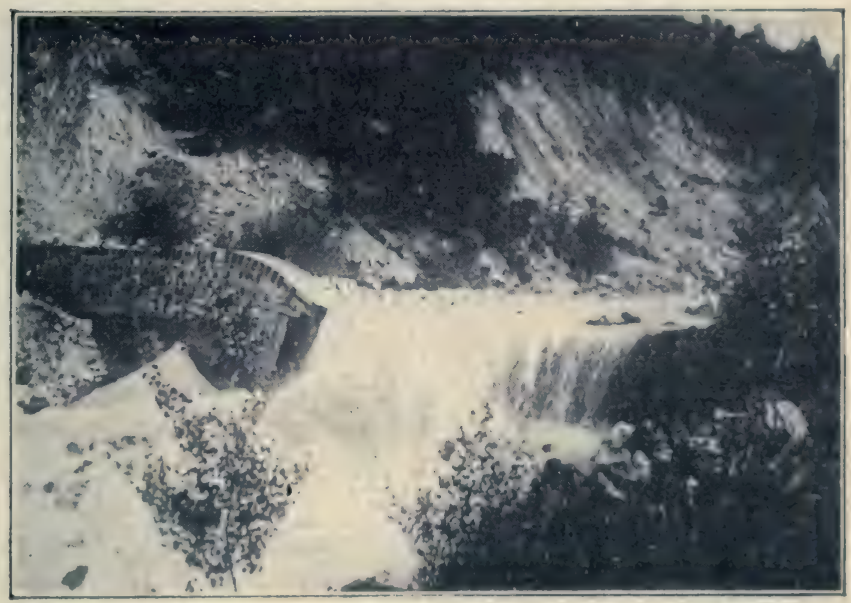

Fig. 89. - Un grand barrage. (Pholographie de M. P. Hirsch.)

première chose à faire, car le torrent, affouillant la base des berges, provoque le glissement de pans énormes de terrain;

$2^{\circ}$ fixer les surfaces des à-dos par une végétation herbacée ou ligneuse qui, arrêtant les eaux de ruissellement dans leur parcours, empêche qu'elles se précipitent en masse dans les ravins oủ leur afflux presque instantané produit le phénomène torrentiel.

Ainsi : consolider les masses, et fixer les surfaces, tel est le but à atteindre. Ajoutons que les torrents accumulent au bas des pentes, en arrivant dans le thalweg principal, des 
masses énormes de détritus, qui constituent le cône de déjection et qu'il importe aussi de boiser.

Les travaux de soutien. - On obtient la consolidation des masses par une série de travaux établis dans le lit même du torrent et qui, depuis le barrage monumental en maçonnerie jusqu'au simple clayonnage, soutiennent la montagne de la base au sommet (fig. 89 et 90).

Le principe est, en général, de couper le lit du torrent par des barrages qui diminuent la vitesse d'écoulement des eaux et provoquent par suite le dépôt d'une partie des matériaux qu'elles charrient: terres délayées, blocs de pierre, etc... Ainsi se forme en amont de chaque barrage un atterrissement, et le profil en long du ravin perd son irrégularité primitive pour se transformer en une série de parties en pente douce séparées par des cascades. Dès lors, les eaux n'affouillent plus le bas des berges, qui sont même rechaussées par l'atterrissement. Ce sont là des travaux d'art; mais le forestier qui les conduit a souvent besoin de faire œuvre de sylviculteur, pour consolider et défendre immédiatement ses travaux.

Il en est ainsi quand il installe des barrages vivants, dans lesquels entrent de fortes boutures ou plançons, qui, tout en remplissant le rôle de pieux dans la carcasse des ouvrages, sont destinés à s'enraciner et augmentent, par leur grossissement, la résistance à la poussée.

S'agit-il de fixer des berges fraîchement décapées ou des talus en voie de réglement, on a recours à des plantations qui prennent alors le caractère de fascinages vivants, dont la disposition varie suivant les circonstances. Le plus souvent, on trace dans les berges une suite de rigoles horizontales' d'une largeur proportionnée à la nature plus ou moins meuble du terrain et éloignées en raison des pentes, qui atteignent parfois 100 à 120 p. 100. Pour établir ces haies de soutien, le regretté M. Couturier, alors qu'il était chef de service dans les Basses-Alpes, imagina le procédé suivant: dans la partie la plus basse de la berge, on creuse une première jauge en rejetant les déblais dans le fond du ravin; sur le plafond réglé en revers, on place horizontalement une série de plants assez rapprochés pour qu'ils puissent remplir 
le rôle de haie vive. Aussitôt ces dispositions prises dans la rigole inférieure, on ouvre immédiatement au-dessus

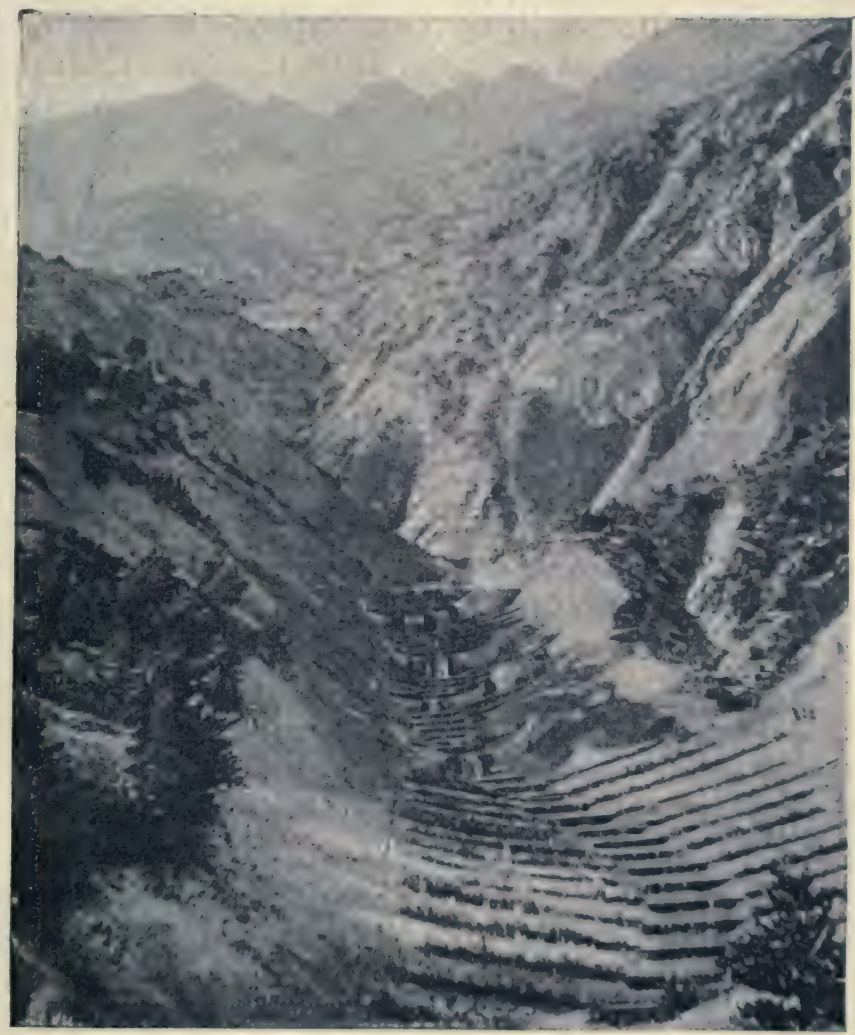

Fig. 90. - Grands et petits barrages : clayonnages vivants en essences feuillues qui consolideront les ouvrages d'art; du torrent Bourget en 1885. (Réduction d'une photographie extraite de la collection due à M. le Conservateur de Gayffer.)

une seconde jauge semblable, dont les déblais sont utilisés pour' combler la première et régler le talus dans sa pente d'équilibre. Ainsi de suite jusqu'au sommet de la berge. Les jeunes plants, disposés dans une terre meuble, poussent avec vigueur, et, de suite, ils retiennent le sol dont on faci- 
lite encore la consolidation par des enherbements en touffes. On peut utiliser, à cet effet, diverses essences feuillues comme le prunier de Briançon (1), le cytise des Alpes, l'aune glutineux, etc., cette dernière espèce, employée en boutures de $0^{\mathrm{m}}, 20$ de longueur et enfoncées de $0^{\mathrm{m}}, 15$ dans le sol, donne malgré l'opinion courante, d'excellents résultats, comme a pu le constater M. l'Inspecteur adjoint Mougin dans le périmètre d'Ugines (Savoie).

Enfin, autour de chaque ouvrage et sur les atterrissements, on plante ou l'on bouture des peupliers, des aunes blancs (A. incana), des saules pourpres ou bleuâtres ( $S$. purpura, cœesia), des hippophaés (H. rhamnoides), etc..., toutes essences, soit drageonnantes, soit susceptibles d'être propagées par marcotte. Le saule marsault lui-même est bouturé dans certaines vallées des Alpes, à l'égal de ses congénères.

Ces plantations, souvent traitées en menus taillis, préservent parfaitement le thalweg contre les érosions et fournissent, à chaque recépage, des matériaux qui seront utilisés avec profit dans tous les chantiers, sous forme de pieux, fascines, etc.

Les boisements proprement dits. - En même temps que l'exécution des ouvrages d'art est poussée vigoureusement, on s'occupe de fixer toutes les surfaces relativement stables dans l'ensemble du périmètre délimité.

Or, on sait que dans les pentes la terre nue n'offre aucune résistance à l'érosion des eaux pluviales ou sauvages, et que la végétation, sous forme de forêts, de broussailles ou d'herbages, est seule capable de rendre au sol son armure protectrice. Ce champ d'action appartient donc exclusivement au forestier reboiseur.

Dans le choix des essences, il importe de s'en tenir à des espèces bien appropriées à l'exposition, à l'altitude, et au terrain. Ainsi, les Alpes méridionales, peuvent se diviser en quatre zones :

(1) Peut-être le cerisier tardif d'Amérique donnerait-il de bons résultats. Ce serait à expérimenter. 
altitude,

10 zone méditerranéenne ou chaude de.

0 à 600 mètres

$2^{\circ}$ zone moyenne ou tempérée de...... 600 à 1,000

30 zone alpestre ou froide de........ 1,000 à 1,800

$4^{\circ}$ zone alpine ou très froide de.......

1,800 à 3,000

Chacune de ces quatre zones peut être caractérisée par l'aspect de sa végétation sauvage.

Dans la première, les prairies proprement dites n'existent que dans les terrains irrigués. Les pâtures nombreuses ne sont autres que des garrigues, ou les plantes, en touffes isolées, ne forment jamais gazon continu ; les graminées sont représentées par des herbes à feuilles rares, dures, ordinairement enroulées.

Dans la seconde, la végétation est encore éparse sur les terrains vagues. Les plantes qui les peuplent sont de familles très diverses; les espèces ligneuses dominent, comme : le buis, la lavande, le thym, le sumac, les genêts, les bruyères, les cistes, les bugranes; le sainfoin s'y rencontre fréquemment.

Dès qu'on pénètre dans la région alpestre, les pâturages peuvent être en gazons continus. Les plantes herbacées y réussissent mieux; différentes familles ont de nombreux représentants, mais les graminées, pour l'importance, sont reléguées au second rang. Les espèces annuelles ont disparu.

Enfin, la zone alpine est la région pastorale par excellence. Les pâturages qui recouvrent de leurs gazons touffus tous les lieux en pente douce, les plateaux, les cols et même les pentes rapides sont peuplés d'espèces vivaces; les graminées prennent plus d'importance que dans la région précédente. Ces pâturages sont parfois fauchés et deviennent alors des prairies.

Les principales essences de boisement appropriées à chacune de ces zones sont indiquées dans le tableau ci-après, en tenant compte des facultés qu'elles présentent pour être employées par semis ou par plantation : 


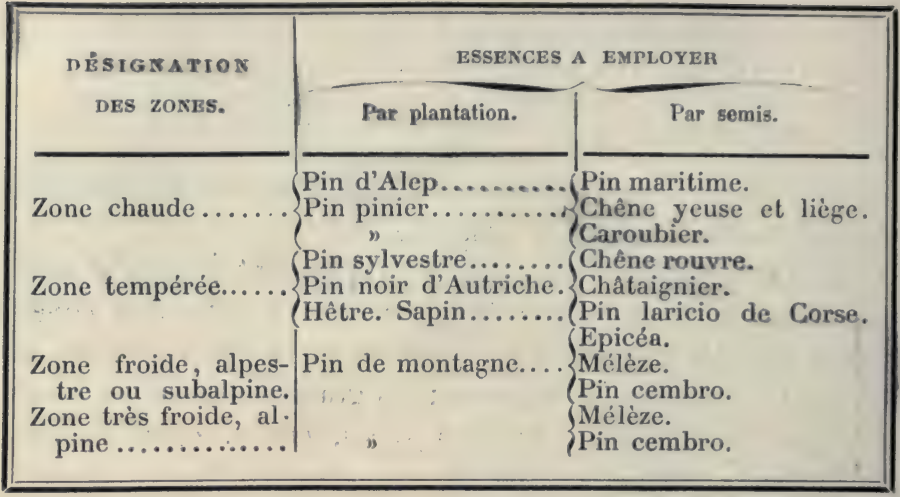

D'ailleurs, à moins qu'on ait des motifs spéciaux d'agir autrement, les résineux s'emploient surtout pour former les massifs dans les surfaces solides en pente régulière, - sur les à-dos en un mot. Les feuillus restent localisés dans le fond des ravins, sur les cônes de déjection et partout où il y a chance de rencontrer un peu de fraîcheur.

Ce n'est pas le moindre talent du directeur des travaux que de savoir faire varier les essences pour mettre chacune à sa place. Le périmètre de Luz-la-Croix-Haute (Drôme), reboisé sous la direction de M. le conservateur Darcy; est particulièrement remarquable à ce point de vue (fig. 91).

Avant de passer à l'exécution, il est de la plus grande importance de déterminer, dès l'origine, le but qu'on se propose d'atteindre : gazonnement, broussaillement ou boisement, sans jamais le perdre de vue dans la suite. Il demeure d'ailleurs entendu que toute surface appartenant au périmètre sera scrupuleusement mise en défend. Dans les terrains stables, cette précaution suffit bien souvent pour ramener une végétation spontanée, dont l'effet est de donner une première protection au sol; mais dans les terrains instables, ce serait une chimère d'attendre aucun résultat utile de ce côté: sans cesse ravinés par les eaux, ils ne s'enherbent pas naturellement, et l'absence de végétation expulse même en tout temps le bétail de ces régions désolées.

Traitement des parties stables. - Pour procéder 
au boisement des parties fixes et conservant encore quelques vestiges de végétation, on commence par receper toutes les broussailles ligneuses fatiguées par la dent du bétail. Puis, on distribue avec soin les ouvriers planteurs ou semeurs; car, dans ces travaux, la bonne organisation des chantiers et leur surveillance constante sont indispensables pour assurer la réussite et éviter le gaspillage. Qu'on opère par semis ou par

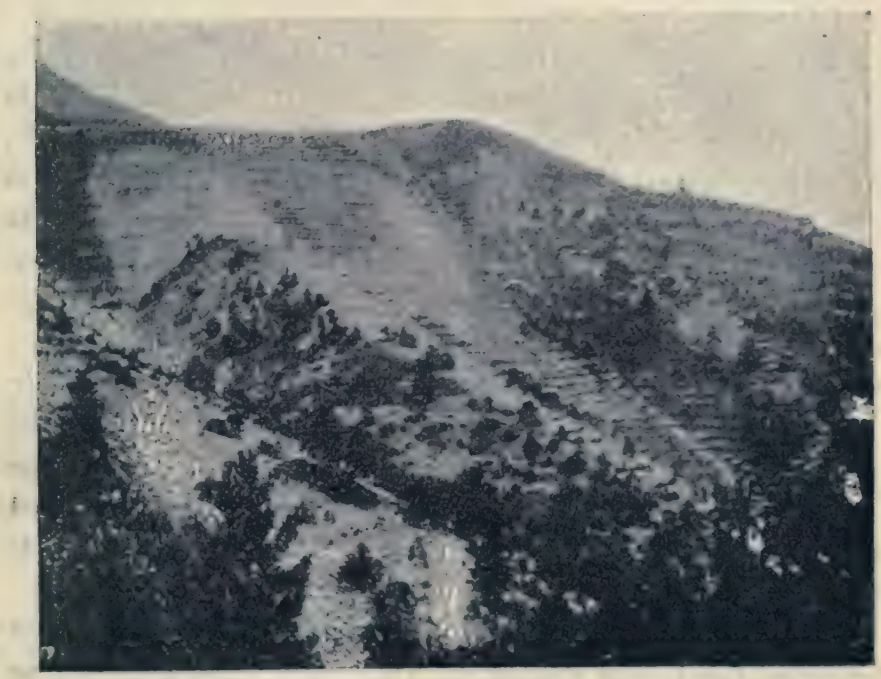

Fig. 91. - Ravins et à-dos reboisés, périmètre de Luz-la-CroixHaute en 1885. (Photographie de M. de Gayffier.)

plantations, le sol doit être préparé par une forte culture avec défoncement dans les formes connues - bandes alternes continues ou brisées, trous, potets, corbeilles, etc... La seule précaution à prendre est de donner au plafond des cultures un léger devers du côté d'amont pour retenir les eaux et éviter les ravinements; quelquefois même, au-dessus des rigoles ou potets, on trace deux sillons de captage destinés à ramener les eaux dans les parties défoncées. La préparation du sol a pour but de prévenir la sécheresse, qui est l'ennemi redoutable dans la plus grande étendue de ces régions. 
L'opération est d'autant plus nécessaire que les stations sont plus chaudes et plus basses ; son importance décroît, en raison de l'altitude, et même il y a lieu de s'abstenir dans les stations froides et très froides, où le déchaussement sous l'influence du gel et du dégel est à craindre ; la limite de l'urgence du défoncement est marquée par la zone où l'herbe reste verte pendant toute l'année.

Les plantations s'exécutent, le plus souvent, au printemps avec des jeunes sujets élevés en pépinières.

En terrain préparé, les semis se font à l'araire pour les grosses graines (glands, châtaignes), en rigoles tracées à la pioche pour les autres semences. En terrain non préparé, quand les circonstances le permettent, on sème parfois sur la neige; mais, généralement, on opère par placeaux dont la surface seule est pelée et ameublic, et que l'on a soin de disposer dans le petit bourrelet de terre végétale accumulé en amont des buissons ligneux ou des touffes de gazon. On peut se contenter, quand le sol est enherbé, de percer des trous au pic en plein gazon; ces trous sont remplis de terre meuble et on y sème quelques graines. Enfin, dans les pelouses, on creuse aussi des sortes de godets en enlevant au hache-pré un petit gazon en forme de coin; on émiette la terre mise à nu et on y jette quelques graines.

Traitement des parties mouvantes. - Parallèlement au boisement des terres solides, on s'occupe des parties nues ou mouvantes. C'est là que se rencontrent les principales difficultés. En semblable circonstance, il s'agit bien moins de créer des forêts et des pâtures que de fixer rapidement le sol; les végétaux robustes, de croissance rapide, traçants ou drageonnants, ligneux ou herbacés, peu importe, mais, en tous cas, vivaces, sont seuls convenables pour atteindre ce but: saule, cytise, sumac, hippophaé, corroyère, brugrane, sainfoin des Alpes, valériane, rumex, tout est bon pour créer un premier revêtement dont on active l'expansion par le marcottage des brins traînants. C'est seulement quand un tel broussaillement aura accompli son œuvre de fixation qu'il sera possible de tenter l'installation définitive de la forêt.

$\mathrm{Si}$, à la rigueur, la réussite de quelques espèces forestières 
semble possible, on a recours à l'enherbement pour fixer la terre entre les potets. On procède par sillons horizontaux,

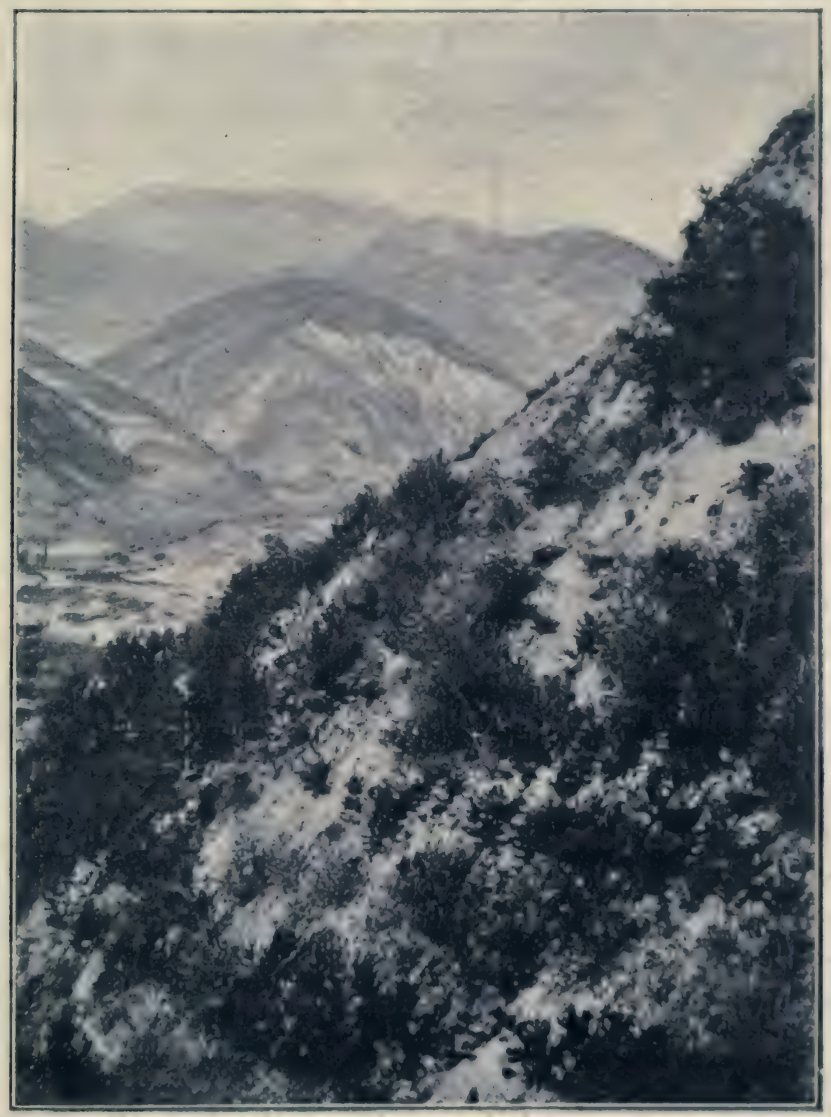

Fig. 92. - Plantations en cordons horizontaux sur un versant rapide. Périmètre de Poyols près de Luc-en-Diois. (Photographie de M. Juvanon du Vachat.)

espacés de $1^{\mathrm{m}}, 50$ à 2 mèlres, suivant les cas. Chacun de ces sillons ne tarde pas à former une petite haie de verdure qui soutient la terre et tue la vitesse d'écoulement des eaux superficielles. 
Ajoutons que dans les chantiers établis sur de pareils terrains, l'ordre et une prudente disposition des ouvriers ne sauraient être trop recommandés : les pierres roulantes parfois de très petites dimensions - que mettent en mouvement les travailleurs placés en amont, peuvent causer des accidents mortels parmi ceux qui, au-dessous d'eux, vaquent à leur besogne. D'ailleurs, à différents points de vue, avant de commencer semis ou plantation, il est bon de parcourir le terrain en faisant écrouler tout ce qui menace ruine, sapant les proéminences ou les replis du sol dont l'équilibre paraît instable, détachant les pierres dont la solidité est douteuse, nivelant, en somme, grossièrement le champ de travail et faisant en un jour, alors qu'aucun dégât n'est à craindre, l'œuvre de plusieurs orages.

Traitement des terres noires. - I.es marnes généralement confondues sous le nom de terres noires, quelles que soient leur coloration et la formation géologique à laquelle elles appartiennent, sont particulièrement réfractaires à la végétation, et leur présence en assises puissantes sur un très grand nombre de points constitue un des principaux obstacles à vaincre dans l'œuvre si difficile du reboisement des Alpes. Ces terres noires, mises à nu par l'érosion des eaux, apparaissent avec leur composition primitive, telles qu'elles ont été déposées en des âges où les conditions de la vie, à la surface du globe, étaient bien différentes de ce qu'elles sont de nos jours; c'est dans leur masse schisteuse affouillable que les torrents les plus dangereux, creusant les gouffres qui leur servent de lit, récoltent les matériaux qui portent avec eux la dévastation.

Outre leur instabilité, ces marnes présentent un grave inconvénient. A cause de la grande quantité de pyrite qu'elles renferment, elles absorbent tout l'oxygène disponible dans le sol pour transformer les sulfures de fer en sulfates; ceux-ci, sans grande fixité, se décomposent; le fer devenu libre s'hydrate, et l'acide sulfurique se combine avec les bases pour former les efflorescences blanches, calcaires ou magnésiennes qui caractérisent la contrée. Aussi, pour acquérir la force productive qui les rende capables de nourrir la flore actuelle. 
est-il nécessaire qu'elles subissent une certaine préparation par l'exposition aux agents atmosphériques : elles ont besoin de s'aérer, de respirer, pour ainsi dire. Une telle transformation ne se fait pas en un jour; d'ailleurs, on la voit s'accomplir lentement, de proche en proche, sur les emplacements que leur peu de déclivité met à l'abri des érosions et des éboulements : leur coloration noire va sans cesse en s'atténuant pour se rapprocher des tons ocreux du fer hydroxydé.

Quoi qu'on fasse, on ne réussira jamais à planter les grandes espèces ligneuses dans une terre noire fraîchement décapée. En cela, il faut encore suivre les indications de la nature, c'est-à-dire commencer par les espèces inférieures les plus rustiques; à mesure que la terre perd son poison, elle accepte des végétaux d'un ordre d'autant plus élevé que la couche désinfectée est elle-même plus profonde. On peut ainsi mesurer l'instant où l'on pourra lui confier des arbres à nourrir.

Dès que les pentes s'accentuent, notamment sur toutes les berges, la surface est entraînée d'autant plus facilement qu'elle est plus ameublie par l'aération; c'est donc toujours la même marne vierge et réfractaire qui affleure. Alors, il faut à tout prix empêcher la bonne terre de s'écrouler au fur et à mesure desa formation. Dans ce but, on installe la végétation dans le fond même de chaque ravin, afin d'arrêter les affouillements; on y parvient à l'aide de fascinages, de barrages rustiques, qui transforment les pentes régulières en une suite de petits ressauts, entre chacun desquels, sur les plates-formes d'atterrissement, on bouture, on plante, on sème, on gazonne suivant les cas. La proximité d'un peuplement forestier rend la chose facile, car on utilise les produits des éclaircies pour recouvrir tout le fond des ravins; les menus brins encore munis de leur feuillage sont disposés bout à bout dans le sens de leur longueur et la cime vers l'amont; la terre végétale fine et meuble, provenant du lavage des berges, s'arrête dans les interstices des feuilles et des ramilles; celles-ci, en pourrissant, se transforment elles-mêmes en terreau, et l'ensemble constitue un compost éminemment favorable au développement des plantes herbacées ou ligneuses qui s'y jettent natu- 
rellement ou qu'on y introduit avec succès. De ces coulées de verdure, suivant l'heureuse expression de M. le Conservateur Carrière, qui a traité de cette façon la combe désolée d'Alavar (périmètre de Seyne, Basses-Alpes), on voit la végétation " monter à l'assaut des berges ", qui sont bientôt fixées à leur tour. Là, comme dans la dune, le problème est résolu par l'installation artificielle d'une couverture mortè, qui prépare et protège les éléments de la forêt future.

Les enherbements. - L'enherbement n'est qu'un auxiliaire du boisement qu'il précéde ou qu'il accompagne toujours. A ce point de vue, il ne faut pas le confondre avec le gazonnement, qui s'entend de la création des herbages d'embouche ou fauchables.

L'enherbement fournit aux jeunes plants naissants un abri tutélaire contre la sécheresse dans les surfaces stables et constitue le meilleur moyen d'éviter le dẹchaussement. Pour tous ces usages, le sainfoin rend les plus utiles services; en terrains préparés, il s'emploie, soit en mélange intime avec la graine forestière, soit pour former des rigoles entre les lignes de plantations ou de semis. Dans les terres noires, on a recours à des mélanges de sainfoin avec de la fenasse : semence d'herbes comprenant généralement les espèces suivantes : le calamagrostis argenté (Calamagrostis argentea), la fétuque bleue (Festuca cœrulea), le chiendent (Triticum repens), la houque molle (Holcus mollis), le fromental (Avena elatior) et la pimprenelle (Eupatorium sanguisorba); on y ajoute souvent du brome des prés (Bromus erectus).

M. Mougin s'est bien trouvé de la formule suivante :

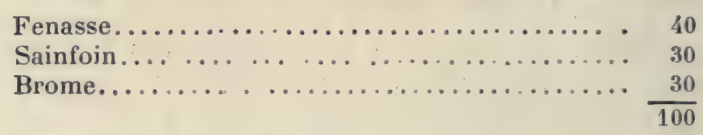

Si la région est chaude ou tempérée, les travaux de semis et d'enherbement peuvent se faire soit à l'automne, soit au commencement du printemps; est-elle froide ou très froide, on n'opère qu'au printemps, aussitôt après la fonte des neiges, quand la terre est imbibée d'eau, de telle sorte que les graines restent le moins longtemps possible sur le sol sans y germer. 
Le gazonnement. - Le gazonnement a pour but, au contraire, de créer des alpages ou pelouses permanentes et n'est pas appli. cable à toutes les altitudes des régions montagneuses. C'est dans les grandes hauteurs avoisinant la limite de la végétation ligneuse, régions purcment pastorales, que ces travaux peuvent fournir le degré d'utilité qu'on leur demande; mais le boisement, qui n'est plus ici l'objectif, lui vient souvent en aide. En effet, bien que le gazonnement intéresse la production pastorale plutôt que la production forestière, il existe une relation nécessaire entre ces deux modes d'exploitation du sol; et, sans sortir de notre cadre, il est permis d'appeler l'attention sur les faits suivants :

Si l'on cherche à se rendre compte de l'origine de ces gazons naturels, de ces pelouses unies qui tapissent les flancs des montagnes au-dessus des forêts actuelles, tout indique qu'elles n'ont pu s'installer que grace à la protection de la végétation ligneuse. Celle-ci a disparu par le fail de l'homme, qui a méconnu les lois de la nature en exploitant les forêts d'une manière désordonnée et en abusant d'elle avec une imprévoyance coupable. C'est ainsi que la limite actuelle des forêts ne doit pas être considérée comme réelle, mais comme artificiclle : les arbres épars et les souches recouvertes qu'on retrouve dans toutes les pàtures en font foi (1).

Ces pelouses ne se reforment plus sur les terrains nus, et celles qui existent encore sont destinées, si l'homme n'y prend garde, à disparaitre à leur tour et à suivre la loi d'abaissement que son égoïsme a déjà imposée aux forêts.

Dans les Alpes de la Provence, par suite du climat sec qui caractérise cette région, la création de nouvelles pelouses sur les terrains supérieurs absolument dénudés ne peut être assurée que par l'intermédiaire de la forêt.

On constate, en effet, que les plantes herbacées qui végètent au-dessus de la limite réelle imposée à la végétation ligneuse par la température du lieu, ne forment pas des gazons sérieusement exploitables' et susceptibles de protéger le sol contre les influences météoriques. C'est en poussant le reboisement jusqu'à cette limite qu'on peut espérer ramener la pelouse partout où le sol est dénudé et assurer la consoliclation des terres dans les régions les plus élevées du bassin de réception.

(1) Rapprochons de ces lignes emprunties à M. Demontzey une phrase de M. Mathey : "Partout oú l'homme n'a pas abaissé par des défrichements les limites supérieures de la forêt naturelle, celle-ci se termine par des arbres épars, le plus souvent stériles, car ils ont dépassé les frontières normales de leur aire d' habitation. Ils proviennent de semences fournies par les massifs inférieurs et apportées par le vent sur des points où ils peuvent encore vivre, sans toutefois pouvoir mùrir leurs graines" (Le páturage en foret). 
Telles sont du moins les conclusions auxquelles s'est arrêté M. Demontzey dans son $\mathrm{xi}^{\mathrm{e}}$ chapitre, intitulé Travaux de gazonnement. Il suffit d'ailleurs de constater la présence du beau gazon qui tapisse le sol de toutes les forêls de mélèzes, même celles de récente création pour être convaincu de ce fait.

Conclusion. - Nous devons nous borner aux simples indications qui précèdent, renvoyant le lecteur désireux de plus amples détails aux nombreux ouvrages publiés sur un aussi vaste sujet, aussi bien en France qu'à l'Étranger. Constatons seulement que l'initiative de l'entreprise et le mérite de la réussite reviennent tout entiers à l'Administration Française des Eaux et Forêts. Les résultats ont dépassé toute espérance et les quelques écoles des premiers jours ne sont rien en comparaison des succès obtenus.

Peut-être, cependant, ne faut-il pas s'endormir dans une quiétude trop confiante. Tout en reconnaissant l'efficacité des moyens d'action et la parfaite exécution des travaux, il est permis d'appeler, une fois encore, l'attention sur une série de faits dont la gravité s'accroît de jour en jour.

En même temps que l'œuvre du reboisement se poursuit, des ennemis jusqu'alors inconnus ou, du moins, méprisés comme inoffensifs, se montrent partout; leurs attaques prennent un caractère inquiétant : du nord au midi, on n'entend plus parler que d'insectes, de champignons, qui détruisent, en totalité ou en partie, les jeunes forêts créées à grands frais et sur lesquelles on fondait les plus belles espérances. Sous une forme animale ou sous une forme végétale, chaque essence artificiellement installée semble porter en elle son parasite. Les espèces exotiques ou étrangères à la région, sont plus particulièrement atteintes; on dirait que le climat, à la rigueur suffisant pour qu'elles puissent s'en accommoder, est particulièrement favorable au développement de leurs ennemis.

Le moment semble venu de couper court au mal par la reconstitution de la forêt spontanée. Sans attendre qu'on y soit contraint par la force majeure, il faut profiter du premier abri, de la première couche de terreau fournis par le boise- 
ment pour installer en mélange les espèces de la région. Le mieux, partout où cela sera possible, sera de créer des sousbois de feuillus, hêtre ou châtaignier; à défaut de hêtre, le sapin formera d'excellents sous-étages dans les stations moyennes. Enfin, dans les plus grandes altitudes, le mélèze, dont les parasites lui appartiennent en propre, sera toujours indiqué pour remettre en état de production les espaces de quelque étendue.

b. La fixation des dunes : les dunes maritimes. - Les moyens d'action. - La dune littorale. - Boisement de la dune blanche. - Les dunes continentales.

Les dunes maritimes. - Le long du littoral de l'Atlantique, de Bayonne à Dunkerque, partout où la mer n'est pas brisée par des falaises, elle dépose sans cesse, sur les plages, des sables siliceux, blancs, fins, provenant de matériaux triturés par les mouvements du flux et du reflux. La tempête soulève ces sables mobiles, les chasse vers l'intérieur où ils s'accumulent en chaînes de collines voyageuses, tantôt parallèles au littoral, tantôt confusément orientées, en tous cas, donnant à la contrée ce relief particulier auquel on a donné le nom de dunes. Entre chacune de ces chaînes s'étendent les lettes, sorte de vallées marécageuses, errantes comme les dunes, et dont les eaux malsaines, refoulées dans le mouvement général de transport, inondent les terres fermes, en même temps qu'elles charrient la fièvre.

L'accumulation des eaux dans les lettes tient à la constitution toute particulière du sable cru qui constitue la dune blanche. Dans leur mouvement de translation, ces grains lavés, presque chimiquement purs, se dessèchent à la façon des poussières atmosphériques et se déposent absolument privés de leur eau globulaire. On sait que de tels milieux restent longtemps imperméables; aussi toutes les eaux pluviales qui tombent sur les pentes roulent-elles rapidement vers le fond des cuvettes où elles sont retenues par des causes semblables. Parfois le desséchement rend les matériaux de transport tellement réfractaires à la capillarité, qu'ils peuvent se déposer en couches assez épaisses à la surface 
des eaux tranquilles pour former les hlouses, dont l'aspect trompeur a été fatal à plus d'un voyageur imprudent.

Aussitôt que le boisement a revêtu la surface de cette couverture forestière dont on connaît les propriétés hygrométriques, en même temps que diffusé la matière organique dans les couches plus profondes, le régime des eaux reprend son cours d'infiltration normal. L'assainissement des lettes devient ainsi la conséquence nécessaire du boisement des dunes.

Les moyens d'action. - Dès la fin du $\mathrm{xvmi}^{\mathrm{e}}$ siècle, on avait compris que le boisement était le seul procédé efficace pour fixer les dunes.

En 1787, l'Ingénieur en chef des Ponts et Chaussées, Brémontier, donnait une réelle impulsion aux travaux de défense qu'il dirigea durant plusieurs années et qui lui valurent, avec le nom de Bienfaiteur des Landes, le monument élevé à sa mémoire à la station de La Bouheyre. Il est juste toutefois de citer les Ruat, les Desbiey, le comte de Montausier et surtout le baron de Villers, ses précurseurs, qui, moins favorisés par les circonstances, ne purent mettre en œuvre les projets qu'ils avaient conçus (1).

Mais, comme pour la restauration des montagnes, la loi devait intervenir en vue de donner à l'opération son caractère obligatoire (2).

Aujourd'hui - et c'est un nouveau point de similitude avec les travaux de restauration des montagnes - on a reconnu la nécessité de se créer une base d'opération, de donner au point d'appui aux premiers boisements exposés à l'assaut des vents et du sable : on a donc encore recours aux mêmes moyens :

$1^{\circ}$ consolider les masses par des travaux d'ordre plutôt technique ;

$2^{\circ}$ fixer les surfaces par la culture forestière.

(1) Pierre Buffault, Étude sur la côte et les dunes du Médoc. Imp. Jehl. Souvigny, 1897.

(2) Décret-loi du 14 décembre 1810, relatif à la plantation des dunes; - ordonnance des 15 juillet 1818-8 mai 1819, contenant règlement des digues et dunes du Pas-de-Celais. 
La dune littorale. - Sans cesse renouvelée par les apports inépuisables de l'Océan, la masse des sables poussés par le vent de mer remonte les pentes assez faibles que présentent les collines du côté du rivage. Elle s'accumule sur les sommets d'où elle s'éboule, en vertu de son poids, suivant des profils atteignant 45 degrés et plus, à cause du manque de cohésion des matériaux. La base de ce talus s'avance ainsi continuellement vers les terres avec des vitesses variables suivant les années et les saisons, mais pouvant atteindre 12 et même 20 mètres en une année. Aucun obstacle ne peut arrêter la marche de ces collines envahissantes dont la hauteur dépasse parfois 70 à 80 mètres: forêts, villages et clochers qui se rencontrent sur leur passage, disparaissent ensablés.

Pour combattre le fléau, il faut l'étouffer à sa naissance; on y arrive par l'édification de la dune littorale (1).

Le baron de Villers, puis Brémontier, semblent avoir conçu l'idée de ce véritable ouvrage de défense dont la construction n'a été commencée que bien après eux, en 1851. Depuis cette époque à nos jours, plusieurs théories se sont succédées quant au profil à lui donner.

L'ancienne méthode, que nous allons décrire, est peut être celle qui donne l'idée la plus nette du but poursuivi et des moyens à employer. Elle a du reste fait bonne besogne pendant de longues années.

A 50 ou 80 mètres de la laisse des hautes eaux, parallèlement au rivage, c'est-à-dire perpendiculairement à la direction du vent, on dispose une palissade formée de madriers d'une largeur de $0^{\mathrm{m}}, 12$ et d'une épaisseur de $0^{\mathrm{m}}, 03$; ces madriers sont profondément enfoncés dans le sable avec une saillie de 1 mètre au-dessus du sol; ils sont espacés les uns des autres de $0^{\mathrm{m}}, 02$ à $0^{\mathrm{m}}, 03$. A la suite de chaque tempête, le sable, poussé par le vent, s'accumule devant la palissade et coule de l'autre côté par les vides laissés entre les planches;

(1) Pour plus de détails, voir les articles et notices publiés par MM. Goursaud, Revue des Eaux et Foréls, 1880; - Grandjean, La dune littorale, Revue des Eaux et Forèls, 1887; - Violelte, Entrelien de la dune littorale, Imp. A. Dupeyron, Mont-de-Marsan, 1899:A. Lafond, Fixation des dunes, Imp. nationale. Paris, 1900. 
suivant la dimension des grains, l'équilibre s'établit plus ou moins facilement entre les dépôts des deux aspects. Mais, contrairement à ce qui se passe dans les dunes naturelles, l'inclinaison est assez forte sur le talus du côté de la mer, tandis qu'à l'opposé la pente s'allonge bien davantage, une partie du sable étant entraînée, par la force du vent, en arrière de la palissade et plus ou moins loin suivant la grosseur des grains. Lorsque les madriers sont enfouis presque jusqu'au sommet, on les relève et l'opération suit son cours jusqu'à ce que la dune ait atteint la hauteur voulue; en général, 10 à 12 mètres au-dessus du niveau de la haute mer.

C'est ainsi que le vent, dompté par l'homme, travaille luimême à élever l'obstacle qui détruira les effets nuisibles de sa force d'entraînement; comme la vapeur, il actionne en même temps les freins et les moteurs.

Tout en exhaussant les madriers, on se préoccupe de la forme la plus favorable à donner au profil de la dune. On surveille la marche des dépôts pour rectifier le travail, parfois peu précis, du vent, par des fascinages, des épis et autres moyens complémentaires. Enfin, le relief normal étant acquis, on fixe la surface en la plantant de touffes de gourbet (Calamagrostis arenacea). Cette précieuse graminée est l'auxiliaire indispensable; elle se propage par voie de boutures comme par voie de semis, lance ses drageons à de grandes distances, et pousse de nouvelles racines au niveau du sol, à mesure que sa tige est ensablée.

La dune littorale étant ainsi achevée et garnie, il ne reste plus qu'à l'entretenir. Une surveillance constante doit assurer la bonne conservation d'un ouvrage qui sera la sauvegarde contre tous les dangers à venir; il faut, sans aucun retard, réparer les brèches ouvertes sur ses flancs par les rafales de vent ou les paquets de mer de la dernière tempête. Dans les départements des Landes et de la Gironde, un personnel de cantonniers est spécialement préposé à la garde de cette dune, dont la plate-forme a été kilométrée pour faciliter la direction du service et la transmission des ordres.

Aujourd'hui une tendance assez générale se manifeste à donner à la dune littorale un profil quasiment inverse du pré- 
cédent. Suivant la pittoresque image employée par $\mathbf{M}$. le Conservateur de Vasselot de Régné (1), la dune naturelle rappelle par son profil un chien assis regardant vers l'Est, vers la terre ferme. On avait pu s'imaginer qu'en retournant le chien, le nez vers l'Océan, il perdrait son humeur vagabonde et ferait bonne garde contre les sables envahissants: d'ou la forme des premières dunes littorales. Mais c'était compter sans le courroux de l'Océan qui, les jours de tempête, se rue sur l'obstacle, y faisant de larges brèches; aussi, a-t-on replacé le chien dans sa position première : comme le roseau de la fable, la dune littorale à pente très douce vers l'Océan, se laisse submerger par les vagues, puis, la tempête finie, quand les eaux se sont retirées, elle reparaît intacte.

Ce nouveau profil s'obtient en disposant, parallèlement au rivage, des lignes successives de petites haies faites de branches de pin, hautes d'environ $0^{\mathrm{m}} 60$ et au pied desquelles le sable s'accumule. Le talent consiste, suivant la forme de la côte et la marche des sables, à disposer ces obstacles au point voulu pour amener la dune à constituer sa ligne de crête à une distance du littoral telle que la pente puisse s'étendre sous l'inclinaison la plus efficace.

Ces mêmes cordons, avec les semis de gourbet sans couverture de branchages, permettent de barrer et d'enrayer les sifflets ou siffle-vent, couloirs que le vent creuse dans la dune et d'aveugler les ventouses.

C'est, d'ailleurs, merveille de voir comme les forestiers compétents savent utiliser les forces de la végétation pour modeler suivant leur gré cette chose d'allures si mobiles et si capricieuses qu'est le sable de la dune : ils s'empressent de fixer par le gourbet les points où le profil est acquis, arrachant ou éclaircissant cette plante, au contraire, quand ils veulent faire déplacer par le vent les amas de sable ou trucs devenus inutiles ou gènants.

Souvent les haies parallèles au littoral sont flanquées d'épis, dont la direction est perpendiculaire à la leur; ceux-ci peuvent eux-mêmes se subdiviser en palte d'oie, ou se ramifier en contre-épis.

(1) De Vasselot de Régné, Notice sur les dunes de la Coubre. Paris, Imprimerie nationale, 1878. 
Quand des côtes sinueuses, en pointe, sont exposées à tous les vents violents, une étude approfondie de la situation peut seule déterminer les points où il faut établir les ouvrages de

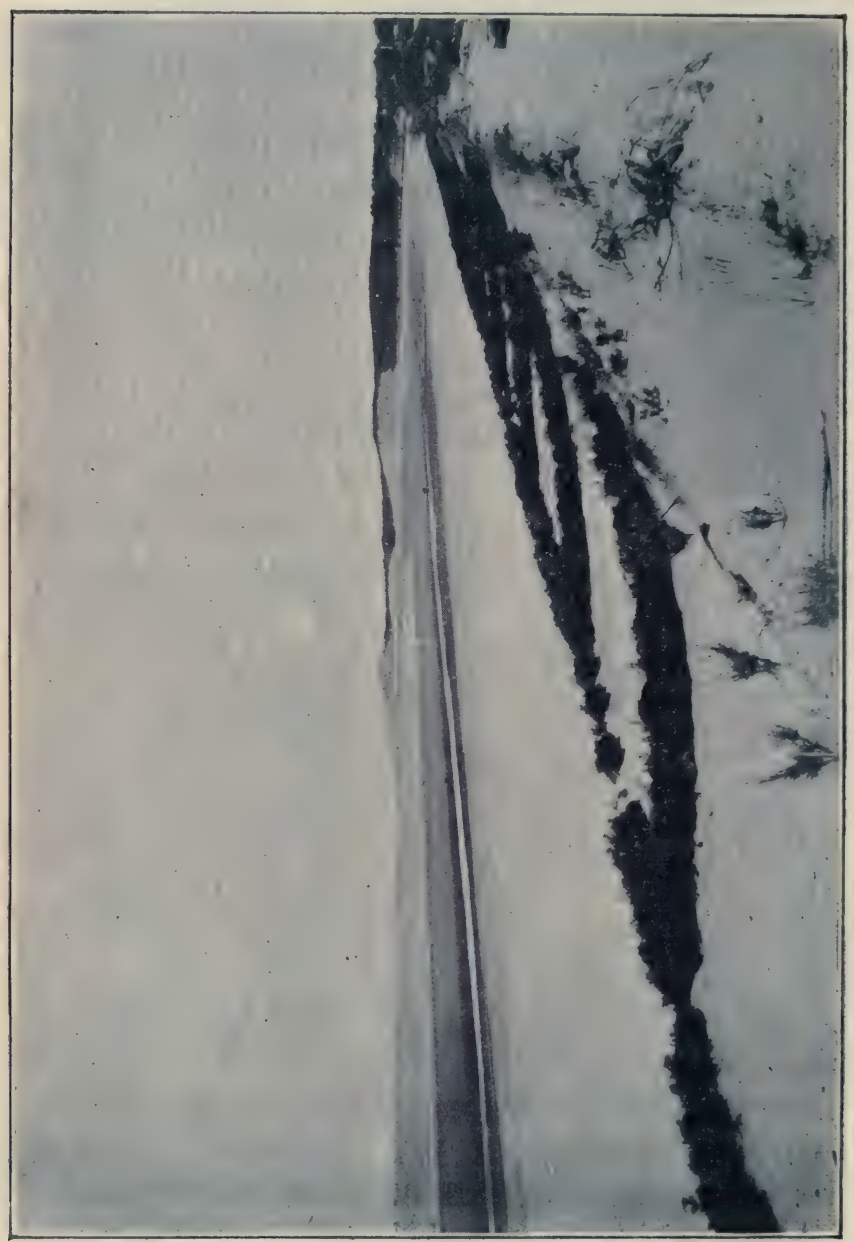

if

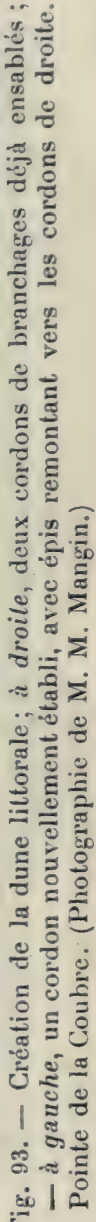

défense, et la direction à leur donner. Il en est ainsi à la dune de la Coubre (Charente-Inférieure) (1); il en sera de

(1) de Vasselot de Régné, loc. cit. 
même, à plus forte raison, quand il s'agira d'établir des dunes contine ita les de défense.

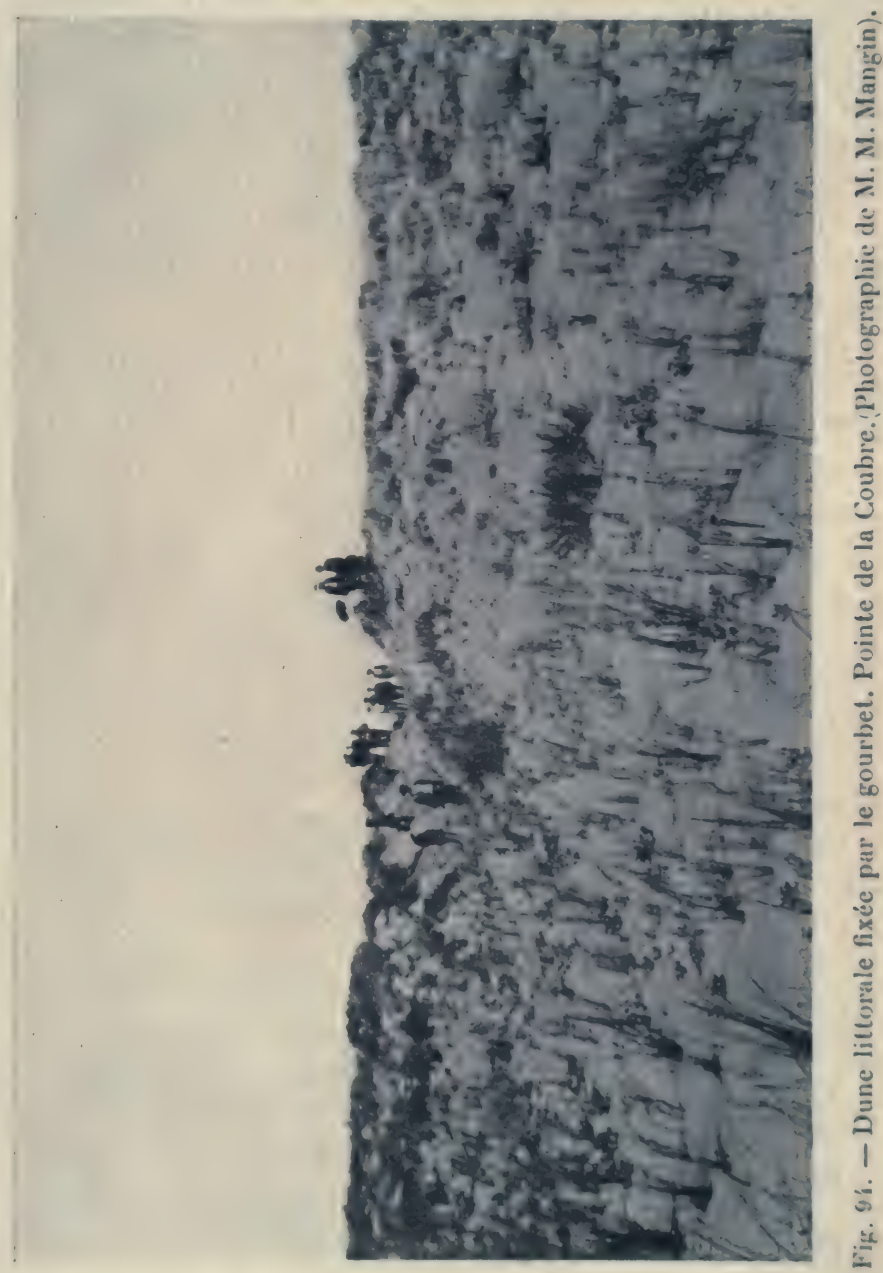

Enfin, sur les points où la mer déferle en rongeant les rives, sans déposer de sable, les matériaux font défaut. On substitue donc à la dune des enrochements et l'on combat la vio- 
lence du flot par la création d'une forêt de solides pieux enfoncés dans le sable et appelés brise-lame. Le tamarix, avec ses longs rameaux flexibles, rend les plus grands services pour consolider toutes ces cuvres mortes par un feutrage vivant. On cherche, en même temps, à remplacer l'ancien profil tracé par le caprice des flots par une véritable plage artificielle dont la pente descende jusquà 5 à 6 p. 100 de façon que la lame puisse s'y étaler en perdant sa force d'érosion.

Ainsi chaque point appelle une solution spéciale; à toutes celles qui sont déjà expérimentées ou entrevues, l'observation de chaque jour en apporte d'autres.

En fait, le profil définitif de la dune n'est pas encore trouvé, peut être même ne le trouvera-t-on jamais. Mais ce que l'on peut dire, c'est que la dune artificielle créée par nos prédécesseurs a accumulé à pied d'œuvre des masses colossales de matériaux plastiques pour les tenir à la disposition du forestier. Celui-ci peut pétrir à son gré les kilomètres cubes de sable suivant le mode ou la formule du jour; il peut en déplacer ou en fixer les masses à l'aide de la force gratuite du vent, sans autre dépense que les petits travaux peu coûteux qui consistent en piochages superficiels quand il veut abaisser les profils ou en plantations des gourbets quand il veut les relever.

Ce qu'on peut dire encore, c'est que le fait dominant de cette œuvre magistrale, a été de permettre le boisement complet de la dune blanche, de la fixer définitivement, de la lande à la mer, et de créer, dans cette large zone qui n'était qu'un désert aussi menaçant qu'improductif, une forêt immense dont la valeur et le rendement sont déjà considérables.

Voici d'ailleurs le procédé de boisement de cette dune blanche.

Boisement de la dune blanche. - Tout le système de fixation repose sur le principe suivant:

Dans la masse de sable nu, susceptible d'être corrodée par le vent, le transport s'opère grain par grain, roulé à mesure que chacun, débarrassé de ceux qui le recouvraient, arrive à la surface pour être transporté à son tour. Dès lors, si l'on parvient à arrêter le déplacement des grains à la superficie, 
il n'y a rien à craindre pour ceux qui sont en dessous, et la masse entière est fixée.

Le moyen consiste à semer le pin maritime sous couverlure. C'est à un propriétaire de la Teste, Pierre Peychan, - on le connait plutôt sous le nom de Maître-Pierre - , que nous devons ce procédé aussi simple que pratique qu'il a conseillé à Brémontier, et que nous employons maintenant encore, presque sans modification.

Pour empêcher les graines d'être entraînées avec le sable, on dispose donc sur toutes les surfaces ensemencées un revêtement de broussailles. Cette précaution est aussi nécessaire pour fixer les semences que pour préserver les jeunes plants contre l'action des sables en mouvement; car les arêtes vives des grains, sans cesse projetés contre les tiges naissantes, les usent au point que celles-ci, ne pouvant plus soutenir leur tête, se renversent; c'est la mort pour le plus grand nombre.

Dans la pratique, on répartit sur les espaces à boiser, des provisions de fagots, à raison de 2.500 par hectare. Ces fagots ont 1 mètre de circonférence mesurée sur le hart, et $1^{\text {” }}, 30$ de longueur. Pour leur confection, l'espèce préférée est l'ajonc; puis vient le genêt, ensuite les brandes, les brnyères, les rouches, les roseaux de marais doux, et les branches de pin; mais ces dernières présentent l'inconvénient que, sous leur couvert, les maladies cryptogamiques évoluent avec trop de fréquence. Les épines, ronces, fougères et bois blancs ne donnent qu'une protection insuffisante au sol, et sont exclus des fournitures.

Immédiatement après le répandage de la graine, on recouvre les parties ensemencées, ou mieux, les deux opérations se font simultanément. On dispose les broussailles en marchant vers la mer, les gros bouts en avant et les ramilles de chaque rangée recouvrant la base de celle qui précède. Puis, à l'effet de maintenir cette couverture, les ouvriers prennent des fortes pelletées de sable dans les parties non ensemencées, et les appliquent sur les ramilles étendues, à environ $0^{\mathrm{m}}, 60$ les unes des autres, mesure prise de milieu en milieu. C'est là le seul perfectionnement apporté au système de Pierre Peychan et de Brémontier, qui fixaient la couverture au moyen de perches placées en 
travers et retenues par des crochets enfoncés dans le sable. Chaque atelier travaille ainsi à reculons, derrière le semeur, sans que personne ait le loisir de chômer; à la fin de la journée, on rechausse solidement la dernière rangée pour que le vent possible de la nuit ne vienne pas ouvrir des ventouses qui détruiraient tout le travail. On répare les avaries, soit en comblant les ventouses, soit en recommençant le semis dans la même forme, sur les parties ensablées.

Les premiers chantiers ont été installés immédiatement en arrière de la dune littorale, en avançant vers la terre, de telle sorte que les travaux en cours d'exécution sont garantis par les massifs déjà constitués. Sur les points où les semis sont exposés à être envahis par des sables de retour chassés par le vent de terre, la section destinée à être ensemencée pendant l'année est entourée de palissades mobiles qu'on déplace au commencement de chaque campagne.

En somme, c'est une véritable couverture morte que l'on a disposée sur le sol. Comme elle ne doit pas durer indéfiniment, on pourvoit dès le début à lui faire succéder une couverture vivante qui continuera son rôle. A cet effet, au lieu d'employer de la graine de pin maritime pure, on répand, par hectare, le mélange suivant:

Pin maritime................... 30 kilogr.

Ajonc ........................... 3 -

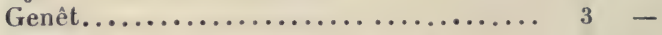

Gourbet........................ 3 -

Graines diverses pour attirer les oiseaux. 3 -

Cette formule est adoptée dans la dune de la Coubre. Dans les chantiers des Landes les quantités sont réduites à :

Pin maritime..................... 10 kilogr.

Genêt............................

Gourbet......................... 4 -

Les pins, les genêts et les ajoncs lèvent en même temps, et l'on remarque que les pins sont d'autant plus beaux que les semis accessoires sont plus nombreux. D'ailleurs les couvertures pourrissent sur place et donnent au sol une première avance de matière organique.

Puisque l'on commence le boisement au pied même de la 
dune, les premiers peuplements créés, sur une profondeur de 200 à 300 mètres, fatigués par le vent de mer, restent le plus souvent chétifs et rabougris; mais, à l'abri de cette zone de protection, les massifs qui les suivent se développent normalement; on constate même que les pins de la dune fournissent, à dimension égale, plus de résine que ceux de la lande.

Ces travaux coûtent nécessairement assez cher. Dans les Landes, le prix de revient moyen a pu être établi à $500 \mathrm{fr}$. par hectare; dans les dunes de la Coubre, il n'a pas dépassé $355 \mathrm{fr}$. Mais qu'est-ce, en somme, que cette dépense comparée à la grandeur des résultats obtenus ?

De Bayonne à la pointe de Grave, la dune littorale est entretenue sur un développement de 300 kilomètres. Plus de 60.000 hectares de sables ont été ainsi reconquis sur les dépendances de la mer, au grand profit de la richesse publique. Malheureusement, les funestes aliénations faites de 1861 à 1865 ont enlevé au domaine de l'État 17.500 hectares, dont la soumission au régime forestier était la meilleure sauvegarde contre les abus du pâturage.

Les dunes continentales. - D'ailleurs, les effets du phénomène éolien qui préside à la formation et au déplacement des dunes ne sont pas limités aux plages maritimes. Sur les différents points de l'immense désert qui s'étend des confins de la Sibérie au cap Vert, le sable s'accumule en dunes. Les oasis du sud de l'Algérie et de la Tunisie sont particulièrement exposées à des invasions de cette origine.

Bien que les mouvements de ces dunes continentales n'affectent pas les allures régulières des dunes marines, les moyens pour les combattre sont à peu près partout les mêmes, et les remarquables travaux exécutés sur les côtes de France peuvent servir de modèles en toutes situations. On se contentera d'utiliser des feuilles de palmier au lieu de madriers, ou d'employer, au lieu de nos espèces européennes, celles qu'on a sous la main (1). Nous ne citerons ici aucun nom d'arbuste ou de plante indigène; mais, dans la flore saharienne, nous

(1) Baraban, A travers la Tunisie. Revue des Eaux et Foréts, 1886. Boppe et Jolyet. 
retrouvons encore le tamarix, tout indiqué pour protéger de l'ensablement les sources ou les puits qui sont la fortune pour ces régions désolées. Il nous rappelle qu'en toute occasion et sous tous les climats, si la nature paraît quelquefois peu hospitalière, du moins, donne-t-elle à l'homme un appui dans les végétaux qui font la forêt, depuis le plus grand arbre jusqu'au plus humble arbrisseau.

\section{ERR A T A}

Page 2, légende de la figure 1; au lieu de: en bas, collet de la racine, lire : audessous $d u$ bois parfat, niveau du collet de la racine.

- 51, ligne 25, au lieu de : a dit quelque part Toussenel, lire : a dit quelque part M. de la Blanchère.

- 61, légende de la figure 26, au lieu de: à gauche chènes de juin, glands de la forêt de Petit-Noir (Jura). - A droite : chênes pédonculés ordinaires, elc... lire : au milieu : chènes de juin, glands de la forêt de Petit-Noir (Jura). A gauche et $\dot{a}$ droite : chênes pédonculés ordinaires, etc...

- 139, légende de la figure 36 , lire : Un chablis. - Sapinière de l'Annuelle (Jura). (Photographie de II. Albert Regad).

- 279, ligne 14, au lieu de : devoir, lire : revoir; devoir et revoir doivent être en italiques.

- 296, ligne 15, au lieu de : quoiqu'il en soit, lire : quoi qu'il en soit. 


\section{TABLE ANALYTIQUE DES MATIËRES}

Avant-propos.

$\mathrm{V}-\mathrm{vi}$

Plan de l'ouvrage.

VII-xI

\section{CHAPITRE PREMIER. - L'ARBRE.}

Article premier. - Parties constitutives d'un arbre.

Partie aérienne, - Enracinement. - Structure du bois. - Écorce. 1-6

\section{Anticle II. - Forme des arbres.}

Élagage naturel. - Forme spécifique. - Influence du sol et du climat. - Forme forestière. - Généralités sur l'accroissement. Accroissement en hauteur. - Accroissement en diamètre; - ses variations suivant les espèces et les individus; - ses variations dans un même arbre $;-$ anomalies diverses................. 6-22

\section{Anticle III. - Reproduction des arbres.}

Brins et rejets. - Fertilité des arbres. - Germination des graines et installation des semis. - Rejets proventifs et rejets adventifs. Influence de l'époque de l'exploitation sur l'évolution des rejets. Influence du mode dabatage. - Drageons. - Rejets de racine. Modes spéciaux d'exploitation...................... 23-34

\section{CHAPITRE II. - LES ESSENGES.}

\section{Anticlo premier. - Généralités.}

Définitions. - Tempérament. - Influence de la lumière; - ... de l'humidité atmosphérique; - ... de la température et autres. Aire forestière. - Influence des qualités chimiques et physiques du sol; - ... de la fertilité. - Essences sociales et disséminées. Dissémination. Longévité....................... 35-55

\section{Anticle II. - Monographie des principales essences.}

Les chênes : C. rouvre et pédonculé, C. tauzin, C. liège, C. yeuse. - Le châtaignier. - Le hêtre. - Le charme. - Le frêne. - Les érables. - Les ormes, - Le micocoulier, - Les fruitiers. - Le bouleau. Les aunes. - Les bois tendres: tilleuls, peupliers, saules. - Le 
sapin. - L'épicéa. - Le mélèze. - Les pins : P. sylvestre, P. de montagne, P. maritime,P. d'Alep, P. laricio (de Corse, des Cévennes, des Pyrénées, de Calabre, noir d'Autriche), P. cembro.... 56-102

\section{CHAPITRE III. - LES PEUPLEMENTS}

\section{Article premier. - Généralités.}

Définition. - Genèse d'un peuplement. - Situation particulière du peuplement forestier.......................... 103-104

\section{Article II. - Action réciproque des arbres les uns sur les autres.}

Les peuplements dans la forêt sauvage. - Perturbations apportées par l'intervention de l'homme. - Origine des peuplements. - Leur forme. - Leur consistance. - Leur constitution en étages de végétation. - Leur composition : purs ou mélangés. - Mélanges rationnels. - Le hêtre. - Conduite des peuplements mélangés. - État de végétation des peuplements................... 104-116

\section{Article III. - Action des arbres sur le sol.}

Fertilité permanente des sols forestiers. - La couverture. - La couverture morte; son rôle physique. - Le terreau; l'ameublissement du sol et la terre à bois. - Rôle chimique de la couverture morte; l'acide phosphorique et la potasse; l'azote. - Les terreaux acides et tourbeux. - Rôle du sylviculteur dans la constitution de la couverture; dans celle du terreau. - La couverture vivante... 116-125

\section{Article IV. - Évolution des peuplements.}

Évolution du peuplement uniforme. - États de développement successifs des futaies régulières; - des taillis simples réguliers. - Érolution des peuplements jardinés ou furetés. - Résumé... 125-128

$$
\text { CHAPITRE IV. - LES FORETS. }
$$

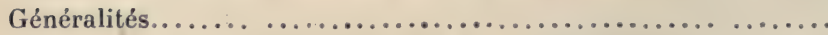

\section{Article premier. - Action du sol.}

Rôle du sol: généralités; classement. - Sols siliceux : propriétés physiques; tapis végétal; allure des forêts; sables à grains fins; alios, tourbières. - Sols argileux : propriétés physiques ; tapis végétal ; allure des forèts; lohm. - Sols à base calcaire : propriétés physiques; tapis végétal; allure des forêts; marne.......... 129-138

\section{Anticle II. - Action du climat.}

I. Aptitude forestière. - II. Les climats de plaine; caractères généraux. - Division en zones. - Zone parisienne. - Zone girondine. - Zone provençale. - III. Le climat de montagne; caractères généraux. - Vosges. - Jura. - Alpes. - Plateau central. - Pyrénées................................ 138-162 


\section{CIIAPITRE V. - LE TRAITEMENT.}

\section{Anticle premier. - Les opérations culturales.}

La régénération. - Les améliorations : les dégagements de semis: les éclaircies; leur but cultural; - leur but économique: - la maniere de les conduire; - leur importance............ 163-174

\section{Article II. - Notions d'aménagement.}

Capital forestier. - Définitions. - Réalisation de la possibilité : par contenance, par volume, par pied d'arbre. - Observations générales................................. 174-181

\section{CHAPITRE VI. - LES DIFFÉRENTS MODES DE TRAITEMENT.}

Article premier. - La futaie régulière.

Principe de la méthode. - Avantages et inconvénients. - La régé. nération. - Procédé par coupe unique. - Procédé par coupes successives. - Soins culturaux. - Application aux principales essences................................... 182-207

\section{Anticle II. - La futaie jardinée.}

Principe de la méthode. - Avantages et inconvénients. - Cas oủ le jardinage doit être maintenu, - Pratique du jardinage. - Les rotations. - Les soins culturaux. - Application aux différentes essences....................................... 207-217

\section{Article III. - Le taillis simple régulier.}

Principe de la méthode. - Avantages et inconvénients. - Régénération. - Soins culturaux. - Application dans les régions tempérées; - ... dans les régions méridionales; $-\ldots$ dans des cas spé-

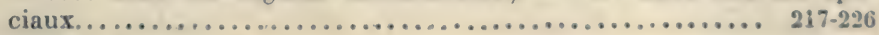

\section{Article IV. - Le taillis simple fureté.}

Forme des peuplements. - Cireonstances dans lesquelles il se justifie. - Régénération. - Réglementation................ 226-229

\section{Article V. - Le taillis sous futaie.}

Constitution. - Solidarité entre la futaie et le taillis. - Avantages et inconvénients. - Régénération du taillis. - Constitution de la futuie. - Les dégagements de semis. - Les éclaircies.......... 229-247

\section{Article VI. - Amélioration des forêts traitées en taillis simple et en taillis sous futaie.}

Transformations et conversions. - Leur peu de raison d'être dans les bois de particuliers. - But à poursuirre. - Améliorations proposées : - allongement des révolutions; - éducation darbres plus nombreux $;$ - substitutions d'eseences.............. 2ii-258 


\section{CHAPITRE VII. - EXPLOITATION ET PROTECTION DES FORETS} Article premier. - Généralités.

\section{Article II. - Dommages causés par l'homme.}

Fait du propriétaire : à l'occasion des exploitations; - des élagages; - des émondages; - des assainissements. - Fait des délinquants : les causes; - la répression. - Les concessions de menus produits: tolérances nécessaires; - menus produits végétaux; - menus produits minéraux. - Le pâturage: la situation actuelle: - nocuité du pâturage suivant les régions; - influence de la nature du bétail introduit; - de l'état des peuplements; - conclusions. - Les incendies: leurs causes et leurs conséquences; - régions montagneuses; - zone parisienne; - régions méridionales..... . 260-297

\section{Article III. - Dégâts des animaux.}

Les mammifères. - Les oiseaux. - Les insectes.......... 297-305

\section{Article IV. - Dommages causés par les végétaux.}

Les plantes sarmenteuses. - Les plantes parasites. - Le gui. - Les champignons. - Les bactéries................... 305-30?

\section{Article V. - Dégâts causés par les météores.}

Généralités. - Le vent: troisième règle d'assiette et massifs de protection. - Le froid et les tares qu'il engendre. - Les coups de soleil. - La foudre. - La neige et les avalanches. - La grêle, le givre et le verglas................................... 310-322

\section{CHAPITRE VIII. - LES PEUPLEMENTS ARTIFIGIELS.}

\section{Anticle premier. - Observations générales.}

But à poursuivre. - Définitions. - Les différents procédés de boise-

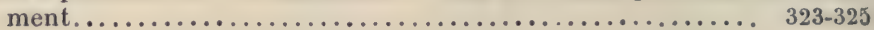

\section{Article II. - Le boisement par semis.}

Qualité des semences. -- Leur récolte. - Leur conservation. - Préparation du sol avant le semis. - Les différents modes de semis : en plein ou à la volée; - par places; - par bandes continues ou brisées; - par trous ou potets; - en terrain non préparé ; - expéditifs. - Répandage de la semence. - Quantité de semence à employer. - Saison favorable pour faire les semis. - Application aux essences sociales. - Soins à donner aux semis............. 326-348

\section{Article III. - Le boisement par plantations.}

Les plants. - Qualité des plants. - Leur origine. - Leur âge et leur dimension........................... 349-351

Les pépinières. - $\$ 1^{\text {er }}$ Installation d'une pépinière centrale. Capital d'installation. - Choix de l'emplacement. - Division du ter- 
rain. - Préparation du sol. - Les engrais. - Le terreau. - Aménagement de la pépinière. - Son étendue. - Les outils. - Les clitures. - Les dangers à combattre............ 351-367

§2. Exploitation de la pépinière. - Kxéculion des semis : en plein ; en rigoles; - en colres; - procédés spéciaux; - quantité de graines à employer; - saison des semis. - Soins a donner aux semis : pralinage des graines; - abris contre la sécheresse ; - abris d'hiver ; - abris contre les gelées printannières ; - abris permanents ; arrosages et irrigations; - sarclages; - binares. - Travaux divers: repiquage des plants; - leur extraction; - leur taille et leur thabillage ; - transport et emballage............... 367-390

$\$$ 3. Pépinières volantes ou locales. - Avantagres. - Installation...................................... 391-392

Exćcution des plantations. - Préparation du sol. - Disposition des plants. - Confection et dimension des trous. - Manipulation des plants. - Plantation à racines nues; - par touffes; - en butte; - en corbeilles. - Plantation en terrain non préparé. - Saison favorable ḋ la plantation. - Application aux principales essences. — Soins à donner aux plantations............... 392-113

\section{Anticle IV. - Procédés spéciaux de boisement.}

Les boutures. - Les plançons. - Les marcottes..........

$113-415$

\section{Article V. - Les essences de boisement.}

1. Généralités. - Chois des essences. - Caractères de la forèt artificielle................................. 415-\$17

2. Les essences indigènes. - Essences résineuses. - Essences feuillues. - Mélanges............................ 417-422

3. Les essences exotiques. - Généralités. - Le choix à faire. - I.es essences les mieux connues. - Conclusions........... $122-131$

\section{Antiche VI. - La mise en valeur par le boisement.}

1. Généralités. - Les boisements facultatiis. - Les boisements obligatoires. - Règle commune à tous deux........... \$31- $\mathbf{4 3 3}$

2. Les boisements facultalifs. - a. Les améliorations on forêt: Considérations générales. - Applications dans les futaies, dans les taillis................................. 433-436

b. Les terres arables abandonnées par l'agriculture: Conditions de déclassement. - Les procédés de boisement. - Boisements spéciaux. - Arboriculture fruitière. - Les têtards; les arbres d'émonde et les ramilles-fourrage........................ 437-144

c. Les terres incultes en pays de plaines et de coteaux : I.es landes. - Les friches. - Les garrigues................... 444-450

d. Les terrains stables en montagne: Les procédés de boisement. Les prés-bois.......................... 450-155 
3. Les boisemenls obligaloires. - a. La restauration des montagnes dégradées: Les causes de la dénudation. - La méthode de travail suivie. - Les travaux de soutien. - Les boisements proprement dits ; - traitement des parties stables; - des parties mouvantes; - des terres noires. - Les enherbements. - Le gazonnement. Conclusion................................ $455-471$

b. La fixation des dunes: Les dunes maritimes, - Les moyens d'action. - La dune littorale. - Boisement de la dune blanche. - Les dunes continentales.............................. $471-\mathbf{1} 82$

Table axalytique des matiéres $\ldots \ldots \ldots \ldots \ldots \ldots \ldots \ldots$. 483-4 $\$ 8$

FiN DE LA TABLE ANALYTIQUe DES Matières. 
$\therefore$ 



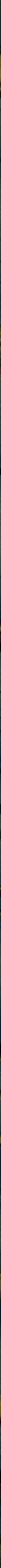





\section{fork}

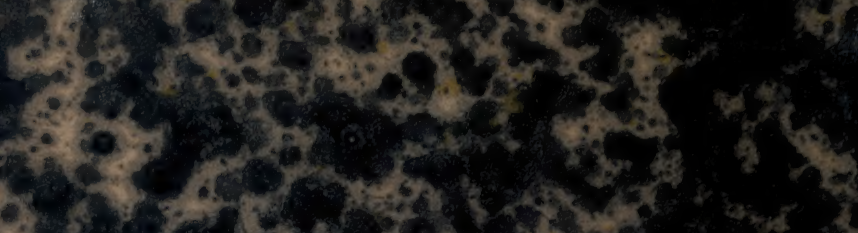

$1+1092$

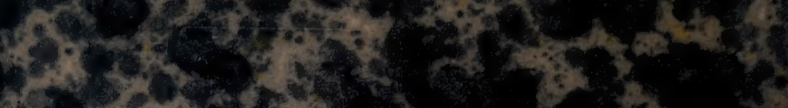
$60+30$ b $01+305$

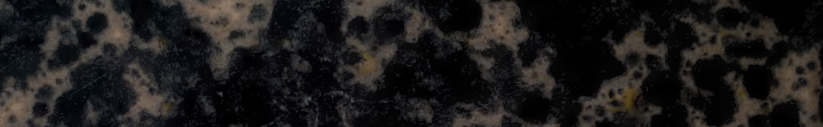

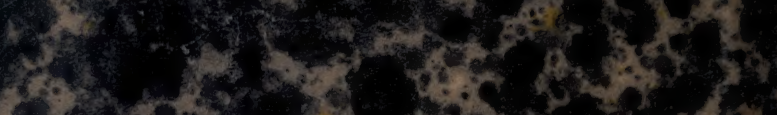

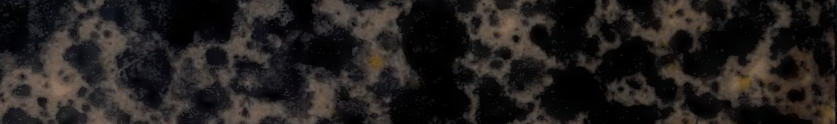
$6+25 x$ ind

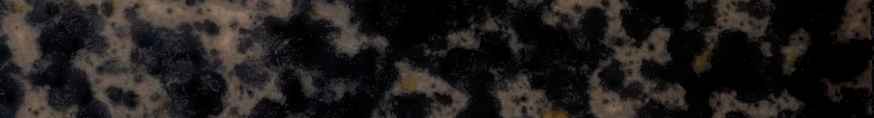
$37 x^{2}+y^{-2}$ $6 x^{2}+2 x$

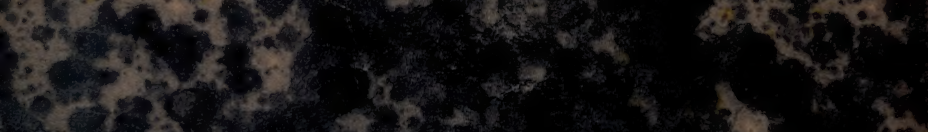

창 tis 1 b is caseds 1. 20.0\%

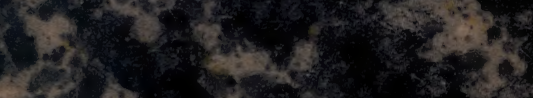
(t) 5

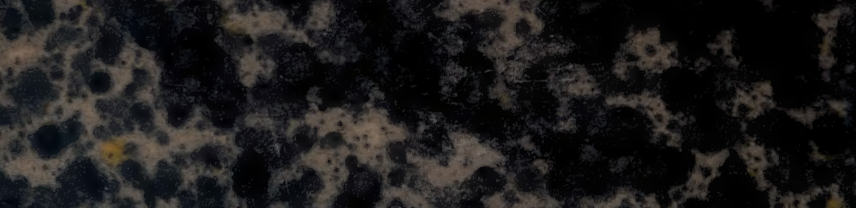

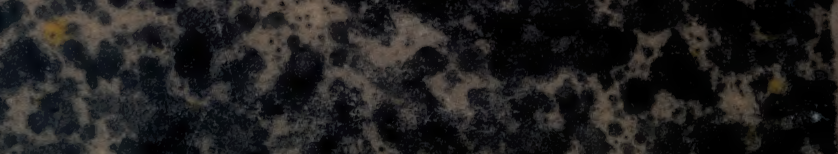

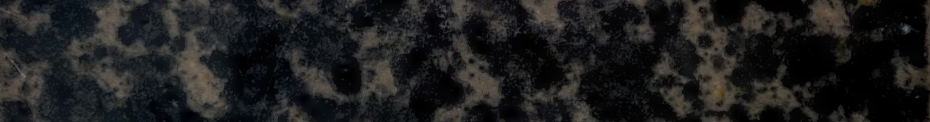

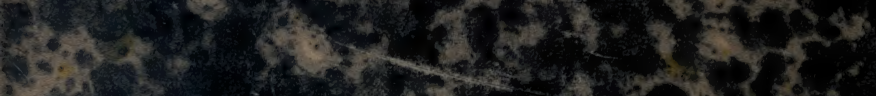
ato $43 x+x^{2}+4 x+2$

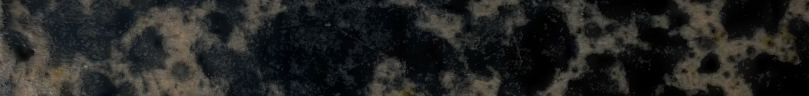

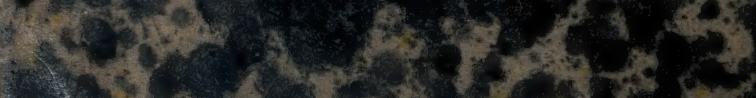

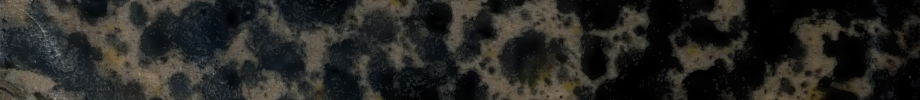

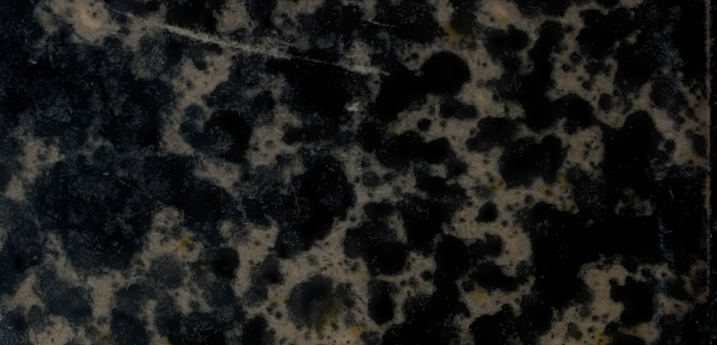

